National Toxicology Program

NTP TECHNICAL REPORT ON THE TOXICOLOGY STUDIES OF

Cobalt Metal

(CASRN 7440-48-4)

IN F344/N RATS AND

B6C3Fl/N MICE

AND TOXICOLOGY AND

CARCINOGENESIS STUDIES OF Cobalt Metal IN

F344/NTAC RATS AND

B6C3Fl/N MICE

(INHALATION STUDIES)

NTP TR 581

DECEMBER 2014 


\title{
NTP Technical Report on the Toxicology Studies of Cobalt Metal (CASRN 7440-48-4) in F344/N Rats and B6C3F1/N Mice and Toxicology and Carcinogenesis Studies of Cobalt Metal in F344/NTac Rats and B6C3F1/N Mice (Inhalation Studies)
}

Technical Report 581

December 2014

\author{
National Toxicology Program \\ Public Health Service \\ U.S. Department of Health and Human Services
}

ISSN: $2378-8925$

Research Triangle Park, North Carolina, USA 


\section{Foreword}

The National Toxicology Program (NTP) is an interagency program within the Public Health Service (PHS) of the Department of Health and Human Services (HHS) and is headquartered at the National Institute of Environmental Health Sciences of the National Institutes of Health (NIEHS/NIH). Three agencies contribute resources to the program: NIEHS/NIH, the National Institute for Occupational Safety and Health of the Centers for Disease Control and Prevention (NIOSH/CDC), and the National Center for Toxicological Research of the Food and Drug Administration (NCTR/FDA). Established in 1978, NTP is charged with coordinating toxicological testing activities, strengthening the science base in toxicology, developing and validating improved testing methods, and providing information about potentially toxic substances to health regulatory and research agencies, scientific and medical communities, and the public.

The Technical Report series began in 1976 with carcinogenesis studies conducted by the National Cancer Institute. In 1981, this bioassay program was transferred to NTP. The studies described in the Technical Report series are designed and conducted to characterize and evaluate the toxicologic potential, including carcinogenic activity, of selected substances in laboratory animals (usually two species, rats and mice). Substances selected for NTP toxicity and carcinogenicity studies are chosen primarily on the basis of human exposure, level of production, and chemical structure. The interpretive conclusions presented in NTP Technical Reports are based only on the results of these NTP studies. Extrapolation of these results to other species, including characterization of hazards and risks to humans, requires analyses beyond the intent of these reports. Selection per se is not an indicator of a substance's carcinogenic potential.

The NTP conducts its studies in compliance with its laboratory health and safety guidelines and FDA Good Laboratory Practice Regulations and must meet or exceed all applicable federal, state, and local health and safety regulations. Animal care and use are in accordance with the Public Health Service Policy on Humane Care and Use of Animals. Studies are subjected to retrospective quality assurance audits before being presented for public review.

The NTP Technical Reports are available free of charge on the NTP website and cataloged in PubMed, a free resource developed and maintained by the National Library of Medicine (part of the National Institutes of Health). Data for these studies are included in NTP's Chemical Effects in Biological Systems database.

For questions about the reports and studies, please email NTP or call 984-287-3211. 


\section{Table of Contents}

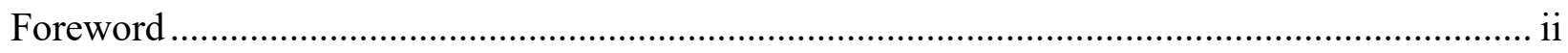

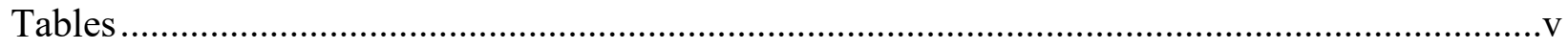

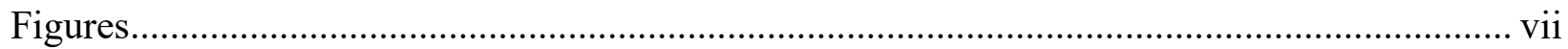

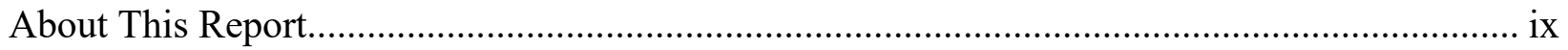

Explanation of Levels of Evidence of Carcinogenic Activity ................................................... xii

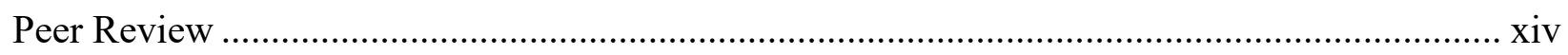

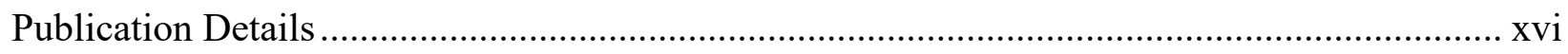

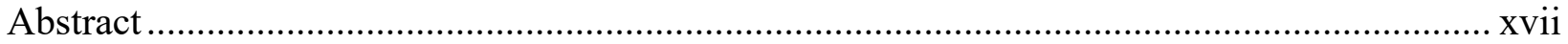

Two-week Study in Rats ........................................................................................ Xvii

Two-week Study in Mice ................................................................................................ xvii

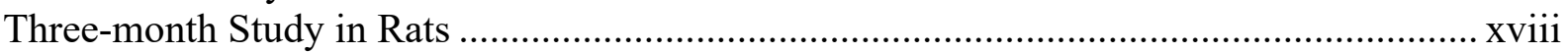

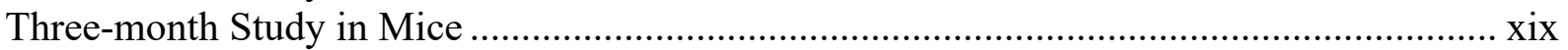

Two-year Study in Rats..................................................................................................

Two-year Study in Mice.............................................................................................

Genetic Toxicology …………………..................................................................... xxii

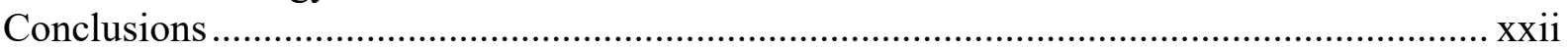

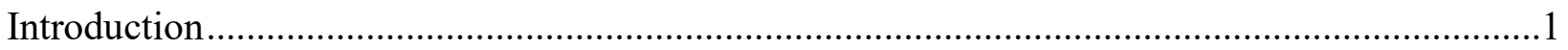

Chemical and Physical Properties .....................................................................................

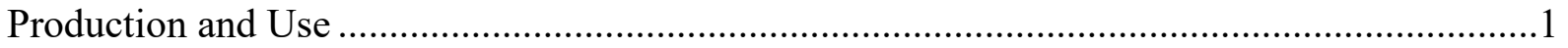

Environmental and Human Exposure …………….......................................................

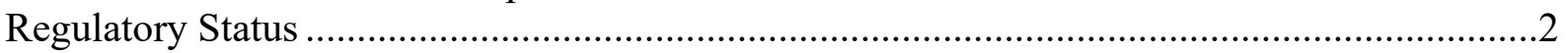

Absorption, Distribution, Metabolism, Excretion, and Toxicokinetics …………......................2

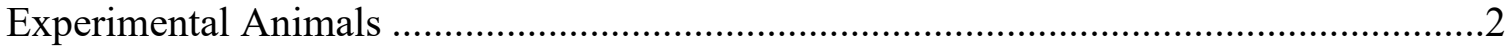

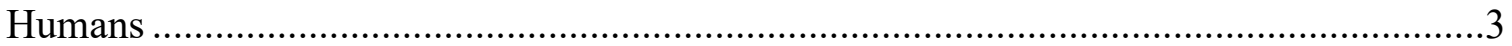

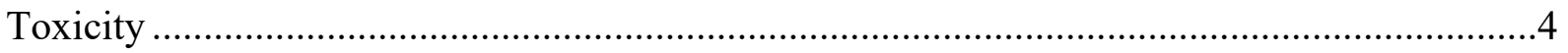

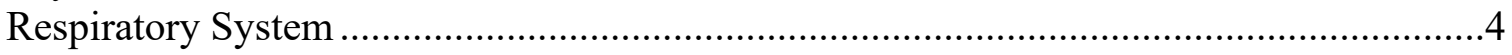

Cardiovascular System...................................................................................

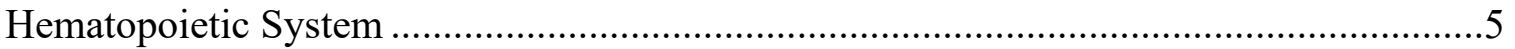

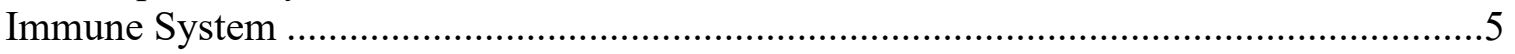

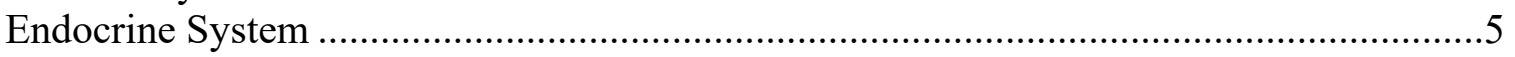

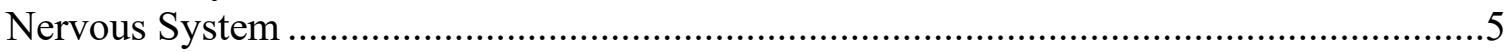

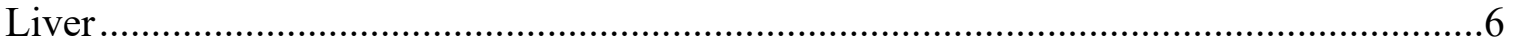

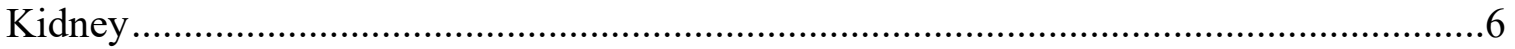

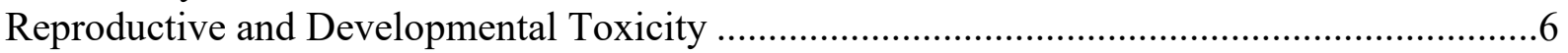

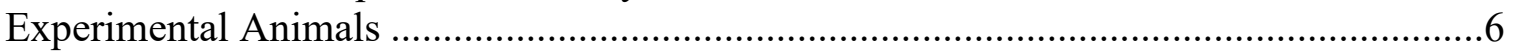

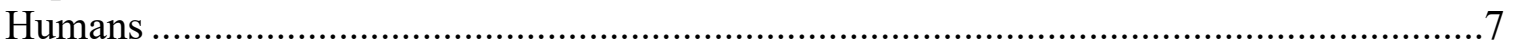

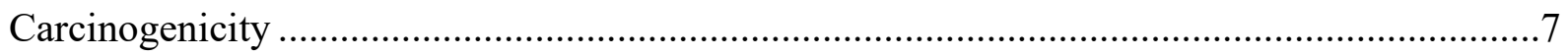

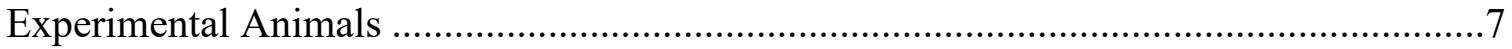

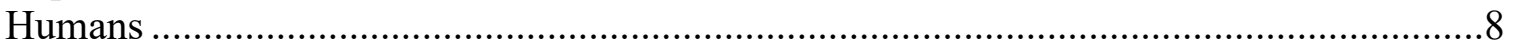

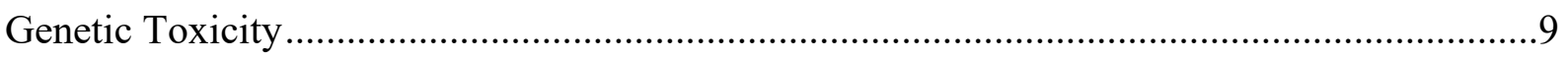

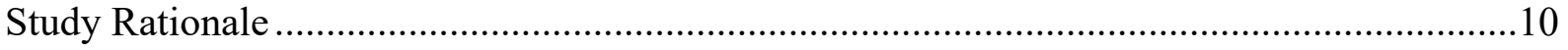




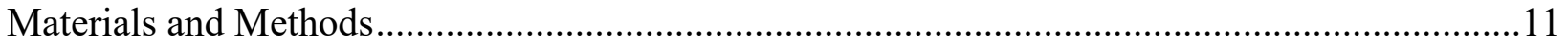

Procurement and Characterization of Cobalt Metal..............................................................11

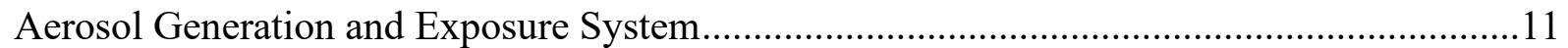

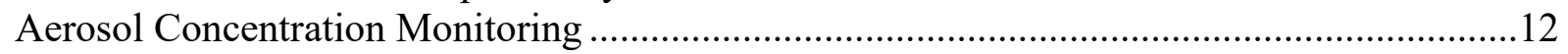

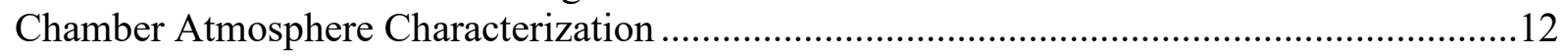

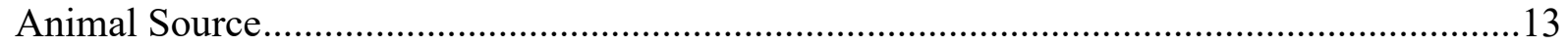

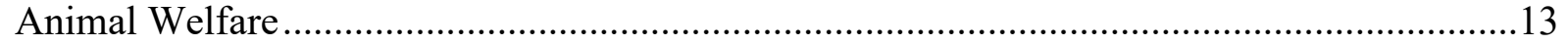

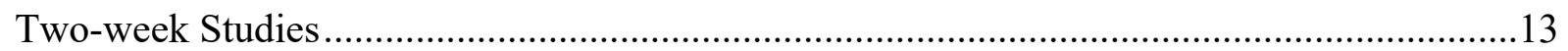

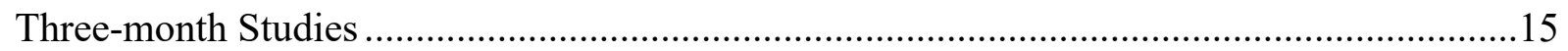

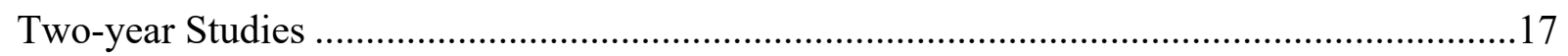

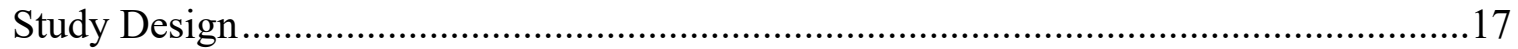

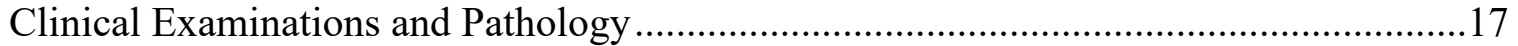

Mutation Analysis of Alveolar/bronchiolar Carcinomas ..................................................18

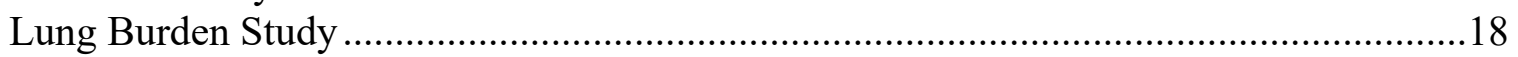

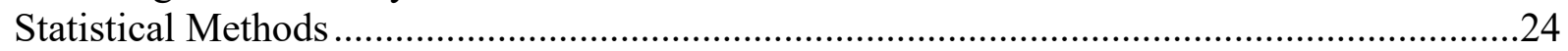

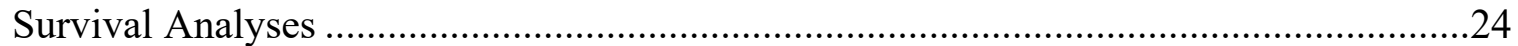

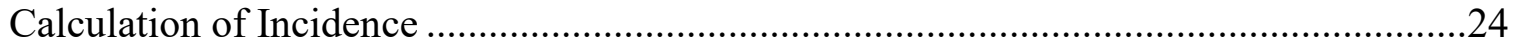

Analysis of Neoplasm and Nonneoplastic Lesion Incidences ..........................................25

Analysis of Continuous Variables ........................................................................25

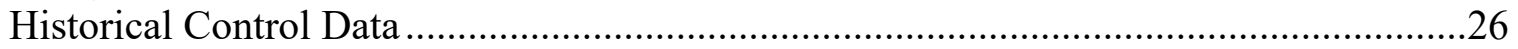

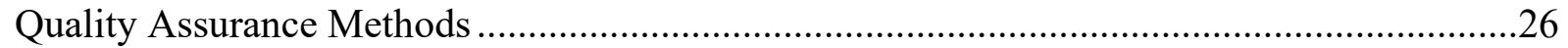

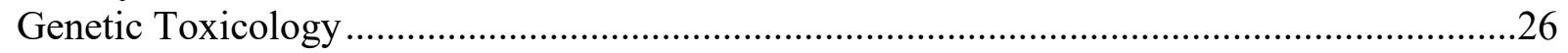

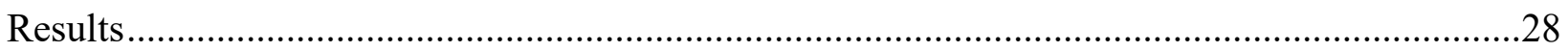

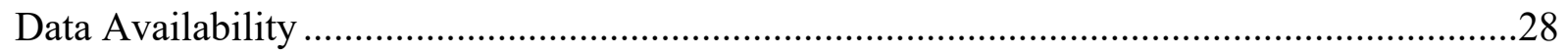

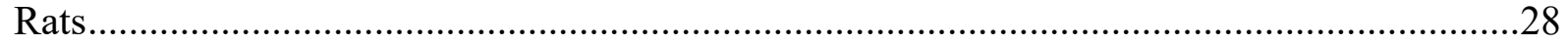

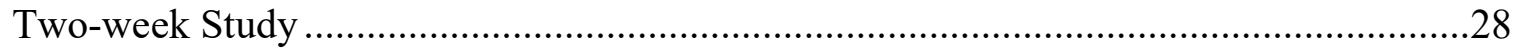

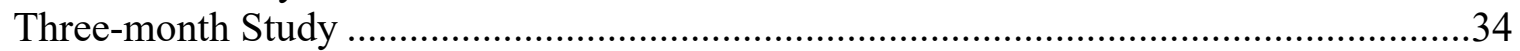

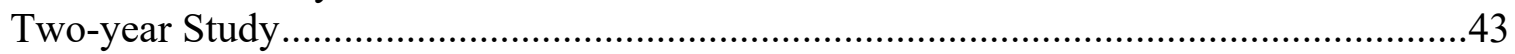

Mice

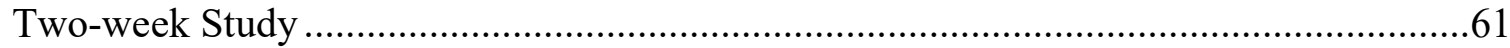

Three-month Study ……………………………………………………………66

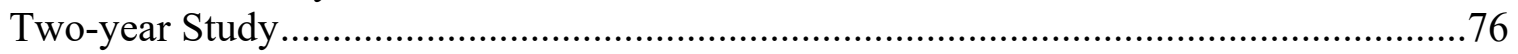

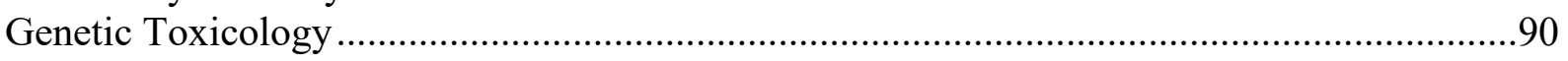

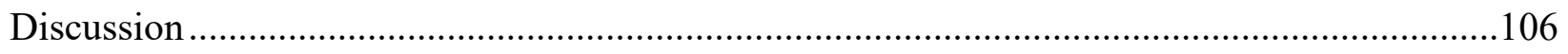

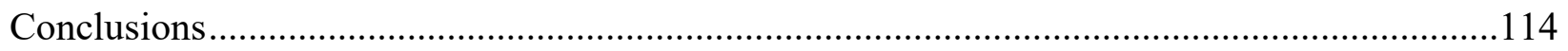

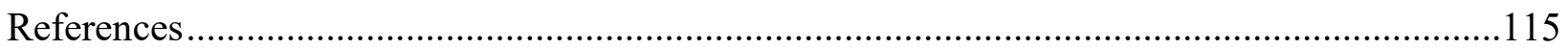

Appendix A. Summary of Lesions in Male Rats in the Two-year Inhalation Study of

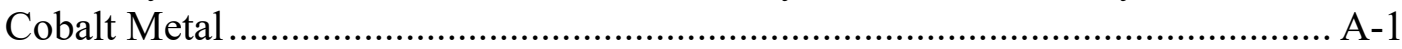

Appendix B. Summary of Lesions in Female Rats in the Two-year Inhalation Study of

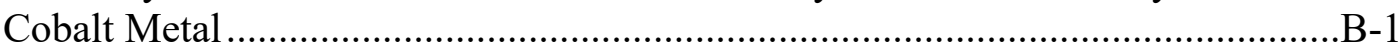

Appendix C. Summary of Lesions in Male Mice in the Two-year Inhalation Study of

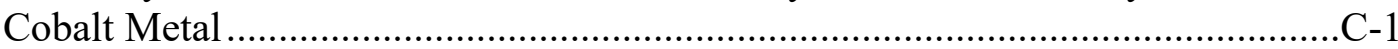


Appendix D. Summary of Lesions in Female Mice in the Two-year Inhalation Study of

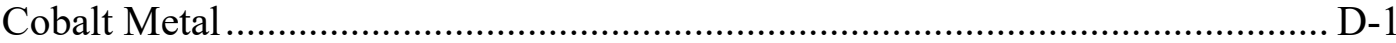

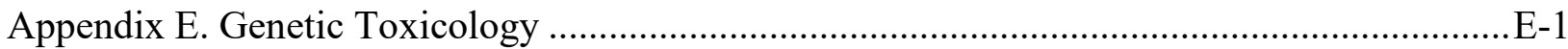

Appendix F. Clinical Pathology Results.........................................................................

Appendix G. Organ Weights and Organ-Weight-to-Body-Weight Ratios ................................. G-1

Appendix H. Reproductive Tissue Evaluations and Estrous Cycle Characterization ................. H-1

Appendix I. Tissue Burden Results ...................................................................................

Appendix J. Liver Cytochrome P450 Data ...........................................................................

Appendix K. Analysis of Kras, Egfr, and Tp53 Mutations in F344/NTac Rat and B6C3F1/N Mouse Alveolar/Bronchiolar Carcinomas Resulting from Chronic Inhalation Exposure to Cobalt Metal.................................................... K-1

Appendix L. Chemical Characterization and Generation of Chamber Concentrations ..................-1

Appendix M. Ingredients, Nutrient Composition, and Contaminant Levels in NTP-2000 Rat and Mouse Ration .................................................................................

Appendix N. Sentinel Animal Program ......................................................................... $\mathrm{N}-1$

Appendix O. Summary of Peer Review Panel Comments ........................................................ O-1

\section{Tables}

Summary of the Two-year Carcinogenesis and Genetic Toxicology Studies of Cobalt Metal xxiii

Table 1. Experimental Design and Materials and Methods in the Inhalation Studies of Cobalt Metal

Table 2. Survival and Body Weights of Rats in the Two-week Inhalation Study of Cobalt Metal

Table 3. Selected Organ Weights and Organ-Weight-to-Body-Weight Ratios for Rats in the Two-week Inhalation Study of Cobalt Metal

Table 4. Incidences of Selected Nonneoplastic Lesions of the Respiratory System in Rats in the Two-week Inhalation Study of Cobalt Metal.....

Table 5. Survival and Body Weights of Rats in the Three-month Inhalation Study of Cobalt Metal

Table 6. Selected Clinical Pathology Data for Rats in the Three-month Inhalation Study of Cobalt Metal.

Table 7. Summary of Reproductive Tissue Evaluations for Male Rats in the Three-month Inhalation Study of Cobalt Metal

Table 8. Incidences of Selected Nonneoplastic Lesions of the Respiratory System in Rats in the Three-month Inhalation Study of Cobalt Metal

Table 9. Survival of Rats in the Two-year Inhalation Study of Cobalt Metal.....

Table 10. Mean Body Weights and Survival of Male Rats in the Two-year Inhalation Study of Cobalt Metal 
Table 11. Mean Body Weights and Survival of Female Rats in the Two-year Inhalation Study of Cobalt Metal

Table 12. Incidences of Neoplasms and Nonneoplastic Lesions of the Lung in Rats in the Two-year Inhalation Study of Cobalt Metal

Table 13. Incidences of Nonneoplastic Lesions of the Nose in Rats in the Two-year Inhalation Study of Cobalt Metal...

Table 14. Incidences of Neoplasms and Nonneoplastic Lesions of the Adrenal Medulla in Rats in the Two-year Inhalation Study of Cobalt Metal

Table 15. Incidences of Neoplasms of the Pancreatic Islets in Rats in the Two-year Inhalation Study of Cobalt Metal

Table 16. Incidences of Neoplasms and Nonneoplastic Lesions of the Kidney in Male Rats in the Two-year Inhalation Study of Cobalt Metal

Table 17. Incidences of Mononuclear Cell Leukemia in Female Rats in the Two-year Inhalation Study of Cobalt Metal.

Table 18. Survival and Body Weights of Mice in the Two-week Inhalation Study of Cobalt Metal

Table 19. Selected Organ Weights and Organ-Weight-to-Body-Weight Ratios for Mice in the Two-week Inhalation Study of Cobalt Metal.

Table 20. Incidences of Selected Nonneoplastic Lesions of the Respiratory System in Mice in the Two-week Inhalation Study of Cobalt Metal.

Table 21. Survival and Body Weights of Mice in the Three-month Inhalation Study of Cobalt Metal....

Table 22. Selected Organ Weights and Organ-Weight-to-Body-Weight Ratios for Mice in the Three-month Inhalation Study of Cobalt Metal....

Table 23. Summary of Reproductive Tissue Evaluations for Male Mice in the Threemonth Inhalation Study of Cobalt Metal.

Table 24. Incidences of Selected Nonneoplastic Lesions of the Respiratory System in Mice in the Three-month Inhalation Study of Cobalt Metal.

Table 25. Incidences of Selected Nonneoplastic Lesions of the Genital System in Male Mice in the Three-month Inhalation Study of Cobalt Metal.

Table 26. Survival of Mice in the Two-year Inhalation Study of Cobalt Metal.

Table 27. Mean Body Weights and Survival of Male Mice in the Two-year Inhalation Study of Cobalt Metal

Table 28. Mean Body Weights and Survival of Female Mice in the Two-year Inhalation Study of Cobalt Metal

Table 29. Incidences of Neoplasms and Nonneoplastic Lesions of the Lung in Mice in the Two-year Inhalation Study of Cobalt Metal

Table 30. Incidences of Nonneoplastic Lesions of the Nose, Larynx, and Trachea in Mice in the Two-year Inhalation Study of Cobalt Metal 


\section{Figures}

Figure 1. Growth Curves for Rats Exposed to Cobalt Metal by Inhalation for Three

Months

Figure 2. Kaplan-Meier Survival Curves for Rats Exposed to Cobalt Metal by Inhalation for Two Years.

Figure 3. Growth Curves for Rats Exposed to Cobalt Metal by Inhalation for Two Years ..........46

Figure 4. Growth Curves for Mice Exposed to Cobalt Metal by Inhalation for Three Months

Figure 5. Kaplan-Meier Survival Curves for Mice Exposed to Cobalt Metal by Inhalation for Two Years.

Figure 6. Growth Curves for Mice Exposed to Cobalt Metal by Inhalation for Two Years .........80

Figure 7. Alveolar/Bronchiolar Adenoma in the Lung of a F344/NTac Female Rat

Exposed to $5 \mathrm{mg} / \mathrm{m}^{3}$ Cobalt Metal by Inhalation for Two Years (H\&E) ......................90

Figure 8. Alveolar/Bronchiolar Carcinoma in the Lung af a F344/NTac Female Rat Exposed to $5 \mathrm{mg} / \mathrm{m}^{3}$ Cobalt Metal by Inhalation for Two Years (H\&E).

Figure 9. Sarcomatous Type Alveolar/Bronchiolar Carcinoma in the Lung of a F344/NTac Female Rat Exposed to $5 \mathrm{mg} / \mathrm{m}^{3}$ Cobalt Metal by Inhalation for Two Years (H\&E)

Figure 10. Cystic Keratinizing Epithelioma in the Lung of a Male F344/NTac Rat Exposed to $1.25 \mathrm{mg} / \mathrm{m}^{3}$ Cobalt Metal by Inhalation for Two Years (H\&E).

Figure 11. Discrete, Focal Alveolar Epithelial Hyperplasia (Arrows) in the Lung of a Female F344/NTac Rat Exposed to $5 \mathrm{mg} / \mathrm{m}^{3}$ Cobalt Metal by Inhalation for Two Years (H\&E)

Figure 12. Focally Extensive Area of Alveolar Epithelial Hyperplasia (Arrows) in the Lung of a Female F344/NTac Rat Exposed to $5 \mathrm{mg} / \mathrm{m}^{3}$ Cobalt Metal by Inhalation for Two Years (H\&E)

Figure 13. Alveolar Proteinosis in the Lung of a Male F344/NTac Rat Exposed to $5 \mathrm{mg} / \mathrm{m}^{3}$ Cobalt Metal by Inhalation for Two Years (H\&E)

Figure 14. An Area of Chronic Active Inflammation (Arrows) in the Lung of a Male F344/NTac Rat Exposed to $5 \mathrm{mg} / \mathrm{m}^{3}$ Cobalt Metal by Inhalation for Two Years (H\&E)

Figure 15. Bronchiolar Epithelial Hyperplasia in the Lung of a Female F344/NTac Rat Exposed to $5 \mathrm{mg} / \mathrm{m}^{3}$ Cobalt Metal by Inhalation for Two Years (H\&E).

Figure 16. Suppurative Inflammation in a Nasal Maxilloturbinate in the Level II Section in the Nose of a Female F344/NTac Rat Exposed to $5 \mathrm{mg} / \mathrm{m}^{3}$ Cobalt Metal by Inhalation for Two Years (H\&E)

Figure 17. Respiratory Epithelial Metaplasia of the Olfactory Epithelium of an Ethmoid Turbinate in the Nose of a Female F344/NTac Rat Exposed to $5 \mathrm{mg} / \mathrm{m}^{3}$ Cobalt Metal by Inhalation for Two Years (H\&E).

Figure 18. Olfactory Epithelial Atrophy and Olfactory Epithelial Hyperplasia in the Ethmoid Turbinate in the Nose of a Male F344/NTac Rat Exposed to $5 \mathrm{mg} / \mathrm{m}^{3}$ Cobalt Metal by Inhalation for Two Years (H\&E)

Figure 19. Respiratory Epithelial Hyperplasia and Squamous Metaplasia in a Maxilloturbinate in the Nose of a Male F344/NTac Rat Exposed to $5 \mathrm{mg} / \mathrm{m}^{3}$ Cobalt Metal by Inhalation for Two Years (H\&E) 
Figure 20. Necrosis of the Respiratory Epithelium in a Maxilloturbinate in the Nose of a Female F344/NTac Rat Exposed to $5 \mathrm{mg} / \mathrm{m}^{3}$ Cobalt Metal by Inhalation for Two Years (H\&E)

Figure 21. Atrophy of the Ethmoid Turbinates in the Nose of a Male F344/NTac Rat Exposed to $5 \mathrm{mg} / \mathrm{m}^{3}$ Cobalt Metal by Inhalation for Two Years (H\&E).

Figure 22. Benign Pheochromocytoma in the Adrenal Medulla of a Male F344/NTac Rat Exposed to $5 \mathrm{mg} / \mathrm{m}^{3}$ Cobalt Metal by Inhalation for Two Years (H\&E).

Figure 23. Malignant Pheochromocytoma in the Adrenal Medulla of a Male F344/NTac Rat Exposed to $5 \mathrm{mg} / \mathrm{m}^{3}$ Cobalt Metal by Inhalation for Two Years (H\&E).

Figure 24. Islet Cell Adenoma in the Pancreas of a Male F344/NTac Rat Exposed to $5 \mathrm{mg} / \mathrm{m}^{3}$ Cobalt Metal by Inhalation for Two Years (H\&E)

Figure 25. Islet Cell Carcinoma in the Pancreas of a Male F344/NTac Rat Exposed to $5 \mathrm{mg} / \mathrm{m}^{3}$ Cobalt Metal by Inhalation for Two Years (H\&E)

Figure 26. Renal Tubule Adenoma in the Kidney of a Male F344/NTac Rat Exposed to $5 \mathrm{mg} / \mathrm{m}^{3}$ Cobalt Metal by Inhalation for Two Years (H\&E)

Figure 27. Renal Tubule Carcinoma in the Kidney of a Male F344/NTac Rat Exposed to $5 \mathrm{mg} / \mathrm{m}^{3}$ Cobalt Metal by Inhalation for Two Years (H\&E)

Figure 28. Low Magnification of an Alveolar/Bronchiolar Adenoma in the Lung of a Male B6C3F1/N Mouse Exposed to $2.5 \mathrm{mg} / \mathrm{m}^{3}$ Cobalt Metal by Inhalation for Two Years (H\&E)

Figure 29. Low Magnification of an Alveolar/Bronchiolar Carcinoma in the Lung of a Male B6C3F1/N Mouse Exposed to $5 \mathrm{mg} / \mathrm{m}^{3}$ Cobalt Metal by Inhalation for Two Years (H\&E)

Figure 30. Higher Magnification of Figure 29 (H\&E)

Figure 31. Alveolar/Bronchiolar Carcinoma in the Lung of a Female B6C3F1/N Mouse Exposed to $5 \mathrm{mg} / \mathrm{m}^{3}$ Cobalt Metal by Inhalation for Two Years (H\&E).

Figure 32. Alveolar/Bronchiolar Epithelial Hyperplasia in the Lung of a Female B6C3F1/N Mouse Exposed to $5 \mathrm{mg} / \mathrm{m}^{3}$ Cobalt Metal by Inhalation for Two Years (H\&E)

Figure 33. Focal Alveolar Epithelial Hyperplasia in the Lung of a Male B6C3F1/N Mouse Exposed to $2.5 \mathrm{mg} / \mathrm{m}^{3}$ Cobalt Metal by Inhalation for Two Years $(\mathrm{H} \& \mathrm{E})$

Figure 34. Focal Bronchiolar Epithelial Hyperplasia in a Terminal Bronchiole (Arrows) of a Male B6C3F1/N Mouse Exposed to $5 \mathrm{mg} / \mathrm{m}^{3}$ Cobalt Metal by Inhalation for Two Years (H\&E) .

Figure 35. Alveolar Histiocytic Infiltrates in the Lung of a Male B6C3F1/N Mouse Exposed to $5 \mathrm{mg} / \mathrm{m}^{3}$ Cobalt Metal by Inhalation for Two Years (H\&E).

Figure 36. Bilateral Atypical Respiratory Epithelial Metaplasia in the Olfactory Epithelium of a Male B6C3F1/N Mouse Exposed to $1.25 \mathrm{mg} / \mathrm{m}^{3}$ Cobalt Metal by Inhalation for Two Years (H\&E)

This report has been reformatted to meet new NTP publishing requirements; its content has not changed. 


\section{About This Report}

National Toxicology Program ${ }^{1}$

${ }^{1}$ Division of the National Toxicology Program, National Institute of Environmental Health

Sciences, Research Triangle Park, North Carolina, USA

\section{Collaborators}

M. Behl, M.J. Hooth, R.A. Herbert, B. Atkinson, C.R. Blystone, A.E. Brix, M.C. Cora, J.A. Dill, P.M. Foster, D.K. Giri, M.M. Gruebbel, S.L. Grumbein, B.K. Hayden, G.D. Hill, A.P. KingHerbert, G.E. Kissling, D.E. Malarkey, B.S. McIntyre, R.A. Miller, R. Moore, B.J.T. Muir, A.R. Pandiri, R.A. Renne, S.L. Smith-Roe, L.M. Staska, M.D. Stout, G.S. Travlos, S. Waidyanatha, N.J. Walker, Y. Wang, K.L. Witt

Division of the National Toxicology Program, National Institute of Environmental Health Sciences, Research Triangle Park, North Carolina, USA

Evaluated and interpreted results and reported findings

M. Behl, Ph.D., Co-Study Scientist

M.J. Hooth, Ph.D., Co-Study Scientist

R.A. Herbert, D.V.M., Ph.D., Study Pathologist

C.R. Blystone, Ph.D.

M.C. Cora, D.V.M.

P.M. Foster, Ph.D.

A.P. King-Herbert, D.V.M.

G.E. Kissling, Ph.D.

D.E. Malarkey, D.V.M., Ph.D.

B.S. McIntyre, Ph.D.

A.R. Pandiri, B.V.Sc. \& A.H., Ph.D., Experimental Pathology Laboratories, Inc.

S.L. Smith-Roe, Ph.D.

M.D. Stout, Ph.D.

G.S. Travlos, D.V.M.

S. Waidyanatha, Ph.D.

N.J. Walker, Ph.D.

K.L. Witt, M.S.

Experimental Pathology Laboratories, Inc., Research Triangle Park, North Carolina, USA Evaluated and interpreted results and reported findings

A.R. Pandiri, B.V.Sc. \& A.H., Ph.D.

\section{Battelle Toxicology Northwest, Richland, Washington, USA}

Conducted studies and evaluated pathology findings

J.A. Dill, Ph.D., Principal Investigator

S.L. Grumbein, D.V.M., Ph.D.

B.K. Hayden

R.A. Renne, D.V.M.

L.M. Staska, D.V.M., Ph.D. 
Experimental Pathology Laboratories, Inc., Research Triangle Park, North Carolina, USA Conducted pathology review

A.E. Brix, D.V.M., Ph.D.

M.M. Gruebbel, D.V.M., Ph.D.

R.A. Miller, D.V.M., Ph.D.

R. Moore, D.V.M.

A.R. Pandiri, B.V.Sc. \& A.H., Ph.D.

ILS, Inc., Research Triangle Park, North Carolina, USA

Coordinated NTP Pathology Working Groups

D.K. Giri, D.V.M., Ph.D, Coordinated 2-year rats (March 29, 2011)

G.D. Hill, D.V.M., Ph.D, Coordinated 2-year mice (March 3, 2011)

Gene Logic Laboratories, Inc.

Provided SMVCE analysis

B.J.T. Muir, Ph.D., Principal Investigator

B. Atkinson, M.Sc.

Y. Wang, M.S.

\section{Contributors}

Experimental Pathology Laboratories, Inc., Research Triangle Park, North Carolina, USA Supervised pathology review

M.H. Hamlin, II, D.V.M., Principal Investigator

NTP Pathology Working Group, National Institute of Environmental Health Sciences, Research Triangle Park, North Carolina, USA

Participated in NTP Pathology Working Group on 2-year rats (March 29, 2011)

A.E. Brix, D.V.M., Ph.D., Experimental Pathology Laboratories, Inc.

S.A. Elmore, D.V.M., M.S., National Toxicology Program

J. Everitt, D.V.M., GlaxoSmithKline

G.P. Flake, M.D., National Toxicology Program

R.A. Herbert, D.V.M., Ph.D., National Toxicology Program

D.E. Malarkey, D.V.M., Ph.D., National Toxicology Program

R.A. Miller, D.V.M., Ph.D., Experimental Pathology Laboratories, Inc.

R. Moore, D.V.M., Experimental Pathology Laboratories, Inc.

A.R. Pandiri, B.V.Sc. \& A.H., Ph.D., Experimental Pathology Laboratories, Inc.

Participated in NTP Pathology Working Group on 2-year mice (March 3, 2011)

S. Chandra, D.V.M., Ph.D., GlaxoSmithKline

S.A. Elmore, D.V.M., M.S., National Toxicology Program

G.P. Flake, M.D., National Toxicology Program

D.K. Giri, D.V.M., Ph.D., ILS, Inc.

M.M. Gruebbel, D.V.M., Ph.D., Experimental Pathology Laboratories, Inc.

R.A. Herbert, D.V.M., Ph.D., National Toxicology Program

D.E. Malarkey, D.V.M., Ph.D., National Toxicology Program

R.A. Miller, D.V.M., Ph.D., Experimental Pathology Laboratories, Inc. 
R. Moore, D.V.M., Experimental Pathology Laboratories, Inc.

A.R. Pandiri, B.V.Sc. \& A.H., Ph.D., Experimental Pathology Laboratories, Inc.

SRA International, Inc., Research Triangle Park, North Carolina, USA

Provided statistical analyses

R.W. Morris, Ph.D., Principal Investigator

L.J. Betz, M.S.

S.F. Harris, B.S.

Dynamac Corporation, Research Triangle Park, North Carolina, USA

Prepared quality assessment audits

S. Brecher, Ph.D., Principal Investigator

S. Iyer, B.S.

V.S. Tharakan, D.V.M.

Biotechnical Services, Inc., Little Rock, Arkansas, USA

Prepared Technical Report

S.R. Gunnels, M.A., Principal Investigator

L.M. Harper, B.S.

D.C. Serbus, Ph.D.

G.E. Simmons, M.A. 


\section{Explanation of Levels of Evidence of Carcinogenic Activity}

The National Toxicology Program describes the results of individual experiments on a chemical agent and notes the strength of the evidence for conclusions regarding each study. Negative results, in which the study animals do not have a greater incidence of neoplasia than control animals, do not necessarily mean that a chemical is not a carcinogen, inasmuch as the experiments are conducted under a limited set of conditions. Positive results demonstrate that a chemical is carcinogenic for laboratory animals under the conditions of the study and indicate that exposure to the chemical has the potential for hazard to humans. Other organizations, such as the International Agency for Research on Cancer, assign a strength of evidence for conclusions based on an examination of all available evidence, including animal studies such as those conducted by NTP, epidemiologic studies, and estimates of exposure. Thus, the actual determination of risk to humans from chemicals found to be carcinogenic in laboratory animals requires a wider analysis that extends beyond the purview of these studies.

Five categories of evidence of carcinogenic activity are used in the Technical Report series to summarize the strength of evidence observed in each experiment: two categories for positive results (clear evidence and some evidence); one category for uncertain findings (equivocal evidence); one category for no observable effects (no evidence); and one category for experiments that cannot be evaluated because of major flaws (inadequate study). These categories of interpretative conclusions were first adopted in June 1983 and then revised on March 1986 for use in the Technical Report series to incorporate more specifically the concept of actual weight of evidence of carcinogenic activity. For each separate experiment (male rats, female rats, male mice, female mice), one of the following five categories is selected to describe the findings. These categories refer to the strength of the experimental evidence and not to potency or mechanism.

- Clear evidence of carcinogenic activity is demonstrated by studies that are interpreted as showing a dose-related (i) increase of malignant neoplasms, (ii) increase of a combination of malignant and benign neoplasms, or (iii) marked increase of benign neoplasms if there is an indication from this or other studies of the ability of such tumors to progress to malignancy.

- Some evidence of carcinogenic activity is demonstrated by studies that are interpreted as showing a chemical-related increased incidence of neoplasms (malignant, benign, or combined) in which the strength of the response is less than that required for clear evidence.

- Equivocal evidence of carcinogenic activity is demonstrated by studies that are interpreted as showing a marginal increase of neoplasms that may be chemical related.

- No evidence of carcinogenic activity is demonstrated by studies that are interpreted as showing no chemical-related increases in malignant or benign neoplasms

- Inadequate study of carcinogenic activity is demonstrated by studies that, because of major qualitative or quantitative limitations, cannot be interpreted as valid for showing either the presence or absence of carcinogenic activity.

For studies showing multiple chemical-related neoplastic effects that if considered individually would be assigned to different levels of evidence categories, the following convention has been 
adopted to convey completely the study results. In a study with clear evidence of carcinogenic activity at some tissue sites, other responses that alone might be deemed some evidence are indicated as "were also related" to chemical exposure. In studies with clear or some evidence of carcinogenic activity, other responses that alone might be termed equivocal evidence are indicated as "may have been" related to chemical exposure.

When a conclusion statement for a particular experiment is selected, consideration must be given to key factors that would extend the actual boundary of an individual category of evidence. Such consideration should allow for incorporation of scientific experience and current understanding of long-term carcinogenesis studies in laboratory animals, especially for those evaluations that may be on the borderline between two adjacent levels. These considerations should include:

- adequacy of the experimental design and conduct;

- occurrence of common versus uncommon neoplasia;

- progression (or lack thereof) from benign to malignant neoplasia as well as from preneoplastic to neoplastic lesions;

- some benign neoplasms have the capacity to regress but others (of the same morphologic type) progress. At current, it is impossible to identify the difference. Therefore, where progression is known to be a possibility, the most prudent course is to assume that benign neoplasms of those types have the potential to become malignant;

- combining benign and malignant tumor incidence known or thought to represent stages of progression in the same organ or tissue;

- latency in tumor induction;

- multiplicity in site-specific neoplasia;

- metastases;

- supporting information from proliferative lesions (hyperplasia) in the same site of neoplasia or other experiments (same lesion in another sex or species);

- presence or absence of dose relationships;

- statistical significance of the observed tumor increase;

- concurrent control tumor incidence as well as the historical control rate and variability for a specific neoplasm;

- survival-adjusted analyses and false positive or false negative concerns;

- structure-activity correlations; and

- in some cases, genetic toxicology. 


\section{Peer Review}

The members of the Peer Review Panel who evaluated the draft NTP Technical Report on the Toxicology Studies of Cobalt Metal (CASRN 7440-48-4) in F344/N Rats and B6C3F1/N Mice and Toxicology and Carcinogenesis Studies of Cobalt Metal in F344/NTAC Rats and B6C3F1/N Mice (Inhalation Studies) on October 29, 2013, are listed below. Panel members served as independent scientists, not as representatives of any institution, company, or governmental agency. In this capacity, reviewers had five major responsibilities in reviewing the NTP studies:

- to ascertain that all relevant literature data have been adequately cited and interpreted,

- to determine if the design and conditions of the NTP studies were appropriate,

- to ensure that the Technical Report presents the experimental results and conclusions fully and clearly,

- to judge the significance of the experimental results by scientific criteria, and

- to assess the evaluation of the evidence of carcinogenic activity and other observed toxic responses.

\section{Peer Reviewers}

John Cullen, V.M.D., Ph.D., Chairperson

College of Veterinary Medicine

North Carolina State University

Raleigh, North Carolina

Norman J. Barlow, M.B.A., M.L.D., D.V.M., Ph.D.

Sanofi

Boston, Massachusetts

Russell C. Cattley, V.M.D., Ph.D.

College of Veterinary Medicine

Auburn University

Auburn, Alabama

Deborah Cory-Slechta, Ph.D., Primary Reviewer

University of Rochester School of Medicine and Dentistry

Rochester, New York

Terry Gordon, Ph.D., Primary Reviewer

New York University Langone Medical Center

New York, New York

\section{Joel Parker, Ph.D.}

University of North Carolina at Chapel Hill

Chapel Hill, North Carolina

\section{Karen Regan, D.V.M., Primary Reviewer}

Regan Pathology/Toxicology Services, Inc.

Ashland, Ohio 
Cobalt Metal, NTP TR 581

Timothy Zacharewski, Ph.D., Primary Reviewer

Michigan State University

East Lansing, Michigan 


\section{Publication Details}

Publisher: National Toxicology Program

Publishing Location: Research Triangle Park, NC

ISSN: $2378-8925$

DOI: https://doi.org/10.22427/NTP-TR-581

Report Series: NTP Technical Report Series

Report Series Number: 581

Official citation: National Toxicology Program (NTP). 2014. NTP technical report on the toxicology studies of cobalt metal (CASRN 7440-48-4) in F344/N rats and B6C3F1/N mice and toxicology and carcinogenesis studies of cobalt metal in F344/NTac rats and B6C3F1/N mice (inhalation studies). Research Triangle Park, NC: National Toxicology Program. Technical Report 581. 


\section{Abstract}

Widespread exposure to cobalt metal dust occurs occupationally through the production of alloys, in the manufacture of cobalt salts, and in nuclear technology. It is an effective catalyst for many organic reactions, particularly in hydrotreating catalysts, which have molybdenum and cobalt sulfides as active components. Concerns have been raised about the occurrence of occupational disease, i.e. hard metal disease, associated with exposure to cobalt and its compounds, including cobalt metal-tungsten carbide. Cobalt metal is also widely dispersed in low concentrations in the environ-ment and the general population may be exposed by breathing air, drinking water, or skin contact with soil, water, cobalt alloys, or other substances that contain cobalt. In addition, cobalt metal is an essential trace element as a component of cyanocobalamin (vitamin $\mathrm{B}_{12}$ ). Cobalt metal dust was nominated for toxicology and carcinogenesis studies by the United Auto Workers and the Cobalt Development Institute based on the widespread occupational exposure and limited availability of data on chronic toxicity and carcinogenic potential of inhaled insoluble cobalt compounds, particularly cobalt metal dust. Inhalation was selected as the route of exposure because this is the most common route of exposure to cobalt metal dust in occupational settings in humans. Male and female F344/N or F344/NTac rats and B6C3F1/N mice were exposed to cobalt metal by inhalation for 2 weeks, 3 months, or 2 years (F344/NTac rats). In addition, genetic toxicology studies were conducted in Salmonella typhimurium, Escherichia coli, and mouse peripheral blood erythrocytes.

\section{Two-week Study in Rats}

Groups of five male and five female rats were exposed to cobalt metal particulate aerosol by inhalation at concentrations of $0,2.5,5,10,20$, or $40 \mathrm{mg} / \mathrm{m}^{3}, 6$ hours plus $\mathrm{T}_{90}$ (12 minutes) per day, 5 days per week for 16 days. Additional groups of five female rats were exposed to the same concentrations for 16 days for tissue burden studies. All rats exposed to $40 \mathrm{mg} / \mathrm{m}^{3}$ and all male and three female rats exposed to $20 \mathrm{mg} / \mathrm{m}^{3}$ died before the end of the study. Mean body weights of males exposed to $10 \mathrm{mg} / \mathrm{m}^{3}$ and of females exposed to 10 or $20 \mathrm{mg} / \mathrm{m}^{3}$ were significantly decreased. Females exposed to $20 \mathrm{mg} / \mathrm{m}^{3}$ lost weight during the study. Exposure-related clinical findings included abnormal breathing, lethargy, and thinness in male rats exposed to 20 or $40 \mathrm{mg} / \mathrm{m}^{3}$ and in females exposed to $40 \mathrm{mg} / \mathrm{m}^{3}$. Dark lungs were observed at necropsy in all rats exposed to $40 \mathrm{mg} / \mathrm{m}^{3}$ and most rats exposed to $20 \mathrm{mg} / \mathrm{m}^{3}$ that died early. Absolute lung weights of females exposed to 10 or $20 \mathrm{mg} / \mathrm{m}^{3}$ and the relative lung weights of both sexes exposed to $10 \mathrm{mg} / \mathrm{m}^{3}$ and females exposed to $20 \mathrm{mg} / \mathrm{m}^{3}$ were significantly greater than those of the chamber controls. Absolute and relative liver weights of males exposed to $2.5 \mathrm{mg} / \mathrm{m}^{3}$ or greater and absolute liver weights of females exposed to $5 \mathrm{mg} / \mathrm{m}^{3}$ or greater were significantly less than those of the chamber controls. The relative liver weight of $20 \mathrm{mg} / \mathrm{m}^{3}$ females was significantly greater than that of the chamber controls. Absolute kidney weights of males exposed to $10 \mathrm{mg} / \mathrm{m}^{3}$ and females exposed to $20 \mathrm{mg} / \mathrm{m}^{3}$ were significantly less than those of the chamber controls. The absolute testis weight of the $10 \mathrm{mg} / \mathrm{m}^{3}$ group was significantly less than that of the chamber controls. Increased incidences of nonneoplastic lesions of the lung occurred in exposed male and female rats and included hemorrhage, acute inflammation, alveolar epi-thelium hyperplasia, histiocytic cellular infiltration of the alveolus, cytoplasmic vacuolization of bronchiolar epithelium, necrosis of the bronchiolar epithelium, and interstitial fibrosis of the alveolar epithelium. Increased incidences of nonneoplastic lesions of the nose occurred in exposed male and female rats and included olfactory epithelium necrosis, olfactory epithelium atrophy, respiratory epithelium necrosis, and respiratory epithelium squamous metaplasia. Tissue 
concentrations of cobalt increased with increasing exposure concentration in all tissues examined.

\section{Two-week Study in Mice}

Groups of five male and five female mice were exposed to cobalt metal particulate aerosol by inhalation at concentrations of $0,2.5,5,10,20$, or $40 \mathrm{mg} / \mathrm{m}^{3}, 6$ hours plus $\mathrm{T}_{90}$ (12 minutes) per day, 5 days per week for 17 days. Additional groups of five female mice were exposed to the same concentrations for 17 days for tissue burden studies. Three male and three female mice exposed to $40 \mathrm{mg} / \mathrm{m}^{3}$ died before the end of the study. Final mean body weights were significantly decreased in male and female mice exposed to 20 or $40 \mathrm{mg} / \mathrm{m}^{3}$, and mean body weight gains of 20 and $40 \mathrm{mg} / \mathrm{m}^{3}$ males and all exposed groups of females were significantly less than those of the chamber controls. Females exposed to $20 \mathrm{mg} / \mathrm{m}^{3}$ and males and females exposed to $40 \mathrm{mg} / \mathrm{m}^{3}$ lost weight during the study. Exposure-related clinical findings included abnormal breathing, lethargy, and thinness in male mice exposed to 20 or $40 \mathrm{mg} / \mathrm{m}^{3}$ and females exposed to $10 \mathrm{mg} / \mathrm{m}^{3}$ or greater. At necropsy, tan lungs were observed in most males and females exposed to 20 or $40 \mathrm{mg} / \mathrm{m}^{3}$. Lung weights of both sexes exposed to $10 \mathrm{mg} / \mathrm{m}^{3}$ or greater were significantly greater than those of the chamber controls. Liver weights of exposed male and female mice were significantly less than those of the chamber controls (except relative weight at $40 \mathrm{mg} / \mathrm{m}^{3}$ ). Increased incidences of nonneoplastic lesions of the lung occurred in exposed male and female mice and included alveolar histiocytic cellular infiltration, cytoplasmic vacuolization of the bronchiolar epithelium, alveolar/bronchiolar epithelium karyomegaly, interstitial fibrosis, and acute inflammation. Increased incidences of nonneoplastic lesions of the nose occurred in exposed groups of male and female mice and included acute inflammation, olfactory epithelium atrophy, olfactory epithelium necrosis, cytoplasmic vacuolization of the respiratory epithelium, and squamous metaplasia of the respiratory epithelium. Tissue concentrations of cobalt increased with increasing exposure concentration in all tissues examined.

\section{Three-month Study in Rats}

Groups of 10 male and 10 female rats were exposed to particulate aerosols of cobalt metal by inhalation at concentrations of $0,0.625,1.25,2.5$, or $5 \mathrm{mg} / \mathrm{m}^{3}, 6$ hours plus $\mathrm{T}_{90}$ (12 minutes) per day, 5 days per week for 14 weeks. Additional groups of 10 male rats (clinical pathology study) and 32 to 36 female rats (special study) were exposed to the same concentrations for 14 weeks. All male and female rats survived to the end of the study. Final mean body weights of males and females exposed to $5 \mathrm{mg} / \mathrm{m}^{3}$ were significantly less than those of the chamber controls, and the mean body weight gain of $5 \mathrm{mg} / \mathrm{m}^{3}$ males was significantly less than that of the chamber controls. At necropsy, pale foci were noted in the lungs of most exposed male and female rats. In male rats, exposure concentration-related increases in the hemoglobin concentration, erythrocyte count, hematocrit value, and manual packed cell volume occurred in the 2.5 and $5 \mathrm{mg} / \mathrm{m}^{3}$ groups on days 3 and 23 and in all exposed groups by week 14; at week 14, female rats also had increases in these parameters. Exposure concentration-related decreases in cholesterol concentrations were observed at all three time points in male and female rats. While this change was not always observed in the lower exposure groups, decreases were consistently observed in the 2.5 and $5 \mathrm{mg} / \mathrm{m}^{3}$ groups of both sexes on day 23 and at week 14. In addition, glucose concentration was decreased in males exposed to $1.25 \mathrm{mg} / \mathrm{m}^{3}$ or greater at week 14. Lung weights of all exposed groups of males and females were significantly greater than those of the chamber controls. Sperm motility was significantly decreased in male rats exposed to cobalt, suggesting a potential for cobalt metal to be a reproductive toxicant in male rats. 
In the lung, chronic active inflammation and alveolar proteinosis occurred in all exposed males and females, and bronchiole epithelium hyperplasia occurred in all males and females exposed to $1.25 \mathrm{mg} / \mathrm{m}^{3}$ or greater. In the nose, incidences of olfactory epithelium degeneration and respiratory epithelium hyperplasia were significantly increased in males and females exposed to 2.5 or $5 \mathrm{mg} / \mathrm{m}^{3}$. The incidences of olfactory epithelium hyperplasia were significantly increased in 2.5 and $5 \mathrm{mg} / \mathrm{m}^{3}$ males and in $5 \mathrm{mg} / \mathrm{m}^{3}$ females. Significantly increased incidences of turbinate atrophy occurred in $2.5 \mathrm{mg} / \mathrm{m}^{3}$ females and $5 \mathrm{mg} / \mathrm{m}^{3}$ males and females. Tissue concentrations of cobalt increased with increasing exposure concentration in all tissues examined.

\section{Three-month Study in Mice}

Groups of 10 male and 10 female mice were exposed to particulate aerosols of cobalt metal by inhalation at concentrations of $0,0.625,1.25,2.5,5$, or $10 \mathrm{mg} / \mathrm{m}^{3}, 6$ hours plus $\mathrm{T}_{90}$ ( 12 minutes) per day, 5 days per week for 14 weeks. Additional groups of 32 to 36 female mice (special study) were exposed to the same concentrations for 14 weeks. One $2.5 \mathrm{mg} / \mathrm{m}^{3}$ female mouse was accidentally killed during the first week of the study; all other mice survived to the end of the study. The mean body weights of males and females exposed to $10 \mathrm{mg} / \mathrm{m}^{3}$ were significantly less than those of the chamber controls. Abnormal breathing was noted in approximately $50 \%$ of males and females exposed to $10 \mathrm{mg} / \mathrm{m}^{3}$. At necropsy, tan lungs were noted in mice exposed to 5 or $10 \mathrm{mg} / \mathrm{m}^{3}$. Lung weights of males exposed to $2.5 \mathrm{mg} / \mathrm{m}^{3}$ or greater and females exposed to 5 or $10 \mathrm{mg} / \mathrm{m}^{3}$ were significantly greater than those of the chamber controls. Liver weights of males exposed to $10 \mathrm{mg} / \mathrm{m}^{3}$ and females exposed to $2.5 \mathrm{mg} / \mathrm{m}^{3}$ or greater were significantly less than those of the chamber controls. Kidney weights of males and females exposed to 5 or $10 \mathrm{mg} / \mathrm{m}^{3}$ were significantly less than those of the chamber controls. Testes weights of males exposed to 5 or $10 \mathrm{mg} / \mathrm{m}^{3}$ were significantly less than those of the chamber controls. Exposure concentration-related decreases in reproductive tissue weights, spermatid and epididymal spermatozoa counts, and sperm motility in combination with histopathologic findings in both the testis and epididymis indicate that cobalt metal is likely to be a reproductive toxicant in male mice.

In the lung, alveolar histiocytic cellular infiltration and bronchiole epithelium cytoplasmic vacuolization occurred in the lung of all exposed male and female mice. Bronchiole epithelium hyperplasia occurred in all mice exposed to $2.5 \mathrm{mg} / \mathrm{m}^{3}$ or greater. Alveolar proteinosis and alveolar/bronchiolar epithelium karyomegaly occurred in all males and females exposed to 5 or $10 \mathrm{mg} / \mathrm{m}^{3}$. The incidences of hemorrhage were significantly increased in $5 \mathrm{mg} / \mathrm{m}^{3}$ females and in 5 and $10 \mathrm{mg} / \mathrm{m}^{3}$ males. In the nose, the incidences of olfactory epithelium degeneration were significantly increased in males and females exposed to $1.25 \mathrm{mg} / \mathrm{m}^{3}$ or greater. Incidences of respiratory epithelium degeneration were significantly increased in males exposed to $1.25 \mathrm{mg} / \mathrm{m}^{3}$ or greater and females exposed to $2.5 \mathrm{mg} / \mathrm{m}^{3}$ or greater. Incidences of respiratory epithelium squamous metaplasia were significantly increased in males and females exposed to $2.5 \mathrm{mg} / \mathrm{m}^{3}$ or greater, and incidences of turbinate atrophy and chronic active inflammation were significantly increased in the 5 and $10 \mathrm{mg} / \mathrm{m}^{3}$ groups of males and females. The incidences of squamous metaplasia were significantly increased in the larynx of all exposed groups of males and females. Tissue concentrations of cobalt increased with increasing exposure concentration in all tissues examined. 


\section{Two-year Study in Rats}

Groups of 50 male and 50 female rats were exposed to cobalt metal particulate aerosol by inhalation at concentrations of $0,1.25,2.5$, or $5 \mathrm{mg} / \mathrm{m}^{3}, 6$ hours plus $\mathrm{T}_{90}$ (12 minutes) per day, 5 days per week for up to 105 weeks. Additional groups of 35 lung burden study female rats were exposed to the same concentrations of cobalt metal for up to 105 weeks. Survival of female rats exposed to $2.5 \mathrm{mg} / \mathrm{m}^{3}$ was significantly less than that of the chamber control group. Mean body weights of 2.5 and $5 \mathrm{mg} / \mathrm{m}^{3}$ males were at least $10 \%$ less than those of the chamber control group after weeks 99 and 12, respectively, and those of 2.5 and $5 \mathrm{mg} / \mathrm{m}^{3}$ females were at least $10 \%$ less after weeks 57 and 21, respectively. Exposure-related clinical findings included abnormal breathing and thinness in male and female rats.

In the lung, the incidences of alveolar/bronchiolar adenoma, alveolar/bronchiolar carcinoma, and alveolar/bronchiolar adenoma or carcinoma (combined) occurred with positive trends in male and female rats and with the exception of the incidence of alveolar/bronchiolar adenoma in $1.25 \mathrm{mg} / \mathrm{m}^{3}$ females, the incidences were significantly greater than those in the chamber controls. The incidences of multiple alveolar/bronchiolar adenoma and carcinoma generally increased with increasing exposure concentration, and the incidences of multiple carcinoma were significantly increased in all exposed groups of males and in $5 \mathrm{mg} / \mathrm{m}^{3}$ females. The incidences of cystic keratinizing epithelioma were increased in exposed groups of female rats; cystic keratinizing epithelioma also occurred in two exposed males. One female rat exposed to $5 \mathrm{mg} / \mathrm{m}^{3}$ had a squamous cell carcinoma. The incidences of alveolar epithelium hyperplasia, alveolar proteinosis, chronic active inflammation, and bronchiole epithelium hyperplasia in all exposed groups were significantly greater than those in the chamber control groups.

There was a higher frequency and different spectrum of point mutations within hot spot regions of Kras, Egfr, and Tp53 genes within alveolar/bronchiolar carcinomas from cobalt metalexposed male and female rats compared to spontaneous alveolar/bronchiolar carcinomas. Kras mutations and $\mathrm{G} \rightarrow \mathrm{T}$ transversions were most frequent in rats chronically exposed to cobalt metal.

A spectrum of nonneoplastic lesions occurred in the nose of exposed male and female rats including chronic active and suppurative inflammation, respiratory metaplasia, atrophy, hyperplasia, basal cell hyperplasia, and necrosis of the olfactory epithelium; hyperplasia, squamous metaplasia, and necrosis of the respiratory epithelium; and atrophy of the turbinate.

In the adrenal medulla, incidences of benign pheochromocytoma, malignant pheochromocytoma, and benign or malignant pheochromocytoma (combined) occurred with positive trends in male and female rats, and with the exception of the incidence of malignant pheochromocytoma in $2.5 \mathrm{mg} / \mathrm{m}^{3}$ females, the incidences in rats exposed to 2.5 or $5 \mathrm{mg} / \mathrm{m}^{3}$ were significantly greater than those in the chamber controls. The incidences of bilateral benign and malignant pheochromocytoma were significantly increased in the $5 \mathrm{mg} / \mathrm{m}^{3}$ groups. Incidences of hyperplasia were significantly increased in female rats exposed to 1.25 or $2.5 \mathrm{mg} / \mathrm{m}^{3}$.

The incidences of carcinoma and adenoma or carcinoma (combined) of the pancreatic islets occurred with positive trends in male rats. The incidences of adenoma in $2.5 \mathrm{mg} / \mathrm{m}^{3}$ males and of adenoma or carcinoma (combined) in males exposed to 2.5 or $5 \mathrm{mg} / \mathrm{m}^{3}$ were significantly greater than those in the chamber controls. Incidences of neoplasms of the pancreatic islets in $5 \mathrm{mg} / \mathrm{m}^{3}$ females were slightly increased. 
The incidences of mononuclear cell leukemia were significantly increased in all exposed groups of female rats.

In the combined standard and extended (step-section) evaluations of the kidney, the incidence of renal tubule adenoma or carcinoma (combined) was increased in male rats exposed to $5 \mathrm{mg} / \mathrm{m}^{3}$.

The incidence of infarct in the testes was significantly increased in male rats exposed to $5 \mathrm{mg} / \mathrm{m}^{3}$.

Cobalt concentrations in the lung increased with increasing exposure concentration.

\section{Two-year Study in Mice}

Groups of 50 male and 50 female mice were exposed to cobalt metal particulate aerosol by inhalation at concentrations of $0,1.25,2.5$, or $5 \mathrm{mg} / \mathrm{m}^{3}, 6$ hours plus $\mathrm{T}_{90}$ (12 minutes) per day, 5 days per week for up to 105 weeks. Additional groups of 35 lung burden study female mice were exposed to the same concentrations of cobalt metal for up to 105 weeks. Survival of males exposed to 2.5 or $5 \mathrm{mg} / \mathrm{m}^{3}$ was significantly less than that of the chamber control group. Mean body weights of $5 \mathrm{mg} / \mathrm{m}^{3}$ males and females were at least $10 \%$ less than those of the chamber control groups after weeks 85 and 21, respectively. Abnormal breathing and thinness were noted in exposed male and female mice.

In the lung, incidences of alveolar/bronchiolar carcinoma and alveolar/bronchiolar adenoma or carcinoma (combined) occurred with positive trends in male and female mice, and the incidences were all significantly greater than those in the chamber controls. The incidences of alveolar/bronchiolar adenoma were significantly increased in $2.5 \mathrm{mg} / \mathrm{m}^{3}$ males and in $5 \mathrm{mg} / \mathrm{m}^{3}$ females. The incidences of multiple alveolar/bronchiolar carcinoma were significantly increased in all exposed groups of males and females.

The incidences of alveolar/bronchiolar epithelium hyperplasia and cytoplasmic vacuolization, alveolar epithelium hyperplasia, proteinosis, and alveolus infiltration cellular histiocyte were significantly increased in all exposed groups of males and females. The incidences of bronchiole epithelium hyperplasia were significantly increased in males exposed to $5 \mathrm{mg} / \mathrm{m}^{3}$ and females exposed to 2.5 or $5 \mathrm{mg} / \mathrm{m}^{3}$. The incidence of bronchiole epithelium erosion was significantly increased in males exposed to $2.5 \mathrm{mg} / \mathrm{m}^{3}$. The incidences of suppurative inflammation were significantly increased in males exposed to 2.5 or $5 \mathrm{mg} / \mathrm{m}^{3}$ and females exposed to $5 \mathrm{mg} / \mathrm{m}^{3}$.

There was a higher frequency and different spectrum of point mutations within hot spot regions of Kras, Egfr, and Tp53 genes within alveolar/bronchiolar carcinomas from cobalt metalexposed male and female mice compared to spontaneous alveolar/bronchiolar carcinomas. Kras mutations and $\mathrm{G} \rightarrow \mathrm{T}$ transversions were most frequent in mice chronically exposed to cobalt metal.

In all groups of exposed male and female mice, significant increases occurred in nasal lesions including suppurative inflammation; olfactory epithelium atrophy, hyperplasia, and respiratory metaplasia; cytoplasmic vacuolization and squamous metaplasia of the respiratory epithelium; and atrophy of the turbinate. The incidences of atypical respiratory metaplasia of the olfactory epithelium and hyaline droplet accumulation of the respiratory epithelium were significantly increased in 1.25 and $2.5 \mathrm{mg} / \mathrm{m}^{3}$ males and females.

The incidences of respiratory epithelium squamous metaplasia and cytoplasmic vacuolization of the larynx in all exposed groups of males and females were significantly greater than those in the 
chamber control groups. The incidences of squamous epithelium hyperplasia were significantly increased in all exposed groups of females and in males exposed to $5 \mathrm{mg} / \mathrm{m}^{3}$. In the trachea, the incidences of epithelium cytoplasmic vacuolization were significantly increased in all exposed groups of males and females.

The incidence of germinal epithelium degeneration in the testes was significantly increased in male mice exposed to $5 \mathrm{mg} / \mathrm{m}^{3}$.

Cobalt concentrations in the lung increased with increasing exposure concentration.

\section{Genetic Toxicology}

Cobalt metal was mutagenic in S. typhimurium strain TA98 in the absence of exogenous metabolic activation (S9); no activity was seen in the presence of S9. Cobalt metal induced a small increase in mutant colonies in strain TA100 in the absence of S9, and no mutagenic activity was seen with S9. No mutagenic activity was detected in E. coli strain WP2 $u v r A / p K M 101$ with or without S9. Results of peripheral blood erythrocyte micronucleus tests in male and female mice in the 3-month study were negative.

\section{Conclusions}

Under the conditions of these 2-year inhalation studies, there was clear evidence of carcinogenic activity of cobalt metal in male F344/NTac rats based on increased incidences of alveolar/bronchiolar adenoma and carcinoma in the lung, including multiples, and on increased incidences of benign and malignant pheochromocytoma of the adrenal medulla, including bilateral neoplasms (see Explanation of Levels of Evidence of Carcinogenic Activity; a summary of the Peer Review Panel comments and the public discussion on this Technical Report appears in Appendix O). The increased incidences of pancreatic islet adenoma or carcinoma (combined) were considered related to exposure. The occurrences of cystic keratinizing epithelioma of the lung and of renal tubule adenoma or carcinoma (combined) may have been related to exposure. There was clear evidence of carcinogenic activity of cobalt metal in female F344/NTac rats based on increased incidences of alveolar/bronchiolar adenoma and carcinoma in the lung, including multiples, and on increased incidences of benign and malignant pheochromocytoma of the adrenal medulla, including bilateral neoplasms. The occurrences of squamous cell neoplasms of the lung (predominantly cystic keratinizing epithelioma), and of mononuclear cell leukemia were considered related to exposure. The occurrences of pancreatic islet carcinoma may have been related to exposure. There was clear evidence of carcinogenic activity of cobalt metal in male and female $\mathrm{B} 6 \mathrm{C} 3 \mathrm{~F} 1 / \mathrm{N}$ mice based on increased incidences of alveolar/bronchiolar neoplasms of the lung (predominantly carcinoma), including multiple carcinoma.

Exposure to cobalt metal resulted in increased incidences of nonneoplastic lesions of the lung and nose in male and female rats, the testes in the male rats and mice, the adrenal medulla in female rats, and the lung, nose, larynx, and trachea in male and female mice.

Synonyms: Cobalt element; super cobalt

Trade name: Aquacat 
Summary of the Two-year Carcinogenesis and Genetic Toxicology Studies of Cobalt Metal

\begin{tabular}{|c|c|c|c|c|}
\hline & $\begin{array}{c}\text { Male } \\
\text { F344/NTac Rats }\end{array}$ & $\begin{array}{c}\text { Female } \\
\text { F344/NTac Rats }\end{array}$ & $\begin{array}{c}\text { Male } \\
\text { B6C3F1/N Mice }\end{array}$ & $\begin{array}{c}\text { Female } \\
\text { B6C3F1/N Mice }\end{array}$ \\
\hline $\begin{array}{l}\text { Concentrations in } \\
\text { Air }\end{array}$ & $\begin{array}{l}0,1.25,2.5, \text { or } \\
5 \mathrm{mg} / \mathrm{m}^{3}\end{array}$ & $\begin{array}{l}0,1.25,2.5, \text { or } \\
5 \mathrm{mg} / \mathrm{m}^{3}\end{array}$ & $\begin{array}{l}0,1.25,2.5, \text { or } \\
5 \mathrm{mg} / \mathrm{m}^{3}\end{array}$ & $0,1.25,2.5$, or $5 \mathrm{mg} / \mathrm{m}^{3}$ \\
\hline Body Weights & $\begin{array}{l}2.5 \text { and } 5 \mathrm{mg} / \mathrm{m}^{3} \\
\text { groups at least } 10 \% \\
\text { less than the chamber } \\
\text { control group after } \\
\text { weeks } 99 \text { and } 12 \text {, } \\
\text { respectively }\end{array}$ & $\begin{array}{l}2.5 \text { and } 5 \mathrm{mg} / \mathrm{m}^{3} \\
\text { groups at least } 10 \% \\
\text { less than the } \\
\text { chamber control } \\
\text { group after } \\
\text { weeks } 57 \text { and } 21, \\
\text { respectively }\end{array}$ & $\begin{array}{l}5 \mathrm{mg} / \mathrm{m}^{3} \text { group at least } \\
10 \% \text { less than } \\
\text { chamber control group } \\
\text { after week } 85\end{array}$ & $\begin{array}{l}5 \mathrm{mg} / \mathrm{m}^{3} \text { group at least } \\
10 \% \text { less than the } \\
\text { chamber control group } \\
\text { after week } 21\end{array}$ \\
\hline Survival Rates & $\begin{array}{l}17 / 50,20 / 50,16 / 50 \\
16 / 50\end{array}$ & $\begin{array}{l}35 / 50,26 / 50,24 / 50 \\
25 / 50\end{array}$ & $\begin{array}{l}39 / 50,31 / 50,29 / 50 \\
25 / 50\end{array}$ & $\begin{array}{l}36 / 50,36 / 50,27 / 50 \\
26 / 50\end{array}$ \\
\hline $\begin{array}{l}\text { Nonneoplastic } \\
\text { Effects }\end{array}$ & $\begin{array}{l}\text { Lung: alveolar } \\
\text { epithelium, } \\
\text { hyperplasia }(3 / 50, \\
\text { 47/50, 49/50, 49/50); } \\
\text { alveolus, proteinosis } \\
(0 / 50,48 / 50,49 / 50, \\
\text { 49/50); } \\
\text { inflammation, } \\
\text { chronic active } \\
\text { (22/50, 50/50, 50/50, } \\
\text { 50/50); bronchiole, } \\
\text { epithelium, } \\
\text { hyperplasia }(0 / 50, \\
\text { 44/50, 47/50, 50/50) } \\
\text { Nose: inflammation, } \\
\text { chronic active } \\
(28 / 48,35 / 47,40 / 45, \\
\text { 49/50); } \\
\text { inflammation, } \\
\text { suppurative }(9 / 48, \\
\text { 12/47, } 24 / 45,46 / 50) \text {; } \\
\text { olfactory epithelium, } \\
\text { metaplasia, } \\
\text { respiratory }(12 / 48, \\
\text { 26/47, 37/45, 50/50); } \\
\text { olfactory epithelium, } \\
\text { atrophy }(2 / 48,21 / 47, \\
\text { 34/45, 29/50); } \\
\text { olfactory epithelium, } \\
\text { hyperplasia }(0 / 48, \\
\text { 1/47, } 2 / 45,7 / 50) ; \\
\text { olfactory epithelium, } \\
\text { hyperplasia, basal } \\
\text { cell }(0 / 48,1 / 47,0 / 45, \\
\text { 13/50); olfactory } \\
\text { epithelium, necrosis } \\
(0 / 48,1 / 47,5 / 45, \\
\text { 5/50); respiratory } \\
\text { epithelium, }\end{array}$ & $\begin{array}{l}\text { Lung: alveolar } \\
\text { epithelium, } \\
\text { hyperplasia }(9 / 50, \\
\text { 49/50, 50/50, } \\
\text { 49/50); alveolus, } \\
\text { proteinosis }(0 / 50, \\
\text { 50/50, 50/50, } \\
\text { 50/50); } \\
\text { inflammation, } \\
\text { chronic active } \\
\text { (20/50, 50/50, } \\
\text { 50/50, 50/50); } \\
\text { bronchiole, } \\
\text { epithelium, } \\
\text { hyperplasia }(0 / 50, \\
\text { 47/50, 46/50, 48/50) } \\
\text { Nose: inflammation, } \\
\text { chronic active } \\
\text { (22/50, 42/50, } \\
\text { 39/49, 50/50); } \\
\text { inflammation, } \\
\text { suppurative }(6 / 50, \\
\text { 4/50, 4/49, 42/50); } \\
\text { olfactory } \\
\text { epithelium, } \\
\text { metaplasia, } \\
\text { respiratory }(6 / 50, \\
\text { 18/50, 24/49, } \\
\text { 47/50); olfactory } \\
\text { epithelium, atrophy } \\
(0 / 50,22 / 50,35 / 49, \\
\text { 35/50): olfactory } \\
\text { epithelium, } \\
\text { hyperplasia }(0 / 50, \\
0 / 50,3 / 49,5 / 50) ; \\
\text { olfactory } \\
\text { epithelium, } \\
\text { hyperplasia, basal } \\
\text { cell }(0 / 50,0 / 50,\end{array}$ & $\begin{array}{l}\text { Lung: } \\
\text { alveolar/bronchiolar } \\
\text { epithelium, } \\
\text { hyperplasia }(0 / 50, \\
\text { 46/49, 49/50, 50/50); } \\
\text { alveolar/bronchiolar } \\
\text { epithelium, } \\
\text { vacuolization } \\
\text { cytoplasmic }(0 / 50, \\
\text { 49/49, 47/50, 48/50); } \\
\text { alveolar epithelium, } \\
\text { hyperplasia }(4 / 50, \\
\text { 29/49, 24/50, 43/50); } \\
\text { bronchiole, } \\
\text { epithelium, } \\
\text { hyperplasia }(4 / 50, \\
\text { 7/49, } 9 / 50,11 / 50) ; \\
\text { bronchiole, } \\
\text { epithelium, erosion } \\
\text { (0/50, 4/49, 10/50, } \\
\text { 2/50); proteinosis } \\
(2 / 50,46 / 49,49 / 50, \\
\text { 50/50); alveolus, } \\
\text { infiltration cellular, } \\
\text { histiocyte }(10 / 50, \\
\text { 49/49, 48/50, 48/50); } \\
\text { inflammation, } \\
\text { suppurative }(1 / 50, \\
\text { 2/49, 6/50, 16/50) } \\
\text { Nose: inflammation, } \\
\text { suppurative }(16 / 50, \\
\text { 32/49, 49/50, 50/50); } \\
\text { olfactory epithelium, } \\
\text { atrophy }(3 / 50,46 / 49, \\
\text { 42/50, 31/50); } \\
\text { olfactory epithelium, } \\
\text { hyperplasia }(0 / 50, \\
\text { 25/49, 17/50, 8/50); } \\
\text { olfactory epithelium, }\end{array}$ & $\begin{array}{l}\text { Lung: } \\
\text { alveolar/bronchiolar } \\
\text { epithelium, hyperplasia } \\
(0 / 49,49 / 50,49 / 50, \\
\text { 50/50); } \\
\text { alveolar/bronchiolar } \\
\text { epithelium, } \\
\text { vacuolization } \\
\text { cytoplasmic }(0 / 49, \\
\text { 48/50, 49/50, 48/50); } \\
\text { alveolar epithelium, } \\
\text { hyperplasia }(2 / 49,27 / 50, \\
\text { 26/50, 41/50); } \\
\text { bronchiole, epithelium, } \\
\text { hyperplasia }(0 / 49,3 / 50, \\
\text { 12/50, } 26 / 50) ; \\
\text { proteinosis }(0 / 49,45 / 50, \\
50 / 50,50 / 50) ; \text { alveolus, } \\
\text { infiltration cellular, } \\
\text { histiocyte }(10 / 49,49 / 50, \\
50 / 50,49 / 50) ; \\
\text { inflammation, } \\
\text { suppurative }(0 / 49,3 / 50, \\
2 / 50,15 / 50) \\
\text { Nose: inflammation, } \\
\text { suppurative }(3 / 50,47 / 50, \\
50 / 50,50 / 50) ; \text { olfactory } \\
\text { epithelium, atrophy } \\
(4 / 50,44 / 50,39 / 50, \\
24 / 50) ; \text { olfactory } \\
\text { epithelium, hyperplasia } \\
(1 / 50,22 / 50,16 / 50, \\
8 / 50) ; \text { olfactory } \\
\text { epithelium, metaplasia, } \\
\text { respiratory }(1 / 50,26 / 50, \\
44 / 50,50 / 50) ; \text { olfactory } \\
\text { epithelium, respiratory } \\
\text { metaplasia, atypical } \\
(0 / 50,18 / 50,14 / 50,\end{array}$ \\
\hline
\end{tabular}




\begin{tabular}{|c|c|c|c|c|}
\hline & $\begin{array}{c}\text { Male } \\
\text { F344/NTac Rats }\end{array}$ & $\begin{array}{c}\text { Female } \\
\text { F344/NTac Rats }\end{array}$ & $\begin{array}{c}\text { Male } \\
\text { B6C3F1/N Mice }\end{array}$ & $\begin{array}{c}\text { Female } \\
\text { B6C3F1/N Mice }\end{array}$ \\
\hline & $\begin{array}{l}\text { hyperplasia }(20 / 48, \\
35 / 47,45 / 45,50 / 50) \text {; } \\
\text { respiratory } \\
\text { epithelium, } \\
\text { metaplasia, } \\
\text { squamous }(0 / 48, \\
1 / 47,11 / 45,35 / 50) \text {; } \\
\text { respiratory } \\
\text { epithelium, necrosis } \\
(1 / 48,4 / 47,5 / 45, \\
13 / 50) ; \text { turbinate, } \\
\text { atrophy }(1 / 48,35 / 47 \text {, } \\
35 / 45,41 / 50) \\
\text { Testes: infarct }(1 / 50, \\
0 / 50,2 / 50,12 / 50)\end{array}$ & $\begin{array}{l}1 / 49,19 / 50) \text {; } \\
\text { respiratory } \\
\text { epithelium, } \\
\text { hyperplasia }(15 / 50, \\
\text { 43/50, 48/49, } \\
\text { 49/50); respiratory } \\
\text { epithelium, } \\
\text { metaplasia, } \\
\text { squamous }(2 / 50, \\
\text { 0/50, 3/49, 45/50); } \\
\text { respiratory } \\
\text { epithelium, necrosis } \\
(1 / 50,1 / 50,1 / 49, \\
\text { 15/50); turbinate, } \\
\text { atrophy }(1 / 50, \\
\text { 38/50, 27/49, 45/50) } \\
\text { Adrenal medulla: } \\
\text { hyperplasia }(12 / 50, \\
\text { 27/50, 27/50, 10/50) }\end{array}$ & $\begin{array}{l}\text { metaplasia, respiratory } \\
(5 / 50,24 / 49,44 / 50, \\
50 / 50) \text {; olfactory } \\
\text { epithelium, respiratory } \\
\text { metaplasia, atypical } \\
(0 / 50,14 / 49,9 / 50, \\
\text { 1/50); respiratory } \\
\text { epithelium, } \\
\text { accumulation, hyaline } \\
\text { droplet }(13 / 50,29 / 49, \\
\text { 29/50, } 7 / 50) ; \\
\text { respiratory epithelium, } \\
\text { vacuolization } \\
\text { cytoplasmic }(0 / 50, \\
41 / 49,39 / 50,37 / 50) \text {; } \\
\text { respiratory epithelium, } \\
\text { metaplasia, squamous } \\
(3 / 50,45 / 49,35 / 50, \\
33 / 50) \text {; turbinate, } \\
\text { atrophy }(3 / 50,25 / 49, \\
49 / 50,50 / 50) \\
\text { Larynx: respiratory } \\
\text { epithelium, } \\
\text { metaplasia, squamous } \\
(7 / 48,47 / 47,49 / 49, \\
49 / 50) \text {; respiratory } \\
\text { epithelium, } \\
\text { vacuolization } \\
\text { cytoplasmic }(0 / 48, \\
\text { 20/47, 24/49, 32/50); } \\
\text { squamous epithelium, } \\
\text { hyperplasia }(2 / 48, \\
5 / 47,5 / 49,8 / 50)\end{array}$ & $\begin{array}{l}1 / 50) \text {; respiratory } \\
\text { epithelium, } \\
\text { accumulation, hyaline } \\
\text { droplet }(12 / 50,38 / 50, \\
\text { 40/50, } 10 / 50) ; \\
\text { respiratory epithelium, } \\
\text { vacuolization } \\
\text { cytoplasmic }(0 / 50, \\
\text { 40/50, 47/50, 47/50); } \\
\text { respiratory epithelium, } \\
\text { metaplasia, squamous } \\
(0 / 50,49 / 50,49 / 50, \\
\text { 50/50); turbinate, } \\
\text { atrophy }(0 / 50,44 / 50, \\
\text { 50/50, 50/50) } \\
\text { Larynx: respiratory } \\
\text { epithelium, metaplasia, } \\
\text { squamous }(2 / 47,49 / 50, \\
\text { 50/50, 47/47); } \\
\text { respiratory epithelium, } \\
\text { vacuolization } \\
\text { cytoplasmic }(0 / 47, \\
24 / 50,31 / 50,34 / 47) ; \\
\text { squamous epithelium, } \\
\text { hyperplasia }(2 / 47,13 / 50, \\
21 / 50,21 / 47) \\
\text { Trachea: epithelium, } \\
\text { vacuolization } \\
\text { cytoplasmic }(0 / 48, \\
26 / 50,37 / 48,39 / 49)\end{array}$ \\
\hline & & & $\begin{array}{l}\text { Trachea: epithelium, } \\
\text { vacuolization } \\
\text { cytoplasmic }(0 / 48, \\
\text { 14/47, } 31 / 48,37 / 50) \\
\text { Testes: germinal } \\
\text { epithelium, } \\
\text { degeneration }(9 / 50, \\
14 / 49,8 / 50,21 / 50)\end{array}$ & \\
\hline Neoplastic Effects & $\begin{array}{l}\text { Lung: } \\
\text { alveolar/bronchiolar } \\
\text { adenoma }(2 / 50, \\
\text { 10/50, 10/50, 14/50); } \\
\text { alveolar/bronchiolar } \\
\text { carcinoma }(0 / 50, \\
\text { 16/50, } 34 / 50,36 / 50) \text {; } \\
\text { alveolar/bronchiolar } \\
\text { adenoma or }\end{array}$ & $\begin{array}{l}\text { Lung: } \\
\text { alveolar/bronchiolar } \\
\text { adenoma }(2 / 50, \\
7 / 50,9 / 50,13 / 50) ; \\
\text { alveolar/bronchiolar } \\
\text { carcinoma }(0 / 50, \\
9 / 50,17 / 50,30 / 50) ; \\
\text { alveolar/bronchiolar } \\
\text { adenoma or }\end{array}$ & $\begin{array}{l}\text { Lung: } \\
\text { alveolar/bronchiolar } \\
\text { adenoma }(7 / 50,11 / 49, \\
\text { 15/50, 3/50); } \\
\text { alveolar/bronchiolar } \\
\text { carcinoma }(11 / 50, \\
38 / 49,42 / 50,46 / 50) ; \\
\text { alveolar/bronchiolar } \\
\text { adenoma or carcinoma }\end{array}$ & $\begin{array}{l}\text { Lung: } \\
\text { alveolar/bronchiolar } \\
\text { adenoma }(3 / 49,9 / 50, \\
8 / 50,10 / 50) ; \\
\text { alveolar/bronchiolar } \\
\text { carcinoma }(5 / 49,25 / 50 \text {, } \\
\text { 38/50, 43/50); } \\
\text { alveolar/bronchiolar } \\
\text { adenoma or carcinoma }\end{array}$ \\
\hline
\end{tabular}


Cobalt Metal, NTP TR 581

\begin{tabular}{|c|c|c|c|c|}
\hline & $\begin{array}{c}\text { Male } \\
\text { F344/NTac Rats }\end{array}$ & $\begin{array}{c}\text { Female } \\
\text { F344/NTac Rats }\end{array}$ & $\begin{array}{c}\text { Male } \\
\text { B6C3F1/N Mice }\end{array}$ & $\begin{array}{c}\text { Female } \\
\text { B6C3F1/N Mice }\end{array}$ \\
\hline & $\begin{array}{l}\text { carcinoma }(2 / 50, \\
25 / 50,39 / 50,44 / 50) \\
\text { Adrenal medulla: } \\
\text { benign } \\
\text { pheochromocytoma } \\
(15 / 50,23 / 50,37 / 50, \\
34 / 50) ; \text { malignant } \\
\text { pheochromocytoma } \\
(2 / 50,2 / 50,9 / 50, \\
16 / 50) \text {; benign or } \\
\text { malignant } \\
\text { pheochromocytoma } \\
(17 / 50,23 / 50,38 / 50, \\
41 / 50) \\
\text { Pancreatic islets: } \\
\text { adenoma or } \\
\text { carcinoma }(2 / 50, \\
2 / 50,10 / 48,9 / 49)\end{array}$ & $\begin{array}{l}\text { carcinoma }(2 / 50, \\
15 / 50,20 / 50, \\
38 / 50) ; \text { cystic } \\
\text { keratinizing } \\
\text { epithelioma }(0 / 50, \\
4 / 50,1 / 50,2 / 50) \\
\\
\text { Adrenal medulla: } \\
\text { benign } \\
\text { pheochromocytoma } \\
(6 / 50,12 / 50,22 / 50, \\
36 / 50) \text {; malignant } \\
\text { pheochromocytoma } \\
(0 / 50,2 / 50,3 / 50, \\
11 / 50) ; \text { benign or } \\
\text { malignant } \\
\text { pheochromocytoma } \\
(6 / 50,13 / 50,23 / 50, \\
40 / 50) \\
\\
\text { Mononuclear cell } \\
\text { leukemia: }(16 / 50, \\
29 / 50,28 / 50,27 / 50)\end{array}$ & $\begin{array}{l}(16 / 50,41 / 49,43 / 50, \\
47 / 50)\end{array}$ & $\begin{array}{l}(8 / 49,30 / 50,41 / 50 \\
45 / 50)\end{array}$ \\
\hline Equivocal Findings & $\begin{array}{l}\text { Lung: cystic } \\
\text { keratinizing } \\
\text { epithelioma }(0 / 50, \\
\text { 1/50, } 0 / 50,1 / 50) \\
\text { Kidney: adenoma or } \\
\text { carcinoma (standard } \\
\text { evaluation - } 0 / 50, \\
1 / 50,0 / 50,4 / 50 ; \\
\text { standard and } \\
\text { extended evaluations } \\
\text { combined }-3 / 50, \\
1 / 50,1 / 50,7 / 50)\end{array}$ & $\begin{array}{l}\text { Pancreatic islets: } \\
\text { carcinoma }(1 / 50, \\
0 / 50,0 / 50,3 / 50)\end{array}$ & None & None \\
\hline $\begin{array}{l}\text { Level of Evidence of } \\
\text { Carcinogenic } \\
\text { Activity }\end{array}$ & Clear evidence & Clear evidence & Clear evidence & Clear evidence \\
\hline \multicolumn{5}{|l|}{ Genetic Toxicology } \\
\hline \multicolumn{2}{|c|}{ Bacterial gene mutations: } & \multicolumn{3}{|c|}{$\begin{array}{l}\text { Positive in S. typhimurium strain TA98 without S9 and negative in } \\
\text { TA98 with S9; equivocal in strain TA100 without S9, and negative } \\
\text { with S9; negative in E. coli strain WP2 uvrA/pKM101 with and } \\
\text { without S9 }\end{array}$} \\
\hline \multicolumn{5}{|c|}{ Micronucleated erythrocytes } \\
\hline \multicolumn{2}{|c|}{ Mouse peripheral blood in vivo: } & \multicolumn{3}{|l|}{ Negative } \\
\hline
\end{tabular}




\section{Introduction}

\section{Chemical and Physical Properties}

Cobalt is a brittle, hard, silver-gray transition metal with ferromagnetic properties. Heating cobalt metal causes oxidation to the mixed oxide, $\mathrm{Co}$ (II, III) oxide $\left(\mathrm{Co}_{3} \mathrm{O}_{4}\right)$; above $900^{\circ} \mathrm{C}$, $\mathrm{Co}$ (II) oxide $(\mathrm{CoO})$ is the end product. Cobalt metal combines with sulfur, phosphorus, and carbon when heated ${ }^{1}$. It readily concentrates under oxidizing conditions with manganese oxides ${ }^{2}$. Cobalt salts have a distinctive brilliant blue color. Cobalt metal exists in two allotropic forms, hexagonal and cubic, both of which are stable in air and water at room temperature ${ }^{3}$.

\section{Production and Use}

Cobalt metal is widely used in the production of cemented tungsten-cobalt (hard metal) and as an alloying element in superalloys, magnetic and hard-facing alloys, cobalt-containing high-strength steels, electrodeposited alloys, and other alloys with special properties ${ }^{1 ; 2}$. Extra fine cobalt metal powder is an important raw material used in production of cemented carbides, diamond tools, and metal welding and spraying components ${ }^{4}$. The major uses of cemented carbide-coated tools are metal-cutting operations and mining and quarrying ${ }^{5}$. It is also used in the manufacture of cobalt salts, and in nuclear medicine, where the isotope ${ }^{60} \mathrm{Co}$ is used as a gamma-ray source ${ }^{3}$. Cobalt is an effective catalyst for many organic reactions, particularly in hydrotreating catalysts, which have molybdenum and cobalt sulfides as active components. It is also used as a target material in electrical $\mathrm{x}$-ray generators ${ }^{1 ; 6-10}$ and in several other military and industrial applications ${ }^{9}$.

Although the United States is the world's largest consumer of cobalt ${ }^{11}$, it does not have well-established primary cobalt mining facilities. In 2006, the aggregated national production volume was between 100 and 500 million pounds ${ }^{12}$, and in 2010 the aggregated national production volume reported to EPA was $23,384,002$ pounds ${ }^{13}$. Comparatively, United States cobalt production between 1964 and 1971 ranged from 690,000 to $1,215,000$ pounds ${ }^{14}$. Import volumes (metric tons) of cobalt into the United States have remained fairly steady during the past years: 10,700 in 2008; 7,680 in 2009; 11,100 in 2010; 10,600 in 2011; and an estimated 11,000 in $2012^{15}$.

\section{Environmental and Human Exposure}

Occupational exposure to cobalt occurring during the production of cobalt powder is a major concern due to the occurrence of hard metal disease and is primarily via inhalation of dusts, fumes, or mists containing cobalt, targeting the skin and the respiratory tract, during the production, processing, and use of hard metal ${ }^{9}$. The USEPA's 2006 IUR records estimated the number of workers likely exposed to cobalt via industrial manufacturing, processing, and use to be $\geq 1,000$ in 1 to 99 worksites $^{12}$.

In the environment, cobalt is the 33rd most abundant element, composing approximately $0.0025 \%$ of the weight of the earth's crust and is present naturally in the soil in varying concentrations ranging from approximately 1 to $40 \mathrm{ppm}$ with an average level of $7 \mathrm{ppm}$. It occurs naturally in the groundwater and sediments and is mainly derived from erosion of volcanic rocks in the mountains. Exposure of the general population is mainly through breathing 
air, drinking water, or skin contact with soil, water, cobalt alloys, or other substances that contain cobalt ${ }^{9}$.

Cobalt is also an essential trace element because it is an integral component of cyanocobalamin (vitamin $\mathrm{B}_{12}$ ), the only metal-containing vitamin. Vitamin $\mathrm{B}_{12}$ is found in meat and dairy products. Vitamin $\mathrm{B}_{12}$ acts as a coenzyme in many enzymatic reactions, most notably in a methyl transfer reaction that converts homocysteine to methionine. It also acts as a coenzyme in a reaction that converts 1-methylmalonylcoenzyme $\mathrm{A}(\mathrm{CoA})$ to succinyl-CoA ${ }^{16}$. Vitamin $\mathrm{B}_{12}$ is also a part of some enzymes involved in hematopoiesis, and a deficiency can lead to pernicious anemia ${ }^{17}$. Conditions such as iron deficiency anemia can lead to increased absorption of cobalt from the gastrointestinal tract, and simultaneous administration of cobalt and iron can reduce the amount of cobalt absorbed ${ }^{18 ; 19}$.

\section{Regulatory Status}

Cobalt is included in the Unregulated Contaminant Monitory Rule contaminant list; its minimum reporting level is $1 \mu \mathrm{g} / \mathrm{L}$ for the reporting period of January 1, 2013, to December 31, $2015^{20}$. Total cobalt is also listed as a hazardous constituent for municipal solid waste landfills ${ }^{21}$, and is on the groundwater monitoring list ${ }^{22}$. Cobalt became regulated under the toxic chemical release reporting: community right-to-know on January $1,1987^{23}$. The American Conference of Governmental Industrial Hygienists (ACGIH) ${ }^{24}$ has given cobalt a classification of A3, confirmed animal carcinogen with unknown relevance to humans, and established an 8-hour time-weighted average (TWA) of $0.02 \mathrm{mg} / \mathrm{m}^{3}$ for occupational exposure. Occupational Safety and Health Administration (OSHA) ${ }^{25}$ has promulgated an 8-hour permissible exposure limit of $0.1 \mathrm{mg} / \mathrm{m}^{3}$, and the National Institute for Occupational Safety and Health recommends an 8-hour TWA of $0.05 \mathrm{mg} / \mathrm{m}^{3}{ }^{26}$. The Minimal Risk Level (MRL) is based on the no-observed-adverseeffect level of $0.0053 \mathrm{mg}$ cobalt $/ \mathrm{m}^{3}$ for decreased respiratory function in exposed workers ${ }^{27}$. A MRL of $1 \times 10^{-4} \mathrm{mg} / \mathrm{kg}$ per day has been derived for chronic-duration inhalation exposure (>365 days) to cobalt 9 .

\section{Absorption, Distribution, Metabolism, Excretion, and Toxicokinetics}

\section{Experimental Animals}

There are numerous studies showing that cobalt is absorbed rapidly following inhalation exposure in animals and distributed to various tissues with significant levels in the lungs ${ }^{28-35}$. Following inhalation exposure of rats to 0.0004 to $0.2 \mathrm{ppm}\left(0.001\right.$ to $\left.0.5 \mathrm{mg} / \mathrm{m}^{3}\right)$ pure cobalt 24 hours per day for 3 months, a dose-dependent distribution and accumulation of cobalt was reported in the thyroid gland, spleen, liver, kidney, and lung ${ }^{35}$. In SD-Jcl rats exposed to $0.880 \mathrm{ppm}\left(2.12 \mathrm{mg} / \mathrm{m}^{3}\right)$ cobalt aerosol 5 hours $/$ day for 4 days, the average cobalt content of the lung and blood 2 hours after the last exposure was $6.42 \mu \mathrm{g} / \mathrm{g}$ and $28.94 \mu \mathrm{g} / \mathrm{L}$, respectively. The values 28 days after exposure were $0.09 \mu \mathrm{g} / \mathrm{g}(1.5 \mathrm{nmol} / \mathrm{g})$ and $0.40 \mu \mathrm{g} / \mathrm{L}(6.8 \mathrm{nM})$, respectively, for lung and blood. The clearance of cobalt in both blood and lung was biphasic with half-lives in the lung of 52.8 and 156 hours and in the blood of 52.8 and 172.8 hours, for the first and second phases, respectively ${ }^{33}$. In miniature swine following inhalation exposure to 0.04 to 
$0.41 \mathrm{ppm}\left(0.1\right.$ to $\left.1.0 \mathrm{mg} / \mathrm{m}^{3}\right)$ pure cobalt powder 6 hours/day, 5 days/week for $3 \mathrm{months}$, cobalt was excreted mostly by the kidney ${ }^{31 ; 36}$.

Cobalt levels in rat urine 24 hours following intratracheal instillation of a tungsten carbide-cobalt mixture were approximately threefold higher compared to instillation of cobalt powder at the same dose ${ }^{37}$. It was later confirmed that this was not due to higher bioavailability but due to rapid urinary excretion following exposure to the tungsten carbide-cobalt mixture ${ }^{38}$. The mean lung cobalt concentration in rats given cobalt was two times more than that of rats given a tungsten carbide-cobalt mixture at 48 hours following exposure; by day 7 , mean levels had decreased significantly to almost the same level in all exposed rats ${ }^{38}$.

The chemical form of a cobalt compound can affect the absorption of cobalt following oral exposure. In rats following oral exposure to cobalt chloride, the absorption was $13 \%$ to $34 \%$, whereas the absorption following administration of insoluble cobalt oxide was in the range of $1 \%$ to $3 \% 19 ; 30 ; 34 ; 39-42$. Although no species difference was observed for absorption of cobalt oxide ${ }^{40}$, absorption of soluble cobalt compounds was greater in rats $(13 \%$ to $34 \%)$ than in cows (1\% to $2 \%$ ) and guinea pigs $(4 \% \text { to } 5 \%)^{19 ; 30 ; 34 ; 39-42}$. Absorption was 3- to 15 -fold greater in younger animals than in adults ${ }^{43}$. The absorbed cobalt was distributed primarily to the liver; appreciable levels were also found in the kidney, heart, stomach, and intestines ${ }^{39}$. In studies where rats were exposed orally to cobalt sulfate or cobalt chloride for longer terms, significant increases in cobalt concentration were reported in the myocardium, muscle and serum, liver, kidney, brain, and testes $^{41 ; 44-47}$. Fecal excretion of cobalt is the primary route of elimination in animals following oral exposure and varies depending on the dose and the type of cobalt given; no difference in elimination has been noted between species $^{30 ; 40 ; 48-51}$.

\section{Humans}

Workers exposed to cobalt dust and fumes in the production of cobalt powder had mean concentrations of 5 to $48 \mu \mathrm{g} / \mathrm{L}$ in blood and 19 to $438 \mu \mathrm{g} / \mathrm{L}$ in urine compared to blood and urine concentrations in the range from 0.1 to $2 \mu \mathrm{g} / \mathrm{L}$ in nonoccupationally exposed persons ${ }^{52}$. Following inhalation exposure to insoluble cobalt compounds such as cobalt metal and cobalt oxide, three-phase elimination kinetics were observed in humans. The half-life for the first phase, likely representing mucociliary clearance in the tracheobronchial region, was approximately 2 to 44 hours ${ }^{53 ; 54}$. The second phase with a half-life of approximately 10 to 78 days may represent macrophage-mediated clearance of cobalt particles from the lung ${ }^{54 ; 55}$. The third phase clearance with a half-life on the order of years may represent long-term clearance from the lung ${ }^{40 ; 54-56}$. Using control-aerosol experiments in humans, it has been shown that about $40 \%$ of the initial lung burden of inhaled cobalt oxide was retained for a period of 6 months after exposure ${ }^{57}$. About $33 \%$ of the initial lung burden was found in the urine with $28 \%$ in feces 6 months after exposure.

The absorption of cobalt in humans following oral exposure varied ( $18 \%$ to $97 \%$ of the dose) depending on the type and dose of the cobalt compound and other nutritional status. Fecal excretion of cobalt is the primary route of elimination in humans following oral exposure and varies depending on the dose and the type of cobalt given $(3 \% \text { to } 99 \%)^{30 ; 40 ; 48-51 ; 58 ; 59}$.

Following dermal exposure to hard metal dust (approximately $5 \%$ to $15 \%$ cobalt metal, $85 \%$ to $95 \%$ tungsten carbide) for 90 minutes, urinary levels of cobalt increased by about an order of 
magnitude compared to preexposure samples, demonstrating that dermal absorption occurs ${ }^{60}$. Cobalt was detected in the fingernails of volunteers who placed their fingers in a cobalt dust solution 10 minutes/day for 7 days $^{61}$.

\section{Toxicity}

The major targets of cobalt toxicity in animals include the respiratory, cardiovascular, hematopoietic, and immune systems. Other targets for cobalt toxicity in animals include the endocrine system, the nervous system, the liver, and the kidney.

\section{Respiratory System}

Inhalation studies of metallic cobalt aerosols and cobalt salts have identified respiratory tract hyperplasia, pulmonary fibrosis, alveolar septa thickening with collagen, elastic tissue, fibroblasts, pleuritis, firm dust lesions, and emphysema as sensitive effects of cobalt on respiratory tissues ${ }^{26 ; 31 ; 33 ; 36 ; 62-67}$. Exposure of rats and mice to cobalt sulfate heptahydrate by inhalation (up to $30 \mathrm{mg} / \mathrm{m}^{3}$ ) for 13 weeks showed degeneration of the olfactory epithelium, squamous metaplasia of the respiratory epithelium, and inflammation in the nose; inflammation, necrosis, and inflammatory polyps (rats) of the larynx; metaplasia of the trachea (mice); and fibrosis, histiocytic infiltrates, bronchiolar epithelial regeneration, and epithelial hyperplasia in the alveoli of the lung ${ }^{66 ; 68}$. The most sensitive tissue was found to be the larynx.

Following inhalation exposure to cobalt-containing particles, the primary target of toxicity in humans is the respiratory tract. Occupational exposure of humans to cobalt metal or cobaltcontaining hard metal primarily affects the respiratory system, including decreased pulmonary function, asthma, interstitial lung disease, wheezing, and dyspnea; these effects were reported at occupational exposure levels ranging from 0.015 to $0.13 \mathrm{mg}$ cobalt $/ \mathrm{m}^{3} 27 ; 69-92$. These effects have been noted in workers employed in cobalt metal refineries, as well as hard metal workers, diamond polishers, and ceramic dish painters.

\section{Cardiovascular System}

Cardiomyopathies appear to be another primary effect of cobalt-induced toxicity in both animals and humans ${ }^{9}$. Rats exposed to cobalt sulfate heptahydrate for 13 weeks showed marginal increases in the severity of cardiomyopathy; no such findings were reported in mice ${ }^{66 ; 68}$. Studies suggest that cardiomyopathies may occur by impairment of oxidation of pyruvate or fatty acids ${ }^{93}$ or by stimulation of carotid body chemoreceptors, thereby mimicking the action of hypoxia ${ }^{94-96}$.

In the 1960s, several breweries added cobalt salts to beer to stabilize foam; this resulted in exposures of 0.04 to $0.14 \mathrm{mg} / \mathrm{kg}^{9}$. Soon after this, an epidemic of "beer-drinkers" cardiomyopathy" occurred in people who drank 8 to 25 pints/day, resulting in severe cardiovascular system effects, including cardiomyopathy and death.

This cardiomyopathy was evidenced by fragmentation and degeneration of myofibers, enlargement of the heart, and aggregates of abnormal mitochondria ${ }^{9 ; 97-99}$. The mitochondrial changes are indicative of disturbances in energy production or utilization possibly related to cobalt effects on lipoic acid. Cobalt irreversibly chelates lipoic acids under aerobic conditions ${ }^{100}$. Lipoic acid is a required cofactor for oxidative decarboxylation of pyruvate to acetyl CoA and of $\alpha$-ketoglutarate to succinate ${ }^{101}$. 
Other observations in people who drank the cobalt-treated beer were gastrointestinal effects (nausea, vomiting) and hepatic necrosis ${ }^{102-104}$. The epidemic ceased when the addition of cobalt salts to beer was discontinued.

\section{Hematopoietic System}

Exposure to cobalt also affects the hematopoietic system by increasing levels of erythrocytes and hemoglobin in both humans and animals ${ }^{9}$. Palmes et al. ${ }^{105}$ demonstrated increased levels of hemoglobin in rats and guinea pigs, but not dogs, exposed to cobalt hydrocarbonyl by inhalation. Polycythemia has been reported in rats, but not mice, exposed to airborne cobalt sulfate heptahydrate ${ }^{66 ; 68}$. Other studies corroborate the above findings of increases in hemoglobin and erythrocyte levels and decreases in blood phospholipids, cholesterol, and $\beta$-lipoproteins in rats exposed to metallic cobalt aerosol via inhalation ${ }^{35}$. Of particular note is an 8 -week study in rats that reported dose- and time-related increases in erythrocyte number following oral administration of cobalt chloride ${ }^{106}$.

The most sensitive endpoint following oral exposure to cobalt in humans appears to be an increase in erythrocyte numbers (polycythemia) ${ }^{9}$. This effect has been noted in both normal subjects and in patients who were anemic as a result of being anephric.

\section{Immune System}

Cobalt may exert its effects through interactions with the immune system, mainly resulting in contact sensitization. In guinea pigs, nickel and cobalt sensitization appear to be interrelated and mutually enhancing, though cross-reactivity has not been reported to occur ${ }^{107}$.

The most commonly observed effect following dermal exposure is dermatitis, as demonstrated by a large number of human studies. Patch tests and intradermal injections demonstrate that the dermatitis is probably caused by an allergic reaction to cobalt, with the cobalt ion functioning as a hapten $^{84 ; 85 ; 108-115}$. Exposure to inhaled cobalt chloride aerosols can precipitate an asthmatic attack in sensitized individuals, suggesting cobalt sensitization is one mechanism by which cobalt-induced asthma may be produced ${ }^{84}$. IgE and IgA antibodies specific to cobalt have been reported in humans ${ }^{84 ; 85 ; 115}$. There is evidence that cobalt sensitivity in humans may be regulated by T-lymphocytes ${ }^{116}$. A human helper T-lymphocyte cell line specific for cobalt $\left(\mathrm{CoCl}_{2}\right)$ has been established ${ }^{117}$. Cobalt may also interact directly with immunologic proteins, such as antibodies or Fc receptors, resulting in immunosensitization ${ }^{118}$.

\section{Endocrine System}

A study in female mice exposed to $26 \mathrm{mg}$ cobalt $/ \mathrm{kg}$ per day in drinking water for up to 45 days produced histopathologic changes to the thyroid ${ }^{119}$. Cobalt significantly stimulated serum testosterone in mice treated orally with $23 \mathrm{mg}$ cobalt chloride $/ \mathrm{kg}$, although no dose-response relationship was present ${ }^{120}$. There have also been reports of increased incidences of pheochromocytoma, a tumor of the adrenal medulla, in female rats exposed to $1.14 \mathrm{mg}$ cobalt $/ \mathrm{m}^{3}$ for 2 years $^{121 ; 122}$.

\section{Nervous System}

In a study in OFA Sprague Dawley rats, exposure-related delays in hearing, swimming ability, and development of muscle strength and locomotor system were reported in the offspring of 
dams that were exposed to $0,25,50$, or $100 \mathrm{mg} / \mathrm{kg}$ body weight cobalt sulfate by gavage throughout gestation ${ }^{123}$. Occupational exposure to cobalt metal in humans has been reported to cause several effects on the nervous system, including memory loss (Wechsler Memory ScaleRevised), nerve deafness, and decreased visual acuity ${ }^{124 ; 125}$. However, these studies had small numbers of subjects ( $\mathrm{n}=38$ or 1 ), and exposure characterization was not reported.

\section{Liver}

No histologic effects on the liver were found in pigs exposed to cobalt metal dust up to $1.0 \mathrm{mg} / \mathrm{m}^{3}$ for 3 months $^{31}$. Hyperemia of the liver and cytoplasmic changes in hepatocytes (clumpy cytoplasm located along the cell membrane) were noted in rats following oral administration of a single dose of $68.2 \mathrm{mg}$ cobalt fluoride $/ \mathrm{kg}$ or a single dose of $157.3 \mathrm{mg}$ cobalt oxide $/ \mathrm{kg}^{126}$. Increased liver weight (17\%) was found in rats exposed to $10 \mathrm{mg}$ cobalt chloride $/ \mathrm{kg}$ per day for 5 months ${ }^{127}$. No morphologic or enzymatic changes were noted in the livers of rats exposed to 2.5 to $30.2 \mathrm{mg}$ cobalt chloride $/ \mathrm{kg}$ by gavage or to cobalt chloride in the drinking water for 3 to 7 months ${ }^{128-130}$. However, in a previous NTP study, rats and mice exposed by inhalation to 50 or $200 \mathrm{mg}$ cobalt sulfate heptahydrate $/ \mathrm{m}^{3}$ or greater for 16 days demonstrated necrosis and congestion in the liver ${ }^{6 ;} ; 68$.

\section{Kidney}

Significant increases were noted in the relative kidney weight in male rats exposed by inhalation to cobalt sulfate heptahydrate $\left(0.3\right.$ to $\left.30 \mathrm{mg} / \mathrm{m}^{3}\right)$ for 13 weeks ${ }^{66 ; 68}$. No effects were observed upon histologic examination of the kidneys in rats or mice following exposure to cobalt sulfate heptahydrate at up to $200 \mathrm{mg} / \mathrm{m}^{3}$ for 16 days, up to $30 \mathrm{mg} / \mathrm{m}^{3}$ for 13 weeks, or up to $3.0 \mathrm{mg} / \mathrm{m}^{3}$ for 104 weeks ${ }^{66 ; 68 ; 121 ; 122}$. No histologic effects on the kidney were found in pigs exposed to up to $1.0 \mathrm{mg}$ cobalt $/ \mathrm{m}^{3}$ for 3 months $^{31}$.

\section{Reproductive and Developmental Toxicity}

\section{Experimental Animals}

Exposure to cobalt-containing aerosols has been shown to affect reproductive endpoints in rats and mice. A decrease in sperm motility in mice was found following exposure to 3 to $30 \mathrm{mg}$ cobalt sulfate heptahydrate $/ \mathrm{m}^{3}$ for 13 weeks, and a significant increase in the length of the estrous cycle was reported in female mice exposed to $30 \mathrm{mg} / \mathrm{m}^{3}$ for 13 weeks ${ }^{66 ; 68}$. Testicular atrophy was reported in rats exposed by inhalation to $50 \mathrm{mg}$ cobalt sulfate heptahydrate $/ \mathrm{m}^{3}$, 6 hours per day for 16 days, but not in rats exposed to $30 \mathrm{mg} / \mathrm{m}^{3}$ for 13 weeks. Testicular atrophy was, however, observed in mice exposed to $30 \mathrm{mg} / \mathrm{m}^{3}$ per day for 13 weeks ${ }^{66 ; 68}$. No testicular effects were observed in rats or mice exposed to cobalt sulfate heptahydrate up to $3.0 \mathrm{mg} / \mathrm{m}^{3}$ for 104 weeks ${ }^{121 ; 122}$. These findings are consistent with studies following oral exposure to soluble cobalt chloride for 2 to 3 months in the diet or drinking water. These studies reported testicular degeneration and atrophy in rats exposed to cobalt chloride in the diet ${ }^{128 ; 131-133}$ and in mice exposed to cobalt chloride for 3 weeks in drinking water ${ }^{134 ; 135}$.

No studies were found in the literature regarding developmental effects in animals following inhalation exposure to cobalt. In a perinatal study design where rat dams were administered 0 , 12,24 , or $48 \mathrm{mg} / \mathrm{kg}$ of cobalt chloride via oral gavage from gestation day 14 through lactation day 21, offspring displayed stunted growth (all cobalt dose levels) and decreased survival (24 
and $48 \mathrm{mg} / \mathrm{kg})^{136}$. No external malformations were observed in the pups. No measurements were collected on the dams, although the authors stated that toxic signs were previously observed in male and female rats administered 24 or $48 \mathrm{mg} / \mathrm{kg}$. The effects on the offspring occurred at levels that also caused maternal toxicity (reduced body weight and food consumption and altered hematological measurements); no teratogenic effects were noted. These authors also reported that rabbits exposed to 20,100 , or $200 \mathrm{mg} / \mathrm{kg}$ cobalt sulfate from gestation days 6 to 20 exhibited excessive dose-related maternal toxicity (death and total resorptions). Fetuses in the $20 \mathrm{mg} / \mathrm{kg}$ group (only cobalt group available for examination) evaluated at term displayed apparent delays in ossification and increases in percentage of fetuses with retarded body weight. Szakmáry et al. ${ }^{123}$ reported that exposure of pregnant rats to cobalt sulfate did not result in changes in fetal death rates, maternal body weight gain, average litter size, or average fetal or placental weights; however, a dose-related trend was seen for the percent of fetuses with retarded body weights. Increased incidences of axial skeletal anomalies were also observed in rat fetuses exposed to 25 to $100 \mathrm{mg} / \mathrm{kg}$ cobalt sulfate ${ }^{123}$. In contrast, no effects on fetal growth or survival were found following exposure of rats to cobalt chloride during gestation days 6 to $15^{137}$. However, the difference in results between the two studies may be explained, at least in part, by exposure concentration, with cobalt sulfate exposure being almost double that of cobalt chloride. There are also reports of preimplantation losses following administration of cobalt in male mice ${ }^{138}$.

Exposure of mice to cobalt chloride during gestation days 8 to 12 was reported to have no effect on fetal growth or mortality ${ }^{139}$. There were no changes in litter size, postimplantation losses, or average fetal or placental weights in a study that exposed pregnant mice to $50 \mathrm{mg}$ cobalt sulfate $/ \mathrm{kg}$ body weight per day; there was an increase in the percent of fetuses with retarded body weights $^{123}$. These authors also reported that rabbits exposed to $20 \mathrm{mg} / \mathrm{kg}$ cobalt sulfate showed nearly complete maternal lethality and complete fetal loss. In addition, there were significant increases in mortality and fetal resorption, as well as an increase in fetuses with retarded body weight.

\section{Humans}

No reports were found in the literature on the reproductive effects in humans following inhalation or oral exposure to cobalt. Pregnant women were treated with cobalt chloride to raise hematocrit and hemoglobin levels that are often depressed during pregnancy. Doses up to $0.6 \mathrm{mg}$ cobalt $/ \mathrm{kg}$ per day for 3 months were given during the final trimester ${ }^{129}$. No developmental effects were reported in the infants, although examination was limited only to the reporting of obvious birth defects.

\section{Carcinogenicity}

\section{Experimental Animals}

In 2-year inhalation studies of soluble cobalt sulfate heptahydrate, pronounced effects on the respiratory tract, including hyperplasia, inflammation, fibrosis, metaplasia, and increased incidences of cancer in rats and mice were reported ${ }^{121 ; 122}$. Increased incidences of alveolar/bronchiolar neoplasms occurred in male rats exposed to $3.0 \mathrm{mg} / \mathrm{m}^{3}$ and in female rats exposed to 1 or $3 \mathrm{mg} / \mathrm{m}^{3}$. Neoplasms occurred in both sexes with significantly positive trends. Male and female mice exposed to $3 \mathrm{mg} / \mathrm{m}^{3}$ showed increases in alveolar/bronchiolar neoplasms; neoplasms in the lung occurred with significantly positive trends. The findings also demonstrated 
an increased incidence of pheochromocytoma, a neoplasm of the adrenal medulla, in female rats exposed to $3.0 \mathrm{mg} / \mathrm{m}^{3}$ for 2 years without any other endocrine effects. The NTP concluded that under the conditions of the 2-year studies, there was some evidence of carcinogenic activity of cobalt sulfate heptahydrate in male F344/N rats based on increased incidences of alveolar/ bronchiolar neoplasms. Marginal increases in the incidences of pheochromocytomas of the adrenal medulla may have been related to exposure to cobalt sulfate heptahydrate. There was clear evidence of carcinogenic activity in female F344/N rats based on increased incidences of alveolar/bronchiolar neoplasms and pheochromocytomas of the adrenal medulla in groups exposed to cobalt sulfate heptahydrate. There was clear evidence of carcinogenic activity of cobalt sulfate heptahydrate in male and female B6C3F1 mice based on increased incidences of alveolar/bronchiolar neoplasms. Molecular analyses of lung neoplasms were also performed in the study; findings showed an exposure concentration-dependent increase in Kras mutations, thereby suggesting a mechanism of tumorigenesis.

Parenteral exposure to cobalt has also been found to induce neoplasms in rodents ${ }^{140-145}$. One study reported sarcomas in rats at the site of injection of cobalt salts or cobalt metal powder ${ }^{1}$. In another study, rats of an unspecified strain were given a single injection of $0.28 \mathrm{mg}$ cobalt metal powder in fowl serum into the thigh muscle ${ }^{142 ; 143}$. Within 2 weeks, atypical myoblasts were observed $^{143}$, and between 5 and 12 months, malignant neoplasms developed at the injection site in 17 of 30 rats; 11 of the neoplasms were rhabdomyosarcomas ${ }^{142}$. Similar neoplastic responses to injections of cobalt sulfide and cobalt oxide were noted in an unspecified strain of rats but not in mice ${ }^{140}$. Shabaan et al. ${ }^{145}$ noted fibrosarcomas in male Wistar rats 8 months to 1 year after administration of $40 \mathrm{mg}$ cobalt chloride/kg per day by subcutaneous injection for 10 days.

\section{Humans}

Few epidemiological studies of cancer risk in cobalt metal-exposed workers exist ${ }^{146}$. A high incidence of pulmonary cancer was found in English cobalt metal miners; however, the etiology was not known ${ }^{65}$. Epidemiological studies of cobalt metal miners in the United States, Canada, Zaire, and other countries found no association between cobalt metal and neoplasm rates; however, cobalt metal was the cause of hard metal respiratory disease ${ }^{147}$. In a mortality study of a cohort of 1,143 workers in an electrochemical plant producing cobalt metal and sodium (110 were engaged in cobalt metal production) for at least a year during 1950 to 1980, an increased number of deaths from lung cancers were observed in those producing cobalt metal; however, smoking may have been a factor ${ }^{148}$. Confounding by nickel and arsenic exposures and the limited size of the exposed population were identified as some limitations ${ }^{146}$. The follow-up (1981 to 1988) did not support the proposed relationship between lung cancer and cobalt metal exposure $^{149}$.

The 12th Report on Carcinogens lists cobalt sulfate and cobalt-tungsten carbide powders and hard metals as reasonably anticipated to be human carcinogens based on sufficient evidence of carcinogenicity from studies in experimental animals ${ }^{150}$. IARC ${ }^{1}$ has listed cobalt compounds as possibly carcinogenic to humans (Group 2B) based on sufficient evidence for cobalt metal and cobalt oxides and limited evidence for cobalt chloride and cobalt sulfate. However, to date, there are no chronic studies reported on cobalt metal dust in rodents. 


\section{Genetic Toxicity}

There is a paucity of data on the genetic toxicity of metallic cobalt, likely due to the assumption that its biological activity is mediated by its ionic forms. However, it has been shown that at least some of the biological activities of the metal cannot be attributed to its ionic forms ${ }^{151}$. The focus of this review is on the genetic toxicity of cobalt metal and not on its ionic or hard metal forms. For reviews of cobalt and cobalt compound toxicities, see De Boeck et al. ${ }^{152 ;}$ Lison et al. ${ }^{153}$; Simonsen et al. ${ }^{154}$.

Two studies published in 1997 showed that cobalt powder causes DNA damage in cultured mammalian cells. In one study, non-cytotoxic doses of cobalt powder were added to cultured human lymphocytes and a dose-dependent increase in DNA single strand breaks was noted at concentrations of $4.5 \mu \mathrm{g} / \mathrm{mL}$ and above ${ }^{155}$. Sodium formate, a hydroxyl radical scavenger, was shown to lower the levels of cobalt-induced DNA damage in this study. The second study examined induction of DNA strand breaks and micronuclei in human lymphocytes exposed in culture to pure cobalt powder (up to $12 \mu \mathrm{g} / \mathrm{mL}$ ) ${ }^{156}$. The authors reported dose-related increases in DNA migration (using the comet assay) and dose-related increases in micronucleated lymphocytes (using the cytokinesis block method). Another study from the same laboratory, focused on measuring the genetic toxicity of cobalt combined with various metallic carbide particles, found induction of DNA strand breaks by cobalt alone to be so variable that results from the testing of the combination materials could not be clearly evaluated ${ }^{157}$. However, induction of micronucleated lymphocytes by cobalt alone or in combination with tungsten carbide, chromium carbide, niobium carbide, and molybdenum carbide occurred in a doserelated fashion ${ }^{157}$; none of the four individual carbides induced micronuclei in the absence of cobalt.

Several lines of investigation suggest that cobalt interferes with DNA repair processes (for a review, see Hartwig et al. ${ }^{158}$ ). One way that proteins interact with DNA is through zinc-finger domains. Proteins in the base excision repair (BER) and nucleotide excision repair (NER) pathways, as well as transcription factors such as p53, contain zinc-finger domains. Biochemical studies have shown that cobalt can take the place of zinc in such domains; for example, cobalt will complex within the bacterial BER enzyme, formamidopyrimidine-DNA glycosylase ${ }^{159}$, and a peptide containing the zinc-finger domain of XPA will accept cobalt ${ }^{160}$. The substitution of cobalt for zinc appears to have negative consequences, as cobalt impaired the ability of XPA purified from mouse cells to bind DNA specifically through its zinc-finger domain ${ }^{161}$. In the NER pathway, XPA plays a critical role in the initial detection of DNA that has been distorted by various chemical modifications that are known to induce mutations, such as oxidized bases and bulky adducts. In another approach, De Boeck et al. ${ }^{162}$ investigated the interference of cobalt with the repair of mutagen-induced DNA damage using the Comet assay. Cultured human lymphocytes were exposed to MMS alone, or to MMS followed by a non-genotoxic dose of $1.2 \mu \mathrm{g} / \mathrm{mL}$ cobalt metal. In the presence of cobalt, there was an increase in the persistence of MMS-induced DNA damage, suggesting that cobalt inhibited the repair of MMS-induced DNA lesions.

Taken together, results from these in vitro studies suggest that cobalt particles can affect the integrity of DNA by producing activated oxygen species and/or by inhibiting DNA repair pathways. 
To assess the genotoxic effects of cobalt dust on workers from cobalt refineries, where the average dust concentration was $20 \mathrm{mg} / \mathrm{m}^{3}, 35$ workers were examined for lymphocyte DNA damage using the comet assay and for lymphocyte micronucleus frequencies. No significant effects were observed for either of these endpoints in exposed workers compared to matched controls ${ }^{163}$.

\section{Study Rationale}

Cobalt metal dust was nominated for toxicology and carcinogenesis studies by the United Auto Workers and the Cobalt Development Institute based on the widespread occupational exposure and the occurrence of occupational disease, i.e. hard metal disease, associated with exposure to cobalt and its compounds, including cobalt metal-tungsten carbide. The carcinogenicity of a soluble cobalt compound, cobalt sulfate heptahydrate, in experimental animals exposed by inhalation was previously assessed by NTP ${ }^{122}$. Limited data were available to assess the chronic toxicity and carcinogenic potential of inhaled insoluble cobalt compounds, particularly cobalt metal dust. Inhalation was selected as the route of exposure because this is the most common route of exposure to cobalt metal dust in occupational settings in humans. 


\section{Materials and Methods}

\section{Procurement and Characterization of Cobalt Metal}

Cobalt metal was produced by OMG Kokkola Chemicals Oy (Kokkola, Finland) and was provided by the Cobalt Development Institute via PEL Technologies in one lot (P32 3040-1) that was used in the 2-week, 3-month, and 2-year studies. Identity and purity analyses were performed by the study laboratory at Battelle Toxicology Northwest [Richland, WA; inductively coupled plasma/atomic emission spectroscopy (ICP/AES) analysis] and by the analytical chemistry laboratories at Pacific Northwest National Laboratory [Richland, WA; X-ray diffraction (XRD) and proton-induced X-ray emission (PIXE) analyses], AMIA Laboratories (The Woodlands, TX; XRD using Rietveld analysis), H\&M Analytical Services, Inc. (Allentown, NJ; XRD with and without Rietveld analysis), Elemental Analysis, Inc. (Lexington, KY; PIXE), and Galbraith Laboratories (Knoxville, TN; coulometry for total carbon) (Appendix L). Reports on analyses performed in support of the cobalt metal studies are on file at the National Institute of Environmental Health Sciences.

Lot P32 3040-1 of the chemical, a silver-gray powder, was identified as cobalt metal by the analytical chemistry laboratories using XRD. XRD patterns were consistent with library reference patterns for cubic and hexagonal phases of cobalt. The purity of lot P32 3040-1 was determined by the analytical chemistry laboratories by determination of the carbon content using combustion/coulometric analysis by induction furnace with a carbon dioxide coulometer and by PIXE analyses to determine the presence of cobalt metal and trace element impurities with atomic numbers from 11 (sodium) to 53 (iodine) or 92 (uranium). The study laboratory quantitated the purity of the bulk chemical using ICP/AES. The carbon content was determined to be $0.09 \% \pm 0.01 \%$. PIXE analysis indicated trace elements of aluminum, sulfur, calcium, chromium, and iron. Chromium was consistently current at approximately $84 \mathrm{ppm}$; the other impurities were below the minimum detection limits. ICP/AES analysis indicated a purity of $98.2 \% \pm 0.6 \%$ relative to a National Institute of Standards and Technology standard reference material [(SRM); cobalt SRM 3113, Gaithersburg, MD]. The overall purity of cobalt metal was determined to be greater than $98 \%$.

To ensure stability, the bulk chemical was stored at room temperature in safety-coated amber glass containers with Teflon ${ }^{\circledR}$-lined caps under a nitrogen headspace. Periodic reanalyses of the bulk chemical were performed by the study laboratory using ICP/AES; no degradation of the bulk chemical was detected.

\section{Aerosol Generation and Exposure System}

During the 2-week studies, an auger feed device was used to meter cobalt metal into a Trost jet mill for aerosolization and particle size reduction. For the 3-month and 2-year studies, the generation system used a linear feed device to meter cobalt metal into the jet mill. Initial particle size reduction was accomplished within the Trost jet mill. From the jet mill, aerosol was directed to the main distribution line where it was diluted with humidified air then conveyed from the exposure control center to the exposure room where it passed through a cyclone separator to further reduce particle size. On exiting the cyclone, the aerosol-laden air was directed to either of two smaller branch lines. From the branch lines, aerosol was delivered to each exposure chamber 
by a sampling tube. The flow through the sampling tube was induced by a stainless steel ejector pump. The aerosol then entered the chamber inlet duct where it was further diluted with conditioned chamber air to achieve the desired exposure concentration.

The study laboratory designed the inhalation exposure chambers so that uniform aerosol concentrations could be maintained throughout the chambers with the catch pans in place. The total volume of the chamber was $2.3 \mathrm{~m}^{3}$ with an active mixing volume of $1.7 \mathrm{~m}^{3}$. Tests showed that aerosol concentration could be reliably maintained homogenous within $8 \%$ throughout the chambers, provided the aerosol was uniformly mixed before passing through the chamber inlet and provided the test material did not react to a significant extent with animals, animal excrement, or the chamber interior ${ }^{164}$.

\section{Aerosol Concentration Monitoring}

Summaries of the chamber aerosol concentrations are given in Table L-1 through Table L-3. The concentration of cobalt metal in the exposure chambers and room air was monitored using three real-time aerosol monitors (RAMs). Each RAM was calibrated by constructing a response curve using the measured RAM voltages (voltage readings were corrected by subtracting the RAM zero-offset voltage from measured RAM voltages) and cobalt metal concentrations that were determined by analyzing tandem Teflon ${ }^{\circledR}$-coated, glass-fiber filters collected daily from the exposure chambers. Cobalt was extracted from the filters and analyzed using ICP/AES. The ICP/AES instrument was calibrated against serially diluted NIST-traceable $10 \mathrm{mg} / \mathrm{mL}$ spectrometric standards of cobalt and the internal standard yttrium.

\section{Chamber Atmosphere Characterization}

Particle size distribution was determined once prior to the 3-month and 2-year studies, once during the 2-week studies, twice during the 3-month studies, and monthly during the 2-year studies. Impactor samples were taken from each exposure chamber using a Mercer-style sevenstage impactor and the stages (glass coverslips lightly coated with silicone to prevent particle bounce) were analyzed using ICP/AES after cobalt was extracted from the slides. The relative mass collected on each stage was analyzed by the CASPACT impactor analysis program developed at Battelle based on probit analysis ${ }^{165}$. The resulting estimates of the mass median aerodynamic particle diameter and the geometric standard deviation of each set of samples are given in Table L-4 through Table L-7.

Buildup and decay rates for chamber aerosol concentrations were determined with (all studies) and without (3-month and 2-year studies) animals present in the chambers. At a chamber airflow rate of 15 air changes per hour, the theoretical value for the time to achieve $90 \%$ of the target concentration after the beginning of aerosol generation $\left(\mathrm{T}_{90}\right)$ and the time for the chamber concentration to decay to $10 \%$ of the target concentration after aerosol generation was terminated $\left(\mathrm{T}_{10}\right)$ was approximately 9.4 minutes. A $\mathrm{T}_{90}$ value of 12 minutes was selected for all studies.

The uniformity of aerosol concentration in the inhalation exposure chambers without animals was evaluated before the 3-month and 2-year studies began; in addition, concentration uniformity with animals present in the chambers was measured once during the 2-week and 3 -month studies and every 3 to 4 months during the 2 -year studies. Chamber concentration uniformity was maintained throughout the studies. The persistence of cobalt metal in the 
exposure chambers after aerosol delivery ended was determined by monitoring the concentration overnight in the $40 \mathrm{mg} / \mathrm{m}^{3}$ rat and mouse chambers in the 2-week studies, the $5 \mathrm{mg} / \mathrm{m}^{3}$ rat and $10 \mathrm{mg} / \mathrm{m}^{3}$ mouse chambers in the 3-month studies, and the $5 \mathrm{mg} / \mathrm{m}^{3}$ rat and mouse chambers in the 2-year studies, with and without (except for the 2-week studies) animals present in the chambers. The average cobalt metal concentration decreased to $1 \%$ of the target concentration within 15 (2-week studies), 17 to 18 (3-month studies), or 19 (2-year studies) minutes.

Stability studies of the test material in the generation and exposure system were performed before and during the studies by the study laboratory and the analytical chemistry laboratories. In these studies, XRD analyses consistently indicated two primary phases of cobalt in the samples, cubic and hexagonal, and minimal detectable concentrations of cobalt oxides. Low and acceptable levels of trace element inorganic impurities were detected in these stability samples using PIXE and ICP/AES assays.

\section{Animal Source}

Male and female F344/N rats and B6C3F1/N mice were obtained from the NTP colony maintained at Taconic Farms, Inc. (Germantown, NY) for the 2-week and 3-month studies. For the 2-year studies, male and female F344/NTac rats were obtained from the commercial colony at Taconic Farms, Inc., and B6C3F1/N mice were obtained from the NTP colony maintained at Taconic Farms, Inc. The rationale for change of rat strain from F344/N to F344/NTac was a programmatic decision. For many years, NTP used the inbred F344/N rat for its toxicity and carcinogenicity studies. Over a period of time, the F344/N rat exhibited sporadic seizures and idiopathic chylothorax, and consistently high rates of mononuclear cell leukemia and testicular neoplasia. Because of these issues in the F344/N rat and NTP's desire to find a more fecund rat model that could be used in both reproductive and carcinogenesis studies for comparative purposes, a change in the rat model was explored. Following a workshop in 2005, the F344 rat from the Taconic commercial colony (F344/NTac) was used for a few NTP studies to allow NTP to evaluate different rat models. The F344/NTac rat was used in four subchronic and two chronic studies (cobalt metal and bromodichloroacetic acid) between 2005 and $2006^{166}$. The current cobalt metal study is the first of the NTP 2-year studies using the F344/NTac rat to be reported.

\section{Animal Welfare}

Animal care and use are in accordance with the Public Health Service Policy on Humane Care and Use of Animals. All animal studies were conducted in an animal facility accredited by the Association for the Assessment and Accreditation of Laboratory Animal Care International. Studies were approved by the Battelle Toxicology Northwest Animal Care and Use Committee and conducted in accordance with all relevant NIH and NTP animal care and use policies and applicable federal, state, and local regulations and guidelines.

\section{Two-week Studies}

On receipt, the rats and mice were approximately 4 weeks old. Animals were quarantined for 12 days and were 5 to 6 weeks old on the first day of the studies. Before the studies began, five male and five female rats and mice were randomly selected for parasite evaluation and gross observation for evidence of disease. At the end of the studies, serologic analyses were performed 
on five male and five female sentinel rats and mice housed in the control chamber using the protocols of the NTP Sentinel Animal Program (Appendix N).

Groups of five male and five female rats and mice were exposed to cobalt metal particulate aerosol by inhalation at concentrations of $0,2.5,5,10,20$, or $40 \mathrm{mg} / \mathrm{m}^{3}, 6$ hours plus $\mathrm{T}_{90}$ (12 minutes) per day, 5 days per week for 16 (rats) or 17 (mice) days. Additional groups of five female rats and mice were exposed to the same concentrations for 16 (rats) or 17 (mice) days for tissue burden studies. Feed and water were available ad libitum, except feed was withheld during exposure periods and urine collection. Rats and mice were housed individually. Clinical findings were recorded once daily and at terminal kill for core study rats and mice. The core study animals were weighed on days 1, 5, and 12 and at the end of the studies. Details of the study design and animal maintenance are summarized in Table 1.

As part of the tissue burden studies, surviving core study rats were placed in metabolism cages on day 12 for a 16-hour urine collection. Urine samples were collected over ice. Volume was measured, and creatine concentration was determined. Remaining samples were stored at approximately $-70^{\circ} \mathrm{C}$ until analyzed for cobalt metal concentration using ICP/AES following open digestion in a heat block. The parameters measured are listed in Table 1.

For tissue burden studies, blood was collected from the retroorbital sinus of all surviving core study rats and mice and two female tissue burden study rats and mice per group on the last day of exposure and from three female tissue burden study rats and mice per group 3 weeks postexposure. Blood was divided between a collection tube containing EDTA and a serum tube without anticoagulant. Blood and serum samples were stored at approximately $-70^{\circ} \mathrm{C}$ for analysis of cobalt metal concentration. Following blood collection, the right femur, heart, right kidney, liver (right lateral and caudate lobes), right lung lobe, and right testis were collected from core study rats and mice and weighed. In addition, whole liver, whole lung, and left lung plus mainstem bronchi were weighed. For tissue burden study rats and mice, the lungs with mainstem bronchi were removed and weighed and the right and left lung lobes were collected and weighed individually. Samples were stored in plastic containers at approximately $-70^{\circ} \mathrm{C}$ until analyzed for cobalt metal. For analyses, blood, serum, femur (femurs were boiled in water, extraneous tissue scraped off, and dried for at least 48 hours before digestion), heart, kidney, and testis samples were prepared in acid-leached Parr bomb digestion liners (Parr Instrument Co., Moline, IL) using an Imperial II radiant heat oven (Lab-line Instruments, Inc., Melrose Park, IL), and liver and lung samples were prepared in microwave digestion liners and a microwave sample preparation station (CEM Corp., Matthews, NC) with internal standard (10 $\mu \mathrm{g}$ yttrium; $0.1 \mu \mathrm{g}$ gallium) and $\mathrm{HNO}_{3}: \mathrm{HCl}(1: 1)$. Digests were quantitatively transferred to plastic containers and diluted to an appropriate volume with deionized water before analysis using ICP/AES (IRIS Intrepid ICP/AES spectrometer, Thermo Elemental, Franklin, MA) (kidney, liver, lung) or ICP/mass spectrometry (MS) (Agilent 7500cc ICP/MS, Agilent Technologies, Palo Alto, CA) (blood, serum, femur, heart, and testis).

Necropsies were performed on all core study rats and mice. The heart, left kidney, liver, lung, left testis, thymus, and thyroid gland were weighed. Tissues for microscopic examination were fixed and preserved in 10\% neutral buffered formalin (except eyes were first fixed in Davidson's solution), processed and trimmed, embedded in paraffin, sectioned, and stained with hematoxylin and eosin. Histopathologic examinations were performed on core study chamber control animals, $20 \mathrm{mg} / \mathrm{m}^{3}$ rats, and $40 \mathrm{mg} / \mathrm{m}^{3}$ rats and mice. The left kidney, left and median liver lobes, and 
thyroid gland of rats and mice, the brain of rats, and the urinary bladder of male rats were examined to a no-effect level; the left lobe of the lung and the nose were examined in all core study groups. Table 1 lists the tissues and organs examined.

\section{Three-month Studies}

The 3-month studies were conducted to evaluate the cumulative toxic effects of repeated exposure to cobalt metal and to determine the appropriate exposure concentrations to be used in the 2-year studies.

On receipt, the rats and mice were approximately 4 weeks old. Animals were quarantined for 12 or 13 days and were 5 to 6 weeks old on the first day of the studies. Before the studies began, five male and five female rats and mice were randomly selected for parasite evaluation and gross observation for evidence of disease. Serologic analyses were performed during the studies using the protocols of the NTP Sentinel Animal Program (Appendix N).

Groups of 10 male and 10 female rats and mice were exposed to particulate aerosols of cobalt metal by inhalation at concentrations of $0,0.625,1.25,2.5,5$, or 10 (mice only) $\mathrm{mg} / \mathrm{m}^{3}, 6$ hours plus $\mathrm{T}_{90}$ (12 minutes) per day, 5 days per week for 14 weeks. Additional groups of 10 male rats (clinical pathology study) and 32 to 36 female rats and mice (special study) were exposed to the same concentrations for 14 weeks. Feed was available ad libitum except during exposure periods; water was available ad libitum. Rats and mice were housed individually. Clinical findings were recorded weekly beginning day 9 (male rats) or 10 and at the end of the studies. Core study animals were weighed initially, weekly beginning day 9 (male rats) or 10, and at the end of the studies. Details of the study design and animal maintenance are summarized in Table 1.

Blood was collected from the retroorbital sinus of male clinical pathology rats and 10 female special study rats on days 3 and 23 and from core study rats and mice at the end of the studies for hematology and clinical chemistry (rats) analyses. Blood for hematology was placed in tubes containing potassium EDTA, and blood for clinical chemistry was placed in tubes containing a separator gel. Hematology analyses were performed on an Abbott Cell-Dyn 3700 analyzer (Abbott Diagnostics Systems, Abbott Park, IL), except manual hematocrit determinations were performed using a microcentrifuge (Hereaus Holding Gmbh., Hanau, Germany) and a Damon/IEC capillary reader (International Equipment Co., Needhum Heights, MA). Platelet, leukocyte, and erythrocyte morphology and nucleated erythrocytes were assessed using smears stained with a Romanowsky-type aqueous stain in a Wescor 7100 aerospray slide stainer (Wescor, Inc., Logan, UT). Reticulocytes were stained with new methylene blue and counted using the Miller disc method ${ }^{167}$. Samples for clinical chemistry were centrifuged, and parameters were measured using a Roche Hitachi 912 system (Roche Diagnostic Corp., Indianapolis, IN). Table 1 lists the clinical pathology parameters measured.

Lungs and blood (retroorbital sinus) were collected from three special study female rats and mice per exposure group on days 5, 12, 26, 40, 61, and 89 and on days 7, 14, 28, and 42 postexposure. On days 26 and 40, livers were also collected. Liver and lungs were weighed. Blood, liver (right lateral and caudate lobes), and lungs were analyzed for cobalt metal concentrations as described for the 2-week studies, except all samples for analysis were placed in microwave sample digestion vessels. 
On days 26 and 40, the remaining liver samples from special study rats and mice were collected and stored at approximately $-70^{\circ} \mathrm{C}$ until analyses for cytochrome $\mathrm{P} 450$ activities. Microsomal suspensions were prepared using the Pearce Method ${ }^{168}$. The concentration of protein in each suspension was determined using the microtiter plate method of the Coomassie ${ }^{\circledR}$ Plus Protein Assay (Pierce Chemical Co., Rockford, IL) with bovine serum albumin as the standard. Acetanilide-4-hydroxylase (A4H), 7-ethoxyresorufin-O-deethylase (EROD), and 7-pentoxyresorufin-O-deethylase (PROD) were determined in microsomal proteins isolated from frozen liver samples according to established procedures.

At the end of the 3-month studies, samples were collected for sperm motility and vaginal cytology evaluations on rats exposed to $0,1.25,2.5$, or $5 \mathrm{mg} / \mathrm{m}^{3}$ and mice exposed to $0,2.5,5$, or $10 \mathrm{mg} / \mathrm{m}^{3}$. The parameters evaluated are listed in Table 1 . For 12 consecutive days prior to scheduled terminal kill, the vaginal vaults of the females were moistened with saline, if necessary, and samples of vaginal fluid and cells were stained. Relative numbers of leukocytes, nucleated epithelial cells, and large squamous epithelial cells were determined and used to ascertain estrous cycle stage (i.e., diestrus, proestrus, estrus, and metestrus). Male animals were evaluated for sperm count and motility. The left testis and left epididymis were isolated and weighed. The tail of the epididymis (cauda epididymis) was then removed from the epididymal body (corpus epididymis) and weighed. Test yolk (rats) or modified Tyrode's buffer (mice) was applied to slides, and a small incision was made at the distal border of the cauda epididymis. The sperm effluxing from the incision were dispersed in the buffer on the slides, and the numbers of motile and nonmotile spermatozoa were counted for five fields per slide by two observers. Following completion of sperm motility estimates, each left cauda epididymis was placed in buffered saline solution. Caudae were finely minced, and the tissue was incubated in the saline solution and then heat fixed at $65^{\circ} \mathrm{C}$. Sperm density was then determined microscopically with the aid of a hemacytometer. To quantify spermatogenesis, the testicular spermatid head count was determined by removing the tunica albuginea and homogenizing the left testis in phosphatebuffered saline containing 10\% dimethyl sulfoxide. Homogenization-resistant spermatid nuclei were counted with a hemacytometer.

Necropsies were performed on all core study and special study animals. The heart, right kidney, liver, lung, right testis, thymus, and thyroid gland (rats) of core study animals were weighed.

Tissues for microscopic examination were fixed and preserved in $10 \%$ neutral buffered formalin (except eyes were first fixed in Davidson's solution), processed and trimmed, embedded in paraffin, sectioned, and stained with hematoxylin and eosin. Complete histopathologic examinations were performed by the study laboratory pathologist on core study 0,5 (rats), and 10 (mice) $\mathrm{mg} / \mathrm{m}^{3}$ groups of rats and mice; tissues were examined to a no-effect level in the remaining groups. Table 1 lists the tissues and organs routinely examined.

After a review of the laboratory reports and selected histopathology slides by a quality assessment (QA) pathologist, the findings and reviewed slides were submitted to a NTP Pathology Working Group (PWG) coordinator for a second independent review. Any inconsistencies in the diagnoses made by the study laboratory and QA pathologists were resolved by the NTP pathology peer review process. Final diagnoses for reviewed lesions represent a consensus of the PWG or a consensus between the study laboratory pathologist, NTP pathologist, QA pathologist(s), and the PWG coordinator. Details of these review procedures have been described, in part, by Maronpot and Boorman ${ }^{169}$ and Boorman et al. ${ }^{170}$. 


\section{Two-year Studies}

\section{Study Design}

Groups of 50 male and 50 female rats and mice were exposed to cobalt metal by inhalation of particulate aerosol at concentrations of $0,1.25,2.5$, or $5 \mathrm{mg} / \mathrm{m}^{3}, 6$ hours plus $\mathrm{T}_{90}$ ( 12 minutes) per day, 5 days per week for up to 105 weeks. Additional groups of 35 lung burden study female rats and mice were exposed to the same concentrations of cobalt metal for up to 79 weeks.

Rats were 3 to 4 weeks old on receipt, and mice were 4 to 5 weeks old. The animals were quarantined for 12 days before the beginning of the studies. Five male and five female rats and mice were randomly selected for parasite evaluation and gross observation of disease. Rats were approximately 5 to 6 weeks old and mice approximately 5 to 6 weeks old at the beginning of the studies. The health of the animals was monitored during the studies according to the protocols of the NTP Sentinel Animal Program (Appendix N).

Rats and mice were housed individually. Feed and water were available ad libitum, except feed was withheld during animal exposures. Chambers and racks were rotated weekly. Further details of animal maintenance are given in Table 1. Information on feed composition and contaminants is provided in Appendix M.

\section{Clinical Examinations and Pathology}

All animals were observed twice daily. Core study animal body weights were recorded on day 1, weekly for the first 13 weeks, every 4 weeks through week 93, every 2 weeks thereafter, and at terminal kill. Clinical findings were recorded every 4 weeks through week 93 , then every 2 weeks, and at terminal kill.

Complete necropsies and microscopic examinations were performed on all core study rats and mice; selected necropsies were performed on lung burden study animals. At necropsy, organs and tissues were examined for grossly visible lesions, and all (core study) major tissues were fixed and preserved in $10 \%$ neutral buffered formalin, processed and trimmed, embedded in paraffin, sectioned to a thickness of 4 to $6 \mu \mathrm{m}$, and stained with hematoxylin and eosin for microscopic examination. For all paired organs (e.g., adrenal gland, kidney, ovary), samples from each organ were examined. Tissues examined microscopically are listed in Table 1. For extended evaluation of renal proliferative lesions in male rats, kidneys were step sectioned at $1 \mathrm{~mm}$ intervals, and three to eight additional sections were obtained from each kidney.

Microscopic evaluations were completed by the study laboratory pathologist, and the pathology data were entered into the Toxicology Data Management System. The report, slides, paraffin blocks, residual wet tissues, and pathology data were sent to the NTP Archives for inventory, slide/block match, wet tissue audit, and storage. The slides, individual animal data records, and pathology tables were evaluated by an independent quality assessment laboratory. The individual animal records and tables were compared for accuracy; the slide and tissue counts were verified, and the histotechnique was evaluated. For the 2-year studies, a quality assessment pathologist evaluated slides from all tumors and all potential target organs, which included larynx, lung, and nose of rats and mice; adrenal medulla and pancreas of rats; kidney and testis of male rats; and spleen of female rats. 
The quality assessment report and the reviewed slides were submitted to the NTP PWG coordinator who reviewed the selected tissues and addressed any inconsistencies in the diagnoses made by the laboratory and quality assessment pathologists. Representative histopathology slides containing examples of lesions related to chemical administration, examples of disagreements in diagnoses between the laboratory and quality assessment pathologists, or lesions of general interest were presented by the coordinator to the PWG for review. The PWG consisted of the quality assessment pathologist and other pathologists experienced in rodent toxicologic pathology. This group examined the tissues without any knowledge of dose groups. When the PWG consensus differed from the opinion of the laboratory pathologist, the diagnosis was changed. Final diagnoses for reviewed lesions represent a consensus between the laboratory pathologist, reviewing pathologist(s), and the PWG. Details of these review procedures have been described, in part, by Maronpot and Boorman ${ }^{169}$ and Boorman et al. ${ }^{170}$. For subsequent analyses of the pathology data, the decision of whether to evaluate the diagnosed lesions for each tissue type separately or combined was generally based on the guidelines of McConnell et al. ${ }^{171}$.

\section{Mutation Analysis of Alveolar/bronchiolar Carcinomas}

After histopathology examination, formalin-fixed, paraffin-embedded (FFPE) blocks from alveolar/bronchiolar carcinomas that arose either spontaneously (in chamber controls) or due to cobalt metal exposure were selected from rats and mice for mutation analysis of commonly altered genes in lung cancer (Kras, Egfr and Tp53). DNA was extracted from the FFPE tissues and subjected to a seminested polymerase chain reaction (PCR) to amplify hot spot regions of Kras, Egfr and Tp53. The lyophilized PCR products were sequenced, and the resulting electropherograms were compared to identify mutations in alveolar/bronchiolar carcinomas that arose spontaneously or due to exposure to cobalt metal. The results are presented in Appendix K.

\section{Lung Burden Study}

Five female lung burden rats and mice per group were randomly selected and sent to necropsy immediately after exposure on days $1,2,3,4,184,366$, and 548. The lungs were removed, weighed, and stored at approximately $-70^{\circ} \mathrm{C}$ until analyzed for cobalt metal concentration using an ICP/AES method similar to that described for the 2-week studies. 
Table 1. Experimental Design and Materials and Methods in the Inhalation Studies of Cobalt Metal

Two-week Studies

Three-month Studies

Two-year Studies

\section{Study Laboratory}

Battelle Toxicology Northwest

(Richland, WA)

Battelle Toxicology Northwest

(Richland, WA)

F344/N rats

$\mathrm{B} 6 \mathrm{C} 3 \mathrm{~F} 1 / \mathrm{N}$ mice

Taconic Farms, Inc. (Germantown, NY) Taconic Farms, Inc.

(Germantown, NY)

Taconic Farms, Inc.

(Germantown, NY)

Time Held Before Studies

12 days

12 or 13 (male rats) days

12 days

Average Age When Studies Began

5 to 6 weeks

5 to 6 weeks

5 to 6 weeks

\section{Date of First Exposure}

August 2, 2004

Rats: March 7 (females) or 8 (males), 2005

Mice: March 7, 2005

Rats: May 8, 2006

Mice: May 15, 2006

\section{Duration of Exposure}

6 hours plus $\mathrm{T}_{90}$ ( 12 minutes) per 6 hours plus $\mathrm{T}_{90}$ ( 12 minutes) per day, day, 5 days per week, for 16 (rats) 5 days per week, for 14 weeks or 17 (mice) days

\section{Date of Last Exposure}

Rats: August 17, 2004

Mice: August 18, 2004

\section{Necropsy Dates}

Rats: August 17, 2004

Mice: August 18, 2004
Rats: June 6 (female) or 7 (males), 2005 Rats: May 5-8, 2008

Mice: June 8 (males) or 9 (females), Mice: May 15, 2008

2005

Rats: June 7 (females) or 8 (males), 2005

Mice: June 9 (males) or 10 (females), 2005

\section{Average Age at Necropsy}

8 weeks

19 to 20 weeks

Rats: May 5-8, 2008
6 hours plus $\mathrm{T}_{90}(12$ minutes $)$ per day, 5 days per week, for 105 weeks

\section{Size of Study Groups}

5 males and 5 females (core study) Rats: 10 males and 10 females (core study) 50 males and 50 females (core study)

5 females (tissue burden study) 10 males (clinical pathology) 35 females (lung burden study)

32 to 36 females (special study)

Mice: May 12-16, 2008

Rats: 109 to 110 weeks Mice: 109 to 111 weeks

Mice: 10 males and 10 females (core study)

32 to 36 females (special study) 


\section{Method of Distribution}

Animals were distributed randomly into groups of approximately equal initial mean body weights.

\section{Animals per Cage}

1

\section{Method of Animal Identification}

Tail tattoo

\section{Diet}

Irradiated NTP-2000 wafer diet (Zeigler Brothers, Inc., Gardners, PA), available ad libitum, except during exposure periods and urine collection, changed weekly

\section{Water}

Tap water (Richland, WA municipal supply) via automatic watering system (Edstrom Industries, Waterford, WI), available ad libitum

\section{Cages}

Stainless steel wire bottom (Lab Products, Inc., Seaford, DE), changed weekly, rotated daily

\section{Cageboard}

Untreated paper cage pan liner (Techboard, Shepherd Specialty Papers, Kalamazoo, MI), changed daily

\section{Chamber Air Supply Filters}

Single HEPA (open stock), charcoal (RSE, Inc., New Baltimore, MI), Purafil (Environmental Systems, Lynnwood, WA), all new at study start

\section{Chambers}

Stainless steel, excreta pan at each Same as 2-week of six levels (Lab Products, Inc., Seaford, DE); chambers changed weekly; excreta pans changed daily
Same as 2-week studies

Same as 2-week studies

Irradiated NTP-2000 wafer diet (Zeigler Same as 3-month studies Brothers, Inc., Gardners, PA), available ad libitum, except during exposure periods, changed weekly

Same as 2-week studies

Same as 2-week studies

Stainless steel wire bottom (Lab Products, Inc., Seaford, DE), changed weekly, rotated weekly in chambers

Same as 2-week studies

Same as 2-week studies, except new at beginning of 2-week studies and changed as needed
Same as 3-month studies

Same as 2-week studies

Same as 2-week studies, except HEPA filter changed annually

Same as 2-week studies 


\section{Chamber Environment}

Temperature: $75^{\circ} \pm 3^{\circ} \mathrm{F}$

Relative humidity: $55 \% \pm 15 \%$

Room fluorescent light:

12 hours/day

Chamber air changes: $15 \pm 2$ /hour
Temperature: $72^{\circ} \pm 3^{\circ} \mathrm{F}$

Relative humidity: $55 \% \pm 15 \%$

Room fluorescent light: 12 hours/day

Chamber air changes: $15 \pm 2$ /hour
Temperature: $72^{\circ} \pm 3^{\circ} \mathrm{F}$

Relative humidity: $55 \% \pm 15 \%$

Room fluorescent light: 12 hours/day

Chamber air changes: $15 \pm 2$ /hour

\section{Exposure Concentrations}
$0,2.5,5,10,20$, or $40 \mathrm{mg} / \mathrm{m}^{3}$
Rats: $0,0.625,1.25,2.5$, or $5 \mathrm{mg} / \mathrm{m}^{3}$
Mice: $0,0.625,1.25,2.5,5$, or $10 \mathrm{mg} / \mathrm{m}^{3}$
$0,1.25,2.5$, or $5 \mathrm{mg} / \mathrm{m}^{3}$

\section{Type and Frequency of Observation}

Observed once daily; core study animals were weighed on days 1 , 5 , and 12 and at the end of the studies; clinical findings were recorded once daily and at terminal kill.

\section{Method of Kill}

Carbon dioxide asphyxiation

\section{Necropsy}

Necropsies were performed on all core study rats and mice.

Following blood collection, the right femur, heart, right kidney, liver (right lateral and caudate lobes), right lung lobe, and right testis were collected from core study rats and mice and weighed. In addition, whole liver, whole lung and left lung plus mainstem bronchi were weighed. For tissue burden study rats and mice, the lungs with mainstem bronchi were removed and weighed and the right and left lung lobes were collected and weighed individually. Samples were stored in plastic containers at approximately $-70^{\circ} \mathrm{C}$ until analyzed for cobalt metal.
Observed twice daily; core study animals were weighed initially, on day 9 (male rats) or 10 , weekly thereafter, and at the end of the studies; clinical findings were recorded on day 9 (male rats) or 10, weekly thereafter, and at the end of the studies.

Same as 2-week studies

Necropsies were performed on core study and special study rats and mice. For core study animals, organs weighed were heart, right kidney, liver, lung, right testis, thymus, and thyroid gland (rats).
Observed twice daily; core study animals weighed initially, weekly for the first 13 weeks, every 4 weeks through week 93 , every 2 weeks thereafter, and at terminal kill; clinical findings were recorded initially and every 4 weeks through week 93, every 2 weeks thereafter, and at terminal kill.

Same as 2-week studies

Necropsies were performed on all animals. 


\section{Clinical Pathology}

None

Blood was collected from the
retroorbital sinus of clinical pathology male rats and 10 special study female rats on days 3 and 23 and from core study animals at the end of the studies for hematology and clinical chemistry (rats).

Hematology: hematocrit; packed cell volume; hemoglobin; erythrocyte, reticulocyte, and platelet counts; total nucleated cells; Howell-Jolly bodies (mice); mean cell volume; mean cell hemoglobin; mean cell hemoglobin concentration; and leukocyte count and differentials

Clinical chemistry: urea nitrogen, creatinine, glucose, total protein, albumin, globulin, cholesterol, triglyceride, alanine aminotransferase, alkaline phosphatase, creatine kinase, sorbitol dehydrogenase, and bile salts
None

\section{Histopathology}

Histopathology was performed on Complete histopathology was performed Complete histopathology was core study 0, 20 (rats), and 40 (rats on core study 0 (rats and mice), 5 (rats), performed on all core study rats and and mice) $\mathrm{mg} / \mathrm{m}^{3}$ animals. In and 10 (mice) $\mathrm{mg} / \mathrm{m}^{3}$ animals. In addition to gross lesions and tissue addition to gross lesions and tissue masses, the following tissues were masses, the following tissues were examined to a no-effect level: left examined to a no-effect level: adrenal kidney, liver (left and median gland, bone with marrow, brain, clitoral lobes), and thyroid gland (rats and gland, esophagus, eye, gallbladder mice), brain (rats), and urinary (mice), Harderian gland, heart, large bladder (male rats). The left lung intestine (cecum, colon, rectum), small and nose were examined in all core intestine (duodenum, jejunum, ileum), study animals.

kidney, larynx, liver, lung, lymph nodes (bronchial, mandibular, mesenteric, and mediastinal), mammary gland, nose, ovary, pancreas, parathyroid gland, pituitary gland, preputial gland, prostate gland, salivary gland, skin, spleen, stomach (forestomach and glandular), testis with epididymis and seminal vesicle, thymus, thyroid gland, trachea, urinary bladder, and uterus. mice. In addition to gross lesions and tissue masses, the following tissues were examined: adrenal gland, bone with marrow, brain, clitoral gland, esophagus, eyes, gallbladder (mice), Harderian gland, heart, large intestine (cecum, colon, rectum), small intestine (duodenum, jejunum, ileum), kidney, larynx, liver, lung, lymph nodes (bronchial, mandibular, mesenteric, and mediastinal), mammary gland, nose, ovary, pancreas, parathyroid gland, pituitary gland, preputial gland, prostate gland, salivary gland, skin, spleen, stomach (forestomach and glandular), testis with epididymis and seminal vesicle, thymus, thyroid gland, trachea, urinary bladder, and uterus. 


\section{Sperm Motility and Vaginal Cytology}

None

\section{Tissue Burden Studies}

Urine was collected from core study rats for 16 hours beginning day 12 ; volume and creatinine and cobalt metal concentrations were determined. Blood was collected from the retroorbital sinus of core study rats and mice and two female tissue burden study rats and mice per group on the last day of exposure and from three female tissue burden study rats and mice per group 3 weeks postexposure; blood and serum were analyzed for cobalt metal concentration.

Following blood collection, the right femur, heart, right kidney, liver (right lateral and caudate lobes), right lung lobe, and right testis were collected from core study animals and weighed. In addition, whole liver, whole lung, and left lung plus mainstem bronchi were removed and weighed and the right and left lung lobes were collected and weighed individually. Tissues were analyzed for cobalt metal concentration.

At the end of the studies, spermatid and None sperm samples were collected from male animals in the $0,1.25$ (rats), $2.5,5$, or 10 (mice) $\mathrm{mg} / \mathrm{m}^{3}$ groups. The following parameters were evaluated: spermatid heads per testis and per gram testis, sperm motility, and sperm per cauda epididymis and per gram cauda epididymis. The left cauda, left epididymis, and left testis were weighed. Vaginal samples were collected for 12 days during the last 2 weeks of the study from females exposed to $0,1.25$ (rats), $2.5,5$, or 10 (mice) $\mathrm{mg} / \mathrm{m}^{3}$.

Lungs and blood (retroorbital sinus) were collected from three special study female rats and mice per exposure group on days $5,12,26,40,61$, and 89 and on days $7,14,28$, and 42 postexposure.

Liver (right lateral and caudate lobes) was also collected on days 26 and 40 . Liver and lungs were weighed; blood, liver, and lungs were analyzed for cobalt metal concentration.
On days $1,2,3,4,184,366$, and 548, lungs were removed from five female lung burden study rats and mice per group, weighed, and analyzed for cobalt metal concentration. 


\section{Cytochrome P450 Activities}

None

\author{
On days 26 and 40, liver samples from None \\ special study rats and mice not used for \\ the tissue burden studies were collected \\ and acetanilide-4-hydroxylase, 7- \\ ethoxyresorufin-O-deethylase, and \\ 7-pentoxyresorufin-O-deethylase \\ activities were determined.
}

Mutation Analysis of Alveolar/bronchiolar Carcinomas
None

\begin{abstract}
DNA was extracted from the formalin-fixed, paraffin-embedded, rat and mouse alveolar/bronchiolar carcinomas. The samples were subjected to semi-nested PCR to amplify hot spot regions of Kras, Egfr, and Tp53. The lyophilized polymerase chain reaction products were sequenced, and the resulting electropherograms were compared to identify mutations in alveolar/bronchiolar carcinomas that either arose spontaneously or due to exposure to cobalt metal.
\end{abstract}

\section{Statistical Methods}

\section{Survival Analyses}

The probability of survival was estimated by the product-limit procedure of Kaplan and Meier ${ }^{172}$ and is presented in the form of graphs. Animals found dead of other than natural causes were censored; animals dying from natural causes were not censored. Statistical analyses for possible dose-related effects on survival used Cox's ${ }^{173}$ method for testing two groups for equality and Tarone's ${ }^{174}$ life table test to identify dose-related trends. All reported $\mathrm{P}$ values for the survival analyses are two sided.

\section{Calculation of Incidence}

The incidences of neoplasms or nonneoplastic lesions are presented in Table A-1, Table A-7, Table B-1, Table B-7, Table C-1, Table C-4, Table D-1, and Table D-4 as the numbers of animals bearing such lesions at a specific anatomic site and the numbers of animals with that site examined microscopically. For calculation of statistical significance, the incidences of most neoplasms (Table A-2, Table B-2, Table C-2, and Table D-2) and all nonneoplastic lesions are given as the numbers of animals affected at each site examined microscopically. However, when macroscopic examination was required to detect neoplasms in certain tissues (e.g., mesentery, pleura, peripheral nerve, skeletal muscle, tongue, tooth, and Zymbal's gland) before microscopic evaluation, the denominators consist of the number of animals that had a gross abnormality. When neoplasms had multiple potential sites of occurrence (e.g., leukemia or lymphoma), the denominators consist of the number of animals on which a necropsy was performed. Table A-2, 
Table B-2, Table C-2, and Table D-2 also give the survival-adjusted neoplasm rate for each group and each site-specific neoplasm. This survival-adjusted rate (based on the Poly-3 method described below) accounts for differential mortality by assigning a reduced risk of neoplasm, proportional to the third power of the fraction of time on study, only to site-specific, lesion-free animals that do not reach terminal kill.

\section{Analysis of Neoplasm and Nonneoplastic Lesion Incidences}

The Poly-k test ${ }^{175-177}$ was used to assess neoplasm and nonneoplastic lesion prevalence. This test is a survival-adjusted quantal-response procedure that modifies the Cochran-Armitage linear trend test to take survival differences into account. More specifically, this method modifies the denominator in the quantal estimate of lesion incidence to approximate more closely the total number of animal years at risk. For analysis of a given site, each animal is assigned a risk weight. This value is one if the animal had a lesion at that site or if it survived until terminal kill; if the animal died prior to terminal kill and did not have a lesion at that site, its risk weight is the fraction of the entire study time that it survived, raised to the kth power.

This method yields a lesion prevalence rate that depends only upon the choice of a shape parameter for a Weibull hazard function describing cumulative lesion incidence over time ${ }^{175}$. Unless otherwise specified, a value of $k=3$ was used in the analysis of site-specific lesions. This value was recommended by Bailer and Portier ${ }^{175}$ following an evaluation of neoplasm onset time distributions for a variety of site-specific neoplasms in control F344/N rats and B6C3F1 mice ${ }^{178}$. Bailer and Portier ${ }^{175}$ showed that the Poly-3 test gave valid results if the true value of $\mathrm{k}$ was anywhere in the range from 1 to 5. A further advantage of the Poly-3 method is that it does not require lesion lethality assumptions. Variation introduced by the use of risk weights, which reflect differential mortality, was accommodated by adjusting the variance of the Poly-3 statistic as recommended by Bieler and Williams ${ }^{179}$.

Tests of significance included pairwise comparisons of each dosed group with controls and a test for an overall dose-related trend. Continuity-corrected Poly-3 tests were used in the analysis of lesion incidence, and reported $\mathrm{P}$ values are one sided. The significance of lower incidences or decreasing trends in lesions is represented as $1-\mathrm{P}$ with the letter $\mathrm{N}$ added (e.g., $\mathrm{P}=0.99$ is presented as $\mathrm{P}=0.01 \mathrm{~N})$.

\section{Analysis of Continuous Variables}

Two approaches were employed to assess the significance of pairwise comparisons between dosed and control groups in the analysis of continuous variables. Organ and body weight data, which historically have approximately normal distributions, were analyzed with the parametric multiple comparison procedures of Dunnett ${ }^{180}$ and Williams ${ }^{181 ; 182}$. Hematology; clinical chemistry; urine, blood, serum, and tissue cobalt metal concentrations; cytochrome P450 activities; spermatid and epididymal spermatozoal data, which have typically skewed distributions, were analyzed using the nonparametric multiple comparison methods of Shirley ${ }^{183}$ (as modified by Williams ${ }^{184}$ ) and Dunn ${ }^{185}$. Jonckheere's test ${ }^{186}$ was used to assess the significance of the dose-related trends and to determine whether a trend-sensitive test (Williams' or Shirley's test) was more appropriate for pairwise comparisons than a test that does not assume a monotonic dose-related trend (Dunnett's or Dunn's test). Prior to statistical analysis, extreme values identified by the outlier test of Dixon and Massey ${ }^{187}$ were examined by NTP personnel, 
and implausible values were eliminated from the analysis. Proportions of regular cycling females in each dosed group were compared to the control group using the Fisher exact test ${ }^{188}$. Tests for extended periods of estrus, diestrus, metestrus, and proestrus, as well as skipped estrus and skipped diestrus, were constructed based on a Markov chain model proposed by Girard and Sager ${ }^{189}$. For each dose group, a transition probability matrix was estimated for transitions among the proestrus, estrus, metestrus, and diestrus stages, with provision for extended stays within each stage as well as for skipping estrus or diestrus within a cycle. Equality of transition matrices among dose groups and between the control group and each dosed group was tested using chi-square statistics.

\section{Historical Control Data}

The concurrent control group represents the most valid comparison to the treated groups and is the only control group analyzed statistically in NTP bioassays. However, historical control data are often helpful in interpreting potential treatment-related effects, particularly for uncommon or rare neoplasm types. For meaningful comparisons, the conditions for studies in the historical control database must be generally similar. Significant factors affecting the background incidences of neoplasms at a variety of sites are diet, sex, strain/stock, and route of exposure. The NTP historical control database contains all 2-year studies for each species, sex, and strain/stock with histopathology findings in control animals completed within the most recent 5-year period $^{190-192}$. In general, the historical control database for a given study includes studies using the same route of administration, and the overall incidences of neoplasms in controls for all routes of administration are included for comparison, including the current mouse study. The current study is the only inhalation study in F344/NTac rats in the historical control database; therefore only historical control incidences for all routes and all vehicles are used for F344/NTac rats in this Technical Report.

\section{Quality Assurance Methods}

The 2-week, 3-month, and 2-year studies were conducted in compliance with Food and Drug Administration Good Laboratory Practice Regulations (21 CFR, Part 58 ${ }^{193}$ ). In addition, as records from the 3-month and 2-year studies were submitted to the NTP Archives, these studies were audited retrospectively by an independent quality assessment contractor. Separate audits covered completeness and accuracy of the pathology data, pathology specimens, final pathology tables, and a draft of this NTP Technical Report. Audit procedures and findings are presented in the reports and are on file at NIEHS. The audit findings were reviewed and assessed by NTP staff, and all comments were resolved or otherwise addressed during the preparation of this Technical Report.

\section{Genetic Toxicology}

The genetic toxicity of cobalt metal was assessed by testing the ability of the chemical to induce mutations in various strains of Salmonella typhimurium and Escherichia coli and increases in the frequency of micronucleated erythrocytes in mouse peripheral blood. Micronuclei (literally "small nuclei" or Howell-Jolly bodies) are biomarkers of induced structural or numerical chromosomal alterations and are formed when acentric fragments or whole chromosomes fail to incorporate into either of two daughter nuclei during cell division ${ }^{194 ;}{ }^{195}$. The protocols for these studies and the results are given in Appendix E. 
The genetic toxicity studies have evolved from an earlier effort by NTP to develop a comprehensive database permitting a critical anticipation of a chemical's carcinogenicity in experimental animals based on numerous considerations, including the molecular structure of the chemical and its observed effects in short-term in vitro and in vivo genetic toxicity tests (structure-activity relationships). The short-term tests were originally developed to clarify proposed mechanisms of chemical-induced DNA damage based on the relationship between electrophilicity and mutagenicity ${ }^{196}$ and the somatic mutation theory of cancer ${ }^{197 ;} 198$. However, it should be noted that not all cancers arise through genotoxic mechanisms.

DNA reactivity combined with Salmonella mutagenicity is highly correlated with induction of carcinogenicity in multiple species/sexes of rodents and at multiple tissue sites ${ }^{199}$. A positive response in the Salmonella test was shown to be the most predictive in vitro indicator for rodent carcinogenicity ( $89 \%$ of the Salmonella mutagens are rodent carcinogens) $)^{200 ; 201}$. Additionally, no battery of tests that included the Salmonella test improved the predictivity of the Salmonella test alone. However, these other tests can provide useful information on the types of DNA and chromosomal damage induced by the chemical under investigation.

The predictivity for carcinogenicity of a positive response in acute in vivo bone marrow chromosome aberration or micronucleus tests appears to be less than that in the Salmonella test ${ }^{202 ; 203}$. However, clearly positive results in long-term peripheral blood micronucleus tests have high predictivity for rodent carcinogenicity; a weak response in one sex only or negative results in both sexes in this assay do not correlate well with either negative or positive results in rodent carcinogenicity studies ${ }^{204}$. Because of the theoretical and observed associations between induced genetic damage and adverse effects in somatic and germ cells, the determination of in vivo genetic effects is important to the overall understanding of the risks associated with exposure to a particular chemical. 


\section{Results}

\section{Data Availability}

The National Toxicology Program (NTP) evaluated all study data. Data relevant for evaluating toxicological findings are presented here. All study data are available in the NTP Chemical Effects in Biological Systems (CEBS) database: https://doi.org/10.22427/NTP-DATA-TR-581.

\section{Rats}

\section{Two-week Study}

The exposure concentrations for the 2-week study were estimated based on exposures of previously studied cobalt sulfate heptahydrate ${ }^{66}$. All rats exposed to $40 \mathrm{mg} / \mathrm{m}^{3}$ and all male and three female rats exposed to $20 \mathrm{mg} / \mathrm{m}^{3}$ died before the end of the study; the majority of deaths occurred by study day 7 (Table 2). Final mean body weights were significantly decreased in male and female rats exposed to $10 \mathrm{mg} / \mathrm{m}^{3}$ and were $20 \%$ and $12 \%$ less than those of the chamber control groups, respectively. In addition, the final mean body weight was significantly decreased in female rats exposed to $20 \mathrm{mg} / \mathrm{m}^{3}$ and was $45 \%$ less than that of the chamber controls. Mean body weight gains of $10 \mathrm{mg} / \mathrm{m}^{3}$ males and females and $20 \mathrm{mg} / \mathrm{m}^{3}$ females were significantly less than those of the chamber controls. Females exposed to $20 \mathrm{mg} / \mathrm{m}^{3}$ lost weight during the study. Exposure-related clinical findings included abnormal breathing, lethargy, and thinness in male rats exposed to 20 or $40 \mathrm{mg} / \mathrm{m}^{3}$, and in females exposed to $40 \mathrm{mg} / \mathrm{m}^{3}$. Dark lungs were observed at necropsy in all early-death rats of both sexes exposed to $40 \mathrm{mg} / \mathrm{m}^{3}$ and most rats exposed to $20 \mathrm{mg} / \mathrm{m}^{3}$. Pale lungs were noted in two females exposed to $20 \mathrm{mg} / \mathrm{m}^{3}$, four males exposed to $10 \mathrm{mg} / \mathrm{m}^{3}$, and one male exposed to $5 \mathrm{mg} / \mathrm{m}^{3}$.

Table 2. Survival and Body Weights of Rats in the Two-week Inhalation Study of Cobalt Metal ${ }^{\mathrm{a}}$

\begin{tabular}{cccccc}
\hline $\begin{array}{c}\text { Concentration } \\
\left(\mathbf{m g} \mathbf{m}^{\mathbf{3}}\right)\end{array}$ & Survival $^{\mathbf{b}}$ & $\begin{array}{c}\text { Initial Body } \\
\text { Weight (g) }\end{array}$ & $\begin{array}{c}\text { Final Body } \\
\text { Weight (g) }\end{array}$ & $\begin{array}{c}\text { Change in Body } \\
\text { Weight (g) }\end{array}$ & $\begin{array}{c}\text { Final Weight } \\
\text { Relative to } \\
\text { Controls (\%) }\end{array}$ \\
\hline Male & & & & & \\
0 & $5 / 5$ & $102 \pm 2$ & $144 \pm 3$ & $41 \pm 2$ & 100 \\
2.5 & $5 / 5$ & $102 \pm 2$ & $144 \pm 2$ & $42 \pm 2$ & 97 \\
5 & $5 / 5$ & $102 \pm 3$ & $140 \pm 4$ & $38 \pm 2$ & 80 \\
10 & $5 / 5$ & $100 \pm 3$ & $155 \pm 6^{* *}$ & $14 \pm 4^{* *}$ & - \\
20 & $0 / 5^{\mathrm{c}}$ & $103 \pm 3$ & - & - & - \\
40 & $0 / 5^{\mathrm{d}}$ & $101 \pm 3$ & - & - & 100 \\
\hline Female & & & & $24 \pm 2$ & 96 \\
0 & $5 / 5$ & $88 \pm 4$ & $112 \pm 4$ & $24 \pm 1$ & 88 \\
2.5 & $5 / 5$ & $88 \pm 2$ & $112 \pm 2$ & $21 \pm 1$ & 55 \\
\hline 10 & $5 / 5$ & $86 \pm 3$ & $107 \pm 3$ & $11 \pm 1^{* *}$ & $-23 \pm 0^{* *}$ \\
\hline
\end{tabular}




\begin{tabular}{cccccc}
\hline $\begin{array}{c}\text { Concentration } \\
\left(\mathbf{m g} / \mathbf{m}^{3}\right)\end{array}$ & Survival $^{\mathrm{b}}$ & $\begin{array}{c}\text { Initial Body } \\
\text { Weight (g) }\end{array}$ & $\begin{array}{c}\text { Final Body } \\
\text { Weight (g) }\end{array}$ & $\begin{array}{c}\text { Change in Body } \\
\text { Weight (g) }\end{array}$ & $\begin{array}{c}\text { Final Weight } \\
\text { Relative to } \\
\text { Controls (\%) }\end{array}$ \\
\hline 40 & $0 / 5^{\mathrm{f}}$ & $86 \pm 3$ & - & - & - \\
\hline
\end{tabular}

**Significantly different $(\mathrm{P} \leq 0.01)$ from the chamber control group by Williams' test.

aweights and weight changes are given as mean \pm standard error. Subsequent calculations are based on animals surviving to the end of the study.

bNumber of animals surviving at 2 weeks/number initially in group.

'Days of deaths: $5,5,5,9,13$.

dDays of deaths: $5,6,6,7,7$.

'Days of deaths: $5,7,13$.

fDays of deaths: 5, 6, 6, 6, 7 .

Absolute lung weights of females exposed to 10 or $20 \mathrm{mg} / \mathrm{m}^{3}$ and the relative lung weights of both sexes exposed to $10 \mathrm{mg} / \mathrm{m}^{3}$ and females exposed to $20 \mathrm{mg} / \mathrm{m}^{3}$ were significantly greater than those of the chamber controls (Table 3 and Table G-1). Absolute and relative liver weights of males exposed to $2.5 \mathrm{mg} / \mathrm{m}^{3}$ or greater and absolute liver weights of females exposed to $5 \mathrm{mg} / \mathrm{m}^{3}$ or greater were significantly less than those of the chamber controls. The relative liver weight of $20 \mathrm{mg} / \mathrm{m}^{3}$ females was significantly greater than that of the chamber controls. Absolute kidney and thymus weights of males exposed to $10 \mathrm{mg} / \mathrm{m}^{3}$ and females exposed to $20 \mathrm{mg} / \mathrm{m}^{3}$.

Table 3. Selected Organ Weights and Organ-Weight-to-Body-Weight Ratios for Rats in the Twoweek Inhalation Study of Cobalt Metal ${ }^{\mathrm{a}}$

\begin{tabular}{|c|c|c|c|c|c|c|}
\hline & $\begin{array}{l}\text { Chamber } \\
\text { Control }\end{array}$ & $2.5 \mathrm{mg} / \mathrm{m}^{3}$ & $5 \mathrm{mg} / \mathrm{m}^{3}$ & $10 \mathrm{mg} / \mathrm{m}^{3}$ & $20 \mathrm{mg} / \mathrm{m}^{3}$ & $40 \mathrm{mg} / \mathrm{m}^{3}$ \\
\hline \multicolumn{7}{|l|}{ Male } \\
\hline $\mathbf{n}$ & 5 & 5 & 5 & 5 & 0 & 0 \\
\hline $\begin{array}{l}\text { Necropsy } \\
\text { body wt }\end{array}$ & $144 \pm 3$ & $144 \pm 2$ & $140 \pm 4$ & $115 \pm 6^{* *}$ & - & - \\
\hline \multicolumn{7}{|l|}{ L. Kidney } \\
\hline Absolute & $0.61 \pm 0.02$ & $0.61 \pm 0.01$ & $0.58 \pm 0.01$ & $0.52 \pm 0.02 * *$ & - & - \\
\hline Relative & $4.25 \pm 0.06$ & $4.26 \pm 0.08$ & $4.12 \pm 0.09$ & $4.57 \pm 0.10^{*}$ & - & - \\
\hline \multicolumn{7}{|l|}{ Liver } \\
\hline Absolute & $5.84 \pm 0.16$ & $5.10 \pm 0.09^{* *}$ & $5.08 \pm 0.15^{* *}$ & $4.29 \pm 0.24 * *$ & - & - \\
\hline Relative & $40.61 \pm 0.46$ & $35.40 \pm 0.28 * *$ & $36.35 \pm 0.63 * *$ & $37.43 \pm 0.86^{* *}$ & - & - \\
\hline \multicolumn{7}{|l|}{ Lung } \\
\hline Absolute & $1.14 \pm 0.10$ & $1.16 \pm 0.08$ & $1.19 \pm 0.04$ & $1.28 \pm 0.12$ & - & - \\
\hline Relative & $7.91 \pm 0.61$ & $8.07 \pm 0.55$ & $8.49 \pm 0.30$ & $11.13 \mathrm{f} \pm 0.50^{* *}$ & - & - \\
\hline \multicolumn{7}{|l|}{ L. Testis } \\
\hline Absolute & $0.886 \pm 0.040$ & $0.928 \pm 0.017$ & $0.852 \pm 0.035$ & $0.590 \pm 0.088^{* *}$ & - & - \\
\hline Relative & $6.165 \pm 0.246$ & $6.446 \pm 0.155$ & $6.103 \pm 0.248$ & $5.053 \pm 0.502$ & - & - \\
\hline
\end{tabular}

Thymus

$\begin{array}{lllll}\text { Absolute } & 0.374 \pm 0.013 & 0.358 \pm 0.025 & 0.358 \pm 0.007 & 0.284 \pm 0.008^{* *}\end{array}$ 


\begin{tabular}{|c|c|c|c|c|c|c|}
\hline & $\begin{array}{l}\text { Chamber } \\
\text { Control }\end{array}$ & $2.5 \mathrm{mg} / \mathrm{m}^{3}$ & $5 \mathrm{mg} / \mathrm{m}^{3}$ & $10 \mathrm{mg} / \mathrm{m}^{3}$ & $20 \mathrm{mg} / \mathrm{m}^{3}$ & $40 \mathrm{mg} / \mathrm{m}^{3}$ \\
\hline Relative & $2.605 \pm 0.054$ & $2.485 \pm 0.161$ & $2.560 \pm 0.023$ & $2.498 \pm 0.112$ & - & - \\
\hline \multicolumn{7}{|l|}{ Female } \\
\hline $\mathbf{n}$ & 5 & 5 & 5 & 5 & 2 & 0 \\
\hline $\begin{array}{l}\text { Necropsy } \\
\text { body wt }\end{array}$ & $112 \pm 4$ & $112 \pm 2$ & $107 \pm 3$ & $98 \pm 4 * *$ & $61 \pm 5^{* *}$ & - \\
\hline \multicolumn{7}{|l|}{ L. Kidney } \\
\hline Absolute & $0.52 \pm 0.02$ & $0.50 \pm 0.01$ & $0.50 \pm 0.02$ & $0.46 \pm 0.01 *$ & $0.35 \pm 0.00^{* *}$ & - \\
\hline Relative & $4.66 \pm 0.11$ & $4.46 \pm 0.05$ & $4.63 \pm 0.08$ & $4.74 \pm 0.12$ & $5.75 \pm 0.42 * *$ & - \\
\hline \multicolumn{7}{|l|}{ Liver } \\
\hline Absolute & $4.07 \pm 0.16$ & $3.77 \pm 0.05$ & $3.61 \pm 0.13^{* *}$ & $3.44 \pm 0.05^{* *}$ & $2.57 \pm 0.06^{* *}$ & - \\
\hline Relative & $36.37 \pm 0.49$ & $33.59 \pm 0.16$ & $33.78 \pm 1.08$ & $35.17 \pm 1.00$ & $42.15 \pm 2.12^{* *}$ & - \\
\hline \multicolumn{7}{|l|}{ Lung } \\
\hline Absolute & $0.86 \pm 0.04$ & $0.83 \pm 0.01$ & $0.91 \pm 0.04$ & $1.03 \pm 0.06^{*}$ & $1.01 \pm 0.04^{*}$ & - \\
\hline Relative & $7.71 \pm 0.36$ & $7.44 \pm 0.07$ & $8.49 \pm 0.34$ & $10.54 \pm 0.69^{* *}$ & $16.54 \pm 0.56^{* *}$ & - \\
\hline \multicolumn{7}{|l|}{ Thymus } \\
\hline Absolute & $0.317 \pm 0.016$ & $0.324 \pm 0.011$ & $0.352 \pm 0.022$ & $0.289 \pm 0.011$ & $0.064 \pm 0.016^{* *}$ & - \\
\hline Relative & $2.842 \pm 0.167$ & $2.895 \pm 0.126$ & $3.289 \pm 0.201$ & $2.948 \pm 0.092$ & $1.024 \pm 0.178^{* *}$ & - \\
\hline
\end{tabular}

*Significantly different $(\mathrm{P} \leq 0.05)$ from the chamber control group by Williams' or Dunnett's test.

$* * \mathrm{P} \leq 0.01$.

${ }^{a}$ Organ weights (absolute weights) and body weights are given in grams; organ-weight-to-body-weight ratios (relative weights) are given as mg organ weight $/ \mathrm{g}$ body weight (mean \pm standard error). No data are available for $20 \mathrm{mg} / \mathrm{m}^{3} \mathrm{males}$ or $40 \mathrm{mg} / \mathrm{m}^{3}$ males or females due to $100 \%$ mortality.and the relative thymus weights of females exposed to $20 \mathrm{mg} / \mathrm{m}^{3}$ were significantly less than those of the chamber controls. Absolute testis weight of the $10 \mathrm{mg} / \mathrm{m}^{3}$ group was significantly less than that of the chamber controls; no histopathologic assessment was performed.

Increased incidences of nonneoplastic lesions of the lung occurred in exposed male and female rats, and the lesions were generally of minimal to mild severity (Table 4). The incidences of minimal to moderate hemorrhage were significantly increased in male rats exposed to 20 or $40 \mathrm{mg} / \mathrm{m}^{3}$ and females exposed to $40 \mathrm{mg} / \mathrm{m}^{3}$. Hemorrhage was observed in rats that died or were killed moribund and consisted of erythrocytes within the alveoli. Incidences of minimal to mild acute inflammation were significantly increased in males exposed to 20 or $40 \mathrm{mg} / \mathrm{m}^{3}$ and females exposed to $40 \mathrm{mg} / \mathrm{m}^{3}$. Inflammation consisted of proteinaceous fluid (edema) and infiltrates of neutrophils within the alveolar septa and spaces. Incidences of minimal to mild alveolar epithelium hyperplasia were increased in male and female rats exposed to 20 or $40 \mathrm{mg} / \mathrm{m}^{3}$; the incidence in $40 \mathrm{mg} / \mathrm{m}^{3}$ males was significantly increased. Hyperplasia consisted of focal, variably sized proliferation of the cuboidal to polygonal epithelial (Type II) cells along the alveolar septa; cell nuclei varied from round to pleomorphic. Incidences of alveolus histiocytic cellular infiltration were generally significantly increased in males exposed to $5 \mathrm{mg} / \mathrm{m}^{3}$ or greater and in females exposed to 20 or $40 \mathrm{mg} / \mathrm{m}^{3}$. Histiocyte infiltration consisted of increased numbers of foamy histiocytes (macrophages) within alveolar spaces. Incidences of cytoplasmic vacuolization of bronchiolar epithelium were significantly increased in $2.5,5$, or $10 \mathrm{mg} / \mathrm{m}^{3}$ males and females. Affected bronchiolar epithelial cells were enlarged and had a foamy appearance due 
to the accumulation of poorly demarcated cytoplasmic vacuoles. Incidences of necrosis of the bronchiolar epithelium were increased in males exposed to 20 or $40 \mathrm{mg} / \mathrm{m}^{3}$ and in females exposed to $10 \mathrm{mg} / \mathrm{m}^{3}$ or greater. Necrosis consisted of segmental to complete loss of the epithelium in terminal bronchioles; in some cases the remaining epithelial cells appeared hypereosinophilic. Minimal to moderate randomly scattered areas of interstial fibrosis of the alveolar septa were associated with areas of inflammation and alveolar epithelium hyperplasia in males and females exposed to 10 or $20 \mathrm{mg} / \mathrm{m}^{3}$. Fibrosis consisted of irregular expansion of the interstium of the alveolar septa by variable amounts of collagen fibers.

Increased incidences of nonneoplastic lesions of the nose occurred in exposed male and female rats (Table 4). Incidences of minimal to moderate olfactory epithelium necrosis were significantly increased in most exposed groups of rats. Necrosis consisted of vacuolization and disorganization of the olfactory epithelium lining the dorsal meatus in Levels II and III nasal sections and the medial surface of ethmoturbinates in Level III; in some cases there was partial or complete loss of the epithelium. The incidences of olfactory epithelium atrophy were significantly increased in all groups of rats exposed to $2.5,5$, or $10 \mathrm{mg} / \mathrm{m}^{3}$ and in females exposed to $20 \mathrm{mg} / \mathrm{m}^{3}$. Atrophy accompanied necrosis and appeared as decreased numbers of layers and density of olfactory epithelial cells in the areas adjacent to necrosis. The remaining olfactory cells were often disorganized with clear spaces between rows of neuronal and sustentacular epithelial cells. The incidences of respiratory epithelium necrosis were significantly increased in $40 \mathrm{mg} / \mathrm{m}^{3}$ males and females exposed to 20 or $40 \mathrm{mg} / \mathrm{m}^{3}$. Necrosis was a subtle lesion characterized by focal degeneration and loss of the mucosal epithelium; in some cases, a decrease in the height (atrophy) of the epithelium resulted. In some areas, a single layer of flat epithelial cells covered areas of degenerated epithelium and was considered an attempt at epithelial regeneration. In a few other areas, a thin layer of squamous epithelium replaced the olfactory and respiratory epithelia and was diagnosed as respiratory epithelium squamous metaplasia.

Table 4. Incidences of Selected Nonneoplastic Lesions of the Respiratory System in Rats in the Two-week Inhalation Study of Cobalt Metal

\begin{tabular}{|c|c|c|c|c|c|c|}
\hline & $\begin{array}{l}\text { Chamber } \\
\text { Control }\end{array}$ & $2.5 \mathrm{mg} / \mathrm{m}^{3}$ & $5 \mathrm{mg} / \mathrm{m}^{3}$ & $10 \mathrm{mg} / \mathrm{m}^{3}$ & $20 \mathrm{mg} / \mathrm{m}^{3}$ & $40 \mathrm{mg} / \mathrm{m}^{3}$ \\
\hline \multicolumn{7}{|l|}{ Male } \\
\hline Lung $^{\mathrm{a}}$ & 5 & 5 & 5 & 5 & 5 & 5 \\
\hline Hemorrhage $^{\mathrm{b}}$ & 0 & 0 & 0 & $1(1.0)^{\mathrm{c}}$ & $5 * *(1.2)$ & $5 * *(3.0)$ \\
\hline Inflammation, Acute & 0 & 0 & 0 & 0 & $4 *(1.3)$ & $5 * *(2.0)$ \\
\hline $\begin{array}{l}\text { Alveolar Epithelium, } \\
\text { Hyperplasia }\end{array}$ & 0 & 0 & 0 & 0 & $3(1.7)$ & $5 * *(1.4)$ \\
\hline $\begin{array}{l}\text { Alveolus, Infiltration } \\
\text { Cellular, Histiocyte }\end{array}$ & 0 & 0 & $4 *(1.0)$ & $3(1.3)$ & $5 * *(2.0)$ & $5 * *(1.2)$ \\
\hline $\begin{array}{l}\text { Bronchiole, Epithelium, } \\
\text { Vacuolization } \\
\text { Cytoplasmic }\end{array}$ & 0 & $5 * *(1.2)$ & $5 * *(1.6)$ & $5 * *(2.0)$ & $1(2.0)$ & 0 \\
\hline $\begin{array}{l}\text { Bronchiole, Epithelium, } \\
\text { Necrosis }\end{array}$ & 0 & 0 & 0 & 0 & $2(1.0)$ & $3(1.0)$ \\
\hline
\end{tabular}


Cobalt Metal, NTP TR 581

\begin{tabular}{|c|c|c|c|c|c|c|}
\hline & $\begin{array}{l}\text { Chamber } \\
\text { Control }\end{array}$ & $2.5 \mathrm{mg} / \mathrm{m}^{3}$ & $5 \mathrm{mg} / \mathrm{m}^{3}$ & $10 \mathrm{mg} / \mathrm{m}^{3}$ & $20 \mathrm{mg} / \mathrm{m}^{3}$ & $40 \mathrm{mg} / \mathrm{m}^{3}$ \\
\hline Interstitium, Fibrosis & 0 & 0 & 0 & $5 * *(1.2)$ & $2(3.0)$ & 0 \\
\hline Nose & 5 & 5 & 5 & 5 & 5 & 5 \\
\hline $\begin{array}{l}\text { Olfactory Epithelium, } \\
\text { Necrosis }\end{array}$ & 0 & $3(1.0)$ & $4 *(1.3)$ & $4 *(1.0)$ & $4 *(2.8)$ & $5 * *(3.0)$ \\
\hline $\begin{array}{l}\text { Olfactory Epithelium, } \\
\text { Atrophy }\end{array}$ & 0 & $5 * *(1.6)$ & $5 * *(1.8)$ & $5 * *(2.4)$ & $3(1.7)$ & $3(1.7)$ \\
\hline $\begin{array}{l}\text { Respiratory Epithelium, } \\
\text { Necrosis }\end{array}$ & 0 & 0 & 0 & $1(1.0)$ & $3(1.3)$ & $5 * *(1.4)$ \\
\hline $\begin{array}{l}\text { Respiratory Epithelium, } \\
\text { Metaplasia, Squamous }\end{array}$ & 0 & 0 & 0 & $1(1.0)$ & $2(1.0)$ & $1(1.0)$ \\
\hline \multicolumn{7}{|l|}{ Female } \\
\hline Lung & 5 & 5 & 5 & 5 & 5 & 5 \\
\hline Hemorrhage & 0 & 0 & 0 & 0 & $3(2.0)$ & $5 * *(2.8)$ \\
\hline Inflammation, Acute & 0 & 0 & 0 & 0 & $2(1.0)$ & $5 * *(1.4)$ \\
\hline $\begin{array}{l}\text { Alveolar Epithelium, } \\
\text { Hyperplasia }\end{array}$ & 0 & 0 & 0 & 0 & $2(1.0)$ & $2(1.0)$ \\
\hline $\begin{array}{l}\text { Alveolus, Infiltration } \\
\text { Cellular, Histiocyte }\end{array}$ & 0 & 0 & 0 & 0 & $5 * *(2.0)$ & $5 * *(1.8)$ \\
\hline $\begin{array}{l}\text { Bronchiole, Epithelium, } \\
\text { Vacuolization } \\
\text { Cytoplasmic }\end{array}$ & 0 & $4 *(1.0)$ & $5 * *(1.0)$ & $5 * *(1.8)$ & $3(1.7)$ & 0 \\
\hline $\begin{array}{l}\text { Bronchiole, Epithelium, } \\
\text { Necrosis }\end{array}$ & 0 & $1(1.0)$ & $1(1.0)$ & $4 *(1.0)$ & $3(1.0)$ & $3(1.0)$ \\
\hline Interstitium, Fibrosis & 0 & 0 & 0 & $4 *(1.0)$ & $3(3.0)$ & 0 \\
\hline Nose & 5 & 5 & 5 & 5 & 5 & 5 \\
\hline $\begin{array}{l}\text { Olfactory Epithelium, } \\
\text { Necrosis }\end{array}$ & 0 & $5 * *(1.0)$ & $3(1.0)$ & $5 * *(1.0)$ & $5 * *(2.0)$ & $5 * *(3.0)$ \\
\hline $\begin{array}{l}\text { Olfactory Epithelium, } \\
\text { Atrophy }\end{array}$ & 0 & $5 * *(1.8)$ & $5 * *(2.0)$ & $5 * *(2.0)$ & $4 *(2.8)$ & $1(2.0)$ \\
\hline $\begin{array}{l}\text { Respiratory Epithelium, } \\
\text { Necrosis }\end{array}$ & 0 & 0 & 0 & 0 & $5 * *(1.4)$ & $5 * *(1.4)$ \\
\hline $\begin{array}{l}\text { Respiratory Epithelium, } \\
\text { Metaplasia, Squamous }\end{array}$ & 0 & 0 & 0 & 0 & $1(1.0)$ & 0 \\
\hline
\end{tabular}

*Significantly different $(\mathrm{P} \leq 0.05)$ from the chamber control group by the Fisher exact test.

$* * \mathrm{P} \leq 0.01$.

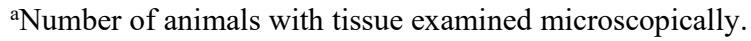

${ }^{b}$ Number of animals with lesion.

${ }^{\mathrm{c}}$ Average severity grade of lesions in affected animals: $1=$ minimal, $2=$ mild, $3=$ moderate, $4=$ marked.

\section{Tissue Burden Studies}

Tissue weights and concentrations were determined in male and female rats at terminal kill and in additional female rats held for 3 weeks postexposure (Appendix I). Data were generated on 
male rats exposed to $10 \mathrm{mg} / \mathrm{m}^{3}$ or less due to mortality at $20 \mathrm{mg} / \mathrm{m}^{3}$. In females, data were generated on all exposure groups; however, a relatively small number of samples ( $n=1$ to 3 ) was available in $20 \mathrm{mg} / \mathrm{m}^{3}$ females due to decreased survival.

Male and female rat lung weights increased with increasing exposure concentration at terminal kill and in females held for the 3-week recovery period (Table I-1); these increases were significant at higher exposure concentrations in females. In general, kidney, liver, heart, and femur weights decreased with increasing exposure concentration in males and females; some of these decreases were significant at higher exposure concentrations. In males exposed to $10 \mathrm{mg} / \mathrm{m}^{3}$, testis weights were decreased in comparison to chamber controls. Because of the significant changes in female lung weights, lung burdens rather than concentrations were evaluated for toxicokinetic parameters.

At terminal kill, cobalt concentrations and burdens increased with increasing exposure concentration in all tissues examined (Table I-1). In general, normalized burdens did not increase with increasing exposure concentration, with the exception of the liver in males and females. Cobalt concentrations in tissues decreased in the order of lung $>$ liver $>$ kidney $>$ femur $>$ heart $>$ serum $>$ blood $\sim$ testes (males). Cobalt burdens in the tissues of male and female rats decreased in the order of liver $>$ lung $>$ kidney $>$ heart $>$ femur $\sim$ testes (males). These data indicate that the tissues examined tended to accumulate cobalt at concentrations greater than could be found in blood and serum, that cobalt was distributed to extra-pulmonary tissues, and that more cobalt accumulated in the liver than in the lung, particularly at the higher concentrations. At 3 weeks postexposure in female rats, cobalt concentrations were markedly reduced in blood, serum, and lung.

Kinetic analysis of data from female rats exposed to $20 \mathrm{mg} / \mathrm{m}^{3}$ or less indicated elimination halflives of 9.2 to 11.1 days (blood), 2.8 to 3.4 days (serum; 10 and $20 \mathrm{mg} / \mathrm{m}^{3}$ only, due to undetectable serum con-centrations of cobalt at lower exposure concentrations at 3 weeks postexposure) and 4.2 to 5.6 days (lung) (Table I-2). Lung cobalt deposition rates and predicted steady-state lung cobalt burdens generally increased less than proportionally across exposure concentrations except when comparing 10 and $20 \mathrm{mg} / \mathrm{m}^{3}$.

In general, the volume of urine collected from male and female rats during the 16-hour collection period after exposure on day 12 decreased with increasing exposure concentration (Table I-3). Increased creatinine concentrations were observed in both sexes in the higher exposure concentration groups. Urinary cobalt concentration increased with increasing exposure concentration in both sexes. When normalized to creatinine, cobalt concentrations increased approximately in proportion to exposure concentration. Total cobalt excreted increased with exposure at lower concentrations before decreasing at higher concentrations.

Exposure Concentration Selection Rationale: Based on significant mortality in male and female rats exposed to 20 and $40 \mathrm{mg} / \mathrm{m}^{3}$ and body weight reductions in the $10 \mathrm{mg} / \mathrm{m}^{3}$ groups coupled with reduced urine volumes with concomitant increases in urine creatinine at the end of the 2week study, $5 \mathrm{mg} / \mathrm{m}^{3}$ was selected as the highest exposure concentration for the 3-month inhalation study in rats. The lesions in the nose were minimal in the $5 \mathrm{mg} / \mathrm{m}^{3}$ group and were not considered sufficiently severe to preclude the use of this concentration. When exposure concentrations for the 3-month studies are different for two species, NTP has elected to have one less exposure concentration for one species as opposed to adding an extra chamber to 
accommodate the differences in species. Hence, only four concentrations were used in the 3month rat studies.

\section{Three-month Study}

All male and female rats survived to the end of the study (Table 5). The final mean body weights of males and females exposed to $5 \mathrm{mg} / \mathrm{m}^{3}$ were significantly less than those of the chamber controls, and the mean body weight gain of $5 \mathrm{mg} / \mathrm{m}^{3}$ males was significantly less than that of the chamber controls (Table 5 and Figure 1). There were no clinical signs related to cobalt metal exposure. At necropsy, pale foci were noted in the lungs of most exposed male and female rats. Based on reports in the literature describing the ability of cobalt to decrease hepatic cytochrome P450 levels and activity ${ }^{9}$, these enzymes were assayed in the current study. On days 26 and 40, microsomal suspensions of liver samples from special study female rats not used for tissue burden studies were prepared and acetanilide-4-hydroxylase $(\mathrm{A} 4 \mathrm{H}), 7$-ethoxyresorufin-Odeethylase (EROD), and 7-pentoxyresorufin- $O$-deethylase (PROD) activities were determined (Table J-1). There were no consistent trends in A4H, EROD, or PROD activities relative to exposure concentrations at either time point.

Table 5. Survival and Body Weights of Rats in the Three-month Inhalation Study of Cobalt Metal ${ }^{\mathrm{a}}$

\begin{tabular}{|c|c|c|c|c|c|}
\hline $\begin{array}{c}\text { Concentration } \\
\left(\mathbf{m g} / \mathrm{m}^{3}\right)\end{array}$ & Survival $^{\mathbf{b}}$ & $\begin{array}{c}\text { Initial Body } \\
\text { Weight } \\
\text { (g) }\end{array}$ & $\begin{array}{l}\text { Final Body } \\
\text { Weight } \\
\text { (g) }\end{array}$ & $\begin{array}{l}\text { Change in Body } \\
\text { Weight } \\
\text { (g) }\end{array}$ & $\begin{array}{c}\text { Final Weight } \\
\text { Relative } \\
\text { to Controls } \\
(\%)\end{array}$ \\
\hline \multicolumn{6}{|l|}{ Male } \\
\hline 0 & $10 / 10$ & $107 \pm 2$ & $319 \pm 5$ & $212 \pm 4$ & \\
\hline 0.625 & $10 / 10$ & $107 \pm 3$ & $336 \pm 6$ & $229 \pm 4$ & 105 \\
\hline 1.25 & $10 / 10$ & $107 \pm 2$ & $327 \pm 7$ & $220 \pm 6$ & 102 \\
\hline 2.5 & $10 / 10$ & $107 \pm 3$ & $326 \pm 6$ & $220 \pm 5$ & 102 \\
\hline 5 & $10 / 10$ & $107 \pm 3$ & $297 \pm 5^{*}$ & $190 \pm 4^{* *}$ & 93 \\
\hline \multicolumn{6}{|l|}{ Female } \\
\hline 0 & $10 / 10$ & $88 \pm 3$ & $201 \pm 3$ & $113 \pm 4$ & \\
\hline 0.625 & $10 / 10$ & $88 \pm 3$ & $205 \pm 4$ & $117 \pm 5$ & 102 \\
\hline 1.25 & $10 / 10$ & $89 \pm 3$ & $198 \pm 4$ & $109 \pm 2$ & 98 \\
\hline 2.5 & $10 / 10$ & $88 \pm 2$ & $199 \pm 4$ & $111 \pm 3$ & 99 \\
\hline 5 & $10 / 10$ & $87 \pm 2$ & $187 \pm 3^{*}$ & $100 \pm 4$ & 93 \\
\hline
\end{tabular}

*Significantly different $(\mathrm{P} \leq 0.05)$ from the chamber control group by Williams' or Dunnett's test.

$* * \mathrm{P} \leq 0.01$.

${ }^{a}$ Weights and weight changes are given as mean \pm standard error.

${ }^{b}$ Number of animals surviving at 3 months/number initially in group. 

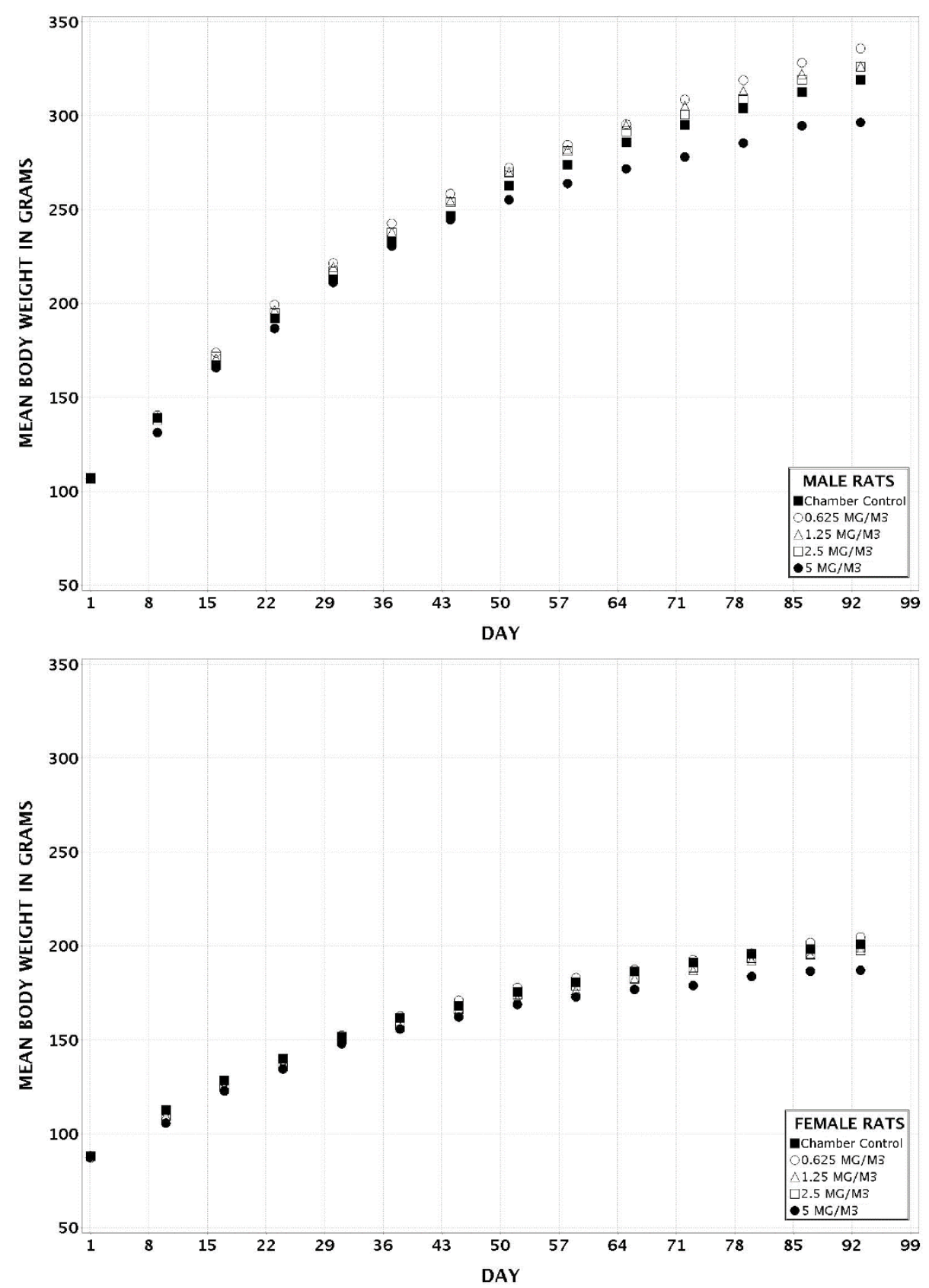

Figure 1. Growth Curves for Rats Exposed to Cobalt Metal by Inhalation for Three Months

The hematology and clinical chemistry data for rats are presented in Table 6 and Table F-1. Erythrocytosis characterized by exposure concentration-related increases in the hemoglobin concentration, erythrocyte count, hematocrit value, and manual packed cell volume occurred in males exposed to 2.5 and $5 \mathrm{mg} / \mathrm{m}^{3}$ on days 3 and 23 and all exposed groups by week 14; at week 14, female rats also had increases in these parameters. Animals in the lower exposure groups demonstrated these increases but less consistently. In addition, reticulocyte counts were increased in $5 \mathrm{mg} / \mathrm{m}^{3}$ males at all three time points, while in the female rats, reticulocyte counts were increased in all exposed groups on day 23 and in the 2.5 and $5 \mathrm{mg} / \mathrm{m}^{3}$ groups at week 14 . At week 14, platelet counts were mildly $(<18 \%)$ decreased in all of the exposed groups of 
females and in males exposed to $1.25 \mathrm{mg} / \mathrm{m}^{3}$ or greater. These platelet count changes may represent an altered peripheral distribution or decreased production. All other hematology changes observed were considered within biological variability and not toxicologically relevant.

At week 14, total protein concentrations were mildly decreased $(<10 \%)$ in 2.5 and $5 \mathrm{mg} / \mathrm{m}^{3}$ females, as well as $5 \mathrm{mg} / \mathrm{m}^{3}$ males. This change was paired with mild decreases in albumin and globulin concentrations in $5 \mathrm{mg} / \mathrm{m}^{3}$ females and a mild decrease in globulin concentration in $5 \mathrm{mg} / \mathrm{m}^{3}$ males. These changes are most likely related to altered food intake compared to concurrent chamber controls and are supported by the mild decreases (7\%) in body weights of $5 \mathrm{mg} / \mathrm{m}^{3}$ males and females. Exposure concentration-dependent decreases in cholesterol concentrations were observed at all three time points in both males and females. While this change was not always observed with the lower exposure concentrations, these decreases were consistently observed in the 2.5 and $5 \mathrm{mg} / \mathrm{m}^{3}$ groups of both sexes on day 23 and at week 14 . In addition, glucose concentration was decreased in $1.25 \mathrm{mg} / \mathrm{m}^{3}$ or greater males at week 14 . All other biochemical changes were transient or inconsistent and not considered toxicologically relevant.

Absolute and relative lung weights of all exposed groups of males and females were significantly greater than those of the chamber controls (Table G-2). The increased lung weights are related to the histopathologic changes observed in the lungs.

Table 6. Selected Clinical Pathology Data for Rats in the Three-month Inhalation Study of Cobalt Metal $^{\mathrm{a}}$

\begin{tabular}{|c|c|c|c|c|c|}
\hline & $\begin{array}{c}\text { Chamber } \\
\text { Control }\end{array}$ & $0.625 \mathrm{mg} / \mathrm{m}^{3}$ & $1.25 \mathrm{mg} / \mathrm{m}^{3}$ & $2.5 \mathrm{mg} / \mathrm{m}^{3}$ & $5 \mathrm{mg} / \mathrm{m}^{3}$ \\
\hline \multicolumn{6}{|l|}{ Male } \\
\hline \multicolumn{6}{|l|}{ Hematology } \\
\hline \multicolumn{6}{|l|}{$\mathbf{n}$} \\
\hline Day 3 & 10 & 10 & 10 & 10 & 10 \\
\hline Day 23 & 10 & 10 & 10 & 9 & 10 \\
\hline Week 14 & 10 & 10 & 10 & 10 & 10 \\
\hline \multicolumn{6}{|c|}{ Hematocrit (spun) (\%) } \\
\hline Day 3 & $46.5 \pm 0.2$ & $46.4 \pm 0.2$ & $47.4 \pm 0.2 *$ & $47.7 \pm 0.3^{* *}$ & $51.2 \pm 0.4^{* *}$ \\
\hline Day 23 & $47.9 \pm 0.4$ & $48.5 \pm 0.4$ & $49.9 \pm 0.5^{* *}$ & $51.1 \pm 0.2^{* *}$ & $53.3 \pm 0.4 * *$ \\
\hline Week 14 & $49.6 \pm 0.4$ & $51.6 \pm 0.4^{* *}$ & $59.2 \pm 0.4^{* *}$ & $61.8 \pm 0.2^{* *}$ & $63.9 \pm 0.3 * *$ \\
\hline \multicolumn{6}{|c|}{ Packed cell volume (mL/dL) } \\
\hline Day 3 & $45.1 \pm 0.2$ & $44.5 \pm 0.3$ & $45.7 \pm 0.3$ & $46.1 \pm 0.4^{*}$ & $49.7 \pm 0.4 * *$ \\
\hline Day 23 & $46.8 \pm 0.4$ & $47.3 \pm 0.4$ & $49.0 \pm 0.6^{* *}$ & $49.7 \pm 0.2^{* *}$ & $51.5 \pm 0.5^{* *}$ \\
\hline Week 14 & $49.6 \pm 0.5$ & $51.7 \pm 0.4 * *$ & $58.3 \pm 0.4^{* *}$ & $60.7 \pm 0.1 * *$ & $62.9 \pm 0.3 * *$ \\
\hline \multicolumn{6}{|c|}{ Hemoglobin (g/dL) } \\
\hline Day 3 & $13.7 \pm 0.1$ & $13.8 \pm 0.1$ & $14.1 \pm 0.1^{* *}$ & $14.2 \pm 0.1^{* *}$ & $15.5 \pm 0.1^{* *}$ \\
\hline Day 23 & $15.0 \pm 0.1$ & $14.9 \pm 0.1$ & $15.4 \pm 0.2$ & $15.8 \pm 0.1^{* *}$ & $16.1 \pm 0.2 * *$ \\
\hline
\end{tabular}




\section{Cobalt Metal, NTP TR 581}

\begin{tabular}{|c|c|c|c|c|c|}
\hline & $\begin{array}{c}\text { Chamber } \\
\text { Control }\end{array}$ & $0.625 \mathrm{mg} / \mathrm{m}^{3}$ & $1.25 \mathrm{mg} / \mathrm{m}^{3}$ & $2.5 \mathrm{mg} / \mathrm{m}^{3}$ & $5 \mathrm{mg} / \mathrm{m}^{3}$ \\
\hline Week 14 & $15.6 \pm 0.1$ & $16.3 \pm 0.1 * *$ & $18.7 \pm 0.1 * *$ & $19.7 \pm 0.1 * *$ & $20.3 \pm 0.1 * *$ \\
\hline \multicolumn{6}{|c|}{ Erythrocytes $\left(10^{6} / \mu \mathrm{L}\right)$} \\
\hline Day 3 & $7.30 \pm 0.06$ & $7.27 \pm 0.09$ & $7.46 \pm 0.05$ & $7.57 \pm 0.08^{*}$ & $8.22 \pm 0.08 * *$ \\
\hline Day 23 & $7.97 \pm 0.09$ & $8.05 \pm 0.09$ & $8.36 \pm 0.11 *$ & $8.44 \pm 0.06^{* *}$ & $9.27 \pm 0.14 * *$ \\
\hline Week 14 & $9.19 \pm 0.10$ & $9.67 \pm 0.09 * *$ & $11.20 \pm 0.06^{* *}$ & $11.80 \pm 0.06^{* *}$ & $11.90 \pm 0.08 * *$ \\
\hline \multicolumn{6}{|c|}{ Reticulocytes $\left(10^{3} / \mu \mathrm{L}\right)$} \\
\hline Day 3 & $612.0 \pm 42.3$ & $562.3 \pm 24.5^{\mathrm{b}}$ & $613.0 \pm 30.8$ & $622.1 \pm 25.6$ & $873.8 \pm 53.4 * *$ \\
\hline Day 23 & $262.9 \pm 13.9$ & $261.3 \pm 17.8$ & $280.9 \pm 14.3$ & $291.6 \pm 18.6$ & $339.6 \pm 25.1^{*}$ \\
\hline Week 14 & $219.8 \pm 16.0$ & $191.7 \pm 19.9$ & $189.3 \pm 13.1$ & $272.7 \pm 18.7$ & $360.0 \pm 35.9^{*}$ \\
\hline \multicolumn{6}{|c|}{ Platelets $\left(10^{3} / \mu \mathrm{L}\right)$} \\
\hline Day 3 & $899.7 \pm 14.0$ & $883.5 \pm 23.9$ & $939.4 \pm 14.4$ & $903.8 \pm 22.8$ & $1,123.7 \pm 34.2 * *$ \\
\hline Day 23 & $740.8 \pm 16.2$ & $712.0 \pm 16.3$ & $732.1 \pm 22.7$ & $682.0 \pm 44.5$ & $796.7 \pm 12.8$ \\
\hline Week 14 & $682.5 \pm 27.7$ & $646.8 \pm 8.7$ & $611.2 \pm 13.8^{*}$ & $548.2 \pm 22.3 * *$ & $573.2 \pm 15.3^{* *}$ \\
\hline
\end{tabular}

\section{Clinical Chemistry}

n

10

10

10

10

10

Total protein $(\mathrm{g} / \mathrm{dL})$

Day 3

Day 23

Week 14

Albumin (g/dL)

Day 3

Day 23

Week 14

Globulin $(\mathrm{g} / \mathrm{dL})$

Day 3

Day 23

Week 14

Cholesterol (mg/dL)

Day 3

Day 23

Week 14

Glucose (mg/dL)

Day 3

Day 23

Week 14
$6.1 \pm 0.1$

$6.5 \pm 0.1$

$7.4 \pm 0.1$

$4.3 \pm 0.0$

$4.6 \pm 0.1$

$4.9 \pm 0.1$

$1.8 \pm 0.0$

$2.0 \pm 0.0$

$2.5 \pm 0.0$

$95 \pm 2$

$79 \pm 1$

$91 \pm 1$

$135 \pm 2$

$132 \pm 3$

$126 \pm 3$
$5.9 \pm 0.0$

$6.4 \pm 0.1$

$7.3 \pm 0.0$

$4.1 \pm 0.0^{* *}$

$4.5 \pm 0.0$

$4.8 \pm 0.0$

$1.7 \pm 0.0$

$2.0 \pm 0.0$

$2.5 \pm 0.0$

$87 \pm 1^{*}$

$76 \pm 2$

$91 \pm 1$

$138 \pm 1$

$132 \pm 4$

$128 \pm 3$
$6.0 \pm 0.0$

$6.5 \pm 0.1$

$7.4 \pm 0.1$

$4.2 \pm 0.0$

$4.5 \pm 0.1$

$4.8 \pm 0.0$

$1.8 \pm 0.0$

$2.1 \pm 0.1$

$2.6 \pm 0.0$

$84 \pm 1^{* *}$

$70 \pm 1 * *$

$88 \pm 2$

$134 \pm 2$

$143 \pm 9$

$118 \pm 5^{*}$
$6.1 \pm 0.1$

$6.3 \pm 0.1 *$

$6.5 \pm 0.1$

$7.3 \pm 0.1$

$6.4 \pm 0.1$

$7.1 \pm 0.1 * *$

$4.3 \pm 0.0$

$4.3 \pm 0.0$

$4.5 \pm 0.0$

$4.4 \pm 0.0$

$4.8 \pm 0.0$

$4.8 \pm 0.0$

$2.0 \pm 0.0^{* *}$

$2.0 \pm 0.0$

$2.0 \pm 0.0$

$2.3 \pm 0.0 * *$

$2.5 \pm 0.0$

$79 \pm 2 * *$

$81 \pm 2^{* *}$

$73 \pm 2 * *$

$63 \pm 1 * *$

$79 \pm 2 * *$

$67 \pm 1^{* *}$

$129 \pm 4$

$120 \pm 2$

$139 \pm 8$

$104 \pm 4 * *$ 
Cobalt Metal, NTP TR 581

\begin{tabular}{|c|c|c|c|c|c|}
\hline & $\begin{array}{c}\text { Chamber } \\
\text { Control }\end{array}$ & $0.625 \mathrm{mg} / \mathrm{m}^{3}$ & $1.25 \mathrm{mg} / \mathrm{m}^{3}$ & $2.5 \mathrm{mg} / \mathrm{m}^{3}$ & $5 \mathrm{mg} / \mathrm{m}^{3}$ \\
\hline \multicolumn{6}{|c|}{ Female } \\
\hline $\mathbf{n}$ & 10 & 10 & 10 & 10 & 10 \\
\hline
\end{tabular}

\section{Hematology}

Hematocrit (spun) (\%)

\begin{tabular}{|c|c|c|c|c|c|}
\hline Day 3 & $49.0 \pm 0.6$ & $47.8 \pm 0.7$ & $47.6 \pm 0.5$ & $48.9 \pm 0.3$ & $49.9 \pm 0.5$ \\
\hline Day 23 & $51.6 \pm 0.5$ & $50.9 \pm 0.3$ & $51.0 \pm 0.3$ & $52.0 \pm 0.2$ & $53.3 \pm 1.9^{* *}$ \\
\hline Week 14 & $48.3 \pm 0.5$ & $48.7 \pm 0.4$ & $52.6 \pm 0.6^{* *}$ & $57.3 \pm 0.5^{* *}$ & $59.5 \pm 0.3^{* *}$ \\
\hline \multicolumn{6}{|c|}{ Packed cell volume (mL/dL) } \\
\hline Day 3 & $47.6 \pm 0.6$ & $46.7 \pm 0.6$ & $46.5 \pm 0.5$ & $47.6 \pm 0.3$ & $48.3 \pm 0.5$ \\
\hline Day 23 & $51.3 \pm 0.5$ & $50.2 \pm 0.3$ & $50.5 \pm 0.3$ & $51.0 \pm 0.3$ & $51.7 \pm 1.7$ \\
\hline Week 14 & $49.1 \pm 0.5$ & $49.5 \pm 0.4$ & $53.1 \pm 0.5^{* *}$ & $57.1 \pm 0.5^{* *}$ & $60.0 \pm 0.4^{* *}$ \\
\hline \multicolumn{6}{|c|}{ Hemoglobin (g/dL) } \\
\hline Day 3 & $14.8 \pm 0.2$ & $14.4 \pm 0.2$ & $14.5 \pm 0.2$ & $14.7 \pm 0.1$ & $15.2 \pm 0.2$ \\
\hline Day 23 & $16.1 \pm 0.1$ & $15.8 \pm 0.1$ & $16.0 \pm 0.1$ & $16.2 \pm 0.1$ & $16.4 \pm 0.6^{*}$ \\
\hline Week 14 & $15.5 \pm 0.2$ & $15.8 \pm 0.1^{*}$ & $16.9 \pm 0.2^{* *}$ & $18.3 \pm 0.1^{* *}$ & $19.1 \pm 0.1 * *$ \\
\hline \multicolumn{6}{|c|}{ Erythrocytes $\left(10^{6} / \mu \mathrm{L}\right)$} \\
\hline Day 3 & $7.79 \pm 0.12$ & $7.62 \pm 0.13$ & $7.65 \pm 0.09$ & $7.77 \pm 0.08$ & $8.07 \pm 0.12$ \\
\hline Day 23 & $8.49 \pm 0.09$ & $8.24 \pm 0.07$ & $8.43 \pm 0.10$ & $8.56 \pm 0.05$ & $8.75 \pm 0.31 *$ \\
\hline Week 14 & $8.55 \pm 0.09$ & $8.69 \pm 0.06$ & $9.30 \pm 0.10^{* *}$ & $10.05 \pm 0.07 * *$ & $10.47 \pm 0.09$ \\
\hline
\end{tabular}

Reticulocytes $\left(10^{3} / \mu \mathrm{L}\right)$

\begin{tabular}{lccccc} 
Day 3 & $489.3 \pm 22.4$ & $497.7 \pm 30.8$ & $544.1 \pm 28.1$ & $511.9 \pm 25.5$ & $520.0 \pm 23.6$ \\
Day 23 & $184.1 \pm 11.1$ & $244.2 \pm 9.9^{* *}$ & $212.9 \pm 12.4^{*}$ & $276.7 \pm 18.9^{* *}$ & $386.6 \pm 20.4^{* *}$ \\
Week 14 & $202.5 \pm 13.0$ & $224.5 \pm 13.0$ & $222.2 \pm 8.8$ & $246.5 \pm 10.8^{*}$ & $316.7 \pm 25.0^{* *}$ \\
Platelets $\left(10^{3} / \mu \mathrm{L}\right)$ & & & & \\
Day 3 & $905.6 \pm 27.8$ & $841.4 \pm 22.5$ & $914.7 \pm 18.8$ & $912.4 \pm 20.1$ & $875.0 \pm 27.4$ \\
Day 23 & $783.1 \pm 20.5$ & $799.2 \pm 13.2$ & $763.0 \pm 25.7$ & $800.7 \pm 21.5$ & $807.9 \pm 22.2$ \\
Week 14 & $702.1 \pm 8.0$ & $660.2 \pm 14.7^{*}$ & $646.6 \pm 17.3^{* *}$ & $575.3 \pm 16.7^{* * \mathrm{~b}}$ & $608.0 \pm 16.8^{* *}$ \\
\hline
\end{tabular}

\section{Clinical Chemistry}

Total protein $(\mathrm{g} / \mathrm{dL})$

\begin{tabular}{lccccc} 
Day 3 & $6.2 \pm 0.1$ & $6.0 \pm 0.1$ & $6.1 \pm 0.1$ & $6.1 \pm 0.1$ & $6.2 \pm 0.0$ \\
Day 23 & $6.5 \pm 0.1$ & $6.5 \pm 0.1$ & $6.6 \pm 0.1$ & $6.5 \pm 0.1$ & $6.5 \pm 0.1$ \\
Week 14 & $7.6 \pm 0.1$ & $7.5 \pm 0.1$ & $7.4 \pm 0.1$ & $7.4 \pm 0.1^{*}$ & $7.0 \pm 0.1^{* *}$ \\
Albumin $(\mathrm{g} / \mathrm{dL})$ & & & & \\
Day 3 & $4.6 \pm 0.1$ & $4.4 \pm 0.0$ & $4.4 \pm 0.1$ & $4.5 \pm 0.0$ & $4.4 \pm 0.0$ \\
\hline
\end{tabular}




\begin{tabular}{|c|c|c|c|c|c|}
\hline & $\begin{array}{c}\text { Chamber } \\
\text { Control }\end{array}$ & $0.625 \mathrm{mg} / \mathrm{m}^{3}$ & $1.25 \mathrm{mg} / \mathrm{m}^{3}$ & $2.5 \mathrm{mg} / \mathrm{m}^{3}$ & $5 \mathrm{mg} / \mathrm{m}^{3}$ \\
\hline Day 23 & $4.7 \pm 0.0$ & $4.7 \pm 0.0$ & $4.7 \pm 0.0$ & $4.7 \pm 0.0$ & $4.7 \pm 0.1$ \\
\hline Week 14 & $5.3 \pm 0.1$ & $5.2 \pm 0.1$ & $5.2 \pm 0.1$ & $5.2 \pm 0.0$ & $5.0 \pm 0.1 * *$ \\
\hline \multicolumn{6}{|c|}{ Globulin (g/dL) } \\
\hline Day 3 & $1.6 \pm 0.0$ & $1.6 \pm 0.0$ & $1.7 \pm 0.1$ & $1.6 \pm 0.0$ & $1.8 \pm 0.0^{* *}$ \\
\hline Day 23 & $1.8 \pm 0.0$ & $1.8 \pm 0.0$ & $1.9 \pm 0.1$ & $1.9 \pm 0.0$ & $1.9 \pm 0.1$ \\
\hline Week 14 & $2.3 \pm 0.0$ & $2.3 \pm 0.0$ & $2.3 \pm 0.1$ & $2.3 \pm 0.0$ & $2.1 \pm 0.1 * *$ \\
\hline \multicolumn{6}{|c|}{ Cholesterol (mg/dL) } \\
\hline Day 3 & $98 \pm 2$ & $95 \pm 2$ & $91 \pm 2^{*}$ & $91 \pm 2 *$ & $93 \pm 2$ \\
\hline Day 23 & $102 \pm 3$ & $97 \pm 2$ & $97 \pm 2$ & $93 \pm 3^{*}$ & $89 \pm 3 * *$ \\
\hline Week 14 & $102 \pm 3$ & $100 \pm 2$ & $92 \pm 3^{*}$ & $91 \pm 3 * *$ & $76 \pm 2 * *$ \\
\hline
\end{tabular}

*Significantly different $(\mathrm{P} \leq 0.05)$ from the chamber control group by Dunn's or Shirley's test.

$* * \mathrm{P} \leq 0.01$.

${ }^{\text {a }}$ Data are presented as mean \pm standard error. Statistical tests were performed on unrounded data. $b_{n}=9$.

Sperm motility was significantly decreased in males exposed to $1.25,2.5$, or $5 \mathrm{mg} / \mathrm{m}^{3}$, and the decrease in the $5 \mathrm{mg} / \mathrm{m}^{3}$ group was approximately $8 \%$ (Table 7 and Table $\mathrm{H}-1$ ). The Markov transition matrix analyses of estrous cyclicity indicated that females in the $5 \mathrm{mg} / \mathrm{m}^{3}$ group had a significantly higher probability of extended diestrus than the chamber control females (Table H-2 and Table H-3; Figure H-1). The toxicologic significance of this subtle alteration in the estrous cycle is unclear and there were no cobalt-related histopathologic findings observed in the female reproductive organs.

In the lung, a spectrum of nonneoplastic lesions was observed that included chronic active inflammation, alveolus proteinosis, bronchiole epithelium hyperplasia, and alveolar epithelium hyperplasia (Table 8). Minimal to mild chronic active inflammation and generally minimal to mild alveolus proteinosis occurred in all exposed males and females, and minimal to mild bronchiole epithelium hyperplasia occurred in all males and females exposed to $1.25 \mathrm{mg} / \mathrm{m}^{3}$ or greater. The severities of these lesions generally increased with increasing exposure concentration. Chronic active inflammation consisted of a mixture of macrophages, neutrophils, and lymphocytes in the alveolar spaces and was sometimes associated with minimal hyperplasia of the alveolar epithelium and minimal fibrosis of the alveolar interstitium. At higher exposure concentrations, the inflammatory cells were more diffusely distributed throughout the lung. However, at the lower exposure concentrations, the inflammatory cells tended to occur in focal, subpleural aggregates. The pale foci noted grossly at necropsy correlated with subpleural inflammation. There were also perivascular and peribronchiolar infiltrates of macrophages, neutrophils, and lymphocytes with extension into the adjacent alveoli. Some males and females exposed to $5 \mathrm{mg} / \mathrm{m}^{3}$ had minimal infiltrates of inflammatory cells in the walls of bronchioles with minimal proliferation of fibrovascular tissue into the lumen. Alveolar proteinosis consisted of clumps of dense amorphous eosinophilic material or less dense, more dispersed accumulations of proteinaceous debris within the alveolar spaces. Alveoli also contained increased numbers of macrophages that contained eosinophilic material similar to that in the alveolar spaces. Hyperplasia of bronchiolar epithelium was characterized by proliferation of the epithelial cells 
lining terminal bronchioles and alveolar ducts. The hyperplastic cells were cuboidal with increased cytoplasm that sometimes contained poorly defined cytoplasmic vacuoles.

Table 7. Summary of Reproductive Tissue Evaluations for Male Rats in the Three-month Inhalation Study of Cobalt Metal ${ }^{\mathrm{a}}$

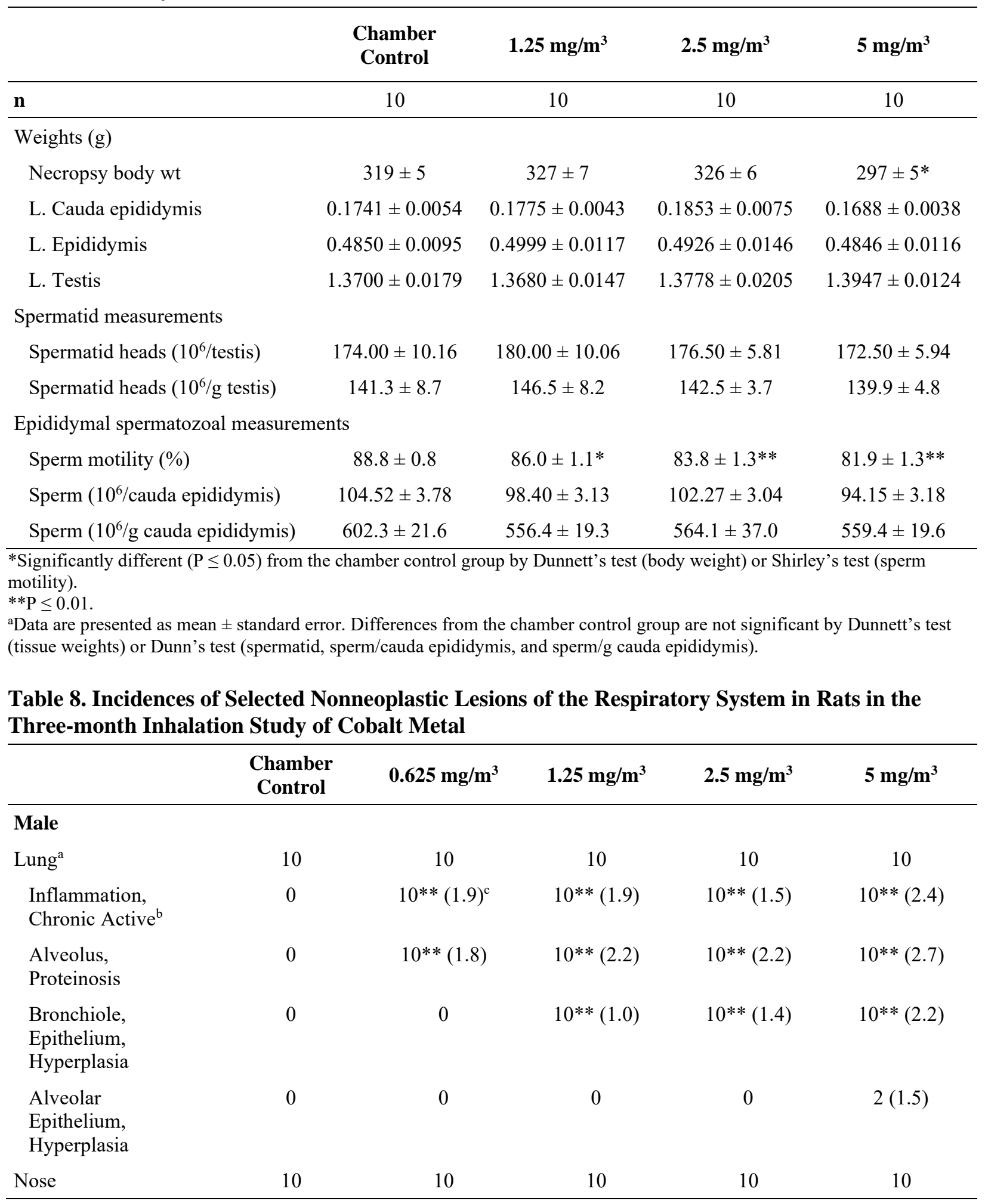


Cobalt Metal, NTP TR 581

\begin{tabular}{|c|c|c|c|c|c|}
\hline & $\begin{array}{c}\text { Chamber } \\
\text { Control }\end{array}$ & $0.625 \mathrm{mg} / \mathrm{m}^{3}$ & $1.25 \mathrm{mg} / \mathrm{m}^{3}$ & $2.5 \mathrm{mg} / \mathrm{m}^{3}$ & $5 \mathrm{mg} / \mathrm{m}^{3}$ \\
\hline $\begin{array}{l}\text { Olfactory } \\
\text { Epithelium, } \\
\text { Degeneration }\end{array}$ & 0 & 0 & $2(1.0)$ & $9 * *(1.0)$ & $10 * *(2.5)$ \\
\hline $\begin{array}{l}\text { Olfactory } \\
\text { Epithelium, } \\
\text { Hyperplasia }\end{array}$ & 0 & 0 & $2(1.0)$ & $6^{* *}(1.2)$ & $10^{* *}(1.7)$ \\
\hline $\begin{array}{l}\text { Respiratory } \\
\text { Epithelium, } \\
\text { Hyperplasia }\end{array}$ & 0 & 0 & $3(1.0)$ & $9 * *(1.0)$ & $10^{* *}(1.8)$ \\
\hline Turbinate, Atrophy & 0 & 0 & 0 & $3(1.0)$ & $9 * *(1.0)$ \\
\hline \multicolumn{6}{|l|}{ Female } \\
\hline Lung & 10 & 10 & 10 & 10 & 10 \\
\hline $\begin{array}{l}\text { Inflammation, } \\
\text { Chronic Active }\end{array}$ & $2(1.0)$ & $10^{* *}(1.9)$ & $10^{* *}(1.5)$ & $10^{* *}(1.6)$ & $10^{* *}(2.4)$ \\
\hline $\begin{array}{l}\text { Alveolus, } \\
\text { Proteinosis }\end{array}$ & 0 & $10^{* *}(1.8)$ & $10 * *(1.9)$ & $10^{* *}(1.9)$ & $10^{* *}(2.1)$ \\
\hline $\begin{array}{l}\text { Bronchiole, } \\
\text { Epithelium, } \\
\text { Hyperplasia }\end{array}$ & 0 & 0 & $10^{* *}(1.0)$ & $10^{* *}(1.3)$ & $10^{* *}(2.0)$ \\
\hline $\begin{array}{l}\text { Alveolar } \\
\text { Epithelium, } \\
\text { Hyperplasia }\end{array}$ & 0 & 0 & 0 & 0 & $1(1.0)$ \\
\hline Nose & 10 & 10 & 10 & 10 & 10 \\
\hline $\begin{array}{l}\text { Olfactory } \\
\text { Epithelium, } \\
\text { Degeneration }\end{array}$ & 0 & 0 & $5 *(1.0)$ & $10^{* *}(1.0)$ & $10^{* *}(2.5)$ \\
\hline $\begin{array}{l}\text { Olfactory } \\
\text { Epithelium, } \\
\text { Hyperplasia }\end{array}$ & 0 & 0 & 0 & $3(1.0)$ & $10^{* *}(2.2)$ \\
\hline $\begin{array}{l}\text { Respiratory } \\
\text { Epithelium, } \\
\text { Hyperplasia }\end{array}$ & 0 & $1(1.0)$ & 0 & $9 * *(1.0)$ & $10^{* *}(1.8)$ \\
\hline Turbinate, Atrophy & 0 & 0 & 0 & $4 *(1.0)$ & $6^{* *}(1.0)$ \\
\hline
\end{tabular}

*Significantly different $(\mathrm{P} \leq 0.05)$ from the chamber control group by the Fisher exact test.

$* * \mathrm{P} \leq 0.01$.

aNumber of animals with tissue examined microscopically.

${ }^{b}$ Number of animals with lesion.

${ }^{\mathrm{c}}$ Average severity grade of lesions in affected animals: $1=$ minimal, $2=$ mild, $3=$ moderate, $4=$ marked.

Minimal alveolar epithelium hyperplasia occurred in low numbers of males and females exposed to $5 \mathrm{mg} / \mathrm{m}^{3}$, but the increased incidences of this lesion were not statistically significant (Table 8). Alveolar epithelium hyperplasia was characterized by randomly scattered, focal, irregular proliferations of cuboidal or sometimes ciliated alveolar epithelial cells. Alveolar epithelial hyperplasia is frequently observed in inhalation studies with particulates and may be considered 
a reparative response and sometimes occurs as a component of inflammatory changes in the lung.

In the nose, a spectrum of nonneoplastic lesions were observed that included olfactory epithelium degeneration and hyperplasia, respiratory epithelium hyperplasia, and turbinate atrophy. The incidences of olfactory epithelium degeneration and minimal to mild respiratory epithelium hyperplasia were significantly increased in males and females exposed to 2.5 or $5 \mathrm{mg} / \mathrm{m}^{3}$; the incidence of olfactory epithelium degeneration was also significantly increased in $1.25 \mathrm{mg} / \mathrm{m}^{3}$ females (Table 8 ). In addition, the incidences of olfactory epithelium hyperplasia were significantly increased in $2.5 \mathrm{mg} / \mathrm{m}^{3}$ males and $5 \mathrm{mg} / \mathrm{m}^{3}$ males and females. Degeneration of the olfactory epithelium was a focal or multifocal lesion that variably involved the epithelium lining the dorsal meatus, ethmoid turbinates, and nasal septa. In affected sites, there was vacuolization and disorganization of the epithelium with variable individual cell necrosis and/or loss of epithelial cells. Olfactory epithelium hyperplasia was characterized by clusters or nests of cells proliferating within or just adjacent to the olfactory epithelium, sometimes with extension into the lamina propria around glandular ducts. The proliferating cells sometimes formed rosettes and had scant cytoplasm and large, round to oval nuclei. Increased incidences of minimal turbinate atrophy occurred in males and females exposed to 2.5 or $5 \mathrm{mg} / \mathrm{m}^{3}$; the increases were statistically significant in $2.5 \mathrm{mg} / \mathrm{m}^{3}$ females and $5 \mathrm{mg} / \mathrm{m}^{3}$ males and females. Respiratory epithelium hyperplasia involved the turbinates and/or lateral walls of the Level I and II nasal sections. Hyperplasia appeared as increased numbers of cells in the respiratory or transitional epithelium resulting in crowding of the cells or an increase in the number of cell layers. The epithelial cells were squamous to cuboidal, usually not ciliated, and some cells were hypertrophied. Turbinate atrophy occurred in all three sections of the nose and was characterized by short and blunt turbinates that had attenuation and/or loss of turbinate bone and interstitial tissue in the lamina propria including the glands, vessels, nerve bundles, and connective tissue, and as a result, the nasal passages appeared wider than normal.

\section{Tissue Burden Studies}

Lung and liver weights and lung, blood, and liver cobalt concentrations were determined in female rats (Table I-4). Lung weights were increased in all exposed groups starting on day 40 $\left(5 \mathrm{mg} / \mathrm{m}^{3}\right)$ or day $61\left(2.5 \mathrm{mg} / \mathrm{m}^{3}\right.$ or less $)$ and remained greater than those in the chamber controls throughout the exposure and postexposure periods. Because of the significant changes in lung weights with exposure concentration, lung cobalt burdens rather than lung cobalt concentrations were evaluated for toxicokinetic parameters.

Liver weights of exposed groups of females were either decreased or similar to chamber controls at each time point (Table I-4).

Lung cobalt concentrations and burdens increased with increasing exposure concentration and were significantly increased over chamber controls with all exposure concentrations at all time points. By day 26, the concentrations and burdens of cobalt in the lung of all exposed groups appeared to reach steady state and did not change significantly through the end of exposure (day 89) before decreasing rapidly during the first week of the postexposure period and then more slowly until the end of the postexposure period (Table I-4). Lung cobalt concentrations in chamber control animals were at or below the limit of detection (LOD) at all time points. Lung cobalt burden data normalized to exposure concentration indicated increases in burden that were proportional to exposure concentration. 
During the 3-month exposure, blood cobalt concentrations in chamber control animals were at or below the LOD at all time points and concentrations in the exposed groups generally increased in proportion to exposure concentration at all time points (Table I-4). Within each exposure concentration, blood cobalt concentrations appeared to be at or near steady state starting from the earliest time point and continuing throughout the exposure period. However, during the recovery period, blood cobalt concentrations fell very rapidly; the largest declines occurred during the first week postexposure. Accordingly, because of the extensive elimination of cobalt from the blood, it was not possible to demonstrate dose proportionality from blood concentration data collected during the recovery period. In addition, it was not possible to fit the blood data to a twocompartment model due to the lack of early sampling times; however, it appears that there were both rapid and slow clearance phases from the blood.

Liver cobalt concentrations in the chamber control group were at or below the LOD, and concentrations and burdens in the exposed groups increased with increasing exposure concentration at both time points (days 26 and 40) (Table I-4). Cobalt concentrations and burdens in the liver of exposed animals were generally lower on day 40 compared to day 26 . The normalized liver cobalt burdens were similar across the exposed groups at both time points. At both time points liver cobalt burdens were similar to and in some cases greater than the corresponding lung cobalt burdens.

Pulmonary clearance of cobalt during the recovery period showed a well-defined two-phase elimination profile (Table I-5). The rapid phase exhibited half-lives ranging from 1.8 to 2.6 days and was followed by a slower lung clearance phase with half-lives of 19 to 23 days. A twocompartment clearance model could not be fit to the lung cobalt burden data collected during the 3-month study due to the lack of data collected prior to 5 days of exposure, but a onecompartment model provided an adequate fit to these data (Table I-6). The results indicated that half-lives ranged from 4.7 to 9.0 days.

Exposure Concentration Selection Rationale: There were no significant effects on survival or body weight in the $5 \mathrm{mg} / \mathrm{m}^{3}$ groups in the 3-month study. In addition, increases in lung weights, and incidences of nonneoplastic lesions in the nose and lung, and alterations of erythroid parameters were not considered sufficiently severe to preclude exposure at this concentration. Hence, $5 \mathrm{mg} / \mathrm{m}^{3}$ was selected as the highest exposure concentration for the 2-year inhalation study in rats.

\section{Two-year Study}

\section{Survival}

Estimates of 2-year survival probabilities for male and female rats are shown in Table 9 and in the Kaplan-Meier survival curves (Figure 2). Survival of female rats exposed to $2.5 \mathrm{mg} / \mathrm{m}^{3}$ was significantly less than that of the chamber control group.

\section{Body Weights and Clinical Findings}

Mean body weights of 2.5 and $5 \mathrm{mg} / \mathrm{m}^{3}$ males were at least $10 \%$ less than those of the chamber control group after weeks 99 and 12, respectively, and those of 2.5 and $5 \mathrm{mg} / \mathrm{m}^{3}$ females were at least 10\% less after weeks 57 and 21, respectively (Figure 3; Table 10 and Table 11). Exposure-related clinical findings included abnormal breathing and thinness in male and female rats. 
Table 9. Survival of Rats in the Two-year Inhalation Study of Cobalt Metal

\begin{tabular}{|c|c|c|c|c|}
\hline & $\begin{array}{c}\text { Chamber } \\
\text { Control }\end{array}$ & $1.25 \mathrm{mg} / \mathrm{m}^{3}$ & $2.5 \mathrm{mg} / \mathrm{m}^{3}$ & $5 \mathrm{mg} / \mathrm{m}^{3}$ \\
\hline \multicolumn{5}{|l|}{ Male } \\
\hline Animals initially in study & 50 & 50 & 50 & 50 \\
\hline Accidental deaths ${ }^{\mathrm{a}}$ & 0 & 0 & 1 & 0 \\
\hline Moribund & 28 & 28 & 27 & 32 \\
\hline Natural deaths & 5 & 2 & 6 & 2 \\
\hline Animals surviving to study termination & 17 & 20 & 16 & 16 \\
\hline Percent probability of survival at end of study & 34 & 40 & 33 & 32 \\
\hline Mean survival (days) ${ }^{\mathrm{c}}$ & 663 & 670 & 677 & 669 \\
\hline Survival analysis ${ }^{\mathrm{d}}$ & $\mathrm{P}=0.808$ & $\mathrm{P}=0.796 \mathrm{~N}$ & $\mathrm{P}=0.988 \mathrm{~N}$ & $\mathrm{P}=1.000$ \\
\hline \multicolumn{5}{|l|}{ Female } \\
\hline Animals initially in study & 50 & 50 & 50 & 50 \\
\hline Moribund & 11 & 20 & 19 & 24 \\
\hline Natural deaths & 4 & 4 & 7 & 1 \\
\hline Animals surviving to study termination & 35 & 26 & 24 & $25^{\mathrm{e}}$ \\
\hline Percent probability of survival at end of study & 70 & 52 & 48 & 50 \\
\hline Mean survival (days) & 688 & 685 & 663 & 672 \\
\hline Survival analysis & $P=0.135$ & $P=0.112$ & $\mathrm{P}=0.038$ & $\mathrm{P}=0.060$ \\
\hline
\end{tabular}

${ }^{a}$ Censored from survival analyses.

baplan-Meier determinations.

'Mean of all deaths (uncensored, censored, and terminal kill).

${ }^{\mathrm{d}}$ The result of the life table trend test ${ }^{174}$ is in the chamber control column, and the results of the life table pairwise comparisons ${ }^{173}$ with the chamber controls are in the exposed group columns. A lower mortality in an exposure group is indicated by $\mathrm{N}$.

eIncludes one animal that died during the last week of the study. 

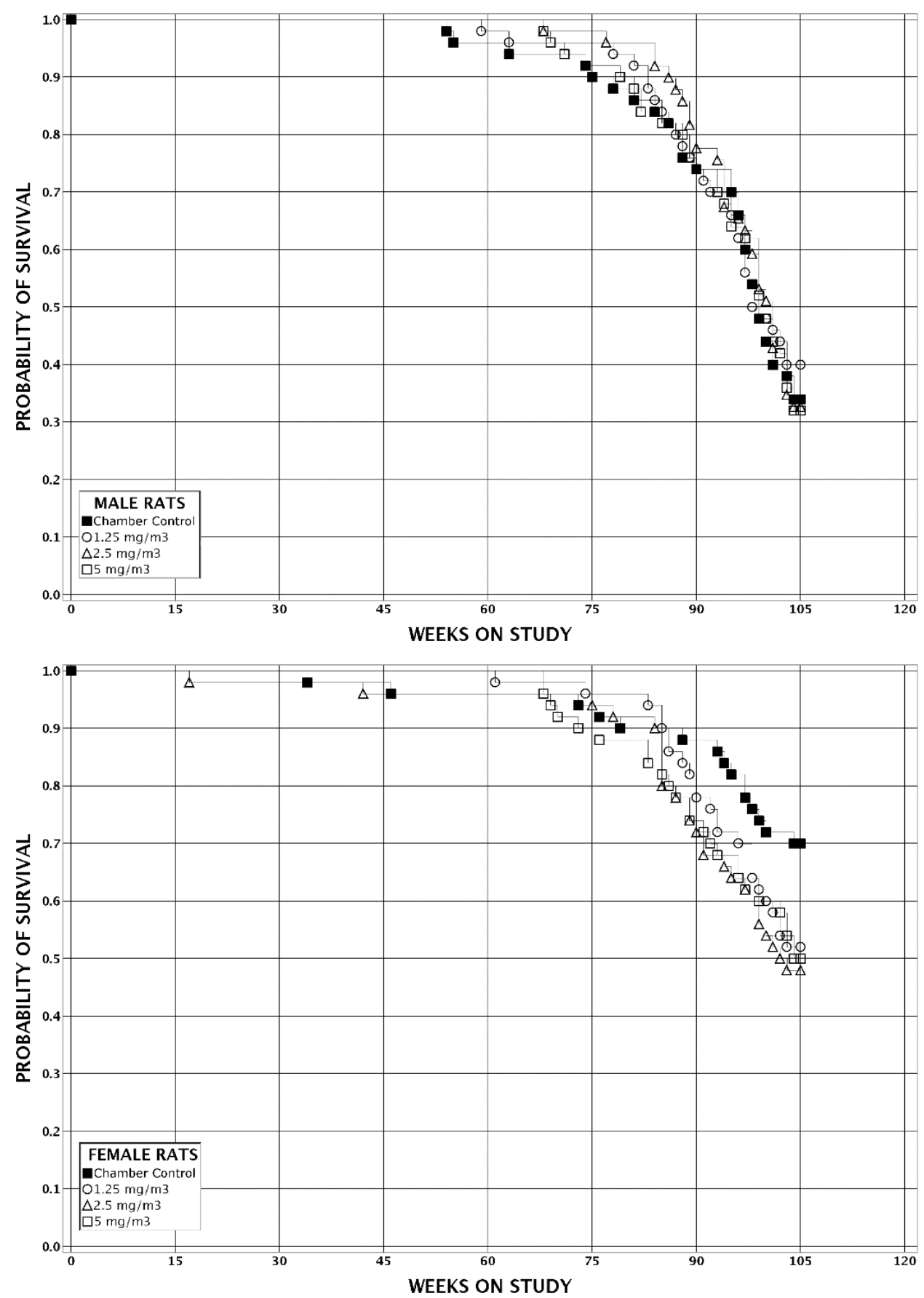

Figure 2. Kaplan-Meier Survival Curves for Rats Exposed to Cobalt Metal by Inhalation for Two Years 

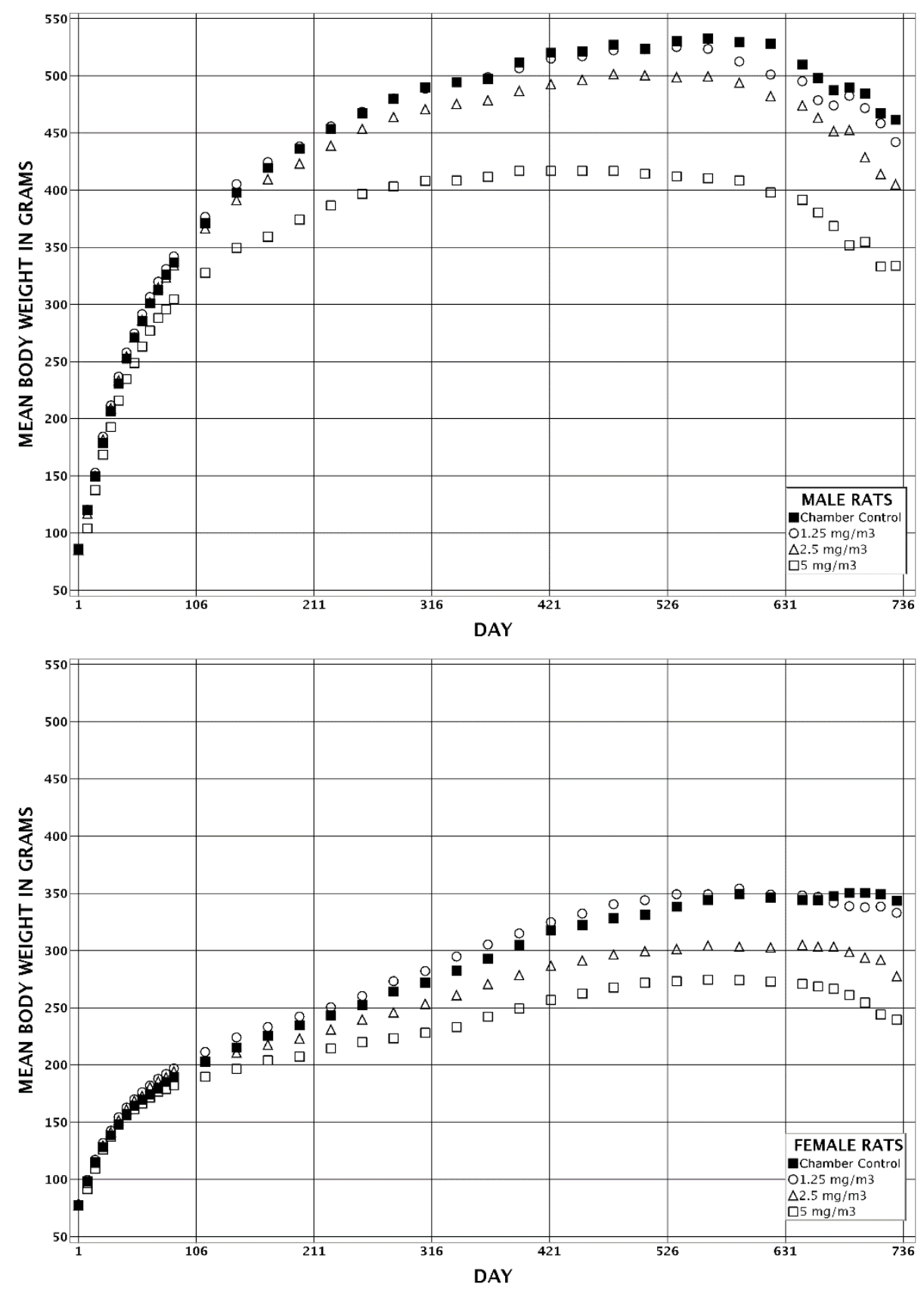

Figure 3. Growth Curves for Rats Exposed to Cobalt Metal by Inhalation for Two Years 
Cobalt Metal, NTP TR 581

Table 10. Mean Body Weights and Survival of Male Rats in the Two-year Inhalation Study of Cobalt Metal

\begin{tabular}{|c|c|c|c|c|c|c|c|c|c|c|c|}
\hline \multirow[b]{2}{*}{ Day } & \multicolumn{2}{|c|}{$\begin{array}{c}\text { Chamber } \\
\text { Control }\end{array}$} & \multicolumn{3}{|c|}{$1.25 \mathrm{mg} / \mathrm{m}^{3}$} & \multicolumn{3}{|c|}{$2.5 \mathrm{mg} / \mathrm{m}^{3}$} & \multicolumn{3}{|c|}{$5 \mathrm{mg} / \mathrm{m}^{3}$} \\
\hline & $\begin{array}{l}\text { Av. } \\
\text { Wt. } \\
\text { (g) }\end{array}$ & $\begin{array}{c}\text { No. of } \\
\text { Survivors }\end{array}$ & $\begin{array}{l}\text { Av. } \\
\text { Wt. } \\
\text { (g) }\end{array}$ & $\begin{array}{l}\text { Wt. (\% of } \\
\text { Controls) }\end{array}$ & $\begin{array}{c}\text { No. of } \\
\text { Survivors }\end{array}$ & $\begin{array}{l}\text { Av. } \\
\text { Wt. } \\
\text { (g) }\end{array}$ & $\begin{array}{l}\text { Wt. (\% of } \\
\text { Controls) }\end{array}$ & $\begin{array}{c}\text { No. of } \\
\text { Survivors }\end{array}$ & $\begin{array}{l}\text { Av. } \\
\text { Wt. } \\
\text { (g) }\end{array}$ & $\begin{array}{l}\text { Wt. (\% of } \\
\text { Controls) }\end{array}$ & $\begin{array}{c}\text { No. of } \\
\text { Survivors }\end{array}$ \\
\hline 1 & 86 & 50 & 86 & 100 & 50 & 85 & 99 & 50 & 86 & 99 & 50 \\
\hline 9 & 120 & 50 & 120 & 100 & 50 & 117 & 97 & 50 & 104 & 87 & 50 \\
\hline 16 & 150 & 50 & 153 & 102 & 50 & 150 & 100 & 50 & 138 & 92 & 50 \\
\hline 23 & 179 & 50 & 184 & 103 & 50 & 182 & 102 & 50 & 169 & 95 & 50 \\
\hline 30 & 207 & 50 & 212 & 103 & 50 & 209 & 101 & 50 & 193 & 93 & 50 \\
\hline 37 & 231 & 50 & 237 & 103 & 50 & 234 & 101 & 50 & 216 & 94 & 50 \\
\hline 44 & 253 & 50 & 258 & 102 & 50 & 254 & 101 & 50 & 235 & 93 & 50 \\
\hline 51 & 271 & 50 & 275 & 101 & 50 & 271 & 100 & 50 & 249 & 92 & 50 \\
\hline 58 & 286 & 50 & 292 & 102 & 50 & 286 & 100 & 50 & 263 & 92 & 50 \\
\hline 65 & 301 & 50 & 307 & 102 & 50 & 302 & 100 & 50 & 277 & 92 & 50 \\
\hline 72 & 313 & 50 & 320 & 102 & 50 & 315 & 101 & 50 & 288 & 92 & 50 \\
\hline 79 & 326 & 50 & 331 & 102 & 50 & 324 & 99 & 50 & 296 & 91 & 50 \\
\hline 86 & 337 & 50 & 342 & 102 & 50 & 334 & 99 & 50 & 305 & 90 & 50 \\
\hline 114 & 371 & 50 & 377 & 102 & 50 & 367 & 99 & 50 & 328 & 88 & 50 \\
\hline 142 & 398 & 50 & 405 & 102 & 50 & 391 & 98 & 50 & 350 & 88 & 50 \\
\hline 170 & 420 & 50 & 425 & 101 & 50 & 410 & 98 & 50 & 359 & 86 & 50 \\
\hline 198 & 436 & 50 & 438 & 100 & 50 & 423 & 97 & 50 & 374 & 86 & 50 \\
\hline 226 & 454 & 50 & 456 & 101 & 50 & 439 & 97 & 50 & 387 & 85 & 50 \\
\hline 254 & 467 & 50 & 469 & 100 & 50 & 454 & 97 & 50 & 397 & 85 & 50 \\
\hline 282 & 480 & 50 & 480 & 100 & 50 & 464 & 97 & 50 & 403 & 84 & 50 \\
\hline 310 & 490 & 50 & 489 & 100 & 50 & 471 & 96 & 50 & 408 & 83 & 50 \\
\hline 338 & 495 & 50 & 495 & 100 & 50 & 475 & 96 & 50 & 409 & 83 & 50 \\
\hline 366 & 498 & 50 & 499 & 100 & 50 & 479 & 96 & 50 & 412 & 83 & 50 \\
\hline 394 & 512 & 48 & 507 & 99 & 50 & 487 & 95 & 50 & 417 & 82 & 50 \\
\hline 422 & 520 & 48 & 515 & 99 & 49 & 493 & 95 & 50 & 417 & 80 & 50 \\
\hline 450 & 522 & 47 & 517 & 99 & 48 & 497 & 95 & 50 & 417 & 80 & 50 \\
\hline 478 & 527 & 47 & 522 & 99 & 48 & 501 & 95 & 49 & 417 & 79 & 48 \\
\hline 506 & 524 & 47 & 523 & 100 & 48 & 500 & 96 & 49 & 415 & 79 & 47 \\
\hline 534 & 531 & 45 & 525 & 99 & 48 & 499 & 94 & 49 & 412 & 78 & 46 \\
\hline 562 & 533 & 43 & 524 & 98 & 46 & 499 & 94 & 47 & 410 & 77 & 44 \\
\hline 590 & 530 & 42 & 513 & 97 & 43 & 494 & 93 & 45 & 409 & 77 & 41 \\
\hline 618 & 528 & 38 & 501 & 95 & 38 & 482 & 91 & 40 & 398 & 75 & 39 \\
\hline 646 & 510 & 37 & 495 & 97 & 35 & 474 & 93 & 37 & 392 & 77 & 35 \\
\hline 660 & 498 & 37 & 479 & 96 & 35 & 463 & 93 & 33 & 381 & 76 & 32 \\
\hline 674 & 488 & 33 & 474 & 97 & 30 & 452 & 93 & 32 & 369 & 76 & 32 \\
\hline 688 & 490 & 25 & 482 & 99 & 25 & 453 & 92 & 27 & 352 & 72 & 30 \\
\hline 702 & 485 & 21 & 472 & 97 & 24 & 429 & 89 & 25 & 355 & 73 & 22 \\
\hline 716 & 467 & 20 & 459 & 98 & 21 & 414 & 89 & 19 & 333 & 71 & 20 \\
\hline \multicolumn{12}{|c|}{ Mean for Weeks } \\
\hline $1-13$ & 235 & - & 240 & 102 & - & 236 & 100 & - & 217 & 92 & - \\
\hline $14-52$ & 446 & - & 448 & 101 & - & 433 & 97 & - & 379 & 85 & - \\
\hline 53-103 & 510 & - & 500 & 98 & - & 476 & 93 & - & 394 & 77 & - \\
\hline
\end{tabular}


Cobalt Metal, NTP TR 581

Table 11. Mean Body Weights and Survival of Female Rats in the Two-year Inhalation Study of Cobalt Metal

\begin{tabular}{|c|c|c|c|c|c|c|c|c|c|c|c|}
\hline \multirow[b]{2}{*}{ Day } & \multicolumn{2}{|c|}{$\begin{array}{c}\text { Chamber } \\
\text { Control }\end{array}$} & \multicolumn{3}{|c|}{$1.25 \mathrm{mg} / \mathrm{m}^{3}$} & \multicolumn{3}{|c|}{$2.5 \mathrm{mg} / \mathrm{m}^{3}$} & \multicolumn{3}{|c|}{$5 \mathrm{mg} / \mathrm{m}^{3}$} \\
\hline & $\begin{array}{l}\text { Av. } \\
\text { Wt. } \\
\text { (g) }\end{array}$ & $\begin{array}{c}\text { No. of } \\
\text { Survivors }\end{array}$ & $\begin{array}{l}\text { Av. } \\
\text { Wt. } \\
\text { (g) }\end{array}$ & $\begin{array}{l}\text { Wt. (\% of } \\
\text { Controls) }\end{array}$ & $\begin{array}{c}\text { No. of } \\
\text { Survivors }\end{array}$ & $\begin{array}{l}\text { Av. } \\
\text { Wt. } \\
\text { (g) }\end{array}$ & $\begin{array}{l}\text { Wt. (\% of } \\
\text { Controls) }\end{array}$ & $\begin{array}{c}\text { No. of } \\
\text { Survivors }\end{array}$ & $\begin{array}{l}\text { Av. } \\
\text { Wt } \\
\text { (g) }\end{array}$ & $\begin{array}{l}\text { Wt. (\% of } \\
\text { Controls) }\end{array}$ & $\begin{array}{c}\text { No. of } \\
\text { Survivors }\end{array}$ \\
\hline 1 & 77 & 50 & 78 & 102 & 50 & 77 & 100 & 50 & 78 & 101 & 50 \\
\hline 9 & 98 & 50 & 100 & 101 & 50 & 97 & 99 & 50 & 92 & 93 & 50 \\
\hline 16 & 115 & 50 & 117 & 102 & 50 & 115 & 100 & 50 & 110 & 95 & 50 \\
\hline 23 & 128 & 50 & 132 & 103 & 50 & 130 & 101 & 50 & 126 & 98 & 50 \\
\hline 30 & 139 & 50 & 143 & 103 & 50 & 141 & 102 & 50 & 138 & 99 & 50 \\
\hline 37 & 148 & 50 & 154 & 104 & 50 & 152 & 103 & 50 & 148 & 100 & 50 \\
\hline 44 & 157 & 50 & 163 & 103 & 50 & 161 & 103 & 50 & 156 & 99 & 50 \\
\hline 51 & 164 & 50 & 170 & 103 & 50 & 169 & 103 & 50 & 161 & 98 & 50 \\
\hline 58 & 170 & 50 & 176 & 104 & 50 & 173 & 102 & 50 & 167 & 98 & 50 \\
\hline 65 & 175 & 50 & 182 & 104 & 50 & 181 & 103 & 50 & 172 & 98 & 50 \\
\hline 72 & 180 & 50 & 188 & 105 & 50 & 186 & 103 & 50 & 177 & 99 & 50 \\
\hline 79 & 185 & 50 & 192 & 104 & 50 & 189 & 102 & 50 & 179 & 97 & 50 \\
\hline 86 & 190 & 50 & 197 & 104 & 50 & 195 & 103 & 50 & 182 & 96 & 50 \\
\hline 114 & 203 & 50 & 211 & 104 & 50 & 203 & 100 & 50 & 190 & 93 & 50 \\
\hline 142 & 215 & 50 & 224 & 104 & 50 & 211 & 98 & 49 & 197 & 91 & 50 \\
\hline 170 & 226 & 50 & 233 & 103 & 50 & 218 & 96 & 49 & 204 & 90 & 50 \\
\hline 198 & 235 & 50 & 242 & 103 & 50 & 223 & 95 & 49 & 208 & 88 & 50 \\
\hline 226 & 243 & 50 & 251 & 103 & 50 & 231 & 95 & 49 & 215 & 88 & 50 \\
\hline 254 & 252 & 49 & 260 & 103 & 50 & 240 & 95 & 49 & 220 & 87 & 50 \\
\hline 282 & 264 & 49 & 273 & 103 & 50 & 246 & 93 & 49 & 224 & 85 & 50 \\
\hline 310 & 272 & 49 & 282 & 104 & 50 & 256 & 93 & 48 & 229 & 84 & 50 \\
\hline 338 & 283 & 48 & 295 & 104 & 50 & 261 & 92 & 48 & 233 & 83 & 50 \\
\hline 366 & 293 & 48 & 305 & 104 & 50 & 271 & 92 & 48 & 242 & 83 & 50 \\
\hline 394 & 305 & 48 & 315 & 103 & 50 & 279 & 91 & 48 & 249 & 82 & 50 \\
\hline 422 & 318 & 48 & 325 & 102 & 49 & 287 & 90 & 48 & 257 & 81 & 50 \\
\hline 450 & 322 & 48 & 332 & 103 & 49 & 291 & 90 & 48 & 263 & 81 & 50 \\
\hline 478 & 328 & 48 & 340 & 104 & 49 & 296 & 90 & 48 & 268 & 82 & 47 \\
\hline 506 & 331 & 48 & 344 & 104 & 49 & 299 & 90 & 48 & 272 & 82 & 45 \\
\hline 534 & 338 & 46 & 349 & 103 & 48 & 302 & 89 & 47 & 273 & 81 & 44 \\
\hline 562 & 344 & 45 & 349 & 101 & 48 & 304 & 88 & 46 & 275 & 80 & 44 \\
\hline 590 & 350 & 45 & 354 & 101 & 46 & 303 & 87 & 42 & 274 & 79 & 41 \\
\hline 618 & 346 & 44 & 349 & 101 & 41 & 303 & 87 & 37 & 273 & 79 & 38 \\
\hline 646 & 344 & 43 & 348 & 101 & 36 & 305 & 89 & 34 & 271 & 79 & 34 \\
\hline 660 & 344 & 42 & 347 & 101 & 36 & 303 & 88 & 33 & 269 & 78 & 34 \\
\hline 674 & 348 & 39 & 342 & 98 & 35 & 303 & 87 & 32 & 267 & 77 & 31 \\
\hline 688 & 350 & 38 & 339 & 97 & 32 & 299 & 85 & 29 & 261 & 75 & 31 \\
\hline 702 & 351 & 36 & 338 & 96 & 29 & 294 & 84 & 26 & 255 & 73 & 30 \\
\hline 716 & 349 & 36 & 338 & 97 & 26 & 292 & 84 & 24 & 244 & 70 & 29 \\
\hline \multicolumn{12}{|c|}{ Mean for Weeks } \\
\hline $1-13$ & 148 & - & 153 & 103 & - & 151 & 102 & - & 145 & 98 & - \\
\hline $14-52$ & 244 & - & 252 & 103 & - & 232 & 95 & - & 213 & 88 & - \\
\hline $53-103$ & 335 & - & 338 & 101 & - & 296 & 88 & - & 263 & 79 & - \\
\hline
\end{tabular}




\section{Pathology and Statistical Analyses}

This section describes the statistically significant or biologically noteworthy changes in the incidences of mononuclear cell leukemia and neoplasms and/or nonneoplastic lesions of the lung, nose, adrenal medulla, pancreatic islets, kidney, liver, and testes. Summaries of the incidences of neoplasms and nonneoplastic lesions, statistical analyses of primary neoplasms that occurred with an incidence of at least 5\% in at least one animal group, and historical incidences for the neoplasms mentioned in this section are presented in Appendix A for male rats and Appendix B for female rats.

Lung: The incidences of alveolar/bronchiolar adenoma, alveolar/bronchiolar carcinoma, and alveolar/ bronchiolar adenoma or carcinoma (combined) occurred with positive trends in male and female rats and with the exception of the incidence of alveolar/bronchiolar adenoma in $1.25 \mathrm{mg} / \mathrm{m}^{3}$ females, the incidences were significantly greater than those in the chamber controls (Table 12, Table A-3, and Table B-3). In addition, the incidences of these neoplasms in all exposed groups exceeded the historical control ranges for all routes of administration. Incidences of multiple alveolar/bronchiolar adenoma generally increased with increasing exposure concentrations in males and females. Significantly increased incidences of multiple alveolar/bronchiolar carcinoma occurred in all exposed groups of males and in females exposed to $5 \mathrm{mg} / \mathrm{m}^{3}$ (Table 12, Table A-1, and Table B-1). Increased incidences of cystic keratinizing epithelioma occurred in exposed groups of female rats; however, the increases were not statistically significant. In male rats, single incidences of cystic keratinizing epithelioma occurred in the 1.25 and $5 \mathrm{mg} / \mathrm{m}^{3}$ exposure groups. One female rat exposed to $5 \mathrm{mg} / \mathrm{m}^{3}$ had a squamous cell carcinoma (Table 12 and Table B-1). Cystic keratinizing epithelioma and squamous cell carcinoma have not been observed in the lung of 100 historical controls for all routes of administration (Table 12).

Alveolar/bronchiolar adenomas were discrete, expansile, densely cellular masses that compressed the surrounding lung parenchyma (Figure 7). They were composed of relatively well differentiated, uniform, cuboidal to columnar cells supported by a fine fibrovascular stroma and arranged in solid nests or papillary fronds that projected into alveolar spaces.

Alveolar/bronchiolar carcinomas were larger, irregular, poorly circumscribed, unencapsulated, expansile, locally invasive masses that effaced the lung parenchyma (Figure 8). They were composed of poorly differentiated, moderately to markedly pleomorphic (anaplastic) cuboidal, columnar, or polygonal cells with pleomorphic nuclei; occasionally, cells had mitotic figures. The cells were arranged in single to multiple layers, formed irregular papillary or acinar structures and/or solid sheets and were supported by fibrovascular stroma. Some carcinomas had areas of squamous differentiation, and many contained extensive areas of necrosis, desmoplastic tissue, and inflammation (Figure 9). Other carcinomas had a core of dense fibrous tissue with embedded islands of malignant cells arranged in irregular cords, clusters, and acini. In several animals, metastases were observed in other tissues. Cystic keratinizing epitheliomas were well circumscribed, unencapsulated, irregularly expansive masses that effaced the lung parenchyma. The epitheliomas consisted of an irregular wall of well differentiated squamous epithelium surrounding a core of concentrically arranged keratin (Figure 10). Invariably the walls of these neoplasms had areas that lacked orderly maturation with foci of basal cell disorganization. The outer portion of the lesion grew by expansion into the adjacent lung, but evidence of invasion was not observed. The squamous cell carcinoma was an infiltrative mass that obliterated the normal lung architecture. The neoplastic cells formed swirling clusters, often around laminated 
keratin, separated by small to moderate amounts of fibrous stroma. The cells were polygonal, variable in size and shape, and contained small to moderate amounts of eosinophilic cytoplasm.

Point mutations in Kras (31\%), Egfr (17\%), and Tp53 (23\%) were noted in the alveolar/bronchiolar carcinomas in rats chronically exposed to cobalt metal dust. Because there were no spontaneous alveolar/bronchiolar carcinomas in the F344/NTac rats in the concurrent cobalt metal study, spontaneous alveolar/bronchiolar carcinomas were evaluated from F344 rat vehicle control groups in previous NTP chronic bioassays. None of these mutations were noted in the controls.

The incidences of alveolar epithelium hyperplasia, alveolar proteinosis, chronic active inflammation, and bronchiole epithelium hyperplasia in all exposed groups of male and female rats were significantly greater than those in the chamber control groups (Table 12, Table A-7, and Table B-7). The severities of these lesions generally increased with increasing exposure concentration. This spectrum of nonneoplastic lesions invariably occurred together and presented as a complex mix of changes, and at times it was difficult to separate the individual components. Alveolar epithelium hyperplasia was a multifocal and sometimes focally extensive, discrete, randomly distributed but frequently subpleural lesion characterized by proliferation of flat to cuboidal to low columnar epithelial cells (presumed to be Type II pneumocytes).

Table 12. Incidences of Neoplasms and Nonneoplastic Lesions of the Lung in Rats in the Two-year Inhalation Study of Cobalt Metal

\begin{tabular}{|c|c|c|c|c|}
\hline & $\begin{array}{c}\text { Chamber } \\
\text { Control }\end{array}$ & $1.25 \mathrm{mg} / \mathrm{m}^{3}$ & $2.5 \mathrm{mg} / \mathrm{m}^{3}$ & $5 \mathrm{mg} / \mathrm{m}^{3}$ \\
\hline \multicolumn{5}{|l|}{ Male } \\
\hline Number Examined Microscopically & 50 & 50 & 50 & 50 \\
\hline Alveolar Epithelium, Hyperplasia ${ }^{a}$ & $3(1.0)^{\mathrm{b}}$ & $47 * *(2.8)$ & $49 * *(3.3)$ & $49^{* *}(3.6)$ \\
\hline Alveolus, Proteinosis & 0 & $48^{* *}(2.6)$ & $49 * *(2.9)$ & $49 * *(3.1)$ \\
\hline Inflammation, Chronic Active & $22(1.1)$ & $50 * *(3.0)$ & $50 * *(2.9)$ & $50 * *(2.9)$ \\
\hline Bronchiole, Epithelium, Hyperplasia & 0 & $44^{* *}(1.5)$ & $47 * *(2.7)$ & $50 * *(3.7)$ \\
\hline Alveolar/bronchiolar Adenoma, Multiple & 1 & 3 & 2 & 6 \\
\hline \multicolumn{5}{|c|}{ Alveolar/bronchiolar Adenoma (includes multiple) ${ }^{c}$} \\
\hline Overall rate $^{\mathrm{d}}$ & $2 / 50(4 \%)$ & $10 / 50(20 \%)$ & $10 / 50(20 \%)$ & $14 / 50(28 \%)$ \\
\hline Adjusted rate ${ }^{\mathrm{e}}$ & $5.0 \%$ & $24.1 \%$ & $23.3 \%$ & $32.5 \%$ \\
\hline Terminal rate ${ }^{f}$ & $1 / 17(6 \%)$ & $6 / 20(30 \%)$ & $2 / 16(13 \%)$ & $4 / 16(25 \%)$ \\
\hline First incidence (days) & 611 & 577 & 535 & 478 \\
\hline Poly-3 test ${ }^{\mathrm{g}}$ & $\mathrm{P}=0.011$ & $\mathrm{P}=0.015$ & $\mathrm{P}=0.018$ & $\mathrm{P}<0.001$ \\
\hline Alveolar/bronchiolar Carcinoma, Multiple & 0 & $6^{*}$ & $14^{* *}$ & $30 * *$ \\
\hline \multicolumn{5}{|c|}{ Alveolar/bronchiolar Carcinoma (includes multiple) ${ }^{\mathrm{h}}$} \\
\hline Overall rate & $0 / 50(0 \%)$ & $16 / 50(32 \%)$ & $34 / 50(68 \%)$ & $36 / 50(72 \%)$ \\
\hline Adjusted rate & $0.0 \%$ & $38.2 \%$ & $76.8 \%$ & $80.6 \%$ \\
\hline Terminal rate & $0 / 17(0 \%)$ & $7 / 20(35 \%)$ & $16 / 16(100 \%)$ & $14 / 16(88 \%)$ \\
\hline
\end{tabular}




\section{Cobalt Metal, NTP TR 581}

\begin{tabular}{|c|c|c|c|c|}
\hline & $\begin{array}{l}\text { Chamber } \\
\text { Control }\end{array}$ & $1.25 \mathrm{mg} / \mathrm{m}^{3}$ & $2.5 \mathrm{mg} / \mathrm{m}^{3}$ & $5 \mathrm{mg} / \mathrm{m}^{3}$ \\
\hline First incidence (days) & $-{ }^{\mathrm{i}}$ & 580 & 472 & 552 \\
\hline Poly-3 test & $\mathrm{P}<0.001$ & $\mathrm{P}<0.001$ & $\mathrm{P}<0.001$ & $\mathrm{P}<0.001$ \\
\hline \multicolumn{5}{|l|}{ Alveolar/bronchiolar Adenoma or Carcinoma ${ }^{\mathrm{j}}$} \\
\hline Overall rate & $2 / 50(4 \%)$ & $25 / 50(50 \%)$ & $39 / 50(78 \%)$ & $44 / 50(88 \%)$ \\
\hline Adjusted rate & $5.0 \%$ & $58.0 \%$ & $84.6 \%$ & $93.6 \%$ \\
\hline Terminal rate & $1 / 17(6 \%)$ & $13 / 20(65 \%)$ & $16 / 16(100 \%)$ & $16 / 16(100 \%)$ \\
\hline First incidence (days) & 611 & 577 & 472 & 478 \\
\hline Poly-3 test & $\mathrm{P}<0.001$ & $\mathrm{P}<0.001$ & $\mathrm{P}<0.001$ & $\mathrm{P}<0.001$ \\
\hline Cystic Keratinizing Epithelioma ${ }^{\mathrm{h}}$ & 0 & 1 & 0 & 1 \\
\hline \multicolumn{5}{|l|}{ Female } \\
\hline Number Examined Microscopically & 50 & 50 & 50 & 50 \\
\hline Alveolar Epithelium, Hyperplasia & $9(1.1)$ & $49 * *(2.8)$ & $50 * *(2.7)$ & $49 * *(3.4)$ \\
\hline Alveolus, Proteinosis & 0 & $50 * *(2.7)$ & $50 * *(2.7)$ & $50 * *(2.9)$ \\
\hline Inflammation, Chronic Active & $20(1.0)$ & $50 * *(3.0)$ & $50 * *(2.9)$ & $50 * *(2.9)$ \\
\hline Bronchiole, Epithelium, Hyperplasia & 0 & $47 * *(1.5)$ & $46^{* *}(2.1)$ & $48 * *(3.8)$ \\
\hline Alveolar/bronchiolar Adenoma, Multiple & 0 & 1 & 3 & 4 \\
\hline \multicolumn{5}{|c|}{ Alveolar/bronchiolar Adenoma (includes multiple) ${ }^{\mathrm{j}}$} \\
\hline Overall rate & $2 / 50(4 \%)$ & $7 / 50(14 \%)$ & $9 / 50(18 \%)$ & $13 / 50(26 \%)$ \\
\hline Adjusted rate & $4.5 \%$ & $16.2 \%$ & $22.1 \%$ & $30.9 \%$ \\
\hline Terminal rate & $1 / 35(3 \%)$ & $5 / 26(19 \%)$ & $6 / 24(25 \%)$ & $8 / 25(32 \%)$ \\
\hline First incidence (days) & 698 & 590 & 587 & 579 \\
\hline Poly-3 test & $\mathrm{P}=0.002$ & $\mathrm{P}=0.072$ & $\mathrm{P}=0.016$ & $\mathrm{P}<0.001$ \\
\hline Alveolar/bronchiolar Carcinoma, Multiple & & 0 & 4 & 3 \\
\hline \multicolumn{5}{|c|}{ Alveolar/bronchiolar Carcinoma (includes multiple) ${ }^{\mathrm{h}}$} \\
\hline Overall rate & $0 / 50(0 \%)$ & $9 / 50(18 \%)$ & $17 / 50(34 \%)$ & $30 / 50(60 \%)$ \\
\hline Adjusted rate & $0.0 \%$ & $21.3 \%$ & $42.0 \%$ & $69.2 \%$ \\
\hline Terminal rate & $0 / 35(0 \%)$ & $9 / 26(35 \%)$ & $14 / 24(58 \%)$ & $20 / 25(80 \%)$ \\
\hline First incidence (days) & - & $730(\mathrm{~T})$ & 690 & 471 \\
\hline Poly-3 test & $\mathrm{P}<0.001$ & $\mathrm{P}<0.001$ & $\mathrm{P}<0.001$ & $\mathrm{P}<0.001$ \\
\hline \multicolumn{5}{|l|}{$\begin{array}{l}\text { Alveolar/bronchiolar Adenoma or Carcinoma } \\
\text { (combined })^{\mathrm{j}}\end{array}$} \\
\hline Overall rate & $2 / 50(4 \%)$ & $15 / 50(30 \%)$ & $20 / 50(40 \%)$ & $38 / 50(76 \%)$ \\
\hline Adjusted rate & $4.5 \%$ & $34.7 \%$ & $48.5 \%$ & $86.2 \%$ \\
\hline Terminal rate & $1 / 35(3 \%)$ & $13 / 26(50 \%)$ & $14 / 24(58 \%)$ & $25 / 25(100 \%)$ \\
\hline First incidence (days) & 698 & 590 & 587 & 471 \\
\hline
\end{tabular}




\begin{tabular}{|c|c|c|c|c|}
\hline & $\begin{array}{l}\text { Chamber } \\
\text { Control }\end{array}$ & $1.25 \mathrm{mg} / \mathrm{m}^{3}$ & $2.5 \mathrm{mg} / \mathrm{m}^{3}$ & $5 \mathrm{mg} / \mathrm{m}^{3}$ \\
\hline Poly-3 test & $\mathrm{P}<0.001$ & $\mathrm{P}<0.001$ & $\mathrm{P}<0.001$ & $\mathrm{P}<0.001$ \\
\hline Cystic Keratinizing Epithelioma ${ }^{\mathrm{h}}$ & 0 & 4 & 1 & 2 \\
\hline Squamous Cell Carcinoma $^{\mathrm{h}}$ & 0 & 0 & 0 & 1 \\
\hline
\end{tabular}

*Significantly different $(\mathrm{P} \leq 0.05)$ from the chamber control group by the Poly-3 test.

$* * \mathrm{P} \leq 0.01$.

(T) Terminal kill.

${ }^{\mathrm{a} N u m b e r}$ of animals with lesion.

${ }^{\mathrm{b}}$ Average severity grade of lesions in affected animals: $1=$ minimal, $2=$ mild, $3=$ moderate, $4=$ marked.

'Historical control incidence for 2-year studies (all routes): 5/100.

${ }^{\mathrm{d} N u m b e r}$ of animals with neoplasm per number of animals with lung examined microscopically.

ePoly-3 estimated neoplasm incidence after adjustment for intercurrent mortality.

fObserved incidence at terminal kill.

${ }^{g}$ Beneath the chamber control incidence is the $\mathrm{P}$ value associated with the trend test. Beneath the exposed group incidence are the

$\mathrm{P}$ values corresponding to pairwise comparisons between the chamber controls and that exposed group. The Poly-3 test accounts

for differential mortality in animals that do not reach terminal kill.

hHistorical control incidence: $0 / 100$.

iNot applicable; no neoplasms in animal group.

${ }^{j}$ Historical control incidence: $2 / 100$.

lining the alveolar septa; however, the underlying alveolar architecture was generally maintained (Figure 11 and Figure 12). The interstitium of the alveolar septa was also variably expanded by increased amounts of collagen. Alveolar proteinosis was characterized by accum-ulations of brightly eosinophilic, wispy to globular, homogeneous, proteinaceous material filling the alveolar spaces (Figure 13); this proteinaceous material frequently contained acicular cholesterol crystals or cleft-like spaces. These lesions were invariably accompanied by chronic active inflammation which consisted of com-plex mixtures of predominantly macrophages and lymphocytes mixed with lesser numbers of neutrophils within the alveolar spaces and septa and low numbers of multinucleated giant cells (Figure 14); clear cleft-like spaces (cholesterol clefts) were frequently present among the inflammatory cells. Also associated with areas of chronic active inflammation, there were frequently variable proliferation of the alveolar epithelial (Type II) cells and variable alveolar septal interstitial fibrosis. Frequently, there were large numbers of inflammatory cell infiltrates, mostly macrophages, accumulated around the alveolar/bronchiolar neoplasms. The alveolar macrophages were frequently engorged with an intensely eosinophilic material similar to that in the alveolar spaces, and many macrophages also contained acicular cholesterol crystals. Multifocal accumulations of plump foamy macrophages within the alveolar spaces were considered a component of the inflammation changes. Bronchiole epithelium hyperplasia was characterized by proliferation and disorganized crowding of ciliated, cuboidal columnar to pleomorphic epithelial cells lining terminal bronchioles with extension onto adjacent alveolar septa (Figure 15). There was often minimal to mildly increased amounts of collagen in the interstitium of the bronchiolar wall.

Nose: A spectrum of nonneoplastic lesions occurred with positive trends in male and female rats, and the incidences were often significantly greater than those in the chamber controls (Table 13, Table A-7, and Table B-7). For some lesions, the severities increased with increasing exposure concentration. Chronic active inflammation was most prominent in Levels I and II and less often in Level III nasal section. Chronic active inflammation consisted of infiltrates of mostly lymphocytes, plasma cells, neutrophils, and fewer macrophages within the lamina propria and overlying epithelium accompanied by cellular debris in the nasal passages. Suppurative 
inflammation occurred primarily in Level II and consisted of accumulations of nondegenerate and degenerate neutrophils mixed with eosinophilic proteinaceous material, occasional macrophages, cellular debris, and sometimes colonies of coccobacilli and foreign material within the nasal passages and adjacent epithelium and lamina propria of the nasal turbinates (Figure 16). More pronounced suppurative inflammation was often accompanied by florid hyperplasia of the adjacent epithelium.

Respiratory metaplasia and/or atrophy of the olfactory epithelium occurred in the dorsal meatuses of Level II and sometimes Level III. When the predominant change in the affected segment was replacement of the olfactory epithelium by respiratory type columnar epithelial cells, olfactory epithelium respiratory metaplasia was diagnosed (Figure 17). When the olfactory epithelium was attenuated due to loss of olfactory epithelial cells, olfactory epithelium atrophy was diagnosed (Figure 18). Olfactory epithelium hyperplasia mostly occurred in rats exposed to $5 \mathrm{mg} / \mathrm{m}^{3}$ and consisted of small, focal, intraepithelial proliferations of epithelial cells that formed clusters or rosettes that sometimes extended into the lamina propria (Figure 18). Olfactory epithelium basal cell hyperplasia was invariably associated with olfactory epithelium hyperplasia and consisted of disorganized proliferation and crowding of the basal olfactory epithelial cells. Necrosis of the olfactory epithelium was a minimal to mild lesion mostly affecting male rats and a few females and was associated with inflammatory lesions. In sites of necrosis, the epithelium was effaced and replaced by cellular and karyorrhectic debris.

Respiratory epithelium hyperplasia occurred in the epithelium lining the tips of the nasoturbinates, maxilloturbinates, and the septa in Levels I and II. In affected sites, the epithelium was thickened by increased numbers of cuboidal to ciliated columnar epithelial cells crowded in multiple layers sometimes forming undulations with invaginations into the underlying lamina propria (Figure 19). This lesion was most prominent in areas of suppurative inflammation. Respiratory epithelium squamous metaplasia was most common at the tips of the nasoturbinates and along the lateral walls of Level I and less often Level II. In affected sites, flattened squamous epithelium of variable thickness replaced the ciliated columnar epithelium normally present in this location (Figure 19). Necrosis of the respiratory epithelium was associated with the inflammatory lesions and primarily affected rats in the $5 \mathrm{mg} / \mathrm{m}^{3}$ groups. In areas of necrosis, the epithelium was effaced and replaced by cellular and karyorrhectic debris (Figure 20); necrosis would sometimes extend into the submucosa and sinuses.

Turbinate atrophy was a minimal to mild change that primarily affected the naso- and maxilloturbinates in Levels I and II and occasionally Level III. Affected turbinates were short, thin, and blunted due to attenuation of the turbinate bone and loss of structures in the lamina propria, including the glands, vessels, nerve bundles, and connective tissue. As a result, the nasal passages appeared wider than normal (Figure 21). The nasal septum was sometimes similarly affected and had a noticeable decrease in width. 
Table 13. Incidences of Nonneoplastic Lesions of the Nose in Rats in the Two-year Inhalation Study of Cobalt Metal

\begin{tabular}{|c|c|c|c|c|}
\hline & $\begin{array}{l}\text { Chamber } \\
\text { Control }\end{array}$ & $1.25 \mathrm{mg} / \mathrm{m}^{3}$ & $2.5 \mathrm{mg} / \mathrm{m}^{3}$ & $5 \mathrm{mg} / \mathrm{m}^{3}$ \\
\hline \multicolumn{5}{|l|}{ Male } \\
\hline Number Examined Microscopically & 48 & 47 & 45 & 50 \\
\hline Inflammation, Chronic Active $^{a}$ & $28(1.2)^{\mathrm{b}}$ & $35^{*}(1.3)$ & $40 * *(1.7)$ & $49 * *(2.6)$ \\
\hline Inflammation, Suppurative & $9(1.0)$ & $12(1.7)$ & $24 * *(2.2)$ & $46^{* *}(2.6)$ \\
\hline $\begin{array}{l}\text { Olfactory Epithelium, Metaplasia, } \\
\text { Respiratory }\end{array}$ & $12(1.1)$ & $26^{* *}(1.7)$ & $37 * *(1.5)$ & $50 * *(2.2)$ \\
\hline Olfactory Epithelium, Atrophy & $2(1.0)$ & $21 * *(1.0)$ & $34 * *(1.0)$ & $29 * *(1.2)$ \\
\hline Olfactory Epithelium, Hyperplasia & 0 & $1(1.0)$ & $2(1.5)$ & $7 * *(1.1)$ \\
\hline $\begin{array}{l}\text { Olfactory Epithelium, Hyperplasia, } \\
\text { Basal Cell }\end{array}$ & 0 & $1(1.0)$ & 0 & $13 * *(1.0)$ \\
\hline Olfactory Epithelium, Necrosis & 0 & $1(1.0)$ & $5 *(1.6)$ & $5 *(1.8)$ \\
\hline Respiratory Epithelium, Hyperplasia & $20(1.3)$ & $35 * *(1.2)$ & $45 * *(1.7)$ & $50 * *(2.2)$ \\
\hline $\begin{array}{l}\text { Respiratory Epithelium, Metaplasia, } \\
\text { Squamous }\end{array}$ & 0 & $1(1.0)$ & $11 * *(1.2)$ & $35 * *(1.3)$ \\
\hline Respiratory Epithelium, Necrosis & $1(1.0)$ & $4(1.8)$ & $5(1.4)$ & $13 * *(1.6)$ \\
\hline Turbinate, Atrophy & $1(1.0)$ & $35 * *(1.0)$ & $35 * *(1.0)$ & $41 * *(1.0)$ \\
\hline \multicolumn{5}{|l|}{ Female } \\
\hline Number Examined Microscopically & 50 & 50 & 49 & 50 \\
\hline Inflammation, Chronic Active & $22(1.3)$ & $42 * *(1.1)$ & $39 * *(1.1)$ & $50 * *(2.4)$ \\
\hline Inflammation, Suppurative & $6(1.2)$ & $4(1.3)$ & $4(1.0)$ & $42 * *(2.2)$ \\
\hline $\begin{array}{l}\text { Olfactory Epithelium, Metaplasia, } \\
\text { Respiratory }\end{array}$ & $6(1.0)$ & $18 * *(1.3)$ & $24 * *(1.2)$ & $47 * *(2.1)$ \\
\hline Olfactory Epithelium, Atrophy & 0 & $22 * *(1.1)$ & $35 * *(1.0)$ & $35 * *(1.2)$ \\
\hline Olfactory Epithelium, Hyperplasia & 0 & 0 & $3(1.0)$ & $5 *(1.0)$ \\
\hline $\begin{array}{l}\text { Olfactory Epithelium, Hyperplasia, } \\
\text { Basal Cell }\end{array}$ & 0 & 0 & $1(1.0)$ & $19 * *(1.0)$ \\
\hline Olfactory Epithelium, Necrosis & 0 & $2(1.5)$ & 0 & $1(3.0)$ \\
\hline Respiratory Epithelium, Hyperplasia & $15(1.2)$ & $43 * *(1.0)$ & $48 * *(1.0)$ & $49 * *(2.1)$ \\
\hline $\begin{array}{l}\text { Respiratory Epithelium, Metaplasia, } \\
\text { Squamous }\end{array}$ & $2(1.0)$ & 0 & $3(1.0)$ & $45 * *(2.0)$ \\
\hline Respiratory Epithelium, Necrosis & $1(3.0)$ & $1(2.0)$ & $1(1.0)$ & $15^{* *}(1.6)$ \\
\hline Turbinate, Atrophy & $1(1.0)$ & $38 * *(1.0)$ & $27 * *(1.0)$ & $45 * *(1.0)$ \\
\hline
\end{tabular}

*Significantly different $(\mathrm{P} \leq 0.05)$ from the chamber control group by the Poly-3 test.

$* * \mathrm{P} \leq 0.01$.

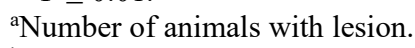

${ }^{\mathrm{b}}$ Average severity grade of lesions in affected animals: $1=$ minimal, $2=$ mild, $3=$ moderate, $4=$ marked 
Adrenal Medulla: The incidences of benign pheochromocytoma, malignant pheochromocytoma, and benign or malignant pheochromocytoma (combined) occurred with positive trends in male and female rats and with the exception of the incidence of malignant pheochromocytoma in $2.5 \mathrm{mg} / \mathrm{m}^{3}$ females, the incidences in rats exposed to 2.5 or $5 \mathrm{mg} / \mathrm{m}^{3}$ were significantly greater than those in the chamber controls and exceeded the historical control incidences for all routes of administration (Table 14, Table A-4, and Table B-4). The incidences of bilateral benign pheochromocytoma were significantly increased in all exposed groups of males and in 2.5 and $5 \mathrm{mg} / \mathrm{m}^{3}$ females, and the incidences of bilateral malignant pheochromocytoma were significantly increased in male and female rats exposed to $5 \mathrm{mg} / \mathrm{m}^{3}$.

Benign pheochromocytoma occurred as variably sized, well-demarcated, expansile proliferations of medullary cells that formed large trabeculae or solid clusters separated by delicate fibrous stroma and/or sinusoids (Figure 22). The cells were polygonal to spindyloid and moderately uniform in size and shape.

Table 14. Incidences of Neoplasms and Nonneoplastic Lesions of the Adrenal Medulla in Rats in the Two-year Inhalation Study of Cobalt Metal

\begin{tabular}{|c|c|c|c|c|}
\hline & $\begin{array}{l}\text { Chamber } \\
\text { Control }\end{array}$ & $1.25 \mathrm{mg} / \mathrm{m}^{3}$ & $2.5 \mathrm{mg} / \mathrm{m}^{3}$ & $5 \mathrm{mg} / \mathrm{m}^{3}$ \\
\hline \multicolumn{5}{|l|}{ Male } \\
\hline Number Examined Microscopically & 50 & 50 & 50 & 50 \\
\hline Hyperplasia $^{\mathrm{a}}$ & $19(2.3)^{b}$ & $21(2.5)$ & $9 *(3.0)$ & $9 * *(2.4)$ \\
\hline $\begin{array}{l}\text { Benign Pheochromocytoma, } \\
\text { Bilateral }\end{array}$ & 4 & $13 *$ & $22 * *$ & $21 * *$ \\
\hline \multicolumn{5}{|l|}{$\begin{array}{l}\text { Benign Pheochromocytoma } \\
\text { (includes bilateral) }^{\mathrm{c}}\end{array}$} \\
\hline Overall rate $^{\mathrm{d}}$ & $15 / 50(30 \%)$ & $23 / 50(46 \%)$ & $37 / 50(74 \%)$ & $34 / 50(68 \%)$ \\
\hline Adjusted rate $e^{e}$ & $35.8 \%$ & $54.3 \%$ & $81.2 \%$ & $76.4 \%$ \\
\hline Terminal rate ${ }^{\mathrm{f}}$ & $3 / 17(18 \%)$ & $12 / 2060 \%)$ & $15 / 16(94 \%)$ & $14 / 16(88 \%)$ \\
\hline First incidence (days) & 519 & 583 & 582 & 572 \\
\hline Poly-3 test ${ }^{\mathrm{g}}$ & $\mathrm{P}<0.001$ & $\mathrm{P}=0.059$ & $\mathrm{P}<0.001$ & $\mathrm{P}<0.001$ \\
\hline $\begin{array}{l}\text { Malignant Pheochromocytoma, } \\
\text { Bilateral }\end{array}$ & 0 & 0 & 0 & $7 * *$ \\
\hline \multicolumn{5}{|l|}{$\begin{array}{l}\text { Malignant Pheochromocytoma } \\
\text { (includes bilateral) }^{\mathrm{h}}\end{array}$} \\
\hline Overall rate & $2 / 50(4 \%)$ & $2 / 50(4 \%)$ & $9 / 50(18 \%)$ & $16 / 50(32 \%)$ \\
\hline Adjusted rate & $5.0 \%$ & $5.0 \%$ & $21.4 \%$ & $39.1 \%$ \\
\hline Terminal rate & $0 / 17(0 \%)$ & $2 / 20(10 \%)$ & $3 / 16(19 \%)$ & $9 / 16(56 \%)$ \\
\hline First incidence (days) & 668 & $729(\mathrm{~T})$ & 628 & 646 \\
\hline Poly-3 test & $\mathrm{P}<0.001$ & $P=0.693 \mathrm{~N}$ & $\mathrm{P}=0.030$ & $\mathrm{P}<0.001$ \\
\hline \multicolumn{5}{|l|}{$\begin{array}{l}\text { Benign or Malignant } \\
\text { Pheochromocytoma }^{\mathrm{i}}\end{array}$} \\
\hline Overall rate & $17 / 50(34 \%)$ & $23 / 50(46 \%)$ & $38 / 50(76 \%)$ & $41 / 50(82 \%)$ \\
\hline Adjusted rate & $40.2 \%$ & $54.3 \%$ & $82.7 \%$ & $90.7 \%$ \\
\hline Terminal rate & $3 / 17(18 \%)$ & $12 / 20(60 \%)$ & $15 / 16(94 \%)$ & $16 / 16(100 \%)$ \\
\hline First incidence (days) & 519 & 583 & 582 & 572 \\
\hline
\end{tabular}


Cobalt Metal, NTP TR 581

\begin{tabular}{|c|c|c|c|c|}
\hline & $\begin{array}{l}\text { Chamber } \\
\text { Control }\end{array}$ & $1.25 \mathrm{mg} / \mathrm{m}^{3}$ & $2.5 \mathrm{mg} / \mathrm{m}^{3}$ & $5 \mathrm{mg} / \mathrm{m}^{3}$ \\
\hline Poly-3 test & $\mathrm{P}<0.001$ & $\mathrm{P}=0.130$ & $\mathrm{P}<0.001$ & $\mathrm{P}<0.001$ \\
\hline \multicolumn{5}{|l|}{ Female } \\
\hline Number Examined Microscopically & 50 & 50 & 50 & 50 \\
\hline Hyperplasia & $12(1.8)$ & $27 * *(2.0)$ & $27 * *(2.3)$ & $10(2.8)$ \\
\hline $\begin{array}{l}\text { Benign Pheochromocytoma, } \\
\text { Bilateral }\end{array}$ & 2 & 4 & $8^{*}$ & $19^{* *}$ \\
\hline \multicolumn{5}{|l|}{$\begin{array}{l}\text { Benign Pheochromocytoma } \\
\text { (includes bilateral) }\end{array}$} \\
\hline Overall rate & $6 / 50(12 \%)$ & $12 / 50(24 \%)$ & $22 / 50(44 \%)$ & $36 / 50(72 \%)$ \\
\hline Adjusted rate & $13.6 \%$ & $27.2 \%$ & $52.1 \%$ & $80.6 \%$ \\
\hline Terminal rate & $6 / 35(17 \%)$ & $5 / 26(19 \%)$ & $13 / 24(54 \%)$ & $21 / 25(84 \%)$ \\
\hline First incidence (days) & $730(\mathrm{~T})$ & 598 & 590 & 579 \\
\hline Poly-3 test & $\mathrm{P}<0.001$ & $\mathrm{P}=0.091$ & $\mathrm{P}<0.001$ & $\mathrm{P}<0.001$ \\
\hline $\begin{array}{l}\text { Malignant Pheochromocytoma, } \\
\text { Bilateral }\end{array}$ & 0 & 1 & 1 & $4^{*}$ \\
\hline \multicolumn{5}{|l|}{$\begin{array}{l}\text { Malignant Pheochromocytoma } \\
\text { (includes bilateral) }^{\mathrm{k}}\end{array}$} \\
\hline Overall rate & $0 / 50(0 \%)$ & $2 / 50(4 \%)$ & $3 / 50(6 \%)$ & $11 / 50(22 \%)$ \\
\hline Adjusted rate & $0.0 \%$ & $4.7 \%$ & $7.5 \%$ & $27.0 \%$ \\
\hline Terminal rate & $0 / 35(0 \%)$ & $2 / 26(8 \%)$ & $2 / 24(8 \%)$ & $9 / 25(36 \%)$ \\
\hline First incidence (days) & $-^{1}$ & $730(\mathrm{~T})$ & 715 & 712 \\
\hline Poly-3 test & $\mathrm{P}<0.001$ & $\mathrm{P}=0.228$ & $\mathrm{P}=0.102$ & $\mathrm{P}<0.001$ \\
\hline \multicolumn{5}{|l|}{$\begin{array}{l}\text { Benign or Malignant } \\
\text { Pheochromocytoma }^{\mathrm{m}}\end{array}$} \\
\hline Overall rate & $6 / 50(12 \%)$ & $13 / 50(26 \%)$ & $23 / 50(46 \%)$ & $40 / 50(80 \%)$ \\
\hline Adjusted rate & $13.6 \%$ & $29.4 \%$ & $54.5 \%$ & $89.4 \%$ \\
\hline Terminal rate & $6 / 35(17 \%)$ & $6 / 26(23 \%)$ & $14 / 24(58 \%)$ & $24 / 25(96 \%)$ \\
\hline First incidence (days) & $730(\mathrm{~T})$ & 598 & 590 & 579 \\
\hline Poly-3 test & $\mathrm{P}<0.001$ & $\mathrm{P}=0.058$ & $\mathrm{P}<0.001$ & $\mathrm{P}<0.001$ \\
\hline
\end{tabular}

*Significantly different $(\mathrm{P} \leq 0.05)$ from the chamber control group by the Poly-3 test.

$* * \mathrm{P} \leq 0.01$.

(T) Terminal kill.

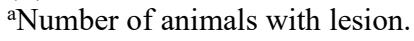

${ }^{\mathrm{b}}$ Average severity grade of lesions in affected animals: $1=$ minimal, $2=$ mild, $3=$ moderate, $4=$ marked.

${ }^{c}$ Historical control incidence for 2-year studies (all routes): 25/100.

${ }^{\mathrm{d}}$ Number of animals with neoplasm per number of animals with adrenal medulla examined microscopically.

ePoly-3 estimated neoplasm incidence after adjustment for intercurrent mortality.

${ }_{\mathrm{f}}$ Observed incidence at terminal kill.

${ }^{g}$ Beneath the chamber control incidence is the $\mathrm{P}$ value associated with the trend test. Beneath the exposed group incidence are the $\mathrm{P}$ values corresponding to pairwise comparisons between the chamber controls and that exposed group. The Poly- 3 test accounts for differential mortality in animals that do not reach terminal kill. A lower incidence in an exposure group is indicated by $\mathrm{N}$.

hHistorical control incidence: $2 / 100$.

${ }^{i}$ Historical control incidence: $27 / 100$.

${ }^{j}$ Historical control incidence: $7 / 100$.

${ }^{\mathrm{k} H i s t o r i c a l ~ c o n t r o l ~ i n c i d e n c e: ~ 1 / 100 . ~}$

${ }^{1}$ Not applicable; no neoplasms in animal group.

${ }^{m}$ Historical control incidence: 8/100. 
Malignant pheochromocytomas were generally larger, irregular, poorly demarcated invasive masses that effaced the adrenal gland extending through the capsule into the periadrenal tissue; the neoplastic cells were poorly differentiated and pleomorphic (Figure 23). In some animals, malignant pheochromocytomas metastasized to other organs. The incidences of medullary hyperplasia in the adrenal gland were significantly increased in female rats exposed to 1.25 or $2.5 \mathrm{mg} / \mathrm{m}^{3}$ (Table 14 and Table B-7); incidences of this lesion were significantly decreased in male rats exposed to 2.5 or $5 \mathrm{mg} / \mathrm{m}^{3}$ (Table 14 and Table A-7). Hyperplasia occurred as focally discrete proliferations of medullary epithelial cells that blended with, but did not compress, the surrounding medullary parenchyma. The cells were generally smaller and more basophilic than the surrounding normal medullary epithelial cells.

Pancreatic Islets: The incidences of carcinoma and adenoma or carcinoma (combined) occurred with positive trends in male rats, and the incidences of adenoma, carcinoma, and adenoma or carcinoma (combined) generally exceeded the historical control incidences for all routes of administration (Table 15, Table A-1, Table A-2, and Table A-5). The incidences of adenoma in $2.5 \mathrm{mg} / \mathrm{m}^{3}$ males and of adenoma or carcinoma (combined) in males exposed to $2.5 \mathrm{or} 5 \mathrm{mg} / \mathrm{m}^{3}$ were significantly greater than those in the chamber controls. Incidences of adenoma, carcinoma, and adenoma or carcinoma (combined) in $5 \mathrm{mg} / \mathrm{m}^{3}$ females were slightly increased; the increases were not statistically significant but did exceed the historical control incidences for all routes of administration (Table 15, Table B-1, Table B-2, and Table B-6). Adenomas were well circumscribed, expansile masses that compressed the acini. The neoplastic cells were well differentiated with minimal to mild cellular atypia and slightly altered growth patterns (Figure 24). Carcinomas were poorly circumscribed, unencapsulated, irregular, expansile, and invasive masses that effaced the parenchyma (Figure 25). Carcinomas had a heterogeneous growth pattern with cells that were moderately to markedly pleomorphic.

Table 15. Incidences of Neoplasms of the Pancreatic Islets in Rats in the Two-year Inhalation Study of Cobalt Metal

\begin{tabular}{|c|c|c|c|c|}
\hline & $\begin{array}{l}\text { Chamber } \\
\text { Control }\end{array}$ & $1.25 \mathrm{mg} / \mathrm{m}^{3}$ & $2.5 \mathrm{mg} / \mathrm{m}^{3}$ & $5 \mathrm{mg} / \mathrm{m}^{3}$ \\
\hline \multicolumn{5}{|l|}{ Male } \\
\hline \multicolumn{5}{|l|}{ Adenoma $^{\mathrm{a}}$} \\
\hline Overall rate ${ }^{\mathrm{b}}$ & $0 / 50(0 \%)$ & $1 / 50(2 \%)$ & $6 / 48(13 \%)$ & $3 / 49(6 \%)$ \\
\hline Adjusted rate ${ }^{c}$ & $0.0 \%$ & $2.5 \%$ & $15.1 \%$ & $7.7 \%$ \\
\hline Terminal rate ${ }^{\mathrm{d}}$ & $0 / 17(0 \%)$ & $0 / 20(0 \%)$ & $1 / 16(6 \%)$ & $3 / 16(19 \%)$ \\
\hline First incidence (days) & $-^{\mathrm{f}}$ & 684 & 618 & $729(\mathrm{~T})$ \\
\hline Poly-3 test ${ }^{\mathrm{e}}$ & $\mathrm{P}=0.052$ & $\mathrm{P}=0.504$ & $\mathrm{P}=0.015$ & $\mathrm{P}=0.116$ \\
\hline \multicolumn{5}{|l|}{ Carcinoma $^{\mathrm{g}}$} \\
\hline Overall rate & $2 / 50(4 \%)$ & $1 / 50(2 \%)$ & $5 / 48(10 \%)$ & $6 / 49(12 \%)$ \\
\hline Adjusted rate & $5.0 \%$ & $2.5 \%$ & $12.6 \%$ & $15.1 \%$ \\
\hline Terminal rate & $0 / 17(0 \%)$ & $0 / 20(0 \%)$ & $3 / 16(19 \%)$ & $2 / 16(13 \%)$ \\
\hline First incidence (days) & 675 & 675 & 618 & 679 \\
\hline Poly-3 test & $\mathrm{P}=0.021$ & $\mathrm{P}=0.496 \mathrm{~N}$ & $\mathrm{P}=0.213$ & $\mathrm{P}=0.129$ \\
\hline Adenoma or Carcinoma & & & & \\
\hline
\end{tabular}




\begin{tabular}{|c|c|c|c|c|}
\hline & $\begin{array}{c}\text { Chamber } \\
\text { Control }\end{array}$ & $1.25 \mathrm{mg} / \mathrm{m}^{3}$ & $2.5 \mathrm{mg} / \mathrm{m}^{3}$ & $5 \mathrm{mg} / \mathrm{m}^{3}$ \\
\hline Overall rate & $2 / 50(4 \%)$ & $2 / 50(4 \%)$ & $10 / 48(21 \%)$ & $9 / 49(18 \%)$ \\
\hline Adjusted rate & $5.0 \%$ & $4.9 \%$ & $24.7 \%$ & $22.6 \%$ \\
\hline Terminal rate & $0 / 17(0 \%)$ & $0 / 20(0 \%)$ & $3 / 16(19 \%)$ & $5 / 16(31 \%)$ \\
\hline First incidence (days) & 675 & 675 & 618 & 679 \\
\hline Poly-3 test & $\mathrm{P}=0.002$ & $\mathrm{P}=0.689 \mathrm{~N}$ & $\mathrm{P}=0.013$ & $\mathrm{P}=0.022$ \\
\hline \multicolumn{5}{|l|}{ Female } \\
\hline Number Examined Microscopically & 50 & 50 & 50 & 50 \\
\hline Adenoma $^{\mathrm{h}}$ & 0 & 0 & 0 & $1(2 \%)$ \\
\hline Carcinoma $^{\mathrm{h}}$ & $1(2 \%)$ & 0 & 0 & $3(6 \%)$ \\
\hline \multicolumn{5}{|l|}{ Adenoma or Carcinoma ${ }^{\mathrm{g}}$} \\
\hline Overall rate & $1 / 50(2 \%)$ & $0 / 50(0 \%)$ & $0 / 50(0 \%)$ & $3 / 50(6 \%)$ \\
\hline Adjusted rate & $2.2 \%$ & $0.0 \%$ & $0.0 \%$ & $7.2 \%$ \\
\hline Terminal rate & $0 / 35(0 \%)$ & $0 / 26(0 \%)$ & $0 / 24(0 \%)$ & $1 / 25(4 \%)$ \\
\hline First incidence (days) & 234 & - & - & 506 \\
\hline Poly- 3 test & $P=0.060$ & $\mathrm{P}=0.512 \mathrm{~N}$ & $\mathrm{P}=0.523 \mathrm{~N}$ & $P=0.279$ \\
\hline
\end{tabular}

(T) Terminal kill.

${ }^{a}$ Historical control incidence for 2-year studies (all routes): 0/100.

${ }^{b}$ Number of animals with neoplasm per number of animals with pancreatic islets examined microscopically.

'Poly-3 estimated neoplasm incidence after adjustment for intercurrent mortality.

dObserved incidence at terminal kill.

${ }^{\mathrm{e} B e n e a t h}$ the chamber control incidence is the $\mathrm{P}$ value associated with the trend test. Beneath the exposed group incidence are the $\mathrm{P}$ values corresponding to pairwise comparisons between the chamber controls and that exposed group. The Poly-3 test accounts for differential mortality in animals that do not reach terminal kill. A lower incidence in an exposure group is indicated by $\mathbf{N}$.

fNot applicable; no neoplasms in animal group.

${ }^{g}$ Historical control incidence for all routes: 2/100.

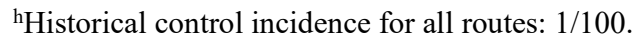

Kidney: In the standard evaluation of the kidney, the incidences of renal tubule adenoma, carcinoma, and adenoma or carcinoma (combined) were slightly increased in male rats exposed to $5 \mathrm{mg} / \mathrm{m}^{3}$ (Table 16, Table A-1, and Table A-2). Although not statistically significant, the incidences in this group exceeded the historical control incidences for all routes of administration (Table 16 and Table A-6). In the standard evaluation, a single section of each kidney is routinely examined microscopically. Because the incidences of renal tubule neoplasms in the standard evaluation suggested the possibility of a treatment-related carcinogenic effect, an extended evaluation of the kidney was performed in male rats to explore this possibility. For the extended evaluation, kidneys of male rats were step-sectioned at $1 \mathrm{~mm}$ intervals to obtain three to four additional sections from each kidney, and these sections were examined microscopically. In the extended evaluation, additional renal tubule adenomas and renal tubule hyperplasias were identified but no additional renal tubule carcinomas (Table 16); a renal tubule oncocytoma was identified in one male exposed to $2.5 \mathrm{mg} / \mathrm{m}^{3}$. In the combined standard and extended evaluations, the incidences of renal tubule hyperplasia in the exposed groups were similar to that in the chamber controls. The incidence of renal tubule adenoma in the $5 \mathrm{mg} / \mathrm{m}^{3}$ group was greater than that in the chamber control group, but the increase was not statistically significant. The incidences of renal tubule carcinomas were unchanged. 
Renal tubule adenomas were small, well circumscribed proliferations of renal tubule epithelial cells with a cross-sectional area greater than five times that of a single, normal renal tubule. The cells were well differentiated and uniform in size and shape and formed poorly defined papillary structures or solid clusters of cells (Figure 26). Renal tubule carcinomas were larger, expansive, and invasive masses that effaced and replaced much of the renal parenchyma (Figure 27).

Table 16. Incidences of Neoplasms and Nonneoplastic Lesions of the Kidney in Male Rats in the Two-year Inhalation Study of Cobalt Metal

\section{$\begin{gathered}\text { Chamber } \\ \text { Control }\end{gathered} \quad 1.25 \mathrm{mg} / \mathrm{m}^{3} \quad 2.5 \mathrm{mg} / \mathrm{m}^{3} \quad 5 \mathrm{mg} / \mathrm{m}^{3}$}

\section{Single Sections (Standard Evaluation)}

Number Examined Microscopically

Renal Tubule, Hyperplasia ${ }^{a}$

50

Renal Tubule, Adenoma, Multiple

Renal Tubule, Adenoma (includes multiple) ${ }^{\mathrm{b}}$

Renal Tubule, Carcinoma ${ }^{c}$

Renal Tubule, Adenoma or Carcinoma ${ }^{b}$

Step Sections (Extended Evaluation)

Number Examined Microscopically

Renal Tubule, Hyperplasia

Renal Tubule, Adenoma

Renal Tubule, Carcinoma

Renal Tubule, Adenoma or Carcinoma

Renal Tubule, Oncocytoma

Single Sections and Step Sections (Combined)

Number Examined Microscopically

Renal Tubule, Hyperplasia

Renal Tubule, Adenoma (includes multiple)

Renal Tubule, Carcinoma

Renal Tubule, Adenoma or Carcinoma

Overall rate ${ }^{\mathrm{d}}$

Adjusted rate

Terminal rate ${ }^{\mathrm{f}}$

First incidence (days)

Poly-3 test ${ }^{\mathrm{g}}$

$\begin{array}{cccc}50 & 50 & 50 & 50 \\ 0 & 0 & 0 & 0 \\ 0 & 0 & 0 & 1 \\ 0 & 1 & 0 & 3 \\ 0 & 0 & 0 & 2 \\ 0 & 1 & 0 & 4\end{array}$

(T) Terminal kill.

aNumber of animals with lesion.

${ }^{b}$ Historical control incidence for 2-year studies (all routes): 1/100.

'Historical control incidence: 0/100.

dNumber of animals with neoplasm per number of animals with kidney examined microscopically.

'Poly-3 estimated neoplasm incidence after adjustment for intercurrent mortality.

fObserved incidence at terminal kill.

${ }^{g}$ Beneath the chamber control incidence is the $\mathrm{P}$ value associated with the trend test. Beneath the exposed group incidence are the $\mathrm{P}$ values corresponding to pairwise comparisons between the chamber controls and that exposed group. The Poly- 3 test accounts for differential mortality in animals that do not reach terminal kill. A lower incidence in an exposure group is indicated by $\mathrm{N}$. 
Mononuclear Cell Leukemia: The incidences of mononuclear cell leukemia were significantly increased in all exposed groups of female rats and exceeded the historical control incidence for all routes of administration (Table 17, Table B-1, Table B-2, and Table B-6).

Table 17. Incidences of Mononuclear Cell Leukemia in Female Rats in the Two-year Inhalation Study of Cobalt Metal

\begin{tabular}{|c|c|c|c|c|}
\hline & $\begin{array}{l}\text { Chamber } \\
\text { Control }\end{array}$ & $1.25 \mathrm{mg} / \mathrm{m}^{3}$ & $2.5 \mathrm{mg} / \mathrm{m}^{3}$ & $5 \mathrm{mg} / \mathrm{m}^{3}$ \\
\hline \multicolumn{5}{|c|}{ All Organs: Mononuclear Cell Leukemia ${ }^{a}$} \\
\hline Overall rate ${ }^{\mathrm{b}}$ & $16 / 50(32 \%)$ & $29 / 50(58 \%)$ & $28 / 50(56 \%)$ & $27 / 50(54 \%)$ \\
\hline Adjusted rate ${ }^{c}$ & $35.7 \%$ & $62.4 \%$ & $60.5 \%$ & $58.9 \%$ \\
\hline Terminal rate ${ }^{\mathrm{d}}$ & $12 / 35(34 \%)$ & $15 / 26(58 \%)$ & $12 / 24(50 \%)$ & $13 / 25(52 \%)$ \\
\hline First incidence (days) & 663 & 590 & 117 & 473 \\
\hline Poly-3 test ${ }^{\mathrm{e}}$ & $\mathrm{P}=0.118$ & $P=0.007$ & $\mathrm{P}=0.013$ & $P=0.019$ \\
\hline
\end{tabular}

${ }^{a}$ Historical control incidence for 2-year studies (all routes): 35/100.

${ }^{b}$ Number of animals with mononuclear cell leukemia per number of animals necropsied.

'Poly-3 estimated neoplasm incidence after adjustment for intercurrent mortality.

dObserved incidence at terminal kill.

${ }^{\mathrm{e} B e n e a t h}$ the chamber control incidence is the $\mathrm{P}$ value associated with the trend test. Beneath the exposed group incidence are the $\mathrm{P}$ values corresponding to pairwise comparisons between the chamber controls and that exposed group. The Poly-3 test accounts for differential mortality in animals that do not reach terminal kill.

Liver: The incidences of basophilic focus occurred with positive trends in male and female rats and in all exposed groups of males (chamber control, $5 / 50 ; 1.25 \mathrm{mg} / \mathrm{m}^{3}, 17 / 50 ; 2.5 \mathrm{mg} / \mathrm{m}^{3}, 17 / 50$; $\left.5 \mathrm{mg} / \mathrm{m}^{3}, 19 / 50\right)$ and in females exposed to $5 \mathrm{mg} / \mathrm{m}^{3}(16 / 50,20 / 50,22 / 50,33 / 50)$, and the incidences were significantly greater than those in the chamber control groups (Table A-7 and Table B-7). Basophilic foci occur spontaneously in rats, and the incidences are sometimes increased with exposure to chemicals. They are considered putative preneoplastic lesions; however, the incidences of hepatocellular neoplasms were not increased in male or female rats exposed to cobalt metal (Table A-1 and Table B-1).

Testes: The incidence of infarct was significantly increased in male rats exposed to $5 \mathrm{mg} / \mathrm{m}^{3}$ $(1 / 50,0 / 50,2 / 50,12 / 50)$ (Table A-4). Infarcts were mostly unilateral, and in affected testes, there was complete effacement of the parenchyma due to necrosis with loss of differential staining (tissue was diffusely hypereosinophilic) and cellular detail. Multifocal intratubular mineralization was current in a few of the affected testes.

\section{Tissue Burden Studies}

Lung weights and lung cobalt burdens were determined in female rats (Table I-7). Lung weights increased in all exposed groups; however, increases in lung weights occurred earlier in the study (day 184) in the 2.5 and $5 \mathrm{mg} / \mathrm{m}^{3}$ groups than in the $1.25 \mathrm{mg} / \mathrm{m}^{3}$ group (day 366). Because of the significant changes in lung weights with increasing exposure concentration, lung cobalt burdens rather than lung cobalt concentrations were evaluated for toxicokinetic parameters.

Cobalt concentrations and burdens in the lung increased with increasing exposure concentration and were significantly increased in all exposed groups of female rats at all time points compared to those in the chamber control group (Table I-7). Cobalt concentrations in the chamber control 
group were at or below the limit of detection (LOD) at all time points except day 548 [one animal had a lung cobalt concentration exceeding the LOD but less than the experimental limit of quantitation (ELOQ)]. By day 184, lung cobalt concentrations for all exposed groups appeared to reach steady state and did not change significantly through day 548; lung cobalt burdens increased rapidly by day 4 , but by day 184 the rate of increase slowed as lung burdens asymptotically approached steady state. Analysis of normalized lung cobalt burdens revealed no tendency toward disproportionate changes and no biologically significant differences in normalized burdens with increasing exposure concentration.

The lung cobalt burden data from the exposure phases of the 3-month and 2-year studies were modeled using a two-compartment model; these data show that steady state was clearly reached at 2.5 and $5 \mathrm{mg} / \mathrm{m}^{3}$ but not at $1.25 \mathrm{mg} / \mathrm{m}^{3}$ (Table I-1). Rapid clearance phase half-lives were between 1.53 days and 2.94 days (Table I-8), while slow clearance phase half-lives were 789 days, 167 days, and 83 days for $1.25 \mathrm{mg} / \mathrm{m}^{3}, 2.5 \mathrm{mg} / \mathrm{m}^{3}$, and $5 \mathrm{mg} / \mathrm{m}^{3}$, respectively. The apparent lack of achievement of steady state and long half-life at $1.25 \mathrm{mg} / \mathrm{m}^{3}$ are likely spurious findings due to uncertainty in the model. Cobalt deposition rates were 1.4, 2.1, and $5.6 \mu \mathrm{g}$ cobalt/day during the rapid clearance phase and $0.018,0.078$, and $0.29 \mu \mathrm{g}$ cobalt/day during the slow clearance phase at $1.25,2.5$, and $5 \mathrm{mg} / \mathrm{m}^{3}$, respectively. Steady-state lung cobalt burdens including both the rapid and slow clearance phases ( $\left.\mathrm{LSSa}_{\mathrm{Sa}}+\mathrm{L}_{\mathrm{SSb}}\right)$ were approximately $25.4,27.8$, and $46.8 \mu \mathrm{g}$ cobalt/lung in animals exposed to $1.25,2.5$, and $5 \mathrm{mg} / \mathrm{m}^{3}$, respectively. The fractions of deposition in the slow clearance phase $\left(\mathrm{F}_{\mathrm{B}}\right)$ for the exposed groups were quite low, increasing from 0.012 to 0.049 as exposure concentrations increased, corresponding to total slow phase lung cobalt clearances of $1.2 \%$ to $4.9 \%$; clearances of total deposited cobalt during the rapid clearance phase ranged from $98.8 \%$ to $95.1 \%\left[\left(1-\mathrm{F}_{\mathrm{B}}\right) \times 100\right]$ with increasing exposure concentration.

\section{Mice}

\section{Two-week Study}

The exposure concentrations for the 2-week study were estimated based on exposures of previously studied cobalt sulfate heptahydrate ${ }^{66}$. Three male and three female mice exposed to $40 \mathrm{mg} / \mathrm{m}^{3}$ died before the end of the study (Table 18). Final mean body weights were significantly decreased in male and female mice exposed to 20 or $40 \mathrm{mg} / \mathrm{m}^{3}$ by $9 \%$ and $27 \%$ (males) or $16 \%$ and $38 \%$ (females), respectively, compared to the chamber control groups. Mean body weight gains of 20 and $40 \mathrm{mg} / \mathrm{m}^{3}$ males and all exposed groups of females were significantly less than those of the chamber controls. Females exposed to $20 \mathrm{mg} / \mathrm{m}^{3}$ and males and females exposed to $40 \mathrm{mg} / \mathrm{m}^{3}$ lost weight during the study. Exposure-related clinical findings included abnormal breathing, lethargy, and thinness in males exposed to 20 or $40 \mathrm{mg} / \mathrm{m}^{3}$ and females exposed to $10 \mathrm{mg} / \mathrm{m}^{3}$ or greater. At necropsy, tan lungs were observed in most males and females exposed to 20 or $40 \mathrm{mg} / \mathrm{m}^{3}$. Dark lung lobes were observed in one early-death male.

Absolute lung weights of both sexes exposed to $5 \mathrm{mg} / \mathrm{m}^{3}$ or greater and relative lung weights of males exposed to $10 \mathrm{mg} / \mathrm{m}^{3}$ or greater and of females exposed to $5 \mathrm{mg} / \mathrm{m}^{3}$ or greater were significantly increased compared to the chamber controls (Table 19 and Table G-3). Absolute liver weights of all exposed groups of males and females and the relative liver weights of both sexes exposed to $2.5,5,10$, or $20 \mathrm{mg} / \mathrm{m}^{3}$ were significantly less than those of the chamber 
controls. Absolute testis weight of the $40 \mathrm{mg} / \mathrm{m}^{3}$ group was significantly less than that of the chamber controls.

Table 18. Survival and Body Weights of Mice in the Two-week Inhalation Study of Cobalt Metal ${ }^{\text {a }}$

\begin{tabular}{|c|c|c|c|c|c|}
\hline $\begin{array}{c}\text { Concentration } \\
\left(\mathbf{m g} / \mathrm{m}^{3}\right)\end{array}$ & Survival ${ }^{\mathbf{b}}$ & $\begin{array}{l}\text { Initial Body } \\
\text { Weight } \\
\text { (g) }\end{array}$ & $\begin{array}{l}\text { Final Body } \\
\text { Weight } \\
\text { (g) }\end{array}$ & $\begin{array}{l}\text { Change in Body } \\
\text { Weight } \\
\text { (g) }\end{array}$ & $\begin{array}{c}\text { Final Weight } \\
\text { Relative to } \\
\text { Controls } \\
(\%) \\
\end{array}$ \\
\hline \multicolumn{6}{|l|}{ Male } \\
\hline 0 & $5 / 5$ & $23.4 \pm 0.3$ & $25.7 \pm 0.5$ & $2.3 \pm 0.3$ & \\
\hline 2.5 & $5 / 5$ & $23.5 \pm 0.3$ & $25.0 \pm 0.5$ & $1.5 \pm 0.2$ & 97 \\
\hline 5 & $5 / 5$ & $23.6 \pm 0.3$ & $25.9 \pm 0.3$ & $2.2 \pm 0.4$ & 101 \\
\hline 10 & $5 / 5$ & $23.8 \pm 0.3$ & $25.3 \pm 0.5$ & $1.5 \pm 0.2$ & 98 \\
\hline 20 & $5 / 5$ & $23.1 \pm 0.4$ & $23.4 \pm 0.4^{* *}$ & $0.2 \pm 0.4^{* *}$ & 91 \\
\hline 40 & $2 / 5^{\mathrm{c}}$ & $23.0 \pm 0.4$ & $18.9 \pm 1.1 * *$ & $-4.7 \pm 1.7^{* *}$ & 73 \\
\hline \multicolumn{6}{|l|}{ Female } \\
\hline 0 & $5 / 5$ & $19.1 \pm 0.3$ & $20.8 \pm 0.1$ & $1.7 \pm 0.3$ & \\
\hline 2.5 & $5 / 5$ & $19.8 \pm 0.5$ & $20.3 \pm 0.5$ & $0.5 \pm 0.2 *$ & 98 \\
\hline 5 & $5 / 5$ & $19.8 \pm 0.5$ & $20.1 \pm 0.5$ & $0.3 \pm 0.4^{*}$ & 97 \\
\hline 10 & $5 / 5$ & $19.4 \pm 0.4$ & $20.0 \pm 0.6$ & $0.6 \pm 0.4^{*}$ & 96 \\
\hline 20 & $5 / 5$ & $19.0 \pm 0.3$ & $17.4 \pm 0.4^{* *}$ & $-1.6 \pm 0.2^{* *}$ & 84 \\
\hline 40 & $2 / 5^{\mathrm{d}}$ & $18.9 \pm 0.2$ & $13.0 \pm 1.6^{* *}$ & $-6.1 \pm 1.1^{* *}$ & 62 \\
\hline
\end{tabular}

*Significantly different $(\mathrm{P} \leq 0.05)$ from the chamber control group by Williams' test.

$* * \mathrm{P} \leq 0.01$.

${ }^{a}$ Weights and weight changes are given as mean \pm standard error. Subsequent calculations are based on animals surviving to the end of the study.

${ }^{b}$ Number of animals surviving at 17 days/number initially in group.

'Days of deaths: $5,5,8$.

${ }^{d}$ Days of deaths: $6,7,9$.

Table 19. Selected Organ Weights and Organ-Weight-to-Body-Weight Ratios for Mice in the Twoweek Inhalation Study of Cobalt Metal ${ }^{\mathrm{a}}$

\begin{tabular}{|c|c|c|c|c|c|c|}
\hline & $\begin{array}{l}\text { Chamber } \\
\text { Control }\end{array}$ & $2.5 \mathrm{mg} / \mathrm{m}^{3}$ & $5 \mathrm{mg} / \mathrm{m}^{3}$ & $10 \mathrm{mg} / \mathrm{m}^{3}$ & $20 \mathrm{mg} / \mathrm{m}^{3}$ & $40 \mathrm{~m} / \mathrm{m}^{3}$ \\
\hline $\mathbf{n}$ & 5 & 5 & 5 & 5 & 5 & 2 \\
\hline \multicolumn{7}{|l|}{ Male } \\
\hline $\begin{array}{l}\text { Necropsy } \\
\text { body wt }\end{array}$ & $25.7 \pm 0.5$ & $25.0 \pm 0.5$ & $25.9 \pm 0.3$ & $25.3 \pm 0.5$ & $23.4 \pm 0.4 * *$ & $18.9 \pm 1.1^{* *}$ \\
\hline \multicolumn{7}{|l|}{ Liver } \\
\hline Absolute & $1.13 \pm 0.04$ & $0.98 \pm 0.04 * *$ & $0.98 \pm 0.04 * *$ & $0.99 \pm 0.02 * *$ & $0.89 \pm 0.02 * *$ & $0.83 \pm 0.01 * *$ \\
\hline Relative & $43.88 \pm 0.80$ & $39.18 \pm 1.35^{*}$ & $\begin{array}{c}37.67 \pm 1.09^{*} \\
*\end{array}$ & $39.32 \pm 0.91^{*}$ & $37.93 \pm 0.51^{* *}$ & $44.20 \pm 2.99$ \\
\hline Lung & & & & & & \\
\hline
\end{tabular}




\begin{tabular}{|c|c|c|c|c|c|c|}
\hline & $\begin{array}{l}\text { Chamber } \\
\text { Control }\end{array}$ & $2.5 \mathrm{mg} / \mathrm{m}^{3}$ & $5 \mathrm{mg} / \mathrm{m}^{3}$ & $10 \mathrm{mg} / \mathrm{m}^{3}$ & $20 \mathrm{mg} / \mathrm{m}^{3}$ & $40 \mathrm{~m} / \mathrm{m}^{3}$ \\
\hline Absolute & $0.18 \pm 0.01$ & $0.21 \pm 0.01$ & $0.23 \pm 0.01 *$ & $0.24 \pm 0.01 * *$ & $0.29 \pm 0.01 * *$ & $0.36 \pm 0.05^{* *}$ \\
\hline Relative & $7.08 \pm 0.15$ & $8.33 \pm 0.32$ & $8.73 \pm 0.33$ & $9.61 \pm 0.62 *$ & $12.62 \pm 0.51 * *$ & $19.31 \pm 3.73 * *$ \\
\hline \multicolumn{7}{|l|}{ L. Testis } \\
\hline Absolute & $0.098 \pm 0.002$ & $0.104 \pm 0.001$ & $0.099 \pm 0.004$ & $0.084 \pm 0.009$ & $0.089 \pm 0.003$ & $0.070 \pm 0.002 * *$ \\
\hline Relative & $3.834 \pm 0.074$ & $4.180 \pm 0.114$ & $3.812 \pm 0.149$ & $3.322 \pm 0.311$ & $3.807 \pm 0.088$ & $3.731 \pm 0.314$ \\
\hline \multicolumn{7}{|l|}{ Female } \\
\hline $\begin{array}{l}\text { Necropsy } \\
\text { body wt }\end{array}$ & $20.8 \pm 0.1$ & $20.3 \pm 0.5$ & $20.1 \pm 0.5$ & $20.0 \pm 0.6$ & $17.4 \pm 0.4^{* *}$ & $13.0 \pm 1.6^{* *}$ \\
\hline \multicolumn{7}{|l|}{ Liver } \\
\hline Absolute & $0.93 \pm 0.03$ & $0.81 \pm 0.02 * *$ & $0.80 \pm 0.03^{* *}$ & $0.75 \pm 0.03 * *$ & $0.69 \pm 0.03 * *$ & $0.61 \pm 0.06^{* *}$ \\
\hline Relative & $44.56 \pm 1.13$ & $\begin{array}{c}40.09 \pm 0.31 * \\
*\end{array}$ & $\begin{array}{c}39.75 \pm 0.82 * \\
*\end{array}$ & $37.40 \pm 1.12 * *$ & $39.73 \pm 0.70 * *$ & $46.88 \pm 1.36$ \\
\hline
\end{tabular}

Lung

\begin{tabular}{lllllll} 
Absolute & $0.19 \pm 0.01$ & $0.19 \pm 0.00$ & $0.22 \pm 0.01 *$ & $0.23 \pm 0.01 * *$ & $0.29 \pm 0.01 * *$ & $0.33 \pm 0.02^{* *}$ \\
Relative & $9.34 \pm 0.38$ & $9.49 \pm 0.37$ & $11.14 \pm 0.24^{*}$ & $11.77 \pm 0.59^{* *}$ & $16.80 \pm 0.58^{* *}$ & $25.67 \pm 1.53^{* *}$ \\
\hline
\end{tabular}

*Significantly different $(\mathrm{P} \leq 0.05)$ from the chamber control group by Williams' or Dunnett's test. $* * \mathrm{P} \leq 0.01$.

${ }^{a}$ Organ weights (absolute weights) and body weights are given in grams; organ-weight-to-body-weight ratios (relative weights) are given as $\mathrm{mg}$ organ weight/g body weight (mean \pm standard error).

Increased incidences of nonneoplastic lesions of the lung occurred in exposed male and female mice (Table 20). The incidences of minimal to moderate alveolar histiocytic cellular infiltration were significantly increased in males and females exposed to $5 \mathrm{mg} / \mathrm{m}^{3}$ or greater and consisted of varying numbers of histiocytes (macrophages) within the alveolar spaces and septa. The incidences of minimal to mild cytoplasmic vacuolization of the bronchiolar epithelium were signify-cantly increased in males exposed to 2.5 or $10 \mathrm{mg} / \mathrm{m}^{3}$ and females exposed to $5 \mathrm{mg} / \mathrm{m}^{3}$. The lesion was characterized by the presence of poorly defined clear spaces within the cytoplasm of bronchiolar epithelial cells. The incidences of minimal to mild alveolar/bronchiolar epithelium karyomegaly were significantly increased in males and females exposed to $5 \mathrm{mg} / \mathrm{m}^{3}$ or greater. Karyomegaly consisted of scattered, hypertrophied epithelial cells with single large and sometimes multiple, atypical nuclei in the terminal bronchioles, alveolar ducts, and alveoli. The incidences of minimal to moderate interstitial fibrosis were increased in all groups of mice exposed to $10 \mathrm{mg} / \mathrm{m}^{3}$ or greater. Fibrosis consisted of multifocal to coalescing areas in which the alveolar architecture was effaced or obscured by fibroblasts and/or collagen within which were accumulations of histiocytes. Minimal to mild acute inflammation occurred in $40 \mathrm{mg} / \mathrm{m}^{3}$ males and females that died before the end of the study. Minimal acute inflammation was also observed in some females exposed to 5,10 , or $20 \mathrm{mg} / \mathrm{m}^{3}$. Acute inflammation consisted of perivascular and, to a lesser extent, peribronchiolar edema mixed with infiltrates of neutrophils.

Increased incidences of nonneoplastic lesions of the nose occurred in exposed groups of male and female mice (Table 20). The incidences of minimal to moderate acute inflammation were significantly increased in males exposed to $10 \mathrm{mg} / \mathrm{m}^{3}$ or greater and females exposed to $5 \mathrm{mg} / \mathrm{m}^{3}$ or greater. There was no increase in severity. 
Table 20. Incidences of Selected Nonneoplastic Lesions of the Respiratory System in Mice in the Two-week Inhalation Study of Cobalt Metal

\begin{tabular}{|c|c|c|c|c|c|c|}
\hline & $\begin{array}{l}\text { Chamber } \\
\text { Control }\end{array}$ & $2.5 \mathrm{mg} / \mathrm{m}^{3}$ & $5 \mathrm{mg} / \mathrm{m}^{3}$ & $10 \mathrm{mg} / \mathrm{m}^{3}$ & $20 \mathrm{mg} / \mathrm{m}^{3}$ & $40 \mathrm{mg} / \mathrm{m}^{3}$ \\
\hline \multicolumn{7}{|l|}{ Male } \\
\hline Lung $^{\mathrm{a}}$ & 5 & 5 & 5 & 5 & 5 & 5 \\
\hline $\begin{array}{l}\text { Alveolus, Infiltration } \\
\text { Cellular, Histiocyte }{ }^{\mathrm{b}}\end{array}$ & 0 & $2(1.0)^{\mathrm{c}}$ & $5 * *(1.0)$ & $5^{* *}(1.4)$ & $5 * *(2.4)$ & $5 * *(2.0)$ \\
\hline $\begin{array}{l}\text { Bronchiole, Epithelium, } \\
\text { Vacuolization, } \\
\text { Cytoplasmic }\end{array}$ & 0 & $4 *(1.0)$ & $3(1.0)$ & $5 * *(1.6)$ & $3(1.0)$ & $3(1.3)$ \\
\hline $\begin{array}{l}\text { Alveolar/bronchiolar } \\
\text { Epithelium, Karyomegaly }\end{array}$ & 0 & 0 & $4 *(1.0)$ & $5^{* *}(1.0)$ & $5^{* *}(1.8)$ & $4 *(1.5)$ \\
\hline Interstitium, Fibrosis & 0 & 0 & 0 & $3(1.0)$ & $5 * *(2.2)$ & $3(2.7)$ \\
\hline Inflammation, Acute & 0 & 0 & 0 & 0 & 0 & $3(1.7)$ \\
\hline Nose & 5 & 5 & 5 & 5 & 5 & 5 \\
\hline Inflammation, Acute & 0 & 0 & $1(1.0)$ & $5 * *(2.4)$ & $5 * *(1.6)$ & $5 * *(1.8)$ \\
\hline $\begin{array}{l}\text { Olfactory Epithelium, } \\
\text { Atrophy }\end{array}$ & 0 & $5 * *(1.0)$ & $5 * *(1.0)$ & $5 * *(1.8)$ & $5^{* *}(1.8)$ & $4 * *(2.0)$ \\
\hline $\begin{array}{l}\text { Olfactory Epithelium, } \\
\text { Necrosis }\end{array}$ & 0 & $2(1.0)$ & $3(1.0)$ & 0 & $5 * *(1.2)$ & $5 * *(1.4)$ \\
\hline $\begin{array}{l}\text { Respiratory Epithelium, } \\
\text { Vacuolization Cytoplasmic }\end{array}$ & 0 & $4 *(1.0)$ & $5 * *(1.0)$ & $4 *(1.0)$ & $5^{* *}(1.2)$ & $5 * *(1.2)$ \\
\hline $\begin{array}{l}\text { Respiratory Epithelium, } \\
\text { Metaplasia, Squamous }\end{array}$ & 0 & 0 & 0 & $4 *(1.0)$ & $4 *(1.0)$ & $2(1.0)$ \\
\hline \multicolumn{7}{|l|}{ Female } \\
\hline Lung & 5 & 5 & 5 & 5 & 5 & 5 \\
\hline $\begin{array}{l}\text { Alveolus, Infiltration } \\
\text { Cellular, Histiocyte }\end{array}$ & 0 & $2(1.0)$ & $5 * *(1.4)$ & $5 * *(1.6)$ & $5 * *(2.6)$ & $5 * *(2.4)$ \\
\hline $\begin{array}{l}\text { Bronchiole, Epithelium, } \\
\text { Vacuolization, } \\
\text { Cytoplasmic }\end{array}$ & 0 & $2(1.0)$ & $4 *(1.0)$ & $3(1.7)$ & $2(1.0)$ & $1(1.0)$ \\
\hline $\begin{array}{l}\text { Alveolar/bronchiolar } \\
\text { Epithelium, Karyomegaly }\end{array}$ & 0 & $3(1.0)$ & $4 *(1.0)$ & $5 * *(1.2)$ & $4 *(1.3)$ & $4 *(1.0)$ \\
\hline Interstitium, Fibrosis & 0 & 0 & 0 & $2(1.0)$ & $5 * *(2.8)$ & $2(3.5)$ \\
\hline Inflammation, Acute & 0 & 0 & $2(1.0)$ & $1(1.0)$ & $3(1.0)$ & $2(1.5)$ \\
\hline Nose & 5 & 5 & 5 & 5 & 5 & 5 \\
\hline Acute Inflammation & 0 & 0 & $5 * *(2.0)$ & $5^{* *}(2.6)$ & $5 * *(2.4)$ & $5 * *(2.2)$ \\
\hline $\begin{array}{l}\text { Olfactory Epithelium, } \\
\text { Atrophy }\end{array}$ & 0 & $5^{* *}(1.4)$ & $5 * *(1.6)$ & $5^{* *}(1.8)$ & $5^{* *}(2.2)$ & $3(2.0)$ \\
\hline $\begin{array}{l}\text { Olfactory Epithelium, } \\
\text { Necrosis }\end{array}$ & 0 & $3(1.0)$ & $5 * *(1.0)$ & $2(1.5)$ & $4 *(1.5)$ & $3(1.7)$ \\
\hline $\begin{array}{l}\text { Respiratory Epithelium, } \\
\text { Vacuolization Cytoplasmic }\end{array}$ & 0 & $5 * *(1.0)$ & $5 * *(1.0)$ & $5 * *(1.2)$ & $4 *(1.0)$ & $4 *(1.0)$ \\
\hline
\end{tabular}




\begin{tabular}{|c|c|c|c|c|c|c|}
\hline & $\begin{array}{l}\text { Chamber } \\
\text { Control }\end{array}$ & $2.5 \mathrm{mg} / \mathrm{m}^{3}$ & $5 \mathrm{mg} / \mathrm{m}^{3}$ & $10 \mathrm{mg} / \mathrm{m}^{3}$ & $20 \mathrm{mg} / \mathrm{m}^{3}$ & $40 \mathrm{mg} / \mathrm{m}^{3}$ \\
\hline $\begin{array}{l}\text { Respiratory Epithelium, } \\
\text { Metaplasia, Squamous }\end{array}$ & 0 & 0 & 0 & $1(1.0)$ & $3(1.0)$ & $1(1.0)$ \\
\hline
\end{tabular}

*Significantly different $(\mathrm{P} \leq 0.05)$ from the chamber control group by the Fisher exact test.

$* * \mathrm{P} \leq 0.01$.

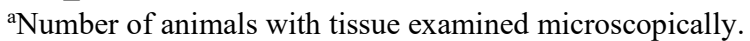

${ }^{b}$ Number of animals with lesion.

${ }^{\mathrm{c}}$ Average severity grade of lesions in affected animals: $1=$ minimal, $2=$ mild, $3=$ moderate, $4=$ marked.

with increasing exposure concentration. Acute inflammation occurred in the respiratory and olfactory regions of the nasal cavity and was characterized by accumulation of proteinaceous fluid, mucus, and neutrophilic cell debris in the nasal passages. Occasionally, there were low numbers of neutrophils within the mucosa and lamina propria. The incidences of minimal to mild olfactory epithelium atrophy were significantly increased in all exposed groups except the $40 \mathrm{mg} / \mathrm{m}^{3}$ females. Atrophy often accompanied necrosis and was characterized by loss of olfactory epithelial cells with decreased height of the epithelium. The remaining olfactory cells were often disorganized, flattened to cuboidal, or respiratory-type ciliated columnar and replaced the olfactory epithelial cells. In some areas, there were clusters or nests of sensory-type cells within the remaining olfactory epithelium; this was interpreted as a regenerative response. The incidences of minimal to mild olfactory epithelium necrosis were significantly increased in males exposed to 20 or $40 \mathrm{mg} / \mathrm{m}^{3}$ and females exposed to 5 or $20 \mathrm{mg} / \mathrm{m}^{3}$. Necrosis was characterized by segmental vacuolization, disorganization, and loss of epithelial cells in the dorsal meatus of the Level II nasal section and the lateral and ventral aspects of the ethmoid turbinates in Level III with full-thickness sloughing of the epithelium in some areas. The incidences of minimal cytoplasmic vacuolization of the respiratory epithelium were significantly increased in all exposed groups of males and females. Cytoplasmic vacuolization was characterized by the presence of poorly defined clear spaces within the cytoplasm of respiratory epithelial cells in Levels I and II. The incidences of minimal squamous metaplasia of the respiratory epithelium were significantly increased in males exposed to 10 or $20 \mathrm{mg} / \mathrm{m}^{3}$. Squamous epithelial metaplasia involved lateral walls, turbinates, and ventral septa in Levels I and II. In affected sites, the normal single layer of tall columnar, ciliated epithelial cells was replaced by one to three layers of flattened (squamous) epithelial cells that lacked cilia.

\section{Tissue Burden Studies}

Tissue weights and concentrations were determined in male and female mice at terminal kill and in additional female mice held for 3 weeks after the exposure. Data were generated on male and female mice in all exposure groups; however, relatively small numbers of samples ( $\mathrm{n}=1$ to 2 ) were available in $40 \mathrm{mg} / \mathrm{m}^{3}$ females due to decreased survival.

Male and female mouse lung weights increased with increasing exposure concentration, reaching weights that were up to 1.5- to 2-fold greater than those of the chamber controls at terminal kill (Table I-9). In female mice that were held for the 3-week recovery period, lung weights of exposed groups recovered such that they were similar to those of the chamber controls at the end of the recovery period. In both males and females, treatment-related decreases in the weights of all other tissues occurred. Because of the significant changes in lung weight, lung cobalt burdens rather than lung concentrations were evaluated for toxicokinetic parameters. 
At terminal kill, cobalt concentrations and burdens increased with exposure concentration in all tissues examined (Table I-9). Cobalt concentrations in tissues decreased in the order of lung $>$ liver $>$ kidney $>$ serum $>$ heart approximately equal to femur $>$ blood $>$ testes (males). Tissue cobalt burdens in male mouse tissues decreased in the order of lung $>$ liver $>$ kidney $>$ heart $>$ femur $>$ testes. With the exception of testes, all tissues examined represented sites where cobalt could accumulate at concentrations greater than observed in the blood or serum. Mice of both sexes accumulated large amounts of cobalt in the liver. While lung cobalt burdens were generally higher than liver cobalt burdens at exposures of $20 \mathrm{mg} / \mathrm{m}^{3}$, liver and lung burdens were similar in females exposed to $20 \mathrm{mg} / \mathrm{m}^{3}$ or less, and liver burdens were greater than lung burdens in $40 \mathrm{mg} / \mathrm{m}^{3}$ males and females. Normalized tissue burdens generally remained the same or decreased with increasing exposure concentration.

Kinetic analysis of data from female mice exposed to $20 \mathrm{mg} / \mathrm{m}^{3}$ or less indicated elimination half-lives of 4.1 to 7.3 days (blood), 2.9 to 3.7 days (serum), or 5.5 to 6.6 days (lung) (Table I-10); in general, half-lives decreased with increasing exposure concentration. Lung cobalt deposition rates and predicted steady-state lung cobalt burdens increased in proportion to exposure concentrations of 2.5 and $5 \mathrm{mg} / \mathrm{m}^{3}$, but the increases were less than proportional at greater exposure concentrations.

Exposure Concentration Selection Rationale: Significant mortality was observed in male and female mice exposed to $40 \mathrm{mg} / \mathrm{m}^{3}$ in the 2-week study. There were significant decreases in body weights in $20 \mathrm{mg} / \mathrm{m}^{3}$ males $(9 \%)$ and females (16\%). Significantly increased incidences of alveolar infiltration (histiocytic) were observed in the lung of males and females exposed to $5 \mathrm{mg} / \mathrm{m}^{3}$ or greater. However, the average severity grade was minimal in the 5 and $10 \mathrm{mg} / \mathrm{m}^{3}$ groups, and the increases in lung weights in the $10 \mathrm{mg} / \mathrm{m}^{3}$ groups were not considered sufficiently severe to preclude the use of this concentration. Hence, $10 \mathrm{mg} / \mathrm{m}^{3}$ was selected as the highest exposure concentration for the 3-month inhalation study in mice.

\section{Three-month Study}

One $2.5 \mathrm{mg} / \mathrm{m}^{3}$ female mouse was accidentally killed during the first week of the study; all other mice survived to the end of the study (Table 21). The final mean body weights and mean body weight gains of males and females exposed to $10 \mathrm{mg} / \mathrm{m}^{3}$ were significantly less than those of the chamber controls (Table 21 and Figure 4). Abnormal breathing was noted in approximately 50\% of males and females exposed to $10 \mathrm{mg} / \mathrm{m}^{3}$. At necropsy, tan lungs were noted in mice exposed to 5 or $10 \mathrm{mg} / \mathrm{m}^{3}$.

On days 26 and 40, microsomal suspensions of liver samples from special study female mice not used for tissue burden studies were prepared and assayed for A4H, EROD, and PROD activities (Table J-2). There were no consistent trends in A4H, EROD, or PROD activities relative to exposure concentrations at either time point or from day 26 to day 40 . A4H activities were significantly increased in the 5 and $10 \mathrm{mg} / \mathrm{m}^{3}$ groups on day 40 . EROD activities were significantly increased in the 5 and $10 \mathrm{mg} / \mathrm{m}^{3}$ groups at both time points.

Similar to effects noted in rats, statistically significant increases were observed in hemoglobin concentration and erythrocyte count of $10 \mathrm{mg} / \mathrm{m}^{3}$ males and in the erythrocyte count of $10 \mathrm{mg} / \mathrm{m}^{3}$ females at 14 weeks (Table F-2). These changes in comparison to the rats, however, were minimal $(<5 \%)$. 
Table 21. Survival and Body Weights of Mice in the Three-month Inhalation Study of Cobalt Metal $^{\mathrm{a}}$

\begin{tabular}{cccccc}
\hline $\begin{array}{c}\text { Concentration } \\
\left(\mathbf{m g} / \mathbf{m}^{3}\right)\end{array}$ & Survival & $\begin{array}{c}\text { Initial Body } \\
\text { Weight } \\
\mathbf{( g )}\end{array}$ & $\begin{array}{c}\text { Final Body } \\
\text { Weight } \\
\mathbf{( g )}\end{array}$ & $\begin{array}{c}\text { Change in Body } \\
\text { Weight } \\
\mathbf{( g )}\end{array}$ & $\begin{array}{c}\text { Final Weight } \\
\text { Relative to } \\
\text { Controls } \\
\mathbf{( \% )}\end{array}$ \\
\hline Male & & & & & \\
0 & $10 / 10$ & $23.7 \pm 0.2$ & $37.7 \pm 0.8$ & $14.0 \pm 0.7$ & \\
0.625 & $10 / 10$ & $23.7 \pm 0.3$ & $38.2 \pm 0.6$ & $14.5 \pm 0.4$ & 101 \\
1.25 & $10 / 10$ & $23.7 \pm 0.2$ & $37.9 \pm 0.8$ & $14.2 \pm 0.8$ & 101 \\
2.5 & $10 / 10$ & $23.8 \pm 0.2$ & $37.0 \pm 0.5$ & $13.3 \pm 0.4$ & 98 \\
5 & $10 / 10$ & $23.7 \pm 0.2$ & $37.0 \pm 0.9$ & $13.4 \pm 0.8$ & 98 \\
10 & $10 / 10$ & $23.8 \pm 0.2$ & $32.5 \pm 0.5^{* *}$ & $8.7 \pm 0.5 * *$ & 86 \\
\hline Female & & & & & \\
0 & $10 / 10$ & $20.5 \pm 0.3$ & $30.9 \pm 1.0$ & $10.4 \pm 1.1$ & 102 \\
0.625 & $10 / 10$ & $20.0 \pm 0.3$ & $31.6 \pm 1.1$ & $11.6 \pm 1.3$ & 102 \\
1.25 & $10 / 10$ & $20.2 \pm 0.4$ & $31.4 \pm 0.9$ & $11.2 \pm 0.7$ & 97 \\
2.5 & $9 / 10^{c}$ & $19.8 \pm 0.2$ & $30.1 \pm 0.7$ & $10.1 \pm 0.7$ & 94 \\
5 & $10 / 10$ & $20.1 \pm 0.3$ & $29.0 \pm 1.1$ & $8.9 \pm 1.0$ & 87 \\
\hline 10 & $10 / 10$ & $19.8 \pm 0.2$ & $26.8 \pm 1.0^{* *}$ & $7.0 \pm 1.0^{*}$ & \\
\hline
\end{tabular}

*Significantly different $(\mathrm{P} \leq 0.05)$ from the chamber control group by Williams' test.

$* * \mathrm{P} \leq 0.01$.

${ }^{\text {a}}$ Weights and weight changes are given as mean \pm standard error. Subsequent calculations are based on animals surviving to the end of the study.

${ }^{b}$ Number of animals surviving at 14 weeks/number initially in group.

${ }^{\mathrm{c}}$ Week of death: 1. 

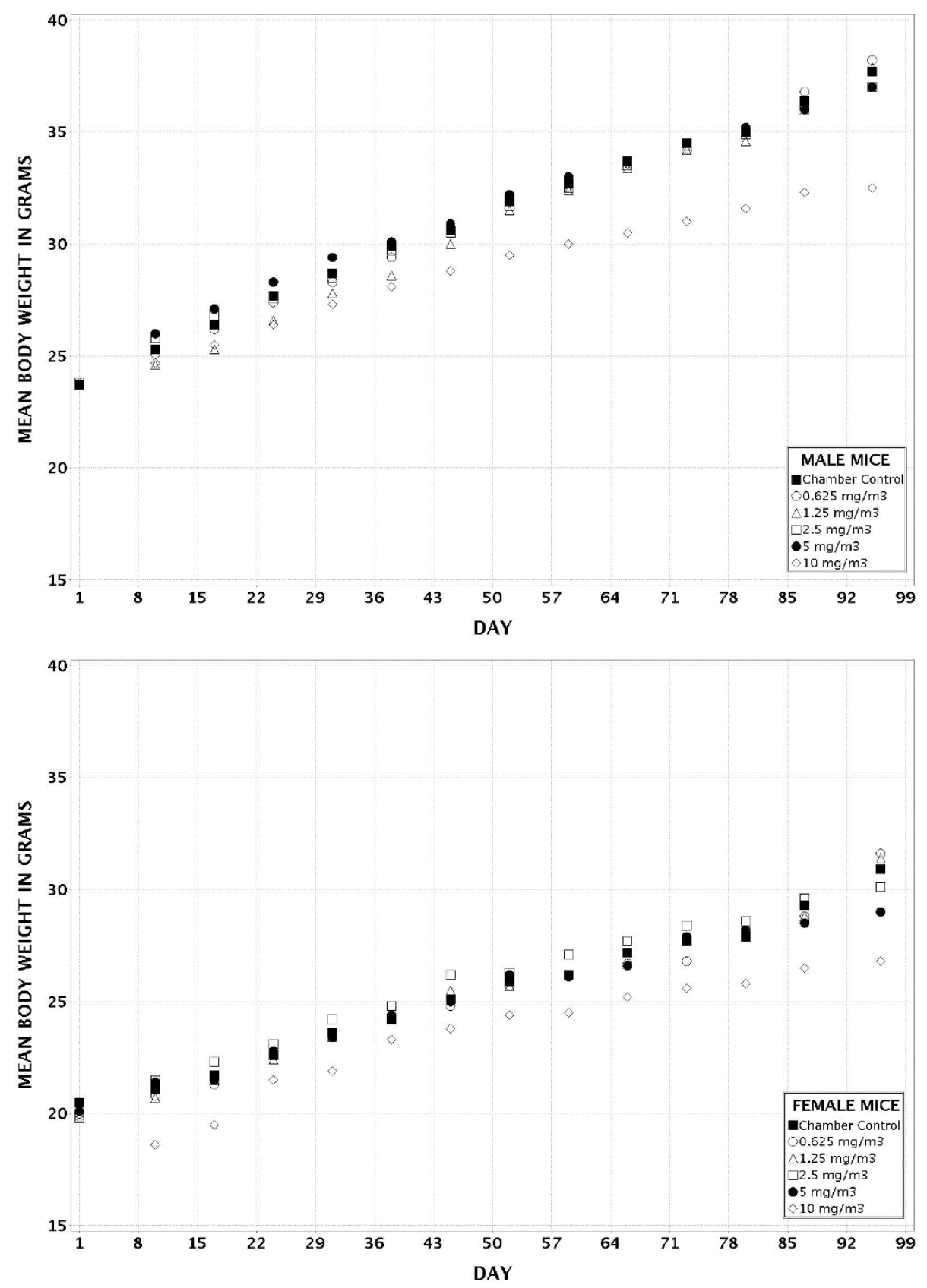

Figure 4. Growth Curves for Mice Exposed to Cobalt Metal by Inhalation for Three Months

Absolute and relative lung weights of males exposed to $2.5 \mathrm{mg} / \mathrm{m}^{3}$ or greater and females exposed to 5 or $10 \mathrm{mg} / \mathrm{m}^{3}$ were significantly greater than those of the chamber controls (Table 22 and Table G-4). Absolute and relative liver weights of males exposed to $10 \mathrm{mg} / \mathrm{m}^{3}$ and females exposed to $2.5 \mathrm{mg} / \mathrm{m}^{3}$ or greater were significantly less than those of the chamber controls. Absolute and relative kidney weights of males and females exposed to 5 or $10 \mathrm{mg} / \mathrm{m}^{3}$ were significantly less than those of the chamber controls. Absolute and relative testes weights of males exposed to 5 or $10 \mathrm{mg} / \mathrm{m}^{3}$ were significantly less than those of the chamber controls. 
Table 22. Selected Organ Weights and Organ-Weight-to-Body-Weight Ratios for Mice in the Three-month Inhalation Study of Cobalt Metal ${ }^{\mathrm{a}}$

\begin{tabular}{|c|c|c|c|c|c|c|}
\hline & $\begin{array}{c}\text { Chamber } \\
\text { Control }\end{array}$ & $0.625 \mathrm{mg} / \mathrm{m}^{3}$ & $1.25 \mathrm{mg} / \mathrm{m}^{3}$ & $2.5 \mathrm{mg} / \mathrm{m}^{3}$ & $5 \mathrm{mg} / \mathrm{m}^{3}$ & $10 \mathrm{mg} / \mathrm{m}^{3}$ \\
\hline \multicolumn{7}{|l|}{ Male } \\
\hline $\mathrm{n}$ & 10 & 10 & 10 & 10 & 10 & 10 \\
\hline Necropsy body wt & $37.7 \pm 0.8$ & $38.2 \pm 0.6$ & $37.6 \pm 0.8$ & $37.0 \pm 0.5$ & $37.0 \pm 0.9$ & $32.5 \pm 0.5^{* *}$ \\
\hline \multicolumn{7}{|l|}{ R. Kidney } \\
\hline Absolute & $0.31 \pm 0.01$ & $0.32 \pm 0.01$ & $0.32 \pm 0.01$ & $0.32 \pm 0.00$ & $0.29 \pm 0.01 * *$ & $0.23 \pm 0.01 * *$ \\
\hline Relative & $8.360 \pm 0.192$ & $8.441 \pm 0.122$ & $8.333 \pm 0.237$ & $8.507 \pm 0.047$ & $7.714 \pm 0.145^{* *}$ & $7.176 \pm 0.131^{* *}$ \\
\hline \multicolumn{7}{|l|}{ Liver } \\
\hline Absolute & $1.48 \pm 0.04$ & $1.53 \pm 0.04$ & $1.51 \pm 0.07$ & $1.49 \pm 0.04$ & $1.42 \pm 0.05$ & $1.15 \pm 0.03 * *$ \\
\hline Relative & $39.217 \pm 0.586$ & $40.049 \pm 0.671$ & $39.753 \pm 1.032$ & $40.301 \pm 0.698$ & $38.159 \pm 0.723$ & $35.457 \pm 0.668^{* *}$ \\
\hline \multicolumn{7}{|l|}{ Lung } \\
\hline Absolute & $0.20 \pm 0.01$ & $0.23 \pm 0.01$ & $0.22 \pm 0.01$ & $0.23 \pm 0.01^{*}$ & $0.27 \pm 0.01^{* *}$ & $0.30 \pm 0.01 * *$ \\
\hline Relative & $5.416 \pm 0.116$ & $6.051 \pm 0.235$ & $5.737 \pm 0.147$ & $6.234 \pm 0.088^{* *}$ & $7.436 \pm 0.262^{* *}$ & $9.142 \pm 0.177^{* *}$ \\
\hline \multicolumn{7}{|l|}{ R. Testis } \\
\hline Absolute & $0.118 \pm 0.002$ & $0.119 \pm 0.002$ & $0.114 \pm 0.002$ & $0.114 \pm 0.002$ & $0.104 \pm 0.003^{* *}$ & $0.033 \pm 0.001^{* *}$ \\
\hline Relative & $3.136 \pm 0.058$ & $3.131 \pm 0.037$ & $3.019 \pm 0.078$ & $3.073 \pm 0.056$ & $2.825 \pm 0.082^{* *}$ & $1.004 \pm 0.025^{* *}$ \\
\hline
\end{tabular}

\section{Female}

$\mathrm{n}$

Necropsy body wt $\quad 30.9 \pm 1.0 \quad 31.6 \pm 1.1 \quad 31.4 \pm 0.9 \quad 30.1 \pm 0.7 \quad 29.0 \pm 1.1 \quad 26.8 \pm 1.0^{* *}$

R. Kidney

$\begin{array}{lcccccc}\text { Absolute } & 0.21 \pm 0.01 & 0.22 \pm 0.00 & 0.21 \pm 0.01 & 0.20 \pm 0.00 & 0.17 \pm 0.00^{* *} & 0.16 \pm 0.00^{* *} \\ \text { Relative } & 6.887 \pm 0.184 & 6.849 \pm 0.155 & 6.661 \pm 0.126 & 6.689 \pm 0.254 & 6.031 \pm 0.132^{* *} & 6.142 \pm 0.185^{* *}\end{array}$

Liver

\begin{tabular}{|c|c|c|c|c|}
\hline Absolute & $1.46 \pm 0.06$ & $1.46 \pm 0.05$ & $1.30 \pm 0.03 *$ & $1.01 \pm 0.03 * *$ \\
\hline Rel & $47.051 \pm 0.8$ & $552 \pm 0.95246 .455 \pm 1.046$ & $\begin{array}{c}43.092 \pm 0.773 * \\
*\end{array}$ & $\begin{array}{l}9.831 \pm 0.459 * 38.045 \pm 1.246 * * \\
*\end{array}$ \\
\hline
\end{tabular}

Lung

\begin{tabular}{lcccccc} 
Absolute & $0.21 \pm 0.01$ & $0.22 \pm 0.00$ & $0.23 \pm 0.01$ & $0.23 \pm 0.01$ & $0.28 \pm 0.01 * *$ & $0.33 \pm 0.01^{* *}$ \\
Relative & $6.904 \pm 0.227$ & $6.884 \pm 0.176$ & $7.300 \pm 0.274$ & $7.555 \pm 0.184$ & $9.787 \pm 0.241^{* *}$ & $12.602 \pm 0.487^{* *}$ \\
\hline
\end{tabular}

*Significantly different $(\mathrm{P} \leq 0.05)$ from the chamber control group by Williams' test.

$* * \mathrm{P} \leq 0.01$.

${ }^{\mathrm{a}}$ Organ weights (absolute weights) and body weights are given in grams; organ-weight-to-body-weight ratios (relative weights) are given as $\mathrm{mg}$ organ weight/g body weight (mean \pm standard error).

Males exposed to $10 \mathrm{mg} / \mathrm{m}^{3}$ exhibited significant decreases in the weights of the cauda epididymis, epididymis, and testis; testis weight was also significantly decreased in the $5 \mathrm{mg} / \mathrm{m}^{3}$ group (Table 23 and Table H-4). Spermatids per testis were significantly decreased in 5 and $10 \mathrm{mg} / \mathrm{m}^{3}$ males, and spermatids per gram testis were significantly decreased in $10 \mathrm{mg} / \mathrm{m}^{3} \mathrm{males}$. 
Sperm motility and total sperm per epididymis and per gram epididymis were significantly decreased in 5 and $10 \mathrm{mg} / \mathrm{m}^{3}$ males; sperm motility was also significantly decreased in $2.5 \mathrm{mg} / \mathrm{m}^{3}$ males. Findings in the $10 \mathrm{mg} / \mathrm{m}^{3}$ males were associated with histopathologic changes. In female mice, the estrous cycle was significantly longer in the $10 \mathrm{mg} / \mathrm{m}^{3}$ group (Table $\mathrm{H}-5$ ). The Markov transition matrix analyses indicated no significant differences in estrous cyclicity between the exposed and chamber control groups of females (Table H-5 and Table H-6; Figure $\mathrm{H}-2$ ).

Table 23. Summary of Reproductive Tissue Evaluations for Male Mice in the Three-month Inhalation Study of Cobalt Metal ${ }^{\mathrm{a}}$

\begin{tabular}{|c|c|c|c|c|}
\hline & $\begin{array}{l}\text { Chamber } \\
\text { Control }\end{array}$ & $2.5 \mathrm{mg} / \mathrm{m}^{3}$ & $5 \mathrm{mg} / \mathrm{m}^{3}$ & $10 \mathrm{mg} / \mathrm{m}^{3}$ \\
\hline $\mathbf{n}$ & 10 & 10 & 10 & 10 \\
\hline \multicolumn{5}{|l|}{ Weights (g) } \\
\hline Necropsy body wt & $37.7 \pm 0.8$ & $37.0 \pm 0.5$ & $37.0 \pm 0.9$ & $32.5 \pm 0.5^{* *}$ \\
\hline L. Cauda epididymis & $0.0217 \pm 0.0014$ & $0.0210 \pm 0.0008$ & $0.0231 \pm 0.0018$ & $0.0168 \pm 0.0006^{*}$ \\
\hline L. Epididymis & $0.0603 \pm 0.0022$ & $0.0578 \pm 0.0019$ & $0.0614 \pm 0.0035$ & $0.0429 \pm 0.0021^{* *}$ \\
\hline L. Testis & $0.1185 \pm 0.0017$ & $0.1132 \pm 0.0023$ & $0.1027 \pm 0.0036^{* *}$ & $0.0316 \pm 0.0014^{* *}$ \\
\hline \multicolumn{5}{|l|}{ Spermatid measurements } \\
\hline Spermatid heads $\left(10^{6} /\right.$ testis $)$ & $22.34 \pm 0.84$ & $22.22 \pm 0.65$ & $18.90 \pm 1.20^{*}$ & $0.53 \pm 0.10^{* *}$ \\
\hline Spermatid heads ( $10^{6} / \mathrm{g}$ testis $)$ & $210.84 \pm 6.85$ & $227.74 \pm 7.16$ & $205.67 \pm 7.43$ & $24.27 \pm 4.78^{* *}$ \\
\hline \multicolumn{5}{|c|}{ Epididymal spermatozoal measurements } \\
\hline Sperm motility (\%) & $86.0 \pm 1.1$ & $82.0 \pm 0.8^{*}$ & $82.2 \pm 1.1^{*}$ & $2.6 \pm 1.2 * *$ \\
\hline Sperm ( $10^{6} /$ cauda epididymis $)$ & $11.55 \pm 0.39$ & $10.53 \pm 0.43$ & $9.62 \pm 0.49^{* *}$ & $0.71 \pm 0.06^{* *}$ \\
\hline Sperm (10\%6 cauda epididymis) & $551.1 \pm 37.9$ & $505.9 \pm 23.3$ & $439.9 \pm 40.3^{*}$ & $43.4 \pm 3.7^{* *}$ \\
\hline
\end{tabular}

Increased incidences of nonneoplastic lesions of the lung in exposed male and female mice included alveolus infiltration cellular, histiocyte; alveolus proteinosis; alveolar/bronchiolar epithelium karyomegaly; bronchiole epithelium hyperplasia and cytoplasmic vacuolization; and hemorrhage; the severities of the lesions increased with increasing exposure concentration (Table 24). Alveolar histiocytic cellular infiltration occurred in the lung of every exposed male and female mouse and was characterized by the presence of low to moderate numbers of histiocytes (macrophages) within alveolar spaces and septa. In some alveoli, the histiocytes were swollen and contained phagocytosed erythrocytes and/or eosinophilic material similar to that in the alveolar spaces. In mice exposed to $2.5 \mathrm{mg} / \mathrm{m}^{3}$ or greater, the intracytoplasmic material appeared golden brown to grey. Minimal to mild alveolar proteinosis occurred in all males and females exposed to 5 or $10 \mathrm{mg} / \mathrm{m}^{3}$ and was characterized by the accumulation of variable amounts of homogenous globular, granular, or fibrillar eosinophilic material within the alveolar spaces. Alveolar/bronchiolar epithelium karyomegaly occurred in all mice exposed to 5 or 
$10 \mathrm{mg} / \mathrm{m}^{3}$ and was characterized by the presence of scattered, hypertrophied cells with single large and, sometimes multiple, atypical nuclei in the epithelium of the terminal bronchioles, alveolar ducts, and immediately adjacent alveoli. Minimal to moderate bronchiole epithelium hyperplasia occurred in every mouse exposed to $2.5 \mathrm{mg} / \mathrm{m}^{3}$ or greater and was characterized by proliferation and hypertrophy of the epithelial cells lining terminal bronchioles with involvement of the alveolar ducts and adjacent alveolar septa in more severe cases. At affected sites, there was piling up of the pleomorphic cells in three to eight disorganized layers. The proliferating epithelial cells generally contained clear cytoplasmic vacuoles; this change was diagnosed as minimal to marked bronchiole epithelium cytoplasmic vacuolization, and it occurred in every exposed mouse. Several males and females exposed to 5 or $10 \mathrm{mg} / \mathrm{m}^{3}$ had minimal hemorrhage characterized by low numbers of red blood cells within a few alveolar spaces.

In the nose, increased incidences of nonneoplastic lesions in exposed male and female mice included chronic active inflammation, olfactory epithelium degeneration and hyperplasia, respiratory epithelium degeneration, and squamous metaplasia, and turbinate atrophy; the severities of these lesions generally increased with increasing exposure concentration (Table 24).

Incidences of chronic active inflammation were significantly increased in the 5 and $10 \mathrm{mg} / \mathrm{m}^{3}$ groups of males and females. Chronic active inflammation was observed in all three nasal sections of the nasal cavity and consisted of infiltrates of neutrophils, lymphocytes, macrophages, and plasma cells within the lamina propria and to a lesser extent the epithelium of the turbinates and nasal septum.

In some cases, the nasal passages contained proteinaceous fluid mixed with variable numbers of degenerate neutrophils, macrophages, and cellular debris. Incidences of olfactory epithelium degeneration were significantly increased in males and females exposed to $1.25 \mathrm{mg} / \mathrm{m}^{3}$ or greater. Degeneration was a focal or multifocal lesion that variably involved the epithelium lining the dorsal meatus, turbinates, or nasal septa. In affected sites, there was disorganization of the epithelium with vacuolization, individual cell death, and/or loss of epithelial cells and decrease in the height of the epithelium and in some cases a decrease in the size of the olfactory nerve bundles in the lamina propria. Scattered incidences of olfactory epithelium hyperplasia occurred primarily in exposed groups of males; the lesion was characterized by clusters or nests of proliferating cells within or just adjacent to the olfactory epithelium and sometimes with extension into the lamina propria around glandular ducts. The cells sometimes formed rosettes and had scant cytoplasm and large, round to oval nuclei. Incidences of minimal to mild respiratory epithelium degeneration were significantly increased in males exposed to $1.25 \mathrm{mg} / \mathrm{m}^{3}$ or greater and females exposed to $2.5 \mathrm{mg} / \mathrm{m}^{3}$ or greater. Respiratory epithelium degeneration involved the turbinates and/or lateral walls of the Level I and II nasal sections. In affected sites, the epithelium appeared disorganized and there was variable epithelial cell vacuolation with loss of cilia, individual cell death, and loss of the epithelial cells. Incidences of minimal to mild respiratory epithelium squamous metaplasia were significantly increased in males and females exposed to $2.5 \mathrm{mg} / \mathrm{m}^{3}$ or greater; the lesion involved the transitional epithelium of the lateral wall and the lateral surface and tips of the nasoturbinates and was often associated with proteinaceous fluid in the nasal passages. In affected sites, the normally ciliated, columnar epithelium was replaced by one or two layers of squamous epithelium. Incidences of turbinate atrophy were significantly increased in the 5 and $10 \mathrm{mg} / \mathrm{m}^{3}$ groups of males and females. Turbinate atrophy occurred in all three histologic sections of the nose. Microscopically, turbinate atrophy appeared as more space in the nasal passages because the individual turbinates were short, blunt, and/or 
narrow due to attenuation and/or loss of turbinate bone and other structures (glands, vessels, nerve bundles, and interstitial tissue) in the lamina propria.

Table 24. Incidences of Selected Nonneoplastic Lesions of the Respiratory System in Mice in the Three-month Inhalation Study of Cobalt Metal

\begin{tabular}{|c|c|c|c|c|c|c|}
\hline & $\begin{array}{l}\text { Chamber } \\
\text { Control }\end{array}$ & $0.625 \mathrm{mg} / \mathrm{m}^{3}$ & $1.25 \mathrm{mg} / \mathrm{m}^{3}$ & $2.5 \mathrm{mg} / \mathrm{m}^{3}$ & $5 \mathrm{mg} / \mathrm{m}^{3}$ & $10 \mathrm{mg} / \mathrm{m}^{3}$ \\
\hline \multicolumn{7}{|l|}{ Male } \\
\hline Lung $^{\mathrm{a}}$ & 10 & 10 & 10 & 10 & 10 & 10 \\
\hline $\begin{array}{l}\text { Alveolus, Infiltration } \\
\text { Cellular, Histiocyte }\end{array}$ & 0 & $10^{* *}(1.0)^{\mathrm{c}}$ & $10 * *(1.0)$ & $10 * *(1.0)$ & $10 * *(2.0)$ & $10 * *(3.0)$ \\
\hline $\begin{array}{l}\text { Bronchiole, Epithelium, } \\
\text { Hyperplasia }\end{array}$ & 0 & 0 & 0 & $10 * *(1.0)$ & $10 * *(1.9)$ & $10 * *(3.0)$ \\
\hline $\begin{array}{l}\text { Bronchiole, Epithelium, } \\
\text { Vacuolization } \\
\text { Cytoplasmic }\end{array}$ & 0 & $10^{* *}(1.0)$ & $10 * *(1.0)$ & $10 * *(1.5)$ & $10 * *(2.7)$ & $10^{* *}(3.9)$ \\
\hline Alveolus, Proteinosis & 0 & 0 & 0 & 0 & $10^{* *}(1.0)^{\mathrm{c}}$ & $10^{* *}(2.0)$ \\
\hline $\begin{array}{l}\text { Alveolar/bronchiolar, } \\
\text { Epithelium, Karyomegaly }\end{array}$ & 0 & 0 & 0 & 0 & $10 * *(1.0)$ & $10 * *(3.0)$ \\
\hline Hemorrhage & 0 & $1(1.0)$ & 0 & $1(1.0)$ & $7 * *(1.1)$ & $6^{* *}(1.0)$ \\
\hline Nose & 10 & 10 & 10 & 10 & 10 & 10 \\
\hline $\begin{array}{l}\text { Inflammation, Chronic } \\
\text { Active }\end{array}$ & 0 & 0 & 0 & 0 & $8 * *(1.4)$ & $10^{* *}(2.5)$ \\
\hline $\begin{array}{l}\text { Olfactory Epithelium, } \\
\text { Degeneration }\end{array}$ & 0 & $2(1.0)$ & $10 * *(1.0)$ & $10 * *(1.0)$ & $10 * *(2.0)$ & $10 * *(3.0)$ \\
\hline $\begin{array}{l}\text { Olfactory Epithelium, } \\
\text { Hyperplasia }\end{array}$ & 0 & 0 & $1(1.0)$ & $5 *(1.0)$ & $2(1.0)$ & $3(1.3)$ \\
\hline $\begin{array}{l}\text { Respiratory Epithelium, } \\
\text { Degeneration }\end{array}$ & 0 & 0 & $6 * *(1.0)$ & $9 * *(1.0)$ & $10 * *(1.9)$ & $10 * *(2.0)$ \\
\hline $\begin{array}{l}\text { Respiratory Epithelium, } \\
\text { Metaplasia, Squamous }\end{array}$ & 0 & 0 & $2(1.0)$ & $5 *(1.0)$ & $10 * *(1.3)$ & $10 * *(1.9)$ \\
\hline Turbinate, Atrophy & 0 & 0 & 0 & 0 & $8 * *(2.1)$ & $10^{* *}(3.0)$ \\
\hline Larynx & 10 & 10 & 10 & 10 & 10 & 10 \\
\hline Metaplasia, Squamous & 0 & $10 * *(1.8)$ & $10 * *(1.8)$ & $10 * *(1.9)$ & $10 * *(1.9)$ & $10 * *(2.1)$ \\
\hline \multicolumn{7}{|l|}{ Female } \\
\hline Lung & 10 & 10 & 10 & 10 & 10 & 10 \\
\hline $\begin{array}{l}\text { Alveolus, Infiltration } \\
\text { Cellular, Histiocyte }\end{array}$ & 0 & $10 * *(1.0)$ & $10 * *(1.0)$ & $10 * *(1.0)$ & $10 * *(2.1)$ & $10 * *(3.0)$ \\
\hline $\begin{array}{l}\text { Bronchiole, Epithelium, } \\
\text { Hyperplasia }\end{array}$ & 0 & 0 & 0 & $10 * *(1.0)$ & $10 * *(1.9)$ & $10 * *(3.0)$ \\
\hline $\begin{array}{l}\text { Bronchiole, Epithelium, } \\
\text { Vacuolization } \\
\text { Cytoplasmic }\end{array}$ & 0 & $10 * *(1.0)$ & $10 * *(1.0)$ & $10 * *(1.1)$ & $10 * *(2.6)$ & $10 * *(3.9)$ \\
\hline
\end{tabular}




\begin{tabular}{|c|c|c|c|c|c|c|}
\hline & $\begin{array}{l}\text { Chamber } \\
\text { Control }\end{array}$ & $0.625 \mathrm{mg} / \mathrm{m}^{3}$ & $1.25 \mathrm{mg} / \mathrm{m}^{3}$ & $2.5 \mathrm{mg} / \mathrm{m}^{3}$ & $5 \mathrm{mg} / \mathrm{m}^{3}$ & $10 \mathrm{mg} / \mathrm{m}^{3}$ \\
\hline Alveolus, Proteinosis & 0 & 0 & 0 & 0 & $10 * *(1.0)$ & $10^{* *}(1.8)$ \\
\hline $\begin{array}{l}\text { Alveolar/bronchiolar, } \\
\text { Epithelium, Karyomegaly }\end{array}$ & 0 & 0 & 0 & 0 & $10 * *(1.7)$ & $10 * *(3.0)$ \\
\hline Hemorrhage & 0 & 0 & 0 & 0 & $8 * *(1.0)$ & $2(1.0)$ \\
\hline Nose & 10 & 10 & 10 & 10 & 10 & 10 \\
\hline $\begin{array}{l}\text { Inflammation, Chronic } \\
\text { Active }\end{array}$ & 0 & 0 & 0 & $1(1.0)$ & $10 * *(2.5)$ & $10 * *(2.4)$ \\
\hline $\begin{array}{l}\text { Olfactory Epithelium, } \\
\text { Degeneration }\end{array}$ & 0 & $1(1.0)$ & $7 * *(1.0)$ & $9 * *(1.0)$ & $10 * *(2.5)$ & $10^{* *}(2.9)$ \\
\hline $\begin{array}{l}\text { Olfactory Epithelium, } \\
\text { Hyperplasia }\end{array}$ & 0 & 0 & 0 & $3(1.0)$ & 0 & 0 \\
\hline $\begin{array}{l}\text { Respiratory Epithelium, } \\
\text { Degeneration }\end{array}$ & 0 & 0 & $1(1.0)$ & $8 * *(1.0)$ & $10 * *(1.9)$ & $10^{* *}(1.9)$ \\
\hline $\begin{array}{l}\text { Respiratory Epithelium, } \\
\text { Metaplasia, Squamous }\end{array}$ & 0 & 0 & 0 & $9 * *(1.0)$ & $10 * *(2.0)$ & $10 * *(2.0)$ \\
\hline Turbinate, Atrophy & 0 & 0 & 0 & 0 & $10 * *(2.2)$ & $10^{* *}(2.9)$ \\
\hline Larynx & 10 & 10 & 10 & 10 & 10 & 10 \\
\hline Metaplasia, Squamous & 0 & $10 * *(1.3)$ & $10 * *(1.4)$ & $10 * *(1.6)$ & $10 * *(1.8)$ & $10^{* *}(2.2)$ \\
\hline
\end{tabular}

*Significantly different $(\mathrm{P} \leq 0.05)$ from the chamber control group by the Fisher exact test.

$* * \mathrm{P} \leq 0.01$.

aNumber of animals with tissue examined microscopically.

${ }^{b}$ Number of animals with lesion.

${ }^{\mathrm{c}}$ Average severity grade of lesions in affected animals: $1=$ minimal, $2=$ mild, $3=$ moderate, $4=$ marked .

The incidences of squamous metaplasia were significantly increased in the larynx of all exposed groups of males and females, and severities of the lesion increased with increasing exposure concentration (Table 24). Squamous metaplasia occurred in the epithelium overlying the submucosal glands at the base of the epiglottis and was characterized by replacement of ciliated columnar epithelium with one to a few layers of flattened, nonciliated, squamous epithelial cells.

The incidence of marked germinal epithelium degeneration in the testes was significantly increased in males exposed to $10 \mathrm{mg} / \mathrm{m}^{3}$ (Table 25). Testes diagnosed with germinal epithelium degeneration had greatly decreased overall cross-sectional diameter compared to chamber control testes. All or most seminiferous tubules had irregular to some-what flattened outlines with markedly decreased or completely absent germinal epithelium, vacuolated Sertoli cells, and aspermia; intraluminal clumps of sloughed germinal cells and amorphous mineralized debris were current in the lumina of the seminiferous tubules.

In the epididymis, the incidences of exfoliated germ cells, hypospermia, cytoplasmic vacuolization, and atrophy were significantly increased in males exposed to $10 \mathrm{mg} / \mathrm{m}^{3}$ (Table 25). Mild to moderate germ cell exfoliation was characterized by sloughed, 
Table 25. Incidences of Selected Nonneoplastic Lesions of the Genital System in Male Mice in the Three-month Inhalation Study of Cobalt Metal

\begin{tabular}{|c|c|c|c|c|c|c|}
\hline & $\begin{array}{l}\text { Chamber } \\
\text { Control }\end{array}$ & $0.625 \mathrm{mg} / \mathrm{m}^{3}$ & $1.25 \mathrm{mg} / \mathrm{m}^{3}$ & $2.5 \mathrm{mg} / \mathrm{m}^{3}$ & $5 \mathrm{mg} / \mathrm{m}^{3}$ & $10 \mathrm{mg} / \mathrm{m}^{3}$ \\
\hline Testes $^{\mathrm{a}}$ & 10 & 10 & 10 & 10 & 10 & 10 \\
\hline $\begin{array}{l}\text { Germinal Epithelium, } \\
\text { Degeneration }^{\mathrm{b}}\end{array}$ & $2(1.0)^{\mathrm{c}}$ & 0 & 0 & 0 & $1(1.0)$ & $10^{* *}(4.0)$ \\
\hline Epididymis & 10 & 10 & 10 & 9 & 10 & 10 \\
\hline Exfoliated Germ Cell & 0 & 0 & 0 & 0 & 0 & $10^{* *}(2.7)$ \\
\hline Hypospermia & 0 & 0 & 0 & 0 & 0 & $10^{* *}(2.9)$ \\
\hline $\begin{array}{l}\text { Vacuolization } \\
\text { Cytoplasmic }\end{array}$ & 0 & 0 & 0 & 0 & 0 & $9 * *(1.0)$ \\
\hline Atrophy & 0 & 0 & 0 & 0 & 0 & $10^{* *}(1.0)$ \\
\hline
\end{tabular}

**Significantly different $(\mathrm{P} \leq 0.01)$ from the chamber control group by the Fisher exact test.

${ }^{a}$ Number of animals with tissue examined microscopically.

${ }^{b}$ Number of animals with lesion.

${ }^{\mathrm{c}}$ Average severity grade of lesions in affected animals: $1=$ minimal, $2=$ mild, $3=$ moderate, $4=$ marked .

degenerate germinal epithelial cells (spermatocytes, spermatogonia, and spermatids), as well as amorphous debris in the body and tail of the epididymal duct. Hypospermia, most likely the consequence of loss of the germinal epithelium, was characterized by mild to moderate decreases in the numbers of mature spermatozoa in the body and tail of the epididymal ducts. Cytoplasmic vacuolization of duct epithelium was a subtle finding of minimal severity noted predominantly in the tail of the epididymis and characterized by increased numbers of ductal epithelial cells that were swollen by a single, large, clear cytoplasmic vacuole. Atrophy was a subtle change noted in the head region of the epididymal duct. In chamber control males, the epididymal duct was lined by densely packed, tall, columnar epithelial cells that appeared pseudostratified due to the occurrence of the basal nuclei at different levels in the cells. In animals exposed to $10 \mathrm{mg} / \mathrm{m}^{3}$, the epithelial cells lining the head were not as tall and the basal nuclei were less numerous and less crowded and generally spaced evenly along the basement membrane in a single layer.

\section{Tissue Burden Studies}

Lung and liver weights and lung, blood, and liver cobalt concentrations were determined in female mice (Table I-11). During the exposure period, lung weights of the 5 and $10 \mathrm{mg} / \mathrm{m}^{3}$ groups were significantly greater than those of the chamber controls starting on study day 12 and generally remained elevated compared to the chamber controls until the end of the postexposure period. Increased lung weights were occasionally observed at $2.5 \mathrm{mg} / \mathrm{m}^{3}$. Because of the significant changes in lung weights with exposure concentration, lung cobalt burdens rather than lung cobalt concentrations were evaluated for toxicokinetic parameters.

Lung cobalt concentrations and burdens increased with increasing exposure concentration and were increased over chamber controls. Lung cobalt concentrations in chamber control animals were near or below the LOD at all time points. By day 40, lung cobalt concentrations in all exposed groups appeared to be approaching steady state and did not change significantly through the end of exposure (day 89) before steadily decreasing during the recovery period (Table I-11). Lung cobalt burdens increased rapidly within the first 5 to 26 days, but by days 12 to 40 , the rate 
of increase slowed as lung burdens asymptotically approached steady state with the higher concentrations taking longer to approach steady state. During the recovery period, lung cobalt burdens decreased very rapidly during the first week, after which lung clearance of cobalt slowed significantly. Normalized lung cobalt burdens tended to increase with exposure concentration up to $5 \mathrm{mg} / \mathrm{m}^{3}$ but were lower in animals exposed to $10 \mathrm{mg} / \mathrm{m}^{3}$ than in animals exposed to $5 \mathrm{mg} / \mathrm{m}^{3}$, indicating a lack of a nonproportional accumulation at $10 \mathrm{mg} / \mathrm{m}^{3}$.

Blood cobalt concentrations in the chamber control animals were at or below the LOD at all time points (Table I-11). During the 3-month exposure, blood cobalt concentrations generally increased in proportion to exposure concentration at all time points and were increased over chamber controls in all groups at all exposure time points and remained elevated through the later postexposure time points. Within each exposure concentration, blood cobalt concentrations appeared to be at or near steady state by study day 12 . However during the recovery period, blood cobalt concentrations fell very rapidly to concentrations that were near or below the LOD in an exposure concentration-related manner. Accordingly, because of the rapid and extensive elimination of cobalt from the blood, it was not possible to demonstrate dose proportionality from blood concentration data collected during the recovery period.

Liver weights of the 5 and $10 \mathrm{mg} / \mathrm{m}^{3}$ groups were significantly less than that of the chamber control group on day 26; similar, although not statistically significant decreased liver weights in these exposed groups were observed on day 40 (Table I-11). Liver cobalt concentrations in chamber control animals were at or below the LOD at both time points. During the 3-month exposure, liver cobalt concentrations and burdens generally increased with exposure concentration and were increased compared to the chamber controls at both time points. Liver cobalt concentrations and total liver cobalt burdens for exposed animals were higher at all exposure concentrations on day 26 compared to day 40 (except for cobalt concentration in animals exposed to $10 \mathrm{mg} / \mathrm{m}^{3}$ ).

Pulmonary clearance of cobalt during the recovery period showed a well-defined two-phase elimination profile (Table I-12). The rapid phase exhibited half-lives ranging from 1.4 to 3.2 days and was followed by a slower lung clearance phase with half-lives of 27 to 39 days; there was no clear relationship to exposure concentration in either phase. A two-compartment clearance model could not be fit to the lung cobalt burden data collected during the 3-month study due to the lack of data collected prior to 5 days of exposure, however a one-compartment model provided an adequate fit to these data (Table I-13). The results indicated that half-lives ranged from 2.4 to 17 days (increased with increasing exposure concentration) for animals exposed to $5 \mathrm{mg} / \mathrm{m}^{3}$ or less. The half-life in animals exposed to $10 \mathrm{mg} / \mathrm{m}^{3}$ was 122 days, but the standard errors for the clearance rate constant and subsequently the calculated half-life were high $(>80 \%)$ making these data unreliable.

Exposure Concentration Selection Rationale: Based on reductions in body weights and moderate severity of nose and lung lesions in the $10 \mathrm{mg} / \mathrm{m}^{3}$ groups in the 3-month study, this exposure concentration was considered too high to be used in the 2-year study. Hence, $5 \mathrm{mg} / \mathrm{m}^{3}$ was selected as the highest exposure concentration for the 2-year inhalation study in mice. 


\section{Two-year Study}

\section{Survival}

Estimates of 2-year survival probabilities for male and female mice are shown in Table 26 and in the Kaplan-Meier survival curves (Figure 5). Survival of males exposed to 2.5 or $5 \mathrm{mg} / \mathrm{m}^{3}$ was significantly less than that of the chamber control group.

\section{Body Weights and Clinical Findings}

Mean body weights of $5 \mathrm{mg} / \mathrm{m}^{3}$ males and females were at least $10 \%$ less than those of the chamber control groups after weeks 85 and 21, respectively (Table 27, Table 28 and Figure 6). Abnormal breathing and thinness were noted in exposed male and female mice.

Table 26. Survival of Mice in the Two-year Inhalation Study of Cobalt Metal

\begin{tabular}{|c|c|c|c|c|}
\hline & $\begin{array}{c}\text { Chamber } \\
\text { Control }\end{array}$ & $1.25 \mathrm{mg} / \mathrm{m}^{3}$ & $2.5 \mathrm{mg} / \mathrm{m}^{3}$ & $5 \mathrm{mg} / \mathrm{m}^{3}$ \\
\hline \multicolumn{5}{|l|}{ Male } \\
\hline Animals initially in study & 50 & 50 & 50 & 50 \\
\hline Moribund & 5 & 13 & 12 & 17 \\
\hline Natural deaths & 6 & 6 & 9 & 8 \\
\hline Animals surviving to study termination & 39 & 31 & $29^{c}$ & $25^{\mathrm{d}}$ \\
\hline Percent probability of survival at end of study ${ }^{a}$ & 78 & 62 & 58 & 50 \\
\hline Mean survival (days) ${ }^{\mathrm{b}}$ & 715 & 695 & 672 & 668 \\
\hline Survival analysis ${ }^{\mathrm{e}}$ & $\mathrm{P}=0.004$ & $\mathrm{P}=0.102$ & $\mathrm{P}=0.035$ & $\mathrm{P}=0.003$ \\
\hline \multicolumn{5}{|l|}{ Female } \\
\hline Animals initially in study & 50 & 50 & 50 & 50 \\
\hline Moribund & 9 & 12 & 19 & 21 \\
\hline Natural deaths & 5 & 2 & 4 & 3 \\
\hline Animals surviving to study termination & 36 & $36^{\mathrm{f}}$ & 27 & 26 \\
\hline Percent probability of survival at end of study & 72 & 70 & 54 & 52 \\
\hline Mean survival (days) & 686 & 695 & 680 & 668 \\
\hline Survival analysis & $P=0.019$ & $\mathrm{P}=1.000$ & $\mathrm{P}=0.110$ & $P=0.061$ \\
\hline \multicolumn{5}{|c|}{ 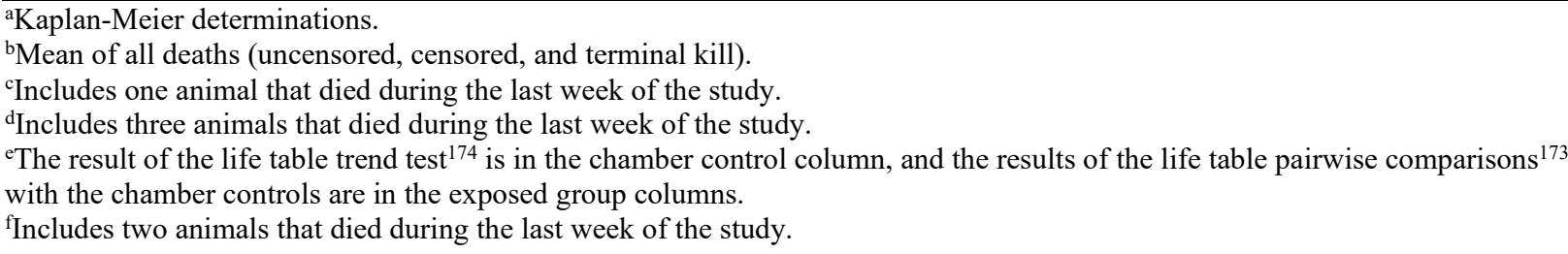 } \\
\hline
\end{tabular}



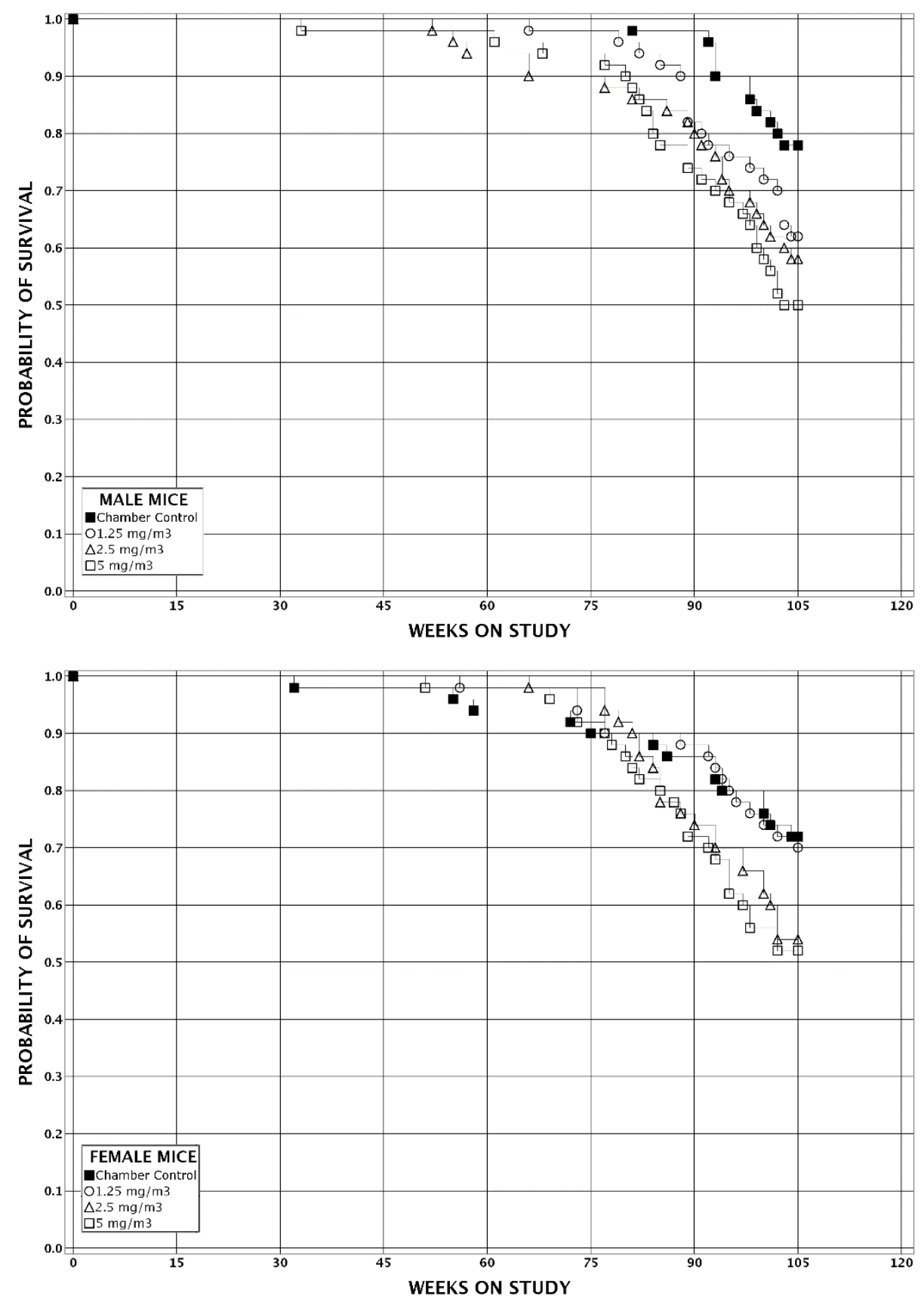

Figure 5. Kaplan-Meier Survival Curves for Mice Exposed to Cobalt Metal by Inhalation for Two Years 
Cobalt Metal, NTP TR 581

Table 27. Mean Body Weights and Survival of Male Mice in the Two-year Inhalation Study of Cobalt Metal

\begin{tabular}{|c|c|c|c|c|c|c|c|c|c|c|c|}
\hline \multirow[b]{2}{*}{ Day } & \multicolumn{2}{|c|}{ Chamber Control } & \multicolumn{3}{|c|}{$1.25 \mathrm{mg} / \mathrm{m}^{3}$} & \multicolumn{3}{|c|}{$2.5 \mathrm{mg} / \mathrm{m}^{3}$} & \multicolumn{3}{|c|}{$5 \mathrm{mg} / \mathrm{m}^{3}$} \\
\hline & $\begin{array}{l}\text { Av. } \\
\text { Wt. } \\
\text { (g) }\end{array}$ & $\begin{array}{c}\text { No. of } \\
\text { Survivors }\end{array}$ & $\begin{array}{l}\text { Av. } \\
\text { Wt. } \\
\text { (g) }\end{array}$ & $\begin{array}{l}\text { Wt. (\% of } \\
\text { Controls) }\end{array}$ & $\begin{array}{c}\text { No. of } \\
\text { Survivors }\end{array}$ & $\begin{array}{l}\text { Av. } \\
\text { Wt. } \\
\text { (g) }\end{array}$ & $\begin{array}{l}\text { Wt. (\% of } \\
\text { Controls) }\end{array}$ & $\begin{array}{c}\text { No. of } \\
\text { Survivors }\end{array}$ & $\begin{array}{l}\text { Av. } \\
\text { Wt. } \\
\text { (g) }\end{array}$ & $\begin{array}{l}\text { Wt. (\% of } \\
\text { Controls) }\end{array}$ & $\begin{array}{c}\text { No. of } \\
\text { Survivors }\end{array}$ \\
\hline 1 & 23.6 & 50 & 23.5 & 99 & 50 & 23.1 & 98 & 50 & 23.2 & 98 & 50 \\
\hline 8 & 25.4 & 50 & 25.1 & 99 & 50 & 24.9 & 98 & 50 & 24.9 & 98 & 50 \\
\hline 15 & 26.5 & 50 & 26.0 & 98 & 50 & 25.5 & 96 & 50 & 26.1 & 98 & 50 \\
\hline 22 & 27.7 & 50 & 27.1 & 98 & 50 & 27.0 & 97 & 50 & 26.9 & 97 & 50 \\
\hline 29 & 28.8 & 50 & 28.3 & 98 & 50 & 28.2 & 98 & 50 & 28.2 & 98 & 50 \\
\hline 36 & 30.1 & 50 & 29.5 & 98 & 50 & 29.3 & 98 & 50 & 29.2 & 97 & 50 \\
\hline 43 & 31.1 & 50 & 30.5 & 98 & 50 & 30.1 & 97 & 50 & 30.0 & 96 & 50 \\
\hline 50 & 32.4 & 50 & 31.8 & 98 & 50 & 31.2 & 96 & 50 & 30.8 & 95 & 50 \\
\hline 57 & 33.2 & 50 & 32.9 & 99 & 50 & 32.3 & 98 & 50 & 31.9 & 96 & 50 \\
\hline 64 & 34.3 & 50 & 34.0 & 99 & 50 & 33.3 & 97 & 50 & 33.0 & 96 & 50 \\
\hline 71 & 35.5 & 50 & 35.1 & 99 & 50 & 34.1 & 96 & 50 & 33.6 & 95 & 50 \\
\hline 78 & 36.4 & 50 & 35.9 & 99 & 50 & 35.0 & 96 & 50 & 34.1 & 94 & 50 \\
\hline 85 & 37.4 & 50 & 37.1 & 99 & 50 & 35.9 & 96 & 50 & 35.0 & 94 & 50 \\
\hline 113 & 40.8 & 50 & 41.1 & 101 & 50 & 39.6 & 97 & 50 & 37.7 & 93 & 50 \\
\hline 141 & 43.5 & 50 & 44.0 & 101 & 50 & 42.7 & 98 & 50 & 40.0 & 92 & 50 \\
\hline 169 & 45.7 & 50 & 45.8 & 100 & 50 & 45.0 & 99 & 50 & 42.3 & 93 & 50 \\
\hline 197 & 47.4 & 50 & 47.8 & 101 & 50 & 47.1 & 100 & 50 & 44.7 & 94 & 50 \\
\hline 225 & 49.1 & 50 & 49.0 & 100 & 50 & 48.6 & 99 & 50 & 45.5 & 93 & 50 \\
\hline 253 & 50.0 & 50 & 50.0 & 100 & 50 & 50.2 & 100 & 50 & 47.5 & 95 & 49 \\
\hline 281 & 51.1 & 50 & 50.8 & 100 & 50 & 50.9 & 100 & 50 & 48.1 & 94 & 49 \\
\hline 309 & 51.5 & 50 & 51.6 & 100 & 50 & 51.8 & 101 & 50 & 49.1 & 95 & 49 \\
\hline 337 & 52.1 & 50 & 52.2 & 100 & 50 & 52.3 & 101 & 50 & 49.7 & 96 & 49 \\
\hline 365 & 52.9 & 50 & 52.8 & 100 & 50 & 52.9 & 100 & 49 & 50.3 & 95 & 49 \\
\hline 393 & 53.3 & 50 & 53.4 & 100 & 50 & 53.3 & 100 & 48 & 50.9 & 96 & 49 \\
\hline 421 & 53.6 & 50 & 53.5 & 100 & 50 & 54.2 & 101 & 47 & 51.3 & 96 & 49 \\
\hline 449 & 53.5 & 50 & 53.6 & 100 & 50 & 54.0 & 101 & 47 & 51.5 & 96 & 48 \\
\hline 477 & 53.8 & 50 & 53.9 & 100 & 49 & 54.5 & 101 & 45 & 51.4 & 96 & 47 \\
\hline 505 & 54.0 & 50 & 53.5 & 99 & 49 & 54.3 & 101 & 45 & 51.1 & 95 & 47 \\
\hline 533 & 54.2 & 50 & 53.4 & 99 & 49 & 54.5 & 101 & 44 & 49.9 & 92 & 47 \\
\hline 561 & 54.3 & 49 & 52.8 & 97 & 48 & 54.1 & 101 & 44 & 49.5 & 91 & 45 \\
\hline 589 & 53.2 & 49 & 51.8 & 97 & 46 & 52.6 & 99 & 43 & 48.8 & 92 & 40 \\
\hline 617 & 53.6 & 49 & 52.2 & 98 & 43 & 51.5 & 96 & 42 & 47.6 & 89 & 38 \\
\hline 645 & 53.4 & 48 & 53.1 & 100 & 39 & 51.3 & 96 & 39 & 46.3 & 87 & 36 \\
\hline 659 & 53.8 & 45 & 52.4 & 98 & 39 & 50.4 & 94 & 36 & 44.2 & 82 & 35 \\
\hline 673 & 52.7 & 45 & 52.3 & 99 & 38 & 50.0 & 95 & 35 & 42.5 & 81 & 34 \\
\hline 687 & 52.4 & 43 & 52.0 & 99 & 37 & 49.0 & 94 & 34 & 40.9 & 78 & 32 \\
\hline 701 & 51.8 & 42 & 51.8 & 100 & 36 & 47.5 & 92 & 32 & 40.1 & 77 & 29 \\
\hline 715 & 51.2 & 40 & 51.8 & 101 & 34 & 47.0 & 92 & 31 & 39.5 & 77 & 26 \\
\hline \multicolumn{12}{|c|}{ Mean forWeeks } \\
\hline $1-13$ & 31.0 & - & 30.5 & 99 & - & 30.0 & 97 & - & 29.8 & 96 & - \\
\hline $14-52$ & 47.9 & - & 48.0 & 100 & - & 47.6 & 99 & - & 45.0 & 94 & - \\
\hline $53-103$ & 53.2 & - & 52.8 & 99 & - & 51.9 & 98 & - & 47.2 & 89 & - \\
\hline
\end{tabular}


Cobalt Metal, NTP TR 581

Table 28. Mean Body Weights and Survival of Female Mice in the Two-year Inhalation Study of Cobalt Metal

\begin{tabular}{|c|c|c|c|c|c|c|c|c|c|c|c|}
\hline \multirow[b]{2}{*}{ Day } & \multicolumn{2}{|c|}{ Chamber Control } & \multicolumn{3}{|c|}{$1.25 \mathrm{mg} / \mathrm{m}^{3}$} & \multicolumn{3}{|c|}{$2.5 \mathrm{mg} / \mathrm{m}^{3}$} & \multicolumn{3}{|c|}{$5 \mathrm{mg} / \mathrm{m}^{3}$} \\
\hline & $\begin{array}{c}\text { Av. Wt. } \\
\text { (g) }\end{array}$ & $\begin{array}{c}\text { No. of } \\
\text { Survivors }\end{array}$ & $\begin{array}{l}\text { Av. } \\
\text { Wt. } \\
\text { (g) }\end{array}$ & $\begin{array}{l}\text { Wt. (\% of } \\
\text { Controls) }\end{array}$ & $\begin{array}{c}\text { No. of } \\
\text { Survivors }\end{array}$ & $\begin{array}{l}\text { Av. } \\
\text { Wt. } \\
\text { (g) }\end{array}$ & $\begin{array}{l}\text { Wt. (\% of } \\
\text { Controls) }\end{array}$ & $\begin{array}{c}\text { No. of } \\
\text { Survivors }\end{array}$ & $\begin{array}{c}\text { Av. } \\
\text { Wt.(g) }\end{array}$ & $\begin{array}{l}\text { Wt. (\% of } \\
\text { Controls) }\end{array}$ & $\begin{array}{c}\text { No. of } \\
\text { Survivors }\end{array}$ \\
\hline 1 & 19.8 & 50 & 19.7 & 99 & 50 & 19.6 & 99 & 50 & 19.6 & 99 & 50 \\
\hline 8 & 21.3 & 50 & 21.0 & 99 & 50 & 21.1 & 99 & 50 & 21.0 & 99 & 50 \\
\hline 15 & 22.1 & 50 & 21.6 & 98 & 50 & 21.9 & 99 & 50 & 21.8 & 99 & 50 \\
\hline 22 & 23.2 & 50 & 22.6 & 97 & 50 & 22.9 & 99 & 50 & 23.0 & 99 & 50 \\
\hline 29 & 23.9 & 50 & 23.7 & 99 & 50 & 23.9 & 100 & 50 & 23.5 & 98 & 50 \\
\hline 36 & 24.9 & 50 & 24.6 & 99 & 50 & 25.0 & 100 & 50 & 24.2 & 97 & 50 \\
\hline 43 & 25.6 & 50 & 25.7 & 100 & 50 & 25.8 & 101 & 50 & 25.0 & 98 & 50 \\
\hline 50 & 26.2 & 50 & 26.7 & 102 & 50 & 26.9 & 103 & 50 & 26.0 & 99 & 50 \\
\hline 57 & 27.3 & 50 & 27.7 & 102 & 50 & 28.0 & 103 & 50 & 26.9 & 98 & 50 \\
\hline 64 & 28.6 & 50 & 28.7 & 100 & 50 & 29.1 & 102 & 50 & 27.4 & 96 & 50 \\
\hline 71 & 29.1 & 50 & 29.9 & 103 & 50 & 30.3 & 104 & 50 & 28.5 & 98 & 50 \\
\hline 78 & 29.7 & 50 & 31.0 & 104 & 50 & 31.3 & 106 & 50 & 29.2 & 98 & 50 \\
\hline 85 & 30.6 & 50 & 32.3 & 105 & 50 & 32.6 & 106 & 50 & 29.5 & 96 & 50 \\
\hline 113 & 34.6 & 50 & 37.0 & 107 & 50 & 37.0 & 107 & 50 & 32.2 & 93 & 50 \\
\hline 141 & 38.6 & 50 & 40.8 & 106 & 50 & 40.9 & 106 & 50 & 35.0 & 91 & 50 \\
\hline 169 & 41.8 & 50 & 43.4 & 104 & 50 & 43.8 & 105 & 50 & 37.6 & 90 & 50 \\
\hline 197 & 44.3 & 50 & 46.2 & 104 & 50 & 46.3 & 105 & 50 & 39.7 & 90 & 50 \\
\hline 225 & 47.4 & 49 & 48.6 & 103 & 50 & 48.0 & 101 & 50 & 41.0 & 87 & 50 \\
\hline 253 & 49.7 & 49 & 50.8 & 102 & 50 & 50.4 & 101 & 50 & 43.1 & 87 & 50 \\
\hline 281 & 53.2 & 49 & 53.1 & 100 & 50 & 51.9 & 98 & 50 & 44.1 & 83 & 50 \\
\hline 309 & 56.5 & 49 & 55.5 & 98 & 50 & 53.9 & 95 & 50 & 45.1 & 80 & 50 \\
\hline 337 & 59.1 & 49 & 57.5 & 97 & 50 & 56.0 & 95 & 50 & 46.7 & 79 & 50 \\
\hline 365 & 60.4 & 49 & 59.2 & 98 & 50 & 57.7 & 96 & 50 & 47.7 & 79 & 49 \\
\hline 393 & 62.6 & 48 & 61.1 & 98 & 49 & 59.2 & 95 & 50 & 48.8 & 78 & 49 \\
\hline 421 & 64.6 & 47 & 62.4 & 97 & 49 & 60.7 & 94 & 50 & 50.1 & 78 & 49 \\
\hline 449 & 65.6 & 47 & 62.5 & 95 & 49 & 61.1 & 93 & 50 & 50.3 & 77 & 49 \\
\hline 477 & 66.7 & 47 & 62.9 & 94 & 49 & 61.2 & 92 & 49 & 49.7 & 75 & 49 \\
\hline 505 & 65.9 & 46 & 62.9 & 95 & 48 & 61.0 & 93 & 49 & 49.7 & 75 & 47 \\
\hline 533 & 66.3 & 45 & 62.8 & 95 & 47 & 60.9 & 92 & 48 & 50.1 & 76 & 45 \\
\hline 561 & 65.1 & 45 & 64.3 & 99 & 45 & 61.1 & 94 & 46 & 49.6 & 76 & 43 \\
\hline 589 & 64.9 & 44 & 62.6 & 96 & 45 & 60.5 & 93 & 42 & 48.5 & 75 & 41 \\
\hline 617 & 63.2 & 43 & 61.9 & 98 & 44 & 60.8 & 96 & 38 & 46.8 & 74 & 38 \\
\hline 645 & 62.0 & 43 & 60.0 & 97 & 43 & 58.2 & 94 & 37 & 47.5 & 77 & 34 \\
\hline 659 & 61.9 & 40 & 60.2 & 97 & 41 & 58.3 & 94 & 35 & 44.7 & 72 & 34 \\
\hline 673 & 60.7 & 40 & 60.7 & 100 & 39 & 56.3 & 93 & 35 & 44.8 & 74 & 30 \\
\hline 687 & 59.1 & 40 & 59.5 & 101 & 38 & 56.2 & 95 & 33 & 43.8 & 74 & 28 \\
\hline 701 & 59.1 & 37 & 58.6 & 99 & 37 & 56.0 & 95 & 31 & 42.0 & 71 & 28 \\
\hline 715 & 58.5 & 37 & 57.3 & 98 & 36 & 56.3 & 96 & 27 & 40.6 & 70 & 26 \\
\hline \multicolumn{12}{|c|}{ Mean for Weeks } \\
\hline $1-13$ & 25.6 & - & 25.6 & 101 & - & 26.0 & 102 & - & 25.0 & 98 & - \\
\hline $14-52$ & 47.2 & - & 48.1 & 102 & - & 47.6 & 101 & - & 40.5 & 87 & - \\
\hline $53-103$ & 62.9 & - & 61.2 & 97 & - & 59.1 & 94 & - & 47.2 & 75 & - \\
\hline
\end{tabular}



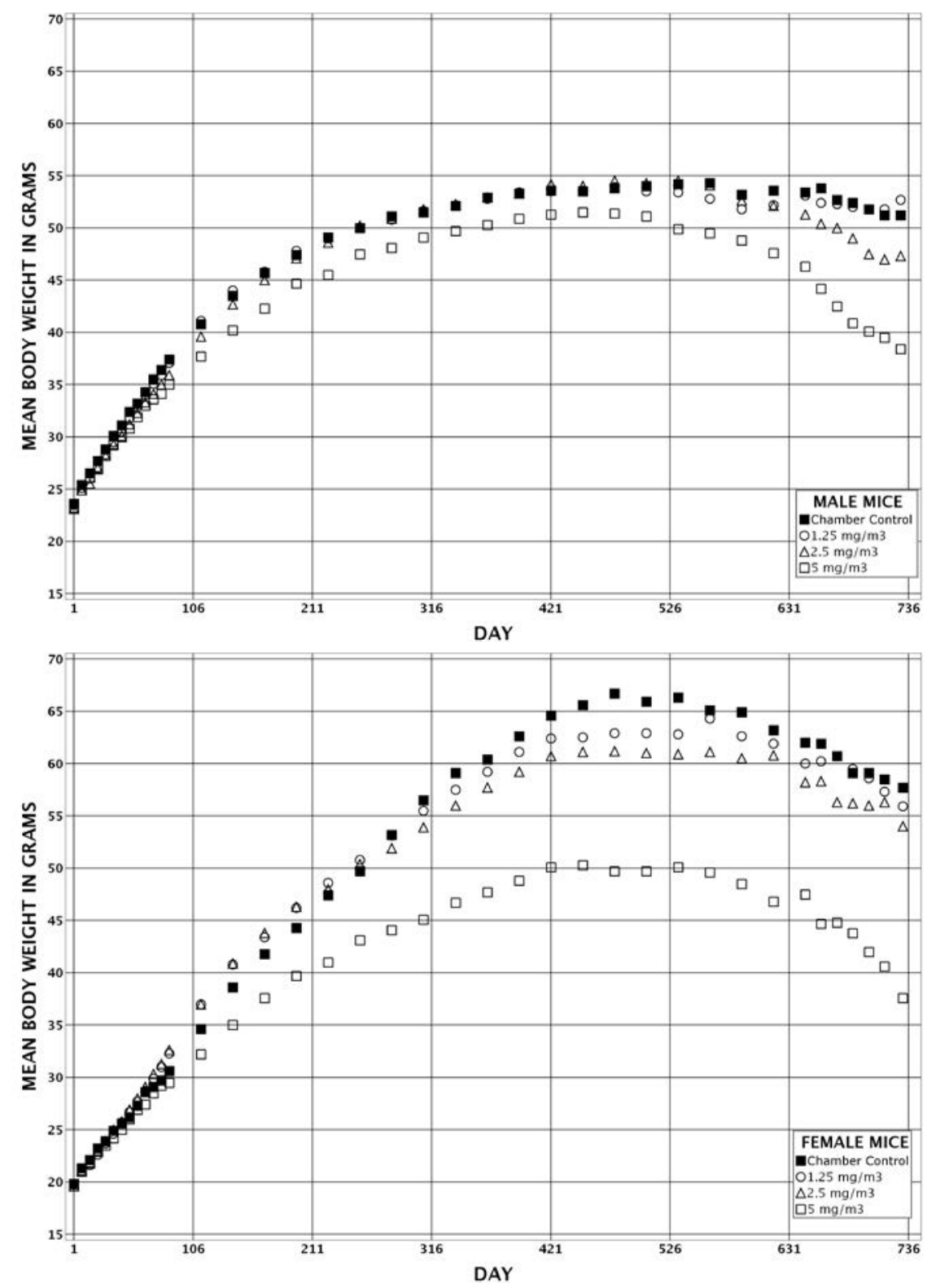

Figure 6. Growth Curves for Mice Exposed to Cobalt Metal by Inhalation for Two Years

\section{Pathology and Statistical Analyses}

This section describes the statistically significant or biologically noteworthy changes in the incidences of neoplasms and/or nonneoplastic lesions of the lung, nose, larynx, trachea, and testes. Summaries of the incidences of neoplasms and nonneoplastic lesions, statistical analyses of primary neoplasms that occurred with an incidence of at least 5\% in at least one animal group, and historical incidences for the neoplasms mentioned in this section are presented in Appendix $\mathrm{C}$ for male mice and Appendix D for female mice.

Lung: The incidences of alveolar/bronchiolar adenoma in males exposed to $2.5 \mathrm{mg} / \mathrm{m}^{3}$ and females exposed to $5 \mathrm{mg} / \mathrm{m}^{3}$ and the incidences of alveolar/bronchiolar carcinoma and alveolar adenoma or carcinoma (combined) in all exposed groups of male and female mice were significantly greater than those in the chamber controls (Table 29, Table C-1, Table C-2, 
Table D-1, and Table D-2). The incidences of alveolar/bronchiolar carcinoma and alveolar/bronchiolar adenoma or carcinoma (combined) occurred with positive trends and exceeded the historical control ranges for inhalation studies and all routes of administration (Table 29, Table C-3, and Table D-3). In addition, significantly increased incidences of multiple alveolar/bronchiolar carcinoma occurred in all exposed groups of males and females.

Alveolar/bronchiolar adenomas were small, generally nodular masses that distorted the alveolar architecture and compressed the adjacent parenchyma. Most adenomas consisted of interlacing papillary fronds and folds which projected into alveolar spaces and were lined by well differentiated cuboidal to low columnar epithelium supported by scant fibrovascular stroma (Figure 28). Other more solid appearing adenomas were composed of closely packed nests or cords of polygonal to cuboidal, lightly eosinophilic cells that completely filled the alveolar spaces. Alveolar/bronchiolar carcinomas were discrete to locally infiltrative, compressive, nodular to irregularly shaped masses that effaced the alveolar parenchyma and ranged in diameter from 1 to over 10 millimeters. Smaller carcinomas were relatively well differentiated neoplasms that were morphologically similar to adenomas but were distinguished by their slightly greater cellular pleomorphism and architectural disorganization. Larger carcinomas were clearly malignant neoplasms which were composed of poorly differentiated, pleomorphic anaplastic cells with a variety of growth patterns ranging from complex papillary, tubular, and/or glandular, and less commonly, solid sheets (Figure 29, Figure 30, and Figure 31). Many lungs that had carcinomas also had multiple, small nests of neoplastic cells randomly scattered throughout the lung parenchyma and occasionally on the pleura. Among the exposed mice with lung carcinomas, metastases occurred in various other organs including the liver, kidney, heart, nose, trachea, pancreas, cecum, adrenal cortex/medulla, coagulating gland, epididymis, testes, lymph nodes, thymus, skin, and skeletal muscle.

Point mutations in Kras (67\%), Egfr (17\%), and Tp53 (19\%) were noted in the alveolar/bronchiolar carcinomas in mice chronically exposed to cobalt metal dust, and none were found in spontaneously arising alveolar/bronchiolar carcinomas in chamber control mice. The results for the molecular analyses are presented in Appendix K.

A spectrum of nonneoplastic lesions occurred in the lungs of male and female mice (Table 29, Table C-4, and Table D-4). The incidences of alveolar/bronchiolar epithelium hyperplasia and cytoplasmic vacuolization, alveolar epithelium hyperplasia, proteinosis, and alveolus infiltration cellular histiocyte occurred with positive trends in male and female mice and were significantly increased in all exposed groups of males and females. The incidences of bronchiole epithelium hyperplasia occurred with positive trends in males and females and were significantly increased in males exposed to $5 \mathrm{mg} / \mathrm{m}^{3}$ and females exposed to 2.5 or $5 \mathrm{mg} / \mathrm{m}^{3}$. The incidence of bronchiole epithelium erosion was significantly increased in males exposed to $2.5 \mathrm{mg} / \mathrm{m}^{3}$. The incidences of suppurative inflammation were significantly increased in males exposed to 2.5 or $5 \mathrm{mg} / \mathrm{m}^{3}$ and females exposed to $5 \mathrm{mg} / \mathrm{m}^{3}$. In general, the severities of these nonneoplastic lesions increased with increasing exposure concentration.

Nonneoplastic lesions invariably occurred together and presented as a complex mixture of lesions that at times were difficult to separate as individual lesions. Alveolar/bronchiolar epithelium hyperplasia and alveolar/bronchiolar epithelium cytoplasmic vacuolization were changes that occurred in the epithelium of the periacinar region of the lung which encompassed the terminal bronchioles, associated alveolar ducts, and immediately adjacent alveoli. The 
primary change throughout the epithelium was cytoplasmic vacuolization of the epithelium of terminal bronchioles and associated alveolar ducts and immediately adjacent alveoli that was also observed to a lesser extent in the larger bronchioles and bronchi. Cells with cytoplasmic vacuolization were swollen, cuboidal to irregularly polygonal, and had finely vacuolated to diffusely clear cytoplasm with small hyperchromic nuclei (Figure 32); generally, there was a decrease or absence of the surface cilia and apical blebbing that are characteristic of the normal ciliated epithelium and mouse Clara cells. Focally to multifocally, the epithelium of the terminal bronchioles appeared thickened by disorganized proliferation and piling up of vacuolated, cuboidal to polygonal epithelial cells that frequently extended into the alveolar ducts and the adjacent alveoli; these changes were diagnosed as alveolar/bronchiolar epithelium hyperplasia (Figure 32). Hyperplasia of the alveolar epithelium occurred as focal, discrete, irregular, noncompressive proliferations of alveolar epithelial (Type II) cells distributed randomly within the parenchyma or adjacent to the terminal bronchioles with preservation of the underlying alveolar architecture. These foci consisted of several contiguous alveolar septa lined by uniformly small cuboidal cells with small, hyperchromatic nuclei (Figure 33); occasional karyomegalic cells were present. Hyperplasia of the bronchiole epithelium was characterized by proliferation of cuboidal to low columnar bronchiolar epithelial cells as few to multiple papillary structures that were supported by scant fibrous stroma and which projected into the lumens of the terminal bronchioles (Figure 34). Erosion of the bronchiole epithelium consisted of minimal, focal denudation of bronchiolar epithelial cells with associated minimal necrosis.

Table 29. Incidences of Neoplasms and Nonneoplastic Lesions of the Lung in Mice in the Two-year Inhalation Study of Cobalt Metal

\begin{tabular}{|c|c|c|c|c|}
\hline & $\begin{array}{l}\text { Chamber } \\
\text { Control }\end{array}$ & $1.25 \mathrm{mg} / \mathrm{m}^{3}$ & $2.5 \mathrm{mg} / \mathrm{m}^{3}$ & $5 \mathrm{mg} / \mathrm{m}^{3}$ \\
\hline \multicolumn{5}{|l|}{ Male } \\
\hline Number Examined Microscopically & 50 & 49 & 50 & 50 \\
\hline $\begin{array}{l}\text { Alveolar/bronchiolar Epithelium, } \\
\text { Hyperplasia }^{\mathrm{a}}\end{array}$ & 0 & $46^{* *}(1.0)^{\mathrm{b}}$ & $49 * *(1.6)$ & $50 * *(2.3)$ \\
\hline $\begin{array}{l}\text { Alveolar/bronchiolar Epithelium, } \\
\text { Vacuolization Cytoplasmic }\end{array}$ & 0 & $49 * *(1.1)$ & $47 * *(1.9)$ & $48 * *(3.1)$ \\
\hline Alveolar Epithelium, Hyperplasia & $4(2.3)$ & $29 * *(1.7)$ & $24 * *(1.8)$ & $43 * *(2.0)$ \\
\hline $\begin{array}{l}\text { Bronchiole, Epithelium, } \\
\text { Hyperplasia }\end{array}$ & $4(2.5)$ & $7(1.3)$ & $9(1.3)$ & $11 *(1.5)$ \\
\hline Bronchiole, Epithelium, Erosion & 0 & $4(1.0)$ & $10 * *(1.3)$ & $2(1.0)$ \\
\hline Proteinosis & $2(1.0)$ & $46^{* *}(1.7)$ & $49 * *(3.1)$ & $50 * *(3.9)$ \\
\hline $\begin{array}{l}\text { Alveolus, Infiltration Cellular, } \\
\text { Histiocyte }\end{array}$ & $10(1.8)$ & $49 * *(1.8)$ & $48 * *(2.5)$ & $48 * *(3.1)$ \\
\hline Inflammation, Suppurative & $1(1.0)$ & $2(2.0)$ & $6^{*}(1.5)$ & $16^{* *}(2.3)$ \\
\hline $\begin{array}{l}\text { Alveolar/bronchiolar Adenoma, } \\
\text { Multiple }\end{array}$ & 0 & 1 & 1 & 0 \\
\hline \multicolumn{5}{|c|}{ Alveolar/bronchiolar Adenoma (includes multiple) ${ }^{\mathrm{c}}$} \\
\hline Overall rate $^{\mathrm{d}}$ & $7 / 50(14 \%)$ & $11 / 49(22 \%)$ & $15 / 50(30 \%)$ & $3 / 50(6 \%)$ \\
\hline Adjusted rate ${ }^{\mathrm{e}}$ & $14.7 \%$ & $24.5 \%$ & $35.9 \%$ & $7.3 \%$ \\
\hline
\end{tabular}


Cobalt Metal, NTP TR 581

\begin{tabular}{|c|c|c|c|c|}
\hline & $\begin{array}{l}\text { Chamber } \\
\text { Control }\end{array}$ & $1.25 \mathrm{mg} / \mathrm{m}^{3}$ & $2.5 \mathrm{mg} / \mathrm{m}^{3}$ & $5 \mathrm{mg} / \mathrm{m}^{3}$ \\
\hline Terminal rate $\mathrm{f}^{\mathrm{f}}$ & $5 / 39(13 \%)$ & $7 / 31(23 \%)$ & $14 / 29(48 \%)$ & $2 / 25(8 \%)$ \\
\hline First incidence (days) & 684 & 571 & 660 & 571 \\
\hline Poly-3 test ${ }^{\mathrm{g}}$ & $\mathrm{P}=0.254 \mathrm{~N}$ & $\mathrm{P}=0.176$ & $\mathrm{P}=0.016$ & $\mathrm{P}=0.226 \mathrm{~N}$ \\
\hline $\begin{array}{l}\text { Alveolar/bronchiolar Carcinoma, } \\
\text { Multiple }\end{array}$ & 3 & $18^{* *}$ & $24 * *$ & $36^{* *}$ \\
\hline \multicolumn{5}{|c|}{ Alveolar/bronchiolar Carcinoma (includes multiple) ${ }^{\mathrm{h}}$} \\
\hline Overall rate & $11 / 50(22 \%)$ & $38 / 49(78 \%)$ & $42 / 50(84 \%)$ & $46 / 50(92 \%)$ \\
\hline Adjusted rate & $22.8 \%$ & $79.4 \%$ & $87.6 \%$ & $93.8 \%$ \\
\hline Terminal rate & $8 / 39(21 \%)$ & $24 / 31(77 \%)$ & $25 / 29(86 \%)$ & $22 / 25(88 \%)$ \\
\hline First incidence (days) & 561 & 551 & 382 & 425 \\
\hline Poly-3 test & $\mathrm{P}<0.001$ & $\mathrm{P}<0.001$ & $\mathrm{P}<0.001$ & $\mathrm{P}<0.001$ \\
\hline \multicolumn{5}{|c|}{ Alveolar/bronchiolar Adenoma or Carcinoma ${ }^{\mathrm{i}}$} \\
\hline Overall rate & $16 / 50(32 \%)$ & $41 / 49(84 \%)$ & $43 / 50(86 \%)$ & $47 / 50(94 \%)$ \\
\hline Adjusted rate & $33.0 \%$ & $85.0 \%$ & $89.7 \%$ & $95.9 \%$ \\
\hline Terminal rate & $11 / 39(28 \%)$ & $26 / 31(84 \%)$ & $26 / 29(90 \%)$ & $23 / 25(92 \%)$ \\
\hline First incidence (days) & 561 & 551 & 382 & 425 \\
\hline Poly-3 test & $\mathrm{P}<0.001$ & $\mathrm{P}<0.001$ & $\mathrm{P}<0.001$ & $\mathrm{P}<0.001$ \\
\hline \multicolumn{5}{|l|}{ Female } \\
\hline Number Examined Microscopically & 49 & 50 & 50 & 50 \\
\hline $\begin{array}{l}\text { Alveolar/bronchiolar Epithelium, } \\
\text { Hyperplasia }\end{array}$ & 0 & $49 * *(1.1)$ & $49 * *(1.9)$ & $50 * *(2.7)$ \\
\hline $\begin{array}{l}\text { Alveolar/bronchiolar Epithelium, } \\
\text { Vacuolization Cytoplasmic }\end{array}$ & 0 & $48 * *(1.1)$ & $49 * *(1.9)$ & $48 * *(3.5)$ \\
\hline Alveolar Epithelium, Hyperplasia & $2(2.5)$ & $27 * *(1.6)$ & $26^{* *}(1.4)$ & $41 * *(1.4)$ \\
\hline $\begin{array}{l}\text { Bronchiole, Epithelium, } \\
\text { Hyperplasia }\end{array}$ & 0 & $3(1.0)$ & $12 * *(1.1)$ & $26^{* *}(1.2)$ \\
\hline Bronchiole, Epithelium, Erosion & 0 & 0 & $4(1.0)$ & $3(1.0)$ \\
\hline Proteinosis & 0 & $45^{* *}(1.4)$ & $50 * *(2.6)$ & $50 * *(3.9)$ \\
\hline $\begin{array}{l}\text { Alveolus, Infiltration Cellular, } \\
\text { Histiocyte }\end{array}$ & $10(1.7)$ & $49 * *(1.6)$ & $50 * *(2.5)$ & $49 * *(3.1)$ \\
\hline Inflammation, Suppurative & 0 & $3(1.3)$ & $2(1.0)$ & $15^{* *}(1.7)$ \\
\hline $\begin{array}{l}\text { Alveolar/bronchiolar Adenoma, } \\
\text { Multiple }\end{array}$ & 0 & 1 & 0 & 1 \\
\hline \multicolumn{5}{|c|}{ Alveolar/bronchiolar Adenoma (includes multiple) $)^{\mathrm{j}}$} \\
\hline Overall rate & $3 / 49(6 \%)$ & $9 / 50(18 \%)$ & $8 / 50(16 \%)$ & $10 / 50(20 \%)$ \\
\hline Adjusted rate & $6.9 \%$ & $19.9 \%$ & $18.9 \%$ & $24.5 \%$ \\
\hline Terminal rate & $3 / 36(8 \%)$ & $7 / 35(20 \%)$ & $6 / 27(22 \%)$ & $6 / 26(23 \%)$ \\
\hline
\end{tabular}


Cobalt Metal, NTP TR 581

\begin{tabular}{|c|c|c|c|c|}
\hline & $\begin{array}{l}\text { Chamber } \\
\text { Control }\end{array}$ & $1.25 \mathrm{mg} / \mathrm{m}^{3}$ & $2.5 \mathrm{mg} / \mathrm{m}^{3}$ & $5 \mathrm{mg} / \mathrm{m}^{3}$ \\
\hline First incidence (days) & $731(\mathrm{~T})$ & 505 & 626 & 593 \\
\hline Poly-3 test & $\mathrm{P}=0.037$ & $P=0.067$ & $\mathrm{P}=0.087$ & $\mathrm{P}=0.024$ \\
\hline $\begin{array}{l}\text { Alveolar/bronchiolar Carcinoma, } \\
\text { Multiple }\end{array}$ & 1 & $7 *$ & $20 * *$ & $24 * *$ \\
\hline \multicolumn{5}{|c|}{ Alveolar/bronchiolar Carcinoma (includes multiple) ${ }^{\mathrm{k}}$} \\
\hline Overall rate & $5 / 49(10 \%)$ & $25 / 50(50 \%)$ & $38 / 50(76 \%)$ & $43 / 50(86 \%)$ \\
\hline Adjusted rate & $11.3 \%$ & $53.8 \%$ & $78.9 \%$ & $87.7 \%$ \\
\hline Terminal rate & $3 / 36(8 \%)$ & $18 / 35(51 \%)$ & $19 / 27(70 \%)$ & $21 / 26(81 \%)$ \\
\hline First incidence (days) & 583 & 537 & 457 & 478 \\
\hline Poly-3 test & $\mathrm{P}<0.001$ & $\mathrm{P}<0.001$ & $\mathrm{P}<0.001$ & $\mathrm{P}<0.001$ \\
\hline \multicolumn{5}{|c|}{ Alveolar/bronchiolar Adenoma or Carcinoma ${ }^{1}$} \\
\hline Overall rate & $8 / 49(16 \%)$ & $30 / 50(60 \%)$ & $41 / 50(82 \%)$ & $45 / 50(90 \%)$ \\
\hline Adjusted rate & $18.0 \%$ & $63.7 \%$ & $84.6 \%$ & $91.6 \%$ \\
\hline Terminal rate & $6 / 36(17 \%)$ & $22 / 35(63 \%)$ & $21 / 27(78 \%)$ & $22 / 26(85 \%)$ \\
\hline First incidence (days) & 583 & 505 & 457 & 478 \\
\hline Poly-3 test & $\mathrm{P}<0.001$ & $\mathrm{P}<0.001$ & $\mathrm{P}<0.001$ & $\mathrm{P}<0.001$ \\
\hline
\end{tabular}

*Significantly different $(\mathrm{P} \leq 0.05)$ from the chamber control group by the Poly- 3 test.

$* * \mathrm{P} \leq 0.01$.

(T) Terminal kill.

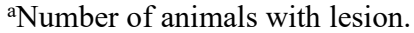

${ }^{\mathrm{b}}$ Average severity grade of lesions in affected animals: $1=$ minimal, $2=$ mild, $3=$ moderate, $4=$ marked.

'Historical incidence for 2-year inhalation studies with chamber control groups (mean \pm standard deviation): $39 / 300$

$(13.0 \% \pm 4.2 \%)$, range $8 \%-20 \%$; all routes: $145 / 950(15.3 \% \pm 6.2 \%)$, range $2 \%-26 \%$.

${ }^{\mathrm{d}}$ Number of animals with neoplasm per number of animals with lung examined microscopically.

ePoly-3 estimated neoplasm incidence after adjustment for intercurrent mortality.

${ }^{f}$ Observed incidence at terminal kill.

gBeneath the chamber control incidence is the $\mathrm{P}$ value associated with the trend test. Beneath the exposed group incidence are the $P$ values corresponding to pairwise comparisons between the chamber controls and that exposed group. The Poly-3 test accounts for differential mortality in animals that do not reach terminal kill. A negative trend or a lower incidence in an exposure group is indicated by $\mathbf{N}$.

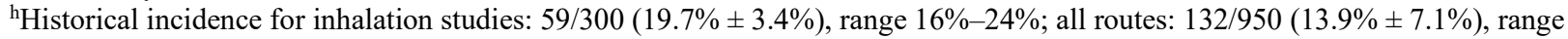
$4 \%-24 \%$.

${ }^{i}$ Historical incidence for inhalation studies: $90 / 300(30.0 \% \pm 5.5 \%)$, range $26 \%-40 \%$; all routes: $263 / 950(27.7 \% \pm 5.7 \%)$, range $16 \%-40 \%$.

${ }^{\mathrm{j} H i s t o r i c a l}$ incidence for inhalation studies: $16 / 299(5.4 \% \pm 3.7 \%)$, range $2 \%-12 \%$; all routes: $54 / 949(5.7 \% \pm 3.6 \%)$, range $0 \%-$ $12 \%$.

${ }^{\mathrm{k}}$ Historical incidence for inhalation studies: $13 / 299(4.4 \% \pm 4.3 \%)$, range $0 \%-10 \%$; all routes: $38 / 949(4.0 \% \pm 3.6 \%)$, range $0 \%-$ $14 \%$.

${ }^{1}$ Historical incidence for inhalation studies: $28 / 299(9.4 \% \pm 4.8 \%)$, range $2 \%-16 \%$; all routes: 90/949 $(9.5 \% \pm 4.8 \%)$, range $2 \%-$ $22 \%$.

Alveoli contained a complex mixture of proteinaceous material (diagnosed as proteinosis) and inflammatory cells. Alveolar proteinosis in mice was morphologically similar to proteinosis in rats and was characterized by accumulation of variable amounts of brightly eosinophilic material within alveolar spaces and ducts with extension into the lumens of the bronchioles in the more extreme cases. The character of this material ranged from pale eosinophilic, flocculent to amorphous aggregates to brightly eosinophilic, dense, round to irregular clumps free within 
alveolar spaces or alveolar macrophages. In the more severe cases of proteinosis, there were single or aggregated slender, elongated, acicular to rectangular fractile, crystalline or spicule-like structures free within the alveoli or alveolar macrophages (Figure 35). Almost diffusely mixed with this proteinaceous material were increased numbers of histiocytes/macrophages that occurred as small, scattered aggregates to massive accumulations that on occasion completely occluded the alveoli in large regions of the lung. The histiocyte/macrophage infiltrates were mixtures of small to swollen macrophages to multinucleated giant cells. Many were distended with proteinaceous material or, especially in the lower exposure concentration groups, had cytoplasm that was lightly eosinophilic to gray, finely granular or foamy (amphophilic). Large histiocytes/macrophages and multinucleated giant cells contained the refractile, crystalline/specular-like structures. Accumulations of small histiocyte/macrophage infiltrates aggregated adjacent to alveolar/bronchiolar neoplasms (especially larger carcinomas). Also considered a component of this lesion were multifocal accumulations of plump foamy histiocytes/macrophages within the alveolar spaces; such cells were more frequent in the lower exposure concentration groups. Together, these histiocyte/macrophage infiltrates were diagnosed as histiocytic cellular infiltration of the alveolus (Figure 35). Mixed with the alveolar proteinaceous material and histiocyte/macrophage infiltrates were areas of prominent neutrophil accumulation that were diagnosed as suppurative inflammation. This occurred primarily in the male and female mice exposed to $5 \mathrm{mg} / \mathrm{m}^{3}$ and consisted of variably sized, localized accumulations of neutrophils and necrotic debris within alveoli. In areas of intense neutrophil accumulation, the alveolar septa were sometimes necrotic or even completely effaced; peribronchiolar edema, intraalveolar hemorrhage, and bacteria were occasionally observed in association with suppurative inflammation.

Nose: A spectrum of nonneoplastic lesions occurred in exposed groups of males and females and the incidences of these lesions were generally significantly greater than those in the chamber control groups (Table 30, Table C-4, and Table D-4). For some lesions, the severities increased with increasing exposure concentration.

Suppurative inflammation in the nasal cavity of mice was morphologically similar to suppurative inflammation in the rats. It occurred primarily in the Level II nasal section and consisted of accumulations of neutrophils, proteinaceous fluid, and cellular debris in the nasal passages at all levels of the nose and was occasionally associated with fragments of plant material. Neutrophils sometimes infiltrated the nasal epithelium and lamina propria, and occasionally, the inflammatory process extended into the nasolacrimal duct, maxillary sinuses, and vomeronasal organ.

Atrophy of the olfactory epithelium was of minimal to mild severity and occurred in the epithelium of the dorsal meatus in Levels II and III and the ethmoturbinates of Level III. In general, olfactory epithelial atrophy in mice was morphologically similar to olfactory epithelial atrophy in the rats and was characterized by focal to diffuse hypocellularity and disorganization of the epithelium, often with increased extent of clear intercellular spaces with or without an overall decrease in height of the epithelium. There were variable decreases in the size and number of the nerve bundles and submucosal glands in the adjacent lamina propria. Hyperplasia of the olfactory epithelium was of minimal to mild severity and consisted of scattered focal proliferation of basal olfactory epithelial cells that extended through the basal lamina into the adjacent lamina propria often associated with Bowman's gland ducts. The cells were sometimes 
clustered in small intraepithelial nests or extended into the lamina propria around the Bowman's gland ducts.

Table 30. Incidences of Nonneoplastic Lesions of the Nose, Larynx, and Trachea in Mice in the Two-year Inhalation Study of Cobalt Metal

\begin{tabular}{|c|c|c|c|c|}
\hline & $\begin{array}{l}\text { Chamber } \\
\text { Control }\end{array}$ & $1.25 \mathrm{mg} / \mathrm{m}^{3}$ & $2.5 \mathrm{mg} / \mathrm{m}^{3}$ & $5 \mathrm{mg} / \mathrm{m}^{3}$ \\
\hline \multicolumn{5}{|l|}{ Male } \\
\hline Nose $^{a}$ & 50 & 49 & 50 & 50 \\
\hline Inflammation, Suppurative ${ }^{\mathrm{b}}$ & $16(1.1)^{\mathrm{c}}$ & $32 * *(1.9)$ & $49 * *(2.7)$ & $50 * *(3.1)$ \\
\hline Olfactory Epithelium, Atrophy & $3(1.0)$ & $46 * *(1.2)$ & $42 * *(1.2)$ & $31 * *(1.2)$ \\
\hline Olfactory Epithelium, Hyperplasia & 0 & $25 * *(1.2)$ & $17 * *(1.0)$ & $8 * *(1.1)$ \\
\hline $\begin{array}{l}\text { Olfactory Epithelium, Metaplasia, } \\
\text { Respiratory }\end{array}$ & $5(1.4)$ & $24 * *(1.3)$ & $44 * *(2.3)$ & $50 * *(3.1)$ \\
\hline $\begin{array}{l}\text { Olfactory Epithelium, Respiratory } \\
\text { Metaplasia, Atypical }\end{array}$ & 0 & $14 * *(2.0)$ & $9 * *(1.1)$ & $1(1.0)$ \\
\hline $\begin{array}{l}\text { Respiratory Epithelium, } \\
\text { Accumulation, Hyaline Droplet }\end{array}$ & $13(1.2)$ & $29 * *(1.1)$ & $29 * *(1.1)$ & $7(1.0)$ \\
\hline $\begin{array}{l}\text { Respiratory Epithelium, } \\
\text { Vacuolization Cytoplasmic }\end{array}$ & 0 & $41 * *(1.2)$ & $39 * *(1.2)$ & $37 * *(1.4)$ \\
\hline $\begin{array}{l}\text { Respiratory Epithelium, } \\
\text { Metaplasia, Squamous }\end{array}$ & $3(1.0)$ & $45 * *(1.0)$ & $35^{* *}(1.1)$ & $33 * *(1.2)$ \\
\hline Turbinate, Atrophy & $3(1.3)$ & $25 * *(1.3)$ & $49 * *(2.1)$ & $50 * *(3.3)$ \\
\hline Larynx & 48 & 47 & 49 & 50 \\
\hline $\begin{array}{l}\text { Respiratory Epithelium, } \\
\text { Metaplasia, Squamous }\end{array}$ & $7(1.0)$ & $47 * *(1.0)$ & $49 * *(1.0)$ & $49 * *(1.0)$ \\
\hline $\begin{array}{l}\text { Respiratory Epithelium, } \\
\text { Vacuolization Cytoplasmic }\end{array}$ & 0 & $20 * *(1.0)$ & $24 * *(1.0)$ & $32 * *(1.1)$ \\
\hline Squamous Epithelium, Hyperplasia & $2(1.0)$ & $5(1.0)$ & $5(1.0)$ & $8 *(1.0)$ \\
\hline Trachea & 48 & 47 & 48 & 50 \\
\hline $\begin{array}{l}\text { Epithelium, Vacuolization } \\
\text { Cytoplasmic }\end{array}$ & 0 & $14 * *(1.4)$ & $31 * *(1.6)$ & $37 * *(1.4)$ \\
\hline \multicolumn{5}{|l|}{ Female } \\
\hline Nose & 50 & 50 & 50 & 50 \\
\hline Inflammation, Suppurative & $3(1.0)$ & $47 * *(2.3)$ & $50 * *(3.1)$ & $50 * *(3.3)$ \\
\hline Olfactory Epithelium, Atrophy & $4(1.0)$ & $44 * *(1.2)$ & $39 * *(1.2)$ & $24 * *(1.2)$ \\
\hline Olfactory Epithelium, Hyperplasia & $1(1.0)$ & $22 * *(1.1)$ & $16^{* *}(1.0)$ & $8 *(1.0)$ \\
\hline $\begin{array}{l}\text { Olfactory Epithelium, Metaplasia, } \\
\text { Respiratory }\end{array}$ & $1(1.0)$ & $26 * *(1.8)$ & $44 * *(2.7)$ & $50 * *(3.3)$ \\
\hline $\begin{array}{l}\text { Olfactory Epithelium, Respiratory } \\
\text { Metaplasia, Atypical }\end{array}$ & 0 & $18 * *(1.6)$ & $14 * *(1.5)$ & $1(1.0)$ \\
\hline
\end{tabular}




\begin{tabular}{|c|c|c|c|c|}
\hline & $\begin{array}{l}\text { Chamber } \\
\text { Control }\end{array}$ & $1.25 \mathrm{mg} / \mathrm{m}^{3}$ & $2.5 \mathrm{mg} / \mathrm{m}^{3}$ & $5 \mathrm{mg} / \mathrm{m}^{3}$ \\
\hline $\begin{array}{l}\text { Respiratory Epithelium, } \\
\text { Accumulation, Hyaline Droplet }\end{array}$ & $12(1.0)$ & $38 * *(1.1)$ & $40 * *(1.2)$ & $10(1.0)$ \\
\hline $\begin{array}{l}\text { Respiratory Epithelium, } \\
\text { Vacuolization Cytoplasmic }\end{array}$ & 0 & $40 * *(1.0)$ & $47 * *(1.1)$ & $47 * *(1.1)$ \\
\hline $\begin{array}{l}\text { Respiratory Epithelium, } \\
\text { Metaplasia, Squamous }\end{array}$ & 0 & $49 * *(1.2)$ & $49 * *(1.4)$ & $50 * *(1.5)$ \\
\hline Turbinate, Atrophy & 0 & $44 * *(2.2)$ & $50 * *(2.9)$ & $50 * *(3.4)$ \\
\hline Larynx & 47 & 50 & 50 & 47 \\
\hline $\begin{array}{l}\text { Respiratory Epithelium, } \\
\text { Metaplasia, Squamous }\end{array}$ & $2(1.0)$ & $49 * *(1.0)$ & $50 * *(1.0)$ & $47 * *(1.1)$ \\
\hline $\begin{array}{l}\text { Respiratory Epithelium, } \\
\text { Vacuolization Cytoplasmic }\end{array}$ & 0 & $24 * *(1.0)$ & $31 * *(1.0)$ & $34 * *(1.0)$ \\
\hline Squamous Epithelium, Hyperplasia & $2(1.0)$ & $13 * *(1.1)$ & $21 * *(1.0)$ & $21 * *(1.0)$ \\
\hline Squamous Epithelium, Erosion & $1(1.0)$ & $2(1.0)$ & $7 *(1.0)$ & $4(1.0)$ \\
\hline Trachea & 48 & 50 & 48 & 49 \\
\hline $\begin{array}{l}\text { Epithelium, Vacuolization } \\
\text { Cytoplasmic }\end{array}$ & 0 & $26 * *(1.4)$ & $37 * *(1.6)$ & $39 * *(1.8)$ \\
\hline
\end{tabular}

Respiratory metaplasia of the olfactory epithelium was of minimal to mild severity and observed more frequently in the dorsal meatus of Level II and on the nasal septum and ethmoturbinates in Level III. These lesions consisted of replacement of olfactory epithelium by ciliated, cuboidal to tall columnar, respiratory-type epithelial cells. The metaplastic epithelium occurred as crypt-like folds and invaginations and extended into the ducts of the submucosal Bowman's glands. In 1.25 and $2.5 \mathrm{mg} / \mathrm{m}^{3}$ animals of both sexes, there were focal, highly site-specific, often bilaterally symmetrical, exophytic lesions located on the dorsal surface of the dorsal scroll. These focal lesions seemingly arose in areas that resembled respiratory metaplasia. The lesions were slightly to prominently elevated above the surface of the ethmoturbinates and in extreme cases, formed synechia with opposing dorsal turbinates and the roof of the dorsal meatus. Morphology ranged from small, single, or few rosette- or gland-like structures, to larger more complex formations of glands lined by flattened to ciliated respiratory type epithelium (Figure 36). These unusual lesions were diagnosed as olfactory epithelium atypical respiratory metaplasia.

Hyaline droplet accumulation in the respiratory epithelium occurred with minimal severity and involved the respiratory epithelium adjacent to the junction with squamous epithelium lining the ventral aspects of the nasal passages in Levels I and II, adjacent to the incisive duct in Level II, and overlying the vomeronasal organ. Hyaline droplet accumulation consisted of intracytoplasmic, homogenous, eosinophilic, globular material in the cytoplasm of the respiratory epithelial cells. 
Cytoplasmic vacuolization of the respiratory epithelium primarily occurred with minimal to mild severity and affected the respiratory epithelium of the dorsal to mid-septum and/or the dorsal meatus of Level I and occasionally Level II (lateral walls and metaplastic region of the dorsal meatus). The morphology of cytoplasmic vacuolization was similar to that observed in the bronchiolar epithelium. In affected sites, the normally tall, pseudostratified, ciliated, columnar epithelium was replaced by plump, variably ciliated, cuboidal to polygonal epithelial cells that had finely vacuolated to diffusely clear cytoplasm.

Squamous metaplasia of the respiratory epithelium of minimal to mild severity was generally similar to this lesion in the rat study. It occurred in the Level I and to a lesser extent, Level II sections along the dorsal to mid-septum and on the tips of the nasoturbinates and maxilloturbinates. Squamous metaplasia was characterized by replacement of the normally current single layer of ciliated columnar respiratory epithelium by nonkeratinized, flat, squamous epithelial cells.

Turbinate atrophy was a minimal to moderate change that was similar to this lesion in the rat study. It was characterized by often prominent attenuation of the bone and structures of the lamina propria including the glands, vessels, nerve bundles, interstitial stroma on the naso-, maxilla-, and ethmoturbinates and the medial septum at Levels I, II, III. This resulted in narrowing, shortening, distortion, and sometimes loss of the turbinates, as well as occasional adhesions of turbinate remnants to each other, the nasal septum, or the lateral walls. The nasal septum was often buckled, bent, and sometimes perforated.

Larynx: The incidences of respiratory epithelium squamous metaplasia and cytoplasmic vacuolization in all exposed groups of males and females were significantly greater than those in the chamber controls (Table 30, Table C-4, and Table D-4). The incidences of squamous epithelium hyperplasia were significantly increased in all exposed groups of females and in males exposed to $5 \mathrm{mg} / \mathrm{m}^{3}$. The incidence of squamous epithelium erosion was significantly increased in females exposed to $2.5 \mathrm{mg} / \mathrm{m}^{3}$. All of these laryngeal lesions were of minimal severity.

Respiratory epithelium squamous metaplasia involved the epithelium at the base of the epiglottis overlying the medial submucosal glands and consisted of one to a few layers of flattened, nonciliated, low-cuboidal to squamous epithelial cells replacing the ciliated, tall, columnar epithelium that normally occurs in this location. Respiratory epithelium cytoplasmic vacuolization was a subtle focal to diffuse change that occurred in the epithelium lining the dorsal aspects and lateral walls of Levels II and III laryngeal sections and was morphologically similar to cytoplasmic vacuolization observed in the bronchiolar epithelium. The ciliated columnar epithelial cells normally seen in the sites were shorter (cuboidal), in general had lost their cilia, and had slightly vacuolated to clear cytoplasm. Squamous epithelium hyperplasia was a focal change most common in the epithelium along the medial aspects and tips of the vocal processes of the arytenoid cartilages and consisted of increased layers of lining epithelial cells from the normal two to three cell layers to four to six cell layers. Erosion was characterized by small focal areas of epithelial necrosis and loss of the superficial epithelium in areas of hyperplastic squamous epithelium.

Trachea: The incidences of minimal to mild epithelium cytoplasmic vacuolization were significantly increased in all exposed groups of males and females (Table 30, Table C-4, and 
Table D-4). Cytoplasmic vacuolization in the epithelium lining the trachea and the submucosal tracheal glands was morphologically similar to that observed in the bronchiolar and laryngeal epithelia.

Testes: The incidence of minimal to mild germinal epithelium degeneration in male mice exposed to $5 \mathrm{mg} / \mathrm{m}^{3}$ was significantly greater than that in the chamber controls (chamber control, $9 / 50 ; 1.25 \mathrm{mg} / \mathrm{m}^{3}, 14 / 49 ; 2.5 \mathrm{mg} / \mathrm{m}^{3}, 8 / 50 ; 5 \mathrm{mg} / \mathrm{m}^{3}, 21 / 50$; Table C-4). Germinal epithelium hyperplasia was generally a minimal to mild lesion usually affecting one to a few scattered seminiferous tubules. Affected tubules were characterized by partial to complete absence of spermatogenic cells often with concurrent swelling of the Sertoli cells with resultant hypocellularity and decreased height of the germinal epithelium. The lumens were generally empty but sometimes contained few spermatozoa, sloughed germinal epithelial cells, or cellular debris.

\section{Tissue Burden Studies}

Lung weights of female mice were significantly increased starting on day 4 in groups exposed to 2.5 or $5 \mathrm{mg} / \mathrm{m}^{3}$ and continuing until day 548 (Table I-14). At $1.25 \mathrm{mg} / \mathrm{m}^{3}$, lung weights were increased on days 366 and 548; because of these increases in lung weights, lung cobalt burdens rather than lung cobalt concentrations were evaluated for toxicokinetic parameters.

Cobalt concentrations and burdens in the lung increased with increasing exposure concentration and were significantly increased in all exposed groups of female mice at all time points compared to those in the chamber control group (Table I-14). Cobalt concentrations in the chamber control group were at or below the LOD at all time points. By day 184, lung cobalt concentrations for all exposed groups appeared to reach steady state and did not change significantly through day 548. Lung cobalt burdens increased rapidly by day 4 , but by day 184 , the rate of increase slowed as lung burdens asymptotically approached steady state. Analysis of lung cobalt burdens normalized to exposure concentration indicated that there were proportional increases between the 1.25 and $2.5 \mathrm{mg} / \mathrm{m}^{3}$ groups, but nonproportional increases were observed between the 2.5 and $5 \mathrm{mg} / \mathrm{m}^{3}$ groups. At the earlier time points, normalized lung cobalt burdens were lower in animals exposed to $5 \mathrm{mg} / \mathrm{m}^{3}$ than in those exposed to $2.5 \mathrm{mg} / \mathrm{m}^{3}$; however the opposite was true at the longer exposure durations, where normalized cobalt burdens were greater than proportional relative to the $2.5 \mathrm{mg} / \mathrm{m}^{3}$ group.

The lung cobalt burden data from the exposure phases of the 3-month and 2-year studies were modeled using a two-compartment model (Figure I-2). Rapid clearance phase half-lives were $1.2,1.1$, and 5.2 days, respectively, for the $1.25,2.5$, and $5 \mathrm{mg} / \mathrm{m}^{3}$ groups, indicating a slightly longer half-life in animals exposed to $5 \mathrm{mg} / \mathrm{m}^{3}$ (Table I-15). Cobalt deposition rates for the rapid clearance phase were $0.87,1.84$, and $1.18 \mu \mathrm{g}$ cobalt/day at $1.25,2.5$, and $5 \mathrm{mg} / \mathrm{m}^{3}$, respectively. Slow clearance phase half-lives revealed the opposite trend, with half-lives of 409,172 , and 118 days with increasing exposure concentration. Cobalt deposition rates for the slow clearance phase were $0.027,0.075$, and $0.25 \mu \mathrm{g}$ cobalt/day. The overall theoretical steady-state lung cobalt burdens, including both the rapid and slow clearance phases $\left(\mathrm{L}_{\mathrm{SSa}}+\mathrm{L}_{\mathrm{SSb}}\right)$, were approximately $17.8,21.4$, and $51.8 \mu \mathrm{g}$ cobalt/lung in the $1.25,2.5$, and $5 \mathrm{mg} / \mathrm{m}^{3}$ groups, respectively; these data support the achievement of steady state in the 2.5 and $5 \mathrm{mg} / \mathrm{m}^{3}$ groups but not in the $1.25 \mathrm{mg} / \mathrm{m}^{3}$ group. The fractions of deposition in the slow clearance phase $\left(\mathrm{F}_{\mathrm{B}}\right)$ for the exposed groups were quite low, increasing from 0.031 to 0.176 as exposure concentration increased, corresponding to total slow phase lung cobalt clearances of $3.1 \%$ to $17.6 \%$; clearances of total deposited cobalt 
during the rapid clearance phase ranged from $96.9 \%$ to $82.4 \%\left[\left(1-\mathrm{F}_{\mathrm{B}}\right) \times 100\right]$ with increasing exposure concentration.

\section{Genetic Toxicology}

Results of the bacterial mutagenicity tests conducted with cobalt metal (the same lot of chemical that was used in the 2-year studies) are presented in Table E-1 and Table E-2. Cobalt metal (100 to $5,000 \mu \mathrm{g} / \mathrm{plate}$ ) gave an equivocal response in Salmonella typhimurium strain TA100 in the absence of S9 activation mix; with $10 \%$ rat liver S9, doses up to 7,500 $\mu \mathrm{g} / \mathrm{plate}$ did not induce an increase in mutant colonies in TA100. In S. typhimurium strain TA98 without S9, cobalt metal (100 to 3,500 $\mu \mathrm{g} /$ plate) was mutagenic, although the responses observed were weak and not well correlated with dose level; with S9, no mutagenic activity was observed. In Escherichia coli strain WP2 uvrA/pKM101, doses of cobalt metal up to $450 \mu \mathrm{g} /$ plate were not associated with mutagenic activity, with or without S9. No increases in the frequencies of micronucleated normochromatic erythrocytes were observed in peripheral blood of male or female mice exposed to cobalt metal $\left(0.625\right.$ to $\left.10 \mathrm{mg} / \mathrm{m}^{3}\right)$ for 3 months by inhalation (Table E-3). No significant alterations in the percentages of reticulocytes (polychromatic erythrocytes) were seen in male or female mice, suggesting that exposure to cobalt metal under these conditions did not cause bone marrow toxicity.

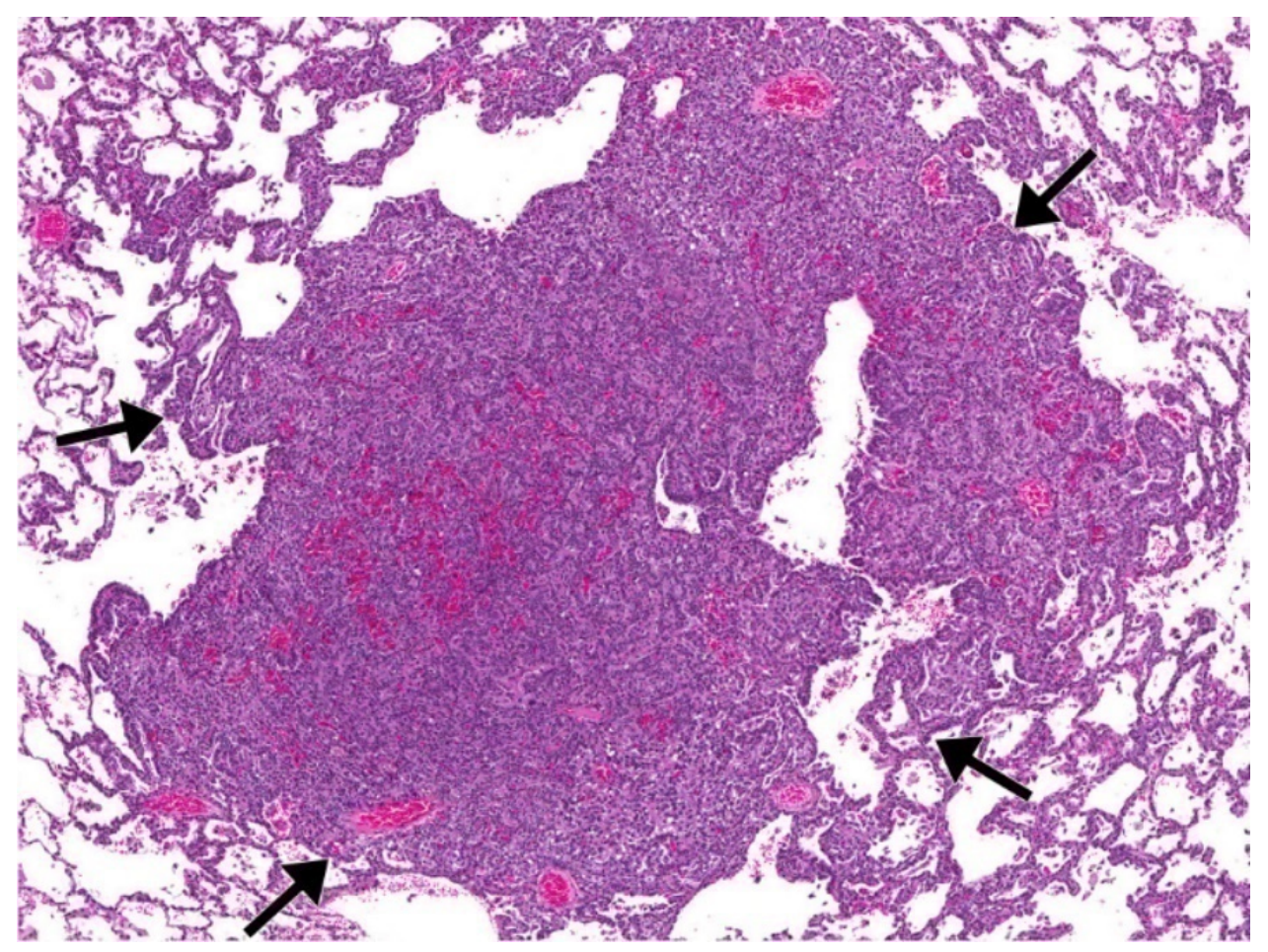

Figure 7. Alveolar/Bronchiolar Adenoma in the Lung of a F344/NTac Female Rat Exposed to $5 \mathrm{mg} / \mathrm{m}^{3}$ Cobalt Metal by Inhalation for Two Years (H\&E)

The adenoma is distinctly demarcated from the surrounding alveolar parenchyma (arrows). 


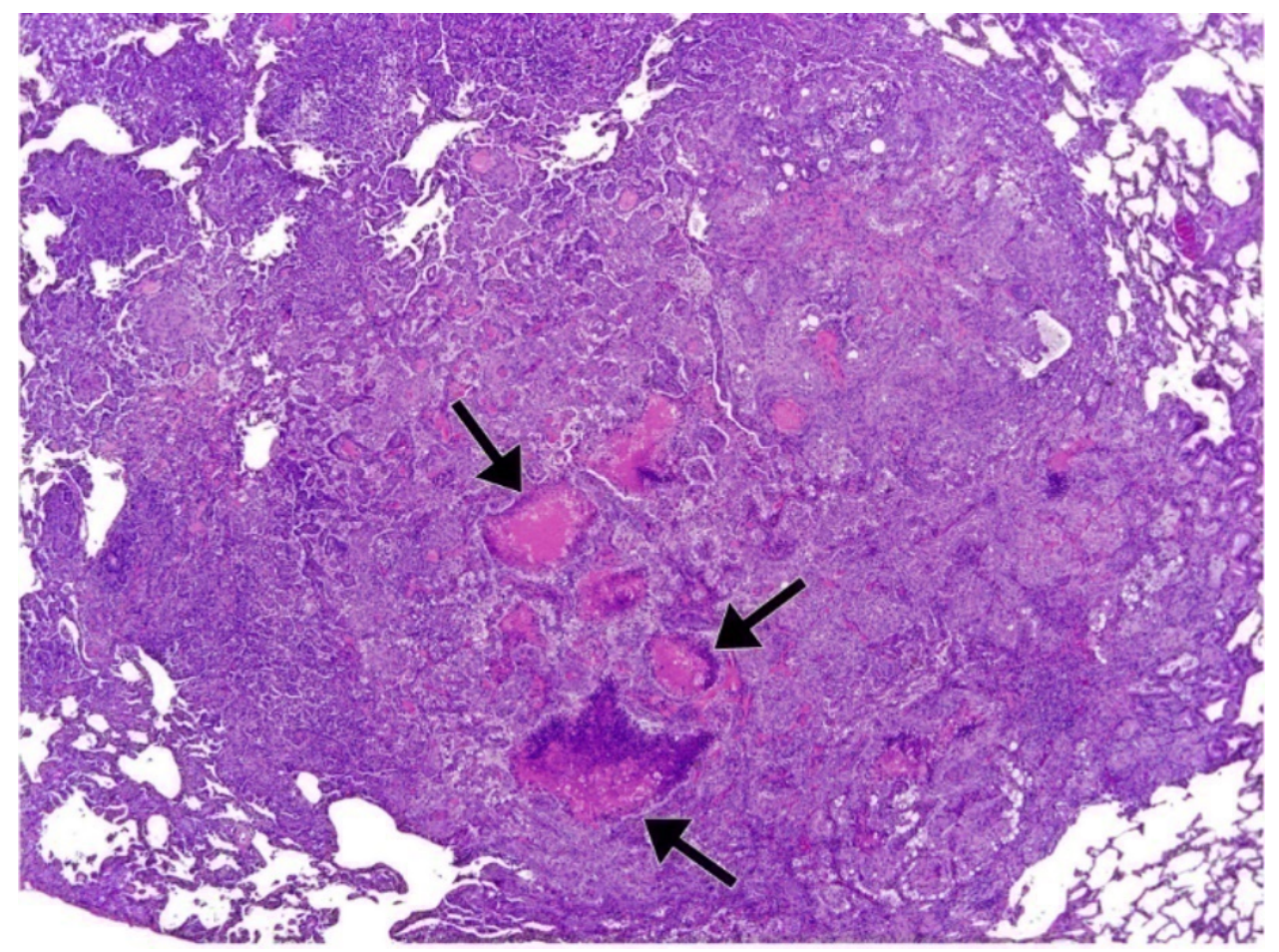

Figure 8. Alveolar/Bronchiolar Carcinoma in the Lung af a F344/NTac Female Rat Exposed to $5 \mathrm{mg} / \mathrm{m}^{3}$ Cobalt Metal by Inhalation for Two Years (H\&E)

The carcinoma is highly invasive and has effaced the lung parenchyma. Note several areas of necrosis (arrows) within the carcinoma.

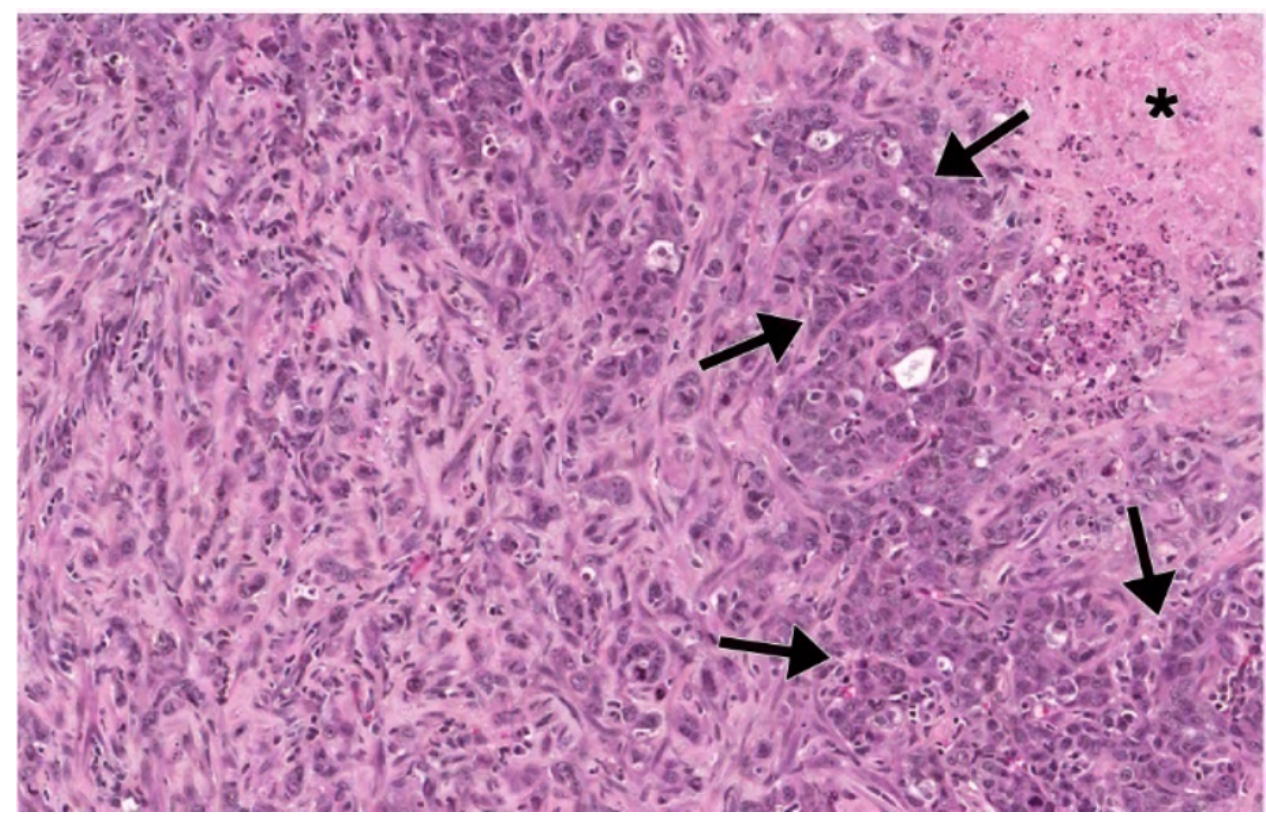

Figure 9. Sarcomatous Type Alveolar/Bronchiolar Carcinoma in the Lung of a F344/NTac Female Rat Exposed to $5 \mathrm{mg} / \mathrm{m}^{3}$ Cobalt Metal by Inhalation for Two Years (H\&E)

The carcinoma is composed mostly of anaplastic spindyloid cells that surround islands of neoplastic epithelial cells (arrows). Note an area of necrosis in the upper right corner of the plate (asterisk). 


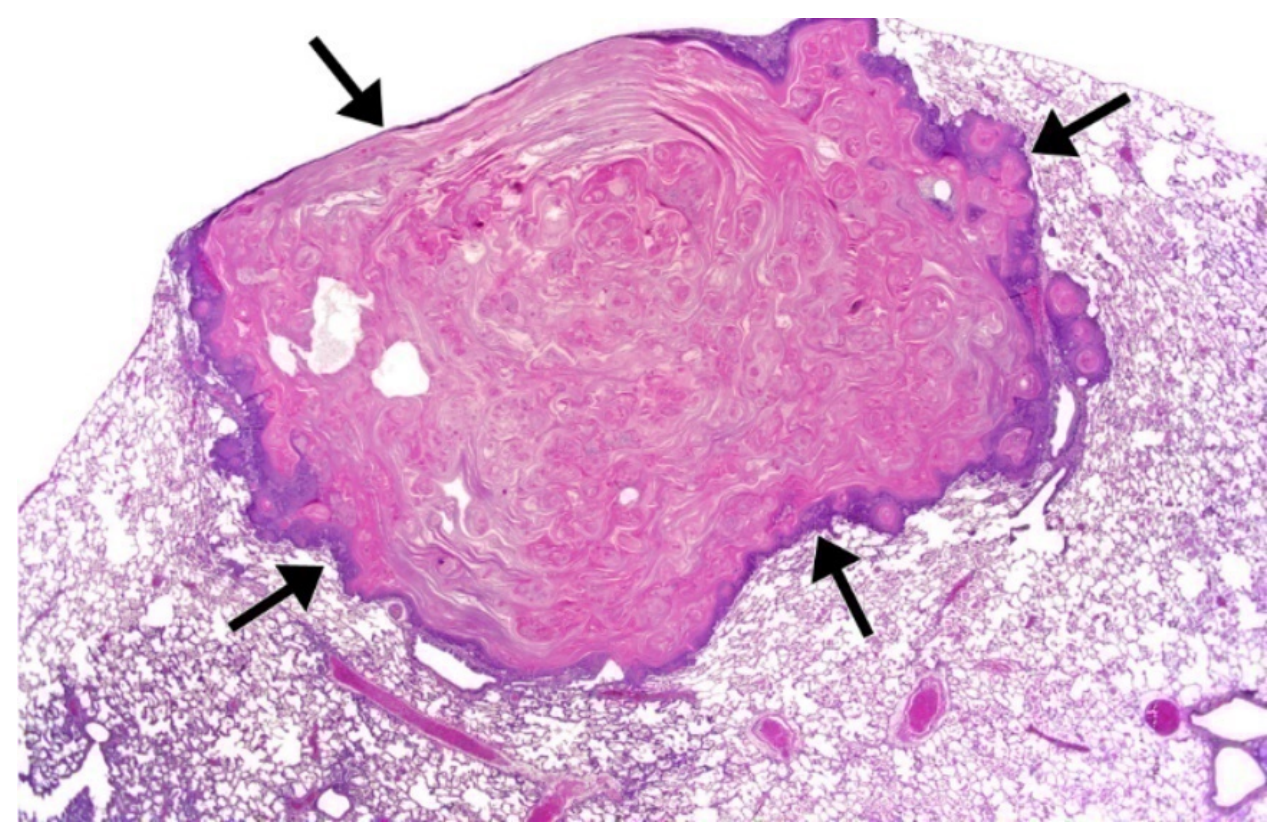

Figure 10. Cystic Keratinizing Epithelioma in the Lung of a Male F344/NTac Rat Exposed to $1.25 \mathrm{mg} / \mathrm{m}^{3}$ Cobalt Metal by Inhalation for Two Years (H\&E)

This neoplasm is characterized by a central mass of concentrically arranged keratin surrounded by wall of squamous epithelium (arrows).

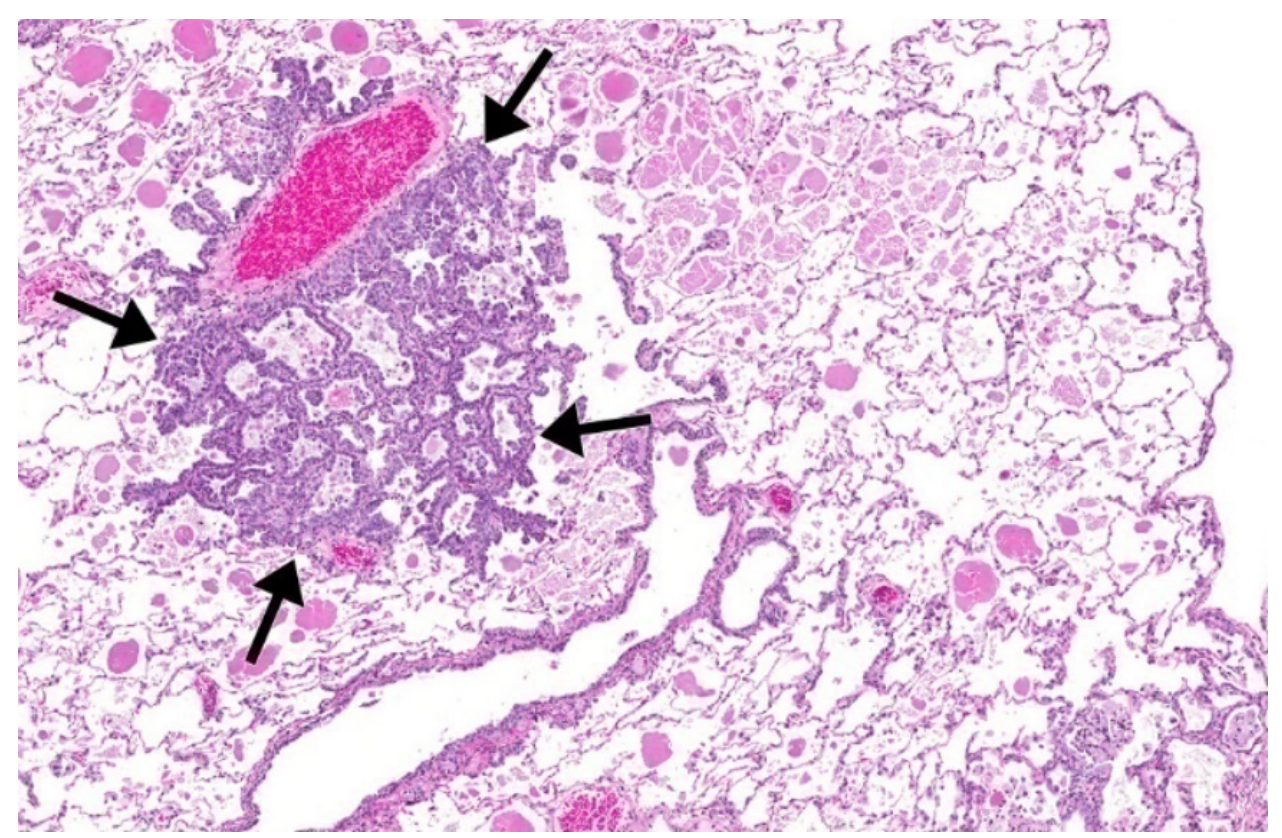

\section{Figure 11. Discrete, Focal Alveolar Epithelial Hyperplasia (Arrows) in the Lung of a Female F344/NTac Rat Exposed to 5 mg/m³ Cobalt Metal by Inhalation for Two Years (H\&E)}

Alveolar epithelial hyperplasia is characterized by proliferation of uniformly cuboidal alveolar epithelial (Type II) cells along the alveolar septa. The architecture of the alveolar parenchyma is generally maintained; however, the proliferation cells are forming papillary structures that project into the alveolar spaces. Note macrophages within the alveolar spaces. 


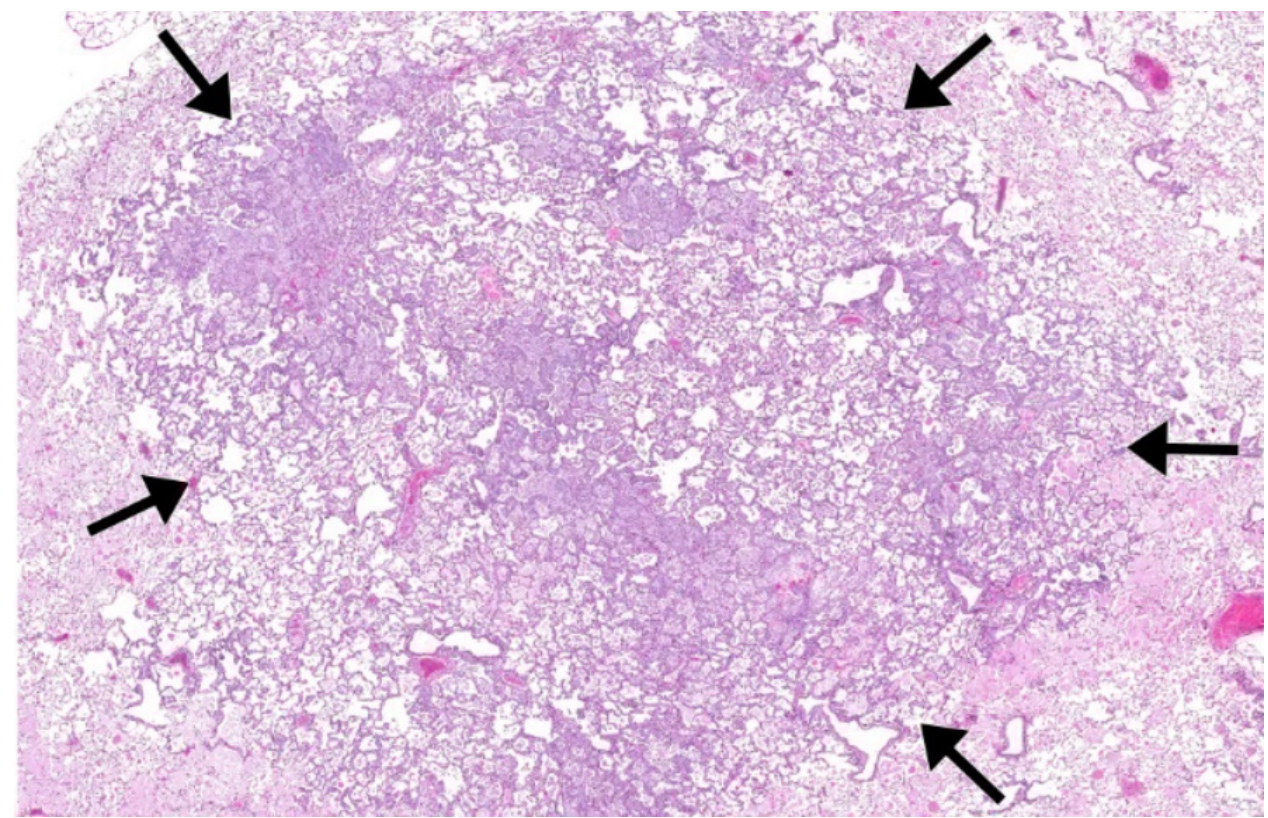

Figure 12. Focally Extensive Area of Alveolar Epithelial Hyperplasia (Arrows) in the Lung of a Female F344/NTac Rat Exposed to $5 \mathrm{mg} / \mathrm{m}^{3}$ Cobalt Metal by Inhalation for Two Years (H\&E)

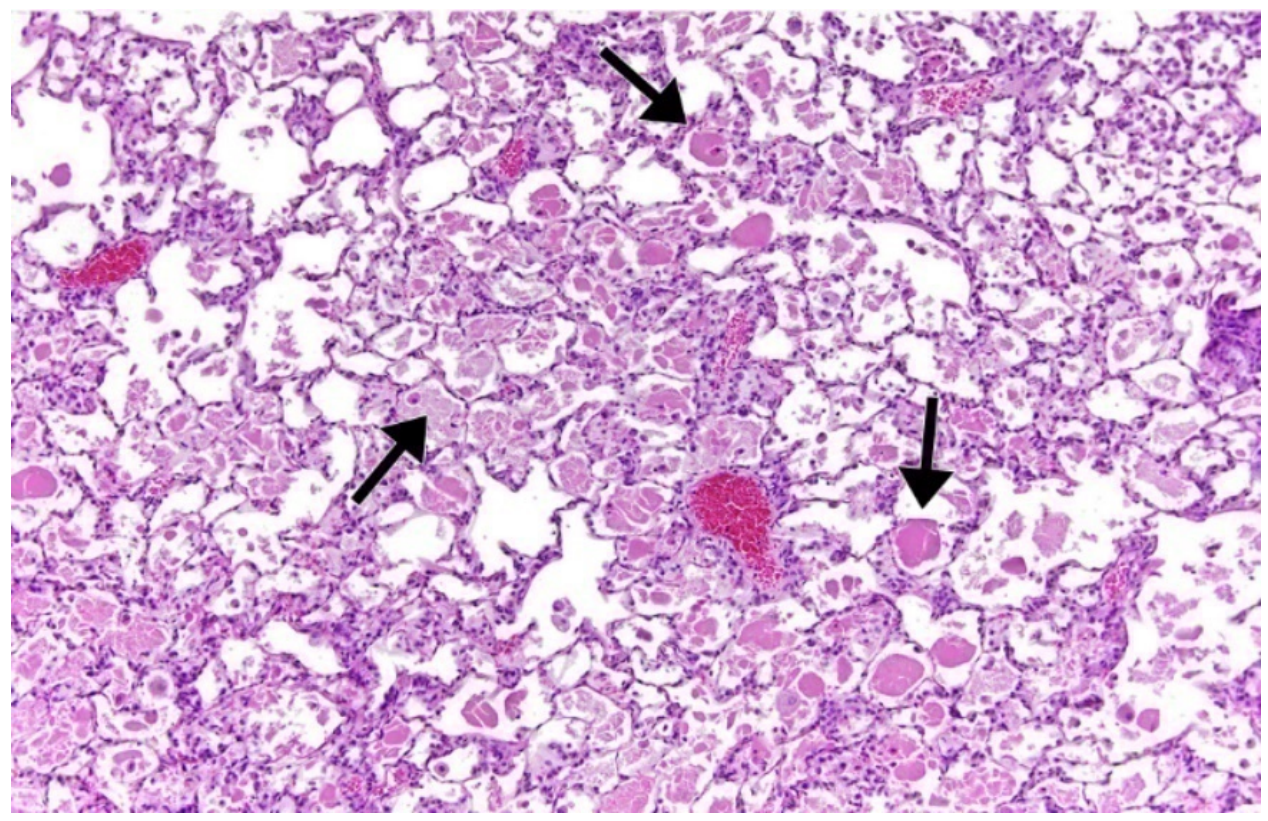

Figure 13. Alveolar Proteinosis in the Lung of a Male F344/NTac Rat Exposed to $5 \mathrm{mg} / \mathrm{m}^{3}$ Cobalt Metal by Inhalation for Two Years (H\&E)

Note the homogenously eosinophilic protein material (arrows) within the alveolar spaces some of which stains brightly eosinophilic. 


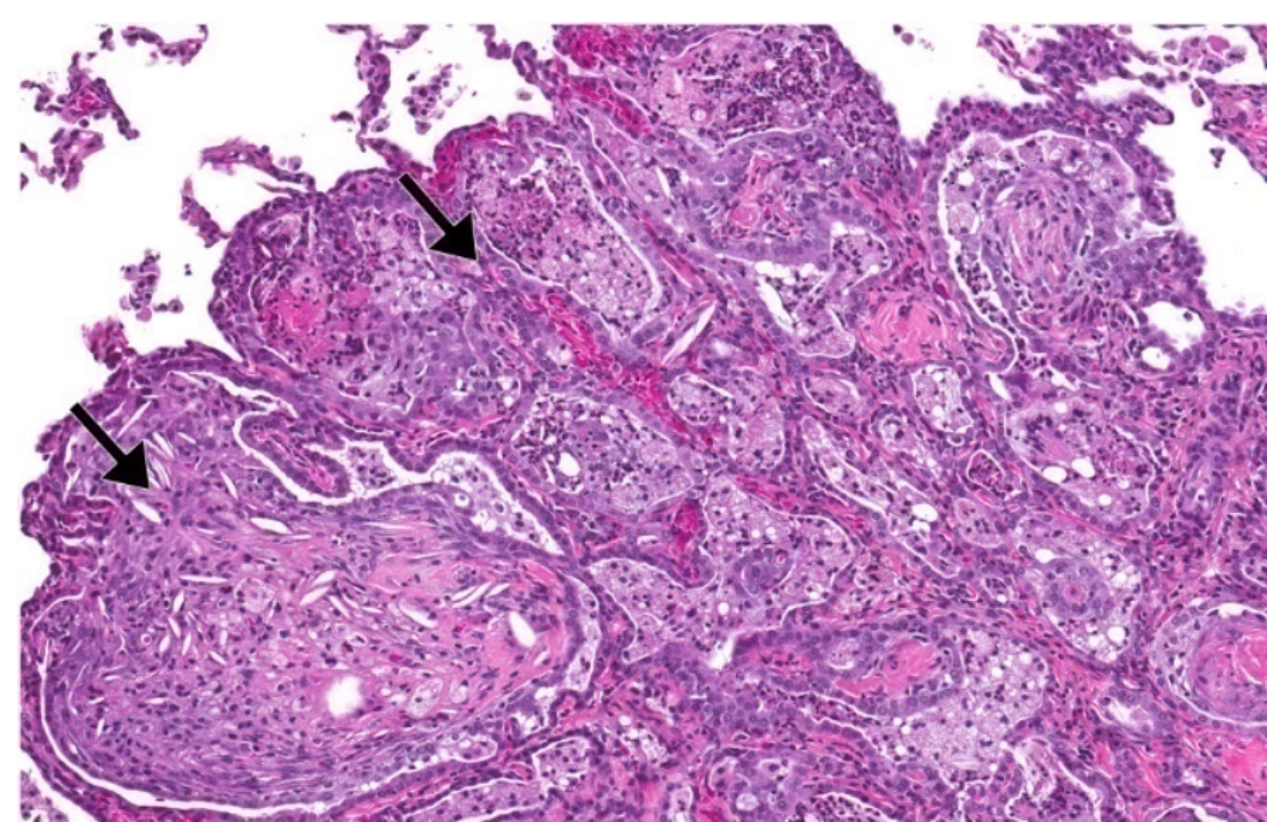

Figure 14. An Area of Chronic Active Inflammation (Arrows) in the Lung of a Male F344/NTac Rat Exposed to $5 \mathrm{mg} / \mathrm{m}^{3}$ Cobalt Metal by Inhalation for Two Years (H\&E)

The alveoli are filled with mostly macrophages, mixed with lesser numbers of neutrophils and degenerate cellular debris (arrows). Note clear angular cleft-like spaces (cholesterol clefts) among the inflammatory cells and debris. Proliferating alveolar epithelial cells line the alveoli.

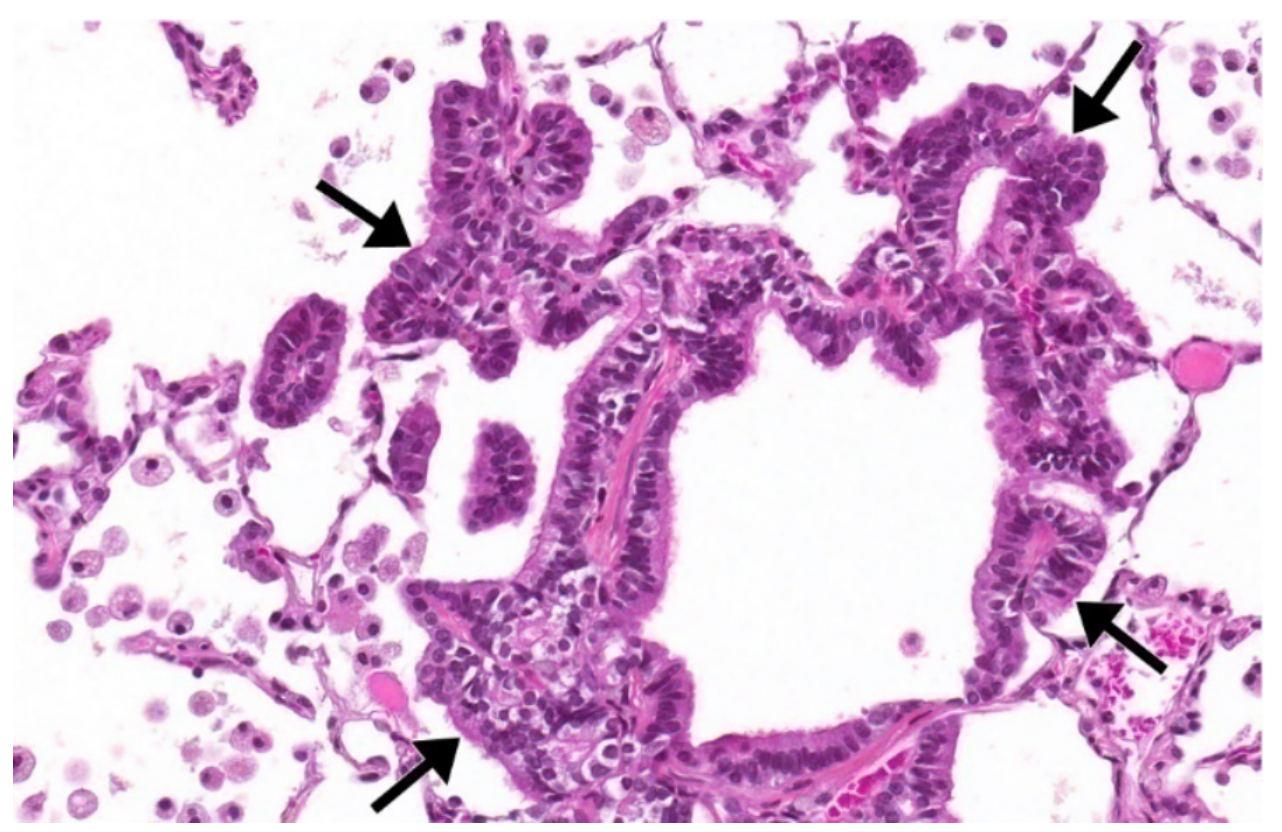

Figure 15. Bronchiolar Epithelial Hyperplasia in the Lung of a Female F344/NTac Rat Exposed to $5 \mathrm{mg} / \mathrm{m}^{3}$ Cobalt Metal by Inhalation for Two Years (H\&E)

Increased numbers of disorganized cuboidal to columnar epithelial cells (arrows), some of which are ciliated, line the terminal bronchiole. The proliferating cells have extended to line alveoli immediately adjacent to the bronchiole. Note macrophages within the alveoli. 


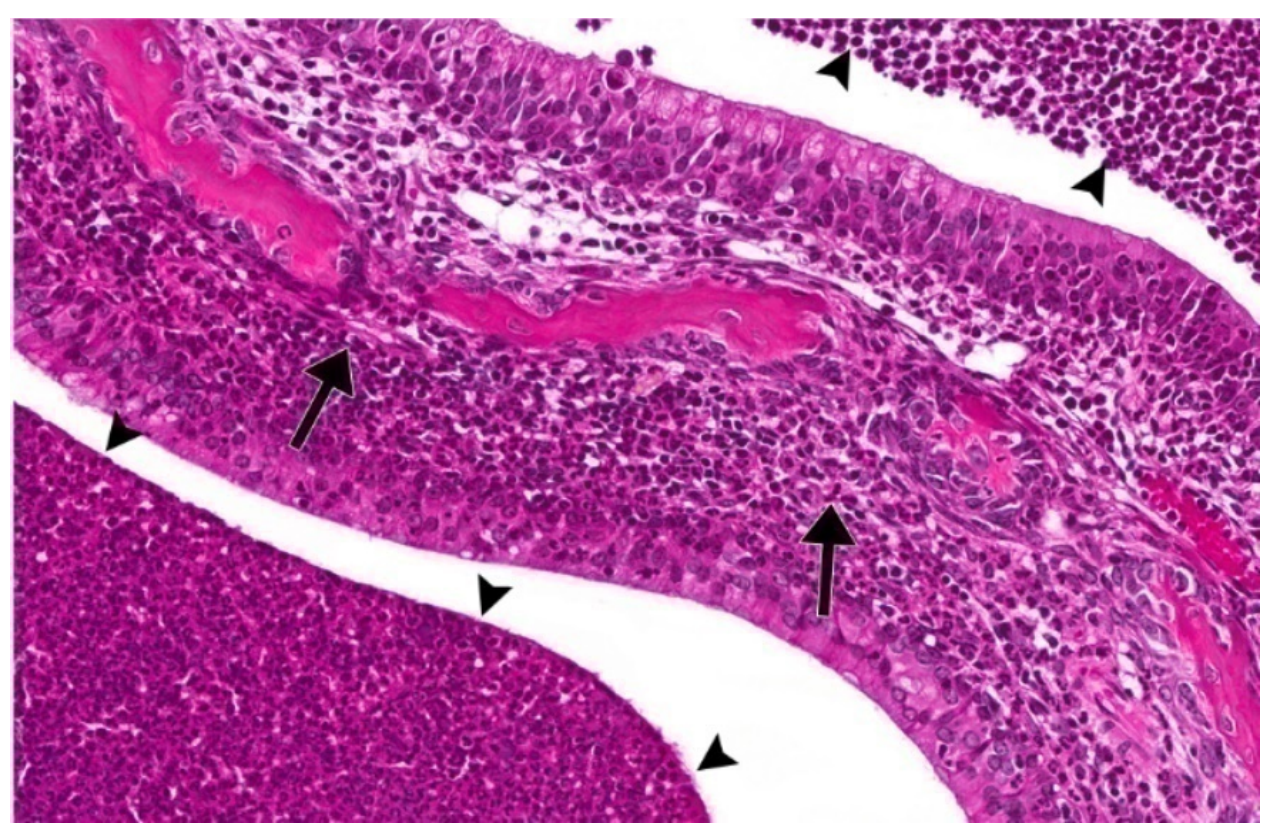

Figure 16. Suppurative Inflammation in a Nasal Maxilloturbinate in the Level II Section in the Nose of a Female F344/NTac Rat Exposed to $5 \mathrm{mg} / \mathrm{m}^{3}$ Cobalt Metal by Inhalation for Two Years (H\&E)

There are numerous neutrophils throughout the submucosal tissue of the turbinate (long arrows) and a purely neutrophilic exudate in the nasal passages (arrowheads).

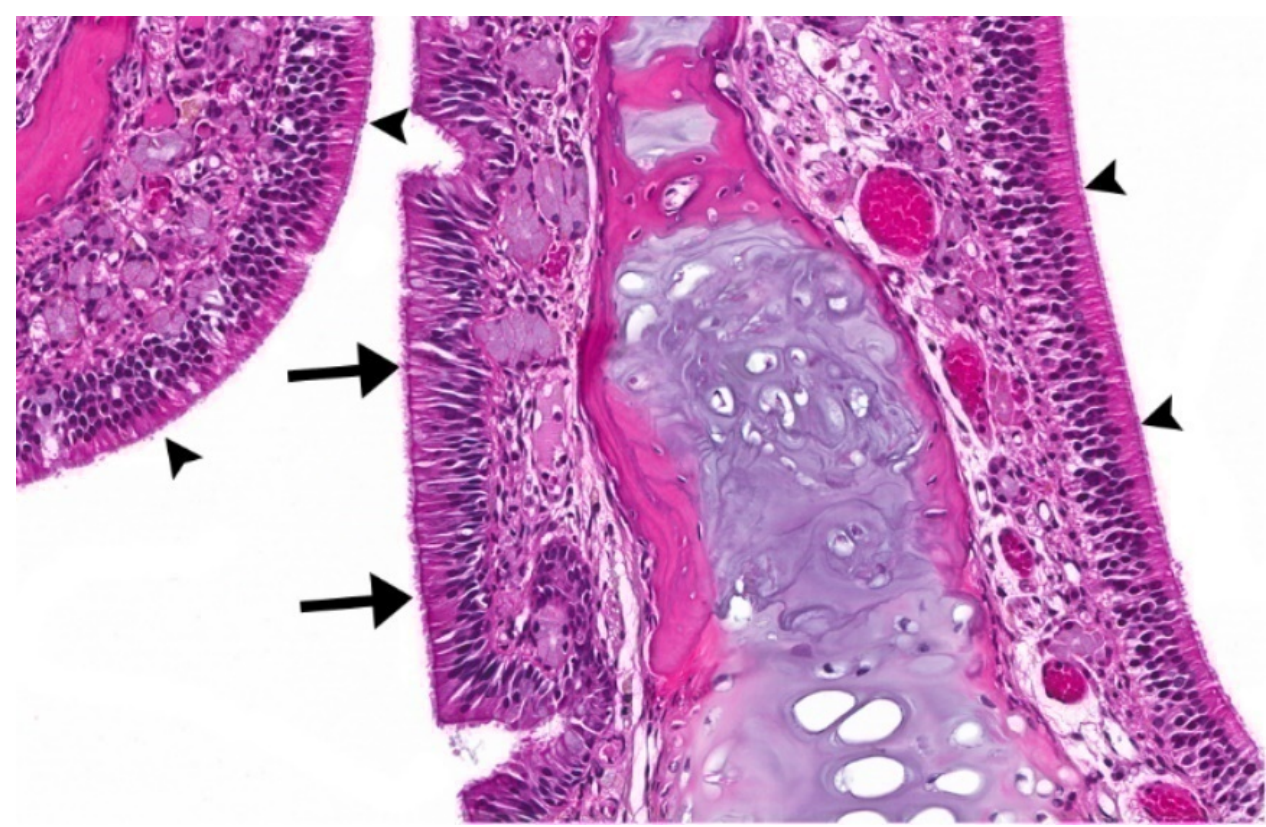

Figure 17. Respiratory Epithelial Metaplasia of the Olfactory Epithelium of an Ethmoid Turbinate in the Nose of a Female F344/NTac Rat Exposed to $5 \mathrm{mg} / \mathrm{m}^{3}$ Cobalt Metal by Inhalation for Two Years (H\&E)

The normal olfactory epithelium (arrowheads) is replaced by tall ciliated columnar epithelial cells (arrows) similar to respiratory epithelium that lines the naso- and maxilloturbinates. 


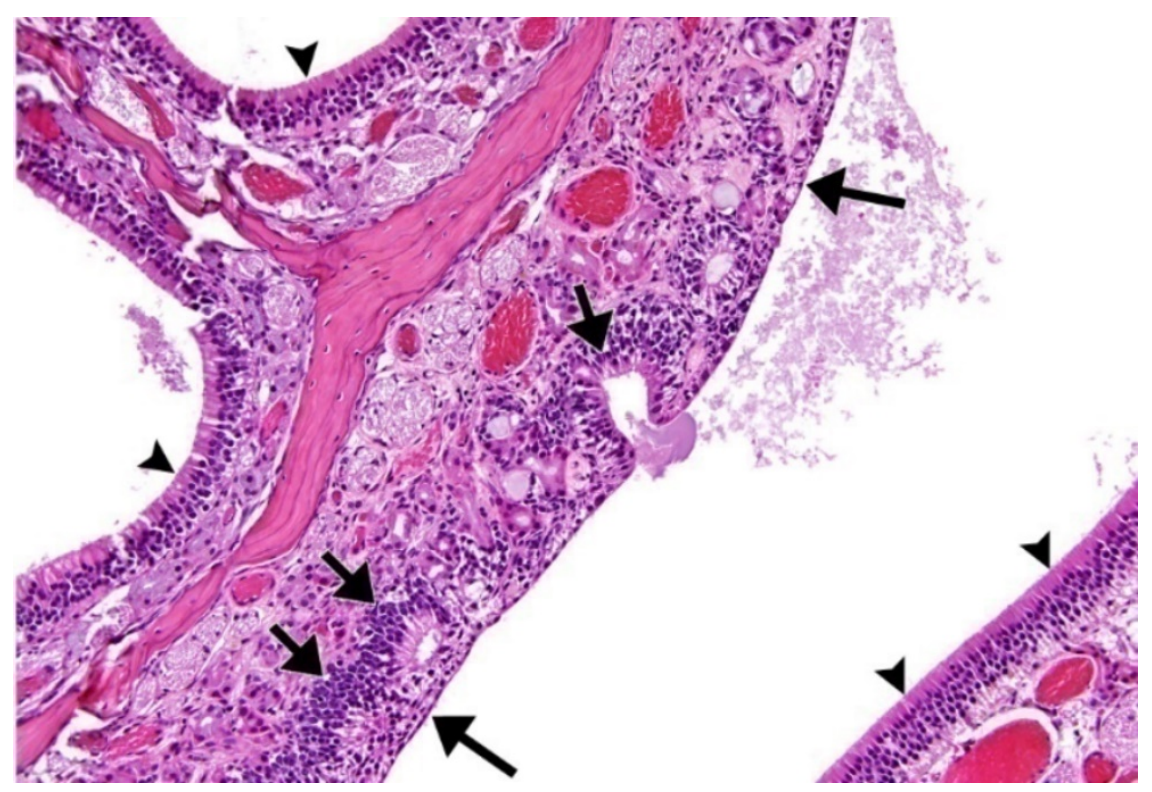

Figure 18. Olfactory Epithelial Atrophy and Olfactory Epithelial Hyperplasia in the Ethmoid Turbinate in the Nose of a Male F344/NTac Rat Exposed to $5 \mathrm{mg} / \mathrm{m}^{3}$ Cobalt Metal by Inhalation for Two Years (H\&E)

In contrast to the normal olfactory epithelium (arrowheads), the atrophic epithelium is attenuated (long arrows). Note hyperplastic olfactory epithelium forming rosette-like structures along the lower margins of the atrophic epithelium (short arrows).

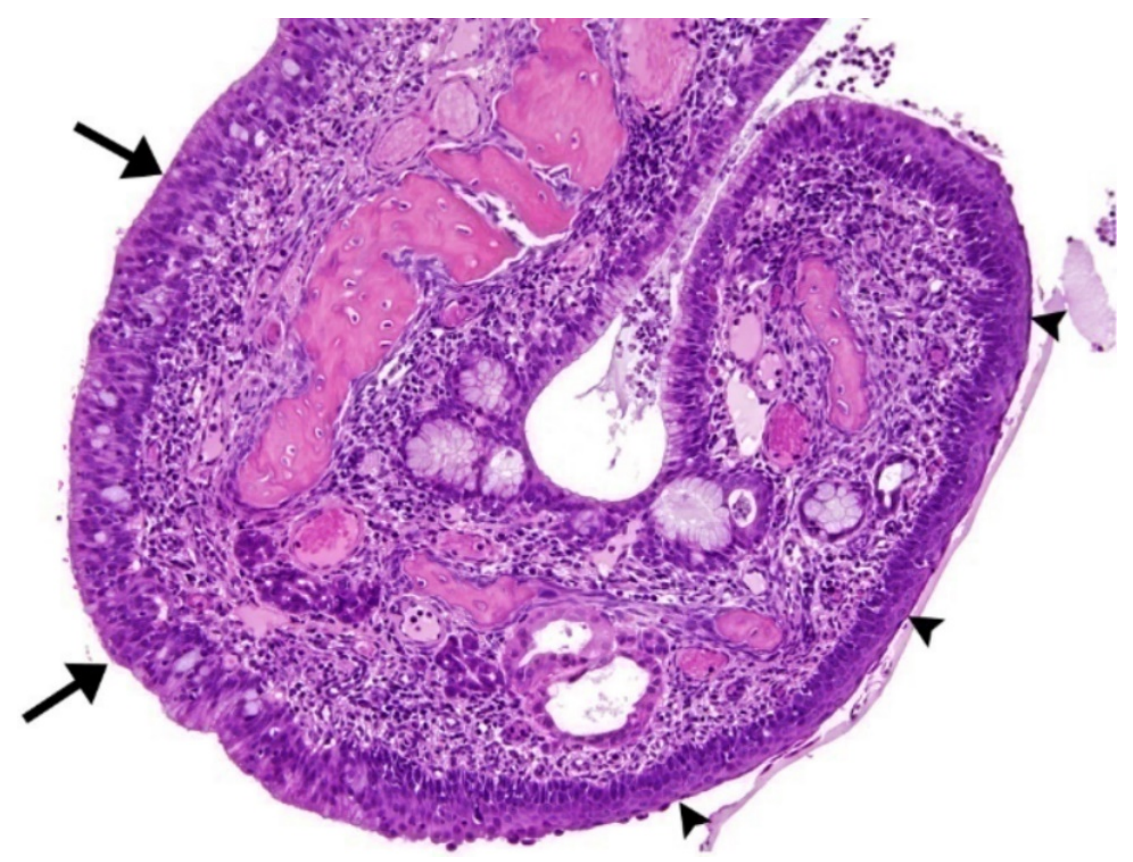

Figure 19. Respiratory Epithelial Hyperplasia and Squamous Metaplasia in a Maxilloturbinate in the Nose of a Male F344/NTac Rat Exposed to $5 \mathrm{mg} / \mathrm{m}^{3}$ Cobalt Metal by Inhalation for Two Years (H\&E)

The normal single layer of tall ciliated columnar epithelial cells is replaced by multiple disorganized layers of proliferating epithelial cells (arrows). Squamous metaplasia is characterized by replacement of the normal respiratory epithelium by flattened squamous epithelial cells (arrowheads). There is chronic active inflammation throughout the submucosal tissue. 


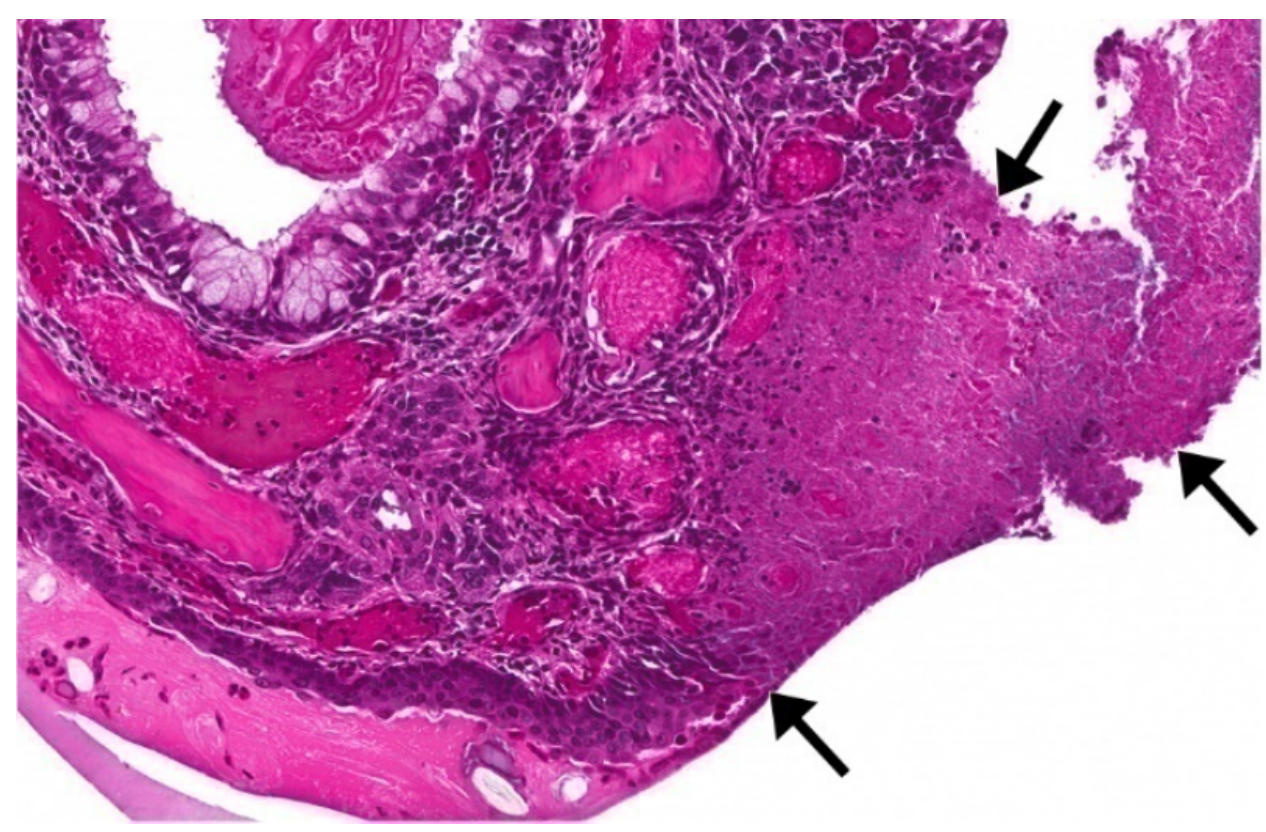

Figure 20. Necrosis of the Respiratory Epithelium in a Maxilloturbinate in the Nose of a Female F344/NTac Rat Exposed to $5 \mathrm{mg} / \mathrm{m}^{3}$ Cobalt Metal by Inhalation for Two Years (H\&E)

The epithelium is replaced by a coagulum of necrotic epithelial cells and cellular debris (arrows).

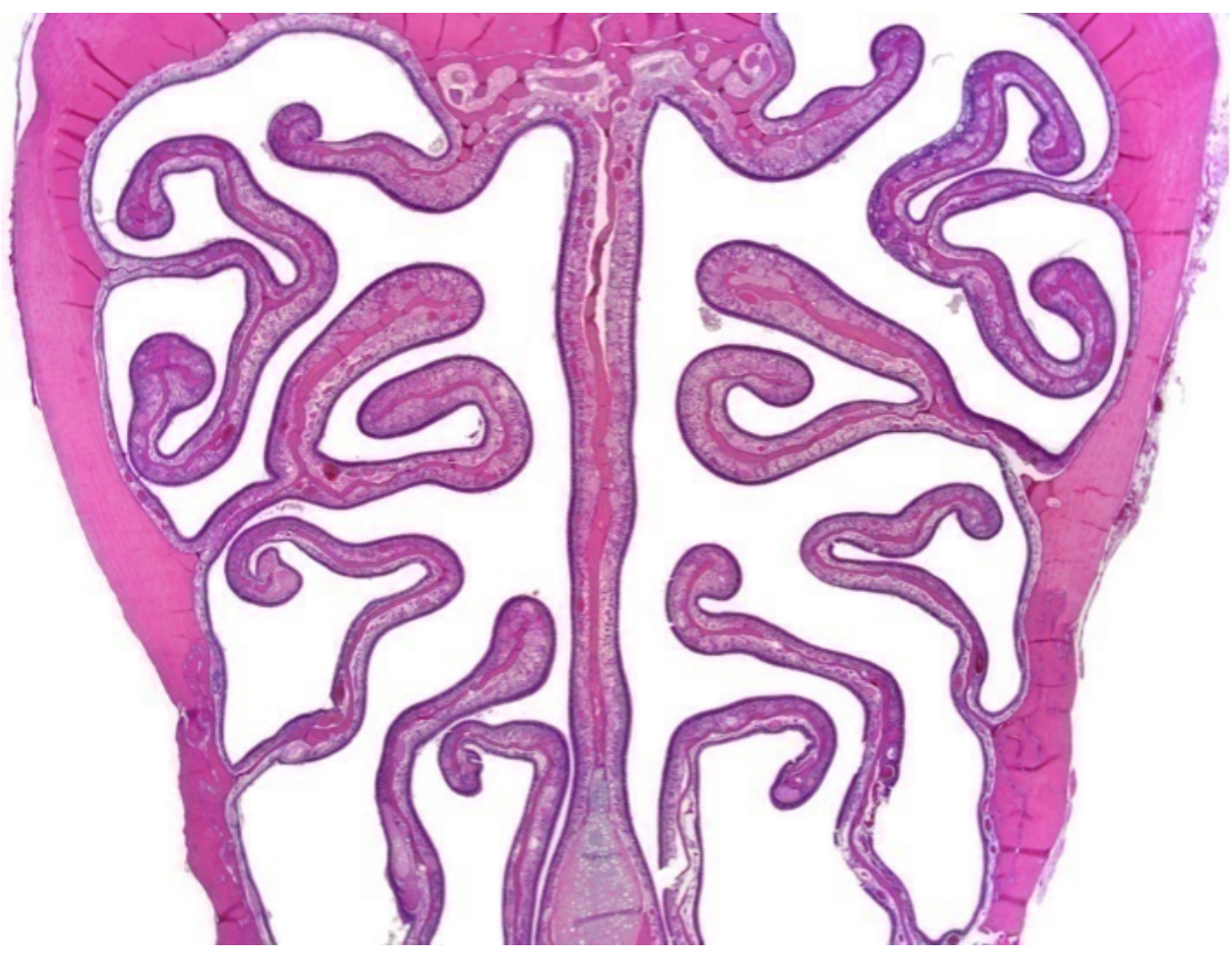

Figure 21. Atrophy of the Ethmoid Turbinates in the Nose of a Male F344/NTac Rat Exposed to $5 \mathrm{mg} / \mathrm{m}^{3}$ Cobalt Metal by Inhalation for Two Years (H\&E)

The turbinate scrolls are short, thin, and somewhat blunted, and there is increased space within the nasal passages. 


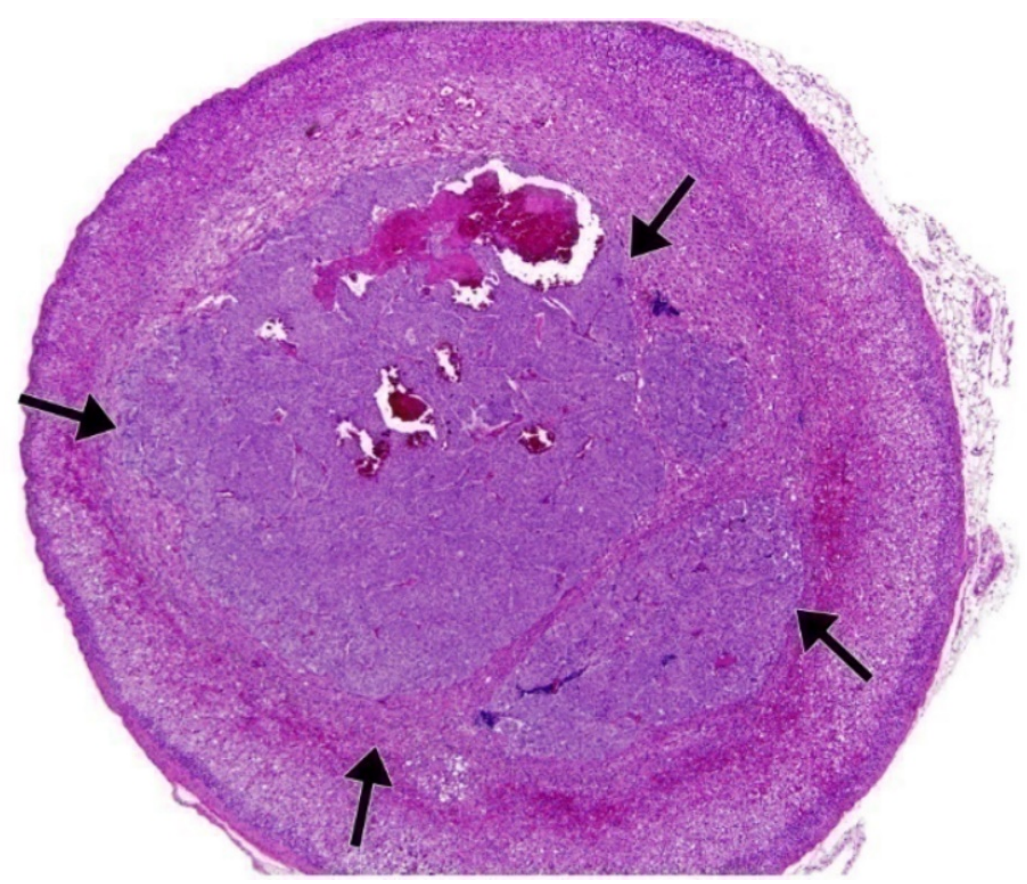

Figure 22. Benign Pheochromocytoma in the Adrenal Medulla of a Male F344/NTac Rat Exposed to $5 \mathrm{mg} / \mathrm{m}^{3}$ Cobalt Metal by Inhalation for Two Years (H\&E)

The neoplasm is well-circumscribed and expansive but still within the confines of the adrenal medulla (arrows).

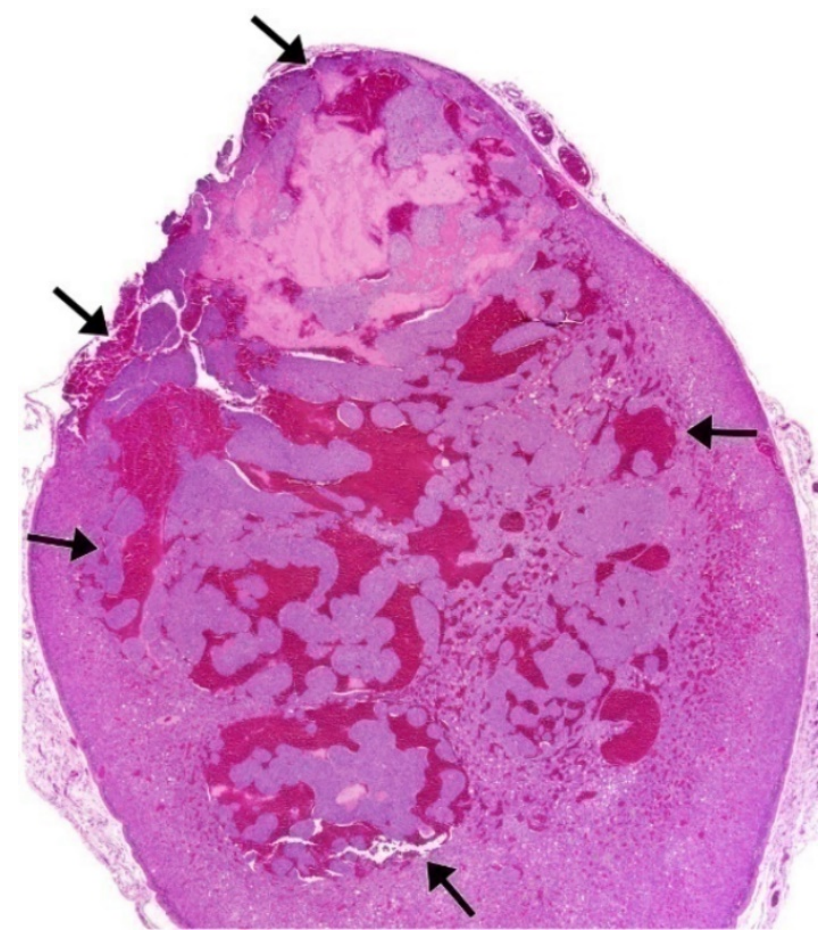

Figure 23. Malignant Pheochromocytoma in the Adrenal Medulla of a Male F344/NTac Rat Exposed to $5 \mathrm{mg} / \mathrm{m}^{3}$ Cobalt Metal by Inhalation for Two Years (H\&E)

The neoplasm (arrows) is expansive, has replaced the adrenal medulla, and has invaded into and through the adrenal cortex. 


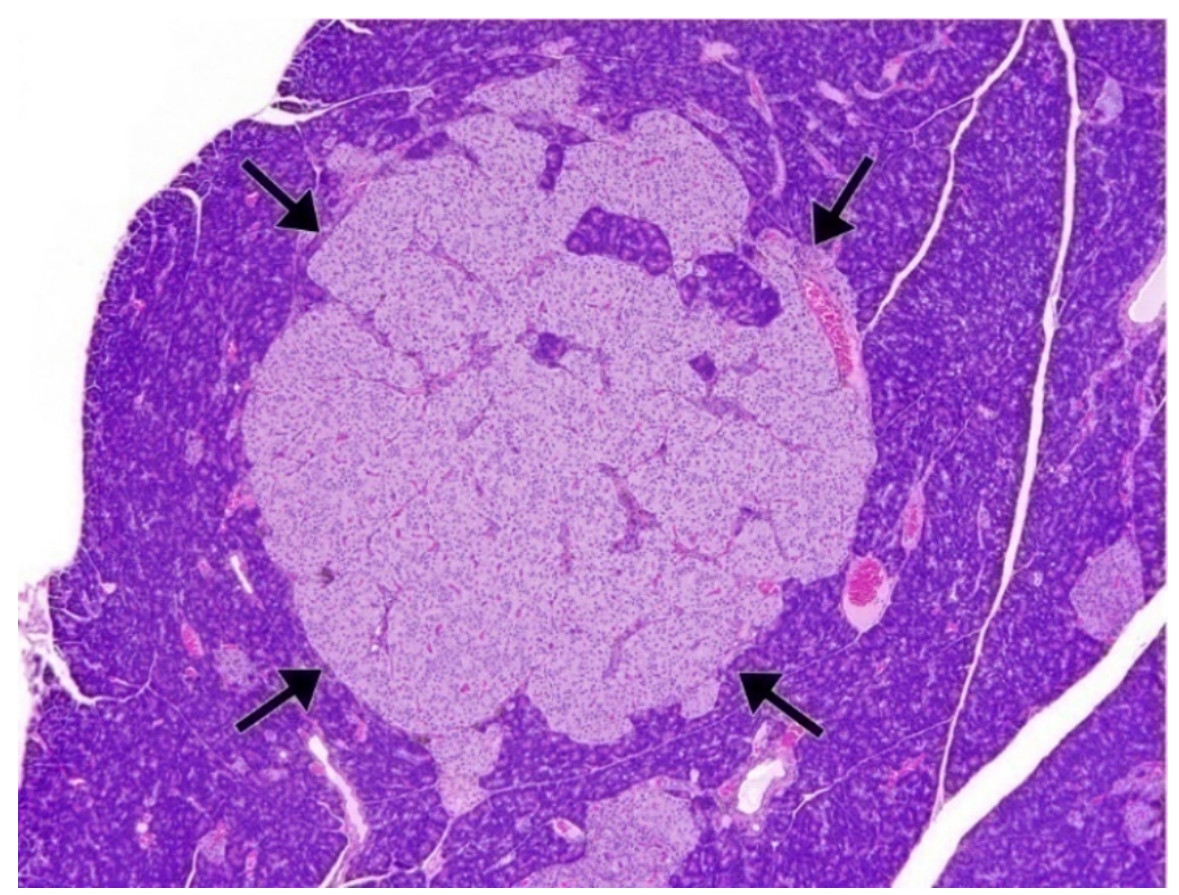

Figure 24. Islet Cell Adenoma in the Pancreas of a Male F344/NTac Rat Exposed to $5 \mathrm{mg} / \mathrm{m}^{3}$ Cobalt Metal by Inhalation for Two Years (H\&E)

The adenoma (arrows) is well-circumscribed and expansive and composed of uniformly normal islet cells partially separated into variably sized packets by a delicate fibrovascular stroma.

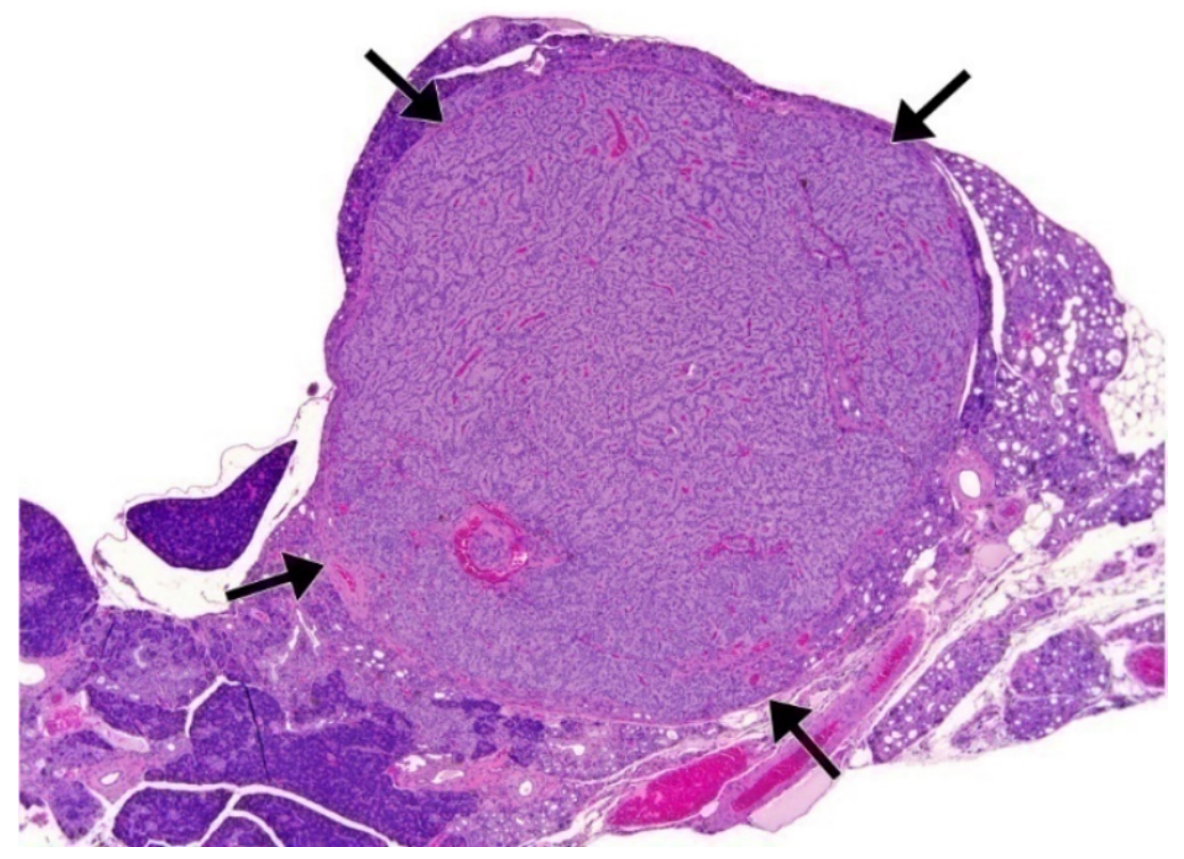

Figure 25. Islet Cell Carcinoma in the Pancreas of a Male F344/NTac Rat Exposed to $5 \mathrm{mg} / \mathrm{m}^{3}$ Cobalt Metal by Inhalation for Two Years (H\&E)

The carcinoma (arrows) has almost completely replaced the pancreas and invaded adjacent tissue. 


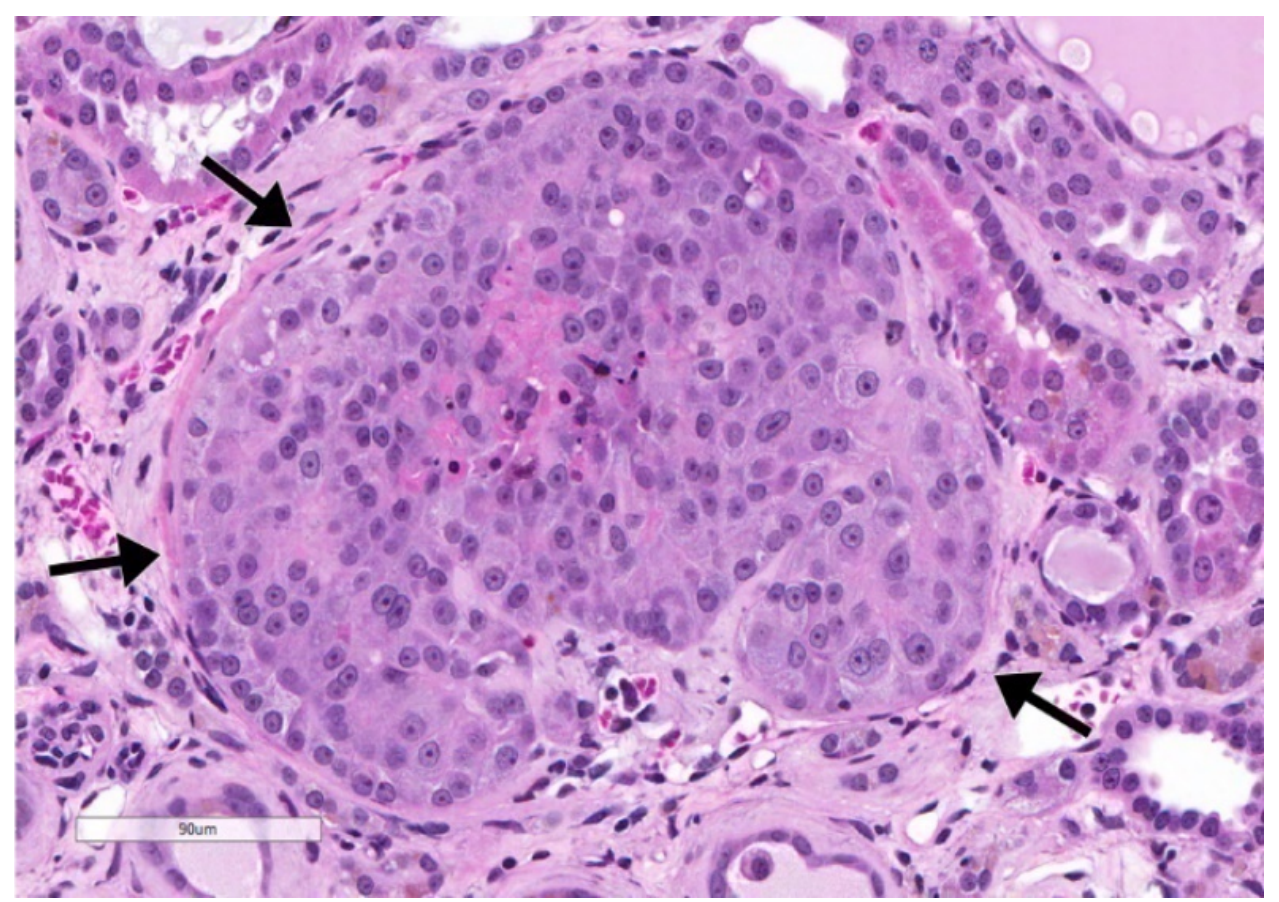

Figure 26. Renal Tubule Adenoma in the Kidney of a Male F344/NTac Rat Exposed to $5 \mathrm{mg} / \mathrm{m}^{3}$ Cobalt Metal by Inhalation for Two Years (H\&E)

The adenoma (arrows) is well-circumscribed and consists of relatively uniform large cells with a granular to glassy cytoplasm.

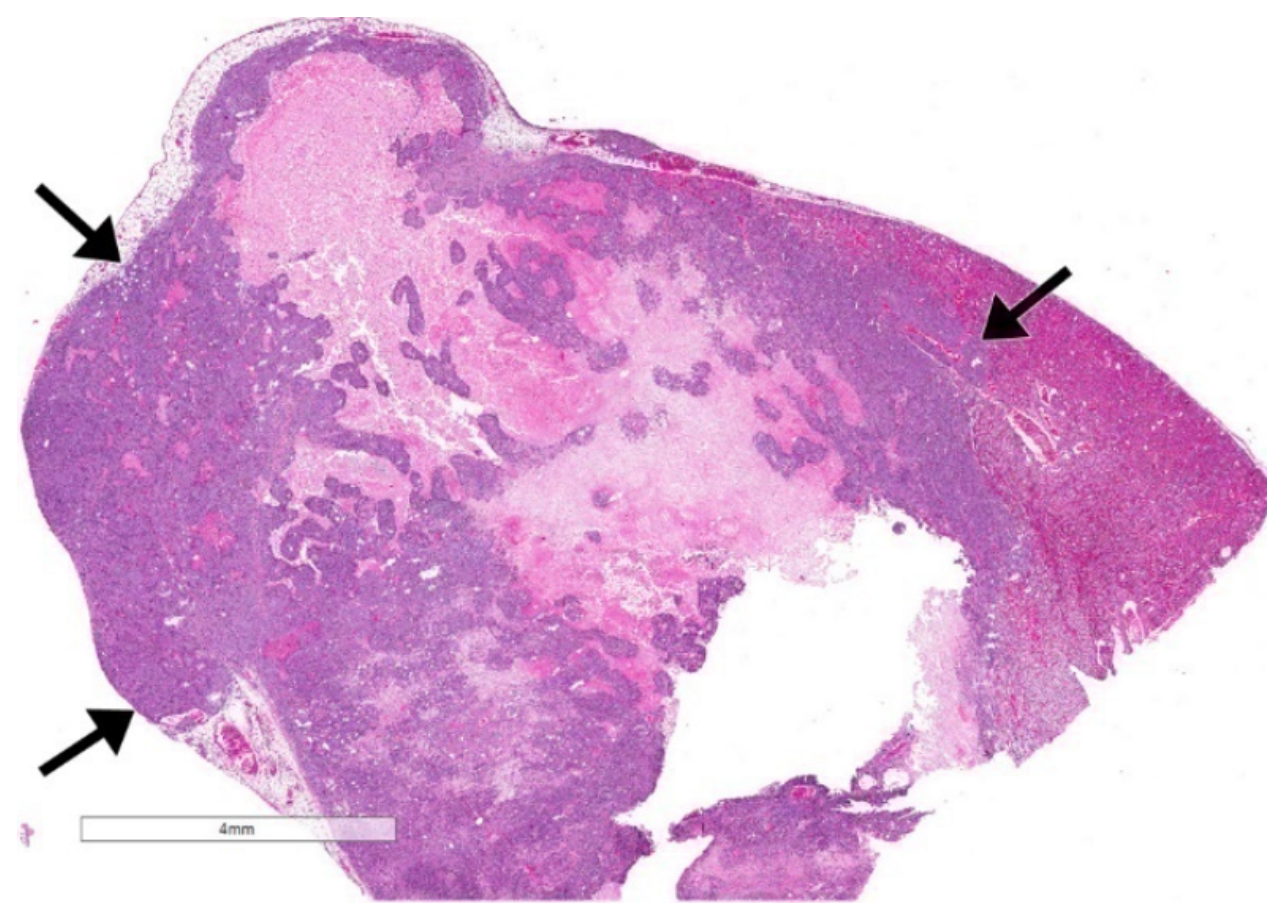

Figure 27. Renal Tubule Carcinoma in the Kidney of a Male F344/NTac Rat Exposed to 5 mg/m ${ }^{3}$ Cobalt Metal by Inhalation for Two Years (H\&E)

The carcinoma (arrows) is invasive and has replaced much of the renal parenchyma. 

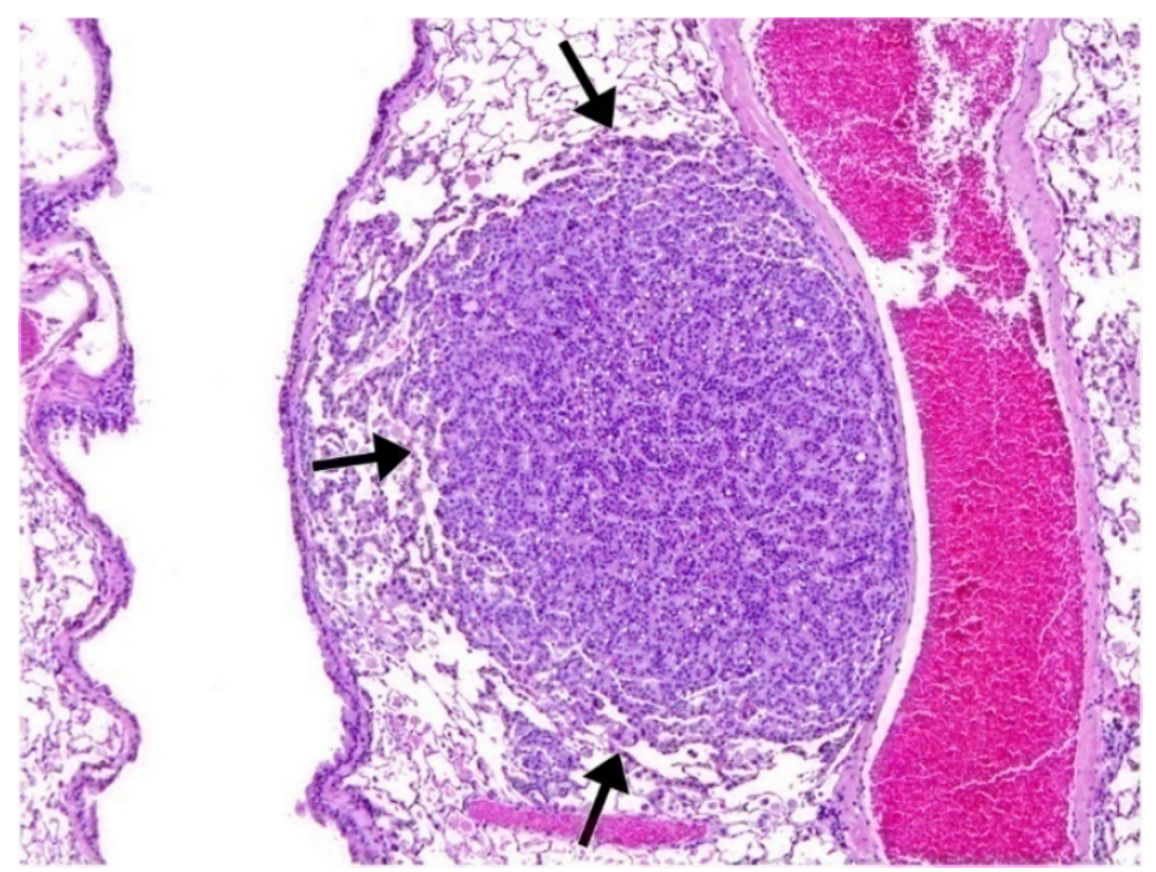

Figure 28. Low Magnification of an Alveolar/Bronchiolar Adenoma in the Lung of a Male B6C3F1/N Mouse Exposed to $2.5 \mathrm{mg} / \mathrm{m}^{3}$ Cobalt Metal by Inhalation for Two Years (H\&E)

The adenoma (arrows) has distinctly demarcated from the surrounding alveolar parenchyma and is composed of uniformly cuboidal cells arranged as papillary structures.

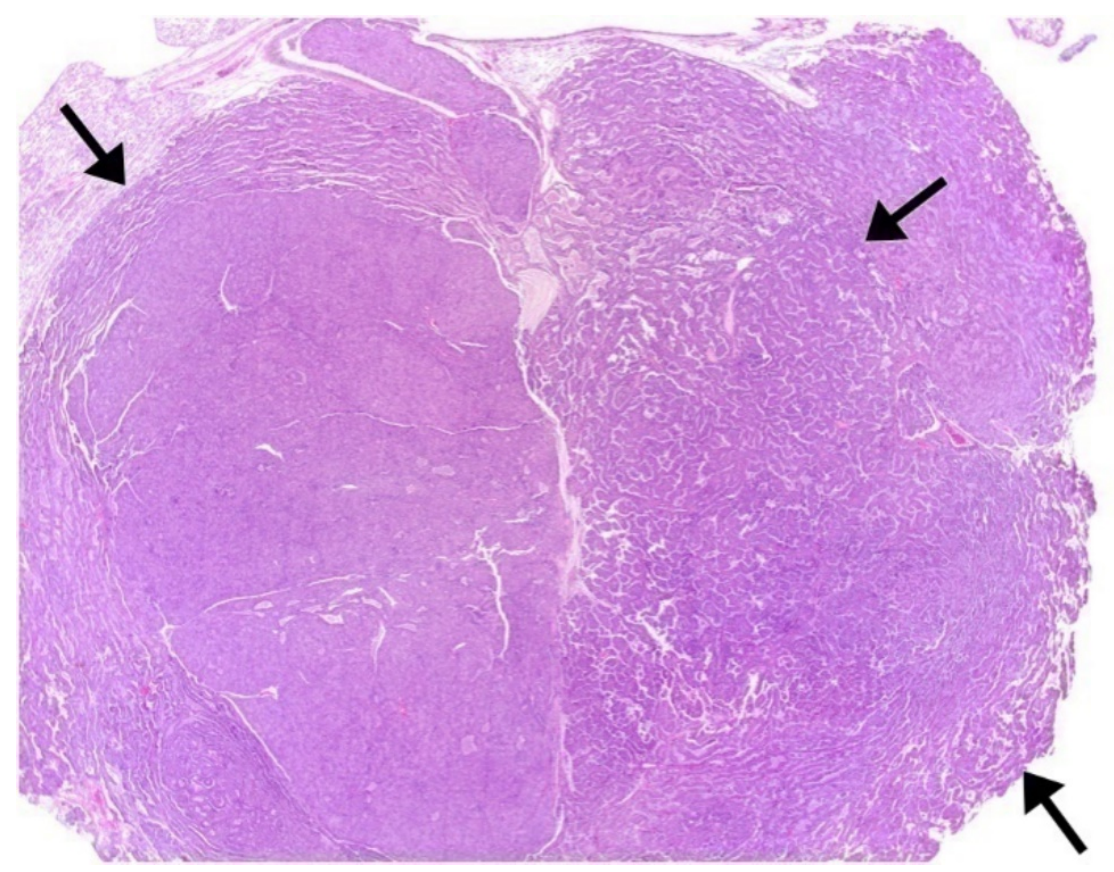

Figure 29. Low Magnification of an Alveolar/Bronchiolar Carcinoma in the Lung of a Male B6C3F1/N Mouse Exposed to $5 \mathrm{mg} / \mathrm{m}^{3}$ Cobalt Metal by Inhalation for Two Years (H\&E)

The carcinoma (arrows) has invaded and almost completely effaced the lung lobe. Note the pleomorphic appearance of the neoplasm. 


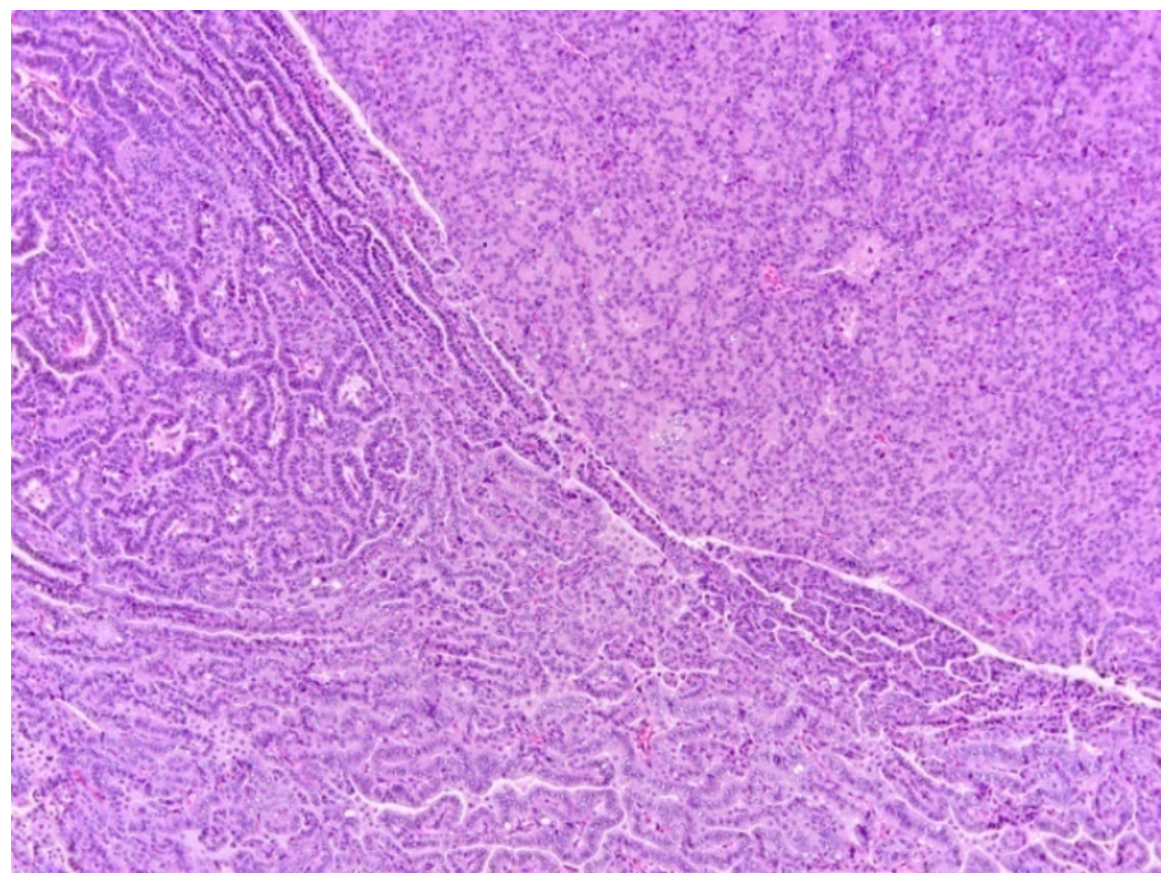

Figure 30. Higher Magnification of Figure 29 (H\&E)

Note the pleomorphic arrangement of the neoplastic cells that form poorly and well-defined papillary structures composed of cuboidal to columnar cells in one area of the neoplasm and in a solid sheet in another area.

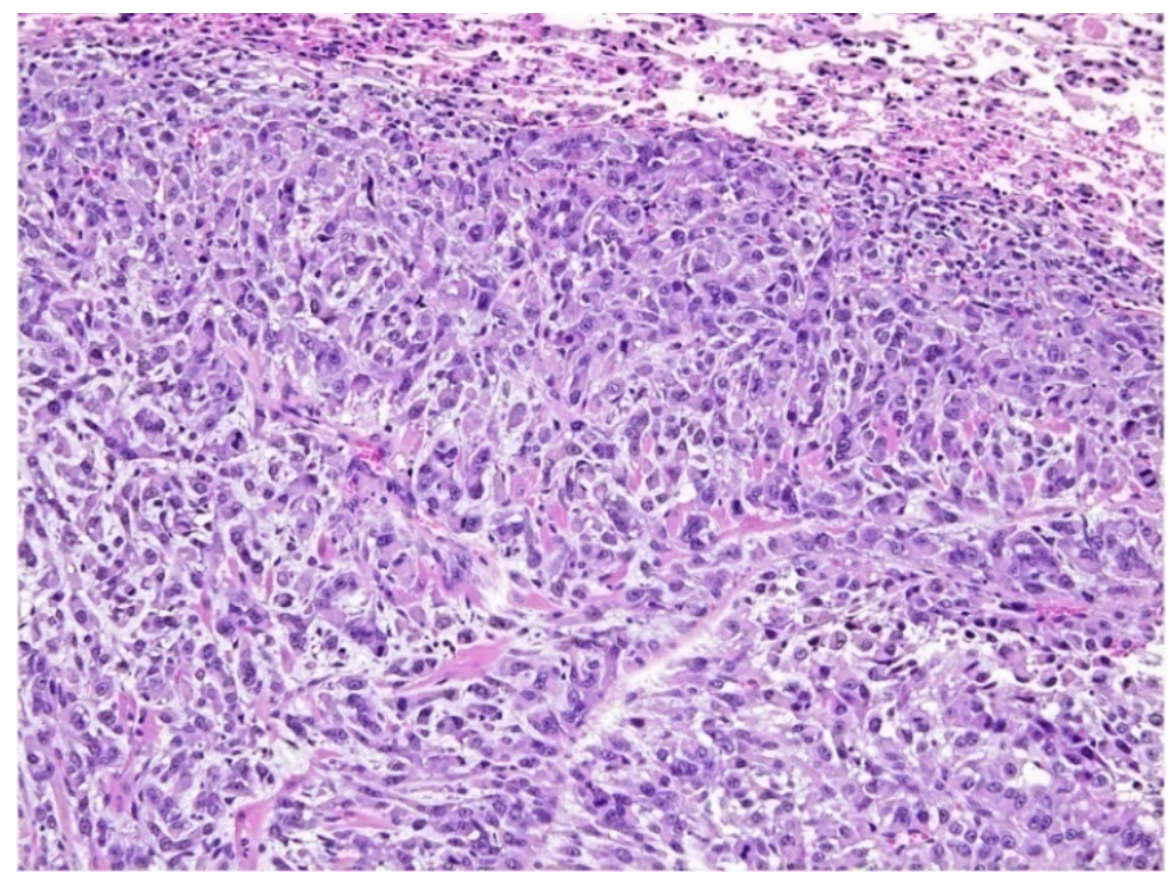

\section{Figure 31. Alveolar/Bronchiolar Carcinoma in the Lung of a Female B6C3F1/N Mouse Exposed to $5 \mathrm{mg} / \mathrm{m}^{3}$ Cobalt Metal by Inhalation for Two Years (H\&E)}

Note the highly anaplastic nature of this carcinoma with marked variation in cellular and size and shape, pleomorphic sometimes bizarre and multilobulated nuclei and numerous mitotic figures. 


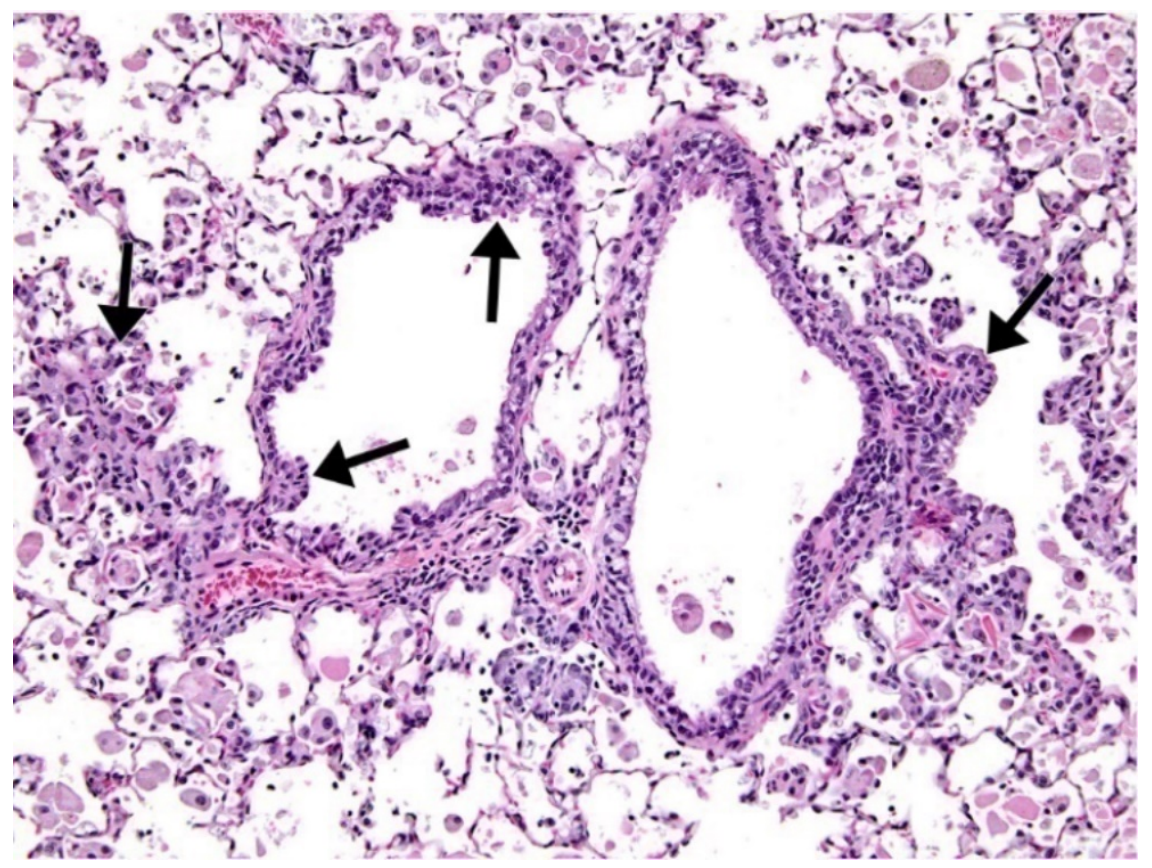

Figure 32. Alveolar/Bronchiolar Epithelial Hyperplasia in the Lung of a Female B6C3F1/N Mouse Exposed to $5 \mathrm{mg} / \mathrm{m}^{3}$ Cobalt Metal by Inhalation for Two Years (H\&E)

There is disorganized proliferation and piling up of the epithelial cells with extension of the proliferating cells along the immediately adjacent alveolar septae (arrows). Note numerous macrophages and proteinaceous material within the alveoli.

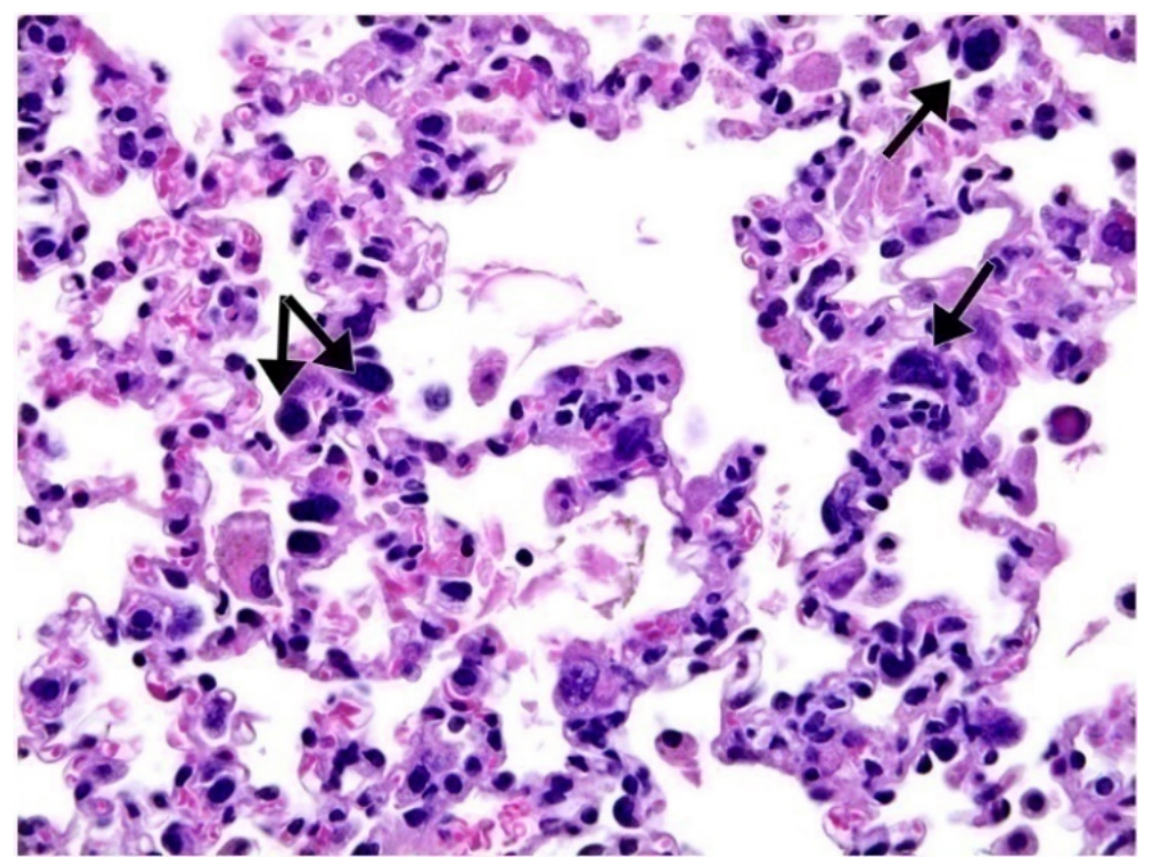

Figure 33. Focal Alveolar Epithelial Hyperplasia in the Lung of a Male B6C3F1/N Mouse Exposed to $2.5 \mathrm{mg} / \mathrm{m}^{3}$ Cobalt Metal by Inhalation for Two Years (H\&E)

The alveolar septa are lined by cuboidal to polygonal alveolar epithelial (Type II) cells; the alveolar architecture is generally maintained. Note epithelial cells with large (karyomegaly) pleomorphic nuclei (arrows). 


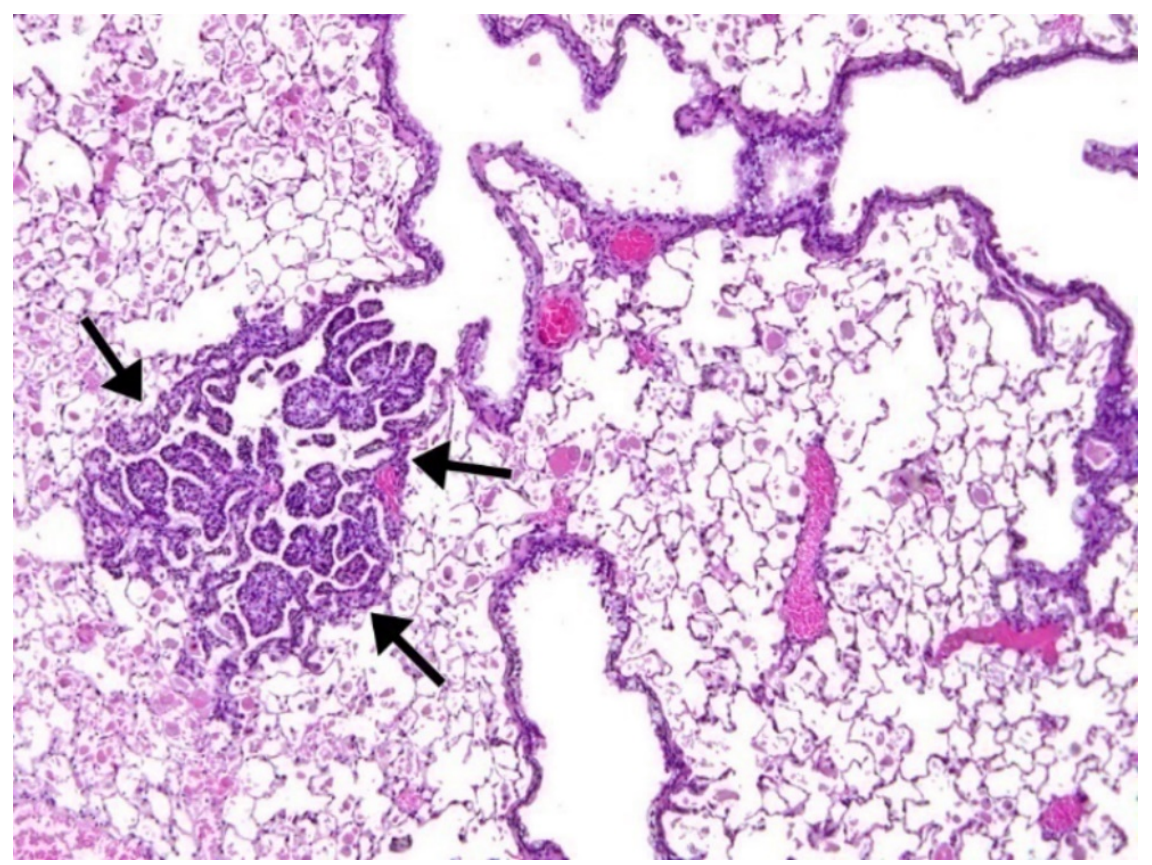

Figure 34. Focal Bronchiolar Epithelial Hyperplasia in a Terminal Bronchiole (Arrows) of a Male B6C3F1/N Mouse Exposed to $5 \mathrm{mg} / \mathrm{m}^{3}$ Cobalt Metal by Inhalation for Two Years (H\&E)

The hyperplastic epithelium (arrows) forms multiple papillary structures that project into the lumen and are lined by cuboidal to low columnar epithelial cells and supported by scant amounts of fibrovascular stroma.

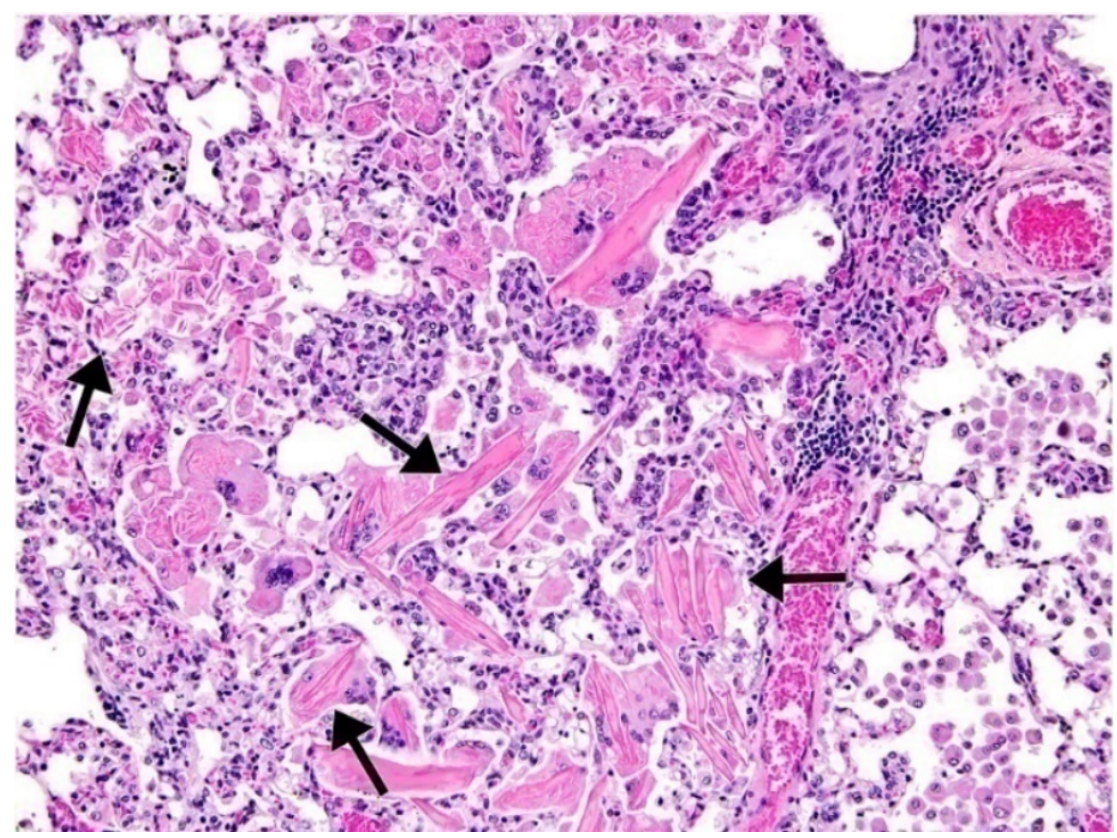

Figure 35. Alveolar Histiocytic Infiltrates in the Lung of a Male B6C3F1/N Mouse Exposed to $5 \mathrm{mg} / \mathrm{m}^{3}$ Cobalt Metal by Inhalation for Two Years (H\&E)

Alveoli contain numerous macrophages many of which are multinucleated and swollen with protein and slender needle-like crystalline material (arrows). Note larger crystals free within the alveoli. Note low numbers of neutrophils and lymphocytes within the alveoli. 


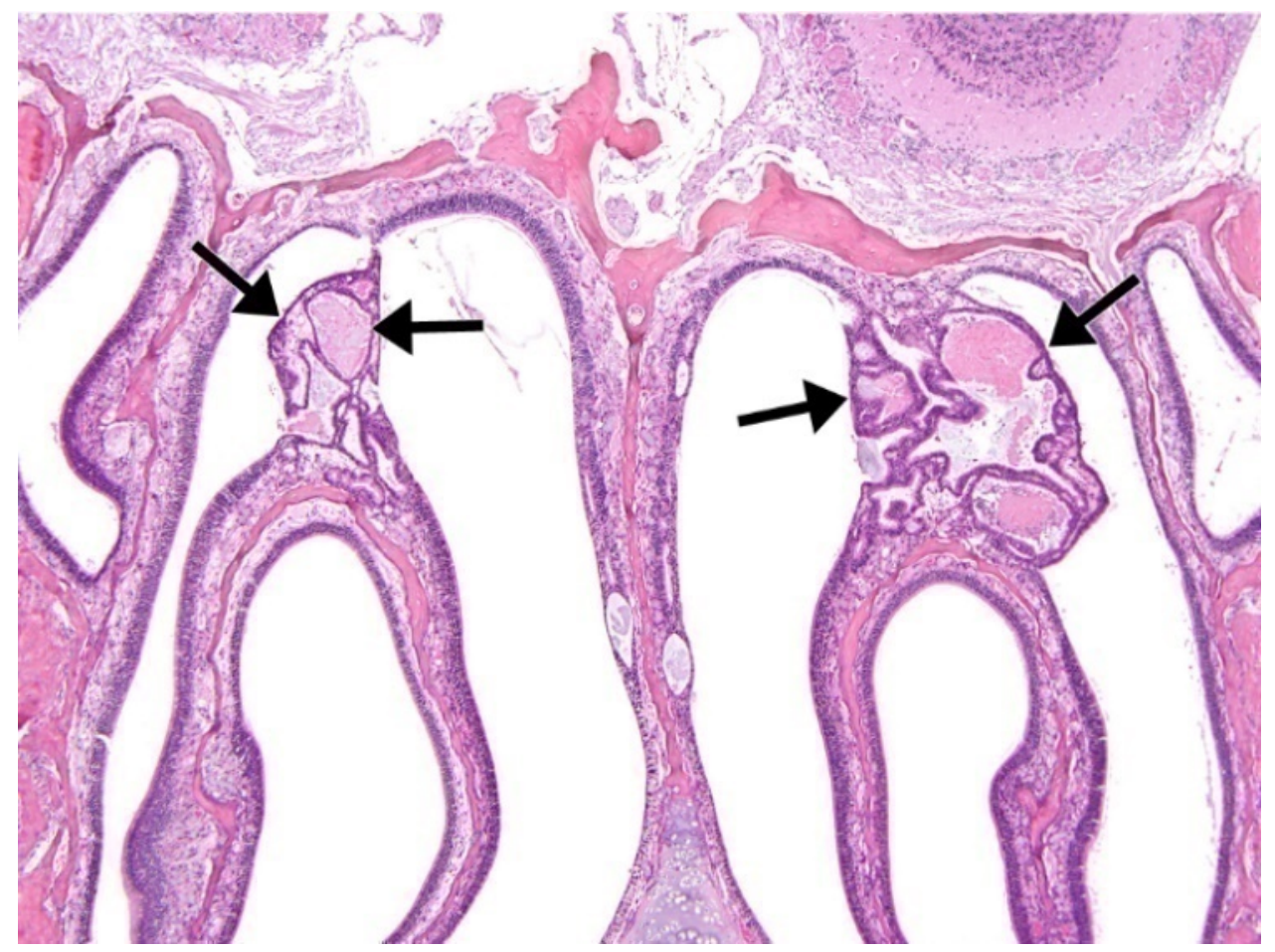

Figure 36. Bilateral Atypical Respiratory Epithelial Metaplasia in the Olfactory Epithelium of a Male B6C3F1/N Mouse Exposed to $1.25 \mathrm{mg} / \mathrm{m}^{3}$ Cobalt Metal by Inhalation for Two Years (H\&E)

There is exophytic proliferation of tall ciliated columnar epithelial cells from the dorsal surface of the dorsal scrolls of the ethmoid turbinates with synechia formation between the turbinate scroll and the epithelium lining the dorsal meatus (arrows). 


\section{Discussion}

Cobalt metal was nominated by the International Union of United Auto Workers and the Cobalt Development Institute for toxicology and carcinogenesis studies based on widespread occupational exposure, the occurrence of hard metal disease, and limited toxicity data. The NTP previously assessed the toxicity and carcinogenicity of a soluble cobalt compound, cobalt sulfate heptahydrate, in experimental animals exposed by inhalation ${ }^{66 ; 68 ; 122}$. Findings from those studies showed lung cancer in rats and mice, thereby raising concerns regarding the carcinogenic potential of cobalt metal dust. Hence, NTP conducted studies on cobalt metal to obtain its toxicity and carcinogenicity profile and to compare and contrast the toxicity of insoluble cobalt metal with a soluble cobalt salt. This Technical Report presents the findings and conclusions of the 2-week, 3-month, and 2-year inhalation studies of rats and mice exposed to cobalt metal.

The exposure concentrations for the 2-week studies were estimated based on exposures of previously studied cobalt sulfate heptahydrate ${ }^{66}$. In the current study, there was significant mortality in male and female rats exposed to 20 and $40 \mathrm{mg} / \mathrm{m}^{3}$ and body weight reductions in the $10 \mathrm{mg} / \mathrm{m}^{3}$ groups coupled with reduced urine volumes and concomitant increases in urine creatinine at the end of 2 weeks. Hence, $5 \mathrm{mg} / \mathrm{m}^{3}$ was selected as the highest exposure concentration for rats in the 3-month studies. In mice, there was significant mortality in males and females exposed to $40 \mathrm{mg} / \mathrm{m}^{3}$ and significant decreases in body weights in the $20 \mathrm{mg} / \mathrm{m}^{3}$ groups. Based on this, $10 \mathrm{mg} / \mathrm{m}^{3}$ was selected as the highest exposure concentration for mice in the 3-month study. Because the exposure concentrations in the mouse study were different from those in the rat study, only four concentrations were used in the rat study in order to optimize the number of inhalation chambers used in the studies.

In the 3-month studies, there was no exposure-related mortality in rats or mice. Consistent with the results of the 2-week studies, the respiratory tract was the primary site of toxicity in both rats and mice. In the lung, nonneoplastic lesions including chronic active lung inflammation, minimal to mild alveolar proteinosis, and minimal to moderate bronchiole epithelium hyperplasia generally occurred in both males and females. In the nose, there were indications of olfactory epithelium degeneration (rats and mice) and minimal to mild respiratory epithelium hyperplasia (rats) in males and females. There were also exposure-related lesions noted in the larynx and trachea of male and female mice. These findings are comparable with the previously conducted inhalation study on cobalt sulfate heptahydrate ${ }^{66 ; 68}$.

Findings from the clinical pathology assessments conducted in the 3-month studies indicated erythrocytosis, as defined by an increase in the red blood cell mass and hemoglobin concentration. There were exposure concentration-related changes on days 3 and 23 and at 14 weeks in male rats and at 14 weeks in female rats with the red blood cell count reaching greater than or equal to $22 \%$ of the concurrent chamber control values in the high concentration groups $\left(5 \mathrm{mg} / \mathrm{m}^{3}\right)$. The differences in the time and magnitude of erythron changes due to cobalt metal exposure between the male and female rats is likely due, in part, to differences in sex hormones as testosterone is a known stimulator of erythropoiesis and estrogen a known suppressor $^{205}$. Minimal hematological effects were seen in the mice. The reason for the species differences in the erythrocytic response to cobalt is not known but has been previously reported for cobalt sulfate heptahydrate ${ }^{66}$. 
Cobalt exposure has long been known to cause an erythrocytosis (independent of oxygen tension) through stimulation of erythropoietin ${ }^{206}$, the mechanisms of which have been recently explained. It is now known that cobalt's effects are due to its ability to stabilize hypoxiainducible factor-1 $\alpha$ (HIF-1 $\alpha$ ), a subunit of hypoxia-inducible factor-1 (HIF-1) a major regulator of oxygen homeostasis. There are a multitude of genes targeted by HIF-1 including erythropoietin, vascular endothelial growth factor, glucose transporter-1 (GLUT-1), and those related to various glycolytic enzymes ${ }^{207}$. During normoxia, ubiquitin- and proteosome-dependent pathways utilize specific proline hydroxylases to continually degrade HIF-1 $\alpha$. By replacing the iron-binding site on these proline hydroxylases, cobalt renders them inactive, thereby indirectly stabilizing HIF-1 $\alpha^{208 ; 209}$. There is also evidence that cobalt directly binds to HIF-1 $\alpha$ thereby preventing its degradation ${ }^{209}$.

Other clinical chemistry indicators including glucose, cholesterol, and triglycerides were assessed in special study rats based on reports in the literature which suggest that cobalt administration affects triglyceride and cholesterol blood levels in rodents ${ }^{68 ; 210}$. Findings from the current studies indicated significant exposure-related decreases in cholesterol concentrations in both male and female rats, as well as significant decreases in glucose concentrations in male rats. Decreases in cholesterol and glucose concentrations are not uncommon findings in toxicology studies and usually represent a combination of decreased food intake and/or altered lipid and carbohydrate metabolism, the mechanisms of which are typically not known. Various studies have revealed several mechanisms by which cobalt may alter lipid and glucose metabolism. As previously mentioned, cobalt stabilizes HIF-1 $\alpha$ which has the potential to activate genes involved in energy metabolism including those of glycolysis. In addition, short-term administration of cobalt to diabetic rats was shown to have a glycemia-lowering effect that may be mediated by reductions in systemic glucose production (modulation of glycogen metabolism and gluconeogenesis), increased tissue glucose uptake by induction of GLUT-1, or a combination of these two mechanisms ${ }^{21-213}$. Administration of cobalt has also been shown to improve insulin sensitivity and glucose tolerance, as well as alter the ratio of HDL to LDL cholesterol in the blood of obese rats and mice by activation of heme-oxygenase-1 and increased secretion of adiponectin with AMK-activated protein kinase activation ${ }^{214-216}$. In the current study, similar exposure-dependent effects were noted in the cobalt metal exposed rats and mice.

Cobalt metal exposure for 3 months had effects on the male reproductive system as indicated by a significant decrease in sperm motility in male rats and mice accompanied by marked germinal epithelium degeneration in the testes of mice. Cobalt has been shown to affect the motility of human sperm in vitro, suggesting a potential direct effect on the spermatozoa ${ }^{217}$. The observed cobalt-related effects in the testes and epididymides of mice are consistent with those that have been previously reported, however the mode of action is unknown. Nevertheless, similar findings are also observed with cadmium chloride, and this finding is believed to be the result of cadmium chloride-induced testicular ischemia resulting in testicular degeneration and atrophy ${ }^{218}$; ${ }^{219}$. Cobalt has been shown to induce hypoxia, resulting in ischemia in a variety of in vitro and in vivo models ${ }^{220}$. Therefore, it is possible that cobalt-related effects on the testis and subsequently on the epididymis were the result of localized hypoxia. In female rats, although there appeared to be a higher probability of extended diestrus in the $5 \mathrm{mg} / \mathrm{m}^{3}$ group the toxicologic significance of this subtle alteration is unclear.

In the current 2-year studies, the survival of female rats was decreased in the top two exposure groups $\left(2.5\right.$ and $\left.5 \mathrm{mg} / \mathrm{m}^{3}\right)$ with a statistically significant reduction in the $2.5 \mathrm{mg} / \mathrm{m}^{3}$ group. Mean 
body weights of both male and female rats were significantly decreased in the 2.5 and $5 \mathrm{mg} / \mathrm{m}^{3}$ groups. In mice, the survival of the males exposed to 2.5 or $5 \mathrm{mg} / \mathrm{m}^{3}$ was significantly less than that of the concurrent chamber controls. Mean body weights were reduced by greater than $10 \%$ in both males and females in the $5 \mathrm{mg} / \mathrm{m}^{3}$ group.

As with the 3-month studies, the respiratory tract was the major target for toxicity in the 2-year studies with the lung as the primary site for carcinogenicity in both rats and mice following cobalt metal exposure. In rats, there were exposure concentration-related significant increases in the incidences of alveolar/bronchiolar adenoma and carcinoma including multiple adenomas and carcinomas, thereby leading to clear evidence of carcinogenicity in male and female rats. In general, the alveolar/bronchiolar adenomas that occurred in rats and mice were morphologically similar to those that occur spontaneously. However, the alveolar/bronchiolar carcinomas tended to be larger, more invasive, pleomorphic masses that sometimes replaced entire lung lobes. In rats, several of the carcinomas had a prom-inent sarcomatous or desmoplastic component which is uncommon in spontaneous alveolar/bronchiolar carcinomas but not uncommon in inhalation studies with particulates ${ }^{122 ; 221 ; 222}$. In male mice, there was clear evidence of carcinogenicity based on statistically significant increases in the incidences of alveolar/bronchiolar carcinoma including multiple carcinomas. In female mice, there was clear evidence of carcinogenicity based on significantly increased incidences of alveolar/bronchiolar adenoma or carcinoma (combined) including multiple carcinomas. In rats and mice, the incidences of hyperplasia in the alveolar and bronchiolar epithelia were significantly increased. In the lung, such hyperplasia is considered a preneoplastic lesion and can progress to neoplasia. In female mice, there was clear evidence of carcino-genicity based on significantly increased incidences of alveolar/bronchiolar neoplasms of the lung (predominantly carcinoma), including multiple carcinomas.

In mice, the lung neoplasms metastasized to other regions such as the cecum, liver, pancreas, heart, adrenal gland, testes, thymus, skin, skeletal muscle, nose, trachea, and kidney as indicated by the presence of neoplasms in these tissues that were morphologically similar to those in the lung.

In rats, exposure to cobalt metal induced cystic keratinizing epitheliomas (CKE) in the lungs with higher occurrences in females compared to males. CKEs are rarely observed spontaneous neoplasms in rats from NTP 2-year inhalation studies and are rarely found in other species ${ }^{223-225}$. CKEs have been induced in the lungs of rats following intratracheal instillation or chronic inhalation exposure to various particulate compounds $223 ; 225-232$. Significantly increased incidences of CKEs were observed in female Sprague Dawley rats following exposure to polychlorinated biphenyls (PCB), dioxin, dioxin-like compounds, and mixtures of dioxin-like compounds by gavage for up to two years ${ }^{233-239}$. CKEs are considered part of a spectrum of lesions that form a continuum considered to progress from squamous metaplasia to keratin cysts to CKE to squamous cell carcinoma. In the current study, few incidences of squamous metaplasia occurred in female rats, and squamous cysts were not observed, although minimal squamous differentiation was observed in some alveolar/bronchiolar carcinomas and a single incidence of a squamous cell carcinoma was observed in a female in the high concentration $5 \mathrm{mg} / \mathrm{m}^{3}$ group. The occurrence of CKEs was considered related to exposure in females based on the rarity of these neoplasms in NTP inhalation studies and the increase over the historical control range for all routes of administration. 
Prolonged exposure to cobalt metal appeared to cause progressive injury to the lungs and nose in both male and female rats as indicated by the increasing incidences and severities of the nonneoplastic lesions relative to the 2-week and 3-month studies. In the 2-year studies, neoplasms that occurred in the lung were accompanied by a spectrum of generally similar inflammatory and nonneoplastic proliferative lesions of the respiratory tract. These lesions generally included epithelial hyperplasia, metaplasia, necrosis, and inflammation in the nose and bronchiolar and alveolar epithelial hyperplasia and inflammation and proteinosis of the lung in male and female rats and mice. A similar spectrum of inflammatory, fibrotic, and proliferative lesions were reported in the respiratory tract of female rats and mice in a previous NTP study on cobalt sulfate heptahydrate ${ }^{122}$.

In rats, another major target organ for carcinogenicity was the adrenal medulla as indicated by exposure concentration-related increased incidences of benign and malignant pheochromocytomas, including bilateral, in both males and females. The results of several NTP inhalation studies with particulate compounds suggest that there may be an association between the occurrence of benign and malignant alveolar/bronchiolar neoplasms and variably extensive chronic pulmonary nonneoplastic lesions of the lung and significantly increased incidences of hyperplasias and benign and malignant pheochromocytomas of the adrenal medulla in exposed male and female rats ${ }^{122 ; 221 ; 222 ; 240-243}$. With varying degrees of statistical significance, in some but not all studies, this relationship appeared to be associated with the severities of lung fibrosis and inflammation ${ }^{244}$. In NTP studies, the mechanism(s) of this association between lung lesions and pheochromocytoma in rats is not understood. However, reduced gas exchange induced by extensive space-occupying neoplasms and nonneoplastic lung lesions such as fibrosis and chronic inflammation may lead to systemic hypoxemia that chronically stimulates catecholamine secretion from the adrenal medulla. This chronic hypersecretory activity may lead to medullary hyperplasia and subsequent neoplasia ${ }^{244}$. In the present studies, abnormal breathing was observed in some animals, however clinical signs of cyanosis were not noted.

Cobalt metal also induced pancreatic islet neoplasms following inhalation exposure in male rats. There were significant increases in the incidences of pancreatic islet adenoma or carcinoma (combined) in the 2.5 and $5 \mathrm{mg} / \mathrm{m}^{3}$ groups and the instances exceeded the historical control range for all routes of administration. Based on the low spontaneous background rate, and significantly increased incidences, it was concluded that there was some evidence of carcinogenicity in the pancreatic islets of male rats. In female rats, there was an increase in the incidence of pancreatic islet carcinoma in the $5 \mathrm{mg} / \mathrm{m}^{3}$ group relative to concurrent chamber controls and historical controls (all routes). However, the absence of statistically significant trends or pairwise comparisons led to the conclusion of equivocal evidence of pancreatic islet carcinoma in female rats. This is the first time that the pancreas has been reported as a target organ of carcinogenicity in NTP inhalation studies.

In female rats, there were statistically significant increases in the incidences of mononuclear cell leukemia at all exposure concentrations compared to the chamber controls; however, no clear exposure-concentration relationship was seen. Although mononuclear cell leukemia is a common spontaneous neoplasm in F344 rats, the increased incidences in females in the current study were considered related to cobalt exposure.

Increased incidences of renal tubule adenoma or carcinoma (combined) occurred in male rats exposed to cobalt metal compared to the chamber control group, but the increases were not 
statistically significant. Since the standard evaluation of the kidney of males suggested the possibility of an exposure-related carcinogenic effect, an extended evaluation was performed by step-sectioning the kidney; however, the findings were not significantly different from the original evaluation. Although there was an overall positive trend in the incidences of renal tubule adenoma or carcinoma (combined) in male rats, no pairwise tests were significant, there was no exposure-concentration response, and three adenomas occurred in chamber control males. In addition, no supporting nonneoplastic findings occurred. However, because these lesions are relatively rare, NTP concluded that they may have been related to cobalt exposure.

Based on reports in the literature, the heart is one of the major target organs following occupational exposure to cobalt in both humans and animals ${ }^{9}$. Occupational exposure of humans to cobalt-containing dust, either as cobalt metal or as hard metal, is believed to result in cardiomyopathy; however, in the majority of these and other reported occupational studies, coexposure to other substances was common ${ }^{9 ; 97}$. However, there was no indication of cardiomyopathy in the current studies.

Toxicokinetic studies were conducted as part of the 2-week, 3-month, and 2-year studies in rats and mice to inform study design, as well as to provide data on pulmonary retention and clearance and systemic distribution. In the 2-week studies, tissues from males and females were weighed and examined for cobalt concentrations and burdens at terminal kill. In females, cobalt was measured in the blood, serum, and lung of additional groups held for 3 weeks after exposure so that clearance from tissues could be evaluated. In the 3-month and 2-year studies, lung weights, lung cobalt concentrations, and lung cobalt burdens were determined in females. In the 3-month studies, the toxicokinetic study included time points during the exposure as well as during a postexposure period. In the 2-year studies, lung cobalt measurements were determined over the first 18 months of the studies. In the 3-month studies, blood cobalt concentrations and liver weights, cobalt concentrations, and cobalt burdens were also determined in females. In all three studies, exposure-related changes in tissue weights, especially exposure-related increases in lung weights which demonstrated greater increases, earlier onsets, and/or greater persistence during the postexposure period in rats and mice (2-week and 3-month studies), prompted the use of tissue cobalt burden (rather than concentration) for determination of cobalt deposition and clearance.

Cobalt concentrations and burdens were increased in all studies in all tissues examined, indicating systemic exposure of rats and mice to cobalt. In particular, liver cobalt concentrations in the 2-week and 3-month studies approached or even exceeded lung cobalt concentrations, particularly at higher exposure concentrations. Normalized lung burden data generally indicated that there were no biologically significant disproportionate changes in cobalt deposition or clearance with increasing exposure concentration; these data are consistent with the modeling data discussed below.

Lung deposition and clearance data from the 2-week studies were used to estimate lung clearance. As there were only two time points, the data were fit to a one-compartment model. Data from the 2-week studies were used to select time points for the 3-month studies (exposure and postexposure phases). Due to a lack of sufficiently early time points during the exposure phase, the data generated during the exposure phase were fit to a one-compartment model, while a two-compartment model was utilized for the postexposure phase. Time points for the 2-year lung deposition and clearance studies were selected using the 3-month data and included early 
time points (days 1, 2, 3, and 4). Following completion of these studies, the lung deposition and clearance data from the exposure phases of the 3-month and 2-year studies were fit to a two-compartment model. Modeling of the lung data generally indicated both rapid (approximately 1 to 5 days for all studies) and slow (longer with increasing exposure duration) clearance phases; the two-phase clearance from the lung probably contributed to the apparent two-phase elimination from the blood. Steady state was reached by the midpoint of the 3-month study in each species. In the 2-year studies, the times required to reach steady state were relatively long (12 to 18 months), due to the slow clearance half-lives. This phenomenon is consistent with previous studies ${ }^{30 ; 32 ; 90}$ and may be caused by binding of dissolved cobalt to tissues or movement of particles to the interstitium and the formation of foci of macrophages on the alveolar wall ${ }^{245}$. In the 2-year studies, slow phase half-lives increased significantly with decreasing exposure concentration. When comparing the rapid and slow clearance phases, the majority ( $>95 \%$ in rats and $>82 \%$ in mice) of the deposited cobalt was cleared with a very short half-life (approximately 1 to 5 days across studies), while the remainder was cleared more slowly. When comparing steady state lung cobalt burdens, it was apparent that burdens were similar in rats and mice, with maximum and steady state lung cobalt burdens (rapid + slow) of approximately $50 \mu \mathrm{g}$ per lung at $5 \mathrm{mg} / \mathrm{m}^{3}$ in both species. These burdens were compared to those that would be required to cause lung overload, which occurs as a result of excessive volume of insoluble particles relative to that of the alveolar macrophage pool. Clearance by macrophages slows when particle volume is $6 \%$ of the macrophage pool volume and then ceases when particle volume is $60 \%$ of the macrophage pool volume, resulting in dramatically increased lung burdens and very long clearance half-lives ${ }^{246-249}$. Overload was originally studied in F344 rats and assumes a density of one; however, for the current studies, the ratio of mouse to rat lung weight at 18 months of the chronic study and the use of the density of the cobalt test article (approximately $8.81 \mathrm{~g} / \mathrm{cm}^{3}$ ) allowed for evaluation of overload specific to rats and mice exposed to cobalt metal. Based on these assumptions, $13.2 \mathrm{mg}$ (rats) or $2.1 \mathrm{mg}$ (mice) per lung would be required to cause overload. These values are 264 (rats) or 42 (mice) times the maximum cobalt lung burdens observed in the 2-year studies, indicating that overload was not approached in these studies.

Multiple lines of evidence, including the rapid clearance of cobalt from the lung and blood, the low lung cobalt burdens, the absence of particle overload, the systemic distribution and elimination of cobalt, and the observed toxicity/carcinogenicity to extrapulmonary sites are consistent with relatively soluble cobalt particles rather than insoluble particles ${ }^{30 ; 32 ; 33}$. Cobalt has been reported to be insoluble in aqueous environments but able to be solubilized by strong mineral acids ${ }^{33 ; 250}$. In vivo studies by $\operatorname{Rae}^{251}$ show that macrophages were able to dissolve a significant amount of cobalt, despite toxicity to the cell. Based on this evidence, alveolar macrophages likely contributed to the solubilizaton and systemic absorption of cobalt via the lung in the current studies. Furthermore, studies by Stopford et al. ${ }^{252}$ using artificial fluids to mimic ingestion and inhalation indicate that lysosomes are likely responsible for dissolving cobalt taken up by macrophages and that any cobalt ingested via grooming or mucocilliary clearance would be solubilized by gastric juices. Because dissolution of cobalt results in toxicity to the macrophages, it is likely that the clearance of cobalt is due primarily to the dissolution and absorption of cobalt, rather than alveolar macrophage mediated clearance of intact particles via mucocilliary clearance. However, gastrointestinal absorption and systemic distribution following grooming or mucocilliary clearance may have also contributed to the tissue distribution of cobalt. 
The mechanisms of cobalt-induced carcinogenesis are not well understood, although the genotoxicity of cobalt compounds has been established in a variety of test systems (reviewed in IARC $^{1}$ ). In the current study, to identify a potential mode of action through which cobalt metal may be inducing its carcinogenic effects, mutation analysis was conducted on the most commonly altered cancer genes in human lung cancer: Kras, Egfr, and Tp53 in lung neoplasms from $\mathrm{F} 344 / \mathrm{NTac}$ rats and $\mathrm{B} 6 \mathrm{C} 3 \mathrm{~F} 1 / \mathrm{N}$ mice exposed to cobalt metal. Chemical-specific genetic mutations have been previously demonstrated with tobacco smoke-induced lung cancer $(\mathrm{C}: \mathrm{G} \rightarrow \mathrm{A}: \mathrm{T})$, ultraviolet light-induced melanoma $(\mathrm{C}: \mathrm{G} \rightarrow \mathrm{T}: \mathrm{A})$, aflatoxin-induced hepatocellular carcinoma $(\mathrm{C}: \mathrm{G} \rightarrow \mathrm{A}: \mathrm{T})$, and aristolochic acid-induced urothelial carcinoma $(\mathrm{A}: \mathrm{T} \rightarrow \mathrm{T}: \mathrm{A})^{253}$. In addition, several cancers also harbor mutations in genes that may have several functions, such as tumor suppressor genes, oncogenes, DNA repair genes, apoptosis genes, and growth factor genes. Several of these mutations are "driver" mutations that are specific to each cancer type. In human lung cancer, the most common driver mutations occur in KRAS, EGFR, and TP53 genes $^{254 ; 255}$. In the current study, hot spot regions in these genes from alveolar/bronchiolar carcinomas from rats and mice chronically exposed to cobalt metal were evaluated.

Findings showed that Kras mutations were more frequent than Tp53 and Egfr mutations within the alveolar/bronchiolar carcinomas from F344/NTac rats and B6C3F1/N mice chronically exposed to cobalt metal. Mutations in KRAS are considered to be an early event, whereas mutations within TP53 are thought to be a late event in the pathogenesis of lung cancer ${ }^{256 ; 257 .}$ KRAS and EGFR mutations are considered to be mutually exclusive in human lung cancer ${ }^{258}$, but unexpectedly, in the current study $38 \%(3 / 8)$ of rats and $25 \%(3 / 12)$ of mice that harbored Egfr mutations also had Kras mutations. The significance of the presence of the independent occurrence of mutations in some alveolar/bronchiolar mutations is unclear but may be related to the repeated exposure to cobalt metal over the 2-year period or due to sampling of genetically heterogeneous tumors from the same lung sample. Alternatively, it may reflect the many pathways that still lead to cancer.

Mutations within codon 12 of Kras were observed in both spontaneous alveolar/bronchiolar carcinomas $\left[27 \%(34 / 124)^{259}\right]$ and alveolar/bronchiolar carcinomas from cobalt metal-exposed mice $[67 \%$ (46/69)]. However, alveolar/bronchiolar carcinomas from cobalt metal-exposed mice had predominantly $\mathrm{G} \rightarrow \mathrm{T}$ transversions [80\% (24/30)], whereas the spontaneous carcinomas had $\mathrm{G} \rightarrow \mathrm{A}$ transitions $[70 \%(14 / 20)]$ in codon 12 . The $\mathrm{G} \rightarrow \mathrm{T}$ transversions were also the most predominant mutations in alveolar/bronchiolar carcinomas from mice chronically exposed to cobalt sulfate heptahydrate aerosols ${ }^{122}$, as well as other chemicals such as ozone, ethylene oxide, and cumene (Appendix K, Table K-17). This suggests that these chemicals target guanine or cytosine bases suggesting that these chemicals induce mutations at multiple sites and tissues by a common mechanism. $\mathrm{G} \rightarrow \mathrm{T}$ transversions are one of the more common Kras mutations in human lung cancer ${ }^{260}$. $\mathrm{G} \rightarrow \mathrm{T}$ Kras mutations were reported to correlate with 8-hydroxydeoxyguanine adducts that result from oxidative stress. In the current study, these transversion mutations were seen almost exclusively in murine alveolar/bronchiolar carcinomas from cobalt exposure but not in spontaneous alveolar/bronchiolar carcinomas.

The results of the NTP bacterial mutagenicity assays lend support to the possibility that cobalt metal induces tumorigenesis by increasing oxidative stress. In bacterial mutagenicity assays conducted by NTP, positive results were seen in Salmonella typhimurium strain TA98 and equivocal results were seen in strain TA100 in the absence of S9 metabolizing enzymes; results for both strains were negative with the addition of S9 mix. The Escherichia coli WP2 
$u v r A / p K M 101$ strain gave negative results in the absence or presence of S9 mix. These observations are of interest considering that cobalt is known to produce reactive oxygen species that could lead to increases in 8-hydroxydeoxyguanine adducts, and the mutations identified in the cancer-related genes sequenced from cobalt metal-induced mouse lung tumors predominately occurred at G: $\mathrm{C}$ base pairs. Strain T98 detects a -1 frameshift that disrupts a dinucleotide run of $(\mathrm{CG})_{4}$ residues; strain TA100 detects reverse mutations at a codon for proline (GGG) in hisG46, and the E. coli WP2 uvrA/pKM101 strain detects reverse mutations at the trpE ochre (TAA) codon. Taken together, the degree to which cobalt metal was mutagenic in the three strains correlated with the ability of each strain to detect mutational events at G:C base pairs. In support of this observation, sequencing of the supF tRNA mutational reporter gene in bacteria exposed to cobalt chloride showed that almost all mutational events (base substitutions and frameshifts) occurred at G:C base pairs ${ }^{261}$. Cobalt metal-induced mutagenicity was not apparent with addition of S9 mix in any bacterial strain that was tested by NTP. Although the composition of S9 mix has not been fully characterized, it contains microsomal and cytosolic enzymes, and could, therefore, contain radical scavenging enzymes such as glutathione peroxidase, glutathione reductase, glutathione-S-transferase, catalase, and superoxide dismutase. The presence of these enzymes in S9 mix may have ameliorated the mutagenic effects of cobalt. Alternatively (or additionally), the absence of cobalt-induced mutagenic activity in the presence of S9 mix might have been due to binding of cobalt to S9 proteins.

The NTP has conducted two 2-year inhalation studies, one on cobalt sulfate heptahydrate ${ }^{122}$ and the current study on cobalt metal. Both compounds induced alveolar/bronchiolar adenomas and carcinomas and nonneoplastic respiratory tract lesions in rats and mice and pheochromocytomas in rats. As there are no tissue burden data available for the cobalt sulfate heptahydrate study, exposure concentrations of cobalt have been used for comparison. When the exposure concentrations of cobalt sulfate heptahydrate are normalized to cobalt metal using the molecular weight ratio of cobalt:cobalt sulfate heptahydrate, the highest concentration of cobalt sulfate heptahydrate $\left(3.0 \mathrm{mg} / \mathrm{m}^{3}\right)$ results in an exposure concentration of cobalt $\left(1.14 \mathrm{mg} / \mathrm{m}^{3}\right)$ that is similar to the lowest exposure concentration $\left(1.25 \mathrm{mg} / \mathrm{m}^{3}\right)$ of cobalt metal in the current study. At the similar cobalt exposure concentration, there were more neoplasms in each sex-species group with cobalt metal, with the exception of female rats; however, significantly increased incidences of lung neoplasms were observed in all four sex-species groups with both cobalt sulfate heptahy-drate and cobalt metal. For pheochromocytomas, the responses at the similar cobalt concentration were also similar. Increased incidences of pancreatic islet neoplasms in male and female rats and mononuclear cell leukemia in female rats exposed to cobalt metal indicated systemic carcinogenicity; however, incidences of these neoplasms were increased at concentrations greater than those used in the cobalt sulfate heptahydrate studies. There were also some differences in the affected tissues, types, incidences, and severities of nonneoplastic respiratory tract lesions between the two studies at the similar exposure concentration. Overall, there was significant toxicity to the respiratory tract in both studies at the similar cobalt exposure concentration, and comparisons of data between the two studies suggest that cobalt is toxic and carcinogenic, at least in the respiratory tract, at a similar exposure concentration. 


\section{Conclusions}

Under the conditions of these 2-year inhalation studies, there was clear evidence of carcinogenic activity of cobalt metal in male F344/NTac rats based on increased incidences of alveolar/bronchiolar adenoma and carcinoma in the lung, including multiples, and on increased incidences of benign and malignant pheochromocytoma of the adrenal medulla, including bilateral neoplasms. ${ }^{\text {a }}$ The increased incidences of pancreatic islet adenoma or carcinoma (combined) were considered related to exposure. The occurrences of cystic keratinizing epithelioma of the lung and of renal tubule adenoma or carcinoma (combined) may have been related to exposure. There was clear evidence of carcinogenic activity of cobalt metal in female F344/NTac rats based on increased incidences of alveolar/bronchiolar adenoma and carcinoma in the lung, including multiples, and on increased incidences of benign and malignant pheochromocytoma of the adrenal medulla, including bilateral neoplasms. The occurrences of squamous cell neoplasms of the lung (predominantly cystic keratinizing epithelioma), and of mononuclear cell leukemia were considered related to exposure. The occurrences of pancreatic islet carcinoma may have been related to exposure. There was clear evidence of carcinogenic activity of cobalt metal in male and female $\mathrm{B} 6 \mathrm{C} 3 \mathrm{~F} 1 / \mathrm{N}$ mice based on increased incidences of alveolar/bronchiolar neoplasms of the lung (predominantly carcinoma), including multiple carcinoma.

Exposure to cobalt metal resulted in increased incidences of nonneoplastic lesions of the lung and nose in male and female rats, the testes in male rats and mice, the adrenal medulla in female rats, and the lung, nose, larynx, and trachea in male and female mice.

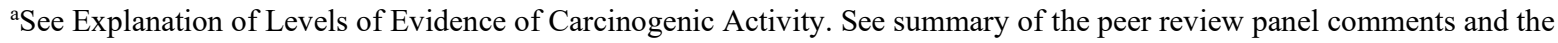
public discussion on this Technical Report in Appendix O. 


\section{References}

1. International Agency for Research on Cancer (IARC). Chlorinated drinking-water; chlorination by-products; some other halogenated compounds; cobalt and cobalt compounds. IARC Monogr Eval Carcinog Risks Hum. Lyon, France: IARC; 1991. p. 363-472.

2. Donaldson JD, Beyersmann D. Cobalt and cobalt compounds. In: Ullmann's Encyclopedia of Industrial Chemistry. New York: Wiley-VCH; 2005. p. http://dx.doi.org/10.1002/14356007.a07_281.pub2

3. The Merck Index. 14th ed. In: O'Neil MJ, editor. Whitehouse Station, NJ: Merck \& Co. Inc; 2006. p. 408-409.

4. Om Group Inc (OMGI). Cobalt powder product descriptions. 2013. https://web.archive.org/web/20101220042624/http://www.omgi.com/product-ampowderspm.html [Accessed: March 2013]

5. Smith IC, Carson BL. Cobalt. In: Trace Metals in the Environment, Vol 6. Ann Arbor, MI: Ann Arbor Science Publishers; 1981.

6. Donaldson JD. Cobalt and cobalt compounds In: Gerhartz W, Yamamoto YS, Campbell FT, Pffeferkorn R, Rounsaville JF, editors. Ullman's Encyclopedia of Industrial Chemistry 5th ed. New York: VCH; 1986. p. 281-313.

7. Hodge FG. Cobalt and cobalt alloys In: Kroschwitz JI, Howe-Grant M, editors. Kirk-Othmer Encyclopedia of Chemical Technology. New York: John Wiley \& Sons; 1993. p. 760-777.

8. United States Geological Survey (USGS). 2002. http://minerals.usgs.gov/minerals/pubs/commodity/cobalt/cobalmyb02.pdf [Accessed: August 2013]

9. Agency for Toxic Substances and Disease Registry (ATSDR). Toxicological profile for cobalt. Atlanta, GA: U.S. Department of Health and Human Services, Public Health Service, Agency for Toxic Substances and Disease Registry; 2004.

10. Cobalt Development Institute (CDI). 2013. https://web.archive.org/web/20200210043406/https://www.cobaltinstitute.org/ [Accessed August 2013]

11. Burstow, C. Cobalt: The changing emphasis: Supply, demand and price outlook for the cobalt market. Proceedings of the Cobalt Conference; May 24-25 2000; Tokyo, Japan. Cobalt Development Institute, United Kingdom.

12. United States Environmental Protection Agency (USEPA). Non-confidential 2006 IUR Records by chemical, including manufacturing, processing and use information. 2010. https://www.epa.gov/chemical-data-reporting/access-cdr-data\#2006 [Accessed: August 2013]

13. United States Environmental Protection Agency (USEPA). Chemical Data Access Tool (CDAT): 2012 Chemical Data Reporting. 2013. 
https://web.archive.org/web/20110808061510/https://java.epa.gov/oppt chemical search/ [Accessed: April 2013]

14. Sibley SF. Cobalt. Mineral Facts and Problems 1975 Edition. Washington, DC: Bureau of Mines; 1975. p. 269-280.

15. Shedd KB. Cobalt. In: Mineral Commodity Summaries. U.S. Geological Survey; 2013. p. 4647. https://s3-us-west-2.amazonaws.com/prd-wret/assets/palladium/production/mineralpubs/mcs/mcs2013.pdf [Accessed: August 2013]

16. Institute of Medicine (IOM). Dietary reference intakes for thiamine, riboflavin, niacin, vitamin B6, folate, vitamin B12, pantothenic acid, biotin and choline. National Academy Press, Washington, DC: Committee on the Scientific Evaluation of Dietary Reference Intakes, Food and Nutrition Board, Institute of Medicine; 1998. http://www.nap.edu/books/0309065542/html/index.html [Accessed: March 11, 2013]

17. Domingo JL. Cobalt in the environment and its toxicological implications. Rev Environ Contam Toxicol. 1989; 108:105-132. http://dx.doi.org/10.1007/978-1-4613-8850-0_3

18. Reuber S, Kreuzer M, Kirchgessner M. Interactions of cobalt and iron in absorption and retention. J Trace Elem Electrolytes Health Dis. 1994; 8:151-158.

19. Schade SG, Felsher BF, Bernier GM, Conrad ME. Interrelationship of cobalt and iron absorption. J Lab Clin Med. 1970; 75(3):435-441.

20. Code of Federal Regulations (CFR). 40:§141.140.

21. Code of Federal Regulations (CFR). 40:Part 258.

22. Code of Federal Regulations (CFR). 40:Part 264.

23. Code of Federal Regulations (CFR). 40:§372.365.

24. American Conference of Governmental Industrial Hygienists (ACGIH). TLVs and BEIs based on the documentation of the threshold limit values for chemical substances and physical agents and biological exposure indices. Cincinnati, OH: ACGIH; 2013. p. 21.

25. Occupational Safety and Health Administration (OSHA). Shipyards, cobalt metal, dust, and fume. 2001.

http://www.osha.gov/pls/oshaweb/owadisp.show document?p table=STANDARDS\&p id=102 $\underline{86}$ [Accessed: March 2013]

26. Registry of Toxic Effects of Chemical Substances (RTECS). Bethesda, MD: National Institute for Occupational Safety and Health, National Library of Medicine; 1991.

27. Nemery B, Casier P, Roosels D, Lahaye D, Demedts M. Survey of cobalt exposure and respiratory health in diamond polishers. Am Rev Respir Dis. 1992; 145(3):610-616. http://dx.doi.org/10.1164/ajrccm/145.3.610

28. Barnes JE, Kanapilly GM, Newton GJ. Cobalt-60 oxide aerosols: Methods of production and short-term retention and distribution kinetics in the beagle dog. Health Phys. 1976; 30(5):391398. http://dx.doi.org/10.1097/00004032-197605000-00003 
29. Brune D, Kjaerheim A, Paulsen G, Beltesbrekke H. Pulmonary deposition following inhalation of chromium-cobalt grinding dust in rats and distribution in other tissues. Scand $\mathrm{J}$ Dent Res. 1980; 88(6):543-551. http://dx.doi.org/10.1111/j.1600-0722.1980.tb01265.x

30. Collier CG, Bailey MR, Hodgson A. An interspecies comparison of the lung clearance of inhaled monodisperse cobalt oxide particles. Part V: Lung clearance of inhaled cobalt oxide particles in hamsters, rats and guinea-pigs. J Aerosol Sci. 1989; 20(2):233-247. http://dx.doi.org/10.1016/0021-8502(89)90046-3

31. Kerfoot EJ, Fredrick WG, Domeier E. Cobalt metal inhalation studies on miniature swine. Am Ind Hyg Assoc J. 1975; 36(1):17-25. http://dx.doi.org/10.1080/0002889758507202

32. Kreyling WG, Ferron GA, Haider B. Metabolic fate of inhaled Co aerosols in beagle dogs. Health Phys. 1986; 51:773-795. http://dx.doi.org/10.1097/00004032-198612000-00007

33. Kyono H, Kusaka Y, Katsunori H, Kubota H, Yoko E, Ichikawa. Reversible lung lesions in rats due to short-term exposure to ultrafine cobalt particles. Ind Health. 1992; 30(3-4):103-118. http://dx.doi.org/10.2486/indhealth.30.103

34. Patrick G, Batchelor AL, Stirling C. An interspecies comparison of the lung clearance of inhaled monodisperse cobalt oxide particles. Part VI: Lung clearance of inhaled cobalt oxide particles in SPF Fischer rats. J Aerosol Sci. 1989; 20(2):249-255. http://dx.doi.org/10.1016/0021$\underline{8502(89) 90047-5}$

35. Popov LN, Kochetkova TA, Gusev MI, Markina NA, Elfimova EV. Accumulation, distribution and morphological changes in the body to the inhalatory exposure to a metallic cobalt aerosol [in Russian]. Gig Sanit. 1977; (6):12-15.

36. Kerfoot EJ. Chronic animal inhalation toxicity to cobalt. Cincinnati, OH: U.S. Department of Health, Education, and Welfare, National Institute for Occupational Safety and Health; 1973.

37. Lasfargues G, Lison D, Maldague P, Lauwerys R. Comparative study of the acute lung toxicity of pure cobalt powder and cobalt-tungsten carbide mixture in rat. Toxicol Appl Pharmacol. 1992; 112(1):41-50. http://dx.doi.org/10.1016/0041-008X(92)90277-Y

38. Lison D, Lauwerys R. Cobalt bioavailability from hard metal particles: Further evidence that cobalt alone is not responsible for the toxicity of hard metal particles. Arch Toxicol. 1994; 68(8):528-531. http://dx.doi.org/10.1007/s002040050108

39. Ayala-Fierro F, Firriolo JM, Carter DE. Disposition, toxicity, and intestinal absorption of cobaltous chloride in male Fischer 344 rats. J Toxicol Environ Health, A. 1999; 56(8):571-591. http://dx.doi.org/10.1080/009841099157908

40. Bailey MR, Kreyling WG, Andre S, Batchelor A, Collier CG, Drosselmeyer E, Ferron GA, Foster P, Haider B, Hodgson A et al. An interspecies comparison of the lung clearance of inhaled monodisperse cobalt oxide particles. Part I: Objectives and summary of results. J Aerosol Sci. 1989; 20(2):169-188. http://dx.doi.org/10.1016/0021-8502(89)90042-6

41. Barnaby CF, Smith T, Thompson BD. Dosimetry of the radioisotopes of cobalt. Phys Med Biol. 1968; 13(3):421-433. http://dx.doi.org/10.1088/0031-9155/13/3/309 
42. Hollins JG, McCullough RS. Radiation dosimetry of internal contamination by inorganic compounds of cobalt: An analysis of cobalt metabolism in rats. Health Phys. 1971; 21(2):233246. http://dx.doi.org/10.1097/00004032-197108000-00010

43. Naylor GP, Harrison JD. Gastrointestinal iron and cobalt absorption and iron status in young rats and guinea pigs. Hum Exp Toxicol. 1995; 14(12):949-954.

http://dx.doi.org/10.1177/096032719501401203

44. Bourg WJ, Nation JR, Clark DE. The effects of chronic cobalt exposure on passiveavoidance performance in the adult rat. Bull Psychon Soc. 1985; 23(6):527-530. http://dx.doi.org/10.3758/BF03329874

45. Clyne N, Lins L-E, Pehrsson SK, Lundberg A, Werner J. Distribution of cobalt in myocardium, skeletal muscle and serum in exposed and unexposed rats. Trace Elem Med. 1988; 5:52-54.

46. Pehrsson SK, Hatori N, Clyne N, Koch J, Lins L-E, Ryden L. The effect of chronic cobalt exposure on cardiac function in rats. Trace Elem Med. 1991; 8:195-198.

47. Thomas RG, Furchner JE, London JE, Drake GA, Wilson JS, Richmond CR. Comparative metabolism of radionuclides in mammals-X. Retention of tracer-level cobalt in the mouse, rat, monkey and dog. Health Phys. 1976; 31(4):323-333. http://dx.doi.org/10.1097/00004032$\underline{197610000-00003}$

48. Andre S, Metivier H, Masse R. An interspecies comparison of the lung clearance of inhaled monodisperse cobalt oxide particles. Part III: Lung clearance of inhaled cobalt oxide particles in baboons. J Aerosol Sci. 1989; 20(2):205-217. http://dx.doi.org/10.1016/0021-8502(89)90044-X

49. Paley KR, Sobel ES, Yalow RS. Effect of oral and intravenous cobaltous chloride on thyroid function. J Clin Endocrinol Metab. 1958; 18(8):850-859. http://dx.doi.org/10.1210/jcem-18-8$\underline{850}$

50. Smith T, Edmonds CJ, Barnaby CF. Absorption and retention of cobalt in man by wholebody counting. Health Phys. 1972; 22(4):359-367. http://dx.doi.org/10.1097/00004032197204000-00007

51. Sorbie J, Olatunbosun D, Corbett WE, Valberg LS. Cobalt excretion test for the assessment of body iron stores. Can Med Assoc J. 1971; 104(9):777-782.

52. Beaugelin-Seiller K, Baudin JP, Brottet D. Use of aquatic mosses for monitoring artificial radionuclides downstream of the nuclear power plant of Bugey (River Rhone, France). J Environ Radioact. 1994; 24(3):217-233. http://dx.doi.org/10.1016/0265-931X(94)90041-8

53. Apostoli P, Porru S, Alessio L. Urinary cobalt excretion in short time occupational exposure to cobalt powders. Sci Total Environ. 1994; 150(1-3):129-132. http://dx.doi.org/10.1016/00489697(94)90139-2

54. Mosconi G, Bacis M, Vitali MT, Leghissa P, Sabbioni E. Cobalt excretion in urine: Results of a study on workers producing diamond grinding tools and on a control group. Sci Total Environ. 1994; 150(1-3):133-139. http://dx.doi.org/10.1016/0048-9697(94)90140-6 
55. Beleznay E, Osvay M. Long-term clearance of accidentally inhaled 60Co aerosols in humans. Health Phys. 1994; 66(4):392-399. http://dx.doi.org/10.1097/00004032-199404000$\underline{00003}$

56. Newton D, Rundo J. The long-term retention of inhaled cobalt-60. Health Phys. 1971; 21(3):377-384. http://dx.doi.org/10.1097/00004032-197109000-00003

57. Foster P, Pearman I, Ramsden D. An interspecies comparison of the lung clearance of inhaled monodisperse cobalt oxide particles. Part II: Lung clearance of inhaled cobalt oxide in man. J Aerosol Sci. 1989; 20(2):189-204. http://dx.doi.org/10.1016/0021-8502(89)90043-8

58. Harp MJ, Scoular FI. Cobalt metabolism of young college women on self-selected diets. J Nutr. 1952; 47(1):67-72. http://dx.doi.org/10.1093/jn/47.1.67

59. Valberg LS, Ludwig J, Olatunbosun D. Alteration in cobalt absorption in patients with disorders of iron metabolism. Gastroenterology. 1969; 56(2):241-251.

http://dx.doi.org/10.1016/S0016-5085(69)80123-X

60. Scansetti G, Botta GC, Spinelli P, Reviglione L, Ponzetti C. Absorption and excretion of cobalt in the hard metal industry. Sci Total Environ. 1994; 150(1-3):141-144.

http://dx.doi.org/10.1016/0048-9697(94)90141-4

61. Nielsen NH, Kristiansen J, Borg L, Christensen JM, Poulsen LK, Menné T. Repeated exposures to cobalt or chromate on the hands of patients with hand eczema and contact allergy to that metal. Contact Derm. 2000; 43(4):212-215. http://dx.doi.org/10.1034/j.1600-

0536.2000.043004212.x

62. Delahant AB. An experimental study of the effects of rare metals on animal lungs. AMA Arch Ind Health. 1955; 12:116-120.

63. Georgiadi GA. Change in the activity of the dehydrogenases and nonspecific enzymes in the respiratory tract mucosa of rats exposed to metallic cobalt dust in a chronic experiment [In Russian]. Zh Ushn Nos Gorl Bolezn. 1978; Jan-Feb(1):63-67.

64. Harding HE. Notes on the toxicology of cobalt metal. Br J Ind Med. 1950; 7(2):76-78. http://dx.doi.org/10.1136/oem.7.2.76

65. Herndon BL, Jacob RA, McCann J. Physiological effects In: Smith IC, Carson BL, editors. Trace Metals in the Environment. 1981. p. 925-1140.

66. National Toxicology Program (NTP). Toxicity studies of cobalt sulfate heptahydrate (CAS No. 10026-24-1) in F344/N rats and B6C3F1 mice (inhalation studies). Research Triangle Park, NC: U.S. Department of Health and Human Services, Public Health Service, National Institutes of Health; 1991. Toxicity Study Report Series No. 5. NIH Publication No. 91-3124.

67. Georgiadi GA, El'kind LA. Morphological changes in the respiratory tract mucosa under the influence of a metallic cobalt aerosol in a chronic experiment [in Russian]. Zh Ushn Nos Gorl Bolezn. 1978; (3):41-45. 
68. Bucher JR, Elwell MR, Thompson MB, Chou BJ, Renne R, Ragan HA. Inhalation toxicity studies of cobalt sulfate in F344/N rats and B6C3F1 mice. Fundam Appl Toxicol. 1990; 15(2):357-372. http://dx.doi.org/10.1016/0272-0590(90)90061-N

69. Anttila S, Sutinen S, Paananen M, Kreus KE, Sivonen SJ, Grekula A, Alapieti T. Hard metal lung disease: A clinical, histological, ultrastructural and X-ray microanalytical study. Eur J Respir Dis. 1986; 69:83-94.

70. Davison AG, Haslam PL, Corrin B, Coutts II, Dewar A, Riding WD, Studdy PR, NewmanTaylor AJ. Interstitial lung disease and asthma in hard-metal workers: Bronchoalveolar lavage, ultrastructural, and analytical findings and results of bronchial provocation tests. Thorax. 1983; 38(2):119-128. http://dx.doi.org/10.1136/thx.38.2.119

71. Demedts M, Gheysens B, Nagels J, Verbeken E, Lauweryns J, van den Eeckhout A, Lahaye D, Gyselen A. Hard-metal lung disease due to cobalt in diamond polishers. Am Rev Respir Dis. 1984; 129:A155.

72. Demedts M, Gheysens B, Nagels J, Verbeken E, Lauweryns J, van den Eeckhout A, Lahaye D, Gyselen A. Cobalt lung in diamond polishers. Am Rev Respir Dis. 1984; 130:130-135.

73. Deng JF, Sinks T, Elliot L, Smith D, Singal M, Fine L. Characterisation of respiratory health and exposures at a sintered permanent magnet manufacturer. Br J Ind Med. 1991; 48(9):609-615. http://dx.doi.org/10.1136/oem.48.9.609

74. Gennart JP, Lauwerys R. Ventilatory function of workers exposed to cobalt and diamond containing dust. Int Arch Occup Environ Health. 1990; 62(4):333-336.

http://dx.doi.org/10.1007/BF00640843

75. Gheysens B, Auwerx J, Van den Eeckhout A, Demedts M. Cobalt-induced bronchial asthma in diamond polishers. Chest. 1985; 88(5):740-744. http://dx.doi.org/10.1378/chest.88.5.740

76. Hartung M, Schaller KH, Brand E. On the question of the pathogenetic importance of cobalt for hard metal fibrosis of the lung. Int Arch Occup Environ Health. 1982; 50(1):53-57. http://dx.doi.org/10.1007/BF00432493

77. Kusaka Y, Ichikawa Y, Shirakawa T, Goto S. Effect of hard metal dust on ventilatory function. Br J Ind Med. 1986; 43(7):486-489. http://dx.doi.org/10.1136/oem.43.7.486

78. Kusaka Y, Iki M, Kumagai S, Goto S. Decreased ventilatory function in hard metal workers. Occup Environ Med. 1996; 53(3):194-199. http://dx.doi.org/10.1136/oem.53.3.194

79. Kusaka Y, Iki M, Kumagai S, Goto S. Epidemiological study of hard metal asthma. Occup Environ Med. 1996; 53(3):188-193. http://dx.doi.org/10.1136/oem.53.3.188

80. Kusaka Y, Yokoyama K, Sera Y, Yamamoto S, Sone S, Kyono H, Shirakawa T, Goto S. Respiratory diseases in hard metal workers: An occupational hygiene study in a factory. Br J Ind Med. 1986; 43(7):474-485. http://dx.doi.org/10.1136/oem.43.7.474

81. Raffn E, Mikkelsen S, Altman DG, M Christensen J, Groth S. Health effects due to occupational exposure to cobalt blue dye among plate painters in a porcelain factory in Denmark. Scand J Work Environ Health. 1988; 14:378-384. http://dx.doi.org/10.5271/sjweh.1903 
82. Rastogi SK, Gupta BN, Husain T, Chandra H, Mathur N, Pangtey BS, Chandra SV, Garg N. A cross-sectional study of pulmonary function among workers exposed to multimetals in the glass bangle industry. Am J Ind Med. 1991; 20(3):391-399.

http://dx.doi.org/10.1002/ajim.4700200311

83. Ruokonen EL, Linnainmaa M, Seuri M, Juhakoski P, Söderström KO. A fatal case of hardmetal disease. Scand J Work Environ Health. 1996; 22(1):62-65.

http://dx.doi.org/10.5271/sjweh.111

84. Shirakawa T, Kusaka Y, Fujimura N, Goto S, Kato M, Heki S, Morimoto K. Occupational asthma from cobalt sensitivity in workers exposed to hard metal dust. Chest. 1989; 95(1):29-37. http://dx.doi.org/10.1378/chest.95.1.29

85. Shirakawa T, Kusaka Y, Fujimura N, Goto S, Morimoto K. The existence of specific antibodies to cobalt in hard metal asthma. Clin Exp Allergy. 1988; 18(5):451-460. http://dx.doi.org/10.1111/j.1365-2222.1988.tb02895.x

86. Sprince NL, Oliver LC, Eisen EA, Greene RE, Chamberlin RI. Cobalt exposure and lung disease in tungsten carbide production: A cross-sectional study of current workers. Am Rev Respir Dis. 1988; 138(5):1220-1226. http://dx.doi.org/10.1164/ajrccm/138.5.1220

87. Sundaram P, Agrawal K, Mandke JV, Joshi JM. Giant cell pneumonitis induced by cobalt. Indian J Chest Dis Allied Sci. 2001; 43:47-49.

88. Swennen B, Buchet J-P, Stánescu D, Lison D, Lauwerys R. Epidemiological survey of workers exposed to cobalt oxides, cobalt salts, and cobalt metal. Br J Ind Med. 1993; 50(9):835842. http://dx.doi.org/10.1136/oem.50.9.835

89. Tabatowski K, Roggli VL, Fulkerson WJ, Langley RL, Benning T, Johnston WW. Giant cell interstitial pneumonia in a hard-metal worker: Cytologic, histologic and analytical electron microscopic investigation. Acta Cytol. 1988; 32:240-246.

90. Talbot RJ, Morgan A. An interspecies comparison of the lung clearance of inhaled monodisperse cobalt oxide particles. Part VIII: Lung clearance of inhaled cobalt oxide particles in mice. J Aerosol Sci. 1989; 20:261-265. http://dx.doi.org/10.1016/0021-8502(89)90049-9

91. Van Cutsem EJ, Ceuppens JL, Lacquet LM, Demedts M. Combined asthma and alveolitis induced by cobalt in a diamond polisher. Eur J Respir Dis. 1987; 70:54-61.

92. Zanelli R, Barbic F, Migliori M, Michetti G. Uncommon evolution of fibrosing alveolitis in a hard metal grinder exposed to cobalt dusts. Sci Total Environ. 1994; 150(1-3):225-229. http://dx.doi.org/10.1016/0048-9697(94)90157-0

93. Wiberg GS. The effect of cobalt ions on energy metabolism in the rat. Can J Biochem. 1968; 46(6):549-554. http://dx.doi.org/10.1139/068-085

94. Di Giulio C, Huang WX, Lahiri S, Mokashi A, Buerk DG. Cobalt stimulates carotid body chemoreceptors. J Appl Physiol. 1990; 68(5):1844-1849.

http://dx.doi.org/10.1152/jappl.1990.68.5.1844 
95. Hatori N, Pehrsson K, Clyne N, Hansson G, Hofman-Bang C, Marklund SL, Rydén L, Sjöqvist P-O, Svensso L. Acute cobalt exposure and oxygen radical scavengers in the rat myocardium. Biochim Biophys Acta. 1993; 1181(3):257-260. http://dx.doi.org/10.1016/09254439(93)90029-Z

96. Morelli L, Di Giulio C, Iezzi M, Data PG. Effect of acute and chronic cobalt administration on carotid body chemoreceptors responses. Sci Total Environ. 1994; 150(1-3):215-216. http://dx.doi.org/10.1016/0048-9697(94)90153-8

97. Barborik M, Dusek J. Cardiomyopathy accompaning industrial cobalt exposure. Br Heart J. 1972; 34(1):113-116. http://dx.doi.org/10.1136/hrt.34.1.113

98. Horowitz SF, Fischbein A, Matza D, Rizzo JN, Stern A, Machac J, Solomon SJ. Evaluation of right and left ventricular function in hard metal workers. Br J Ind Med. 1988; 45(11):742-746. http://dx.doi.org/10.1136/oem.45.11.742

99. Jarvis JQ, Hammond E, Meier R, Robinson C. Cobalt cardiomyopathy: A report of two cases from mineral assay laboratories and a review of the literature. J Occup Med. 1992; 34(6):620626.

100. Webb M. The biological action of cobalt and other metals III. Chelation of cations by dihydrolipoic acid. Biochim Biophys Acta. 1962; 65(1):47-65. http://dx.doi.org/10.1016/0006$\underline{3002(62) 90148-8}$

101. Lehninger AL. Principles of biochemistry. New York, NY: Worth Publishers, Inc; 1982. p. 361-466.

102. Alexander CS. Cobalt-beer cardiomyopathy: A clinical and pathologic study of twentyeight cases. Am J Med. 1972; 53(4):395-417. http://dx.doi.org/10.1016/0002-9343(72)90136-2

103. Alexander CS. Cobalt and the heart. Ann Intern Med. 1969; 70(2):411-413. http://dx.doi.org/10.7326/0003-4819-70-2-411

104. Morin Y, Têtu A, Mercier G. Cobalt cardiomyopathy: Clinical aspects. Br Heart J. 1971; 33(Suppl):175-178. http://dx.doi.org/10.1136/hrt.33.Suppl.175

105. Palmes ED, Nelson N, Laskin S, Kuschner M. Inhalation toxicity of cobalt hydrocarbonyl. Am Ind Hyg Assoc J. 1959; 20(6):453-468. http://dx.doi.org/10.1080/00028895909343751

106. Stanley AJ, Hopps HC, Shideler AM. Cobalt polycythemia relative effects of oral and subcutaneous administration of cobaltous chloride. Proc Soc Exp Biol Med. 1947; 66(1):19-20. http://dx.doi.org/10.3181/00379727-66-15968

107. Lammintausta K, Pitkänen O-P, Kalimo K, Jansen CT. Interrelationship of nickel and cobalt contact sensitization. Contact Derm. 1985; 13(3):148-152. http://dx.doi.org/10.1111/j.1600$\underline{0536.1985 . t b 02527 . x}$

108. Alomar A, Conde-Salazar L, Romaguera C. Occupational dermatoses from cutting oils. Contact Derm. 1985; 12(3):129-138. http://dx.doi.org/10.1111/j.1600-0536.1985.tb01081.x 
109. Dooms-Goossens A, Ceuterick A, Vanmaele N, Degreef H. Follow-up study of patients with contact dermatitis caused by chromates, nickel, and cobalt. Dermatologica. 1980; 160:249260. http://dx.doi.org/10.1159/000250501

110. Fischer T, Rystedt I. Cobalt allergy in hard metal workers. Contact Dermatitis. 1983; 9(2):115-121. http://dx.doi.org/10.1111/j.1600-0536.1983.tb04316.x

111. Goh CL, Gan SL, Ngui SJ. Occupational dermatitis in a prefabrication construction factory. Contact Derm. 1986; 15:235-240. http://dx.doi.org/10.1111/j.1600-0536.1986.tb01343.x

112. Kanerva L, Estlander T, Jolanki R. Occupational skin disease in Finland: An analysis of 10 years of statistics from an occupational dermatology clinic. Int Arch Occup Environ Health. 1988; 60(2):89-94. http://dx.doi.org/10.1007/BF00381486

113. Marcussen PV. Cobalt dermatitis. Clinical picture. Acta Derm Venereol. 1963; 43:231-234.

114. Valér M, Somogyi Z, Racz I. Studies concerning the sensitizing effect of cobalt.

Dermatologica. 1967; 134:36-50. http://dx.doi.org/10.1159/000254236

115. Bencko V, Wagner V, Wagnerová M, Reichrtová E. Immuno-biochemical findings in groups of individuals occupationally and non-occupationally exposed to emissions containing nickel and cobalt. J Hyg Epidemiol Microbiol Immunol. 1983; 27(4):387-394.

116. Katsarou A, Baxevanis C, Armenaka M, Volonakis M, Balamotis A, Papamihail M. Study of persistence and loss of patch test reactions to dichromate and cobalt. Contact Derm. 1997; 36(2):87-90. http://dx.doi.org/10.1111/j.1600-0536.1997.tb00419.x

117. Löfström A, Wigzell H. Antigen specific human T cell lines for cobalt chloride. Acta Derm Venereol. 1986; 66:200-206.

118. Cirla AM. Cobalt-related asthma: Clinical and immunological aspects. Sci Total Environ. 1994; 150(1-3):85-94. http://dx.doi.org/10.1016/0048-9697(94)90133-3

119. Shrivastava VK, David CV, Khare N, Khare N. Cobalt chloride induced histopathological changes in thyroid gland of female mice, Mus musculus. Pollut Res. 1996; 15:307-309.

120. Pedigo NG, George WJ, Anderson MB. Effects of acute and chronic exposure to cobalt on male reproduction in mice. Reprod Toxicol. 1988; 2(1):45-53. http://dx.doi.org/10.1016/S0890$\underline{6238(88) 80008-X}$

121. Bucher JR, Hailey JR, Roycroft JR, Haseman JK, Sills RC, Grumbein SL, Mellick PW, Chou BJ. Inhalation toxicity and carcinogenicity studies of cobalt sulfate. Toxicol Sci. 1999; 49(1):56-67. http://dx.doi.org/10.1093/toxsci/49.1.56

122. National Toxicology Program (NTP). Toxicology and carcinogenesis studies of cobalt sulfate heptahydrate (CAS No. 10026-24-1) in F344/N rats and B6C3F1 mice (inhalation studies). Research Triangle Park, NC: U.S. Department of Health and Human Services, Public Health Service, National Institutes of Health; 1998. Technical Report Series No. 471. NIH Publication No. 98-3961. 
123. Szakmáry É, Ungváry G, Hudák A, Tátrai E, Náray M, Morvai V. Effects of cobalt sulfate on prenatal development of mice, rats, and rabbits, and on early postnatal development of rats. $\mathrm{J}$ Toxicol Environ Health, A. 2001; 62(5):367-386.

http://dx.doi.org/10.1080/152873901300018110

124. Jordan C, Whitman RD, Harbut M, Tanner B. Memory deficits in workers suffering from hard metal disease. Toxicol Lett. 1990; 54(2-3):241-243. http://dx.doi.org/10.1016/03784274(90)90190-W

125. Meecham HM, Humphrey P. Industrial exposure to cobalt causing optic atrophy and nerve deafness: A case report. J Neurol Neurosurg Psychiatry. 1991; 54(4):374-375.

http://dx.doi.org/10.1136/jnnp.54.4.374

126. Speijers GJA, Krajnc EI, Berkvens JM, Van Logten MJ. Acute oral toxicity of inorganic cobalt compounds in rats. Food Chem Toxicol. 1982; 20(3):311-314.

http://dx.doi.org/10.1016/S0278-6915(82)80298-6

127. Murdock HR, Jr. Studies on the pharmacology of cobalt chloride. J Am Pharm Assoc. 1959; 48(3):140-142. http://dx.doi.org/10.1002/jps.3030480303

128. Domingo JL, Llobet JM, Bernat R. A study of the effects of cobalt administered orally to rats. Arch Farmacol Toxicol. 1984; 10(1):13-20.

129. Holly RG. Studies on iron and cobalt metabolism. J Am Med Assoc. 1955; 158(15):13491352. http://dx.doi.org/10.1001/jama.1955.02960150019005

130. Krasovskii GN, Fridlyand SA. Experimental data for the validation of the maximum permissible concentration of cobalt in water bodies. Hyg Sanit. 1971; 26:277-279.

131. Corrier DE, Mollenhauer HH, Clark DE, Hare MF, Elissalde MH. Testicular degeneration and necrosis induced by dietary cobalt. Vet Pathol. 1985; 22(6):610-616.

http://dx.doi.org/10.1177/030098588502200616

132. Mollenhauer HH, Corrier DE, Clark DE, Hare MF, Elissalde MH. Effects of dietary cobalt on testicular structure. Virchows Archiv B. 1985; 49(1):241-248.

http://dx.doi.org/10.1007/BF02912101

133. Nation JR, Bourgeois AE, Clark DE, Hare MF. The effects of chronic cobalt exposure on behavior and metallothionein levels in the adult rat. Neurobehav Toxicol Teratol. 1983; (5):9-15. http://dx.doi.org/10.1016/0378-4274(83)90357-0

134. Anderson MB, Lepak K, Farinas V, George WJ. Protective action of zinc against cobaltinduced testicular damage in the mouse. Reprod Toxicol. 1993; 7(1):49-54.

http://dx.doi.org/10.1016/0890-6238(93)90009-V

135. Anderson MB, Pedigo NG, Katz RP, George WJ. Histopathology of testes from mice chronically treated with cobalt. Reprod Toxicol. 1992; 6(1):41-50.

http://dx.doi.org/10.1016/0890-6238(92)90019-P 
136. Domingo JL, Paternain JL, Llobet JM, Corbella J. Effects of cobalt on postnatal development and late gestation in rats upon oral administration. Rev Esp Fisiol. 1985; 41:293298.

137. Paternain JL, Domingo JL, Corbella J. Developmental toxicity of cobalt in the rat. J Toxicol Environ Health. 1988; 24(2):193-200. http://dx.doi.org/10.1080/15287398809531153

138. Pedigo NG, Vernon MW. Embryonic losses after 10-week administration of cobalt to male mice. Reprod Toxicol. 1993; 7(2):111-116. http://dx.doi.org/10.1016/0890-6238(93)90244-2

139. Seidenberg JM, Anderson DG, Becker RA. Validation of an in vivo developmental toxicity screen in the mouse. Teratog Carcinog Mutagen. 1986; 6(5):361-374.

http://dx.doi.org/10.1002/tcm.1770060503

140. Gilman JPW. Metal carcinogenesis: II. A study on the carcinogenic activity of cobalt, copper, iron, and nickel compounds. Cancer Res. 1962; 22(2):158-162.

141. Gilman JPW, Ruckerbauer GM. Metal carcinogenesis: I. Observations on the carcinogenicity of a refinery dust, cobalt oxide, and colloidal thorium dioxide. Cancer Res. 1962; 22(2):152-157.

142. Heath JC. The production of malignant tumours by cobalt in the rat. Br J Cancer. 1956; 10:668-673. http://dx.doi.org/10.1038/bjc.1956.80

143. Heath JC. The histogenesis of malignant tumours induced by cobalt in the rat. Br J Cancer. 1960; 14:478-482. http://dx.doi.org/10.1038/bjc.1960.52

144. Heath JC, Daniel MR. The production of malignant tumours by cobalt in the rat: Intrathoracic tumours. Br J Cancer. 1962; 16(3):473-478. http://dx.doi.org/10.1038/bjc.1962.53

145. Shabaan AA, Marks V, Lancaster MC, Dufeu GN. Fibrosarcomas induced by cobalt chloride (CoCl2) in rats. Lab Anim. 1977; 11(1):43-46.

146. Jensen AA, Tüchsen F. Cobalt exposure and cancer risk. Crit Rev Toxicol. 1990; 20(6):427439. http://dx.doi.org/10.3109/10408449009029330

147. Payne LR. The hazards of cobalt. J Soc Occup Med. 1977; 27(1):20-25. http://dx.doi.org/10.1093/occmed/27.1.20

148. Mur JM, Moulin JJ, Charruyer-Seinerra MP, Lafitte J. A cohort mortality study among cobalt and sodium workers in an electrochemical plant. Am J Ind Med. 1987; 11(1):75-81. http://dx.doi.org/10.1002/ajim.4700110108

149. Moulin JJ, Wild P, Mur JM, Fournier-Betz M, Mercier-Gallay M. A mortality study of cobalt production workers: An extension of the follow-up. Am J Ind Med. 1993; 23(2):281-288. http://dx.doi.org/10.1002/ajim.4700230205

150. National Toxicology Program (NTP). 12th Report on Carcinogens. Research Triangle Park, NC: U.S. Department of Health and Human Services, Public Health Service, National Institutes of Health; 2011. 
151. Lison D, Lauwerys R. Study of the mechanism responsible for the elective toxicity of tungsten carbide-cobalt powder toward macrophages. Toxicol Lett. 1992; 60(2):203-210. http://dx.doi.org/10.1016/0378-4274(92)90275-O

152. De Boeck M, Kirsch-Volders M, Lison D. Cobalt and antimony: Genotoxicity and carcinogenicity. Mutat Res. 2003; 533(1-2):135-152.

http://dx.doi.org/10.1016/j.mrfmmm.2003.07.012

153. Lison D, De Boeck M, Verougstraete V, Kirsch-Volders M. Update on the genotoxicity and carcinogenicity of cobalt compounds. Occup Environ Med. 2001; 58(10):619-625. http://dx.doi.org/10.1136/oem.58.10.619

154. Simonsen LO, Harbak H, Bennekou P. Cobalt metabolism and toxicology: A brief update. Sci Total Environ. 2012; 432:210-215. http://dx.doi.org/10.1016/j.scitotenv.2012.06.009

155. Anard D, Kirsch-Volders M, Elhajouji A, Belpaeme K, Lison D. In vitro genotoxic effects of hard metal particles assessed by alkaline single cell gel and elution assays. Carcinogenesis. 1997; 18:177-184. http://dx.doi.org/10.1093/carcin/18.1.177

156. Van Goethem F, Lison D, Kirsch-Volders M. Comparative evaluation of the in vitro micronucleus test and the alkaline single cell gel electrophoresis assay for the detection of DNA damaging agents: Genotoxic effects of cobalt powder, tungsten carbide and cobalt-tungsten carbide. Mutat Res Genet Toxicol Environ Mutagen. 1997; 392(1-2):31-43. http://dx.doi.org/10.1016/S0165-1218(97)00043-8

157. De Boeck M, Lombaert N, De Backer S, Finsy R, Lison D, Kirsch-Volders M. In vitro genotoxic effects of different combinations of cobalt and metallic carbide particles. Mutagenesis. 2003; 18(2):177-186. http://dx.doi.org/10.1093/mutage/18.2.177

158. Hartwig A, Asmuss M, Blessing H, Hoffmann S, Jahnke G, Khandelwal S, Pelzer A, Bürkle A. Interference by toxic metal ions with zinc-dependent proteins involved in maintaining genomic stability. Food Chem Toxicol. 2002; 40(8):1179-1184. http://dx.doi.org/10.1016/S0278$\underline{6915(02) 00043-1}$

159. Buchko GW, Hess NJ, Bandaru V, Wallace SS, Kennedy MA. Spectroscopic studies of zinc(II)- and cobalt(II)- associated escherichia coli formamidopyrimidine-DNA glycosylase: Extended x-ray absorption fine structure evidence for a metal-binding domain. Biochemistry. 2000; 39(40):12441-12449. http://dx.doi.org/10.1021/bi001377k

160. Kopera E, Schwerdtle T, Hartwig A, Bal W. Co(II) and Cd(II) substitute for Zn(II) in the zinc finger derived from the DNA repair protein XPA, demonstrating a variety of potential mechanisms of toxicity. Chem Res Toxicol. 2004; 17(11):1452-1458.

http://dx.doi.org/10.1021/tx049842s

161. Asmuss M, Mullenders LHF, Eker A, Hartwig A. Differential effects of toxic metal compounds on the activities of Fpg and XPA, two zinc finger proteins involved in DNA repair. Carcinogenesis. 2000; 21:2097-2104. http://dx.doi.org/10.1093/carcin/21.11.2097

162. De Boeck M, Lison D, Kirsch-Volders M. Evaluation of the in vitro direct and indirect genotoxic effects of cobalt compounds using the alkaline comet assay. Influence of interdonor 
and interexperimental variability. Carcinogenesis. 1998; 19(11):2021-2029. http://dx.doi.org/10.1093/carcin/19.11.2021

163. De Boeck M, Lardau S, Buchet J-P, Kirsch-Volders M, Lison D. Absence of significant genotoxicity in lymphocytes and urine from workers exposed to moderate levels of cobaltcontaining dust: A cross-sectional study. Environ Mol Mutag. 2000; 36(2):151-160. http://dx.doi.org/10.1002/1098-2280(2000)36:2<151::AID-EM10>3.0.CO;2-V

164. Griffis LC, Wolff RK, Beethe RL, Hobbs CH, McClellan RO. Evaluation of a multitiered inhalation exposure chamber. Toxicol Sci. 1981; 1(1):8-12. http://dx.doi.org/10.1093/toxsci/1.1.8

165. Hill MA, Watson CR, Moss OR. An interactive computer program for particle size analysis. Richland, WA: Pacific Northwest Laboratory; 1977. PNL-2405. UC-32.

166. King-Herbert A, Thayer K. NTP workshop: Animal models for the NTP rodent cancer bioassay: Stocks and strains--should we switch? Toxicol Pathol. 2006; 34(6):802-805. http://dx.doi.org/10.1080/01926230600935938

167. Brecher G, Schneiderman M. A time-saving device for the counting of reticulocytes. Am J Clin Pathol. 1950; 20(11_ts):1079-1083. http://dx.doi.org/10.1093/ajcp/20.11 ts.1079

168. Pearce RE, McIntyre CJ, Madan A, Sanzgiri U, Draper AJ, Bullock PL, Cook DC, Burton LA, Latham J, Nevins C et al. Effects of freezing, thawing, and storing human liver microsomes on cytochrome P450 activity. Arch Biochem Biophys. 1996; 331(2):145-169.

http://dx.doi.org/10.1006/abbi.1996.0294

169. Maronpot RR, Boorman GA. Interpretation of rodent hepatocellular proliferative alterations and hepatocellular tumors in chemical safety assessment. Toxicol Pathol. 1982; 10(2):71-78. http://dx.doi.org/10.1177/019262338201000210

170. Boorman GA, Montgomery CA, Jr., Eustis SL, Wolfe MJ, McConnell EE, Hardisty JF. Quality assurance in pathology for rodent carcinogenicity studies In: Milman HA, Weisburger EK, editors. Handbook of Carcinogen Testing. Park Ridge, NJ: Noyes Publications; 1985. p. 345-357.

171. McConnell EE, Solleveld HA, Swenberg JA, Boorman GA. Guidelines for combining neoplasms for evaluation of rodent carcinogenesis studies. JNCI. 1986; 76(2):283-289.

172. Kaplan EL, Meier P. Nonparametric estimation from incomplete observations. J Am Stat Assoc. 1958; 53(282):457-481. http://dx.doi.org/10.1080/01621459.1958.10501452

173. Cox DR. Regression models and life-tables. J R Stat Soc Ser B. 1972; 34(2):187-220.

174. Tarone RE. Tests for trend in life table analysis. Biometrika. 1975; 62:679-682. http://dx.doi.org/10.1093/biomet/62.3.679

175. Bailer AJ, Portier C. Effects of treatment-induced mortality and tumor-induced mortality on tests for carcinogenicity in small samples. Biometrics. 1988; 44:417-431.

http://dx.doi.org/10.2307/2531856 
176. Piegorsch W, Bailer AJ. Statistics for environmental biology and toxicology. Section 6.3.2. London, UK: Chapman and Hall; 1997.

177. Portier CJ, Bailer AJ. Testing for increased carcinogenicity using a survival-adjusted quantal response test. Fundam Appl Toxicol. 1989; 12(4):731-737.

http://dx.doi.org/10.1016/0272-0590(89)90004-3

178. Portier CJ, Hedges JC, Hoel DG. Age-specific models of mortality and tumor on-set for historical control animals in the National Toxicology Program's carcinogenicity experiments. Cancer Res. 1986; 46(9):4372-4378.

179. Bieler GS, Williams RL. Ratio estimates, the delta method, and quantal response tests for increased carcinogenicity. Biometrics. 1993; 49:793-801. http://dx.doi.org/10.2307/2532200

180. Dunnett CW. A multiple comparison procedure for comparing several treatments with a control. J Am Stat Assoc. 1955; 50(272):1096-1121. http://dx.doi.org/10.1080/01621459.1955.10501294

181. Williams DA. A test for differences between treatment means when several dose levels are compared with a zero dose control. Biometrics. 1971; 27:103-117.

http://dx.doi.org/10.2307/2528930

182. Williams DA. The comparison of several dose levels with a zero dose control. Biometrics. 1972; 28:519-531. http://dx.doi.org/10.2307/2556164

183. Shirley E. A non-parametric equivalent of Williams' Test for contrasting increasing dose levels of a treatment. Biometrics. 1977; 33:386-389. http://dx.doi.org/10.2307/2529789

184. Williams DA. A note on Shirley's nonparametric test for comparing several dose levels with a zero-dose control. Biometrics. 1986; 42:183-186. http://dx.doi.org/10.2307/2531254

185. Dunn OJ. Multiple comparisons using rank sums. Technometrics. 1964; 6:241-252. http://dx.doi.org/10.1080/00401706.1964.10490181

186. Jonckheere AR. A distribution-free k-sample test against ordered alternatives. Biometrika. 1954; 41(1-2):133-145. http://dx.doi.org/10.1093/biomet/41.1-2.133

187. Dixon WJ, Massey FJ, Jr. Introduction to statistical analysis. 2nd ed. New York, NY: McGraw-Hill Book Company, Inc; 1957. http://dx.doi.org/10.2307/2332898

188. Gart JJ, Chu KC, Tarone RE. Statistical issues in interpretation of chronic bioassay tests for carcinogenicity. JNCI. 1979; 62(4):957-974.

189. Girard DM, Sager DB. The use of Markov chains to detect subtle variation in reproductive cycling. Biometrics. 1987; 43:225-234. http://dx.doi.org/10.2307/2531963

190. Haseman JK. Value of historical controls in the interpretation of rodent tumor data. Drug Inf J. 1992; 26(2):191-200. http://dx.doi.org/10.1177/009286159202600210

191. Haseman JK. Data analysis: Statistical analysis and use of historical control data. Regul Toxicol Pharm. 1995; 21(1):52-59. http://dx.doi.org/10.1006/rtph.1995.1009 
192. Haseman JK, Rao GN. Effects of corn oil, time-related changes, and inter-laboratory variability on tumor occurrence in control Fischer $344(\mathrm{~F} 344 / \mathrm{N})$ rats. Toxicol Pathol. 1992; 20(1):52-60. http://dx.doi.org/10.1177/019262339202000107

193. Code of Federal Regulations (CFR). 21:Part 58.

194. Heddle JA, Hite M, Kirkhart B, Mavournin K, MacGregor JT, Newell GW, Salamone MF. The induction of micronuclei as a measure of genotoxicity: A report of the U.S. Environmental Protection Agency Gene-Tox program. Mutat Res. 1983; 123(1):61-118.

http://dx.doi.org/10.1016/0165-1110(83)90047-7

195. Schmid W. The micronucleus test. Mutat Res. 1975; 31(1):9-15.

http://dx.doi.org/10.1016/0165-1161(75)90058-8

196. Miller JA, Miller EC. Ultimate chemical carcinogens as reactive mutagenic electrophiles In: Hiatt HH, Watson JD, Winsten JA, editors. Origins of Human Cancer. Cold Spring Harbor, NY: Cold Spring Harbor Laboratory; 1977. p. 605-627.

197. Crawford BD. Perspectives on the somatic mutation model of carcinogenesis In: Mehlman MA, Flamm WG, Lorentzen RJ, editors. Advances in Modern Environmental Toxicology Mechanisms and Toxicity of Chemical Carcinogens and Mutagens. Princeton, NJ: Princeton Scientific Publishing Co. Inc; 1985. p. 13-59.

198. Straus DS. Somatic mutation, cellular differentiation, and cancer causation. JNCI. 1981; 67:233-241.

199. Ashby J, Tennant RW. Definitive relationships among chemical structure, carcinogenicity and mutagenicity for 301 chemicals tested by the U.S. NTP. Mutat Res. 1991; 257(3):229-306. http://dx.doi.org/10.1016/0165-1110(91)90003-E

200. Tennant RW, Margolin BH, Shelby MD, Zeiger E, Haseman JK, Spalding J, Caspary W, Resnick M, Stasiewicz S, Anderson B et al. Prediction of chemical carcinogenicity in rodents from in vitro genetic toxicity assays. Science. 1987; 236(4804):933-941.

http://dx.doi.org/10.1126/science.3554512

201. Zeiger E, Haseman JK, Shelby MD, Margolin BH, Tennant RW, Holden HE. Evaluation of four in vitro genetic toxicity tests for predicting rodent carcinogenicity: Confirmation of earlier results with 41 additional chemicals. Environ Mol Mutag. 1990; 16(S18):1-14. http://dx.doi.org/10.1002/em.2850160502

202. Shelby MD, Erexson GL, Hook GJ, Tice RR. Evaluation of a three-exposure mouse bone marrow micronucleus protocol: Results with 49 chemicals. Environ Mol Mutag. 1993; 21(2):160-179. http://dx.doi.org/10.1002/em.2850210210

203. Shelby MD, Witt KL. Comparison of results from mouse bone marrow chromosome aberration and micronucleus tests. Environ Mol Mutag. 1995; 25(4):302-313. http://dx.doi.org/10.1002/em.2850250407

204. Witt KL, Knapton A, Wehr CM, Hook GJ, Mirsalis J, Shelby MD, MacGregor JT. Micronucleated erythrocyte frequency in peripheral blood of $\mathrm{B} 6 \mathrm{C} 3 \mathrm{~F} 1$ mice from short-term, prechronic, and chronic studies of the NTP carcinogenesis bioassay program. Environ Mol 
Mutag. 2000; 36(3):163-194. http://dx.doi.org/10.1002/1098-2280(2000)36:3<163::AIDEM1>3.0.CO;2-P

205. Jain NC. Schalm's veterinary hematology. 4th ed. Philadelphia: Lea \& Febiger; 1986. p. 507-508.

206. Goldwasser E, Jacobson LO, Fried W, Plzak LF. Studies on erythropoiesis: V. The effect of cobalt on the production of erythropoietin. Blood. 1958; 13(1):55.

207. Schofield CJ, Ratcliffe PJ. Oxygen sensing by HIF hydroxylases. Nat Rev Mol Cell Biol. 2004; 5:343-354. http://dx.doi.org/10.1038/nrm1366

208. Epstein ACR, Gleadle JM, McNeill LA, Hewitson KS, O'Rourke J, Mole DR, Mukherji M, Metzen E, Wilson MI, Dhanda A et al. C. elegans EGL-9 and mammalian homologs define a family of dioxygenases that regulate HIF by prolyl hydroxylation. Cell. 2001; 107(1):43-54. http://dx.doi.org/10.1016/S0092-8674(01)00507-4

209. Yuan Y, Hilliard G, Ferguson T, Millhorn DE. Cobalt inhibits the interaction between hypoxia-inducible factor-alpha and von Hippel-Lindau protein by direct binding to hypoxiainducible factor alpha. J Biol Chem. 2003; 278:15911-15916.

http://dx.doi.org/10.1074/jbc.M300463200

210. Taylor A, Marks V. Cobalt: A review. J Hum Nutr. 1978; 32:165-177.

211. Nomura Y, Okamoto S-i, Sakamoto M, Feng Z, Nakamura T. Effect of cobalt on the liver glycogen content in the streptozotocin-induced diabetic rats. Mol Cell Biochem. 2005; 277:127130. http://dx.doi.org/10.1007/s11010-005-5777-y

212. Saker F, Ybarra J, Leahy P, Hanson RW, Kalhan SC, Ismail-Beigi F. Glycemia-lowering effect of cobalt chloride in the diabetic rat: Role of decreased gluconeogenesis. Am J Physiol. 1998; 274(6):E984-E991. http://dx.doi.org/10.1152/ajpendo.1998.274.6.E984

213. Ybarra J, Behrooz A, Gabriel A, Koseoglu MH, Ismail-Beigi F. Glycemia-lowering effect of cobalt chloride in the diabetic rat: Increased GLUT1 mRNA expression. Mol Cell Endocrinol. 1997; 133(2):151-160. http://dx.doi.org/10.1016/S0303-7207(97)00162-7

214. Kawakami T, Hanao N, Nishiyama K, Kadota Y, Inoue M, Sato M, Suzuki S. Differential effects of cobalt and mercury on lipid metabolism in the white adipose tissue of high-fat dietinduced obesity mice. Toxicol Appl Pharmacol. 2012; 258(1):32-42.

http://dx.doi.org/10.1016/j.taap.2011.10.004

215. Li M, Kim DH, Tsenovoy PL, Peterson SJ, Rezzani R, Rodella LF, Aronow WS, Ikehara S, Abraham NG. Treatment of obese diabetic mice with a heme oxygenase inducer reduces visceral and subcutaneous adiposity, increases adiponectin levels, and improves insulin sensitivity and glucose tolerance. Diabetes. 2008; 57(6):1526-1535. http://dx.doi.org/10.2337/db07-1764

216. Nicolai A, Li M, Kim DH, Peterson SJ, Vanella L, Positano V, Gastaldelli A, Rezzani R, Rodella LF, Drummond G et al. Heme oxygenase-1 induction remodels adipose tissue and improves insulin sensitivity in obesity-induced diabetic rats. Hypertension. 2009; 53(3):508-515. http://dx.doi.org/10.1161/HYPERTENSIONAHA.108.124701 
217. Kumar GP, Laloraya M, Laloraya MM. Powerful anti-sperm motility action of cobaltous ion and its recovery by a sulfhydryl compound. Contraception. 1990; 41(6):633-639. http://dx.doi.org/10.1016/S0010-7824(09)91008-3

218. Gray J, Nicholson JK, Creasy DM, Timbrell JA. Studies on the relationship between acute testicular damage and urinary and plasma creatine concentration. Arch Toxicol. 1990; 64(6):443450. http://dx.doi.org/10.1007/BF01977625

219. Härkönen M, Kormano M. Acute cadmium-induced changes in the energy metabolism of the rat testis. J Repord Fertil. 1970; 21(2):221-226.

220. Caltana L, Merelli A, Lazarowski A, Brusco A. Neuronal and glial alterations due to focal cortical hypoxia induced by direct cobalt chloride $(\mathrm{CoCl} 2)$ brain injection. Neurotox Res. 2009; 15(4):348-358. http://dx.doi.org/10.1007/s12640-009-9038-9

221. National Toxicology Program (NTP). Toxicology and carcinogenesis studies of gallium arsenide (CAS No. 1303-00-0) in F344/N rats and B6C3F1 mice (inhalation studies). Research Triangle Park, NC: U.S. Department of Health and Human Services, Public Health Service, National Institutes of Health; 2000. Technical Report Series No. 492. NIH Publication No. 003951.

222. National Toxicology Program (NTP). Toxicology and carcinogenesis studies of indium phosphide (CAS No. 22398-80-7) in F344/N rats and B6C3F1 mice (inhalation studies). Research Triangle Park, NC.: U.S. Department of Health and Human Services, Public Health Service, National Institutes of Health; 2001. Technical Report Series No. 499. NIH Publication No. 01-4433.

223. Boorman GA, Brockmann M, Carlton WW, Davis JMG, Dungworth DL, Hahn FF, Mohr U, Reichhelm H-BR, Turusov VS, Wagner BM. Classification of cystic keratinizing squamous lesions of the rat lung: Report of a workshop. Toxicol Pathol. 1996; 24(5):564-572. http://dx.doi.org/10.1177/019262339602400505

224. Rittinghausen S, Dungworth DL, Ernst H, Mohr U. Primary pulmonary tumors In: Mohr U, Dungworth DL, Capen CC, editors. Pathobiology of the Aging Rat. Washington, DC: ILSI Press; 1992. p. 161-172.

225. Rittinghausen S, Kaspareit J. Spontaneous cystic keratinizing epithelioma in the lung of a Sprague-Dawley rat. Toxicol Pathol. 1998; 26(2):298-300.

http://dx.doi.org/10.1177/019262339802600218

226. Hobbs CH, Abdo KM, Hahn FF, Gillett NA, Eustis SL, Jones RK, Benson JM, Barr EB, Dieter MP, Pickrell JA et al. Summary of the chronic inhalation toxicity of talc in F344/N rats and B6C3F1 mice In: Mohr U, Dungworth DL, Mauderly JL, Oberdorster G, editors. Toxic and Carcinogenic Effects of Solid Particles in the Respiratory Tract. Washington, D.C.: ILSI Press; 1993. p. 525-528.

227. Lee KP, Trochimowicz HJ, Reinhardt CF. Pulmonary response of rats exposed to titanium dioxide (TiO2) by inhalation for two years. Toxicol Appl Pharmacol. 1985; 79(2):179-192. http://dx.doi.org/10.1016/0041-008X(85)90339-4 
228. Martin JC, Daniel H, LeBouffant L. Short- and long- term experimental study of the toxicity of coal-mine dust and some of its constituents In: Walton WH, editor. Inhaled Particles IV (Part I) Proceedings of an International Symposium organized by the British Occupational Hygiene Society, Edinburg, September 22-26, 1975. Pergamon Press; 1977. p. 361-370.

229. Muhle H, Takenaka S, Mohr U, Dasenbrock C, Mermelstein R. Lung tumor induction upon long-term low-level inhalation of crystalline Silica. Am J Ind Med. 1989; 15(3):343-346. http://dx.doi.org/10.1002/ajim.4700150309

230. Pott F, Dungworth DL, Heinrich U, Muhle H, Kamino K, Germann PG, Roller M, Rippe $\mathrm{RM}$, Mohr U. Lung tumours in rats after intratracheal instillation of dusts. Ann Occup Hyg. 1994; 38:357-363.

231. Mauderly JL, Snipes MB, Barr EB, Belinsky SA, Bond JA, Brooks AL, Chang IY, Cheng YS, Gillett NA, Griffith WC et al. Pulmonary toxicity of inhaled diesel exhaust and carbon black in chronically exposed rats. Part I: Neoplastic and nonneoplastic lung lesions. Res Rep Health Eff Inst. 1994; (68 Pt 1):1-75; 77-97.

232. Dixon D, Herbert RA, Kissling GE, Brix AE, Miller RA, Maronpot RR. Summary of chemically induced pulmonary lesions in the National Toxicology Program (NTP) toxicology and carcinogenesis studies. Toxicol Pathol. 2008; 36(3):428-439.

http://dx.doi.org/10.1177/0192623308315360

233. National Toxicology Program (NTP). Toxicology and carcinogenesis studies of 2,2',4,4',5,5'-hexachlorobiphenyl (PCB 153) (CAS No. 35065-27-1) in female Harlan SpragueDawley rats (gavage studies). Research Triangle Park, NC: U.S. Department of Health and Human Services, Public Health Service, National Institutes of Health; 2006. Technical Report Series No. 529. NIH Publication No. 06-4465.

234. National Toxicology Program (NTP). Toxicology and carcinogenesis studies of 3,3',4,4',5pentachlorobiphenyl (PCB 126) (CAS No. 57465-28-8) in female Harlan Sprague-Dawley rats (gavage studies). Research Triangle Park, NC: U.S. Department of Health and Human Services, Public Health Service, National Institutes of Health; 2006. Technical Report Series No. 520. NIH Publication No. 06-4454.

235. National Toxicology Program (NTP). Toxicology and carcinogenesis studies of 2,3,7,8tetrachlorodibenzo-p-dioxin (TCDD) (CAS No. 1746-01-6) in female Harlan Sprague-Dawley rats (gavage studies). Research Triangle Park, NC: U.S. Department of Health and Human Services, Public Health Service, National Institutes of Health; 2006. Technical Report Series No. 521. NIH Publication No. 06-4468.

236. National Toxicology Program (NTP). Toxicology and carcinogenesis studies of a binary mixture PCB 126, (CAS Nos. 57465-28-8 and 35065-27-1) in female Harlan Sprague-Dawley rats (gavage studies). Research Triangle Park, NC: U.S. Department of Health and Human Services, Public Health Service, National Institutes of Health; 2006. Technical Report Series No. 530. NIH Publication No. 06-4466.

237. National Toxicology Program (NTP). Toxicology and carcinogenesis studies of a binary mixture PCB 126 and PCB 118, (CAS Nos. 57465-28-8 and 31508-00-6) in female Harlan Sprague-Dawley rats (gavage studies). Research Triangle Park, NC: U.S. Department of Health 
and Human Services, Public Health Service, National Institutes of Health; 2006. Technical Report Series No. 531. NIH Publication No. 07-4467.

238. National Toxicology Program (NTP). Toxicology and carcinogenesis studies of 3,3',4,4'tetrachloroazobenzene (TCAB) (CAS No. 14047-09-7) in Harlan Sprague-Dawley rats and B6C3F1 mice (gavage studies). Research Triangle Park, NC: U.S. Department of Health and Human Services, Public Health Service, National Institutes of Health; 2010. Technical Report Series No. 558. NIH Publication No. 11-5899.

239. National Toxicology Program (NTP). Toxicology and carcinogenesis studies of 2,3',4,4',5pentachlorobiphenyl (PCB 118) (CAS No. 31508-00-6) in female Harlan Sprague-Dawley rats (gavage studies). Research Triangle Park, NC: U.S. Department of Health and Human Services, Public Health Service, National Institutes of Health; 2010. Technical Report Series No. 559. NIH Publication No. 11-5900.

240. National Toxicology Program (NTP). Toxicology and carcinogenesis studies of talc (CAS No. 14807-96-6) in F344/N rats and B6C3F1 mice (inhalation studies). Research Triangle Park, NC: U.S. Department of Health and Human Services, Public Health Service, National Institutes of Health; 1993. Technical Report Series No. 421. NIH Publication No. 93-3152.

241. National Toxicology Program (NTP). Toxicology and carcinogenesis studies of nickel subsulfide (CAS No. 12035-72-2) in F344/N rats and B6C3F1 mice (inhalation studies). Research Triangle Park, NC: U.S. Department of Health and Human Services, Public Health Service, National Institutes of Health; 1996. Technical Report Series No. 453. NIH Publication No. 96-3369.

242. National Toxicology Program (NTP). Toxicology and carcinogenesis studies of nickel sulfate hexahydrate (CAS No. 10101-97-0) in F344/N rats and B6C3F1 mice (inhalation studies). National Institutes of Health, Research Triangle Park, NC: U.S. Department of Health and Human Services, Public Health Service; 1996. Technical Report Series No. 454. NIH Publication No. 96-3370.

243. National Toxicology Program (NTP). Toxicology and carcinogenesis studies of molybdenum trioxide (CAS No. 1313-27-5) in F344/N rats and B6C3F1 mice (inhalation studies). Research Triangle Park, NC: U.S. Department of Health and Human Services, Public Health Service, National Institutes of Health; 1997. Technical Report Series No. 462. NIH Publication No. 97-3378.

244. Ozaki K, Haseman JK, Hailey JR, Maronpot RR, Nyska A. Association of adrenal pheochromocytoma and lung pathology in inhalation studies with particulate vompounds in the male F344 rat: The National Toxicology Program experience. Toxicol Pathol. 2002; 30(2):263270. http://dx.doi.org/10.1080/019262302753559605

245. Collier CG, Hodgson A, Gray SA, Moody JC, Ball A. The lung clearance kinetics of

${ }^{57} \mathrm{Co}_{3} \mathrm{O}_{4}$ in rats of various ages. J Aerosol Sci. 1991; 22(4):537-549. http://dx.doi.org/10.1016/0021-8502(91)90009-7

246. Lehnert BE, Morrow PE. The initial lag in phagocytic rate by macrdphages in monolayer is related to particle encounters and binding. Immunol Invest. 1985; 14(6):515-521.

http://dx.doi.org/10.3109/08820138509022680 
247. Lehnert BE, Morrow PE. Characteristics of alveolar macrophages following the deposition of a low burden of iron oxide in the lung. J Toxicol Environ Health. 1985; 16(6):855-868. http://dx.doi.org/10.1080/15287398509530793

248. Morrow PE. Possible mechanisms to explain dust overloading of the lungs. Fundam Appl Toxicol. 1988; 10:369-384. http://dx.doi.org/10.1016/0272-0590(88)90284-9

249. Morrow PE. Dust overloading of the lungs: Update and appraisal. Toxicol Appl Pharmacol. 1992; 113(1):1-12. http://dx.doi.org/10.1016/0041-008X(92)90002-A

250. Takahashi H, Koshi K. Solubility and cell toxicity of cobalt, zinc and lead. Ind Health. 1981; 19:47-59. http://dx.doi.org/10.2486/indhealth.19.47

251. Rae T. A study on the effects of particulate metals of orthopaedic interest on murine macrophages in vitro. J Bone Joint Surg Br. 1975; 57:444-450. http://dx.doi.org/10.1302/0301620X.57B 4.444

252. Stopford W, Turner J, Cappellini D, Brock T. Bioaccessibility testing of cobalt compounds. J Environ Monit. 2003; 5(4):675-680. http://dx.doi.org/10.1039/b302257a

253. Lee W, Ladanyi M. Genomics traces carcinogen fingerprints. Sci Transl Med. 2013; 5(197):197fs31. http://dx.doi.org/10.1126/scitranslmed.3006810

254. Rodin SN, Rodin AS. Human lung cancer and p53: The interplay between mutagenesis and selection. Proc Natl Acad Sci USA. 2000; 97(22):12244-12249.

http://dx.doi.org/10.1073/pnas.180320897

255. Suda K, Tomizawa K, Mitsudomi T. Biological and clinical significance of KRAS mutations in lung cancer: An oncogenic driver that contrasts with EGFR mutation. Cancer Metastasis Rev. 2010; 29(1):49-60. http://dx.doi.org/10.1007/s10555-010-9209-4

256. Horio Y, Chen A, Rice P, Roth JA, Malkinson AM, Schrump DS. Ki-ras and p53 mutations are early and late events, respectively, in urethane-induced pulmonary carcinogenesis in $\mathrm{A} / \mathrm{J}$ mice. Mol Carcinog. 1996; 17(4):217-223. http://dx.doi.org/10.1002/(SICI)10982744(199612)17:4<217::AID-MC5>3.0.CO;2-A

257. Westra WH. Early glandular neoplasia of the lung. Respir Res. 2000; 1(3):163-169. http://dx.doi.org/10.1186/rr28

258. Wistuba II, Gazdar AF. Lung cancer preneoplasia. Annu Rev Pathol Mech Dis. 2006; 1(1):331-348. http://dx.doi.org/10.1146/annurev.pathol.1.110304.100103

259. Hong H-HL, Ton T-VT, Kim Y, Wakamatsu N, Clayton NP, Chan P-C, Sills RC, Lahousse SA. Genetic alterations in K-ras and p53 cancer genes in lung neoplasms from B6C3F1 mice exposed to cumene. Toxicol Pathol. 2008; 36(5):720-726.

http://dx.doi.org/10.1177/0192623308320280

260. Rodenhuis S, van de Wetering ML, Mooi WJ, Evers SG, van Zandwijk N, Bos JL. Mutational activation of the K-ras oncogene. N Engl J Med. 1987; 317(15):929-935. http://dx.doi.org/10.1056/NEJM198710083171504 
261. Ogawa HI, Ohyama Y, Ohsumi Y, Kakimoto K, Kato Y, Shirai Y, Nunoshibaand T, Yamamoto K. Cobaltous chloride-induced mutagenesis in the supF tRNA gene of Escherichia coli. Mutagenesis. 1999; 14(2):249-253. http://dx.doi.org/10.1093/mutage/14.2.249

262. Zeiger E, Anderson B, Haworth S, Lawlor T, Mortelmans K. Salmonella mutagenicity tests: V. Results from the testing of 311 chemicals. Environ Mol Mutag. 1992; 19(S21):2-141. http://dx.doi.org/10.1002/em.2850190603

263. MacGregor JT, Wehr CM, Henika PR, Shelby MD. The in vivo erythrocyte micronucleus test: Measurement at steady state increases assay efficiency and permits integration with toxicity studies. Fundam Appl Toxicol. 1990; 14(3):513-522. http://dx.doi.org/10.1016/0272$\underline{0590(90) 90255-\mathrm{I}}$

264. Tuveson DA, Jacks T. Modeling human lung cancer in mice: Similarities and shortcomings. Oncogene. 1999; 18(38):5318-5324. http://dx.doi.org/10.1038/sj.onc.1203107

265. Malarkey DE, Hoenerhoff M, Maronpot RR. Carcinogenesis: Mechanisms and manifestations In: Haschek WM, Rousseaux CG, Wallig MA, editors. Haschek and Rousseaux's Handbook of Toxicologic Pathology. Academic Press, Waltham, MA.; 2013. p. 107. http://dx.doi.org/10.1016/B978-0-12-415759-0.00005-4

266. Pao W, Girard N. New driver mutations in non-small-cell lung cancer. Lancet Oncol. 2011; 12(2):175-180. http://dx.doi.org/10.1016/S1470-2045(10)70087-5

267. Boch C, Kollmeier J, Roth A, Stephan-Falkenau S, Misch D, Grüning W, Bauer TT, Mairinger T. The frequency of EGFR and KRAS mutations in non-small cell lung cancer (NSCLC): Routine screening data for central Europe from a cohort study. BMJ Open. 2013; 3(4):e002560. http://dx.doi.org/10.1136/bmjopen-2013-002560

268. Ellis CA, Clark G. The importance of being K-Ras. Cell Signal. 2000; 12(7):425-434. http://dx.doi.org/10.1016/S0898-6568(00)00084-X

269. Roberts PJ, Der CJ. Targeting the Raf-MEK-ERK mitogen-activated protein kinase cascade for the treatment of cancer. Oncogene. 2007; 26(22):3291-3310.

http://dx.doi.org/10.1038/sj.onc.1210422

270. Johnson BE, Jänne PA. Epidermal growth factor receptor mutations in patients with nonsmall cell lung cancer. Cancer Res. 2005; 65(17):7525-7529. http://dx.doi.org/10.1158/00085472.CAN-05-1257

271. Husgafvel-Pursiainen K, Kannio A. Cigarette smoking and p53 mutations in lung cancer and bladder cancer. Environ Health Perspect. 1996; 104(Suppl 3):553-556.

272. Hernandez-Boussard TM, Hainaut P. A specific spectrum of p53 mutations in lung cancer from smokers: Review of mutations compiled in the IARC p53 database. Environ Health Perspect. 1998; 106(7):385-391. http://dx.doi.org/10.1289/ehp.98106385

273. Toyooka S, Tsuda T, Gazdar AF. The TP53 gene, tobacco exposure, and lung cancer. Hum Mutat. 2003; 21(3):229-239. http://dx.doi.org/10.1002/humu.10177 
274. Herbst RS, Heymach JV, Lippman SM. Lung cancer. N Engl J Med. 2008; 359:1367-1380. http://dx.doi.org/10.1056/NEJMra0802714

275. Hong HH, Ton TV, Kim Y, Wakamatsu N, Clayton NP, Chan PC, Sills RC, Lahousse SA. Genetic alterations in K-ras and p53 cancer genes in lung neoplasms from $\mathrm{B} 6 \mathrm{C} 3 \mathrm{~F} 1$ mice exposed to cumene. Toxicol Pathol. 2008; 36(5):720-726. http://dx.doi.org/10.1177/0192623308320280

276. Bialkowska A, Bialkowski K, Gerschenson M, Diwan BA, Jones AB, Olivero OA, Poirier MC, Anderson LM, Kasprzak KS, Sipowicz MA. Oxidative DNA damage in fetal tissues after transplacental exposure to 3'-azido-3'-deoxythymidine (AZT). Carcinogenesis. 2000; 21(5):10591062. http://dx.doi.org/10.1093/carcin/21.5.1059

277. Donnelly PJ, Devereux TR, Foley JF, Maronpot RR, Anderson MW, Massey TE. Activation of K-ras in aflatoxin B 1-induced lung tumors from AC3F1 $(\mathrm{A} / \mathrm{J} \times \mathrm{C} 3 \mathrm{H} / \mathrm{HeJ})$ mice. Carcinogenesis. 1996; 17(8):1735-1740. http://dx.doi.org/10.1093/carcin/17.8.1735

278. Ronai ZA, Gradia S, Peterson LA, Hecht SS. G to A transitions and G to T transversions in codon 12 of the Ki-ras oncogene isolated from mouse lung tumors induced by $4-$ (methylnitrosamino)-1-(3-pyridyl)-1-butanone (NNK) and relati DNA methylating and pyridyloxobutylating agents. Carcinogenesis. 1993; 14(11):2419-2422.

http://dx.doi.org/10.1093/carcin/14.11.2419

279. Sills RC, Hong HL, Greenwell A, Herbert RA, Boornian GA, Devereux TR. Increased frequency of K-ras mutations in lung neoplasms from female B6C3F1 mice exposed to ozone for 24 or 30 months. Carcinogenesis. 1995; 16(7):1623-1628.

http://dx.doi.org/10.1093/carcin/16.7.1623 


\section{Appendix A. Summary of Lesions in Male Rats in the Two- year Inhalation Study of Cobalt Metal}

\section{Tables}

Table A-1. Summary of the Incidence of Neoplasms in Male Rats in the Two-year Inhalation Study of Cobalt Metal

Table A-2. Statistical Analysis of Primary Neoplasms in Male Rats in the Two-year Inhalation Study of Cobalt Metal

Table A-3. Historical Incidence of Alveolar/bronchiolar Neoplasms in Control Male F344/NTac Rats

Table A-4. Historical Incidence of Pheochromocytoma of the Adrenal Medulla in Control Male F344/NTac Rats

Table A-5. Historical Incidence of Pancreatic Islet Neoplasms in Control Male F344/NTac Rats.

Table A-6. Historical Incidence of Renal Tubule Neoplasms in Control Male F344/NTac Rats

Table A-7. Summary of the Incidence of Nonneoplastic Lesions in Male Rats in the Twoyear Inhalation Study of Cobalt Metal 
Table A-1. Summary of the Incidence of Neoplasms in Male Rats in the Two-year Inhalation Study of Cobalt Metal ${ }^{\mathbf{a}}$

$\begin{gathered}\text { Chamber } \\ \text { Control }\end{gathered} \quad 1.25 \mathrm{mg} / \mathrm{m}^{3} \quad 2.5 \mathrm{mg} / \mathrm{m}^{3} \quad 5 \mathrm{mg} / \mathrm{m}^{3}$

\section{Disposition Summary}

Animals initially in study

50

50

50

50

Early deaths

Accidental death

Moribund

$-$

Natural deaths

28

5

28

1

27

32

Survivors

Terminal kill
Animals examined microscopically

17

50

2

6

2

\section{Alimentary System}

Esophagus

Intestine large, cecum

Intestine large, colon

Carcinoma

Intestine large, rectum

Intestine small, duodenum

Intestine small, ileum

Intestine small, jejunum

Liver

Alveolar/bronchiolar carcinoma, metastatic, lung

Carcinoma, metastatic, kidney

Hemangiosarcoma

Hepatocellular adenoma

Mesentery

Lipoma

Pancreas

Carcinoma, metastatic, kidney

Mixed tumor malignant

Acinus, adenoma

Salivary glands

Adenoma, tubular

Schwannoma malignant

Stomach, forestomach
$-$

$$
-
$$$$
-
$$$$
-
$$

$$
-
$$

$1(2 \%)$

$1(2 \%)$

$-$

(50)
$1(2 \%)$

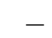

$\begin{array}{ll}- & 1(2 \%) \\ - & 1(2 \%)\end{array}$

$-$

(9)

(3)

$1(11 \%)$

$$
-
$$

$1(2 \%)$

$-$

(50)

(50)

(50)

$1(2 \%)$

(50)

(50) 
Cobalt Metal, NTP TR 581

\begin{tabular}{|c|c|c|c|c|}
\hline & $\begin{array}{l}\text { Chamber } \\
\text { Control }\end{array}$ & $1.25 \mathrm{mg} / \mathrm{m}^{3}$ & $2.5 \mathrm{mg} / \mathrm{m}^{3}$ & $5 \mathrm{mg} / \mathrm{m}^{3}$ \\
\hline Squamous cell papilloma & - & $1(2 \%)$ & - & - \\
\hline Stomach, glandular & $(50)$ & $(50)$ & (49) & $(50)$ \\
\hline Tooth & (0) & (2) & (0) & $(0)$ \\
\hline \multicolumn{5}{|l|}{$\begin{array}{l}\text { Cardiovascular System } \\
\text {. }\end{array}$} \\
\hline Blood vessel & (1) & (1) & $(0)$ & (0) \\
\hline Heart & (50) & (50) & (50) & (50) \\
\hline Alveolar/bronchiolar carcinoma, metastatic & - & - & $1(2 \%)$ & $2(4 \%)$ \\
\hline $\begin{array}{l}\text { Alveolar/bronchiolar carcinoma, metastatic, } \\
\text { lung }\end{array}$ & - & $2(4 \%)$ & - & $1(2 \%)$ \\
\hline $\begin{array}{l}\text { Pheochromocytoma malignant, metastatic, } \\
\text { adrenal medulla }\end{array}$ & - & - & - & $1(2 \%)$ \\
\hline Schwannoma malignant & $1(2 \%)$ & $1(2 \%)$ & $1(2 \%)$ & $2(4 \%)$ \\
\hline \multicolumn{5}{|l|}{ Endocrine System } \\
\hline Adrenal cortex & $(50)$ & (50) & $(50)$ & (50) \\
\hline Adrenal medulla & $(50)$ & $(50)$ & $(50)$ & $(50)$ \\
\hline Pheochromocytoma benign & $11(22 \%)$ & $10(20 \%)$ & $15(30 \%)$ & $13(26 \%)$ \\
\hline Pheochromocytoma malignant & $2(4 \%)$ & $2(4 \%)$ & $9(18 \%)$ & $9(18 \%)$ \\
\hline Bilateral, pheochromocytoma benign & $4(8 \%)$ & $13(26 \%)$ & $22(44 \%)$ & $21(42 \%)$ \\
\hline Bilateral, pheochromocytoma malignant & - & - & - & $7(14 \%)$ \\
\hline Islets, pancreatic & $(50)$ & $(50)$ & (48) & (49) \\
\hline Adenoma & - & $1(2 \%)$ & $6(13 \%)$ & $3(6 \%)$ \\
\hline Carcinoma & $2(4 \%)$ & $1(2 \%)$ & $5(10 \%)$ & $6(12 \%)$ \\
\hline Parathyroid gland & $(45)$ & (45) & (47) & (46) \\
\hline Pituitary gland & $(50)$ & (50) & (49) & (49) \\
\hline Pars distalis, adenoma & $27(54 \%)$ & $32(64 \%)$ & $31(63 \%)$ & $24(49 \%)$ \\
\hline Pars intermedia, adenoma & - & $1(2 \%)$ & - & - \\
\hline Thyroid gland & (49) & (50) & (50) & (49) \\
\hline Bilateral, C-cell, adenoma & - & - & - & $1(2 \%)$ \\
\hline C-cell, adenoma & $4(8 \%)$ & $8(16 \%)$ & $8(16 \%)$ & $6(12 \%)$ \\
\hline C-cell, carcinoma & $1(2 \%)$ & $2(4 \%)$ & $3(6 \%)$ & $1(2 \%)$ \\
\hline Follicular cell, carcinoma & $2(4 \%)$ & $2(4 \%)$ & - & - \\
\hline \multicolumn{5}{|l|}{ General Body System } \\
\hline Peritoneum & $(0)$ & $(0)$ & $(0)$ & (1) \\
\hline \multicolumn{5}{|l|}{ Genital System } \\
\hline Epididymis & $(50)$ & (50) & $(50)$ & $(50)$ \\
\hline Penis & (0) & (1) & (0) & $(0)$ \\
\hline
\end{tabular}


Cobalt Metal, NTP TR 581

\begin{tabular}{|c|c|c|c|c|}
\hline & $\begin{array}{l}\text { Chamber } \\
\text { Control }\end{array}$ & $1.25 \mathrm{mg} / \mathrm{m}^{3}$ & $2.5 \mathrm{mg} / \mathrm{m}^{3}$ & $5 \mathrm{mg} / \mathrm{m}^{3}$ \\
\hline Preputial gland & (50) & $(50)$ & (49) & $(48)$ \\
\hline Adenoma & $1(2 \%)$ & $1(2 \%)$ & - & - \\
\hline Carcinoma & $2(4 \%)$ & - & $1(2 \%)$ & $3(6 \%)$ \\
\hline Prostate gland & (50) & $(50)$ & (50) & $(50)$ \\
\hline Adenoma & $1(2 \%)$ & - & - & - \\
\hline $\begin{array}{l}\text { Pheochromocytoma malignant, metastatic, } \\
\text { adrenal medulla }\end{array}$ & - & - & $1(2 \%)$ & - \\
\hline Seminal vesicle & $(50)$ & $(50)$ & $(50)$ & $(50)$ \\
\hline Carcinoma, metastatic, kidney & - & - & - & $1(2 \%)$ \\
\hline Testes & $(50)$ & $(50)$ & (50) & $(50)$ \\
\hline Bilateral, interstitial cell, adenoma & $23(46 \%)$ & $21(42 \%)$ & $17(34 \%)$ & $12(24 \%)$ \\
\hline Interstitial cell, adenoma & $12(24 \%)$ & $18(36 \%)$ & $17(34 \%)$ & $17(34 \%)$ \\
\hline \multicolumn{5}{|l|}{ Hematopoietic System } \\
\hline Bone marrow & $(50)$ & $(50)$ & $(50)$ & $(50)$ \\
\hline Lymph node & (12) & (9) & (5) & (9) \\
\hline $\begin{array}{l}\text { Renal, pheochromocytoma malignant, } \\
\text { metastatic, adrenal medulla }\end{array}$ & - & - & - & $1(11 \%)$ \\
\hline Lymph node, bronchial & (27) & (25) & (21) & $(20)$ \\
\hline Lymph node, mandibular & (48) & $(49)$ & $(50)$ & $(48)$ \\
\hline Carcinoma, metastatic, Zymbal's gland & - & $1(2 \%)$ & - & - \\
\hline Lymph node, mediastinal & (46) & $(48)$ & (46) & $(48)$ \\
\hline $\begin{array}{l}\text { Alveolar/bronchiolar carcinoma, metastatic, } \\
\text { lung }\end{array}$ & - & - & - & $3(6 \%)$ \\
\hline Lymph node, mesenteric & (49) & $(50)$ & (49) & $(49)$ \\
\hline Spleen & $(50)$ & $(50)$ & $(50)$ & $(50)$ \\
\hline Thymus & (46) & (44) & (47) & $(46)$ \\
\hline $\begin{array}{l}\text { Alveolar/bronchiolar carcinoma, metastatic, } \\
\text { lung }\end{array}$ & - & - & - & $2(4 \%)$ \\
\hline \multicolumn{5}{|l|}{ Integumentary System } \\
\hline Mammary gland & (31) & (32) & (30) & $(31)$ \\
\hline Carcinoma & $1(3 \%)$ & - & - & - \\
\hline Fibroadenoma & $1(3 \%)$ & $1(3 \%)$ & - & $1(3 \%)$ \\
\hline Skin & $(50)$ & $(50)$ & $(50)$ & $(50)$ \\
\hline Basal cell adenoma & $2(4 \%)$ & - & $1(2 \%)$ & $3(6 \%)$ \\
\hline Basal cell carcinoma & - & - & $1(2 \%)$ & - \\
\hline Hematoma & $1(2 \%)$ & - & - & - \\
\hline Keratoacanthoma & $4(8 \%)$ & $6(12 \%)$ & $7(14 \%)$ & $2(4 \%)$ \\
\hline
\end{tabular}


Cobalt Metal, NTP TR 581

\begin{tabular}{|c|c|c|c|c|}
\hline & $\begin{array}{l}\text { Chamber } \\
\text { Control }\end{array}$ & $1.25 \mathrm{mg} / \mathrm{m}^{3}$ & $2.5 \mathrm{mg} / \mathrm{m}^{3}$ & $5 \mathrm{mg} / \mathrm{m}^{3}$ \\
\hline Squamous cell carcinoma & $1(2 \%)$ & - & - & - \\
\hline Squamous cell papilloma & - & $1(2 \%)$ & - & - \\
\hline Trichoepithelioma & $2(4 \%)$ & - & - & $1(2 \%)$ \\
\hline Lip, squamous cell papilloma & - & $1(2 \%)$ & - & - \\
\hline Pinna, neural crest tumor & - & $1(2 \%)$ & - & - \\
\hline Sebaceous gland, adenoma & - & - & $1(2 \%)$ & - \\
\hline Subcutaneous tissue, fibroma & $2(4 \%)$ & $3(6 \%)$ & $3(6 \%)$ & - \\
\hline Subcutaneous tissue, fibrous histiocytoma & - & - & - & $2(4 \%)$ \\
\hline Subcutaneous tissue, lipoma & $2(4 \%)$ & $1(2 \%)$ & - & - \\
\hline \multicolumn{5}{|l|}{ Musculoskeletal System } \\
\hline Bone & (50) & $(50)$ & $(50)$ & $(50)$ \\
\hline $\begin{array}{l}\text { Alveolar/bronchiolar carcinoma, metastatic, } \\
\text { lung }\end{array}$ & - & - & - & $1(2 \%)$ \\
\hline Skeletal muscle & (1) & $(0)$ & $(0)$ & (4) \\
\hline $\begin{array}{l}\text { Alveolar/bronchiolar carcinoma, metastatic, } \\
\text { lung }\end{array}$ & - & - & - & $2(50 \%)$ \\
\hline Carcinoma, metastatic, kidney & - & - & - & $1(25 \%)$ \\
\hline Fibrous histiocytoma, metastatic, skin & - & - & - & $1(25 \%)$ \\
\hline \multicolumn{5}{|l|}{ Nervous System } \\
\hline Brain & $(50)$ & $(50)$ & $(50)$ & $(50)$ \\
\hline Astrocytoma malignant & $1(2 \%)$ & - & - & - \\
\hline Carcinoma, metastatic, Zymbal's gland & - & $1(2 \%)$ & - & - \\
\hline Oligodendroglioma malignant & - & $2(4 \%)$ & - & - \\
\hline Spinal cord & (1) & $(0)$ & $(0)$ & $(0)$ \\
\hline \multicolumn{5}{|l|}{ Respiratory System } \\
\hline Larynx & $(50)$ & (50) & $(50)$ & $(50)$ \\
\hline Lung & (50) & (50) & (50) & (50) \\
\hline Alveolar/bronchiolar adenoma & $1(2 \%)$ & $7(14 \%)$ & $8(16 \%)$ & $8(16 \%)$ \\
\hline Alveolar/bronchiolar adenoma, multiple & $1(2 \%)$ & $3(6 \%)$ & $2(4 \%)$ & $6(12 \%)$ \\
\hline Alveolar/bronchiolar carcinoma & - & $10(20 \%)$ & $20(40 \%)$ & $6(12 \%)$ \\
\hline Alveolar/bronchiolar carcinoma, multiple & - & $6(12 \%)$ & $14(28 \%)$ & $30(60 \%)$ \\
\hline Carcinoma, metastatic, kidney & - & - & - & $1(2 \%)$ \\
\hline Carcinoma, metastatic, Zymbal's gland & - & $1(2 \%)$ & - & $1(2 \%)$ \\
\hline Cystic keratinizing epithelioma & - & $1(2 \%)$ & - & $1(2 \%)$ \\
\hline $\begin{array}{l}\text { Pheochromocytoma malignant, metastatic, } \\
\text { adrenal medulla }\end{array}$ & $1(2 \%)$ & - & - & $1(2 \%)$ \\
\hline
\end{tabular}


Cobalt Metal, NTP TR 581

\begin{tabular}{|c|c|c|c|c|}
\hline & $\begin{array}{c}\text { Chamber } \\
\text { Control }\end{array}$ & $1.25 \mathrm{mg} / \mathrm{m}^{3}$ & $2.5 \mathrm{mg} / \mathrm{m}^{3}$ & $5 \mathrm{mg} / \mathrm{m}^{3}$ \\
\hline Squamous cell carcinoma, metastatic, skin & $1(2 \%)$ & - & - & - \\
\hline $\begin{array}{l}\text { Mediastinum, alveolar/bronchiolar } \\
\text { carcinoma, metastatic, lung }\end{array}$ & - & $2(4 \%)$ & $5(10 \%)$ & $3(6 \%)$ \\
\hline Mediastinum, carcinoma, metastatic, kidney & - & - & - & $1(2 \%)$ \\
\hline $\begin{array}{l}\text { Mediastinum, carcinoma, metastatic, } \\
\text { uncertain primary site }\end{array}$ & - & - & - & $1(2 \%)$ \\
\hline Nose & (48) & $(47)$ & $(45)$ & $(50)$ \\
\hline Trachea & $(50)$ & $(50)$ & $(50)$ & $(50)$ \\
\hline \multicolumn{5}{|l|}{ Special Senses System } \\
\hline Ear & (1) & (5) & $(0)$ & $(0)$ \\
\hline Eye & $(50)$ & $(50)$ & (50) & (50) \\
\hline Harderian gland & $(50)$ & $(50)$ & $(50)$ & $(50)$ \\
\hline Zymbal's gland & (4) & (3) & $(0)$ & (1) \\
\hline Adenoma & $1(25 \%)$ & - & - & - \\
\hline Carcinoma & $3(75 \%)$ & $3(100 \%)$ & - & $1(100 \%)$ \\
\hline \multicolumn{5}{|l|}{ Urinary System } \\
\hline Kidney & $(50)$ & $(50)$ & $(50)$ & (50) \\
\hline $\begin{array}{l}\text { Alveolar/bronchiolar carcinoma, metastatic, } \\
\text { lung }\end{array}$ & - & - & $1(2 \%)$ & $1(2 \%)$ \\
\hline Renal tubule, adenoma & - & $1(2 \%)$ & - & $2(4 \%)$ \\
\hline Renal tubule, adenoma, multiple & - & - & - & $1(2 \%)$ \\
\hline Renal tubule, carcinoma & - & - & - & $2(4 \%)$ \\
\hline Urinary bladder & $(50)$ & $(50)$ & $(50)$ & $(50)$ \\
\hline \multicolumn{5}{|l|}{ Systemic Lesions } \\
\hline Multiple organs ${ }^{\mathrm{b}}$ & (50) & $(50)$ & $(50)$ & $(50)$ \\
\hline Histiocytic sarcoma & - & - & $1(2 \%)$ & - \\
\hline Leukemia mononuclear & $21(42 \%)$ & $25(50 \%)$ & $22(44 \%)$ & $22(44 \%)$ \\
\hline Mesothelioma malignant & $2(4 \%)$ & $3(6 \%)$ & - & $1(2 \%)$ \\
\hline \multicolumn{5}{|l|}{ Neoplasm Summary } \\
\hline Total animals with primary neoplasms ${ }^{\mathrm{c}}$ & 50 & 50 & 49 & 50 \\
\hline Total primary neoplasms & 141 & 191 & 218 & 216 \\
\hline Total animals with benign neoplasms & 47 & 49 & 49 & 47 \\
\hline Total benign neoplasms & 101 & 133 & 139 & 123 \\
\hline Total animals with malignant neoplasms & 31 & 37 & 42 & 48 \\
\hline Total malignant neoplasms & 40 & 57 & 79 & 93 \\
\hline Total animals with metastatic neoplasms & 2 & 4 & 6 & 12 \\
\hline
\end{tabular}


Cobalt Metal, NTP TR 581

\begin{tabular}{|c|c|c|c|c|}
\hline & $\begin{array}{l}\text { Chamber } \\
\text { Control }\end{array}$ & $1.25 \mathrm{mg} / \mathrm{m}^{3}$ & $2.5 \mathrm{mg} / \mathrm{m}^{3}$ & $5 \mathrm{mg} / \mathrm{m}^{3}$ \\
\hline Total metastatic neoplasms & 2 & 7 & 8 & 28 \\
\hline $\begin{array}{l}\text { Total animals with malignant neoplasms } \\
\text { of uncertain primary site }\end{array}$ & - & - & - & 1 \\
\hline $\begin{array}{l}\text { Total animals with uncertain neoplasms- } \\
\text { benign or malignant }\end{array}$ & - & 1 & - & - \\
\hline Total uncertain neoplasms & - & 1 & - & - \\
\hline
\end{tabular}

${ }^{a}$ Number of animals examined microscopically at the site and the number of animals with neoplasm.

${ }^{b}$ Number of animals with any tissue examined microscopically.

cPrimary neoplasms: all neoplasms except metastatic neoplasms. 
Table A-2. Statistical Analysis of Primary Neoplasms in Male Rats in the Two-year Inhalation Study of Cobalt Metal

\begin{tabular}{|c|c|c|c|c|}
\hline & $\begin{array}{l}\text { Chamber } \\
\text { Control }\end{array}$ & $1.25 \mathrm{mg} / \mathrm{m}^{3}$ & $2.5 \mathrm{mg} / \mathrm{m}^{3}$ & $5 \mathrm{mg} / \mathrm{m}^{3}$ \\
\hline \multicolumn{5}{|c|}{ Adrenal Medulla: Benign Pheochromocytoma } \\
\hline Overall rate ${ }^{a}$ & $15 / 50(30 \%)$ & $23 / 50(46 \%)$ & $37 / 50(74 \%)$ & $34 / 50(68 \%)$ \\
\hline Adjusted rate ${ }^{b}$ & $35.8 \%$ & $54.3 \%$ & $81.2 \%$ & $76.4 \%$ \\
\hline Terminal rate ${ }^{c}$ & $3 / 17(18 \%)$ & $12 / 20(60 \%)$ & $15 / 16(94 \%)$ & $14 / 16(88 \%)$ \\
\hline First incidence (days) & 519 & 583 & 582 & 572 \\
\hline Poly-3 test ${ }^{\mathrm{d}}$ & $\mathrm{P}<0.001$ & $\mathrm{P}=0.059$ & $\mathrm{P}<0.001$ & $\mathrm{P}<0.001$ \\
\hline \multicolumn{5}{|c|}{ Adrenal Medulla: Malignant Pheochromocytoma } \\
\hline Overall rate & $2 / 50(4 \%)$ & $2 / 50(4 \%)$ & $9 / 50(18 \%)$ & $16 / 50(32 \%)$ \\
\hline Adjusted rate & $5.0 \%$ & $5.0 \%$ & $21.4 \%$ & $39.1 \%$ \\
\hline Terminal rate & $0 / 17(0 \%)$ & $2 / 20(10 \%)$ & $3 / 16(19 \%)$ & $9 / 16(56 \%)$ \\
\hline First incidence (days) & 668 & $729(\mathrm{~T})$ & 628 & 646 \\
\hline Poly-3 test & $\mathrm{P}<0.001$ & $\mathrm{P}=0.693 \mathrm{~N}$ & $\mathrm{P}=0.030$ & $\mathrm{P}<0.001$ \\
\hline \multicolumn{5}{|c|}{ Adrenal Medulla: Benign or Malignant Pheochromocytoma } \\
\hline Overall rate & $17 / 50(34 \%)$ & $23 / 50(46 \%)$ & $38 / 50(76 \%)$ & $41 / 50(82 \%)$ \\
\hline Adjusted rate & $40.2 \%$ & $54.3 \%$ & $82.7 \%$ & $90.7 \%$ \\
\hline Terminal rate & $3 / 17(18 \%)$ & $12 / 20(60 \%)$ & $15 / 16(94 \%)$ & $16 / 16(100 \%)$ \\
\hline First incidence (days) & 519 & 583 & 582 & 572 \\
\hline Poly-3 test & $\mathrm{P}<0.001$ & $\mathrm{P}=0.130$ & $\mathrm{P}<0.001$ & $\mathrm{P}<0.001$ \\
\hline \multicolumn{5}{|c|}{ Kidney (Renal Tubule): Adenoma (Single Sections) } \\
\hline Overall rate & $0 / 50(0 \%)$ & $1 / 50(2 \%)$ & $0 / 50(0 \%)$ & $3 / 50(6 \%)$ \\
\hline Adjusted rate & $0.0 \%$ & $2.5 \%$ & $0.0 \%$ & $7.5 \%$ \\
\hline Terminal rate & $0 / 17(0 \%)$ & $1 / 20(5 \%)$ & $0 / 16(0 \%)$ & $2 / 16(13 \%)$ \\
\hline First incidence (days) & $-^{\mathrm{e}}$ & $729(\mathrm{~T})$ & - & 696 \\
\hline Poly-3 test & $P=0.061$ & $\mathrm{P}=0.503$ & $--^{\mathrm{f}}$ & $P=0.120$ \\
\hline \multicolumn{5}{|c|}{ Kidney (Renal Tubule): Adenoma (Step Sections) } \\
\hline Overall rate & $3 / 50(6 \%)$ & $1 / 50(2 \%)$ & $1 / 50(2 \%)$ & $3 / 50(6 \%)$ \\
\hline Adjusted rate & $7.5 \%$ & $2.5 \%$ & $2.4 \%$ & $7.5 \%$ \\
\hline Terminal rate & $0 / 17(0 \%)$ & $1 / 20(5 \%)$ & $1 / 16(6 \%)$ & $1 / 16(6 \%)$ \\
\hline First incidence (days) & 678 & $729(\mathrm{~T})$ & $729(\mathrm{~T})$ & 691 \\
\hline Poly-3 test & $P=0.424$ & $\mathrm{P}=0.302 \mathrm{~N}$ & $\mathrm{P}=0.294 \mathrm{~N}$ & $P=0.660 \mathrm{~N}$ \\
\hline \multicolumn{5}{|c|}{ Kidney (Renal Tubule): Adenoma (Single and Step Sections) } \\
\hline Overall rate & $3 / 50(6 \%)$ & $1 / 50(2 \%)$ & $1 / 50(2 \%)$ & $6 / 50(12 \%)$ \\
\hline Adjusted rate & $7.5 \%$ & $2.5 \%$ & $2.4 \%$ & $14.9 \%$ \\
\hline Terminal rate & 0/17 (0\%) & $1 / 20(5 \%)$ & $1 / 16(6 \%)$ & 3/16 (19\%) \\
\hline
\end{tabular}


Cobalt Metal, NTP TR 581

\begin{tabular}{|c|c|c|c|c|}
\hline & $\begin{array}{c}\text { Chamber } \\
\text { Control }\end{array}$ & $1.25 \mathrm{mg} / \mathrm{m}^{3}$ & $2.5 \mathrm{mg} / \mathrm{m}^{3}$ & $5 \mathrm{mg} / \mathrm{m}^{3}$ \\
\hline First incidence (days) & 678 & $729(\mathrm{~T})$ & $729(\mathrm{~T})$ & 691 \\
\hline Poly-3 test & $\mathrm{P}=0.056$ & $\mathrm{P}=0.302 \mathrm{~N}$ & $\mathrm{P}=0.294 \mathrm{~N}$ & $\mathrm{P}=0.244$ \\
\hline \multicolumn{5}{|c|}{ Kidney (Renal Tubule): Adenoma or Carcinoma (Single Sections) } \\
\hline Overall rate & $0 / 50(0 \%)$ & $1 / 50(2 \%)$ & $0 / 50(0 \%)$ & $4 / 50(8 \%)$ \\
\hline Adjusted rate & $0.0 \%$ & $2.5 \%$ & $0.0 \%$ & $10.0 \%$ \\
\hline Terminal rate & $0 / 17(0 \%)$ & $1 / 20(5 \%)$ & $0 / 16(0 \%)$ & $3 / 16(19 \%)$ \\
\hline First incidence (days) & - & $729(\mathrm{~T})$ & - & 696 \\
\hline Poly-3 test & $\mathrm{P}=0.018$ & $P=0.503$ & - & $P=0.061$ \\
\hline \multicolumn{5}{|c|}{ Kidney (Renal Tubule): Adenoma or Carcinoma (Step Sections) } \\
\hline Overall rate & $3 / 50(6 \%)$ & $1 / 50(2 \%)$ & $1 / 50(2 \%)$ & $5 / 50(10 \%)$ \\
\hline Adjusted rate & $7.5 \%$ & $2.5 \%$ & $2.4 \%$ & $12.4 \%$ \\
\hline Terminal rate & $0 / 17(0 \%)$ & $1 / 20(5 \%)$ & $1 / 16(6 \%)$ & $2 / 16(13 \%)$ \\
\hline First incidence (days) & 678 & $729(\mathrm{~T})$ & $729(\mathrm{~T})$ & 691 \\
\hline Poly-3 test & $\mathrm{P}=0.123$ & $\mathrm{P}=0.302 \mathrm{~N}$ & $\mathrm{P}=0.294 \mathrm{~N}$ & $\mathrm{P}=0.361$ \\
\hline \multicolumn{5}{|c|}{ Kidney (Renal Tubule): Adenoma or Carcinoma (Single and Step Sections) } \\
\hline Overall rate & $3 / 50(6 \%)$ & $1 / 50(2 \%)$ & $1 / 50(2 \%)$ & $7 / 50(14 \%)$ \\
\hline Adjusted rate & $7.5 \%$ & $2.5 \%$ & $2.4 \%$ & $17.4 \%$ \\
\hline Terminal rate & $0 / 17(0 \%)$ & $1 / 20(5 \%)$ & $1 / 16(6 \%)$ & $4 / 16(25 \%)$ \\
\hline First incidence (days) & 678 & $729(\mathrm{~T})$ & $729(\mathrm{~T})$ & 691 \\
\hline Poly-3 test & $\mathrm{P}=0.023$ & $\mathrm{P}=0.302 \mathrm{~N}$ & $\mathrm{P}=0.294 \mathrm{~N}$ & $\mathrm{P}=0.158$ \\
\hline \multicolumn{5}{|c|}{ Lung: Alveolar/bronchiolar Adenoma } \\
\hline Overall rate & $2 / 50(4 \%)$ & $10 / 50(20 \%)$ & $10 / 50(20 \%)$ & $14 / 50(28 \%)$ \\
\hline Adjusted rate & $5.0 \%$ & $24.1 \%$ & $23.3 \%$ & $32.5 \%$ \\
\hline Terminal rate & $1 / 17(6 \%)$ & $6 / 20(30 \%)$ & $2 / 16(13 \%)$ & $4 / 16(25 \%)$ \\
\hline First incidence (days) & 611 & 577 & 535 & 478 \\
\hline Poly-3 test & $\mathrm{P}=0.011$ & $\mathrm{P}=0.015$ & $\mathrm{P}=0.018$ & $\mathrm{P}<0.001$ \\
\hline \multicolumn{5}{|c|}{ Lung: Alveolar/bronchiolar Carcinoma } \\
\hline Overall rate & $0 / 50(0 \%)$ & $16 / 50(32 \%)$ & $34 / 50(68 \%)$ & $36 / 50(72 \%)$ \\
\hline Adjusted rate & $0.0 \%$ & $38.2 \%$ & $76.8 \%$ & $80.6 \%$ \\
\hline Terminal rate & $0 / 17(0 \%)$ & $7 / 20(35 \%)$ & $16 / 16(100 \%)$ & $14 / 16(88 \%)$ \\
\hline First incidence (days) & - & 580 & 472 & 552 \\
\hline Poly-3 test & $\mathrm{P}<0.001$ & $\mathrm{P}<0.001$ & $\mathrm{P}<0.001$ & $\mathrm{P}<0.001$ \\
\hline \multicolumn{5}{|c|}{ Lung: Alveolar/bronchiolar Adenoma or Carcinoma } \\
\hline Overall rate & $2 / 50(4 \%)$ & $25 / 50(50 \%)$ & $39 / 50(50 \%)$ & $44 / 50(88 \%)$ \\
\hline Adjusted rate & $5.0 \%$ & $58.0 \%$ & $84.6 \%$ & $93.6 \%$ \\
\hline
\end{tabular}


Cobalt Metal, NTP TR 581

\begin{tabular}{|c|c|c|c|c|}
\hline & $\begin{array}{l}\text { Chamber } \\
\text { Control }\end{array}$ & $1.25 \mathrm{mg} / \mathrm{m}^{3}$ & $2.5 \mathrm{mg} / \mathrm{m}^{3}$ & $5 \mathrm{mg} / \mathrm{m}^{3}$ \\
\hline Terminal rate & $1 / 17(6 \%)$ & $13 / 20(65 \%)$ & $16 / 16(100 \%)$ & $16 / 16(100 \%)$ \\
\hline First incidence (days) & 611 & 577 & 472 & 478 \\
\hline Poly-3 test & $\mathrm{P}<0.001$ & $\mathrm{P}<0.001$ & $\mathrm{P}<0.001$ & $\mathrm{P}<0.001$ \\
\hline \multicolumn{5}{|c|}{ Pancreatic Islets: Adenoma } \\
\hline Overall rate & $0 / 50(0 \%)$ & $1 / 50(2 \%)$ & $6 / 48(13 \%)$ & $3 / 49(6 \%)$ \\
\hline Adjusted rate & $0.0 \%$ & $2.5 \%$ & $15.1 \%$ & $7.7 \%$ \\
\hline Terminal rate & $0 / 17(0 \%)$ & $0 / 20(0 \%)$ & $1 / 16(6 \%)$ & $3 / 16(19 \%)$ \\
\hline First incidence (days) & - & 684 & 618 & $729(\mathrm{~T})$ \\
\hline Poly-3 test & $\mathrm{P}=0.052$ & $\mathrm{P}=0.504$ & $\mathrm{P}=0.015$ & $\mathrm{P}=0.116$ \\
\hline \multicolumn{5}{|c|}{ Pancreatic Islets: Carcinoma } \\
\hline Overall rate & $2 / 50(4 \%)$ & $1 / 50(2 \%)$ & $5 / 48(10 \%)$ & $6 / 49(12 \%)$ \\
\hline Adjusted rate & $5.0 \%$ & $2.5 \%$ & $12.6 \%$ & $15.1 \%$ \\
\hline Terminal rate & $0 / 17(0 \%)$ & $0 / 20(0 \%)$ & $3 / 16(19 \%)$ & $2 / 16(13 \%)$ \\
\hline First incidence (days) & 675 & 675 & 618 & 679 \\
\hline Poly-3 test & $\mathrm{P}=0.021$ & $\mathrm{P}=0.496 \mathrm{~N}$ & $\mathrm{P}=0.213$ & $\mathrm{P}=0.129$ \\
\hline \multicolumn{5}{|c|}{ Pancreatic Islets: Adenoma or Carcinoma } \\
\hline Overall rate & $2 / 50(4 \%)$ & $2 / 50(4 \%)$ & $10 / 48(21 \%)$ & $9 / 49(18 \%)$ \\
\hline Adjusted rate & $5.0 \%$ & $4.9 \%$ & $24.7 \%$ & $22.6 \%$ \\
\hline Terminal rate & $0 / 17(0 \%)$ & $0 / 20(0 \%)$ & $3 / 16(19 \%)$ & $5 / 16(31 \%)$ \\
\hline First incidence (days) & 675 & 675 & 618 & 679 \\
\hline Poly-3 test & $\mathrm{P}=0.002$ & $\mathrm{P}=0.689 \mathrm{~N}$ & $\mathrm{P}=0.013$ & $\mathrm{P}=0.022$ \\
\hline \multicolumn{5}{|c|}{ Pituitary Gland (Pars Distalis): Adenoma } \\
\hline Overall rate & $27 / 50(54 \%)$ & $32 / 50(64 \%)$ & $31 / 49(63 \%)$ & $24 / 49(49 \%)$ \\
\hline Adjusted rate & $60.5 \%$ & $67.7 \%$ & $68.2 \%$ & $55.1 \%$ \\
\hline Terminal rate & $8 / 17(47 \%)$ & $10 / 20(50 \%)$ & $10 / 15(67 \%)$ & $6 / 15(40 \%)$ \\
\hline First incidence (days) & 441 & 413 & 472 & 470 \\
\hline Poly-3 test & $\mathrm{P}=0.219 \mathrm{~N}$ & $\mathrm{P}=0.305$ & $\mathrm{P}=0.289$ & $\mathrm{P}=0.377 \mathrm{~N}$ \\
\hline \multicolumn{5}{|c|}{ Preputial Gland: Carcinoma } \\
\hline Overall rate & $2 / 50(4 \%)$ & $0 / 50(0 \%)$ & $1 / 49(2 \%)$ & $3 / 48(6 \%)$ \\
\hline Adjusted rate & $5.0 \%$ & $0.0 \%$ & $2.5 \%$ & $7.8 \%$ \\
\hline Terminal rate & $0 / 17(0 \%)$ & $0 / 20(0 \%)$ & $0 / 16(0 \%)$ & $1 / 15(7 \%)$ \\
\hline First incidence (days) & 562 & - & 598 & 572 \\
\hline Poly-3 test & $\mathrm{P}=0.155$ & $\mathrm{P}=0.237 \mathrm{~N}$ & $\mathrm{P}=0.497 \mathrm{~N}$ & $\mathrm{P}=0.483$ \\
\hline \multicolumn{5}{|c|}{ Preputial Gland: Adenoma or Carcinoma } \\
\hline Overall rate & $3 / 50(6 \%)$ & $1 / 50(2 \%)$ & $1 / 49(2 \%)$ & $3 / 48(6 \%)$ \\
\hline
\end{tabular}


Cobalt Metal, NTP TR 581

\begin{tabular}{|c|c|c|c|c|}
\hline & $\begin{array}{c}\text { Chamber } \\
\text { Control }\end{array}$ & $1.25 \mathrm{mg} / \mathrm{m}^{3}$ & $2.5 \mathrm{mg} / \mathrm{m}^{3}$ & $5 \mathrm{mg} / \mathrm{m}^{3}$ \\
\hline Adjusted rate & $7.5 \%$ & $2.5 \%$ & $2.5 \%$ & $7.8 \%$ \\
\hline Terminal rate & $1 / 17(6 \%)$ & $1 / 20(5 \%)$ & $0 / 16(0 \%)$ & $1 / 15(7 \%)$ \\
\hline First incidence (days) & 562 & $729(\mathrm{~T})$ & 598 & 572 \\
\hline Poly-3 test & $\mathrm{P}=0.406$ & $\mathrm{P}=0.305 \mathrm{~N}$ & $\mathrm{P}=0.301 \mathrm{~N}$ & $P=0.646$ \\
\hline \multicolumn{5}{|c|}{ Skin: Keratoacanthoma } \\
\hline Overall rate & $4 / 50(8 \%)$ & $6 / 50(12 \%)$ & $7 / 50(14 \%)$ & $2 / 50(4 \%)$ \\
\hline Adjusted rate & $9.9 \%$ & $14.7 \%$ & $16.4 \%$ & $5.0 \%$ \\
\hline Terminal rate & $3 / 17(18 \%)$ & $3 / 20(15 \%)$ & $0 / 16(0 \%)$ & $1 / 16(6 \%)$ \\
\hline First incidence (days) & 376 & 580 & 582 & 652 \\
\hline Poly-3 test & $\mathrm{P}=0.212 \mathrm{~N}$ & $P=0.376$ & $\mathrm{P}=0.292$ & $\mathrm{P}=0.337 \mathrm{~N}$ \\
\hline \multicolumn{5}{|c|}{ Skin: Basal Cell Adenoma } \\
\hline Overall rate & $2 / 50(4 \%)$ & $0 / 50(0 \%)$ & $1 / 50(2 \%)$ & $3 / 50(6 \%)$ \\
\hline Adjusted rate & $5.0 \%$ & $0.0 \%$ & $2.4 \%$ & $7.5 \%$ \\
\hline Terminal rate & $1 / 17(6 \%)$ & $0 / 20(0 \%)$ & $0 / 16(0 \%)$ & $1 / 16(6 \%)$ \\
\hline First incidence (days) & 542 & - & 705 & 689 \\
\hline Poly-3 test & $\mathrm{P}=0.165$ & $\mathrm{P}=0.236 \mathrm{~N}$ & $\mathrm{P}=0.492 \mathrm{~N}$ & $\mathrm{P}=0.502$ \\
\hline \multicolumn{5}{|c|}{ Skin: Trichoepithelioma or Basal Cell Adenoma } \\
\hline Overall rate & $4 / 50(8 \%)$ & $0 / 50(0 \%)$ & $1 / 50(2 \%)$ & $4 / 50(8 \%)$ \\
\hline Adjusted rate & $9.9 \%$ & $0.0 \%$ & $2.4 \%$ & $9.9 \%$ \\
\hline Terminal rate & $1 / 17(6 \%)$ & $0 / 20(0 \%)$ & $0 / 16(0 \%)$ & $1 / 16(6 \%)$ \\
\hline First incidence (days) & 542 & - & 705 & 689 \\
\hline Poly-3 test & $\mathrm{P}=0.248$ & $\mathrm{P}=0.060 \mathrm{~N}$ & $\mathrm{P}=0.172 \mathrm{~N}$ & $\mathrm{P}=0.643$ \\
\hline \multicolumn{5}{|c|}{ Skin: Trichoepithelioma, Basal Cell Adenoma, or Basal Cell Carcinoma } \\
\hline Overall rate & $4 / 50(8 \%)$ & $0 / 50(0 \%)$ & $2 / 50(4 \%)$ & $4 / 50(8 \%)$ \\
\hline Adjusted rate & $9.9 \%$ & $0.0 \%$ & $4.8 \%$ & $9.9 \%$ \\
\hline Terminal rate & $1 / 17(6 \%)$ & $0 / 20(0 \%)$ & $0 / 16(0 \%)$ & $1 / 16(6 \%)$ \\
\hline First incidence (days) & 542 & - & 652 & 689 \\
\hline Poly-3 test & $\mathrm{P}=0.229$ & $\mathrm{P}=0.060 \mathrm{~N}$ & $\mathrm{P}=0.326 \mathrm{~N}$ & $\mathrm{P}=0.643$ \\
\hline \multicolumn{5}{|c|}{ Skin: Squamous Cell Papilloma or Keratoacanthoma } \\
\hline Overall rate & $4 / 50(8 \%)$ & $8 / 50(16 \%)$ & $7 / 50(14 \%)$ & $2 / 50(4 \%)$ \\
\hline Adjusted rate & $9.9 \%$ & $19.3 \%$ & $16.4 \%$ & $5.0 \%$ \\
\hline Terminal rate & $3 / 17(18 \%)$ & $3 / 20(15 \%)$ & $0 / 16(0 \%)$ & $1 / 16(6 \%)$ \\
\hline First incidence (days) & 376 & 580 & 582 & 652 \\
\hline Poly-3 test & $\mathrm{P}=0.120 \mathrm{~N}$ & $\mathrm{P}=0.186$ & $\mathrm{P}=0.292$ & $\mathrm{P}=0.337 \mathrm{~N}$ \\
\hline
\end{tabular}


Cobalt Metal, NTP TR 581

\begin{tabular}{|c|c|c|c|c|}
\hline & $\begin{array}{l}\text { Chamber } \\
\text { Control }\end{array}$ & $1.25 \mathrm{mg} / \mathrm{m}^{3}$ & $2.5 \mathrm{mg} / \mathrm{m}^{3}$ & $5 \mathrm{mg} / \mathrm{m}^{3}$ \\
\hline \multicolumn{5}{|c|}{ Skin: Squamous Cell Papilloma, Keratoacanthoma, or Squamous Cell Carcinoma } \\
\hline Overall rate & $5 / 50(10 \%)$ & $8 / 50(16 \%)$ & $7 / 50(14 \%)$ & $2 / 50(4 \%)$ \\
\hline Adjusted rate & $12.4 \%$ & $19.3 \%$ & $16.4 \%$ & $5.0 \%$ \\
\hline Terminal rate & $3 / 17(18 \%)$ & $3 / 20(15 \%)$ & $0 / 16(0 \%)$ & $1 / 16(6 \%)$ \\
\hline First incidence (days) & 376 & 580 & 582 & 652 \\
\hline Poly-3 test & $\mathrm{P}=0.086 \mathrm{~N}$ & $\mathrm{P}=0.290$ & $\mathrm{P}=0.418$ & $\mathrm{P}=0.215 \mathrm{~N}$ \\
\hline \multicolumn{5}{|c|}{$\begin{array}{l}\text { Skin: Squamous Cell Papilloma, Keratoacanthoma, Trichoepithelioma, Basal Cell Adenoma, Basal Cell } \\
\text { Carcinoma, or Squamous Cell Carcinoma }\end{array}$} \\
\hline Overall rate & $8 / 50(16 \%)$ & $8 / 50(16 \%)$ & $8 / 50(16 \%)$ & $6 / 50(12 \%)$ \\
\hline Adjusted rate & $19.4 \%$ & $19.3 \%$ & $18.6 \%$ & $14.8 \%$ \\
\hline Terminal rate & $3 / 17(18 \%)$ & $3 / 20(15 \%)$ & $0 / 16(0 \%)$ & $2 / 16(13 \%)$ \\
\hline First incidence (days) & 376 & 580 & 582 & 652 \\
\hline Poly-3 test & $\mathrm{P}=0.322 \mathrm{~N}$ & $P=0.605 \mathrm{~N}$ & $\mathrm{P}=0.574 \mathrm{~N}$ & $\mathrm{P}=0.398 \mathrm{~N}$ \\
\hline \multicolumn{5}{|c|}{ Skin (Subcutaneous Tissue): Fibroma } \\
\hline Overall rate & $2 / 50(4 \%)$ & $3 / 50(6 \%)$ & $3 / 50(6 \%)$ & $0 / 50(0 \%)$ \\
\hline Adjusted rate & $5.0 \%$ & $7.5 \%$ & $7.2 \%$ & $0.0 \%$ \\
\hline Terminal rate & $0 / 17(0 \%)$ & $2 / 20(10 \%)$ & $1 / 16(6 \%)$ & $0 / 16(0 \%)$ \\
\hline First incidence (days) & 682 & 674 & 628 & - \\
\hline Poly-3 test & $\mathrm{P}=0.137 \mathrm{~N}$ & $\mathrm{P}=0.506$ & $\mathrm{P}=0.519$ & $\mathrm{P}=0.236 \mathrm{~N}$ \\
\hline \multicolumn{5}{|c|}{ Skin (Subcutaneous Tissue): Fibrous Histiocytoma or Fibroma } \\
\hline Overall rate & $2 / 50(4 \%)$ & $3 / 50(6 \%)$ & $3 / 50(6 \%)$ & $2 / 50(4 \%)$ \\
\hline Adjusted rate & $5.0 \%$ & $7.5 \%$ & $7.2 \%$ & $4.9 \%$ \\
\hline Terminal rate & $0 / 17(0 \%)$ & $2 / 20(10 \%)$ & $1 / 16(6 \%)$ & $0 / 16(0 \%)$ \\
\hline First incidence (days) & 682 & 674 & 628 & 516 \\
\hline Poly-3 test & $\mathrm{P}=0.487 \mathrm{~N}$ & $\mathrm{P}=0.506$ & $\mathrm{P}=0.519$ & $\mathrm{P}=0.683 \mathrm{~N}$ \\
\hline \multicolumn{5}{|l|}{ Testes: Adenoma } \\
\hline Overall rate & $35 / 50(70 \%)$ & $39 / 50(78 \%)$ & $34 / 50(68 \%)$ & $29 / 50(58 \%)$ \\
\hline Adjusted rate & $78.6 \%$ & $87.3 \%$ & $76.3 \%$ & $65.2 \%$ \\
\hline Terminal rate & $15 / 17(88 \%)$ & $19 / 20(95 \%)$ & $15 / 16(94 \%)$ & $10 / 16(63 \%)$ \\
\hline First incidence (days) & 441 & 540 & 548 & 552 \\
\hline Poly-3 test & $\mathrm{P}=0.008 \mathrm{~N}$ & $\mathrm{P}=0.176$ & $\mathrm{P}=0.499 \mathrm{~N}$ & $\mathrm{P}=0.103 \mathrm{~N}$ \\
\hline \multicolumn{5}{|c|}{ Thyroid Gland (C-Cell): Adenoma } \\
\hline Overall rate & $4 / 49(8 \%)$ & $8 / 50(16 \%)$ & $8 / 50(16 \%)$ & $7 / 49(14 \%)$ \\
\hline Adjusted rate & $10.2 \%$ & $19.5 \%$ & $18.9 \%$ & $17.5 \%$ \\
\hline Terminal rate & $2 / 17(12 \%)$ & $5 / 20(25 \%)$ & $2 / 16(13 \%)$ & $4 / 16(25 \%)$ \\
\hline First incidence (days) & 613 & 562 & 548 & 618 \\
\hline
\end{tabular}


Cobalt Metal, NTP TR 581

\begin{tabular}{|c|c|c|c|c|}
\hline & $\begin{array}{c}\text { Chamber } \\
\text { Control }\end{array}$ & $1.25 \mathrm{mg} / \mathrm{m}^{3}$ & $2.5 \mathrm{mg} / \mathrm{m}^{3}$ & $5 \mathrm{mg} / \mathrm{m}^{3}$ \\
\hline Poly-3 test & $\mathrm{P}=0.379$ & $\mathrm{P}=0.196$ & $\mathrm{P}=0.213$ & $\mathrm{P}=0.271$ \\
\hline \multicolumn{5}{|c|}{ Thyroid Gland (C-Cell): Carcinoma } \\
\hline Overall rate & $1 / 49(2 \%)$ & $2 / 50(4 \%)$ & $3 / 50(6 \%)$ & $1 / 49(2 \%)$ \\
\hline Adjusted rate & $2.6 \%$ & $5.0 \%$ & $7.3 \%$ & $2.5 \%$ \\
\hline Terminal rate & $0 / 17(0 \%)$ & $2 / 20(10 \%)$ & $2 / 16(13 \%)$ & $0 / 16(0 \%)$ \\
\hline First incidence (days) & 670 & $729(\mathrm{~T})$ & 618 & 717 \\
\hline Poly-3 test & $\mathrm{P}=0.545 \mathrm{~N}$ & $\mathrm{P}=0.512$ & $\mathrm{P}=0.328$ & $\mathrm{P}=0.757 \mathrm{~N}$ \\
\hline \multicolumn{5}{|c|}{ Thyroid Gland (C-Cell): Adenoma or Carcinoma } \\
\hline Overall rate & $5 / 49(10 \%)$ & $10 / 50(20 \%)$ & $11 / 50(22 \%)$ & $8 / 49(16 \%)$ \\
\hline Adjusted rate & $12.7 \%$ & $24.4 \%$ & $25.8 \%$ & $20.0 \%$ \\
\hline Terminal rate & $2 / 17(12 \%)$ & $7 / 20(35 \%)$ & $4 / 16(25 \%)$ & $4 / 16(25 \%)$ \\
\hline First incidence (days) & 613 & 562 & 548 & 618 \\
\hline Poly-3 test & $\mathrm{P}=0.411$ & $\mathrm{P}=0.143$ & $\mathrm{P}=0.110$ & $\mathrm{P}=0.284$ \\
\hline \multicolumn{5}{|c|}{ Zymbal's Gland: Carcinoma } \\
\hline Overall rate & $3 / 50(6 \%)$ & $3 / 50(6 \%)$ & $0 / 50(0 \%)$ & $1 / 50(2 \%)$ \\
\hline Adjusted rate & $7.4 \%$ & $7.4 \%$ & $0.0 \%$ & $2.5 \%$ \\
\hline Terminal rate & $1 / 17(6 \%)$ & $1 / 20(5 \%)$ & $0 / 16(0 \%)$ & $0 / 16(0 \%)$ \\
\hline First incidence (days) & 383 & 603 & - & 717 \\
\hline Poly-3 test & $\mathrm{P}=0.102 \mathrm{~N}$ & $\mathrm{P}=0.662$ & $\mathrm{P}=0.117 \mathrm{~N}$ & $\mathrm{P}=0.311 \mathrm{~N}$ \\
\hline \multicolumn{5}{|c|}{ Zymbal's Gland: Adenoma or Carcinoma } \\
\hline Overall rate & $4 / 50(8 \%)$ & $3 / 50(6 \%)$ & $0 / 50(0 \%)$ & $1 / 50(2 \%)$ \\
\hline Adjusted rate & $9.7 \%$ & $7.4 \%$ & $0.0 \%$ & $2.5 \%$ \\
\hline Terminal rate & $1 / 17(6 \%)$ & $1 / 20(5 \%)$ & $0 / 16(0 \%)$ & $0 / 16(0 \%)$ \\
\hline First incidence (days) & 383 & 603 & - & 717 \\
\hline Poly-3 test & $\mathrm{P}=0.063 \mathrm{~N}$ & $\mathrm{P}=0.508 \mathrm{~N}$ & $\mathrm{P}=0.060 \mathrm{~N}$ & $\mathrm{P}=0.186 \mathrm{~N}$ \\
\hline \multicolumn{5}{|c|}{ All Organs: Malignant Mesothelioma } \\
\hline Overall rate & $2 / 50(4 \%)$ & $3 / 50(6 \%)$ & $0 / 50(0 \%)$ & $1 / 50(2 \%)$ \\
\hline Adjusted rate & $5.0 \%$ & $7.4 \%$ & $0.0 \%$ & $2.5 \%$ \\
\hline Terminal rate & $1 / 17(6 \%)$ & $1 / 20(5 \%)$ & $0 / 16(0 \%)$ & $1 / 16(6 \%)$ \\
\hline First incidence (days) & 441 & 663 & - & $729(\mathrm{~T})$ \\
\hline Poly-3 test & $\mathrm{P}=0.163 \mathrm{~N}$ & $\mathrm{P}=0.503$ & $\mathrm{P}=0.233 \mathrm{~N}$ & $\mathrm{P}=0.503 \mathrm{~N}$ \\
\hline \multicolumn{5}{|c|}{ All Organs: Mononuclear Cell Leukemia } \\
\hline Overall rate & $21 / 50(42 \%)$ & $25 / 50(50 \%)$ & $22 / 50(44 \%)$ & $22 / 50(44 \%)$ \\
\hline Adjusted rate & $48.9 \%$ & $58.0 \%$ & $50.2 \%$ & $47.9 \%$ \\
\hline Terminal rate & $7 / 17(41 \%)$ & $15 / 20(75 \%)$ & $7 / 16(44 \%)$ & $4 / 16(25 \%)$ \\
\hline
\end{tabular}


Cobalt Metal, NTP TR 581

\begin{tabular}{|c|c|c|c|c|}
\hline & $\begin{array}{c}\text { Chamber } \\
\text { Control }\end{array}$ & $1.25 \mathrm{mg} / \mathrm{m}^{3}$ & $2.5 \mathrm{mg} / \mathrm{m}^{3}$ & $5 \mathrm{mg} / \mathrm{m}^{3}$ \\
\hline First incidence (days) & 519 & 540 & 582 & 478 \\
\hline Poly-3 test & $\mathrm{P}=0.296 \mathrm{~N}$ & $\mathrm{P}=0.257$ & $\mathrm{P}=0.541$ & $\mathrm{P}=0.547 \mathrm{~N}$ \\
\hline \multicolumn{5}{|c|}{ All Organs: Benign Neoplasms } \\
\hline Overall rate & $47 / 50(94 \%)$ & $49 / 50(98 \%)$ & $49 / 50(98 \%)$ & $47 / 50(94 \%)$ \\
\hline Adjusted rate & $97.4 \%$ & $98.9 \%$ & $98.8 \%$ & $96.9 \%$ \\
\hline Terminal rate & $17 / 17(100 \%)$ & $20 / 20(100 \%)$ & $16 / 16(100 \%)$ & $16 / 16(100 \%)$ \\
\hline First incidence (days) & 376 & 413 & 472 & 470 \\
\hline Poly-3 test & $\mathrm{P}=0.436 \mathrm{~N}$ & $\mathrm{P}=0.613$ & $P=0.627$ & $\mathrm{P}=0.719 \mathrm{~N}$ \\
\hline \multicolumn{5}{|c|}{ All Organs: Malignant Neoplasms } \\
\hline Overall rate & $31 / 50(62 \%)$ & $37 / 50(74 \%)$ & $42 / 50(84 \%)$ & $48 / 50(96 \%)$ \\
\hline Adjusted rate & $66.3 \%$ & $82.4 \%$ & $89.7 \%$ & $97.4 \%$ \\
\hline Terminal rate & $7 / 17(41 \%)$ & $18 / 20(90 \%)$ & $16 / 16(100 \%)$ & $15 / 16(94 \%)$ \\
\hline First incidence (days) & 383 & 540 & 472 & 478 \\
\hline Poly-3 test & $\mathrm{P}<0.001$ & $\mathrm{P}=0.051$ & $\mathrm{P}=0.003$ & $\mathrm{P}<0.001$ \\
\hline \multicolumn{5}{|c|}{ All Organs: Benign or Malignant Neoplasms } \\
\hline Overall rate & $50 / 50(100 \%)$ & $50 / 50(100 \%)$ & $49 / 50(98 \%)$ & $50 / 50(100 \%)$ \\
\hline Adjusted rate & $100.0 \%$ & $100.0 \%$ & $98.8 \%$ & $100.0 \%$ \\
\hline Terminal rate & $17 / 17(100 \%)$ & $20 / 20(100 \%)$ & $16 / 16(100 \%)$ & $16 / 16(100 \%)$ \\
\hline First incidence (days) & 376 & 413 & 472 & 470 \\
\hline Poly-3 test & $\mathrm{P}=0.776 \mathrm{~N}$ & - & $\mathrm{P}=0.761 \mathrm{~N}$ & - \\
\hline
\end{tabular}

(T) Terminal kill.

${ }^{a}$ Number of neoplasm-bearing animals/number of animals examined. Denominator is number of animals examined microscopically for adrenal gland, kidney, lung, pancreatic islets, pituitary gland, preputial gland, testes, and thyroid gland; for other tissues, denominator is number of animals necropsied.

bPoly-3 estimated neoplasm incidence after adjustment for intercurrent mortality.

'Observed incidence at terminal kill.

${ }^{\mathrm{d}}$ Beneath the chamber control incidence is the $\mathrm{P}$ value associated with the trend test. Beneath the exposed group incidence are the $\mathrm{P}$ values corresponding to pairwise comparisons between the chamber controls and that exposed group. The Poly-3 test accounts for differential mortality in animals that do not reach terminal kill. A negative trend or a lower incidence in an exposure group is indicated by $\mathbf{N}$.

eNot applicable; no neoplasms in animal group.

fValue of statistic cannot be computed. 
Table A-3. Historical Incidence of Alveolar/bronchiolar Neoplasms in Control Male F344/NTac Rats $^{\text {a }}$

\begin{tabular}{lccc}
\hline & Adenoma & Carcinoma & Adenoma or Carcinoma \\
\hline Overall Historical Incidence: All Routes & & $5 / 100(5.0 \%)$ \\
Total $(\%)$ & $5 / 100(5.0 \%)$ & $0 / 100$ & $5.0 \% \pm 1.4 \%$ \\
Mean \pm standard deviation & $5.0 \% \pm 1.4 \%$ & - & $4 \%-6 \%$ \\
Range & $4 \%-6 \%$ & - & \\
\hline
\end{tabular}

Data as of June 2013.

Table A-4. Historical Incidence of Pheochromocytoma of the Adrenal Medulla in Control Male F344/NTac Rats ${ }^{\text {a }}$

\begin{tabular}{lccc}
\hline & Benign & Malignant & Benign or Malignant \\
\hline Overall Historical Incidence: All Routes & & $27 / 100(27.0 \%)$ \\
Total (\%) & $25 / 100(25.0 \%)$ & $2 / 100(2.0 \%)$ & $27.0 \% \pm 9.9 \%$ \\
Mean \pm standard deviation & $25.0 \% \pm 7.1 \%$ & $2.0 \% \pm 2.8 \%$ & $20 \%-34 \%$ \\
Range & $20 \%-30 \%$ & $0 \%-4 \%$ & \\
\hline
\end{tabular}

Data as of June 2013.

Table A-5. Historical Incidence of Pancreatic Islet Neoplasms in Control Male F344/NTac Rats ${ }^{\text {a }}$

\begin{tabular}{lccc} 
& Adenoma & Carcinoma & Adenoma or Carcinoma \\
\hline Overall Historical Incidence: All Routes & & \\
Total (\%) & $0 / 100$ & $2 / 100(2.0 \%)$ & $2 / 100(2.0 \%)$ \\
Mean \pm standard deviation & - & $2.0 \% \pm 2.8 \%$ & $2.0 \% \pm 2.8 \%$ \\
Range & - & $0 \%-4 \%$ & $0 \%-4 \%$ \\
\hline
\end{tabular}

${ }^{\mathrm{a} D a t a}$ as of June 2013.

Table A-6. Historical Incidence of Renal Tubule Neoplasms in Control Male F344/NTac Rats ${ }^{\mathrm{a}}$

\begin{tabular}{|c|c|c|c|}
\hline & Adenoma & Carcinoma & Adenoma or Carcinoma \\
\hline \multicolumn{4}{|c|}{ Overall Historical Incidence: All Routes } \\
\hline Total (\%) & $1 / 100(1.0 \%)$ & $0 / 100$ & $1 / 100(1.0 \%)$ \\
\hline Mean \pm standard deviation & $1.0 \% \pm 1.41 \%$ & - & $1.0 \% \pm 1.41 \%$ \\
\hline Range & $0 \%-2 \%$ & - & $0 \%-2 \%$ \\
\hline
\end{tabular}

Data as of June 2013. 
Table A-7. Summary of the Incidence of Nonneoplastic Lesions in Male Rats in the Two-year Inhalation Study of Cobalt Metal ${ }^{\mathrm{a}}$

\begin{tabular}{|c|c|c|c|c|}
\hline & $\begin{array}{c}\text { Chamber } \\
\text { Control }\end{array}$ & $1.25 \mathrm{mg} / \mathrm{m}^{3}$ & $2.5 \mathrm{mg} / \mathrm{m}^{3}$ & $5 \mathrm{mg} / \mathrm{m}^{3}$ \\
\hline \multicolumn{5}{|l|}{ Disposition Summary } \\
\hline Animals initially in study & 50 & 50 & 50 & 50 \\
\hline \multicolumn{5}{|l|}{ Early deaths } \\
\hline Accidental death & - & - & 1 & - \\
\hline Moribund & 28 & 28 & 27 & 32 \\
\hline Natural deaths & 5 & 2 & 6 & 2 \\
\hline \multicolumn{5}{|l|}{ Survivors } \\
\hline Terminal kill & 17 & 20 & 16 & 16 \\
\hline Animals examined microscopically & 50 & 50 & 50 & 50 \\
\hline \multicolumn{5}{|l|}{ Alimentary System } \\
\hline Esophagus & $(50)$ & $(50)$ & (48) & $(48)$ \\
\hline Inflammation, suppurative & $1(2 \%)$ & - & - & - \\
\hline Intestine large, cecum & (49) & (50) & (49) & $(50)$ \\
\hline Inflammation, granulomatous & - & $1(2 \%)$ & - & - \\
\hline Epithelium, hyperplasia & - & - & $1(2 \%)$ & - \\
\hline Intestine large, colon & $(50)$ & (50) & (49) & (50) \\
\hline Epithelium, hyperplasia & - & - & $1(2 \%)$ & - \\
\hline Intestine large, rectum & $(50)$ & (49) & (49) & (49) \\
\hline Intestine small, duodenum & $(50)$ & $(50)$ & (49) & $(50)$ \\
\hline Inflammation, chronic active & - & - & $5(10 \%)$ & - \\
\hline Necrosis & - & - & $1(2 \%)$ & $1(2 \%)$ \\
\hline Intestine small, ileum & (48) & (50) & (47) & (50) \\
\hline Inflammation, suppurative & - & - & $1(2 \%)$ & - \\
\hline Epithelium, hyperplasia & - & - & $1(2 \%)$ & - \\
\hline Intestine small, jejunum & $(48)$ & (49) & (48) & (49) \\
\hline Epithelium, hyperplasia & - & - & $1(2 \%)$ & - \\
\hline Liver & $(50)$ & (50) & (50) & $(50)$ \\
\hline Atrophy & $1(2 \%)$ & - & - & $2(4 \%)$ \\
\hline Basophilic focus & $5(10 \%)$ & $17(34 \%)$ & $17(34 \%)$ & $19(38 \%)$ \\
\hline Clear cell focus & $9(18 \%)$ & $11(22 \%)$ & $7(14 \%)$ & $9(18 \%)$ \\
\hline Degeneration, cystic & - & $5(10 \%)$ & - & $1(2 \%)$ \\
\hline Eosinophilic focus & $3(6 \%)$ & $2(4 \%)$ & - & $1(2 \%)$ \\
\hline Fatty change & $2(4 \%)$ & $4(8 \%)$ & $3(6 \%)$ & $4(8 \%)$ \\
\hline
\end{tabular}


Cobalt Metal, NTP TR 581

\begin{tabular}{|c|c|c|c|c|}
\hline & $\begin{array}{c}\text { Chamber } \\
\text { Control }\end{array}$ & $1.25 \mathrm{mg} / \mathrm{m}^{3}$ & $2.5 \mathrm{mg} / \mathrm{m}^{3}$ & $5 \mathrm{mg} / \mathrm{m}^{3}$ \\
\hline Hepatodiaphragmatic nodule & $4(8 \%)$ & $9(18 \%)$ & $11(22 \%)$ & $9(18 \%)$ \\
\hline Inflammation, chronic active & $1(2 \%)$ & - & - & $1(2 \%)$ \\
\hline Mixed cell focus & - & $1(2 \%)$ & $1(2 \%)$ & $1(2 \%)$ \\
\hline Necrosis & $5(10 \%)$ & $6(12 \%)$ & $4(8 \%)$ & $2(4 \%)$ \\
\hline Regeneration & - & - & $1(2 \%)$ & - \\
\hline Thrombosis & $1(2 \%)$ & - & - & - \\
\hline Bile duct, hyperplasia & $1(2 \%)$ & - & - & - \\
\hline Mesentery & $(18)$ & (4) & (9) & (3) \\
\hline Infiltration cellular & - & - & $1(11 \%)$ & - \\
\hline Artery, inflammation, chronic active & - & - & - & $1(33 \%)$ \\
\hline Artery, thrombosis & $1(6 \%)$ & - & - & - \\
\hline Fat, necrosis & $14(78 \%)$ & $4(100 \%)$ & $8(89 \%)$ & $1(33 \%)$ \\
\hline Pancreas & $(50)$ & $(50)$ & (49) & $(50)$ \\
\hline Acinus, atrophy & $23(46 \%)$ & $35(70 \%)$ & $28(57 \%)$ & $30(60 \%)$ \\
\hline Acinus, hyperplasia & $1(2 \%)$ & - & - & - \\
\hline Artery, mineralization & - & - & - & $1(2 \%)$ \\
\hline Salivary glands & $(50)$ & $(50)$ & $(50)$ & $(50)$ \\
\hline Degeneration & - & - & - & $1(2 \%)$ \\
\hline Stomach, forestomac ${ }^{\mathrm{h}}$ & $(50)$ & (50) & (50) & $(50)$ \\
\hline Edema & $2(4 \%)$ & - & - & - \\
\hline Hyperplasia, squamous & $10(20 \%)$ & $14(28 \%)$ & $13(26 \%)$ & $11(22 \%)$ \\
\hline Inflammation, chronic active & $2(4 \%)$ & $4(8 \%)$ & $2(4 \%)$ & $1(2 \%)$ \\
\hline Mineralization & $2(4 \%)$ & - & - & - \\
\hline Ulcer & $4(8 \%)$ & $4(8 \%)$ & $10(20 \%)$ & $2(4 \%)$ \\
\hline Stomach, glandular & $(50)$ & $(50)$ & (49) & $(50)$ \\
\hline Edema & $1(2 \%)$ & - & - & - \\
\hline Erosion & - & $1(2 \%)$ & $2(4 \%)$ & - \\
\hline Inflammation, chronic active & - & $1(2 \%)$ & $1(2 \%)$ & $1(2 \%)$ \\
\hline Mineralization & $1(2 \%)$ & - & - & - \\
\hline Ulcer & $1(2 \%)$ & $2(4 \%)$ & $2(4 \%)$ & $1(2 \%)$ \\
\hline Tooth & $(0)$ & (2) & $(0)$ & (0) \\
\hline Inflammation, chronic active & - & $2(100 \%)$ & - & - \\
\hline \multicolumn{5}{|l|}{ Cardiovascular System } \\
\hline Blood vessel & (1) & (1) & $(0)$ & (0) \\
\hline Aorta, mineralization & $1(100 \%)$ & - & - & - \\
\hline
\end{tabular}


Cobalt Metal, NTP TR 581

\begin{tabular}{|c|c|c|c|c|}
\hline & $\begin{array}{c}\text { Chamber } \\
\text { Control }\end{array}$ & $1.25 \mathrm{mg} / \mathrm{m}^{3}$ & $2.5 \mathrm{mg} / \mathrm{m}^{3}$ & $5 \mathrm{mg} / \mathrm{m}^{3}$ \\
\hline Heart & $(50)$ & $(50)$ & $(50)$ & $(50)$ \\
\hline Cardiomyopathy & $45(90 \%)$ & $44(88 \%)$ & $46(92 \%)$ & $39(78 \%)$ \\
\hline Inflammation, suppurative & - & $1(2 \%)$ & $2(4 \%)$ & - \\
\hline Thrombosis & $4(8 \%)$ & $2(4 \%)$ & $2(4 \%)$ & - \\
\hline Artery, inflammation, chronic active & - & $1(2 \%)$ & - & $1(2 \%)$ \\
\hline Atrium, congestion & - & $1(2 \%)$ & $1(2 \%)$ & - \\
\hline Epicardium, hyperplasia & - & $1(2 \%)$ & - & - \\
\hline \multicolumn{5}{|l|}{ Endocrine System } \\
\hline Adrenal cortex & $(50)$ & $(50)$ & $(50)$ & $(50)$ \\
\hline Accessory adrenal cortical nodule & $3(6 \%)$ & - & $2(4 \%)$ & $3(6 \%)$ \\
\hline Atrophy & - & $1(2 \%)$ & - & - \\
\hline Degeneration & $1(2 \%)$ & - & - & - \\
\hline Hyperplasia & $35(70 \%)$ & $24(48 \%)$ & $23(46 \%)$ & $29(58 \%)$ \\
\hline Necrosis & - & $1(2 \%)$ & $1(2 \%)$ & - \\
\hline Adrenal medulla & $(50)$ & $(50)$ & (50) & (50) \\
\hline Hyperplasia & $19(38 \%)$ & $21(42 \%)$ & $9(18 \%)$ & $9(18 \%)$ \\
\hline Necrosis & - & $1(2 \%)$ & - & - \\
\hline Islets, pancreatic & $(50)$ & $(50)$ & (48) & (49) \\
\hline Hyperplasia & - & $1(2 \%)$ & $1(2 \%)$ & $3(6 \%)$ \\
\hline Parathyroid gland & $(45)$ & $(45)$ & (47) & $(46)$ \\
\hline Pituitary gland & $(50)$ & $(50)$ & (49) & (49) \\
\hline Angiectasis & $1(2 \%)$ & - & - & $1(2 \%)$ \\
\hline Cyst & $1(2 \%)$ & - & - & - \\
\hline Hemorrhage & - & $1(2 \%)$ & $1(2 \%)$ & - \\
\hline Pars distalis, hyperplasia & $18(36 \%)$ & $12(24 \%)$ & $14(29 \%)$ & $22(45 \%)$ \\
\hline Thyroid gland & (49) & $(50)$ & (50) & (49) \\
\hline Hemorrhage & $1(2 \%)$ & - & - & - \\
\hline C-cell, hyperplasia & $7(14 \%)$ & $8(16 \%)$ & $5(10 \%)$ & $9(18 \%)$ \\
\hline Follicle, cyst & - & - & - & $1(2 \%)$ \\
\hline \multicolumn{5}{|l|}{ General Body System } \\
\hline Peritoneum & $(0)$ & $(0)$ & $(0)$ & (1) \\
\hline Inflammation, chronic active & - & - & - & $1(100 \%)$ \\
\hline \multicolumn{5}{|l|}{ Genital System } \\
\hline Epididymis & $(50)$ & $(50)$ & (50) & $(50)$ \\
\hline Cyst & $1(2 \%)$ & - & - & - \\
\hline
\end{tabular}




\section{Cobalt Metal, NTP TR 581}

\begin{tabular}{|c|c|c|c|c|}
\hline & $\begin{array}{l}\text { Chamber } \\
\text { Control }\end{array}$ & $1.25 \mathrm{mg} / \mathrm{m}^{3}$ & $2.5 \mathrm{mg} / \mathrm{m}^{3}$ & $5 \mathrm{mg} / \mathrm{m}^{3}$ \\
\hline Penis & (0) & (1) & (0) & (0) \\
\hline Concretion & - & $1(100 \%)$ & - & - \\
\hline Preputial gland & (50) & (50) & $(49)$ & (48) \\
\hline Atrophy & - & - & - & $1(2 \%)$ \\
\hline Ectasia & $3(6 \%)$ & $1(2 \%)$ & $1(2 \%)$ & - \\
\hline Inflammation, chronic active & $5(10 \%)$ & $4(8 \%)$ & $3(6 \%)$ & $3(6 \%)$ \\
\hline Prostate gland & $(50)$ & $(50)$ & $(50)$ & $(50)$ \\
\hline Fibrosis & - & - & $1(2 \%)$ & - \\
\hline Hyperplasia & $1(2 \%)$ & $6(12 \%)$ & $1(2 \%)$ & $1(2 \%)$ \\
\hline Inflammation, chronic active & $36(72 \%)$ & $39(78 \%)$ & $42(84 \%)$ & $28(56 \%)$ \\
\hline Seminal vesicle & (50) & $(50)$ & $(50)$ & (50) \\
\hline Inflammation, chronic active & $1(2 \%)$ & $1(2 \%)$ & - & $1(2 \%)$ \\
\hline Testes & (50) & $(50)$ & $(50)$ & (50) \\
\hline Atrophy & $35(70 \%)$ & $34(68 \%)$ & $36(72 \%)$ & $39(78 \%)$ \\
\hline Infarct & $1(2 \%)$ & - & $2(4 \%)$ & $12(24 \%)$ \\
\hline Arteriole, inflammation, chronic active & $2(4 \%)$ & - & $1(2 \%)$ & - \\
\hline Interstitial cell, hyperplasia & $7(14 \%)$ & $10(20 \%)$ & $12(24 \%)$ & $12(24 \%)$ \\
\hline \multicolumn{5}{|l|}{ Hematopoietic System } \\
\hline Bone marrow & $(50)$ & $(50)$ & $(50)$ & $(50)$ \\
\hline Necrosis & - & - & $1(2 \%)$ & - \\
\hline Lymph node & (12) & (9) & (5) & (9) \\
\hline Iliac, ectasia & $1(8 \%)$ & - & - & - \\
\hline Iliac, lumbar, ectasia & - & $1(11 \%)$ & - & - \\
\hline Iliac, renal, ectasia & - & $1(11 \%)$ & - & - \\
\hline Lumbar, hyperplasia & - & $1(11 \%)$ & - & - \\
\hline Pancreatic, ectasia & $1(8 \%)$ & - & - & $1(11 \%)$ \\
\hline Pancreatic, infiltration cellular, histiocyte & - & - & $1(20 \%)$ & - \\
\hline Pancreatic, necrosis & - & $1(11 \%)$ & - & - \\
\hline Renal, ectasia & $2(17 \%)$ & $3(33 \%)$ & $1(20 \%)$ & $1(11 \%)$ \\
\hline Renal, hemorrhage & - & - & - & $1(11 \%)$ \\
\hline Lymph node, bronchial & (27) & $(25)$ & $(21)$ & (20) \\
\hline Lymph node, mandibular & (48) & (49) & (50) & (48) \\
\hline Atrophy & $1(2 \%)$ & - & - & - \\
\hline Congestion & - & $1(2 \%)$ & - & - \\
\hline Ectasia & $1(2 \%)$ & $2(4 \%)$ & $1(2 \%)$ & $2(4 \%)$ \\
\hline
\end{tabular}


Cobalt Metal, NTP TR 581

\begin{tabular}{|c|c|c|c|c|}
\hline & $\begin{array}{c}\text { Chamber } \\
\text { Control }\end{array}$ & $1.25 \mathrm{mg} / \mathrm{m}^{3}$ & $2.5 \mathrm{mg} / \mathrm{m}^{3}$ & $5 \mathrm{mg} / \mathrm{m}^{3}$ \\
\hline Hyperplasia & $1(2 \%)$ & $2(4 \%)$ & $1(2 \%)$ & $1(2 \%)$ \\
\hline Necrosis & $1(2 \%)$ & $1(2 \%)$ & - & - \\
\hline Lymph node, mediastinal & $(46)$ & $(48)$ & (46) & (48) \\
\hline Hyperplasia, lymphoid & - & - & $1(2 \%)$ & - \\
\hline Lymph node, mesenteric & $(49)$ & (50) & (49) & (49) \\
\hline Hemorrhage & $1(2 \%)$ & - & - & - \\
\hline Infiltration cellular, histiocyte & - & - & - & $1(2 \%)$ \\
\hline Spleen & $(50)$ & $(50)$ & $(50)$ & $(50)$ \\
\hline Congestion & - & $1(2 \%)$ & - & - \\
\hline Fibrosis & $8(16 \%)$ & $8(16 \%)$ & $7(14 \%)$ & $4(8 \%)$ \\
\hline Hematopoietic cell proliferation & $3(6 \%)$ & $2(4 \%)$ & - & $3(6 \%)$ \\
\hline Hemorrhage & $1(2 \%)$ & $1(2 \%)$ & - & - \\
\hline Necrosis & $3(6 \%)$ & $2(4 \%)$ & $1(2 \%)$ & $6(12 \%)$ \\
\hline Stromal hyperplasia & - & - & $1(2 \%)$ & - \\
\hline Capsule, angiectasis & $1(2 \%)$ & - & - & - \\
\hline Thymus & (46) & (44) & (47) & $(46)$ \\
\hline \multicolumn{5}{|l|}{ Integumentary System } \\
\hline Mammary gland & (31) & (32) & (30) & $(31)$ \\
\hline Galactocele & $2(6 \%)$ & $1(3 \%)$ & $1(3 \%)$ & - \\
\hline Hyperplasia & $2(6 \%)$ & $1(3 \%)$ & $1(3 \%)$ & - \\
\hline Inflammation, chronic active & $1(3 \%)$ & - & - & - \\
\hline Skin & (50) & (50) & $(50)$ & (50) \\
\hline Cyst epithelial inclusion & $1(2 \%)$ & - & - & $1(2 \%)$ \\
\hline Hemorrhage & - & $1(2 \%)$ & - & - \\
\hline Hyperplasia, squamous & $2(4 \%)$ & $2(4 \%)$ & $5(10 \%)$ & $1(2 \%)$ \\
\hline Inflammation & $7(14 \%)$ & $5(10 \%)$ & $9(18 \%)$ & $4(8 \%)$ \\
\hline Inflammation, granulomatous & - & $1(2 \%)$ & - & - \\
\hline \multicolumn{5}{|l|}{ Musculoskeletal System } \\
\hline Bone & $(50)$ & $(50)$ & $(50)$ & $(50)$ \\
\hline Cranium, fibrosis & - & $1(2 \%)$ & - & - \\
\hline Cranium, fracture & - & - & $1(2 \%)$ & - \\
\hline Cranium, inflammation, chronic active & - & $1(2 \%)$ & - & - \\
\hline Maxilla, inflammation, chronic active & - & - & - & $1(2 \%)$ \\
\hline Vertebra, degeneration & - & $1(2 \%)$ & - & - \\
\hline Skeletal muscle & (1) & (0) & $(0)$ & (4) \\
\hline
\end{tabular}




\section{Cobalt Metal, NTP TR 581}

\begin{tabular}{|c|c|c|c|c|}
\hline & $\begin{array}{c}\text { Chamber } \\
\text { Control }\end{array}$ & $1.25 \mathrm{mg} / \mathrm{m}^{3}$ & $2.5 \mathrm{mg} / \mathrm{m}^{3}$ & $5 \mathrm{mg} / \mathrm{m}^{3}$ \\
\hline \multicolumn{5}{|l|}{ Nervous System } \\
\hline Brain & $(50)$ & (50) & $(50)$ & $(50)$ \\
\hline Compression & $15(30 \%)$ & $14(28 \%)$ & $18(36 \%)$ & $7(14 \%)$ \\
\hline Edema & $2(4 \%)$ & - & - & $1(2 \%)$ \\
\hline Hemorrhage & $2(4 \%)$ & $3(6 \%)$ & $4(8 \%)$ & $3(6 \%)$ \\
\hline Infiltration cellular, mononuclear cell & $1(2 \%)$ & - & - & $1(2 \%)$ \\
\hline Metaplasia, osseous & $1(2 \%)$ & - & - & - \\
\hline Meninges, inflammation, suppurative & $1(2 \%)$ & - & - & - \\
\hline Spinal cord & (1) & $(0)$ & $(0)$ & $(0)$ \\
\hline Infiltration cellular & $1(100 \%)$ & - & - & - \\
\hline \multicolumn{5}{|l|}{ Respiratory System } \\
\hline Larynx & $(50)$ & $(50)$ & $(50)$ & $(50)$ \\
\hline Autolysis & - & - & $1(2 \%)$ & - \\
\hline Foreign body & $3(6 \%)$ & - & $2(4 \%)$ & - \\
\hline Hyperkeratosis & - & - & - & $1(2 \%)$ \\
\hline Inflammation & $28(56 \%)$ & $18(36 \%)$ & $18(36 \%)$ & $16(32 \%)$ \\
\hline Metaplasia, squamous & $3(6 \%)$ & $1(2 \%)$ & $1(2 \%)$ & $2(4 \%)$ \\
\hline Ulcer & $1(2 \%)$ & - & - & - \\
\hline Respiratory epithelium, hyperplasia & - & - & - & $1(2 \%)$ \\
\hline Squamous epithelium, hyperplasia & - & - & - & $1(2 \%)$ \\
\hline Lung & $(50)$ & $(50)$ & $(50)$ & $(50)$ \\
\hline Inflammation, suppurative & - & $1(2 \%)$ & $1(2 \%)$ & - \\
\hline Inflammation, chronic active & $22(44 \%)$ & $50(100 \%)$ & $50(100 \%)$ & $50(100 \%)$ \\
\hline Metaplasia, osseous & $3(6 \%)$ & $3(6 \%)$ & - & - \\
\hline Mineralization & $1(2 \%)$ & - & - & - \\
\hline Thrombosis & $2(4 \%)$ & - & - & - \\
\hline Alveolar epithelium, hyperplasia & $3(6 \%)$ & $47(94 \%)$ & $49(98 \%)$ & $49(98 \%)$ \\
\hline Alveolus, proteinosis & - & $48(96 \%)$ & $49(98 \%)$ & $49(98 \%)$ \\
\hline Artery, mediastinum, inflammation, chronic active & - & $1(2 \%)$ & - & - \\
\hline Bronchiole, epithelium, hyperplasia & - & $44(88 \%)$ & $47(94 \%)$ & $50(100 \%)$ \\
\hline Mediastinum, inflammation, suppurative & - & - & $1(2 \%)$ & - \\
\hline Mediastinum, metaplasia, osseous & - & $1(2 \%)$ & - & - \\
\hline Nose & $(48)$ & $(47)$ & (45) & $(50)$ \\
\hline Foreign body & $5(10 \%)$ & $2(4 \%)$ & $4(9 \%)$ & $5(10 \%)$ \\
\hline Hemorrhage & - & - & $1(2 \%)$ & - \\
\hline
\end{tabular}


Cobalt Metal, NTP TR 581

\begin{tabular}{|c|c|c|c|c|}
\hline & $\begin{array}{c}\text { Chamber } \\
\text { Control }\end{array}$ & $1.25 \mathrm{mg} / \mathrm{m}^{3}$ & $2.5 \mathrm{mg} / \mathrm{m}^{3}$ & $5 \mathrm{mg} / \mathrm{m}^{3}$ \\
\hline Inflammation, suppurative & $9(19 \%)$ & $12(26 \%)$ & $24(53 \%)$ & $46(92 \%)$ \\
\hline Inflammation, chronic active & $28(58 \%)$ & $35(74 \%)$ & $40(89 \%)$ & $49(98 \%)$ \\
\hline Thrombosis & $7(15 \%)$ & $2(4 \%)$ & $4(9 \%)$ & $2(4 \%)$ \\
\hline Olfactory epithelium, accumulation, hyaline droplet & $2(4 \%)$ & $4(9 \%)$ & $1(2 \%)$ & $2(4 \%)$ \\
\hline Olfactory epithelium, atrophy & $2(4 \%)$ & $21(45 \%)$ & $34(76 \%)$ & $29(58 \%)$ \\
\hline Olfactory epithelium, hyperplasia & - & $1(2 \%)$ & $2(4 \%)$ & $7(14 \%)$ \\
\hline Olfactory epithelium, hyperplasia, basal cell & - & $1(2 \%)$ & - & $13(26 \%)$ \\
\hline Olfactory epithelium, metaplasia, respiratory & $12(25 \%)$ & $26(55 \%)$ & $37(82 \%)$ & $50(100 \%)$ \\
\hline Olfactory epithelium, necrosis & - & $1(2 \%)$ & $5(11 \%)$ & $5(10 \%)$ \\
\hline Respiratory epithelium, hyperplasia & $20(42 \%)$ & $35(74 \%)$ & $45(100 \%)$ & $50(100 \%)$ \\
\hline Respiratory epithelium, metaplasia, squamous & - & $1(2 \%)$ & $11(24 \%)$ & $35(70 \%)$ \\
\hline Respiratory epithelium, necrosis & $1(2 \%)$ & $4(9 \%)$ & $5(11 \%)$ & $13(26 \%)$ \\
\hline Turbinate, atrophy & $1(2 \%)$ & $35(74 \%)$ & $35(78 \%)$ & $41(82 \%)$ \\
\hline Turbinate, hyperostosis & $2(4 \%)$ & - & - & - \\
\hline Trachea & $(50)$ & $(50)$ & $(50)$ & $(50)$ \\
\hline Inflammation, suppurative & - & - & - & $1(2 \%)$ \\
\hline Metaplasia, squamous & - & - & - & $1(2 \%)$ \\
\hline \multicolumn{5}{|l|}{ Special Senses System } \\
\hline Ear & (1) & (5) & $(0)$ & $(0)$ \\
\hline Inflammation, chronic active & $1(100 \%)$ & $5(100 \%)$ & - & - \\
\hline Eye & (50) & $(50)$ & $(50)$ & (50) \\
\hline Cataract & $3(6 \%)$ & $9(18 \%)$ & $9(18 \%)$ & $7(14 \%)$ \\
\hline Inflammation, suppurative & $1(2 \%)$ & - & - & - \\
\hline Metaplasia, osseous & $31(62 \%)$ & $36(72 \%)$ & $33(66 \%)$ & $31(62 \%)$ \\
\hline Harderian gland & (50) & $(50)$ & $(50)$ & $(50)$ \\
\hline Zymbal's gland & (4) & (3) & $(0)$ & (1) \\
\hline \multicolumn{5}{|l|}{ Urinary System } \\
\hline Kidney & $(50)$ & $(50)$ & $(50)$ & (50) \\
\hline Accumulation, hyaline droplet & - & $1(2 \%)$ & $4(8 \%)$ & $1(2 \%)$ \\
\hline Congestion & - & - & $1(2 \%)$ & - \\
\hline Cyst & - & $3(6 \%)$ & - & $1(2 \%)$ \\
\hline Fibrosis & - & - & - & $1(2 \%)$ \\
\hline Infarct & $2(4 \%)$ & - & - & $1(2 \%)$ \\
\hline Metaplasia, osseous & - & - & - & $1(2 \%)$ \\
\hline Nephropathy & $49(98 \%)$ & $49(98 \%)$ & $50(100 \%)$ & $50(100 \%)$ \\
\hline
\end{tabular}




\section{Cobalt Metal, NTP TR 581}

\begin{tabular}{lcccc}
\hline & $\begin{array}{c}\text { Chamber } \\
\text { Control }\end{array}$ & $\mathbf{1 . 2 5} \mathbf{~ \mathbf { g } / \mathbf { m } ^ { \mathbf { 3 } }}$ & $\mathbf{2 . 5} \mathbf{~ \mathbf { g } / \mathbf { m } ^ { \mathbf { 3 } }}$ & $\mathbf{5} \mathbf{~ \mathbf { g } / \mathbf { m } ^ { \mathbf { 3 } }}$ \\
\hline Thrombosis & - & $1(2 \%)$ & - & - \\
Artery, inflammation, chronic active & - & - & - & $1(2 \%)$ \\
Pelvis, inflammation, suppurative & - & - & $1(2 \%)$ & - \\
Urinary bladder & $(50)$ & $(50)$ & $(50)$ & $(50)$ \\
Hemorrhage & $1(2 \%)$ & - & - & - \\
Inflammation & & - & $2(4 \%)$ & $1(2 \%)$ \\
Inflammation, granulomatous & $2(4 \%)$ & - & - & - \\
Transitional epithelium, hyperplasia & $1(2 \%)$ & $1(2 \%)$ & $1(2 \%)$ & - \\
\hline
\end{tabular}

${ }^{a}$ Number of animals examined microscopically at the site and the number of animals with lesion. 


\section{Appendix B. Summary of Lesions in Female Rats in the Two- year Inhalation Study of Cobalt Metal}

\section{Tables}

Table B-1. Summary of the Incidence of Neoplasms in Female Rats in the Two-year Inhalation Study of Cobalt Metal

Table B-2. Statistical Analysis of Primary Neoplasms in Female Rats in the Two-year Inhalation Study of Cobalt Metal

Table B-3. Historical Incidence of Alveolar/bronchiolar Neoplasms in Control Female F344/NTac Rats

Table B-4. Historical Incidence of Pheochromocytoma of the Adrenal Medulla in Control

Female F344/NTac Rats

Table B-5. Historical Incidence of Pancreatic Islet Neoplasms in Control Female F344/NTac Rats.

Table B-6. Historical Incidence of Mononuclear Cell Leukemia in Control Female F344/NTac Rats

Table B-7. Summary of the Incidence of Nonneoplastic Lesions in Female Rats in the Two-year Inhalation Study of Cobalt Metal. 
Table B-1. Summary of the Incidence of Neoplasms in Female Rats in the Two-year Inhalation Study of Cobalt Metal ${ }^{\text {a }}$

\begin{tabular}{|c|c|c|c|c|}
\hline & $\begin{array}{l}\text { Chamber } \\
\text { Control }\end{array}$ & $1.25 \mathrm{mg} / \mathrm{m}^{3}$ & $2.5 \mathrm{mg} / \mathrm{m}^{3}$ & $5 \mathrm{mg} / \mathrm{m}^{3}$ \\
\hline \multicolumn{5}{|l|}{ Disposition Summary } \\
\hline Animals initially in study & 50 & 50 & 50 & 50 \\
\hline \multicolumn{5}{|l|}{ Early deaths } \\
\hline Moribund & 11 & 20 & 19 & 24 \\
\hline Natural deaths & 4 & 4 & 7 & 1 \\
\hline \multicolumn{5}{|l|}{ Survivors } \\
\hline Died last week of study & - & - & - & 1 \\
\hline Terminal kill & 35 & 26 & 24 & 24 \\
\hline Animals examined microscopically & 50 & 50 & 50 & 50 \\
\hline \multicolumn{5}{|l|}{ Alimentary System } \\
\hline Esophagus & $(50)$ & $(50)$ & (49) & (48) \\
\hline Intestine large, cecum & (50) & $(50)$ & (49) & $(50)$ \\
\hline Carcinoma, metastatic, islets, pancreatic & $1(2 \%)$ & - & - & - \\
\hline Intestine large, colon & $(50)$ & (50) & (49) & $(50)$ \\
\hline Carcinoma, metastatic, islets, pancreatic & $1(2 \%)$ & - & - & - \\
\hline Intestine large, rectum & $(50)$ & (50) & (50) & (49) \\
\hline Intestine small, duodenum & $(48)$ & $(50)$ & $(50)$ & $(50)$ \\
\hline Intestine small, ileum & (49) & $(50)$ & $(48)$ & $(50)$ \\
\hline Carcinoma & $1(2 \%)$ & - & - & - \\
\hline Carcinoma, metastatic, islets, pancreatic & $1(2 \%)$ & - & - & - \\
\hline Fibrous histiocytoma, metastatic, skin & $1(2 \%)$ & - & - & - \\
\hline Intestine small, jejunum & $(50)$ & (50) & (47) & $(50)$ \\
\hline Carcinoma & $1(2 \%)$ & - & - & - \\
\hline Carcinoma, metastatic, islets, pancreatic & $1(2 \%)$ & - & - & - \\
\hline Liver & $(50)$ & $(50)$ & $(50)$ & $(50)$ \\
\hline Carcinoma, metastatic, islets, pancreatic & $1(2 \%)$ & - & - & - \\
\hline Fibrous histiocytoma, metastatic, skin & $1(2 \%)$ & $1(2 \%)$ & - & - \\
\hline Hepatocellular adenoma & - & $1(2 \%)$ & - & - \\
\hline Hepatocellular carcinoma & - & $1(2 \%)$ & - & - \\
\hline Mesentery & (13) & (15) & (8) & (9) \\
\hline Carcinoma, metastatic, islets, pancreatic & $1(8 \%)$ & - & - & - \\
\hline Pancreas & $(50)$ & $(50)$ & $(50)$ & $(50)$ \\
\hline $\begin{array}{l}\text { Acinus, carcinoma, metastatic, islets, } \\
\text { pancreatic }\end{array}$ & $1(2 \%)$ & - & - & - \\
\hline
\end{tabular}


Cobalt Metal, NTP TR 581

\begin{tabular}{|c|c|c|c|c|}
\hline & $\begin{array}{l}\text { Chamber } \\
\text { Control }\end{array}$ & $1.25 \mathrm{mg} / \mathrm{m}^{3}$ & $2.5 \mathrm{mg} / \mathrm{m}^{3}$ & $5 \mathrm{mg} / \mathrm{m}^{3}$ \\
\hline Salivary glands & $(50)$ & $(50)$ & $(50)$ & $(50)$ \\
\hline Fibrous histiocytoma, metastatic, skin & $1(2 \%)$ & - & - & - \\
\hline Stomach, forestomach & $(50)$ & $(50)$ & $(50)$ & $(50)$ \\
\hline Squamous cell papilloma & $1(2 \%)$ & - & - & $1(2 \%)$ \\
\hline Stomach, glandular & $(50)$ & $(50)$ & (50) & (50) \\
\hline Tongue & (1) & $(0)$ & (1) & (0) \\
\hline Squamous cell carcinoma & - & - & $1(100 \%)$ & - \\
\hline Squamous cell papilloma & $1(100 \%)$ & - & - & - \\
\hline Tooth & (1) & (0) & (2) & (2) \\
\hline \multicolumn{5}{|l|}{ Cardiovascular System } \\
\hline Blood vessel & (0) & (1) & $(0)$ & (0) \\
\hline $\begin{array}{l}\text { Alveolar/bronchiolar carcinoma, metastatic, } \\
\text { lung }\end{array}$ & - & $1(100 \%)$ & - & - \\
\hline $\begin{array}{l}\text { Aorta, alveolar/bronchiolar carcinoma, } \\
\text { metastatic, lung }\end{array}$ & - & $1(100 \%)$ & - & - \\
\hline Heart & $(50)$ & $(50)$ & $(50)$ & $(50)$ \\
\hline Carcinoma, metastatic, islets, pancreatic & $1(2 \%)$ & - & - & - \\
\hline Fibrous histiocytoma, metastatic, skin & $1(2 \%)$ & - & - & - \\
\hline $\begin{array}{l}\text { Pheochromocytoma malignant, metastatic, } \\
\text { adrenal medulla }\end{array}$ & - & - & - & $1(2 \%)$ \\
\hline Schwannoma malignant & - & - & $1(2 \%)$ & - \\
\hline $\begin{array}{l}\text { Pericardium, rhabdomyosarcoma, metastatic, } \\
\text { uncertain primary site }\end{array}$ & - & - & - & $1(2 \%)$ \\
\hline \multicolumn{5}{|l|}{ Endocrine System } \\
\hline Adrenal cortex & $(50)$ & $(50)$ & $(50)$ & $(50)$ \\
\hline Carcinoma, metastatic, islets, pancreatic & $1(2 \%)$ & - & - & - \\
\hline Adrenal medulla & $(50)$ & $(50)$ & $(50)$ & $(50)$ \\
\hline Pheochromocytoma benign & $4(8 \%)$ & $8(16 \%)$ & $14(28 \%)$ & $17(34 \%)$ \\
\hline Pheochromocytoma malignant & - & $1(2 \%)$ & $2(4 \%)$ & $7(14 \%)$ \\
\hline Bilateral, fibrous histiocytoma, metastatic, skin & $1(2 \%)$ & - & - & - \\
\hline Bilateral, pheochromocytoma benign & $2(4 \%)$ & $4(8 \%)$ & $8(16 \%)$ & $19(38 \%)$ \\
\hline Bilateral, pheochromocytoma malignant & - & $1(2 \%)$ & $1(2 \%)$ & $4(8 \%)$ \\
\hline Islets, pancreatic & $(50)$ & $(50)$ & $(50)$ & $(50)$ \\
\hline Adenoma & - & - & - & $1(2 \%)$ \\
\hline Carcinoma & $1(2 \%)$ & - & - & $3(6 \%)$ \\
\hline Parathyroid gland & $(42)$ & $(45)$ & (38) & $(45)$ \\
\hline Pituitary gland & $(50)$ & $(50)$ & $(49)$ & $(50)$ \\
\hline
\end{tabular}


Cobalt Metal, NTP TR 581

\begin{tabular}{|c|c|c|c|c|}
\hline & $\begin{array}{l}\text { Chamber } \\
\text { Control }\end{array}$ & $1.25 \mathrm{mg} / \mathrm{m}^{3}$ & $2.5 \mathrm{mg} / \mathrm{m}^{3}$ & $5 \mathrm{mg} / \mathrm{m}^{3}$ \\
\hline Pars distalis, adenoma & $29(58 \%)$ & $23(46 \%)$ & $26(53 \%)$ & $22(44 \%)$ \\
\hline Pars distalis, carcinoma & - & $3(6 \%)$ & $1(2 \%)$ & $1(2 \%)$ \\
\hline Thyroid gland & (50) & (49) & (49) & $(50)$ \\
\hline Bilateral, C-cell, adenoma & $1(2 \%)$ & - & $2(4 \%)$ & - \\
\hline C-cell, adenoma & $7(14 \%)$ & $6(12 \%)$ & $2(4 \%)$ & $2(4 \%)$ \\
\hline C-cell, carcinoma & $1(2 \%)$ & $2(4 \%)$ & $1(2 \%)$ & $1(2 \%)$ \\
\hline Follicular cell, adenoma & - & - & $1(2 \%)$ & $1(2 \%)$ \\
\hline Follicular cell, carcinoma & $1(2 \%)$ & - & - & - \\
\hline \multicolumn{5}{|l|}{ General Body System } \\
\hline Peritoneum & (1) & (1) & $(0)$ & $(0)$ \\
\hline Carcinoma, metastatic, islets, pancreatic & $1(100 \%)$ & - & - & - \\
\hline Fibrous histiocytoma, metastatic, skin & - & $1(100 \%)$ & - & - \\
\hline \multicolumn{5}{|l|}{ Genital System } \\
\hline Clitoral gland & (49) & (47) & (47) & (46) \\
\hline Adenoma & $2(4 \%)$ & $4(9 \%)$ & $6(13 \%)$ & $2(4 \%)$ \\
\hline Carcinoma & $4(8 \%)$ & $5(11 \%)$ & $3(6 \%)$ & $4(9 \%)$ \\
\hline Bilateral, adenoma & - & $1(2 \%)$ & - & - \\
\hline Ovary & $(50)$ & $(50)$ & $(50)$ & $(50)$ \\
\hline Carcinoma, metastatic, islets, pancreatic & $1(2 \%)$ & - & - & - \\
\hline Cystadenocarcinoma & - & $1(2 \%)$ & - & - \\
\hline Granulosa cell tumor malignant & - & - & $1(2 \%)$ & - \\
\hline Thecoma benign & - & - & - & $1(2 \%)$ \\
\hline Uterus & $(50)$ & (50) & $(50)$ & $(50)$ \\
\hline Carcinoma & - & $1(2 \%)$ & $1(2 \%)$ & - \\
\hline Carcinoma, metastatic, islets, pancreatic & $1(2 \%)$ & - & - & - \\
\hline Hemangiosarcoma & $1(2 \%)$ & - & - & - \\
\hline Leiomyoma & $1(2 \%)$ & $1(2 \%)$ & - & - \\
\hline Polyp stromal & $10(20 \%)$ & $7(14 \%)$ & $6(12 \%)$ & $10(20 \%)$ \\
\hline \multicolumn{5}{|l|}{ Hematopoietic System } \\
\hline Bone marrow & $(50)$ & (50) & (50) & (50) \\
\hline Lymph node & (4) & (3) & (7) & $(5)$ \\
\hline $\begin{array}{l}\text { Deep cervical, fibrous histiocytoma, } \\
\text { metastatic, skin }\end{array}$ & $1(25 \%)$ & - & - & - \\
\hline $\begin{array}{l}\text { Lumbar, renal, carcinoma, metastatic, islets, } \\
\text { pancreatic }\end{array}$ & $1(25 \%)$ & - & - & - \\
\hline Lymph node, bronchial & $(26)$ & (18) & (22) & (17) \\
\hline
\end{tabular}




\begin{tabular}{|c|c|c|c|c|}
\hline & $\begin{array}{l}\text { Chamber } \\
\text { Control }\end{array}$ & $1.25 \mathrm{mg} / \mathrm{m}^{3}$ & $2.5 \mathrm{mg} / \mathrm{m}^{3}$ & $5 \mathrm{mg} / \mathrm{m}^{3}$ \\
\hline $\begin{array}{l}\text { Alveolar/bronchiolar carcinoma, metastatic, } \\
\text { lung }\end{array}$ & - & - & - & $1(6 \%)$ \\
\hline Fibrous histiocytoma, metastatic, skin & $1(4 \%)$ & - & - & - \\
\hline Lymph node, mandibular & $(48)$ & (48) & (47) & $(47)$ \\
\hline Fibrous histiocytoma, metastatic, skin & $1(2 \%)$ & - & - & - \\
\hline Lymph node, mediastinal & $(42)$ & (43) & (48) & $(48)$ \\
\hline Carcinoma, metastatic, islets, pancreatic & $1(2 \%)$ & - & - & - \\
\hline Lymph node, mesenteric & $(50)$ & $(50)$ & (50) & $(50)$ \\
\hline Carcinoma, metastatic, islets, pancreatic & $1(2 \%)$ & - & - & - \\
\hline Spleen & $(50)$ & $(50)$ & $(50)$ & $(50)$ \\
\hline Carcinoma, metastatic, islets, pancreatic & $1(2 \%)$ & - & - & - \\
\hline Fibrous histiocytoma, metastatic, skin & $1(2 \%)$ & - & - & - \\
\hline Thymus & (47) & (46) & (46) & (47) \\
\hline $\begin{array}{l}\text { Alveolar/bronchiolar carcinoma, metastatic, } \\
\text { lung }\end{array}$ & - & - & - & $1(2 \%)$ \\
\hline \multicolumn{5}{|l|}{ Integumentary System } \\
\hline Mammary gland & $(50)$ & $(50)$ & (50) & $(50)$ \\
\hline Adenocarcinoma & - & - & $1(2 \%)$ & - \\
\hline Adenoma & $1(2 \%)$ & $2(4 \%)$ & $1(2 \%)$ & $1(2 \%)$ \\
\hline Carcinoma & $2(4 \%)$ & $5(10 \%)$ & - & $3(6 \%)$ \\
\hline Fibroadenoma & $17(34 \%)$ & $21(42 \%)$ & $15(30 \%)$ & $5(10 \%)$ \\
\hline Fibroadenoma, multiple & $1(2 \%)$ & $1(2 \%)$ & - & - \\
\hline Skin & $(50)$ & (50) & (50) & $(50)$ \\
\hline Basal cell adenoma & $1(2 \%)$ & - & - & - \\
\hline Keratoacanthoma & - & - & $2(4 \%)$ & $1(2 \%)$ \\
\hline Papilloma & - & $1(2 \%)$ & - & - \\
\hline Squamous cell papilloma & $1(2 \%)$ & - & $1(2 \%)$ & - \\
\hline Subcutaneous tissue, fibroma & $2(4 \%)$ & $1(2 \%)$ & - & $1(2 \%)$ \\
\hline Subcutaneous tissue, fibrous histiocytoma & $1(2 \%)$ & $2(4 \%)$ & - & - \\
\hline Subcutaneous tissue, lipoma & $1(2 \%)$ & - & - & - \\
\hline Subcutaneous tissue, schwannoma malignant & $1(2 \%)$ & - & - & $1(2 \%)$ \\
\hline \multicolumn{5}{|l|}{ Musculoskeletal System } \\
\hline Bone & $(50)$ & $(50)$ & (50) & $(50)$ \\
\hline Skeletal muscle & (1) & (1) & $(0)$ & (1) \\
\hline Carcinoma, metastatic, islets, pancreatic & $1(100 \%)$ & - & - & - \\
\hline Lipoma & - & $1(100 \%)$ & - & - \\
\hline
\end{tabular}




\begin{tabular}{|c|c|c|c|c|}
\hline & $\begin{array}{l}\text { Chamber } \\
\text { Control }\end{array}$ & $1.25 \mathrm{mg} / \mathrm{m}^{3}$ & $2.5 \mathrm{mg} / \mathrm{m}^{3}$ & $5 \mathrm{mg} / \mathrm{m}^{3}$ \\
\hline Schwannoma malignant, metastatic, skin & - & - & - & $1(100 \%)$ \\
\hline \multicolumn{5}{|l|}{ Nervous System } \\
\hline Brain & $(50)$ & $(50)$ & $(50)$ & $(50)$ \\
\hline Carcinoma, metastatic, pituitary gland & - & $3(6 \%)$ & $1(2 \%)$ & $1(2 \%)$ \\
\hline Glioma malignant & - & - & $1(2 \%)$ & - \\
\hline \multicolumn{5}{|l|}{ Respiratory System } \\
\hline Larynx & $(50)$ & $(50)$ & $(50)$ & $(50)$ \\
\hline Lung & $(50)$ & $(50)$ & $(50)$ & $(50)$ \\
\hline Alveolar/bronchiolar adenoma & $2(4 \%)$ & $6(12 \%)$ & $6(12 \%)$ & $9(18 \%)$ \\
\hline Alveolar/bronchiolar adenoma, multiple & - & $1(2 \%)$ & $3(6 \%)$ & $4(8 \%)$ \\
\hline Alveolar/bronchiolar carcinoma & - & $5(10 \%)$ & $14(28 \%)$ & $12(24 \%)$ \\
\hline Alveolar/bronchiolar carcinoma, multiple & - & $4(8 \%)$ & $3(6 \%)$ & $18(36 \%)$ \\
\hline Carcinoma, metastatic, islets, pancreatic & $1(2 \%)$ & - & - & - \\
\hline Cystic keratinizing epithelioma & - & $4(8 \%)$ & $1(2 \%)$ & $2(4 \%)$ \\
\hline Fibrous histiocytoma, metastatic, skin & $1(2 \%)$ & - & - & - \\
\hline $\begin{array}{l}\text { Pheochromocytoma malignant, metastatic, } \\
\text { adrenal medulla }\end{array}$ & - & - & - & $1(2 \%)$ \\
\hline Squamous cell carcinoma & - & - & - & $1(2 \%)$ \\
\hline $\begin{array}{l}\text { Mediastinum, alveolar/bronchiolar carcinoma, } \\
\text { metastatic, lung }\end{array}$ & - & $2(4 \%)$ & - & $1(2 \%)$ \\
\hline $\begin{array}{l}\text { Mediastinum, fibrous histiocytoma, metastatic, } \\
\text { skin }\end{array}$ & $1(2 \%)$ & - & - & - \\
\hline Nose & $(50)$ & $(50)$ & (49) & $(50)$ \\
\hline Trachea & $(50)$ & $(50)$ & $(50)$ & $(50)$ \\
\hline \multicolumn{5}{|l|}{ Special Senses System } \\
\hline Ear & (4) & (4) & $(0)$ & (3) \\
\hline Eye & $(50)$ & $(50)$ & (49) & $(50)$ \\
\hline Harderian gland & $(50)$ & $(50)$ & $(50)$ & $(50)$ \\
\hline Zymbal's gland & (0) & (1) & $(0)$ & $(0)$ \\
\hline Adenoma & - & $1(100 \%)$ & - & - \\
\hline \multicolumn{5}{|l|}{ Urinary System } \\
\hline Kidney & $(50)$ & $(50)$ & $(50)$ & $(50)$ \\
\hline Transitional epithelium, papilloma & - & $1(2 \%)$ & - & - \\
\hline Urinary bladder & $(50)$ & $(50)$ & $(50)$ & $(50)$ \\
\hline Carcinoma, metastatic, islets, pancreatic & $1(2 \%)$ & - & - & - \\
\hline Schwannoma malignant, metastatic, skin & - & - & - & $1(2 \%)$ \\
\hline
\end{tabular}


Cobalt Metal, NTP TR 581

\begin{tabular}{|c|c|c|c|c|}
\hline & $\begin{array}{l}\text { Chamber } \\
\text { Control }\end{array}$ & $1.25 \mathrm{mg} / \mathrm{m}^{3}$ & $2.5 \mathrm{mg} / \mathrm{m}^{3}$ & $5 \mathrm{mg} / \mathrm{m}^{3}$ \\
\hline \multicolumn{5}{|l|}{ Systemic Lesions } \\
\hline Multiple organs ${ }^{\mathrm{b}}$ & $(50)$ & (50) & $(50)$ & $(50)$ \\
\hline Leukemia mononuclear & $16(32 \%)$ & $29(58 \%)$ & $28(56 \%)$ & $27(54 \%)$ \\
\hline \multicolumn{5}{|l|}{ Neoplasm Summary } \\
\hline Total animals with primary neoplasms ${ }^{c}$ & 48 & 50 & 50 & 49 \\
\hline Total primary neoplasms & 114 & 155 & 153 & 181 \\
\hline Total animals with benign neoplasms & 44 & 44 & 43 & 43 \\
\hline Total benign neoplasms & 84 & 95 & 94 & 99 \\
\hline Total animals with malignant neoplasms & 25 & 42 & 42 & 48 \\
\hline Total malignant neoplasms & 30 & 60 & 59 & 82 \\
\hline Total animals with metastatic neoplasms & 2 & 5 & 1 & 6 \\
\hline Total metastatic neoplasms & 30 & 9 & 1 & 9 \\
\hline $\begin{array}{l}\text { Total animals with malignant neoplasms of } \\
\text { uncertain primary site }\end{array}$ & - & - & - & 1 \\
\hline
\end{tabular}

${ }^{a}$ Number of animals examined microscopically at the site and the number of animals with neoplasm.

${ }^{b}$ Number of animals with any tissue examined microscopically.

cPrimary neoplasms: all neoplasms except metastatic neoplasms. 
Table B-2. Statistical Analysis of Primary Neoplasms in Female Rats in the Two-year Inhalation Study of Cobalt Metal

\begin{tabular}{|c|c|c|c|c|}
\hline & $\begin{array}{l}\text { Chamber } \\
\text { Control }\end{array}$ & $1.25 \mathrm{mg} / \mathrm{m}^{3}$ & $2.5 \mathrm{mg} / \mathrm{m}^{3}$ & $5 \mathrm{mg} / \mathrm{m}^{3}$ \\
\hline \multicolumn{5}{|c|}{ Adrenal Medulla: Benign Pheochromocytoma } \\
\hline Overall rate $^{\mathrm{a}}$ & $6 / 50(12 \%)$ & $12 / 50(24 \%)$ & $22 / 50(44 \%)$ & $36 / 50(72 \%)$ \\
\hline Adjusted rate ${ }^{b}$ & $13.6 \%$ & $27.2 \%$ & $52.1 \%$ & $80.6 \%$ \\
\hline Terminal rate ${ }^{\mathrm{c}}$ & $6 / 35(17 \%)$ & $5 / 26(19 \%)$ & $13 / 24(54 \%)$ & $21 / 25(84 \%)$ \\
\hline First incidence (days) & $730(\mathrm{~T})$ & 598 & 590 & 579 \\
\hline Poly-3 test ${ }^{\mathrm{d}}$ & $\mathrm{P}<0.001$ & $\mathrm{P}=0.091$ & $\mathrm{P}<0.001$ & $\mathrm{P}<0.001$ \\
\hline \multicolumn{5}{|c|}{ Adrenal Medulla: Malignant Pheochromocytoma } \\
\hline Overall rate & $0 / 50(0 \%)$ & $2 / 50(4 \%)$ & $3 / 50(6 \%)$ & $11 / 50(22 \%)$ \\
\hline Adjusted rate & $0.0 \%$ & $4.7 \%$ & $7.5 \%$ & $27.0 \%$ \\
\hline Terminal rate & $0 / 35(0 \%)$ & $2 / 26(8 \%)$ & $2 / 24(8 \%)$ & $9 / 25(36 \%)$ \\
\hline First incidence (days) & $-^{\mathrm{e}}$ & $730(\mathrm{~T})$ & 715 & 712 \\
\hline Poly-3 test & $\mathrm{P}<0.001$ & $\mathrm{P}=0.228$ & $\mathrm{P}=0.102$ & $\mathrm{P}<0.001$ \\
\hline \multicolumn{5}{|c|}{ Adrenal Medulla: Benign or Malignant Pheochromocytoma } \\
\hline Overall rate & $6 / 50(12 \%)$ & $13 / 50(26 \%)$ & $23 / 50(46 \%)$ & $40 / 50(80 \%)$ \\
\hline Adjusted rate & $13.6 \%$ & $29.4 \%$ & $54.5 \%$ & $89.4 \%$ \\
\hline Terminal rate & $6 / 35(17 \%)$ & $6 / 26(23 \%)$ & $14 / 24(58 \%)$ & $24 / 25(96 \%)$ \\
\hline First incidence (days) & $730(\mathrm{~T})$ & 598 & 590 & 579 \\
\hline Poly-3 test & $\mathrm{P}<0.001$ & $\mathrm{P}=0.058$ & $\mathrm{P}<0.001$ & $\mathrm{P}<0.001$ \\
\hline \multicolumn{5}{|c|}{ Clitoral Gland: Adenoma } \\
\hline Overall rate & $2 / 49(4 \%)$ & $5 / 47(11 \%)$ & 6/47 (13\%) & $2 / 46(4 \%)$ \\
\hline Adjusted rate & $4.5 \%$ & $12.3 \%$ & $16.1 \%$ & $5.4 \%$ \\
\hline Terminal rate & $1 / 35(3 \%)$ & $1 / 23(4 \%)$ & $6 / 22(27 \%)$ & $1 / 22(5 \%)$ \\
\hline First incidence (days) & 674 & 593 & $730(\mathrm{~T})$ & 691 \\
\hline Poly-3 test & $\mathrm{P}=0.517 \mathrm{~N}$ & $\mathrm{P}=0.181$ & $\mathrm{P}=0.083$ & $\mathrm{P}=0.631$ \\
\hline \multicolumn{5}{|c|}{ Clitoral Gland: Carcinoma } \\
\hline Overall rate & $4 / 49(8 \%)$ & $5 / 47(11 \%)$ & $3 / 47(6 \%)$ & $4 / 46(9 \%)$ \\
\hline Adjusted rate & $9.1 \%$ & $12.5 \%$ & $8.0 \%$ & $10.6 \%$ \\
\hline Terminal rate & $3 / 35(9 \%)$ & $1 / 23(4 \%)$ & $1 / 22(5 \%)$ & $2 / 22(9 \%)$ \\
\hline First incidence (days) & 722 & 646 & 618 & 478 \\
\hline Poly-3 test & $\mathrm{P}=0.524 \mathrm{~N}$ & $\mathrm{P}=0.438$ & $\mathrm{P}=0.585 \mathrm{~N}$ & $\mathrm{P}=0.558$ \\
\hline \multicolumn{5}{|c|}{ Clitoral Gland: Adenoma or Carcinoma } \\
\hline Overall rate & $6 / 49(12 \%)$ & $10 / 47(21 \%)$ & 9/47 (19\%) & $6 / 46(13 \%)$ \\
\hline Adjusted rate & $13.5 \%$ & $24.3 \%$ & $23.9 \%$ & $15.8 \%$ \\
\hline Terminal rate & $4 / 35(11 \%)$ & $2 / 23(9 \%)$ & $7 / 22(32 \%)$ & $3 / 22(14 \%)$ \\
\hline
\end{tabular}




\begin{tabular}{|c|c|c|c|c|}
\hline & $\begin{array}{l}\text { Chamber } \\
\text { Control }\end{array}$ & $1.25 \mathrm{mg} / \mathrm{m}^{3}$ & $2.5 \mathrm{mg} / \mathrm{m}^{3}$ & $5 \mathrm{mg} / \mathrm{m}^{3}$ \\
\hline First incidence (days) & 674 & 593 & 618 & 478 \\
\hline Poly-3 test & $\mathrm{P}=0.480 \mathrm{~N}$ & $\mathrm{P}=0.159$ & $P=0.179$ & $\mathrm{P}=0.511$ \\
\hline \multicolumn{5}{|c|}{ Lung: Cystic Keratinizing Epithelioma } \\
\hline Overall rate & $0 / 50(0 \%)$ & $4 / 50(8 \%)$ & $1 / 50(2 \%)$ & $2 / 50(4 \%)$ \\
\hline Adjusted rate & $0.0 \%$ & $9.5 \%$ & $2.5 \%$ & $4.9 \%$ \\
\hline Terminal rate & $0 / 35(0 \%)$ & $4 / 26(15 \%)$ & $1 / 24(4 \%)$ & $1 / 25(4 \%)$ \\
\hline First incidence (days) & - & $730(\mathrm{~T})$ & $730(\mathrm{~T})$ & 603 \\
\hline Poly-3 test & $\mathrm{P}=0.582$ & $\mathrm{P}=0.055$ & $\mathrm{P}=0.481$ & $\mathrm{P}=0.222$ \\
\hline \multicolumn{5}{|c|}{ Lung: Alveolar/bronchiolar Adenoma } \\
\hline Overall rate & $2 / 50(4 \%)$ & $7 / 50(14 \%)$ & $9 / 50(18 \%)$ & $13 / 50(26 \%)$ \\
\hline Adjusted rate & $4.5 \%$ & $16.2 \%$ & $22.1 \%$ & $30.9 \%$ \\
\hline Terminal rate & $1 / 35(3 \%)$ & $5 / 26(19 \%)$ & $6 / 24(25 \%)$ & $8 / 25(32 \%)$ \\
\hline First incidence (days) & 698 & 590 & 587 & 579 \\
\hline Poly-3 test & $\mathrm{P}=0.002$ & $\mathrm{P}=0.072$ & $P=0.016$ & $\mathrm{P}<0.001$ \\
\hline \multicolumn{5}{|c|}{ Lung: Alveolar/bronchiolar Carcinoma } \\
\hline Overall rate & $0 / 50(0 \%)$ & $9 / 50(18 \%)$ & $17 / 50(34 \%)$ & $30 / 50(60 \%)$ \\
\hline Adjusted rate & $0.0 \%$ & $21.3 \%$ & $42.0 \%$ & $69.2 \%$ \\
\hline Terminal rate & $0 / 35(0 \%)$ & $9 / 26(35 \%)$ & $14 / 24(58 \%)$ & $20 / 25(80 \%)$ \\
\hline First incidence (days) & - & $730(\mathrm{~T})$ & 690 & 471 \\
\hline Poly-3 test & $\mathrm{P}<0.001$ & $\mathrm{P}<0.001$ & $\mathrm{P}<0.001$ & $\mathrm{P}<0.001$ \\
\hline \multicolumn{5}{|c|}{ Lung: Alveolar/bronchiolar Adenoma or Carcinoma } \\
\hline Overall rate & $2 / 50(4 \%)$ & $15 / 50(30 \%)$ & $20 / 50(40 \%)$ & $38 / 50(76 \%)$ \\
\hline Adjusted rate & $4.5 \%$ & $34.7 \%$ & $48.5 \%$ & $86.2 \%$ \\
\hline Terminal rate & $1 / 35(3 \%)$ & $13 / 26(50 \%)$ & $14 / 24(58 \%)$ & $25 / 25(100 \%)$ \\
\hline First incidence (days) & 698 & 590 & 587 & 471 \\
\hline Poly-3 test & $\mathrm{P}<0.001$ & $\mathrm{P}<0.001$ & $\mathrm{P}<0.001$ & $\mathrm{P}<0.001$ \\
\hline \multicolumn{5}{|c|}{ Mammary Gland: Fibroadenoma } \\
\hline Overall rate & $18 / 50(36 \%)$ & $22 / 50(44 \%)$ & $15 / 50(30 \%)$ & $5 / 50(10 \%)$ \\
\hline Adjusted rate & $39.3 \%$ & $49.5 \%$ & $36.0 \%$ & $12.3 \%$ \\
\hline Terminal rate & $14 / 35(40 \%)$ & $16 / 26(62 \%)$ & $9 / 24(38 \%)$ & $5 / 25(20 \%)$ \\
\hline First incidence (days) & 316 & 516 & 590 & $730(\mathrm{~T})$ \\
\hline Poly-3 test & $\mathrm{P}<0.001 \mathrm{~N}$ & $\mathrm{P}=0.220$ & $\mathrm{P}=0.459 \mathrm{~N}$ & $\mathrm{P}=0.003 \mathrm{~N}$ \\
\hline \multicolumn{5}{|c|}{ Mammary Gland: Fibroadenoma or Adenoma } \\
\hline Overall rate & $19 / 50(38 \%)$ & $23 / 50(46 \%)$ & $16 / 50(32 \%)$ & $6 / 50(12 \%)$ \\
\hline Adjusted rate & $41.5 \%$ & $51.3 \%$ & $38.4 \%$ & $14.7 \%$ \\
\hline
\end{tabular}




\begin{tabular}{|c|c|c|c|c|}
\hline & $\begin{array}{c}\text { Chamber } \\
\text { Control }\end{array}$ & $1.25 \mathrm{mg} / \mathrm{m}^{3}$ & $2.5 \mathrm{mg} / \mathrm{m}^{3}$ & $5 \mathrm{mg} / \mathrm{m}^{3}$ \\
\hline Terminal rate & $15 / 35(43 \%)$ & $16 / 26(62 \%)$ & $10 / 24(42 \%)$ & $6 / 25(24 \%)$ \\
\hline First incidence (days) & 316 & 516 & 590 & $730(\mathrm{~T})$ \\
\hline Poly-3 test & $\mathrm{P}<0.001 \mathrm{~N}$ & $\mathrm{P}=0.231$ & $\mathrm{P}=0.467 \mathrm{~N}$ & $\mathrm{P}=0.005 \mathrm{~N}$ \\
\hline \multicolumn{5}{|c|}{ Mammary Gland: Carcinoma } \\
\hline Overall rate & $2 / 50(4 \%)$ & $5 / 50(10 \%)$ & $1 / 50(2 \%)$ & $3 / 50(6 \%)$ \\
\hline Adjusted rate & $4.5 \%$ & $11.7 \%$ & $2.5 \%$ & $7.4 \%$ \\
\hline Terminal rate & $1 / 35(3 \%)$ & $3 / 26(12 \%)$ & $1 / 24(4 \%)$ & $3 / 25(12 \%)$ \\
\hline First incidence (days) & 698 & 578 & $730(\mathrm{~T})$ & $730(\mathrm{~T})$ \\
\hline Poly-3 test & $\mathrm{P}=0.446 \mathrm{~N}$ & $\mathrm{P}=0.203$ & $\mathrm{P}=0.534 \mathrm{~N}$ & $\mathrm{P}=0.462$ \\
\hline \multicolumn{5}{|c|}{ Mammary Gland: Adenoma or Carcinoma } \\
\hline Overall rate & $3 / 50(6 \%)$ & $7 / 50(14 \%)$ & $1 / 50(2 \%)$ & $4 / 50(8 \%)$ \\
\hline Adjusted rate & $6.8 \%$ & $16.2 \%$ & $2.5 \%$ & $9.8 \%$ \\
\hline Terminal rate & $2 / 35(6 \%)$ & $4 / 26(15 \%)$ & $1 / 24(4 \%)$ & $4 / 25(16 \%)$ \\
\hline First incidence (days) & 698 & 578 & $730(\mathrm{~T})$ & $730(\mathrm{~T})$ \\
\hline Poly-3 test & $\mathrm{P}=0.345 \mathrm{~N}$ & $\mathrm{P}=0.147$ & $\mathrm{P}=0.341 \mathrm{~N}$ & $\mathrm{P}=0.455$ \\
\hline \multicolumn{5}{|c|}{ Mammary Gland: Fibroadenoma, Adenoma, or Carcinoma } \\
\hline Overall rate & $20 / 50(40 \%)$ & $26 / 50(52 \%)$ & $16 / 50(32 \%)$ & $9 / 50(18 \%)$ \\
\hline Adjusted rate & $43.6 \%$ & $57.2 \%$ & $38.4 \%$ & $22.1 \%$ \\
\hline Terminal rate & $15 / 35(43 \%)$ & $17 / 26(65 \%)$ & $10 / 24(42 \%)$ & $9 / 25(36 \%)$ \\
\hline First incidence (days) & 316 & 516 & 590 & $730(\mathrm{~T})$ \\
\hline Poly-3 test & $\mathrm{P}<0.001 \mathrm{~N}$ & $\mathrm{P}=0.131$ & $\mathrm{P}=0.389 \mathrm{~N}$ & $\mathrm{P}=0.026 \mathrm{~N}$ \\
\hline \multicolumn{5}{|c|}{ Pancreatic Islets: Carcinoma } \\
\hline Overall rate & $1 / 50(2 \%)$ & $0 / 50(0 \%)$ & $0 / 50(0 \%)$ & $3 / 50(6 \%)$ \\
\hline Adjusted rate & $2.2 \%$ & $0.0 \%$ & $0.0 \%$ & $7.2 \%$ \\
\hline Terminal rate & $0 / 35(0 \%)$ & $0 / 26(0 \%)$ & $0 / 24(0 \%)$ & $1 / 25(4 \%)$ \\
\hline First incidence (days) & 234 & - & - & 506 \\
\hline Poly-3 test & $\mathrm{P}=0.060$ & $\mathrm{P}=0.512 \mathrm{~N}$ & $\mathrm{P}=0.523 \mathrm{~N}$ & $\mathrm{P}=0.279$ \\
\hline \multicolumn{5}{|c|}{ Pancreatic Islets: Adenoma or Carcinoma } \\
\hline Overall rate & $1 / 50(2 \%)$ & $0 / 50(0 \%)$ & $0 / 50(0 \%)$ & $3 / 50(6 \%)$ \\
\hline Adjusted rate & $2.2 \%$ & $0.0 \%$ & $0.0 \%$ & $7.2 \%$ \\
\hline Terminal rate & $0 / 35(0 \%)$ & $0 / 26(0 \%)$ & $0 / 24(0 \%)$ & $1 / 25(4 \%)$ \\
\hline First incidence (days) & 234 & - & - & 506 \\
\hline Poly-3 test & $\mathrm{P}=0.060$ & $\mathrm{P}=0.512 \mathrm{~N}$ & $\mathrm{P}=0.523 \mathrm{~N}$ & $\mathrm{P}=0.279$ \\
\hline \multicolumn{5}{|c|}{ Pituitary Gland (Pars Distalis): Adenoma } \\
\hline Overall rate & $29 / 50(58 \%)$ & $23 / 50(46 \%)$ & $26 / 49(53 \%)$ & $22 / 50(44 \%)$ \\
\hline
\end{tabular}




\begin{tabular}{|c|c|c|c|c|}
\hline & $\begin{array}{l}\text { Chamber } \\
\text { Control }\end{array}$ & $1.25 \mathrm{mg} / \mathrm{m}^{3}$ & $2.5 \mathrm{mg} / \mathrm{m}^{3}$ & $5 \mathrm{mg} / \mathrm{m}^{3}$ \\
\hline Adjusted rate & $61.5 \%$ & $51.6 \%$ & $59.9 \%$ & $50.1 \%$ \\
\hline Terminal rate & $20 / 35(57 \%)$ & $14 / 26(54 \%)$ & $15 / 24(63 \%)$ & $11 / 25(44 \%)$ \\
\hline First incidence (days) & 511 & 593 & 291 & 506 \\
\hline Poly-3 test & $\mathrm{P}=0.287 \mathrm{~N}$ & $\mathrm{P}=0.223 \mathrm{~N}$ & $\mathrm{P}=0.525 \mathrm{~N}$ & $\mathrm{P}=0.183 \mathrm{~N}$ \\
\hline \multicolumn{5}{|c|}{ Pituitary Gland (Pars Distalis): Carcinoma } \\
\hline Overall rate & $0 / 50(0 \%)$ & $3 / 50(6 \%)$ & $1 / 49(2 \%)$ & $1 / 50(2 \%)$ \\
\hline Adjusted rate & $0.0 \%$ & $6.9 \%$ & $2.5 \%$ & $2.5 \%$ \\
\hline Terminal rate & $0 / 35(0 \%)$ & $1 / 26(4 \%)$ & $1 / 24(4 \%)$ & $1 / 25(4 \%)$ \\
\hline First incidence (days) & - & 578 & $730(\mathrm{~T})$ & $730(\mathrm{~T})$ \\
\hline Poly-3 test & $\mathrm{P}=0.497 \mathrm{~N}$ & $\mathrm{P}=0.115$ & $\mathrm{P}=0.478$ & $\mathrm{P}=0.484$ \\
\hline \multicolumn{5}{|c|}{ Pituitary Gland (Pars Distalis): Adenoma or Carcinoma } \\
\hline Overall rate & $29 / 50(58 \%)$ & $26 / 50(52 \%)$ & $27 / 49(55 \%)$ & $23 / 50(46 \%)$ \\
\hline Adjusted rate & $61.5 \%$ & $57.1 \%$ & $62.2 \%$ & $52.3 \%$ \\
\hline Terminal rate & $20 / 35(57 \%)$ & $15 / 26(58 \%)$ & $16 / 24(67 \%)$ & $12 / 25(48 \%)$ \\
\hline First incidence (days) & 511 & 578 & 291 & 506 \\
\hline Poly-3 test & $\mathrm{P}=0.279 \mathrm{~N}$ & $\mathrm{P}=0.412 \mathrm{~N}$ & $\mathrm{P}=0.559$ & $\mathrm{P}=0.247 \mathrm{~N}$ \\
\hline \multicolumn{5}{|c|}{ Skin: Papilloma, Squamous Cell Papilloma, or Keratoacanthoma } \\
\hline Overall rate & $1 / 50(2 \%)$ & $1 / 50(2 \%)$ & $3 / 50(6 \%)$ & $1 / 50(2 \%)$ \\
\hline Adjusted rate & $2.3 \%$ & $2.4 \%$ & $7.5 \%$ & $2.5 \%$ \\
\hline Terminal rate & $1 / 35(3 \%)$ & $1 / 26(4 \%)$ & $3 / 24(13 \%)$ & $1 / 25(4 \%)$ \\
\hline First incidence (days) & $730(\mathrm{~T})$ & $730(\mathrm{~T})$ & $730(\mathrm{~T})$ & $730(\mathrm{~T})$ \\
\hline Poly-3 test & $\mathrm{P}=0.469$ & $\mathrm{P}=0.752$ & $\mathrm{P}=0.272$ & $\mathrm{P}=0.743$ \\
\hline \multicolumn{5}{|c|}{ Skin: Papilloma, Squamous Cell Papilloma, Keratoacanthoma, or Basal Cell Adenoma } \\
\hline Overall rate & $2 / 50(4 \%)$ & $1 / 50(2 \%)$ & $3 / 50(6 \%)$ & $1 / 50(2 \%)$ \\
\hline Adjusted rate & $4.5 \%$ & $2.4 \%$ & $7.5 \%$ & $2.5 \%$ \\
\hline Terminal rate & $2 / 35(6 \%)$ & $1 / 26(4 \%)$ & $3 / 24(13 \%)$ & $1 / 25(4 \%)$ \\
\hline First incidence (days) & $730(\mathrm{~T})$ & $730(\mathrm{~T})$ & $730(\mathrm{~T})$ & $730(\mathrm{~T})$ \\
\hline Poly-3 test & $\mathrm{P}=0.570 \mathrm{~N}$ & $\mathrm{P}=0.514 \mathrm{~N}$ & $\mathrm{P}=0.457$ & $\mathrm{P}=0.528 \mathrm{~N}$ \\
\hline \multicolumn{5}{|c|}{ Skin (Subcutaneous Tissue): Fibroma or Fibrous Histiocytoma } \\
\hline Overall rate & $3 / 50(6 \%)$ & $3 / 50(6 \%)$ & $0 / 50(0 \%)$ & $1 / 50(2 \%)$ \\
\hline Adjusted rate & $6.6 \%$ & $7.0 \%$ & $0.0 \%$ & $2.5 \%$ \\
\hline Terminal rate & $1 / 35(3 \%)$ & $1 / 26(4 \%)$ & $0 / 24(0 \%)$ & $1 / 25(4 \%)$ \\
\hline First incidence (days) & 316 & 422 & - & $730(\mathrm{~T})$ \\
\hline Poly-3 test & $\mathrm{P}=0.118 \mathrm{~N}$ & $P=0.641$ & $\mathrm{P}=0.141 \mathrm{~N}$ & $\mathrm{P}=0.344 \mathrm{~N}$ \\
\hline
\end{tabular}




\begin{tabular}{|c|c|c|c|c|}
\hline & $\begin{array}{l}\text { Chamber } \\
\text { Control }\end{array}$ & $1.25 \mathrm{mg} / \mathrm{m}^{3}$ & $2.5 \mathrm{mg} / \mathrm{m}^{3}$ & $5 \mathrm{mg} / \mathrm{m}^{3}$ \\
\hline \multicolumn{5}{|c|}{ Thyroid Gland (C-Cell): Adenoma } \\
\hline Overall rate & $8 / 50(16 \%)$ & $6 / 49(12 \%)$ & $4 / 49(8 \%)$ & $2 / 50(4 \%)$ \\
\hline Adjusted rate & $18.1 \%$ & $14.5 \%$ & $10.2 \%$ & $4.8 \%$ \\
\hline Terminal rate & $7 / 35(20 \%)$ & $5 / 25(20 \%)$ & $4 / 24(17 \%)$ & $1 / 25(4 \%)$ \\
\hline First incidence (days) & 674 & 684 & $730(\mathrm{~T})$ & 506 \\
\hline Poly-3 test & $\mathrm{P}=0.036 \mathrm{~N}$ & $\mathrm{P}=0.437 \mathrm{~N}$ & $\mathrm{P}=0.237 \mathrm{~N}$ & $\mathrm{P}=0.056 \mathrm{~N}$ \\
\hline \multicolumn{5}{|c|}{ Thyroid Gland (C-Cell): Adenoma or Carcinoma } \\
\hline Overall rate & $9 / 50(18 \%)$ & $8 / 49(16 \%)$ & $5 / 49(10 \%)$ & $3 / 50(6 \%)$ \\
\hline Adjusted rate & $20.3 \%$ & $19.3 \%$ & $12.7 \%$ & $7.2 \%$ \\
\hline Terminal rate & $8 / 35(23 \%)$ & $7 / 25(28 \%)$ & $5 / 24(21 \%)$ & $1 / 25(4 \%)$ \\
\hline First incidence (days) & 674 & 684 & $730(\mathrm{~T})$ & 506 \\
\hline Poly-3 test & $\mathrm{P}=0.035 \mathrm{~N}$ & $\mathrm{P}=0.560 \mathrm{~N}$ & $\mathrm{P}=0.262 \mathrm{~N}$ & $\mathrm{P}=0.072 \mathrm{~N}$ \\
\hline \multicolumn{5}{|l|}{ Uterus: Stromal Polyp } \\
\hline Overall rate & $10 / 50(20 \%)$ & $7 / 50(14 \%)$ & $6 / 50(12 \%)$ & $10 / 50(20 \%)$ \\
\hline Adjusted rate & $22.3 \%$ & $16.2 \%$ & $14.5 \%$ & $24.1 \%$ \\
\hline Terminal rate & $7 / 35(20 \%)$ & $4 / 26(15 \%)$ & $3 / 24(13 \%)$ & $6 / 25(24 \%)$ \\
\hline First incidence (days) & 646 & 624 & 541 & 646 \\
\hline Poly-3 test & $\mathrm{P}=0.377$ & $\mathrm{P}=0.326 \mathrm{~N}$ & $\mathrm{P}=0.253 \mathrm{~N}$ & $\mathrm{P}=0.524$ \\
\hline \multicolumn{5}{|c|}{ All Organs: Mononuclear Cell Leukemia } \\
\hline Overall rate & $16 / 50(32 \%)$ & $29 / 50(58 \%)$ & $28 / 50(56 \%)$ & $27 / 50(54 \%)$ \\
\hline Adjusted rate & $35.7 \%$ & $62.4 \%$ & $60.5 \%$ & $58.9 \%$ \\
\hline Terminal rate & $12 / 35(34 \%)$ & $15 / 26(58 \%)$ & $12 / 24(50 \%)$ & $13 / 25(52 \%)$ \\
\hline First incidence (days) & 663 & 590 & 117 & 473 \\
\hline Poly-3 test & $\mathrm{P}=0.118$ & $\mathrm{P}=0.007$ & $\mathrm{P}=0.013$ & $\mathrm{P}=0.019$ \\
\hline \multicolumn{5}{|c|}{ All Organs: Benign Neoplasms } \\
\hline Overall rate & $44 / 50(88 \%)$ & $44 / 50(88 \%)$ & $43 / 50(86 \%)$ & $43 / 50(86 \%)$ \\
\hline Adjusted rate & $89.7 \%$ & $91.4 \%$ & $92.5 \%$ & $92.6 \%$ \\
\hline Terminal rate & $30 / 35(86 \%)$ & $24 / 26(92 \%)$ & $24 / 24(100 \%)$ & $24 / 25(96 \%)$ \\
\hline First incidence (days) & 316 & 516 & 291 & 506 \\
\hline Poly-3 test & $\mathrm{P}=0.373$ & $\mathrm{P}=0.525$ & $\mathrm{P}=0.454$ & $\mathrm{P}=0.447$ \\
\hline \multicolumn{5}{|c|}{ All Organs: Malignant Neoplasms } \\
\hline Overall rate & $25 / 50(50 \%)$ & $42 / 50(84 \%)$ & $42 / 50(84 \%)$ & $49 / 50(98 \%)$ \\
\hline Adjusted rate & $52.5 \%$ & $86.6 \%$ & $89.0 \%$ & $98.7 \%$ \\
\hline Terminal rate & $16 / 35(46 \%)$ & $23 / 26(89 \%)$ & $22 / 24(92 \%)$ & $25 / 25(100 \%)$ \\
\hline First incidence (days) & 234 & 422 & 117 & 471 \\
\hline
\end{tabular}


Cobalt Metal, NTP TR 581

\begin{tabular}{|c|c|c|c|c|}
\hline & $\begin{array}{c}\text { Chamber } \\
\text { Control }\end{array}$ & $1.25 \mathrm{mg} / \mathrm{m}^{3}$ & $2.5 \mathrm{mg} / \mathrm{m}^{3}$ & $5 \mathrm{mg} / \mathrm{m}^{3}$ \\
\hline Poly-3 test & $\mathrm{P}<0.001$ & $\mathrm{P}<0.001$ & $\mathrm{P}<0.001$ & $\mathrm{P}<0.001$ \\
\hline \multicolumn{5}{|c|}{ All Organs: Benign or Malignant Neoplasms } \\
\hline Overall rate & $48 / 50(96 \%)$ & $50 / 50(100 \%)$ & $50 / 50(100 \%)$ & $50 / 50(100 \%)$ \\
\hline Adjusted rate & $96.0 \%$ & $100.0 \%$ & $100.0 \%$ & $100.0 \%$ \\
\hline Terminal rate & $33 / 35(94 \%)$ & $26 / 26(100 \%)$ & $24 / 24(100 \%)$ & $25 / 25(100 \%)$ \\
\hline First incidence (days) & 234 & 422 & 117 & 471 \\
\hline Poly-3 test & $\mathrm{P}=0.185$ & $P=0.237$ & $P=0.237$ & $P=0.237$ \\
\hline
\end{tabular}

(T) Terminal kill.

${ }^{a}$ Number of neoplasm-bearing animals/number of animals examined. Denominator is number of animals examined

microscopically for adrenal gland, clitoral gland, lung, pancreatic islets, pituitary gland, and thyroid gland; for other tissues, denominator is number of animals necropsied.

bPoly-3 estimated neoplasm incidence after adjustment for intercurrent mortality.

'Observed incidence at terminal kill.

${ }^{\mathrm{d}}$ Beneath the chamber control incidence is the $\mathrm{P}$ value associated with the trend test. Beneath the exposed group incidence are the $P$ values corresponding to pairwise comparisons between the chamber controls and that exposed group. The Poly- 3 test accounts for differential mortality in animals that do not reach terminal kill. A negative trend or a lower incidence in an exposure group is indicated by $\mathbf{N}$.

${ }^{\mathrm{e} N o t}$ applicable; no neoplasms in animal group. 
Table B-3. Historical Incidence of Alveolar/bronchiolar Neoplasms in Control Female F344/NTac Rats $^{\text {a }}$

\begin{tabular}{lccc}
\hline & Adenoma & Carcinoma & Adenoma or Carcinoma \\
\hline Overall Historical Incidence: All Routes & & \\
Total $(\%)$ & $2 / 100(2.0 \%)$ & $0 / 100$ & $2 / 100(2.0 \%)$ \\
Mean \pm standard deviation & $2.0 \% \pm 2.8 \%$ & - & $2.0 \% \pm 2.8 \%$ \\
Range & $0 \%-4 \%$ & - & $0 \%-4 \%$ \\
\hline
\end{tabular}

${ }^{\mathrm{a} D a t a}$ as of June 2013.

Table B-4. Historical Incidence of Pheochromocytoma of the Adrenal Medulla in Control Female F344/NTac Rats ${ }^{\mathrm{a}}$

\begin{tabular}{lccc}
\hline & Benign & Malignant & Benign or Malignant \\
\hline Overall Historical Incidence: All Routes & & \\
Total ( $\%)$ & $7 / 100(7.0 \%)$ & $1 / 100(1.0 \%)$ & $8 / 100(8.0 \%)$ \\
Mean \pm standard deviation & $7.0 \% \pm 7.1 \%$ & $1.0 \% \pm 1.4 \%$ & $8.0 \% \pm 5.7 \%$ \\
Range & $2 \%-12 \%$ & $0 \%-2 \%$ & $4 \%-12 \%$ \\
\hline
\end{tabular}

${ }^{\mathrm{a} D a t a}$ as of June 2013.

Table B-5. Historical Incidence of Pancreatic Islet Neoplasms in Control Female F344/NTac Rats ${ }^{\mathrm{a}}$

Adenoma Carcinoma Adenoma or Carcinoma

\begin{tabular}{lccc}
\hline Overall Historical Incidence: All Routes & & \\
Total (\%) & $1 / 100(1.0 \%)$ & $1 / 100(1.0 \%)$ & $2 / 100(2.0 \%)$ \\
Mean \pm standard deviation & $1.0 \% \pm 1.4 \%$ & $1.0 \% \pm 1.4 \%$ & $2.0 \% \pm 0.0 \%$ \\
Range & $0 \%-2 \%$ & $0 \%-2 \%$ & $2 \%$ \\
\hline
\end{tabular}

${ }^{\text {aData as of June } 2013 .}$

Table B-6. Historical Incidence of Mononuclear Cell Leukemia in Control Female F344/NTac Rats ${ }^{\mathrm{a}}$ Incidence in Controls

\begin{tabular}{lc}
\hline Overall Historical Incidence: All Routes & \\
Total (\%) & $35 / 100(35.0 \%)$ \\
Mean \pm standard deviation & $35.0 \% \pm 4.2 \%$ \\
Range & $32 \%-38 \%$ \\
\hline
\end{tabular}

Data as of June 2013. 
Table B-7. Summary of the Incidence of Nonneoplastic Lesions in Female Rats in the Two-year Inhalation Study of Cobalt Metal ${ }^{\mathrm{a}}$

\begin{tabular}{|c|c|c|c|c|}
\hline & $\begin{array}{c}\text { Chamber } \\
\text { Control }\end{array}$ & $1.25 \mathrm{mg} / \mathrm{m}^{3}$ & $2.5 \mathrm{mg} / \mathrm{m}^{3}$ & $5 \mathrm{mg} / \mathrm{m}^{3}$ \\
\hline \multicolumn{5}{|l|}{ Disposition Summary } \\
\hline Animals initially in study & 50 & 50 & 50 & 50 \\
\hline \multicolumn{5}{|l|}{ Early deaths } \\
\hline Moribund & 11 & 20 & 19 & 24 \\
\hline Natural deaths & 4 & 4 & 7 & 1 \\
\hline \multicolumn{5}{|l|}{ Survivors } \\
\hline Died last week of study & - & - & - & 1 \\
\hline Terminal kill & 35 & 26 & 24 & 24 \\
\hline Animals examined microscopically & 50 & 50 & 50 & 50 \\
\hline \multicolumn{5}{|l|}{ Alimentary System } \\
\hline Esophagus & $(50)$ & $(50)$ & $(49)$ & (48) \\
\hline Intestine large, cecum & $(50)$ & (50) & (49) & $(50)$ \\
\hline Hemorrhage & - & - & $1(2 \%)$ & - \\
\hline Inflammation, chronic active & - & - & - & $1(2 \%)$ \\
\hline Intestine large, colon & $(50)$ & (50) & (49) & $(50)$ \\
\hline Mucosa, hyperplasia & - & $2(4 \%)$ & - & - \\
\hline Intestine large, rectum & (50) & (50) & $(50)$ & (49) \\
\hline Intestine small, duodenum & $(48)$ & $(50)$ & $(50)$ & (50) \\
\hline Infiltration cellular, chronic active & - & $1(2 \%)$ & - & - \\
\hline Intestine small, ileum & (49) & $(50)$ & $(48)$ & $(50)$ \\
\hline Intestine small, jejunum & $(50)$ & $(50)$ & $(47)$ & $(50)$ \\
\hline Congestion & - & - & $1(2 \%)$ & - \\
\hline Hemorrhage & - & - & $1(2 \%)$ & - \\
\hline Inflammation, suppurative & - & - & $1(2 \%)$ & - \\
\hline Necrosis & - & - & $1(2 \%)$ & - \\
\hline Liver & $(50)$ & $(50)$ & $(50)$ & (50) \\
\hline Angiectasis & $1(2 \%)$ & $1(2 \%)$ & - & $1(2 \%)$ \\
\hline Atrophy & $1(2 \%)$ & - & - & - \\
\hline Basophilic focus & $16(32 \%)$ & $20(40 \%)$ & $22(44 \%)$ & $33(66 \%)$ \\
\hline Clear cell focus & $12(24 \%)$ & $6(12 \%)$ & $3(6 \%)$ & $6(12 \%)$ \\
\hline Eosinophilic focus & - & $1(2 \%)$ & $2(4 \%)$ & $2(4 \%)$ \\
\hline Fatty change & $13(26 \%)$ & $9(18 \%)$ & $6(12 \%)$ & $3(6 \%)$ \\
\hline Fibrosis & - & $1(2 \%)$ & - & - \\
\hline
\end{tabular}


Cobalt Metal, NTP TR 581

\begin{tabular}{|c|c|c|c|c|}
\hline & $\begin{array}{c}\text { Chamber } \\
\text { Control }\end{array}$ & $1.25 \mathrm{mg} / \mathrm{m}^{3}$ & $2.5 \mathrm{mg} / \mathrm{m}^{3}$ & $5 \mathrm{mg} / \mathrm{m}^{3}$ \\
\hline Hepatodiaphragmatic nodule & $6(12 \%)$ & $11(22 \%)$ & $12(24 \%)$ & $15(30 \%)$ \\
\hline Inflammation, chronic active & $1(2 \%)$ & - & - & $2(4 \%)$ \\
\hline Mixed cell focus & $1(2 \%)$ & $4(8 \%)$ & $2(4 \%)$ & - \\
\hline Necrosis & $6(12 \%)$ & $3(6 \%)$ & $2(4 \%)$ & $2(4 \%)$ \\
\hline Regeneration & $2(4 \%)$ & $3(6 \%)$ & $5(10 \%)$ & $4(8 \%)$ \\
\hline Bile duct, cyst & - & - & $1(2 \%)$ & - \\
\hline Serosa, thrombosis & $1(2 \%)$ & - & - & - \\
\hline Mesentery & (13) & (15) & (8) & (9) \\
\hline Fibrosis & - & $1(7 \%)$ & - & - \\
\hline Fat, necrosis & $12(92 \%)$ & $14(93 \%)$ & $8(100 \%)$ & $9(100 \%)$ \\
\hline Pancreas & $(50)$ & $(50)$ & $(50)$ & (50) \\
\hline Basophilic focus & - & $1(2 \%)$ & - & - \\
\hline Acinus, atrophy & $18(36 \%)$ & $23(46 \%)$ & $27(54 \%)$ & $32(64 \%)$ \\
\hline Acinus, hyperplasia & - & - & $1(2 \%)$ & - \\
\hline Salivary glands & $(50)$ & (50) & (50) & $(50)$ \\
\hline Atrophy & $1(2 \%)$ & - & $1(2 \%)$ & - \\
\hline Inflammation, chronic active & - & - & $1(2 \%)$ & - \\
\hline Duct, hyperplasia & - & $1(2 \%)$ & - & - \\
\hline Stomach, forestomach & $(50)$ & $(50)$ & (50) & $(50)$ \\
\hline Edema & - & - & $1(2 \%)$ & $1(2 \%)$ \\
\hline Erosion & - & - & $1(2 \%)$ & - \\
\hline Fibrosis & - & $1(2 \%)$ & - & - \\
\hline Hyperplasia, squamous & $5(10 \%)$ & $10(20 \%)$ & $2(4 \%)$ & $3(6 \%)$ \\
\hline Inflammation, chronic active & - & $1(2 \%)$ & $1(2 \%)$ & - \\
\hline Ulcer & $1(2 \%)$ & $5(10 \%)$ & $2(4 \%)$ & $2(4 \%)$ \\
\hline Stomach, glandular & $(50)$ & $(50)$ & $(50)$ & $(50)$ \\
\hline Edema & $2(4 \%)$ & - & - & - \\
\hline Erosion & $1(2 \%)$ & - & $1(2 \%)$ & - \\
\hline Inflammation, suppurative & - & - & - & $1(2 \%)$ \\
\hline Necrosis & - & - & - & $1(2 \%)$ \\
\hline Thrombosis & - & - & $1(2 \%)$ & - \\
\hline Ulcer & - & $3(6 \%)$ & $1(2 \%)$ & $1(2 \%)$ \\
\hline Tongue & (1) & (0) & (1) & (0) \\
\hline Tooth & (1) & (0) & (2) & (2) \\
\hline Inflammation, chronic active & $1(100 \%)$ & - & $2(100 \%)$ & $2(100 \%)$ \\
\hline
\end{tabular}


Cobalt Metal, NTP TR 581

\begin{tabular}{|c|c|c|c|c|}
\hline & $\begin{array}{l}\text { Chamber } \\
\text { Control }\end{array}$ & $1.25 \mathrm{mg} / \mathrm{m}^{3}$ & $2.5 \mathrm{mg} / \mathrm{m}^{3}$ & $5 \mathrm{mg} / \mathrm{m}^{3}$ \\
\hline \multicolumn{5}{|l|}{ Cardiovascular System } \\
\hline Blood vessel & (0) & (1) & $(0)$ & $(0)$ \\
\hline Heart & $(50)$ & (50) & (50) & $(50)$ \\
\hline Cardiomyopathy & $41(82 \%)$ & $34(68 \%)$ & $42(84 \%)$ & $35(70 \%)$ \\
\hline Thrombosis & $2(4 \%)$ & $4(8 \%)$ & $2(4 \%)$ & - \\
\hline Artery, inflammation, chronic active & $1(2 \%)$ & - & $1(2 \%)$ & - \\
\hline Atrium, congestion & $1(2 \%)$ & - & - & - \\
\hline Pericardium, hyperplasia & - & - & - & $1(2 \%)$ \\
\hline \multicolumn{5}{|l|}{ Endocrine System } \\
\hline Adrenal cortex & $(50)$ & $(50)$ & (50) & $(50)$ \\
\hline Accessory adrenal cortical nodule & $5(10 \%)$ & - & $3(6 \%)$ & $4(8 \%)$ \\
\hline Atrophy & $1(2 \%)$ & - & $1(2 \%)$ & - \\
\hline Degeneration & $3(6 \%)$ & $1(2 \%)$ & $3(6 \%)$ & $3(6 \%)$ \\
\hline Hemorrhage & $1(2 \%)$ & - & - & - \\
\hline Hyperplasia & $25(50 \%)$ & $27(54 \%)$ & $28(56 \%)$ & $28(56 \%)$ \\
\hline Necrosis & $1(2 \%)$ & - & $1(2 \%)$ & $2(4 \%)$ \\
\hline Adrenal medulla & $(50)$ & (50) & $(50)$ & $(50)$ \\
\hline Degeneration, cystic & $1(2 \%)$ & - & - & - \\
\hline Hyperplasia & $12(24 \%)$ & $27(54 \%)$ & $27(54 \%)$ & $10(20 \%)$ \\
\hline Infiltration cellular, mononuclear cell & $1(2 \%)$ & - & - & - \\
\hline Islets, pancreatic & $(50)$ & $(50)$ & $(50)$ & $(50)$ \\
\hline Hyperplasia & $1(2 \%)$ & $1(2 \%)$ & - & $1(2 \%)$ \\
\hline Parathyroid gland & $(42)$ & $(45)$ & $(38)$ & $(45)$ \\
\hline Pituitary gland & $(50)$ & (50) & $(49)$ & (50) \\
\hline Angiectasis & - & - & $1(2 \%)$ & $2(4 \%)$ \\
\hline Cyst & - & - & - & $1(2 \%)$ \\
\hline Pars distalis, hyperplasia & $19(38 \%)$ & $20(40 \%)$ & $18(37 \%)$ & $19(38 \%)$ \\
\hline Thyroid gland & $(50)$ & (49) & $(49)$ & (50) \\
\hline Hemorrhage & - & - & - & $1(2 \%)$ \\
\hline C-cell, hyperplasia & $9(18 \%)$ & $9(18 \%)$ & $8(16 \%)$ & $6(12 \%)$ \\
\hline Follicular cell, hyperplasia & $1(2 \%)$ & - & - & - \\
\hline \multicolumn{5}{|l|}{ General Body System } \\
\hline Peritoneum & (1) & (1) & $(0)$ & $(0)$ \\
\hline \multicolumn{5}{|l|}{ Genital System } \\
\hline Clitoral gland & (49) & (47) & (47) & $(46)$ \\
\hline
\end{tabular}


Cobalt Metal, NTP TR 581

\begin{tabular}{|c|c|c|c|c|}
\hline & $\begin{array}{l}\text { Chamber } \\
\text { Control }\end{array}$ & $1.25 \mathrm{mg} / \mathrm{m}^{3}$ & $2.5 \mathrm{mg} / \mathrm{m}^{3}$ & $5 \mathrm{mg} / \mathrm{m}^{3}$ \\
\hline Cyst & - & - & - & $1(2 \%)$ \\
\hline Hyperplasia & $2(4 \%)$ & $1(2 \%)$ & $1(2 \%)$ & - \\
\hline Inflammation, suppurative & $2(4 \%)$ & - & - & $1(2 \%)$ \\
\hline Inflammation, chronic active & - & $4(9 \%)$ & - & $2(4 \%)$ \\
\hline Ovary & $(50)$ & $(50)$ & $(50)$ & (50) \\
\hline Cyst & $5(10 \%)$ & $6(12 \%)$ & $7(14 \%)$ & $5(10 \%)$ \\
\hline Inflammation, suppurative & - & - & $1(2 \%)$ & - \\
\hline Inflammation, chronic active & $1(2 \%)$ & - & - & - \\
\hline Necrosis & - & $1(2 \%)$ & - & - \\
\hline Uterus & (50) & $(50)$ & $(50)$ & $(50)$ \\
\hline Angiectasis & $1(2 \%)$ & - & - & - \\
\hline Cyst & $1(2 \%)$ & - & - & - \\
\hline Inflammation, suppurative & $1(2 \%)$ & $1(2 \%)$ & - & - \\
\hline Inflammation, chronic active & $1(2 \%)$ & $2(4 \%)$ & $1(2 \%)$ & $1(2 \%)$ \\
\hline Necrosis & $2(4 \%)$ & - & - & - \\
\hline Thrombosis & - & - & $1(2 \%)$ & - \\
\hline Endometrium, hyperplasia, cystic & $4(8 \%)$ & $2(4 \%)$ & $1(2 \%)$ & $4(8 \%)$ \\
\hline \multicolumn{5}{|l|}{ Hematopoietic System } \\
\hline Bone marrow & $(50)$ & $(50)$ & $(50)$ & $(50)$ \\
\hline Fibrosis & - & - & - & $1(2 \%)$ \\
\hline Lymph node & (4) & (3) & (7) & $(5)$ \\
\hline Deep cervical, infiltration cellular & - & $1(33 \%)$ & - & - \\
\hline Iliac, ectasia & - & $1(33 \%)$ & - & $1(20 \%)$ \\
\hline Inguinal, fibrosis & - & - & - & $1(20 \%)$ \\
\hline Renal, ectasia & $1(25 \%)$ & - & $1(14 \%)$ & - \\
\hline Lymph node, bronchial & $(26)$ & $(18)$ & $(22)$ & (17) \\
\hline Lymph node, mandibular & $(48)$ & $(48)$ & $(47)$ & (47) \\
\hline Atrophy & $1(2 \%)$ & - & - & - \\
\hline Ectasia & - & - & $1(2 \%)$ & - \\
\hline Fibrosis & - & $1(2 \%)$ & - & - \\
\hline Necrosis & - & $1(2 \%)$ & - & - \\
\hline Lymph node, mediastinal & $(42)$ & (43) & $(48)$ & (48) \\
\hline Atrophy & $1(2 \%)$ & - & - & - \\
\hline Ectasia & $1(2 \%)$ & $1(2 \%)$ & - & - \\
\hline Hyperplasia & - & - & - & $1(2 \%)$ \\
\hline
\end{tabular}


Cobalt Metal, NTP TR 581

\begin{tabular}{|c|c|c|c|c|}
\hline & $\begin{array}{l}\text { Chamber } \\
\text { Control }\end{array}$ & $1.25 \mathrm{mg} / \mathrm{m}^{3}$ & $2.5 \mathrm{mg} / \mathrm{m}^{3}$ & $5 \mathrm{mg} / \mathrm{m}^{3}$ \\
\hline Infiltration cellular, histiocyte & - & - & - & $1(2 \%)$ \\
\hline Lymph node, mesenteric & $(50)$ & (50) & $(50)$ & (50) \\
\hline Ectasia & - & $1(2 \%)$ & - & - \\
\hline Fibrosis & - & - & - & $1(2 \%)$ \\
\hline Infiltration cellular, histiocyte & $1(2 \%)$ & - & - & - \\
\hline Spleen & $(50)$ & (50) & (50) & $(50)$ \\
\hline Fibrosis & - & $1(2 \%)$ & $5(10 \%)$ & $3(6 \%)$ \\
\hline Hematopoietic cell proliferation & $2(4 \%)$ & $5(10 \%)$ & $3(6 \%)$ & $2(4 \%)$ \\
\hline Hemorrhage & - & - & $3(6 \%)$ & - \\
\hline Infiltration cellular, histiocyte & $1(2 \%)$ & - & - & - \\
\hline Necrosis & - & $1(2 \%)$ & - & $2(4 \%)$ \\
\hline Thymus & (47) & (46) & $(46)$ & (47) \\
\hline Thrombosis & - & $1(2 \%)$ & - & - \\
\hline \multicolumn{5}{|l|}{ Integumentary System } \\
\hline Mammary gland & $(50)$ & $(50)$ & $(50)$ & $(50)$ \\
\hline Galactocele & $1(2 \%)$ & $1(2 \%)$ & $4(8 \%)$ & $1(2 \%)$ \\
\hline Hyperplasia & $3(6 \%)$ & $2(4 \%)$ & $1(2 \%)$ & $5(10 \%)$ \\
\hline Inflammation, chronic active & - & $1(2 \%)$ & - & - \\
\hline Skin & (50) & (50) & (50) & (50) \\
\hline Cyst epithelial inclusion & $1(2 \%)$ & $1(2 \%)$ & - & - \\
\hline Hyperkeratosis & $1(2 \%)$ & - & - & - \\
\hline Hyperplasia, squamous & $1(2 \%)$ & $1(2 \%)$ & - & $2(4 \%)$ \\
\hline Inflammation & - & $1(2 \%)$ & $1(2 \%)$ & - \\
\hline Ulcer & - & - & - & $1(2 \%)$ \\
\hline Subcutaneous tissue, inflammation & - & $1(2 \%)$ & - & - \\
\hline \multicolumn{5}{|l|}{ Musculoskeletal System } \\
\hline Bone & $(50)$ & $(50)$ & $(50)$ & $(50)$ \\
\hline Fracture & $1(2 \%)$ & - & - & - \\
\hline Femur, hyperostosis & $4(8 \%)$ & $2(4 \%)$ & $5(10 \%)$ & $2(4 \%)$ \\
\hline Maxilla, inflammation, chronic active & $2(4 \%)$ & - & - & $1(2 \%)$ \\
\hline Skeletal muscle & (1) & (1) & $(0)$ & (1) \\
\hline \multicolumn{5}{|l|}{ Nervous System } \\
\hline Brain & $(50)$ & $(50)$ & $(50)$ & $(50)$ \\
\hline Compression & $16(32 \%)$ & $14(28 \%)$ & $14(28 \%)$ & $17(34 \%)$ \\
\hline Hemorrhage & - & $2(4 \%)$ & - & $3(6 \%)$ \\
\hline
\end{tabular}


Cobalt Metal, NTP TR 581

\begin{tabular}{|c|c|c|c|c|}
\hline & $\begin{array}{l}\text { Chamber } \\
\text { Control }\end{array}$ & $1.25 \mathrm{mg} / \mathrm{m}^{3}$ & $2.5 \mathrm{mg} / \mathrm{m}^{3}$ & $5 \mathrm{mg} / \mathrm{m}^{3}$ \\
\hline Infiltration cellular, mononuclear cell & $1(2 \%)$ & - & - & - \\
\hline Necrosis & - & - & $1(2 \%)$ & - \\
\hline \multicolumn{5}{|l|}{ Respiratory System } \\
\hline Larynx & $(50)$ & $(50)$ & $(50)$ & $(50)$ \\
\hline Foreign body & $1(2 \%)$ & $3(6 \%)$ & $6(12 \%)$ & $2(4 \%)$ \\
\hline Inflammation & $22(44 \%)$ & $23(46 \%)$ & $14(28 \%)$ & $13(26 \%)$ \\
\hline Metaplasia, squamous & $4(8 \%)$ & $4(8 \%)$ & $3(6 \%)$ & $5(10 \%)$ \\
\hline Respiratory epithelium, hyperplasia & - & $4(8 \%)$ & - & $1(2 \%)$ \\
\hline Lung & $(50)$ & $(50)$ & (50) & $(50)$ \\
\hline Hemorrhage & - & - & - & $1(2 \%)$ \\
\hline Inflammation, suppurative & - & - & - & $1(2 \%)$ \\
\hline Inflammation, chronic active & $20(40 \%)$ & $50(100 \%)$ & $50(100 \%)$ & $50(100 \%)$ \\
\hline Necrosis & - & - & - & $1(2 \%)$ \\
\hline Pigmentation & - & - & - & $1(2 \%)$ \\
\hline Alveolar epithelium, hyperplasia & $9(18 \%)$ & $49(98 \%)$ & $50(100 \%)$ & $49(98 \%)$ \\
\hline $\begin{array}{l}\text { Alveolar epithelium, metaplasia, } \\
\text { squamous }\end{array}$ & - & $2(4 \%)$ & $1(2 \%)$ & - \\
\hline Alveolus, proteinosis & - & $50(100 \%)$ & $50(100 \%)$ & $50(100 \%)$ \\
\hline Bronchiole, epithelium, hyperplasia & - & $47(94 \%)$ & $46(92 \%)$ & $48(96 \%)$ \\
\hline Interstitium, fibrosis & $1(2 \%)$ & - & - & - \\
\hline Mediastinum, inflammation & $1(2 \%)$ & - & - & - \\
\hline Nose & $(50)$ & $(50)$ & (49) & (50) \\
\hline Foreign body & $5(10 \%)$ & $3(6 \%)$ & - & - \\
\hline Inflammation & - & - & $1(2 \%)$ & - \\
\hline Inflammation, suppurative & $6(12 \%)$ & $4(8 \%)$ & $4(8 \%)$ & $42(84 \%)$ \\
\hline Inflammation, chronic active & $22(44 \%)$ & $42(84 \%)$ & $39(80 \%)$ & $50(100 \%)$ \\
\hline Thrombosis & $1(2 \%)$ & $4(8 \%)$ & $6(12 \%)$ & $3(6 \%)$ \\
\hline $\begin{array}{l}\text { Olfactory epithelium, accumulation, } \\
\text { hyaline droplet }\end{array}$ & $8(16 \%)$ & $2(4 \%)$ & - & - \\
\hline Olfactory epithelium, atrophy & - & $22(44 \%)$ & $35(71 \%)$ & $35(70 \%)$ \\
\hline Olfactory epithelium, hyperplasia & - & - & $3(6 \%)$ & $5(10 \%)$ \\
\hline $\begin{array}{l}\text { Olfactory epithelium, hyperplasia, } \\
\text { basal cell }\end{array}$ & - & - & $1(2 \%)$ & $19(38 \%)$ \\
\hline $\begin{array}{l}\text { Olfactory epithelium, metaplasia, } \\
\text { respiratory }\end{array}$ & $6(12 \%)$ & $18(36 \%)$ & $24(49 \%)$ & $47(94 \%)$ \\
\hline Olfactory epithelium, necrosis & - & $2(4 \%)$ & - & $1(2 \%)$ \\
\hline
\end{tabular}


Cobalt Metal, NTP TR 581

\begin{tabular}{|c|c|c|c|c|}
\hline & $\begin{array}{l}\text { Chamber } \\
\text { Control }\end{array}$ & $1.25 \mathrm{mg} / \mathrm{m}^{3}$ & $2.5 \mathrm{mg} / \mathrm{m}^{3}$ & $5 \mathrm{mg} / \mathrm{m}^{3}$ \\
\hline $\begin{array}{l}\text { Respiratory epithelium, accumulation, } \\
\text { hyaline droplet }\end{array}$ & $1(2 \%)$ & - & - & - \\
\hline Respiratory epithelium, hyperplasia & $15(30 \%)$ & $43(86 \%)$ & $48(98 \%)$ & $49(98 \%)$ \\
\hline $\begin{array}{l}\text { Respiratory epithelium, metaplasia, } \\
\text { squamous }\end{array}$ & $2(4 \%)$ & - & $3(6 \%)$ & $45(90 \%)$ \\
\hline Respiratory epithelium, necrosis & $1(2 \%)$ & $1(2 \%)$ & $1(2 \%)$ & $15(30 \%)$ \\
\hline Turbinate, atrophy & $1(2 \%)$ & $38(76 \%)$ & $27(55 \%)$ & $45(90 \%)$ \\
\hline Turbinate, hyperostosis & $3(6 \%)$ & $2(4 \%)$ & $5(10 \%)$ & $2(4 \%)$ \\
\hline Trachea & $(50)$ & $(50)$ & $(50)$ & $(50)$ \\
\hline \multicolumn{5}{|l|}{ Special Senses System } \\
\hline Ear & (4) & (4) & $(0)$ & (3) \\
\hline Inflammation, chronic active & $4(100 \%)$ & $4(100 \%)$ & - & $3(100 \%)$ \\
\hline Eye & $(50)$ & $(50)$ & $(49)$ & (50) \\
\hline Cataract & $8(16 \%)$ & $10(20 \%)$ & $11(22 \%)$ & $3(6 \%)$ \\
\hline Hemorrhage & - & $1(2 \%)$ & - & - \\
\hline Metaplasia, osseous & $24(48 \%)$ & $19(38 \%)$ & $26(53 \%)$ & $13(26 \%)$ \\
\hline Cornea, inflammation, chronic active & - & $1(2 \%)$ & - & - \\
\hline Harderian gland & $(50)$ & $(50)$ & $(50)$ & $(50)$ \\
\hline Atrophy & - & $1(2 \%)$ & - & - \\
\hline Hyperplasia & - & - & $1(2 \%)$ & - \\
\hline Inflammation, chronic active & $3(6 \%)$ & - & - & $2(4 \%)$ \\
\hline Zymbal's gland & $(0)$ & (1) & $(0)$ & $(0)$ \\
\hline \multicolumn{5}{|l|}{ Urinary System } \\
\hline Kidney & $(50)$ & $(50)$ & $(50)$ & $(50)$ \\
\hline Cyst & - & $1(2 \%)$ & - & $1(2 \%)$ \\
\hline Infarct & - & $1(2 \%)$ & - & $2(4 \%)$ \\
\hline Mineralization & - & - & $1(2 \%)$ & - \\
\hline Necrosis, focal & $1(2 \%)$ & - & - & - \\
\hline Nephropathy & $48(96 \%)$ & $48(96 \%)$ & $47(94 \%)$ & $48(96 \%)$ \\
\hline Renal tubule, hyperplasia & - & $2(4 \%)$ & - & - \\
\hline Renal tubule, necrosis & - & - & $1(2 \%)$ & - \\
\hline Urinary bladder & $(50)$ & $(50)$ & $(50)$ & $(50)$ \\
\hline
\end{tabular}

${ }^{a}$ Number of animals examined microscopically at the site and the number of animals with lesion. 


\section{Appendix C. Summary of Lesions in Male Mice in the Two- year Inhalation Study of Cobalt Metal}

\section{Tables}

Table C-1. Summary of the Incidence of Neoplasms in Male Mice in the Two-year Inhalation Study of Cobalt Metal

Table C-2. Statistical Analysis of Primary Neoplasms in Male Mice in the Two-year Inhalation Study of Cobalt Metal

Table C-3. Historical Incidence of Alveolar/bronchiolar Neoplasms in Control Male B6C3F1/N Mice

Table C-4. Summary of the Incidence of Nonneoplastic Lesions in Male Mice in the Two-year Inhalation Study of Cobalt Metal. 
Table C-1. Summary of the Incidence of Neoplasms in Male Mice in the Two-year Inhalation Study of Cobalt Metal ${ }^{\mathbf{a}}$

\begin{tabular}{|c|c|c|c|c|}
\hline & $\begin{array}{l}\text { Chamber } \\
\text { Control }\end{array}$ & $1.25 \mathrm{mg} / \mathrm{m}^{3}$ & $2.5 \mathrm{mg} / \mathrm{m}^{3}$ & $5 \mathrm{mg} / \mathrm{m}^{3}$ \\
\hline \multicolumn{5}{|l|}{ Disposition Summary } \\
\hline Animals initially in study & 50 & 50 & 50 & 50 \\
\hline \multicolumn{5}{|l|}{ Early deaths } \\
\hline Moribund & 5 & 13 & 12 & 17 \\
\hline Natural deaths & 6 & 6 & 9 & 8 \\
\hline \multicolumn{5}{|l|}{ Survivors } \\
\hline Died last week of study & - & - & 1 & 3 \\
\hline Terminal kill & 39 & 31 & 28 & 22 \\
\hline Animals examined microscopically & 50 & 50 & 50 & 50 \\
\hline \multicolumn{5}{|l|}{ Alimentary System } \\
\hline Esophagus & (50) & (50) & (50) & $(50)$ \\
\hline Gallbladder & (43) & (37) & (34) & $(35)$ \\
\hline Intestine large, cecum & (45) & (45) & (44) & $(45)$ \\
\hline $\begin{array}{l}\text { Alveolar/bronchiolar carcinoma, } \\
\text { metastatic, lung }\end{array}$ & - & - & - & $1(2 \%)$ \\
\hline Intestine large, colon & (47) & (45) & $(45)$ & $(46)$ \\
\hline Intestine large, rectum & (44) & (44) & (45) & $(48)$ \\
\hline Intestine small, duodenum & (45) & (44) & (44) & (44) \\
\hline Adenoma & $1(2 \%)$ & - & - & - \\
\hline Intestine small, ileum & (45) & (45) & (43) & (44) \\
\hline Adenoma & $1(2 \%)$ & - & - & - \\
\hline Intestine small, jejunum & (45) & (45) & (43) & (44) \\
\hline Liver & (50) & (50) & (50) & $(50)$ \\
\hline $\begin{array}{l}\text { Alveolar/bronchiolar carcinoma, } \\
\text { metastatic, lung }\end{array}$ & - & $1(2 \%)$ & - & $1(2 \%)$ \\
\hline Cholangiocarcinoma & - & $1(2 \%)$ & - & $1(2 \%)$ \\
\hline Hemangiosarcoma & $3(6 \%)$ & $1(2 \%)$ & $3(6 \%)$ & - \\
\hline Hemangiosarcoma, multiple & $1(2 \%)$ & $1(2 \%)$ & - & - \\
\hline Hepatoblastoma & $2(4 \%)$ & $2(4 \%)$ & - & - \\
\hline Hepatocellular adenoma & $15(30 \%)$ & $9(18 \%)$ & $13(26 \%)$ & $11(22 \%)$ \\
\hline Hepatocellular adenoma, multiple & $13(26 \%)$ & $16(32 \%)$ & $8(16 \%)$ & $1(2 \%)$ \\
\hline Hepatocellular carcinoma & $15(30 \%)$ & $15(30 \%)$ & $11(22 \%)$ & $8(16 \%)$ \\
\hline Hepatocellular carcinoma, multiple & $10(20 \%)$ & $6(12 \%)$ & $3(6 \%)$ & $1(2 \%)$ \\
\hline Sarcoma, metastatic, mesentery & - & $1(2 \%)$ & - & - \\
\hline Mesentery & (5) & $(5)$ & $(5)$ & (3) \\
\hline
\end{tabular}


Cobalt Metal, NTP TR 581

\begin{tabular}{|c|c|c|c|c|}
\hline & $\begin{array}{l}\text { Chamber } \\
\text { Control }\end{array}$ & $1.25 \mathrm{mg} / \mathrm{m}^{3}$ & $2.5 \mathrm{mg} / \mathrm{m}^{3}$ & $5 \mathrm{mg} / \mathrm{m}^{3}$ \\
\hline Sarcoma & - & $1(20 \%)$ & - & - \\
\hline Pancreas & $(50)$ & $(47)$ & $(50)$ & $(50)$ \\
\hline $\begin{array}{l}\text { Alveolar/bronchiolar carcinoma, } \\
\text { metastatic, lung }\end{array}$ & - & - & - & $1(2 \%)$ \\
\hline Carcinoma, metastatic, islets, pancreatic & - & $1(2 \%)$ & - & - \\
\hline Salivary glands & $(50)$ & (49) & $(50)$ & $(50)$ \\
\hline Stomach, forestomach & $(50)$ & $(48)$ & $(50)$ & $(50)$ \\
\hline Sarcoma, metastatic, mesentery & - & $1(2 \%)$ & - & - \\
\hline Stomach, glandular & $(48)$ & (46) & $(48)$ & (49) \\
\hline Sarcoma, metastatic, mesentery & - & $1(2 \%)$ & - & - \\
\hline Tooth & (8) & (2) & (2) & (1) \\
\hline \multicolumn{5}{|l|}{ Cardiovascular System } \\
\hline Heart & $(50)$ & (50) & $(50)$ & $(50)$ \\
\hline $\begin{array}{l}\text { Alveolar/bronchiolar carcinoma, } \\
\text { metastatic, lung }\end{array}$ & - & $1(2 \%)$ & $1(2 \%)$ & $2(4 \%)$ \\
\hline Hemangiosarcoma & $1(2 \%)$ & - & $1(2 \%)$ & - \\
\hline \multicolumn{5}{|l|}{ Endocrine System } \\
\hline Adrenal cortex & (50) & (49) & $(50)$ & $(50)$ \\
\hline $\begin{array}{l}\text { Alveolar/bronchiolar carcinoma, } \\
\text { metastatic, lung }\end{array}$ & - & - & $1(2 \%)$ & - \\
\hline $\begin{array}{l}\text { Hepatocellular carcinoma, } \\
\text { metastatic, liver }\end{array}$ & $1(2 \%)$ & - & - & - \\
\hline Bilateral, subcapsular, adenoma & $1(2 \%)$ & - & - & - \\
\hline Subcapsular, adenoma & - & $4(8 \%)$ & $1(2 \%)$ & $1(2 \%)$ \\
\hline Adrenal medulla & $(50)$ & (49) & $(50)$ & (48) \\
\hline $\begin{array}{l}\text { Alveolar/bronchiolar carcinoma, } \\
\text { metastatic, lung }\end{array}$ & - & $1(2 \%)$ & - & - \\
\hline Islets, pancreatic & $(49)$ & (47) & $(50)$ & (50) \\
\hline Adenoma & - & $1(2 \%)$ & - & - \\
\hline Carcinoma & - & $2(4 \%)$ & - & - \\
\hline Parathyroid gland & (29) & (25) & $(26)$ & (27) \\
\hline Pituitary gland & (47) & (49) & (48) & (48) \\
\hline Thyroid gland & (49) & (49) & $(50)$ & (49) \\
\hline
\end{tabular}

\section{General Body System}

Tissue NOS
(0)

(0)

(0)

(1)

\section{Genital System}

Coagulating gland

(0)

(1)

(0)

(1)

Adenoma

$1(100 \%)$ 
Cobalt Metal, NTP TR 581

\begin{tabular}{|c|c|c|c|c|}
\hline & $\begin{array}{c}\text { Chamber } \\
\text { Control }\end{array}$ & $1.25 \mathrm{mg} / \mathrm{m}^{3}$ & $2.5 \mathrm{mg} / \mathrm{m}^{3}$ & $5 \mathrm{mg} / \mathrm{m}^{3}$ \\
\hline $\begin{array}{l}\text { Alveolar/bronchiolar carcinoma, } \\
\text { metastatic, lung }\end{array}$ & - & - & - & $1(100 \%)$ \\
\hline Epididymis & $(50)$ & $(49)$ & (50) & $(50)$ \\
\hline $\begin{array}{l}\text { Alveolar/bronchiolar carcinoma, } \\
\text { metastatic, lung }\end{array}$ & - & $1(2 \%)$ & - & $1(2 \%)$ \\
\hline Hemangiosarcoma & - & $1(2 \%)$ & - & - \\
\hline Penis & (0) & (0) & (0) & (1) \\
\hline Preputial gland & $(49)$ & $(50)$ & (49) & $(50)$ \\
\hline Prostate & $(50)$ & $(49)$ & (48) & $(50)$ \\
\hline Seminal vesicle & $(48)$ & $(48)$ & (49) & $(50)$ \\
\hline Testes & $(50)$ & (49) & (50) & $(50)$ \\
\hline $\begin{array}{l}\text { Alveolar/bronchiolar carcinoma, } \\
\text { metastatic, lung }\end{array}$ & - & $1(2 \%)$ & - & $1(2 \%)$ \\
\hline Interstitial cell, adenoma & $1(2 \%)$ & - & - & - \\
\hline \multicolumn{5}{|l|}{ Hematopoietic System } \\
\hline Bone marrow & $(50)$ & $(49)$ & $(50)$ & (49) \\
\hline Hemangiosarcoma & $1(2 \%)$ & $1(2 \%)$ & $2(4 \%)$ & - \\
\hline Lymph node & (2) & (0) & (1) & (1) \\
\hline Lymph node, bronchial & $(26)$ & $(32)$ & (20) & (24) \\
\hline $\begin{array}{l}\text { Alveolar/bronchiolar carcinoma, } \\
\text { metastatic, lung }\end{array}$ & - & $2(6 \%)$ & $1(5 \%)$ & $2(8 \%)$ \\
\hline $\begin{array}{l}\text { Hepatocellular carcinoma, } \\
\text { metastatic, liver }\end{array}$ & - & $1(3 \%)$ & - & - \\
\hline Lymph node, mandibular & $(37)$ & $(23)$ & (27) & (37) \\
\hline Lymph node, mediastinal & $(34)$ & $(34)$ & (36) & (44) \\
\hline $\begin{array}{l}\text { Alveolar/bronchiolar carcinoma, } \\
\text { metastatic, lung }\end{array}$ & - & $2(6 \%)$ & $3(8 \%)$ & - \\
\hline $\begin{array}{l}\text { Hepatocellular carcinoma, } \\
\text { metastatic, liver }\end{array}$ & - & $1(3 \%)$ & $1(3 \%)$ & - \\
\hline Lymph node, mesenteric & $(47)$ & $(44)$ & $(44)$ & $(42)$ \\
\hline Hemangiosarcoma & - & - & $1(2 \%)$ & - \\
\hline Spleen & (50) & $(48)$ & (49) & (48) \\
\hline Hemangioma & - & $1(2 \%)$ & - & $1(2 \%)$ \\
\hline Hemangiosarcoma & $3(6 \%)$ & - & $4(8 \%)$ & $3(6 \%)$ \\
\hline Thymus & $(42)$ & $(44)$ & (40) & (38) \\
\hline $\begin{array}{l}\text { Alveolar/bronchiolar carcinoma, } \\
\text { metastatic, lung }\end{array}$ & - & - & $1(3 \%)$ & $1(3 \%)$ \\
\hline Hemangiosarcoma & - & - & $1(3 \%)$ & - \\
\hline
\end{tabular}


Cobalt Metal, NTP TR 581

\begin{tabular}{|c|c|c|c|c|}
\hline & $\begin{array}{c}\text { Chamber } \\
\text { Control }\end{array}$ & $1.25 \mathrm{mg} / \mathrm{m}^{3}$ & $2.5 \mathrm{mg} / \mathrm{m}^{3}$ & $5 \mathrm{mg} / \mathrm{m}^{3}$ \\
\hline $\begin{array}{l}\text { Hepatocellular carcinoma, } \\
\text { metastatic, liver }\end{array}$ & - & $1(2 \%)$ & - & - \\
\hline \multicolumn{5}{|l|}{ Integumentary System } \\
\hline Skin & $(50)$ & $(50)$ & $(50)$ & $(49)$ \\
\hline $\begin{array}{l}\text { Alveolar/bronchiolar carcinoma, } \\
\text { metastatic, lung }\end{array}$ & - & $1(2 \%)$ & - & - \\
\hline $\begin{array}{l}\text { Subcutaneous tissue, fibrous } \\
\text { histiocytoma }\end{array}$ & - & $1(2 \%)$ & - & - \\
\hline Subcutaneous tissue, hemangiosarcoma & - & - & $1(2 \%)$ & - \\
\hline Subcutaneous tissue, neural crest tumor & $1(2 \%)$ & - & $1(2 \%)$ & - \\
\hline \multicolumn{5}{|l|}{ Musculoskeletal System } \\
\hline Bone & $(50)$ & (49) & $(50)$ & $(50)$ \\
\hline Skeletal muscle & (0) & (3) & (3) & (1) \\
\hline $\begin{array}{l}\text { Head, alveolar/bronchiolar carcinoma, } \\
\text { metastatic, lung }\end{array}$ & - & - & $1(33 \%)$ & - \\
\hline $\begin{array}{l}\text { Thoracic, alveolar/bronchiolar } \\
\text { carcinoma, metastatic, lung }\end{array}$ & - & $3(100 \%)$ & $3(100 \%)$ & $1(100 \%)$ \\
\hline \multicolumn{5}{|l|}{ Nervous System } \\
\hline Brain & $(50)$ & $(50)$ & (50) & $(50)$ \\
\hline Peripheral nerve & (1) & (0) & (0) & (1) \\
\hline Spinal cord & (1) & (0) & (0) & (1) \\
\hline \multicolumn{5}{|l|}{ Respiratory System } \\
\hline Larynx & $(48)$ & $(47)$ & $(49)$ & $(50)$ \\
\hline Lung & $(50)$ & $(49)$ & $(50)$ & $(50)$ \\
\hline Alveolar/bronchiolar adenoma & $7(14 \%)$ & $10(20 \%)$ & $14(28 \%)$ & $3(6 \%)$ \\
\hline Alveolar/bronchiolar adenoma, multiple & - & $1(2 \%)$ & $1(2 \%)$ & - \\
\hline Alveolar/bronchiolar carcinoma & $8(16 \%)$ & $20(41 \%)$ & $18(36 \%)$ & $10(20 \%)$ \\
\hline $\begin{array}{l}\text { Alveolar/bronchiolar carcinoma, } \\
\text { multiple }\end{array}$ & $3(6 \%)$ & $18(37 \%)$ & $24(48 \%)$ & $36(72 \%)$ \\
\hline Carcinoma, metastatic, islets, pancreatic & - & $1(2 \%)$ & - & - \\
\hline Cholangiocarcinoma, metastatic, liver & - & - & - & $1(2 \%)$ \\
\hline Hepatoblastoma, metastatic, liver & - & $2(4 \%)$ & - & - \\
\hline $\begin{array}{l}\text { Hepatocellular carcinoma, metastatic, } \\
\text { liver }\end{array}$ & $9(18 \%)$ & $6(12 \%)$ & $7(14 \%)$ & $1(2 \%)$ \\
\hline Nose & $(50)$ & $(49)$ & $(50)$ & $(50)$ \\
\hline $\begin{array}{l}\text { Alveolar/bronchiolar carcinoma, } \\
\text { metastatic, lung }\end{array}$ & - & - & - & $1(2 \%)$ \\
\hline Glands, olfactory epithelium, adenoma & - & $1(2 \%)$ & - & - \\
\hline Trachea & $(48)$ & $(47)$ & (48) & $(50)$ \\
\hline
\end{tabular}


Cobalt Metal, NTP TR 581

\begin{tabular}{|c|c|c|c|c|}
\hline & $\begin{array}{l}\text { Chamber } \\
\text { Control }\end{array}$ & $1.25 \mathrm{mg} / \mathrm{m}^{3}$ & $2.5 \mathrm{mg} / \mathrm{m}^{3}$ & $5 \mathrm{mg} / \mathrm{m}^{3}$ \\
\hline $\begin{array}{l}\text { Alveolar/bronchiolar carcinoma, } \\
\text { metastatic, lung }\end{array}$ & - & - & $1(2 \%)$ & - \\
\hline \multicolumn{5}{|l|}{ Special Senses System } \\
\hline Eye & (47) & (46) & (43) & (45) \\
\hline Harderian gland & (49) & (48) & (48) & (50) \\
\hline Adenoma & $6(12 \%)$ & $3(6 \%)$ & $4(8 \%)$ & $4(8 \%)$ \\
\hline Carcinoma & $2(4 \%)$ & $1(2 \%)$ & - & - \\
\hline Zymbal's gland & (0) & (1) & (0) & $(0)$ \\
\hline \multicolumn{5}{|l|}{ Urinary System } \\
\hline Kidney & (50) & (49) & (49) & (50) \\
\hline $\begin{array}{l}\text { Alveolar/bronchiolar carcinoma, } \\
\text { metastatic, lung }\end{array}$ & - & $3(6 \%)$ & $1(2 \%)$ & $2(4 \%)$ \\
\hline $\begin{array}{l}\text { Hepatocellular carcinoma, metastatic, } \\
\text { liver }\end{array}$ & - & $1(2 \%)$ & - & - \\
\hline Urinary bladder & (48) & (48) & (50) & (47) \\
\hline Hemangiosarcoma & - & - & $1(2 \%)$ & - \\
\hline \multicolumn{5}{|l|}{ Systemic Lesions } \\
\hline Multiple organs ${ }^{\mathrm{b}}$ & (50) & (50) & $(50)$ & $(50)$ \\
\hline Histiocytic sarcoma & $1(2 \%)$ & - & - & - \\
\hline Lymphoma malignant & $4(8 \%)$ & $1(2 \%)$ & $1(2 \%)$ & $3(6 \%)$ \\
\hline \multicolumn{5}{|l|}{ Neoplasm Summary } \\
\hline Total animals with primary neoplasmsc & 45 & 49 & 50 & 49 \\
\hline Total primary neoplasms & 100 & 119 & 113 & 83 \\
\hline Total animals with benign neoplasms & 35 & 32 & 34 & 19 \\
\hline Total benign neoplasms & 45 & 47 & 41 & 21 \\
\hline Total animals with malignant neoplasms & 36 & 46 & 46 & 48 \\
\hline Total malignant neoplasms & 54 & 72 & 71 & 62 \\
\hline Total animals with metastatic neoplasms & 9 & 13 & 11 & 5 \\
\hline Total metastatic neoplasms & 10 & 33 & 21 & 17 \\
\hline $\begin{array}{l}\text { Total animals with uncertain neoplasms- } \\
\text { benign or malignant }\end{array}$ & 1 & - & 1 & - \\
\hline Total uncertain neoplasms & 1 & - & 1 & - \\
\hline
\end{tabular}

${ }^{a}$ Number of animals examined microscopically at the site and the number of animals with neoplasm.

${ }^{b}$ Number of animals with any tissue examined microscopically.

'Primary neoplasms: all neoplasms except metastatic neoplasms. 
Table C-2. Statistical Analysis of Primary Neoplasms in Male Mice in the Two-year Inhalation Study of Cobalt Metal

\begin{tabular}{|c|c|c|c|c|}
\hline & $\begin{array}{l}\text { Chamber } \\
\text { Control }\end{array}$ & $1.25 \mathrm{mg} / \mathrm{m}^{3}$ & $2.5 \mathrm{mg} / \mathrm{m}^{3}$ & $5 \mathrm{mg} / \mathrm{m}^{3}$ \\
\hline \multicolumn{5}{|c|}{ Adrenal Cortex: Adenoma } \\
\hline Overall rate ${ }^{\mathrm{a}}$ & $1 / 50(2 \%)$ & $4 / 49(8 \%)$ & $1 / 50(2 \%)$ & $1 / 50(2 \%)$ \\
\hline Adjusted rate ${ }^{b}$ & $2.1 \%$ & $9.1 \%$ & $2.4 \%$ & $2.5 \%$ \\
\hline Terminal rate ${ }^{c}$ & $1 / 39(3 \%)$ & $3 / 31(10 \%)$ & $1 / 29(3 \%)$ & $1 / 25(4 \%)$ \\
\hline First incidence (days) & $729(\mathrm{~T})$ & 715 & $729(\mathrm{~T})$ & $729(\mathrm{~T})$ \\
\hline Poly-3 test ${ }^{\mathrm{d}}$ & $\mathrm{P}=0.444 \mathrm{~N}$ & $\mathrm{P}=0.157$ & $\mathrm{P}=0.730$ & $\mathrm{P}=0.724$ \\
\hline \multicolumn{5}{|c|}{ Harderian Gland: Adenoma } \\
\hline Overall rate & $6 / 50(12 \%)$ & $3 / 50(6 \%)$ & $4 / 50(8 \%)$ & $4 / 50(8 \%)$ \\
\hline Adjusted rate & $12.6 \%$ & $6.8 \%$ & $9.6 \%$ & $9.7 \%$ \\
\hline Terminal rate & $5 / 39(13 \%)$ & $3 / 31(10 \%)$ & $3 / 29(10 \%)$ & $2 / 25(8 \%)$ \\
\hline First incidence (days) & 651 & $729(\mathrm{~T})$ & 706 & 635 \\
\hline Poly-3 test & $\mathrm{P}=0.454 \mathrm{~N}$ & $\mathrm{P}=0.281 \mathrm{~N}$ & $\mathrm{P}=0.460 \mathrm{~N}$ & $\mathrm{P}=0.465 \mathrm{~N}$ \\
\hline \multicolumn{5}{|c|}{ Harderian Gland: Adenoma or Carcinoma } \\
\hline Overall rate & $8 / 50(16 \%)$ & $4 / 50(8 \%)$ & $4 / 50(8 \%)$ & $4 / 50(8 \%)$ \\
\hline Adjusted rate & $16.6 \%$ & $9.0 \%$ & $9.6 \%$ & $9.7 \%$ \\
\hline Terminal rate & $6 / 39(15 \%)$ & $4 / 31(13 \%)$ & $3 / 29(10 \%)$ & $2 / 25(8 \%)$ \\
\hline First incidence (days) & 646 & $729(\mathrm{~T})$ & 706 & 635 \\
\hline Poly-3 test & $\mathrm{P}=0.224 \mathrm{~N}$ & $\mathrm{P}=0.220 \mathrm{~N}$ & $\mathrm{P}=0.255 \mathrm{~N}$ & $\mathrm{P}=0.260 \mathrm{~N}$ \\
\hline \multicolumn{5}{|c|}{ Liver: Hemangiosarcoma } \\
\hline Overall rate & $4 / 50(8 \%)$ & $2 / 50(4 \%)$ & $3 / 50(6 \%)$ & $0 / 50(0 \%)$ \\
\hline Adjusted rate & $8.4 \%$ & $4.5 \%$ & $7.1 \%$ & $0.0 \%$ \\
\hline Terminal rate & $2 / 39(5 \%)$ & $1 / 31(3 \%)$ & $0 / 29(0 \%)$ & $0 / 25(0 \%)$ \\
\hline First incidence (days) & 682 & 611 & 597 & $-^{\mathrm{e}}$ \\
\hline Poly-3 test & $\mathrm{P}=0.083 \mathrm{~N}$ & $P=0.369 \mathrm{~N}$ & $\mathrm{P}=0.563 \mathrm{~N}$ & $\mathrm{P}=0.084 \mathrm{~N}$ \\
\hline \multicolumn{5}{|c|}{ Liver: Hepatocellular Adenoma } \\
\hline Overall rate & $28 / 50(56 \%)$ & $25 / 50(50 \%)$ & $21 / 50(42 \%)$ & $12 / 50(24 \%)$ \\
\hline Adjusted rate & $57.5 \%$ & $56.0 \%$ & $46.7 \%$ & $29.1 \%$ \\
\hline Terminal rate & $22 / 39(56 \%)$ & $20 / 31(65 \%)$ & $14 / 29(48 \%)$ & $8 / 25(32 \%)$ \\
\hline First incidence (days) & 646 & 684 & 361 & 635 \\
\hline Poly-3 test & $\mathrm{P}=0.002 \mathrm{~N}$ & $\mathrm{P}=0.527 \mathrm{~N}$ & $\mathrm{P}=0.199 \mathrm{~N}$ & $\mathrm{P}=0.005 \mathrm{~N}$ \\
\hline \multicolumn{5}{|c|}{ Liver: Hepatocellular Carcinoma } \\
\hline Overall rate & $25 / 50(50 \%)$ & $21 / 50(42 \%)$ & $14 / 50(28 \%)$ & $9 / 50(18 \%)$ \\
\hline Adjusted rate & $50.0 \%$ & $43.6 \%$ & $31.5 \%$ & $21.9 \%$ \\
\hline
\end{tabular}


Cobalt Metal, NTP TR 581

\begin{tabular}{|c|c|c|c|c|}
\hline & $\begin{array}{l}\text { Chamber } \\
\text { Control }\end{array}$ & $1.25 \mathrm{mg} / \mathrm{m}^{3}$ & $2.5 \mathrm{mg} / \mathrm{m}^{3}$ & $5 \mathrm{mg} / \mathrm{m}^{3}$ \\
\hline Terminal rate & $14 / 39(36 \%)$ & $9 / 31(29 \%)$ & $5 / 29(17 \%)$ & $6 / 25(24 \%)$ \\
\hline First incidence (days) & 561 & 457 & 394 & 649 \\
\hline Poly-3 test & $\mathrm{P}=0.002 \mathrm{~N}$ & $\mathrm{P}=0.334 \mathrm{~N}$ & $\mathrm{P}=0.052 \mathrm{~N}$ & $\mathrm{P}=0.004 \mathrm{~N}$ \\
\hline \multicolumn{5}{|c|}{ Liver: Hepatocellular Adenoma or Carcinoma } \\
\hline Overall rate & $38 / 50(76 \%)$ & $38 / 50(76 \%)$ & $32 / 50(64 \%)$ & $18 / 50(36 \%)$ \\
\hline Adjusted rate & $76.0 \%$ & $78.7 \%$ & $68.4 \%$ & $43.1 \%$ \\
\hline Terminal rate & $27 / 39(69 \%)$ & $23 / 31(74 \%)$ & $19 / 29(66 \%)$ & $11 / 25(44 \%)$ \\
\hline First incidence (days) & 561 & 457 & 361 & 635 \\
\hline Poly-3 test & $\mathrm{P}<0.001 \mathrm{~N}$ & $\mathrm{P}=0.469$ & $\mathrm{P}=0.269 \mathrm{~N}$ & $\mathrm{P}<0.001 \mathrm{~N}$ \\
\hline \multicolumn{5}{|c|}{ Liver: Hepatocellular Carcinoma or Hepatoblastoma } \\
\hline Overall rate & $27 / 50(54 \%)$ & $22 / 50(44 \%)$ & $14 / 50(28 \%)$ & $9 / 50(18 \%)$ \\
\hline Adjusted rate & $54.0 \%$ & $45.7 \%$ & $31.5 \%$ & $21.9 \%$ \\
\hline Terminal rate & $16 / 39(41 \%)$ & $10 / 31(32 \%)$ & $5 / 29(17 \%)$ & $6 / 25(24 \%)$ \\
\hline First incidence (days) & 561 & 457 & 394 & 649 \\
\hline Poly-3 test & $\mathrm{P}<0.001 \mathrm{~N}$ & $\mathrm{P}=0.268 \mathrm{~N}$ & $\mathrm{P}=0.021 \mathrm{~N}$ & $\mathrm{P}<0.001 \mathrm{~N}$ \\
\hline \multicolumn{5}{|c|}{ Liver: Hepatocellular Adenoma, Hepatocellular Carcinoma, or Hepatoblastoma } \\
\hline Overall rate & $39 / 50(78 \%)$ & $39 / 50(78 \%)$ & $32 / 50(64 \%)$ & $18 / 50(36 \%)$ \\
\hline Adjusted rate & $78.0 \%$ & $80.8 \%$ & $68.4 \%$ & $43.1 \%$ \\
\hline Terminal rate & $28 / 39(72 \%)$ & $24 / 31(77 \%)$ & $19 / 29(66 \%)$ & $11 / 25(44 \%)$ \\
\hline First incidence (days) & 561 & 457 & 361 & 635 \\
\hline Poly-3 test & $\mathrm{P}<0.001 \mathrm{~N}$ & $\mathrm{P}=0.464$ & $\mathrm{P}=0.197 \mathrm{~N}$ & $\mathrm{P}<0.001 \mathrm{~N}$ \\
\hline \multicolumn{5}{|c|}{ Lung: Alveolar/bronchiolar Adenoma } \\
\hline Overall rate & $7 / 50(14 \%)$ & $11 / 49(22 \%)$ & $15 / 50(30 \%)$ & $3 / 50(6 \%)$ \\
\hline Adjusted rate & $14.7 \%$ & $24.5 \%$ & $35.9 \%$ & $7.3 \%$ \\
\hline Terminal rate & $5 / 39(13 \%)$ & $7 / 31(23 \%)$ & $14 / 29(48 \%)$ & $2 / 25(8 \%)$ \\
\hline First incidence (days) & 684 & 571 & 660 & 571 \\
\hline Poly-3 test & $\mathrm{P}=0.254 \mathrm{~N}$ & $\mathrm{P}=0.176$ & $\mathrm{P}=0.016^{*}$ & $\mathrm{P}=0.226 \mathrm{~N}$ \\
\hline \multicolumn{5}{|c|}{ Lung: Alveolar/bronchiolar Carcinoma } \\
\hline Overall rate & $11 / 50(22 \%)$ & $38 / 49(78 \%)$ & $42 / 50(84 \%)$ & $46 / 50(92 \%)$ \\
\hline Adjusted rate & $22.8 \%$ & $79.4 \%$ & $87.6 \%$ & $93.8 \%$ \\
\hline Terminal rate & $8 / 39(21 \%)$ & $24 / 31(77 \%)$ & $25 / 29(86 \%)$ & $22 / 25(88 \%)$ \\
\hline First incidence (days) & 561 & 551 & 382 & 425 \\
\hline Poly-3 test & $\mathrm{P}<0.001$ & $\mathrm{P}<0.001$ & $\mathrm{P}<0.001$ & $\mathrm{P}<0.001$ \\
\hline \multicolumn{5}{|c|}{ Lung: Alveolar/bronchiolar Adenoma or Carcinoma } \\
\hline Overall rate & $16 / 50(32 \%)$ & $41 / 49(84 \%)$ & $43 / 50(86 \%)$ & $47 / 50(94 \%)$ \\
\hline
\end{tabular}


Cobalt Metal, NTP TR 581

\begin{tabular}{|c|c|c|c|c|}
\hline & $\begin{array}{l}\text { Chamber } \\
\text { Control }\end{array}$ & $1.25 \mathrm{mg} / \mathrm{m}^{3}$ & $2.5 \mathrm{mg} / \mathrm{m}^{3}$ & $5 \mathrm{mg} / \mathrm{m}^{3}$ \\
\hline Adjusted rate & $33.0 \%$ & $85.0 \%$ & $89.7 \%$ & $95.9 \%$ \\
\hline Terminal rate & $11 / 39(28 \%)$ & $26 / 31(84 \%)$ & $26 / 29(90 \%)$ & $23 / 25(92 \%)$ \\
\hline First incidence (days) & 561 & 551 & 382 & 425 \\
\hline Poly-3 test & $\mathrm{P}<0.001$ & $\mathrm{P}<0.001$ & $\mathrm{P}<0.001$ & $\mathrm{P}<0.001$ \\
\hline \multicolumn{5}{|c|}{ Pancreatic Islets: Adenoma or Carcinoma } \\
\hline Overall rate & $0 / 49(0 \%)$ & $3 / 47(6 \%)$ & $0 / 50(0 \%)$ & $0 / 50(0 \%)$ \\
\hline Adjusted rate & $0.0 \%$ & $7.0 \%$ & $0.0 \%$ & $0.0 \%$ \\
\hline Terminal rate & $0 / 39(0 \%)$ & $0 / 31(0 \%)$ & $0 / 29(0 \%)$ & $0 / 25(0 \%)$ \\
\hline First incidence (days) & - & 589 & - & - \\
\hline Poly-3 test & $\mathrm{P}=0.357 \mathrm{~N}$ & $\mathrm{P}=0.104$ & $-{ }^{f}$ & - \\
\hline \multicolumn{5}{|c|}{ Spleen: Hemangiosarcoma } \\
\hline Overall rate & $3 / 50(6 \%)$ & $0 / 48(0 \%)$ & $4 / 49(8 \%)$ & $3 / 48(6 \%)$ \\
\hline Adjusted rate & $6.3 \%$ & $0.0 \%$ & $9.6 \%$ & $7.7 \%$ \\
\hline Terminal rate & $2 / 39(5 \%)$ & $0 / 31(0 \%)$ & $1 / 29(3 \%)$ & $2 / 25(8 \%)$ \\
\hline First incidence (days) & 708 & - & 652 & 649 \\
\hline Poly-3 test & $\mathrm{P}=0.292$ & $\mathrm{P}=0.137 \mathrm{~N}$ & $\mathrm{P}=0.431$ & $\mathrm{P}=0.568$ \\
\hline \multicolumn{5}{|c|}{ All Organs: Hemangiosarcoma } \\
\hline Overall rate & $7 / 50(14 \%)$ & $3 / 50(6 \%)$ & $7 / 50(14 \%)$ & $3 / 50(6 \%)$ \\
\hline Adjusted rate & $14.7 \%$ & $6.7 \%$ & $16.4 \%$ & $7.3 \%$ \\
\hline Terminal rate & $5 / 39(13 \%)$ & $2 / 31(7 \%)$ & $2 / 29(7 \%)$ & $2 / 25(8 \%)$ \\
\hline First incidence (days) & 682 & 611 & 597 & 649 \\
\hline Poly-3 test & $\mathrm{P}=0.291 \mathrm{~N}$ & $\mathrm{P}=0.186 \mathrm{~N}$ & $P=0.526$ & $\mathrm{P}=0.228 \mathrm{~N}$ \\
\hline \multicolumn{5}{|c|}{ All Organs: Hemangioma or Hemangiosarcoma } \\
\hline Overall rate & $7 / 50(14 \%)$ & $3 / 50(6 \%)$ & $7 / 50(14 \%)$ & $4 / 50(8 \%)$ \\
\hline Adjusted rate & $14.7 \%$ & $6.7 \%$ & $16.4 \%$ & $9.8 \%$ \\
\hline Terminal rate & $5 / 39(13 \%)$ & $2 / 31(7 \%)$ & $2 / 29(7 \%)$ & $3 / 25(12 \%)$ \\
\hline First incidence (days) & 682 & 611 & 597 & 649 \\
\hline Poly-3 test & $\mathrm{P}=0.428 \mathrm{~N}$ & $P=0.186 \mathrm{~N}$ & $P=0.526$ & $\mathrm{P}=0.357 \mathrm{~N}$ \\
\hline \multicolumn{5}{|c|}{ All Organs: Malignant Lymphoma } \\
\hline Overall rate & $4 / 50(8 \%)$ & $1 / 50(2 \%)$ & $1 / 50(2 \%)$ & $3 / 50(6 \%)$ \\
\hline Adjusted rate & $8.3 \%$ & $2.3 \%$ & $2.4 \%$ & $7.2 \%$ \\
\hline Terminal rate & $1 / 39(3 \%)$ & $1 / 31(3 \%)$ & $0 / 29(0 \%)$ & $1 / 25(4 \%)$ \\
\hline First incidence (days) & 646 & $729(\mathrm{~T})$ & 627 & 227 \\
\hline Poly-3 test & $\mathrm{P}=0.551 \mathrm{~N}$ & $\mathrm{P}=0.207 \mathrm{~N}$ & $\mathrm{P}=0.226 \mathrm{~N}$ & $\mathrm{P}=0.578 \mathrm{~N}$ \\
\hline
\end{tabular}


Cobalt Metal, NTP TR 581

\begin{tabular}{|c|c|c|c|c|}
\hline & $\begin{array}{l}\text { Chamber } \\
\text { Control }\end{array}$ & $1.25 \mathrm{mg} / \mathrm{m}^{3}$ & $2.5 \mathrm{mg} / \mathrm{m}^{3}$ & $5 \mathrm{mg} / \mathrm{m}^{3}$ \\
\hline \multicolumn{5}{|c|}{ All Organs: Benign Neoplasms } \\
\hline Overall rate & $35 / 50(70 \%)$ & $32 / 50(64 \%)$ & $34 / 50(68 \%)$ & $19 / 50(38 \%)$ \\
\hline Adjusted rate & $71.8 \%$ & $70.3 \%$ & $75.1 \%$ & $45.2 \%$ \\
\hline Terminal rate & $28 / 39(72 \%)$ & $24 / 31(77 \%)$ & $25 / 29(86 \%)$ & $13 / 25(52 \%)$ \\
\hline First incidence (days) & 646 & 571 & 361 & 571 \\
\hline Poly-3 test & $\mathrm{P}=0.004 \mathrm{~N}$ & $\mathrm{P}=0.528 \mathrm{~N}$ & $\mathrm{P}=0.446$ & $\mathrm{P}=0.006 \mathrm{~N}$ \\
\hline \multicolumn{5}{|c|}{ All Organs: Malignant Neoplasms } \\
\hline Overall rate & $36 / 50(72 \%)$ & $46 / 50(92 \%)$ & $46 / 50(92 \%)$ & $48 / 50(96 \%)$ \\
\hline Adjusted rate & $72.0 \%$ & $92.0 \%$ & $93.7 \%$ & $96.0 \%$ \\
\hline Terminal rate & $25 / 39(64 \%)$ & $27 / 31(87 \%)$ & $26 / 29(90 \%)$ & $23 / 25(92 \%)$ \\
\hline First incidence (days) & 561 & 457 & 382 & 227 \\
\hline Poly-3 test & $\mathrm{P}<0.001$ & $\mathrm{P}=0.008$ & $\mathrm{P}=0.004$ & $\mathrm{P}<0.001$ \\
\hline \multicolumn{5}{|c|}{ All Organs: Benign or Malignant Neoplasms } \\
\hline Overall rate & $45 / 50(90 \%)$ & $49 / 50(98 \%)$ & $50 / 50(100 \%)$ & $49 / 50(98 \%)$ \\
\hline Adjusted rate & $90.0 \%$ & $98.0 \%$ & $100.0 \%$ & $98.0 \%$ \\
\hline Terminal rate & $34 / 39(87 \%)$ & $30 / 31(97 \%)$ & $29 / 29(100 \%)$ & $24 / 25(96 \%)$ \\
\hline First incidence (days) & 561 & 457 & 361 & 227 \\
\hline Poly-3 test & $\mathrm{P}=0.050$ & $\mathrm{P}=0.102$ & $\mathrm{P}=0.031$ & $\mathrm{P}=0.102$ \\
\hline
\end{tabular}

(T) Terminal kill.

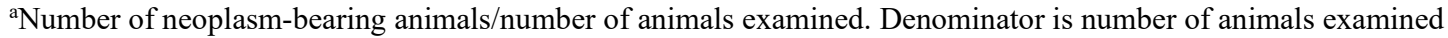
microscopically for adrenal gland, liver, lung, pancreatic islets, and spleen; for other tissues, denominator is number of animals necropsied.

bPoly-3 estimated neoplasm incidence after adjustment for intercurrent mortality.

'Observed incidence at terminal kill.

${ }^{\mathrm{d}}$ Beneath the chamber control incidence is the $\mathrm{P}$ value associated with the trend test. Beneath the exposed group incidence are the $\mathrm{P}$ values corresponding to pairwise comparisons between the chamber controls and that exposed group. The Poly-3 test accounts for differential mortality in animals that do not reach terminal kill. A negative trend or a lower incidence in an exposure group is indicated by $\mathbf{N}$.

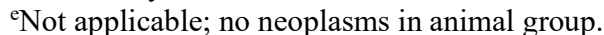

fValue of statistic cannot be computed. 
Table C-3. Historical Incidence of Alveolar/bronchiolar Neoplasms in Control Male B6C3F1/N Mice $^{\mathrm{a}}$

\begin{tabular}{lccc}
\hline \multicolumn{1}{c}{ Study (Study Start) } & Adenoma & Carcinoma & $\begin{array}{c}\text { Adenoma or } \\
\text { Carcinoma }\end{array}$ \\
\hline Historical Incidence: Inhalation Studies & & & \\
1-Bromopropane (July 2003) & $6 / 50$ & $8 / 50$ & $13 / 50$ \\
CIMSTAR 3800 (May 2008) & $5 / 50$ & $8 / 50$ & $13 / 50$ \\
Cobalt metal (May 2006) & $7 / 50$ & $11 / 50$ & $16 / 50$ \\
Diethylamine (August 2003) & $4 / 50$ & $12 / 50$ & $15 / 50$ \\
Tetralin (June 2003) & $10 / 50$ & $11 / 50$ & $20 / 50$ \\
Vinylidene chloride (June 2005) & $7 / 50$ & $9 / 50$ & $13 / 50$ \\
Total (\%) & $39 / 300(13.0 \%)$ & $59 / 300(19.7 \%)$ & $90 / 300(30.0 \%)$ \\
Mean \pm standard deviation & $13.0 \% \pm 4.2 \%$ & $19.7 \% \pm 3.4 \%$ & $30.0 \% \pm 5.5 \%$ \\
Range & $8 \%-20 \%$ & $16 \%-24 \%$ & $26 \%-40 \%$ \\
Overall Historical Incidence: All Routes & & & \\
Total (\%) & $145 / 950(15.3 \%)$ & $132 / 950(13.9 \%)$ & $263 / 950(27.7 \%)$ \\
Mean \pm standard deviation & $15.3 \% \pm 6.2 \%$ & $13.9 \% \pm 7.1 \%$ & $27.7 \% \pm 5.7 \%$ \\
Range & $2 \%-26 \%$ & $4 \%-24 \%$ & $16 \%-40 \%$ \\
\hline ata as of May 2013. & & &
\end{tabular}


Table C-4. Summary of the Incidence of Nonneoplastic Lesions in Male Mice in the Two-year Inhalation Study of Cobalt Metal ${ }^{\mathrm{a}}$

\begin{tabular}{|c|c|c|c|c|}
\hline & $\begin{array}{c}\text { Chamber } \\
\text { Control }\end{array}$ & $1.25 \mathrm{mg} / \mathrm{m}^{3}$ & $2.5 \mathrm{mg} / \mathrm{m}^{3}$ & $5 \mathrm{mg} / \mathrm{m}^{3}$ \\
\hline \multicolumn{5}{|l|}{ Disposition Summary } \\
\hline Animals initially in study & 50 & 50 & 50 & 50 \\
\hline \multicolumn{5}{|l|}{ Early deaths } \\
\hline Moribund & 5 & 13 & 12 & 17 \\
\hline Natural deaths & 6 & 6 & 9 & 8 \\
\hline \multicolumn{5}{|l|}{ Survivors } \\
\hline Died last week of study & - & - & 1 & 3 \\
\hline Terminal kill & 39 & 31 & 28 & 22 \\
\hline Animals examined microscopically & 50 & 50 & 50 & 50 \\
\hline \multicolumn{5}{|l|}{ Alimentary System } \\
\hline Esophagus & $(50)$ & $(50)$ & $(50)$ & $(50)$ \\
\hline Arteriole, inflammation, acute & - & - & $1(2 \%)$ & - \\
\hline Gallbladder & (43) & (37) & $(34)$ & (35) \\
\hline Degeneration, hyaline & - & - & $1(3 \%)$ & - \\
\hline Intestine large, cecum & (45) & (45) & (44) & (45) \\
\hline Intestine large, colon & (47) & (45) & $(45)$ & (46) \\
\hline Intestine large, rectum & (44) & (44) & $(45)$ & (48) \\
\hline Intestine small, duodenum & (45) & (44) & (44) & (44) \\
\hline Inflammation, chronic active & $1(2 \%)$ & - & - & - \\
\hline Ulcer & - & - & $1(2 \%)$ & - \\
\hline Intestine small, ileum & (45) & (45) & (43) & (44) \\
\hline Inflammation, acute & - & $1(2 \%)$ & - & - \\
\hline Inflammation, chronic active & - & - & $1(2 \%)$ & - \\
\hline Intestine small, jejunum & (45) & (45) & (43) & (44) \\
\hline Liver & (50) & (50) & (50) & (50) \\
\hline Angiectasis & - & $1(2 \%)$ & - & $1(2 \%)$ \\
\hline Basophilic focus & $4(8 \%)$ & $4(8 \%)$ & $6(12 \%)$ & $4(8 \%)$ \\
\hline Clear cell focus & $17(34 \%)$ & $17(34 \%)$ & $8(16 \%)$ & - \\
\hline Eosinophilic focus & $6(12 \%)$ & $5(10 \%)$ & $4(8 \%)$ & $2(4 \%)$ \\
\hline Fatty change & - & - & $1(2 \%)$ & - \\
\hline Hemorrhage & - & - & $1(2 \%)$ & - \\
\hline Hepatodiaphragmatic nodule & - & $3(6 \%)$ & - & $3(6 \%)$ \\
\hline Inflammation, chronic & - & - & $1(2 \%)$ & - \\
\hline
\end{tabular}


Cobalt Metal, NTP TR 581

\begin{tabular}{|c|c|c|c|c|}
\hline & $\begin{array}{c}\text { Chamber } \\
\text { Control }\end{array}$ & $1.25 \mathrm{mg} / \mathrm{m}^{3}$ & $2.5 \mathrm{mg} / \mathrm{m}^{3}$ & $5 \mathrm{mg} / \mathrm{m}^{3}$ \\
\hline Mixed cell focus & $1(2 \%)$ & - & - & - \\
\hline Necrosis & $2(4 \%)$ & $3(6 \%)$ & $1(2 \%)$ & $1(2 \%)$ \\
\hline Tension lipidosis & $2(4 \%)$ & - & $2(4 \%)$ & - \\
\hline Mesentery & (5) & (5) & (5) & (3) \\
\hline Fat, necrosis & $5(100 \%)$ & $4(80 \%)$ & $5(100 \%)$ & $3(100 \%)$ \\
\hline Pancreas & $(50)$ & (47) & $(50)$ & (50) \\
\hline Atrophy & - & - & $1(2 \%)$ & $1(2 \%)$ \\
\hline Salivary glands & $(50)$ & (49) & $(50)$ & $(50)$ \\
\hline Inflammation, chronic & - & $1(2 \%)$ & - & - \\
\hline Stomach, forestomach & $(50)$ & (48) & (50) & $(50)$ \\
\hline Hyperplasia, squamous & $1(2 \%)$ & $2(4 \%)$ & $2(4 \%)$ & $4(8 \%)$ \\
\hline Inflammation, chronic active & - & $1(2 \%)$ & - & $3(6 \%)$ \\
\hline Ulcer & $1(2 \%)$ & $5(10 \%)$ & $3(6 \%)$ & $1(2 \%)$ \\
\hline Stomach, glandular & $(48)$ & (46) & $(48)$ & (49) \\
\hline Necrosis & $1(2 \%)$ & $1(2 \%)$ & $1(2 \%)$ & - \\
\hline Ulcer & - & $1(2 \%)$ & - & $1(2 \%)$ \\
\hline Arteriole, inflammation, acute & - & - & $1(2 \%)$ & - \\
\hline Tooth & (8) & (2) & (2) & (1) \\
\hline Dysplasia & $8(100 \%)$ & $1(50 \%)$ & $2(100 \%)$ & $1(100 \%)$ \\
\hline Inflammation, chronic active & - & $1(50 \%)$ & - & - \\
\hline \multicolumn{5}{|l|}{ Cardiovascular System } \\
\hline Heart & $(50)$ & (50) & (50) & (50) \\
\hline Cardiomyopathy & $13(26 \%)$ & $5(10 \%)$ & $12(24 \%)$ & $8(16 \%)$ \\
\hline Hemorrhage & - & - & - & $1(2 \%)$ \\
\hline Inflammation, suppurative & - & $1(2 \%)$ & - & - \\
\hline Mineralization & $1(2 \%)$ & $1(2 \%)$ & - & - \\
\hline Necrosis & - & - & $1(2 \%)$ & - \\
\hline Thrombosis & $1(2 \%)$ & - & - & - \\
\hline Pericardium, inflammation, chronic & - & - & - & $1(2 \%)$ \\
\hline \multicolumn{5}{|l|}{ Endocrine System } \\
\hline Adrenal cortex & $(50)$ & $(49)$ & $(50)$ & (50) \\
\hline Hyperplasia & $6(12 \%)$ & $10(20 \%)$ & $2(4 \%)$ & $1(2 \%)$ \\
\hline Hypertrophy & $18(36 \%)$ & $9(18 \%)$ & $12(24 \%)$ & $2(4 \%)$ \\
\hline Necrosis & $1(2 \%)$ & - & - & - \\
\hline Adrenal medulla & $(50)$ & (49) & $(50)$ & (48) \\
\hline
\end{tabular}


Cobalt Metal, NTP TR 581

\begin{tabular}{|c|c|c|c|c|}
\hline & $\begin{array}{l}\text { Chamber } \\
\text { Control }\end{array}$ & $1.25 \mathrm{mg} / \mathrm{m}^{3}$ & $2.5 \mathrm{mg} / \mathrm{m}^{3}$ & $5 \mathrm{mg} / \mathrm{m}^{3}$ \\
\hline Hyperplasia & $2(4 \%)$ & $2(4 \%)$ & - & - \\
\hline Islets, pancreatic & (49) & (47) & (50) & (50) \\
\hline Hyperplasia & $2(4 \%)$ & $3(6 \%)$ & - & $2(4 \%)$ \\
\hline Parathyroid gland & (29) & (25) & (26) & (27) \\
\hline Pituitary gland & (47) & (49) & $(48)$ & (48) \\
\hline Pars distalis, hyperplasia & $1(2 \%)$ & $2(4 \%)$ & $1(2 \%)$ & - \\
\hline Thyroid gland & (49) & (49) & (50) & (49) \\
\hline \multicolumn{5}{|l|}{ General Body System } \\
\hline Tissue NOS & $(0)$ & $(0)$ & $(0)$ & $(1)$ \\
\hline \multicolumn{5}{|l|}{ Genital System } \\
\hline Coagulating gland & $(0)$ & (1) & (0) & (1) \\
\hline Epididymis & (50) & (49) & $(50)$ & (50) \\
\hline Granuloma sperm & $2(4 \%)$ & - & $1(2 \%)$ & - \\
\hline Penis & (0) & (0) & (0) & (1) \\
\hline Inflammation, acute & - & - & - & $1(100 \%)$ \\
\hline Preputial gland & (49) & (50) & (49) & (50) \\
\hline Ectasia & $1(2 \%)$ & $1(2 \%)$ & - & - \\
\hline Inflammation, chronic active & $1(2 \%)$ & $1(2 \%)$ & - & - \\
\hline Prostate gland & $(50)$ & (49) & (48) & (50) \\
\hline Inflammation, acute & - & $1(2 \%)$ & - & - \\
\hline Seminal vesicle & (48) & (48) & (49) & (50) \\
\hline Congestion & - & $1(2 \%)$ & - & - \\
\hline Inflammation, chronic active & - & $1(2 \%)$ & - & $1(2 \%)$ \\
\hline Testes & $(50)$ & (49) & $(50)$ & $(50)$ \\
\hline Germinal epithelium, degeneration & $9(18 \%)$ & $14(29 \%)$ & $8(16 \%)$ & $21(42 \%)$ \\
\hline Interstitial cell, hyperplasia & - & - & - & $1(2 \%)$ \\
\hline \multicolumn{5}{|l|}{ Hematopoietic System } \\
\hline Bone marrow & (50) & (49) & $(50)$ & (49) \\
\hline Angiectasis & - & - & - & $1(2 \%)$ \\
\hline Thrombosis & - & - & - & $1(2 \%)$ \\
\hline Lymph node & (2) & (0) & (1) & (1) \\
\hline Renal, hemorrhage & $1(50 \%)$ & - & - & - \\
\hline Lymph node, bronchial & (26) & (32) & (20) & (24) \\
\hline Lymph node, mandibular & (37) & (23) & (27) & (37) \\
\hline Lymph node, mediastinal & (34) & (34) & (36) & (44) \\
\hline
\end{tabular}


Cobalt Metal, NTP TR 581

\begin{tabular}{|c|c|c|c|c|}
\hline & $\begin{array}{l}\text { Chamber } \\
\text { Control }\end{array}$ & $1.25 \mathrm{mg} / \mathrm{m}^{3}$ & $2.5 \mathrm{mg} / \mathrm{m}^{3}$ & $5 \mathrm{mg} / \mathrm{m}^{3}$ \\
\hline Hyperplasia, lymphoid & - & - & - & $1(2 \%)$ \\
\hline Lymph node, mesenteric & (47) & (44) & (44) & $(42)$ \\
\hline Hemorrhage & $1(2 \%)$ & $1(2 \%)$ & - & - \\
\hline Hyperplasia, lymphoid & - & - & $1(2 \%)$ & - \\
\hline Arteriole, inflammation, chronic active & - & - & - & $1(2 \%)$ \\
\hline Spleen & (50) & (48) & $(49)$ & (48) \\
\hline Hematopoietic cell proliferation & $5(10 \%)$ & - & - & - \\
\hline Infarct & - & $1(2 \%)$ & - & - \\
\hline Thymus & (42) & (44) & $(40)$ & (38) \\
\hline \multicolumn{5}{|l|}{ Integumentary System } \\
\hline Skin & $(50)$ & $(50)$ & $(50)$ & (49) \\
\hline Inflammation, chronic active & $1(2 \%)$ & $1(2 \%)$ & $2(4 \%)$ & $2(4 \%)$ \\
\hline Metaplasia, osseous & - & - & $1(2 \%)$ & - \\
\hline Subcutaneous tissue, edema & $1(2 \%)$ & - & - & - \\
\hline \multicolumn{5}{|l|}{ Musculoskeletal System } \\
\hline Bone & $(50)$ & (49) & (50) & (50) \\
\hline Skeletal muscle & (0) & (3) & (3) & (1) \\
\hline \multicolumn{5}{|l|}{ Nervous System } \\
\hline Brain & (50) & (50) & (50) & $(50)$ \\
\hline Infiltration cellular, histiocyte & - & $1(2 \%)$ & - & - \\
\hline Necrosis & - & $1(2 \%)$ & - & - \\
\hline Peripheral nerve & (1) & $(0)$ & (0) & (1) \\
\hline Degeneration & $1(100 \%)$ & - & - & - \\
\hline Spinal cord & (1) & $(0)$ & $(0)$ & (1) \\
\hline \multicolumn{5}{|l|}{ Respiratory System } \\
\hline Larynx & (48) & (47) & (49) & (50) \\
\hline Inflammation, suppurative & $7(15 \%)$ & $2(4 \%)$ & $2(4 \%)$ & $4(8 \%)$ \\
\hline Inflammation, chronic & $1(2 \%)$ & $1(2 \%)$ & - & - \\
\hline Arteriole, inflammation, acute & - & - & $1(2 \%)$ & - \\
\hline Respiratory epithelium, erosion & - & - & - & $1(2 \%)$ \\
\hline $\begin{array}{l}\text { Respiratory epithelium, metaplasia, } \\
\text { squamous }\end{array}$ & $7(15 \%)$ & $47(100 \%)$ & $49(100 \%)$ & $49(98 \%)$ \\
\hline $\begin{array}{l}\text { Respiratory epithelium, } \\
\text { vacuolization cytoplasmic }\end{array}$ & - & $20(43 \%)$ & $24(49 \%)$ & $32(64 \%)$ \\
\hline Squamous epithelium, erosion & $1(2 \%)$ & $3(6 \%)$ & - & $1(2 \%)$ \\
\hline
\end{tabular}


Cobalt Metal, NTP TR 581

\begin{tabular}{|c|c|c|c|c|}
\hline & $\begin{array}{c}\text { Chamber } \\
\text { Control }\end{array}$ & $1.25 \mathrm{mg} / \mathrm{m}^{3}$ & $2.5 \mathrm{mg} / \mathrm{m}^{3}$ & $5 \mathrm{mg} / \mathrm{m}^{3}$ \\
\hline Squamous epithelium, hyperplasia & $2(4 \%)$ & $5(11 \%)$ & $5(10 \%)$ & $8(16 \%)$ \\
\hline Lung & $(50)$ & (49) & $(50)$ & (50) \\
\hline Inflammation, suppurative & $1(2 \%)$ & $2(4 \%)$ & $6(12 \%)$ & $16(32 \%)$ \\
\hline Proteinosis & $2(4 \%)$ & $46(94 \%)$ & $49(98 \%)$ & $50(100 \%)$ \\
\hline $\begin{array}{l}\text { Alveolar/bronchiolar epithelium, } \\
\text { hyperplasia }\end{array}$ & - & $46(94 \%)$ & $49(98 \%)$ & $50(100 \%)$ \\
\hline $\begin{array}{l}\text { Alveolar/bronchiolar epithelium, } \\
\text { vacuolization cytoplasmic }\end{array}$ & - & $49(100 \%)$ & $47(94 \%)$ & $48(96 \%)$ \\
\hline Alveolar epithelium, hyperplasia & $4(8 \%)$ & $29(59 \%)$ & $24(48 \%)$ & $43(86 \%)$ \\
\hline $\begin{array}{l}\text { Alveolus, infiltration cellular, } \\
\text { histiocyte }\end{array}$ & $10(20 \%)$ & $49(100 \%)$ & $48(96 \%)$ & $48(96 \%)$ \\
\hline Bronchiole, epithelium, erosion & - & $4(8 \%)$ & $10(20 \%)$ & $2(4 \%)$ \\
\hline Bronchiole, epithelium, hyperplasia & $4(8 \%)$ & $7(14 \%)$ & $9(18 \%)$ & $11(22 \%)$ \\
\hline Nose & $(50)$ & (49) & (50) & $(50)$ \\
\hline Inflammation, suppurative & $16(32 \%)$ & $32(65 \%)$ & $49(98 \%)$ & $50(100 \%)$ \\
\hline Olfactory epithelium, atrophy & $3(6 \%)$ & $46(94 \%)$ & $42(84 \%)$ & $31(62 \%)$ \\
\hline Olfactory epithelium, hyperplasia & - & $25(51 \%)$ & $17(34 \%)$ & $8(16 \%)$ \\
\hline $\begin{array}{l}\text { Olfactory epithelium, metaplasia, } \\
\text { respiratory }\end{array}$ & $5(10 \%)$ & $24(49 \%)$ & $44(88 \%)$ & $50(100 \%)$ \\
\hline $\begin{array}{l}\text { Olfactory epithelium, respiratory } \\
\text { metaplasia, atypical }\end{array}$ & - & $14(29 \%)$ & $9(18 \%)$ & $1(2 \%)$ \\
\hline $\begin{array}{l}\text { Respiratory epithelium, accumulation, } \\
\text { hyaline droplet }\end{array}$ & $13(26 \%)$ & $29(59 \%)$ & $29(58 \%)$ & $7(14 \%)$ \\
\hline Respiratory epithelium, erosion & - & $1(2 \%)$ & - & - \\
\hline Respiratory epithelium, hyperplasia & $44(88 \%)$ & $41(84 \%)$ & $36(72 \%)$ & $19(38 \%)$ \\
\hline $\begin{array}{l}\text { Respiratory epithelium, metaplasia, } \\
\text { squamous }\end{array}$ & $3(6 \%)$ & $45(92 \%)$ & $35(70 \%)$ & $33(66 \%)$ \\
\hline $\begin{array}{l}\text { Respiratory epithelium, } \\
\text { vacuolization cytoplasmic }\end{array}$ & - & $41(84 \%)$ & $39(78 \%)$ & $37(74 \%)$ \\
\hline Squamous epithelium, erosion & $1(2 \%)$ & $1(2 \%)$ & $2(4 \%)$ & - \\
\hline Turbinate, atrophy & $3(6 \%)$ & $25(51 \%)$ & $49(98 \%)$ & $50(100 \%)$ \\
\hline Trachea & (48) & (47) & (48) & $(50)$ \\
\hline Inflammation, suppurative & - & - & - & $1(2 \%)$ \\
\hline Epithelium, vacuolization cytoplasmic & - & $14(30 \%)$ & $31(65 \%)$ & $37(74 \%)$ \\
\hline \multicolumn{5}{|l|}{ Special Senses System } \\
\hline Eye & (47) & $(46)$ & (43) & (45) \\
\hline Cataract & $1(2 \%)$ & - & - & $1(2 \%)$ \\
\hline Cornea, inflammation, chronic active & $4(9 \%)$ & $1(2 \%)$ & $1(2 \%)$ & - \\
\hline
\end{tabular}




\section{Cobalt Metal, NTP TR 581}

\begin{tabular}{|c|c|c|c|c|}
\hline & $\begin{array}{l}\text { Chamber } \\
\text { Control }\end{array}$ & $1.25 \mathrm{mg} / \mathrm{m}^{3}$ & $2.5 \mathrm{mg} / \mathrm{m}^{3}$ & $5 \mathrm{mg} / \mathrm{m}^{3}$ \\
\hline Harderian gland & (49) & (48) & (48) & $(50)$ \\
\hline Hyperplasia & $1(2 \%)$ & - & - & $2(4 \%)$ \\
\hline Zymbal's gland & $(0)$ & (1) & (0) & (0) \\
\hline Inflammation, suppurative & - & $1(100 \%)$ & - & - \\
\hline \multicolumn{5}{|l|}{ Urinary System } \\
\hline Kidney & (50) & (49) & (49) & (50) \\
\hline Cyst & $1(2 \%)$ & $1(2 \%)$ & - & - \\
\hline Infarct & $2(4 \%)$ & $4(8 \%)$ & $1(2 \%)$ & $3(6 \%)$ \\
\hline Nephropathy & $46(92 \%)$ & $44(90 \%)$ & $42(86 \%)$ & $37(74 \%)$ \\
\hline Thrombosis & - & - & $1(2 \%)$ & - \\
\hline Arteriole, inflammation, chronic active & - & - & - & $1(2 \%)$ \\
\hline Capsule, hemorrhage & $1(2 \%)$ & - & - & - \\
\hline Pelvis, inflammation, suppurative & $1(2 \%)$ & - & - & - \\
\hline Renal tubule, hyperplasia & - & $1(2 \%)$ & $1(2 \%)$ & $1(2 \%)$ \\
\hline Renal tubule, necrosis & - & - & $1(2 \%)$ & - \\
\hline Urinary bladder & (48) & (48) & $(50)$ & (47) \\
\hline Hemorrhage & - & - & - & $1(2 \%)$ \\
\hline Inflammation, chronic active & $1(2 \%)$ & - & - & - \\
\hline Arteriole, inflammation, chronic active & - & - & - & $1(2 \%)$ \\
\hline Transitional epithelium, hyperplasia & $1(2 \%)$ & - & $1(2 \%)$ & - \\
\hline
\end{tabular}

${ }^{a}$ Number of animals examined microscopically at the site and the number of animals with lesion. 


\section{Appendix D. Summary of Lesions in Female Mice in the Two- year Inhalation Study of Cobalt Metal}

\section{Tables}

Table D-1. Summary of the Incidence of Neoplasms in Female Mice in the Two-year Inhalation Study of Cobalt Metal

Table D-2. Statistical Analysis of Primary Neoplasms in Female Mice in the Two-year Inhalation Study of Cobalt Metal

Table D-3. Historical Incidence of Alveolar/bronchiolar Neoplasms in Control Female B6C3F1/N Mice ....

Table D-4. Summary of the Incidence of Nonneoplastic Lesions in Female Mice in the Two-year Inhalation Study of Cobalt Metal. 
Table D-1. Summary of the Incidence of Neoplasms in Female Mice in the Two-year Inhalation Study of Cobalt Metal ${ }^{\text {a }}$

\begin{tabular}{|c|c|c|c|c|}
\hline & $\begin{array}{c}\text { Chamber } \\
\text { Control }\end{array}$ & $1.25 \mathrm{mg} / \mathrm{m}^{3}$ & $2.5 \mathrm{mg} / \mathrm{m}^{3}$ & $5 \mathrm{mg} / \mathrm{m}^{3}$ \\
\hline \multicolumn{5}{|l|}{ Disposition Summary } \\
\hline Animals initially in study & 50 & 50 & 50 & 50 \\
\hline \multicolumn{5}{|l|}{ Early deaths } \\
\hline Moribund & 9 & 12 & 19 & 21 \\
\hline Natural deaths & 5 & 2 & 4 & 3 \\
\hline \multicolumn{5}{|l|}{ Survivors } \\
\hline Died last week of study & - & 2 & - & - \\
\hline Terminal kill & 36 & 34 & 27 & 26 \\
\hline Animals examined microscopically & 50 & 50 & 50 & 50 \\
\hline \multicolumn{5}{|l|}{ Alimentary System } \\
\hline Esophagus & $(50)$ & $(50)$ & (50) & $(50)$ \\
\hline Gallbladder & (44) & $(41)$ & (38) & $(46)$ \\
\hline Intestine large, cecum & $(46)$ & $(47)$ & (49) & $(48)$ \\
\hline Intestine large, colon & (46) & $(48)$ & (49) & (49) \\
\hline Intestine large, rectum & $(46)$ & $(48)$ & (48) & $(49)$ \\
\hline Intestine small, duodenum & $(45)$ & $(47)$ & (48) & (49) \\
\hline Intestine small, ileum & $(45)$ & $(46)$ & (49) & (49) \\
\hline Carcinoma & $1(2 \%)$ & - & - & - \\
\hline Intestine small, jejunum & $(45)$ & $(47)$ & (49) & $(49)$ \\
\hline Carcinoma & - & $1(2 \%)$ & - & - \\
\hline Liver & $(50)$ & $(50)$ & $(50)$ & $(50)$ \\
\hline Hemangiosarcoma & $2(4 \%)$ & $1(2 \%)$ & - & - \\
\hline Hepatocellular adenoma & $9(18 \%)$ & $13(26 \%)$ & $6(12 \%)$ & $2(4 \%)$ \\
\hline Hepatocellular adenoma, multiple & $10(20 \%)$ & $5(10 \%)$ & $4(8 \%)$ & \\
\hline Hepatocellular carcinoma & $6(12 \%)$ & $4(8 \%)$ & $5(10 \%)$ & $2(4 \%)$ \\
\hline Hepatocellular carcinoma, multiple & $4(8 \%)$ & $3(6 \%)$ & - & - \\
\hline Hepatocholangiocarcinoma & - & $1(2 \%)$ & - & - \\
\hline Mast cell tumor malignant & $1(2 \%)$ & - & - & - \\
\hline Mesentery & (18) & (13) & $(10)$ & $(8)$ \\
\hline $\begin{array}{l}\text { Alveolar/bronchiolar carcinoma, } \\
\text { metastatic, lung }\end{array}$ & - & - & - & $1(13 \%)$ \\
\hline Sarcoma & - & $1(8 \%)$ & - & - \\
\hline Pancreas & $(50)$ & $(50)$ & (50) & (49) \\
\hline Sarcoma, metastatic, skin & $1(2 \%)$ & - & - & - \\
\hline
\end{tabular}


Cobalt Metal, NTP TR 581

\begin{tabular}{|c|c|c|c|c|}
\hline & $\begin{array}{l}\text { Chamber } \\
\text { Control }\end{array}$ & $1.25 \mathrm{mg} / \mathrm{m}^{3}$ & $2.5 \mathrm{mg} / \mathrm{m}^{3}$ & $5 \mathrm{mg} / \mathrm{m}^{3}$ \\
\hline Salivary glands & (50) & (50) & (50) & (50) \\
\hline Stomach, forestomach & (50) & (50) & $(50)$ & $(50)$ \\
\hline Squamous cell papilloma & $1(2 \%)$ & - & - & - \\
\hline Stomach, glandular & (48) & (50) & (49) & (49) \\
\hline \multicolumn{5}{|l|}{ Cardiovascular System } \\
\hline Heart & (50) & (50) & (50) & (50) \\
\hline $\begin{array}{l}\text { Alveolar/bronchiolar carcinoma, } \\
\text { metastatic, lung }\end{array}$ & $1(2 \%)$ & - & - & $2(4 \%)$ \\
\hline Hemangiosarcoma & - & - & $1(2 \%)$ & - \\
\hline \multicolumn{5}{|l|}{ Endocrine System } \\
\hline Adrenal cortex & (50) & (50) & $(50)$ & (48) \\
\hline Subcapsular, carcinoma & - & - & $2(4 \%)$ & - \\
\hline Adrenal medulla & $(50)$ & $(50)$ & (49) & (48) \\
\hline Pheochromocytoma benign & $1(2 \%)$ & $1(2 \%)$ & - & - \\
\hline Islets, pancreatic & $(50)$ & $(50)$ & $(50)$ & (49) \\
\hline Parathyroid gland & (28) & (28) & $(24)$ & (32) \\
\hline Pituitary gland & (47) & (50) & $(48)$ & (49) \\
\hline Pars distalis, adenoma & $3(6 \%)$ & $4(8 \%)$ & $1(2 \%)$ & - \\
\hline Pars intermedia, adenoma & - & $1(2 \%)$ & - & - \\
\hline Thyroid gland & (50) & $(50)$ & $(50)$ & (49) \\
\hline Follicular cell, adenoma & $1(2 \%)$ & - & - & $1(2 \%)$ \\
\hline \multicolumn{5}{|l|}{ General Body System } \\
\hline None & - & - & - & - \\
\hline \multicolumn{5}{|l|}{ Genital System } \\
\hline Clitoral gland & (43) & (44) & $(41)$ & (43) \\
\hline Ovary & (48) & $(50)$ & $(50)$ & $(50)$ \\
\hline Cystadenoma & $1(2 \%)$ & $4(8 \%)$ & - & $3(6 \%)$ \\
\hline Hemangiosarcoma & - & $1(2 \%)$ & $1(2 \%)$ & - \\
\hline Osteosarcoma, metastatic, bone & - & - & $1(2 \%)$ & - \\
\hline Yolk sac carcinoma & $1(2 \%)$ & - & - & - \\
\hline Uterus & $(49)$ & (50) & $(50)$ & $(50)$ \\
\hline Hemangiosarcoma & - & $2(4 \%)$ & - & - \\
\hline Polyp stromal & $2(4 \%)$ & $1(2 \%)$ & $1(2 \%)$ & $1(2 \%)$ \\
\hline Endometrium, carcinoma & - & $1(2 \%)$ & - & - \\
\hline Vagina & (0) & (0) & (1) & (0) \\
\hline
\end{tabular}




\begin{tabular}{|c|c|c|c|c|}
\hline & $\begin{array}{l}\text { Chamber } \\
\text { Control }\end{array}$ & $1.25 \mathrm{mg} / \mathrm{m}^{3}$ & $2.5 \mathrm{mg} / \mathrm{m}^{3}$ & $5 \mathrm{mg} / \mathrm{m}^{3}$ \\
\hline \multicolumn{5}{|l|}{ Hematopoietic System } \\
\hline Bone marrow & $(50)$ & (50) & $(50)$ & (50) \\
\hline Mast cell tumor malignant & $1(2 \%)$ & - & - & - \\
\hline Lymph node & (11) & (8) & (7) & (1) \\
\hline Lumbar, carcinoma, metastatic, kidney & $1(9 \%)$ & - & - & - \\
\hline Lymph node, bronchial & (22) & (33) & (34) & (22) \\
\hline $\begin{array}{l}\text { Alveolar/bronchiolar carcinoma, } \\
\text { metastatic, lung }\end{array}$ & $2(9 \%)$ & $1(3 \%)$ & $1(3 \%)$ & $1(5 \%)$ \\
\hline Lymph node, mandibular & (40) & (41) & $(38)$ & (31) \\
\hline Mast cell tumor malignant & $1(3 \%)$ & - & - & - \\
\hline Lymph node, mediastinal & $(42)$ & (39) & (44) & (46) \\
\hline $\begin{array}{l}\text { Alveolar/bronchiolar carcinoma, } \\
\text { metastatic, lung }\end{array}$ & $2(5 \%)$ & $1(3 \%)$ & $3(7 \%)$ & $3(7 \%)$ \\
\hline Mast cell tumor malignant & $1(2 \%)$ & - & - & - \\
\hline Lymph node, mesenteric & (49) & $(49)$ & $(47)$ & (47) \\
\hline Sarcoma, metastatic, skin & $1(2 \%)$ & $1(2 \%)$ & - & - \\
\hline Spleen & (49) & (49) & $(48)$ & $(49)$ \\
\hline Hemangioma & - & $1(2 \%)$ & - & - \\
\hline Hemangiosarcoma & $2(4 \%)$ & - & $1(2 \%)$ & - \\
\hline Mast cell tumor malignant & $1(2 \%)$ & - & - & - \\
\hline Thymus & (46) & (46) & (46) & (46) \\
\hline $\begin{array}{l}\text { Alveolar/bronchiolar carcinoma, } \\
\text { metastatic, lung }\end{array}$ & $1(2 \%)$ & - & - & $2(4 \%)$ \\
\hline Neoplasm NOS & $1(2 \%)$ & - & - & - \\
\hline Osteosarcoma, metastatic, bone & - & - & $1(2 \%)$ & - \\
\hline \multicolumn{5}{|l|}{ Integumentary System } \\
\hline Mammary gland & (48) & (50) & $(50)$ & $(50)$ \\
\hline Carcinoma & $1(2 \%)$ & - & $1(2 \%)$ & - \\
\hline Skin & (50) & (50) & (49) & $(50)$ \\
\hline Subcutaneous tissue, hemangioma & - & - & - & $1(2 \%)$ \\
\hline Subcutaneous tissue, hemangiosarcoma & - & $2(4 \%)$ & - & - \\
\hline Subcutaneous tissue, neural crest tumor & $1(2 \%)$ & - & - & - \\
\hline Subcutaneous tissue, sarcoma & $2(4 \%)$ & $2(4 \%)$ & $2(4 \%)$ & - \\
\hline Subcutaneous tissue, sarcoma, multiple & - & $1(2 \%)$ & - & - \\
\hline \multicolumn{5}{|l|}{ Musculoskeletal System } \\
\hline Bone & $(50)$ & $(50)$ & $(50)$ & $(50)$ \\
\hline
\end{tabular}


Cobalt Metal, NTP TR 581

\begin{tabular}{|c|c|c|c|c|}
\hline & $\begin{array}{l}\text { Chamber } \\
\text { Control }\end{array}$ & $1.25 \mathrm{mg} / \mathrm{m}^{3}$ & $2.5 \mathrm{mg} / \mathrm{m}^{3}$ & $5 \mathrm{mg} / \mathrm{m}^{3}$ \\
\hline Osteosarcoma & - & - & $1(2 \%)$ & - \\
\hline $\begin{array}{l}\text { Vertebra, schwannoma malignant, } \\
\text { metastatic, peripheral nerve }\end{array}$ & - & - & $1(2 \%)$ & - \\
\hline Skeletal muscle & (0) & (2) & (1) & $(0)$ \\
\hline $\begin{array}{l}\text { Thoracic, alveolar/bronchiolar carcinoma, } \\
\text { metastatic, lung }\end{array}$ & - & $1(50 \%)$ & $1(100 \%)$ & - \\
\hline \multicolumn{5}{|l|}{ Nervous System } \\
\hline Brain & $(50)$ & $(50)$ & $(50)$ & $(50)$ \\
\hline Peripheral nerve & (0) & (0) & (1) & $(0)$ \\
\hline Schwannoma malignant & - & - & $1(100 \%)$ & - \\
\hline \multicolumn{5}{|l|}{ Respiratory System } \\
\hline Larynx & $(47)$ & $(50)$ & $(50)$ & (47) \\
\hline Lung & (49) & $(50)$ & $(50)$ & $(50)$ \\
\hline Alveolar/bronchiolar adenoma & $3(6 \%)$ & $8(16 \%)$ & $8(16 \%)$ & $9(18 \%)$ \\
\hline Alveolar/bronchiolar adenoma, multiple & - & $1(2 \%)$ & - & $1(2 \%)$ \\
\hline Alveolar/bronchiolar carcinoma & $4(8 \%)$ & $18(36 \%)$ & $18(36 \%)$ & $19(38 \%)$ \\
\hline Alveolar/bronchiolar carcinoma, multiple & $1(2 \%)$ & $7(14 \%)$ & $20(40 \%)$ & $24(48 \%)$ \\
\hline $\begin{array}{l}\text { Hepatocellular carcinoma, metastatic, } \\
\text { liver }\end{array}$ & $5(10 \%)$ & $2(4 \%)$ & $1(2 \%)$ & - \\
\hline Osteosarcoma, metastatic, bone & - & - & $1(2 \%)$ & - \\
\hline Nose & (50) & (50) & $(50)$ & (50) \\
\hline Pleura & (0) & (1) & (0) & (1) \\
\hline $\begin{array}{l}\text { Alveolar/bronchiolar carcinoma, } \\
\text { metastatic, lung }\end{array}$ & - & $1(100 \%)$ & - & $1(100 \%)$ \\
\hline Trachea & $(48)$ & $(50)$ & $(48)$ & (49) \\
\hline $\begin{array}{l}\text { Alveolar/bronchiolar carcinoma, } \\
\text { metastatic, lung }\end{array}$ & - & - & $1(2 \%)$ & - \\
\hline \multicolumn{5}{|l|}{ Special Senses System } \\
\hline Eye & (46) & (46) & $(48)$ & $(48)$ \\
\hline Carcinoma, metastatic, Harderian gland & - & $1(2 \%)$ & - & - \\
\hline Harderian gland & (49) & (49) & (49) & (50) \\
\hline Adenoma & $3(6 \%)$ & $5(10 \%)$ & $4(8 \%)$ & $3(6 \%)$ \\
\hline Carcinoma & - & $2(4 \%)$ & - & - \\
\hline Zymbal's gland & (0) & (1) & (1) & $(0)$ \\
\hline \multicolumn{5}{|l|}{ Urinary System } \\
\hline Kidney & (50) & $(50)$ & $(50)$ & (50) \\
\hline
\end{tabular}




\section{Cobalt Metal, NTP TR 581}

\begin{tabular}{|c|c|c|c|c|}
\hline & $\begin{array}{l}\text { Chamber } \\
\text { Control }\end{array}$ & $1.25 \mathrm{mg} / \mathrm{m}^{3}$ & $2.5 \mathrm{mg} / \mathrm{m}^{3}$ & $5 \mathrm{mg} / \mathrm{m}^{3}$ \\
\hline $\begin{array}{l}\text { Alveolar/bronchiolar carcinoma, } \\
\text { metastatic, lung }\end{array}$ & - & - & - & $2(4 \%)$ \\
\hline Renal tubule, adenoma & - & - & - & $1(2 \%)$ \\
\hline Transitional epithelium, carcinoma & $1(2 \%)$ & - & - & - \\
\hline Urinary bladder & (49) & $(49)$ & $(48)$ & (49) \\
\hline \multicolumn{5}{|l|}{ Systemic Lesions } \\
\hline Multiple organs ${ }^{\mathrm{b}}$ & $(50)$ & $(50)$ & $(50)$ & $(50)$ \\
\hline Histiocytic sarcoma & $1(2 \%)$ & - & $1(2 \%)$ & $1(2 \%)$ \\
\hline Lymphoma malignant & $14(28 \%)$ & $14(28 \%)$ & $12(24 \%)$ & $4(8 \%)$ \\
\hline \multicolumn{5}{|l|}{ Neoplasm Summary } \\
\hline Total animals with primary neoplasms ${ }^{\mathrm{c}}$ & 40 & 47 & 49 & 47 \\
\hline Total primary neoplasms & 81 & 105 & 90 & 72 \\
\hline Total animals with benign neoplasms & 29 & 31 & 20 & 17 \\
\hline Total benign neoplasms & 34 & 44 & 24 & 22 \\
\hline Total animals with malignant neoplasms & 31 & 41 & 46 & 44 \\
\hline Total malignant neoplasms & 45 & 61 & 66 & 50 \\
\hline Total animals with metastatic neoplasms & 8 & 5 & 8 & 4 \\
\hline Total metastatic neoplasms & 14 & 8 & 11 & 12 \\
\hline $\begin{array}{l}\text { Total animals with uncertain neoplasms- } \\
\text { benign or malignant }\end{array}$ & 2 & - & - & - \\
\hline Total uncertain neoplasms & 2 & - & - & - \\
\hline
\end{tabular}

${ }^{a}$ Number of animals examined microscopically at the site and the number of animals with neoplasm.

${ }^{b}$ Number of animals with any tissue examined microscopically.

cPrimary neoplasms: all neoplasms except metastatic neoplasms. 
Table D-2. Statistical Analysis of Primary Neoplasms in Female Mice in the Two-year Inhalation Study of Cobalt Metal

\begin{tabular}{|c|c|c|c|c|}
\hline & $\begin{array}{l}\text { Chamber } \\
\text { Control }\end{array}$ & $1.25 \mathrm{mg} / \mathrm{m}^{3}$ & $2.5 \mathrm{mg} / \mathrm{m}^{3}$ & $5 \mathrm{mg} / \mathrm{m}^{3}$ \\
\hline \multicolumn{5}{|c|}{ Harderian Gland: Adenoma } \\
\hline Overall rate ${ }^{a}$ & $3 / 50(6 \%)$ & $5 / 50(10 \%)$ & $4 / 50(8 \%)$ & $3 / 50(6 \%)$ \\
\hline Adjusted rate ${ }^{\mathrm{b}}$ & $6.8 \%$ & $11.2 \%$ & $9.3 \%$ & $7.4 \%$ \\
\hline Terminal rate $\mathrm{c}^{\mathrm{C}}$ & $2 / 36(6 \%)$ & $4 / 35(11 \%)$ & $1 / 27(4 \%)$ & $1 / 26(4 \%)$ \\
\hline First incidence (days) & 697 & 712 & 562 & 618 \\
\hline Poly-3 test ${ }^{\mathrm{d}}$ & $\mathrm{P}=0.535 \mathrm{~N}$ & $\mathrm{P}=0.363$ & $\mathrm{P}=0.487$ & $P=0.626$ \\
\hline \multicolumn{5}{|c|}{ Harderian Gland: Adenoma or Carcinoma } \\
\hline Overall rate & $3 / 50(6 \%)$ & $7 / 50(14 \%)$ & $4 / 50(8 \%)$ & $3 / 50(6 \%)$ \\
\hline Adjusted rate & $6.8 \%$ & $15.7 \%$ & $9.3 \%$ & $7.4 \%$ \\
\hline Terminal rate & $2 / 36(6 \%)$ & $5 / 35(14 \%)$ & $1 / 27(4 \%)$ & $1 / 26(4 \%)$ \\
\hline First incidence (days) & 697 & 671 & 562 & 618 \\
\hline Poly-3 test & $\mathrm{P}=0.438 \mathrm{~N}$ & $P=0.164$ & $P=0.487$ & $P=0.626$ \\
\hline \multicolumn{5}{|c|}{ Liver: Hepatocellular Adenoma } \\
\hline Overall rate & $19 / 50(38 \%)$ & $18 / 50(36 \%)$ & $10 / 50(20 \%)$ & $2 / 50(4 \%)$ \\
\hline Adjusted rate & $42.6 \%$ & $40.5 \%$ & $23.7 \%$ & $5.0 \%$ \\
\hline Terminal rate & $16 / 36(44 \%)$ & $17 / 35(49 \%)$ & $7 / 27(26 \%)$ & $2 / 26(8 \%)$ \\
\hline First incidence (days) & 499 & 712 & 646 & $731(\mathrm{~T})$ \\
\hline Poly-3 test & $\mathrm{P}<0.001 \mathrm{~N}$ & $\mathrm{P}=0.505 \mathrm{~N}$ & $\mathrm{P}=0.046 \mathrm{~N}$ & $\mathrm{P}<0.001 \mathrm{~N}$ \\
\hline \multicolumn{5}{|c|}{ Liver: Hepatocellular Carcinoma } \\
\hline Overall rate & $10 / 50(20 \%)$ & $7 / 50(14 \%)$ & $5 / 50(10 \%)$ & $2 / 50(4 \%)$ \\
\hline Adjusted rate & $22.2 \%$ & $15.7 \%$ & $11.9 \%$ & $4.9 \%$ \\
\hline Terminal rate & $7 / 36(19 \%)$ & $6 / 35(17 \%)$ & $3 / 27(11 \%)$ & $0 / 26(0 \%)$ \\
\hline First incidence (days) & 499 & 661 & 646 & 506 \\
\hline Poly-3 test & $\mathrm{P}=0.013 \mathrm{~N}$ & $\mathrm{P}=0.302 \mathrm{~N}$ & $\mathrm{P}=0.162 \mathrm{~N}$ & $\mathrm{P}=0.020 \mathrm{~N}$ \\
\hline \multicolumn{5}{|c|}{ Liver: Hepatocellular Adenoma or Carcinoma } \\
\hline Overall rate & $25 / 50(50 \%)$ & $21 / 50(42 \%)$ & $13 / 50(26 \%)$ & $4 / 50(8 \%)$ \\
\hline Adjusted rate & $55.3 \%$ & $46.9 \%$ & $30.7 \%$ & $9.8 \%$ \\
\hline Terminal rate & $20 / 36(56 \%)$ & $19 / 35(54 \%)$ & $9 / 27(33 \%)$ & $2 / 26(8 \%)$ \\
\hline First incidence (days) & 499 & 661 & 646 & 506 \\
\hline Poly-3 test & $\mathrm{P}<0.001 \mathrm{~N}$ & $\mathrm{P}=0.277 \mathrm{~N}$ & $\mathrm{P}=0.014 \mathrm{~N}$ & $\mathrm{P}<0.001 \mathrm{~N}$ \\
\hline \multicolumn{5}{|c|}{ Lung: Alveolar/bronchiolar Adenoma } \\
\hline Overall rate & $3 / 49(6 \%)$ & $9 / 50(18 \%)$ & $8 / 50(16 \%)$ & $10 / 50(20 \%)$ \\
\hline Adjusted rate & $6.9 \%$ & $19.9 \%$ & $18.9 \%$ & $24.5 \%$ \\
\hline Terminal rate & $3 / 36(8 \%)$ & $7 / 35(20 \%)$ & $6 / 27(22 \%)$ & $6 / 26(23 \%)$ \\
\hline
\end{tabular}




\begin{tabular}{|c|c|c|c|c|}
\hline & $\begin{array}{c}\text { Chamber } \\
\text { Control }\end{array}$ & $1.25 \mathrm{mg} / \mathrm{m}^{3}$ & $2.5 \mathrm{mg} / \mathrm{m}^{3}$ & $5 \mathrm{mg} / \mathrm{m}^{3}$ \\
\hline First incidence (days) & $731(\mathrm{~T})$ & 505 & 626 & 593 \\
\hline Poly-3 test & $P=0.037$ & $\mathrm{P}=0.067$ & $P=0.087$ & $\mathrm{P}=0.024$ \\
\hline \multicolumn{5}{|c|}{ Lung: Alveolar/bronchiolar Carcinoma } \\
\hline Overall rate & $5 / 49(10 \%)$ & $25 / 50(50 \%)$ & $38 / 50(76 \%)$ & $43 / 50(86 \%)$ \\
\hline Adjusted rate & $11.3 \%$ & $53.8 \%$ & $78.9 \%$ & $87.7 \%$ \\
\hline Terminal rate & $3 / 36(8 \%)$ & $18 / 35(51 \%)$ & $19 / 27(70 \%)$ & $21 / 26(81 \%)$ \\
\hline First incidence (days) & 583 & 537 & 457 & 478 \\
\hline Poly-3 test & $\mathrm{P}<0.001$ & $\mathrm{P}<0.001$ & $\mathrm{P}<0.001$ & $\mathrm{P}<0.001$ \\
\hline \multicolumn{5}{|c|}{ Lung: Alveolar/bronchiolar Adenoma or Carcinoma } \\
\hline Overall rate & $8 / 49(16 \%)$ & $30 / 50(60 \%)$ & $41 / 50(82 \%)$ & $45 / 50(90 \%)$ \\
\hline Adjusted rate & $18.0 \%$ & $63.7 \%$ & $84.6 \%$ & $91.6 \%$ \\
\hline Terminal rate & $6 / 36(17 \%)$ & $22 / 35(63 \%)$ & $21 / 27(78 \%)$ & $22 / 26(85 \%)$ \\
\hline First incidence (days) & 583 & 505 & 457 & 478 \\
\hline Poly-3 test & $\mathrm{P}<0.001$ & $\mathrm{P}<0.001$ & $\mathrm{P}<0.001$ & $\mathrm{P}<0.001$ \\
\hline \multicolumn{5}{|l|}{ Ovary: Cystadenoma } \\
\hline Overall rate & $1 / 48(2 \%)$ & $4 / 50(8 \%)$ & $0 / 50(0 \%)$ & $3 / 50(6 \%)$ \\
\hline Adjusted rate & $2.3 \%$ & $8.9 \%$ & $0.0 \%$ & $7.4 \%$ \\
\hline Terminal rate & $1 / 35(3 \%)$ & $3 / 35(9 \%)$ & $0 / 27(0 \%)$ & $2 / 26(8 \%)$ \\
\hline First incidence (days) & $731(\mathrm{~T})$ & 614 & $-^{\mathrm{e}}$ & 618 \\
\hline Poly-3 test & $\mathrm{P}=0.374$ & $\mathrm{P}=0.194$ & $\mathrm{P}=0.505 \mathrm{~N}$ & $\mathrm{P}=0.285$ \\
\hline \multicolumn{5}{|c|}{ Pituitary Gland (Pars Distalis): Adenoma } \\
\hline Overall rate & $3 / 47(6 \%)$ & $4 / 50(8 \%)$ & $1 / 48(2 \%)$ & $0 / 49(0 \%)$ \\
\hline Adjusted rate & $7.2 \%$ & $9.0 \%$ & $2.5 \%$ & $0.0 \%$ \\
\hline Terminal rate & $2 / 34(6 \%)$ & $3 / 35(9 \%)$ & $0 / 27(0 \%)$ & $0 / 25(0 \%)$ \\
\hline First incidence (days) & 649 & 671 & 646 & - \\
\hline Poly-3 test & $\mathrm{P}=0.052 \mathrm{~N}$ & $\mathrm{P}=0.534$ & $\mathrm{P}=0.321 \mathrm{~N}$ & $\mathrm{P}=0.132 \mathrm{~N}$ \\
\hline \multicolumn{5}{|c|}{ Skin (Subcutaneous Tissue): Sarcoma } \\
\hline Overall rate & $2 / 50(4 \%)$ & $3 / 50(6 \%)$ & $2 / 50(4 \%)$ & $0 / 50(0 \%)$ \\
\hline Adjusted rate & $4.5 \%$ & $6.6 \%$ & $4.8 \%$ & $0.0 \%$ \\
\hline Terminal rate & $1 / 36(3 \%)$ & $0 / 35(0 \%)$ & $0 / 27(0 \%)$ & $0 / 26(0 \%)$ \\
\hline First incidence (days) & 649 & 506 & 626 & - \\
\hline Poly-3 test & $\mathrm{P}=0.163 \mathrm{~N}$ & $\mathrm{P}=0.517$ & $\mathrm{P}=0.677$ & $\mathrm{P}=0.260 \mathrm{~N}$ \\
\hline \multicolumn{5}{|c|}{ All Organs: Hemangiosarcoma } \\
\hline Overall rate & $3 / 50(6 \%)$ & $6 / 50(12 \%)$ & $3 / 50(6 \%)$ & $0 / 50(0 \%)$ \\
\hline Adjusted rate & $6.9 \%$ & $13.5 \%$ & $7.2 \%$ & $0.0 \%$ \\
\hline
\end{tabular}




\begin{tabular}{|c|c|c|c|c|}
\hline & $\begin{array}{c}\text { Chamber } \\
\text { Control }\end{array}$ & $1.25 \mathrm{mg} / \mathrm{m}^{3}$ & $2.5 \mathrm{mg} / \mathrm{m}^{3}$ & $5 \mathrm{mg} / \mathrm{m}^{3}$ \\
\hline Terminal rate & $2 / 36(6 \%)$ & $5 / 35(14 \%)$ & $2 / 27(7 \%)$ & $0 / 26(0 \%)$ \\
\hline First incidence (days) & 725 & 712 & 709 & - \\
\hline Poly-3 test & $\mathrm{P}=0.070 \mathrm{~N}$ & $\mathrm{P}=0.249$ & $P=0.639$ & $\mathrm{P}=0.136 \mathrm{~N}$ \\
\hline \multicolumn{5}{|c|}{ All Organs: Hemangioma or Hemangiosarcoma } \\
\hline Overall rate & $3 / 50(6 \%)$ & $7 / 50(14 \%)$ & $3 / 50(6 \%)$ & $1 / 50(2 \%)$ \\
\hline Adjusted rate & $6.9 \%$ & $15.6 \%$ & $7.2 \%$ & $2.5 \%$ \\
\hline Terminal rate & $2 / 36(6 \%)$ & $5 / 35(14 \%)$ & $2 / 27(7 \%)$ & $0 / 26(0 \%)$ \\
\hline First incidence (days) & 725 & 649 & 709 & 660 \\
\hline Poly-3 test & $\mathrm{P}=0.140 \mathrm{~N}$ & $\mathrm{P}=0.165$ & $P=0.639$ & $\mathrm{P}=0.336 \mathrm{~N}$ \\
\hline \multicolumn{5}{|c|}{ All Organs: Malignant Lymphoma } \\
\hline Overall rate & $14 / 50(28 \%)$ & $14 / 50(28 \%)$ & $12 / 50(24 \%)$ & $4 / 50(8 \%)$ \\
\hline Adjusted rate & $31.7 \%$ & $29.8 \%$ & $28.0 \%$ & $10.0 \%$ \\
\hline Terminal rate & $11 / 36(31 \%)$ & $8 / 35(23 \%)$ & $8 / 27(30 \%)$ & $3 / 26(12 \%)$ \\
\hline First incidence (days) & 697 & 391 & 537 & 673 \\
\hline Poly-3 test & $\mathrm{P}=0.011 \mathrm{~N}$ & $\mathrm{P}=0.513 \mathrm{~N}$ & $\mathrm{P}=0.444 \mathrm{~N}$ & $\mathrm{P}=0.013 \mathrm{~N}$ \\
\hline \multicolumn{5}{|c|}{ All Organs: Benign Neoplasms } \\
\hline Overall rate & $29 / 50(58 \%)$ & $31 / 50(62 \%)$ & $20 / 50(40 \%)$ & $17 / 50(34 \%)$ \\
\hline Adjusted rate & $63.0 \%$ & $66.1 \%$ & $45.8 \%$ & $40.5 \%$ \\
\hline Terminal rate & $23 / 36(64 \%)$ & $24 / 35(69 \%)$ & $14 / 27(52 \%)$ & $10 / 26(39 \%)$ \\
\hline First incidence (days) & 223 & 505 & 562 & 593 \\
\hline Poly-3 test & $\mathrm{P}=0.005 \mathrm{~N}$ & $\mathrm{P}=0.460$ & $\mathrm{P}=0.070 \mathrm{~N}$ & $\mathrm{P}=0.024 \mathrm{~N}$ \\
\hline \multicolumn{5}{|c|}{ All Organs: Malignant Neoplasms } \\
\hline Overall rate & $31 / 50(62 \%)$ & $41 / 50(82 \%)$ & $46 / 50(92 \%)$ & $44 / 50(88 \%)$ \\
\hline Adjusted rate & $66.1 \%$ & $82.0 \%$ & $92.0 \%$ & $89.8 \%$ \\
\hline Terminal rate & $21 / 36(58 \%)$ & $26 / 35(74 \%)$ & $23 / 27(85 \%)$ & $22 / 26(85 \%)$ \\
\hline First incidence (days) & 499 & 391 & 457 & 478 \\
\hline Poly-3 test & $\mathrm{P}=0.002$ & $\mathrm{P}=0.057$ & $\mathrm{P}<0.001$ & $\mathrm{P}=0.004$ \\
\hline \multicolumn{5}{|c|}{ All Organs: Benign or Malignant Neoplasms } \\
\hline Overall rate & $40 / 50(80 \%)$ & $47 / 50(94 \%)$ & $49 / 50(98 \%)$ & $47 / 50(94 \%)$ \\
\hline Adjusted rate & $83.6 \%$ & $94.0 \%$ & $98.0 \%$ & $95.7 \%$ \\
\hline Terminal rate & $29 / 36(81 \%)$ & $32 / 35(91 \%)$ & $26 / 27(96 \%)$ & $24 / 26(92 \%)$ \\
\hline First incidence (days) & 223 & 391 & 457 & 478 \\
\hline Poly-3 test & $\mathrm{P}=0.026$ & $\mathrm{P}=0.088$ & $\mathrm{P}=0.012$ & $\mathrm{P}=0.045$ \\
\hline
\end{tabular}

(T) Terminal kill.

${ }^{a}$ Number of neoplasm-bearing animals/number of animals examined. Denominator is number of animals examined microscopically for liver, lung, ovary, and pituitary gland; for other tissues, denominator is number of animals necropsied. bPoly-3 estimated neoplasm incidence after adjustment for intercurrent mortality. 
${ }^{\mathrm{c}}$ Observed incidence at terminal kill.

${ }^{\mathrm{d} B e n e a t h}$ the chamber control incidence is the $\mathrm{P}$ value associated with the trend test. Beneath the exposed group incidence are the $P$ values corresponding to pairwise comparisons between the chamber controls and that exposed group. The Poly-3 test accounts for differential mortality in animals that do not reach terminal kill. A negative trend or a lower incidence in an exposure group is indicated by $\mathbf{N}$

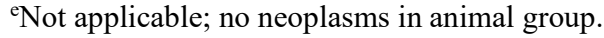

Table D-3. Historical Incidence of Alveolar/bronchiolar Neoplasms in Control Female B6C3F1/N Mice $^{a}$

$\begin{array}{lcc}\text { Study (Study Start) } \quad \text { Adenoma } & \text { Carcinoma } & \begin{array}{c}\text { Adenoma or } \\ \text { Carcinoma }\end{array}\end{array}$

\begin{tabular}{lccc}
\hline Historical Incidence: Inhalation Studies & & & \\
1-Bromopropane (July 2003) & $1 / 50$ & $0 / 50$ & $1 / 50$ \\
CIMSTAR 3800 (May 2008) & $1 / 50$ & $4 / 50$ & $4 / 50$ \\
Cobalt metal (May 2006) & $3 / 49$ & $5 / 49$ & $8 / 49$ \\
Diethylamine (August 2003) & $2 / 50$ & $3 / 50$ & $5 / 50$ \\
Tetralin (June 2003) & $6 / 50$ & $0 / 50$ & $6 / 50$ \\
Vinylidene chloride (June 2005) & $3 / 50$ & $1 / 50$ & $4 / 50$ \\
Total (\%) & $16 / 299(5.4 \%)$ & $13 / 299(4.4 \%)$ & $28 / 299(9.4 \%)$ \\
Mean \pm standard deviation & $5.4 \% \pm 3.7 \%$ & $4.4 \% \pm 4.3 \%$ & $9.4 \% \pm 4.8 \%$ \\
Range & $2 \%-12 \%$ & $0 \%-10 \%$ & $2 \%-16 \%$ \\
Overall Historical Incidence: All Routes & & $90 / 949(9.5 \%)$ \\
Total (\%) & $54 / 949(5.7 \%)$ & $38 / 949(4.0 \%)$ & $9.5 \% \pm 4.8 \%$ \\
Mean \pm standard deviation & $5.7 \% \pm 3.6 \%$ & $4.0 \% \pm 3.6 \%$ & $2 \%-22 \%$ \\
Range & $0 \%-12 \%$ & $0 \%-14 \%$ & \\
\hline ata as of May 2013. & & &
\end{tabular}


Table D-4. Summary of the Incidence of Nonneoplastic Lesions in Female Mice in the Two-year Inhalation Study of Cobalt Metal ${ }^{\mathrm{a}}$

\begin{tabular}{|c|c|c|c|c|}
\hline & $\begin{array}{l}\text { Chamber } \\
\text { Control }\end{array}$ & $1.25 \mathrm{mg} / \mathrm{m}^{3}$ & $2.5 \mathrm{mg} / \mathrm{m}^{3}$ & $5 \mathrm{mg} / \mathrm{m}^{3}$ \\
\hline \multicolumn{5}{|l|}{ Disposition Summary } \\
\hline Animals initially in study & 50 & 50 & 50 & 50 \\
\hline \multicolumn{5}{|l|}{ Early deaths } \\
\hline Moribund & 9 & 12 & 19 & 21 \\
\hline Natural deaths & 5 & 2 & 4 & 3 \\
\hline \multicolumn{5}{|l|}{ Survivors } \\
\hline Died last week of study & - & 2 & - & - \\
\hline Terminal kill & 36 & 34 & 27 & 26 \\
\hline Animals examined microscopically & 50 & 50 & 50 & 50 \\
\hline \multicolumn{5}{|l|}{ Alimentary System } \\
\hline Esophagus & $(50)$ & $(50)$ & (50) & $(50)$ \\
\hline Gallbladder & (44) & $(41)$ & (38) & (46) \\
\hline Intestine large, cecum & $(46)$ & (47) & (49) & (48) \\
\hline Hemorrhage & $1(2 \%)$ & - & - & - \\
\hline Intestine large, colon & $(46)$ & $(48)$ & (49) & (49) \\
\hline Intestine large, rectum & $(46)$ & $(48)$ & (48) & (49) \\
\hline Intestine small, duodenum & $(45)$ & $(47)$ & (48) & (49) \\
\hline Inflammation, acute & - & $1(2 \%)$ & - & - \\
\hline Necrosis & - & $1(2 \%)$ & - & - \\
\hline Intestine small, ileum & $(45)$ & $(46)$ & (49) & (49) \\
\hline Intestine small, jejunum & $(45)$ & $(47)$ & (49) & (49) \\
\hline Liver & $(50)$ & $(50)$ & (50) & (50) \\
\hline Angiectasis & $1(2 \%)$ & - & - & - \\
\hline Basophilic focus & $1(2 \%)$ & $2(4 \%)$ & $2(4 \%)$ & $1(2 \%)$ \\
\hline Clear cell focus & $1(2 \%)$ & $1(2 \%)$ & $1(2 \%)$ & - \\
\hline Eosinophilic focus & $3(6 \%)$ & $2(4 \%)$ & $4(8 \%)$ & - \\
\hline Fatty change & - & - & $1(2 \%)$ & - \\
\hline Hematopoietic cell proliferation & $1(2 \%)$ & - & - & - \\
\hline Hepatodiaphragmatic nodule & - & - & $1(2 \%)$ & $1(2 \%)$ \\
\hline Mixed cell focus & - & $1(2 \%)$ & $1(2 \%)$ & - \\
\hline Necrosis & $6(12 \%)$ & $3(6 \%)$ & $2(4 \%)$ & $1(2 \%)$ \\
\hline Tension lipidosis & $5(10 \%)$ & $5(10 \%)$ & $3(6 \%)$ & $2(4 \%)$ \\
\hline Vacuolization cytoplasmic & $1(2 \%)$ & $1(2 \%)$ & - & $1(2 \%)$ \\
\hline
\end{tabular}


Cobalt Metal, NTP TR 581

\begin{tabular}{|c|c|c|c|c|}
\hline & $\begin{array}{c}\text { Chamber } \\
\text { Control }\end{array}$ & $1.25 \mathrm{mg} / \mathrm{m}^{3}$ & $2.5 \mathrm{mg} / \mathrm{m}^{3}$ & $5 \mathrm{mg} / \mathrm{m}^{3}$ \\
\hline Bile duct, cyst & - & - & - & $1(2 \%)$ \\
\hline Mesentery & $(18)$ & (13) & (10) & (8) \\
\hline Congestion & - & - & $1(10 \%)$ & - \\
\hline Inflammation, chronic active & - & $1(8 \%)$ & - & - \\
\hline Fat, hemorrhage & $1(6 \%)$ & - & - & - \\
\hline Fat, necrosis & $18(100 \%)$ & $11(85 \%)$ & $10(100 \%)$ & $8(100 \%)$ \\
\hline Pancreas & $(50)$ & $(50)$ & $(50)$ & (49) \\
\hline Atrophy & $1(2 \%)$ & - & $2(4 \%)$ & $1(2 \%)$ \\
\hline Salivary glands & $(50)$ & $(50)$ & (50) & (50) \\
\hline Stomach, forestomach & $(50)$ & $(50)$ & $(50)$ & $(50)$ \\
\hline Hyperplasia, squamous & - & - & $3(6 \%)$ & $1(2 \%)$ \\
\hline Metaplasia, hepatocyte & - & - & - & $1(2 \%)$ \\
\hline Ulcer & $2(4 \%)$ & $2(4 \%)$ & $1(2 \%)$ & - \\
\hline Stomach, glandular & $(48)$ & $(50)$ & (49) & (49) \\
\hline Metaplasia, hepatocyte & - & - & $1(2 \%)$ & - \\
\hline Ulcer & $1(2 \%)$ & $2(4 \%)$ & - & - \\
\hline \multicolumn{5}{|l|}{ Cardiovascular System } \\
\hline Heart & $(50)$ & $(50)$ & $(50)$ & $(50)$ \\
\hline Cardiomyopathy & $6(12 \%)$ & $3(6 \%)$ & $11(22 \%)$ & $9(18 \%)$ \\
\hline Inflammation, suppurative & - & - & - & $1(2 \%)$ \\
\hline Mineralization & $1(2 \%)$ & $1(2 \%)$ & - & - \\
\hline Necrosis & - & $1(2 \%)$ & - & $1(2 \%)$ \\
\hline Thrombosis & $1(2 \%)$ & - & - & $1(2 \%)$ \\
\hline Capillary, hyperplasia & $1(2 \%)$ & - & - & - \\
\hline \multicolumn{5}{|l|}{ Endocrine System } \\
\hline Adrenal cortex & $(50)$ & $(50)$ & $(50)$ & (48) \\
\hline Hyperplasia & $1(2 \%)$ & $1(2 \%)$ & $1(2 \%)$ & $3(6 \%)$ \\
\hline Hypertrophy & - & $2(4 \%)$ & - & - \\
\hline Adrenal medulla & $(50)$ & $(50)$ & (49) & (48) \\
\hline Hyperplasia & $2(4 \%)$ & $1(2 \%)$ & - & $1(2 \%)$ \\
\hline Islets, pancreatic & $(50)$ & $(50)$ & $(50)$ & (49) \\
\hline Hyperplasia & - & $2(4 \%)$ & - & $1(2 \%)$ \\
\hline Parathyroid gland & $(28)$ & (28) & (24) & (32) \\
\hline Pituitary gland & (47) & $(50)$ & $(48)$ & (49) \\
\hline Pars distalis, angiectasis & $2(4 \%)$ & $1(2 \%)$ & - & - \\
\hline
\end{tabular}


Cobalt Metal, NTP TR 581

\begin{tabular}{|c|c|c|c|c|}
\hline & $\begin{array}{l}\text { Chamber } \\
\text { Control }\end{array}$ & $1.25 \mathrm{mg} / \mathrm{m}^{3}$ & $2.5 \mathrm{mg} / \mathrm{m}^{3}$ & $5 \mathrm{mg} / \mathrm{m}^{3}$ \\
\hline Pars distalis, hyperplasia & $12(26 \%)$ & $5(10 \%)$ & $6(13 \%)$ & $5(10 \%)$ \\
\hline Thyroid gland & $(50)$ & $(50)$ & $(50)$ & (49) \\
\hline Follicular cell, hyperplasia & - & $1(2 \%)$ & - & - \\
\hline \multicolumn{5}{|l|}{ General Body System } \\
\hline None & - & - & - & - \\
\hline \multicolumn{5}{|l|}{ Genital System } \\
\hline Clitoral gland & (43) & (44) & (41) & (43) \\
\hline Ovary & $(48)$ & $(50)$ & $(50)$ & $(50)$ \\
\hline Angiectasis & - & - & - & $1(2 \%)$ \\
\hline Cyst & $8(17 \%)$ & $2(4 \%)$ & $11(22 \%)$ & $10(20 \%)$ \\
\hline Inflammation, suppurative & - & - & - & $1(2 \%)$ \\
\hline Thrombosis & $1(2 \%)$ & - & $1(2 \%)$ & - \\
\hline Uterus & (49) & $(50)$ & (50) & $(50)$ \\
\hline Angiectasis & - & $4(8 \%)$ & - & - \\
\hline Inflammation, chronic active & $2(4 \%)$ & - & $1(2 \%)$ & - \\
\hline Thrombosis & - & $2(4 \%)$ & - & $1(2 \%)$ \\
\hline Endometrium, hyperplasia, cystic & $41(84 \%)$ & $38(76 \%)$ & $40(80 \%)$ & $34(68 \%)$ \\
\hline Vagina & $(0)$ & (0) & (1) & $(0)$ \\
\hline \multicolumn{5}{|l|}{ Hematopoietic System } \\
\hline Bone marrow & $(50)$ & $(50)$ & $(50)$ & $(50)$ \\
\hline Lymph node & (11) & (8) & (7) & (1) \\
\hline Iliac, ectasia & $3(27 \%)$ & - & $1(14 \%)$ & - \\
\hline Lumbar, ectasia & - & $1(13 \%)$ & - & - \\
\hline Renal, ectasia & $2(18 \%)$ & $2(25 \%)$ & - & - \\
\hline Renal, erythrophagocytosis & - & $1(13 \%)$ & - & - \\
\hline Renal, hyperplasia, lymphoid & $1(9 \%)$ & - & - & - \\
\hline Lymph node, bronchial & $(22)$ & $(33)$ & $(34)$ & (22) \\
\hline Inflammation, suppurative & - & - & - & $1(5 \%)$ \\
\hline Lymph node, mandibular & $(40)$ & (41) & (38) & (31) \\
\hline Hyperplasia, lymphoid & - & $1(2 \%)$ & - & - \\
\hline Lymph node, mediastinal & $(42)$ & (39) & (44) & (46) \\
\hline Hyperplasia, lymphoid & $1(2 \%)$ & - & - & $1(2 \%)$ \\
\hline Inflammation, suppurative & - & - & - & $1(2 \%)$ \\
\hline Lymph node, mesenteric & (49) & (49) & (47) & (47) \\
\hline Ectasia & - & $1(2 \%)$ & - & - \\
\hline
\end{tabular}


Cobalt Metal, NTP TR 581

\begin{tabular}{|c|c|c|c|c|}
\hline & $\begin{array}{l}\text { Chamber } \\
\text { Control }\end{array}$ & $1.25 \mathrm{mg} / \mathrm{m}^{3}$ & $2.5 \mathrm{mg} / \mathrm{m}^{3}$ & $5 \mathrm{mg} / \mathrm{m}^{3}$ \\
\hline Hyperplasia, lymphoid & $1(2 \%)$ & $1(2 \%)$ & - & - \\
\hline Spleen & (49) & (49) & (48) & (49) \\
\hline Hematopoietic cell proliferation & $5(10 \%)$ & $4(8 \%)$ & $4(8 \%)$ & $1(2 \%)$ \\
\hline Hyperplasia, lymphoid & - & $1(2 \%)$ & $1(2 \%)$ & - \\
\hline Necrosis & $1(2 \%)$ & - & - & - \\
\hline Thymus & (46) & (46) & (46) & (46) \\
\hline Hyperplasia, lymphoid & - & - & - & $1(2 \%)$ \\
\hline \multicolumn{5}{|l|}{ Integumentary System } \\
\hline Mammary gland & $(48)$ & $(50)$ & (50) & (50) \\
\hline Hyperplasia & $1(2 \%)$ & - & $1(2 \%)$ & - \\
\hline Skin & $(50)$ & $(50)$ & (49) & (50) \\
\hline Inflammation, chronic active & $2(4 \%)$ & $1(2 \%)$ & $2(4 \%)$ & - \\
\hline \multicolumn{5}{|l|}{ Musculoskeletal System } \\
\hline Bone & $(50)$ & $(50)$ & (50) & $(50)$ \\
\hline Hyperostosis & $1(2 \%)$ & - & - & - \\
\hline Skeletal muscle & $(0)$ & (2) & (1) & $(0)$ \\
\hline \multicolumn{5}{|l|}{ Nervous System } \\
\hline Brain & (50) & (50) & $(50)$ & $(50)$ \\
\hline $\begin{array}{l}\text { Meninges, infiltration cellular, } \\
\text { mononuclear cell }\end{array}$ & - & $2(4 \%)$ & - & - \\
\hline Peripheral nerve & $(0)$ & $(0)$ & (1) & $(0)$ \\
\hline \multicolumn{5}{|l|}{ Respiratory System } \\
\hline Larynx & $(47)$ & $(50)$ & (50) & (47) \\
\hline Inflammation, suppurative & - & $1(2 \%)$ & - & $2(4 \%)$ \\
\hline Inflammation, chronic & - & - & $1(2 \%)$ & - \\
\hline Inflammation, chronic active & - & $1(2 \%)$ & - & $1(2 \%)$ \\
\hline $\begin{array}{l}\text { Respiratory epithelium, metaplasia, } \\
\text { squamous }\end{array}$ & $2(4 \%)$ & $49(98 \%)$ & $50(100 \%)$ & $47(100 \%)$ \\
\hline $\begin{array}{l}\text { Respiratory epithelium, } \\
\text { vacuolization cytoplasmic }\end{array}$ & - & $24(48 \%)$ & $31(62 \%)$ & $34(72 \%)$ \\
\hline Squamous epithelium, erosion & $1(2 \%)$ & $2(4 \%)$ & $7(14 \%)$ & $4(9 \%)$ \\
\hline Squamous epithelium, hyperplasia & $2(4 \%)$ & $13(26 \%)$ & $21(42 \%)$ & $21(45 \%)$ \\
\hline Lung & $(49)$ & $(50)$ & (50) & (50) \\
\hline Infiltration cellular, histiocyte & - & - & $1(2 \%)$ & - \\
\hline Inflammation, suppurative & - & $3(6 \%)$ & $2(4 \%)$ & $15(30 \%)$ \\
\hline Proteinosis & - & $45(90 \%)$ & $50(100 \%)$ & $50(100 \%)$ \\
\hline
\end{tabular}


Cobalt Metal, NTP TR 581

\begin{tabular}{|c|c|c|c|c|}
\hline & $\begin{array}{l}\text { Chamber } \\
\text { Control }\end{array}$ & $1.25 \mathrm{mg} / \mathrm{m}^{3}$ & $2.5 \mathrm{mg} / \mathrm{m}^{3}$ & $5 \mathrm{mg} / \mathrm{m}^{3}$ \\
\hline Thrombosis & - & - & $1(2 \%)$ & - \\
\hline $\begin{array}{l}\text { Alveolar/bronchiolar epithelium, } \\
\text { hyperplasia }\end{array}$ & - & $49(98 \%)$ & $49(98 \%)$ & $50(100 \%)$ \\
\hline $\begin{array}{l}\text { Alveolar/bronchiolar epithelium, } \\
\text { vacuolization cytoplasmic }\end{array}$ & - & $48(96 \%)$ & $49(98 \%)$ & $48(96 \%)$ \\
\hline Alveolar epithelium, hyperplasia & $2(4 \%)$ & $27(54 \%)$ & $26(52 \%)$ & $41(82 \%)$ \\
\hline $\begin{array}{l}\text { Alveolar epithelium, metaplasia, } \\
\text { squamous }\end{array}$ & - & - & - & $1(2 \%)$ \\
\hline $\begin{array}{l}\text { Alveolus, infiltration cellular, } \\
\text { histiocyte }\end{array}$ & $10(20 \%)$ & $49(98 \%)$ & $50(100 \%)$ & $49(98 \%)$ \\
\hline Bronchiole, epithelium, erosion & - & - & $4(8 \%)$ & $3(6 \%)$ \\
\hline Bronchiole, epithelium, hyperplasia & - & $3(6 \%)$ & $12(24 \%)$ & $26(52 \%)$ \\
\hline Nose & $(50)$ & $(50)$ & (50) & (50) \\
\hline Inflammation, suppurative & $3(6 \%)$ & $47(94 \%)$ & $50(100 \%)$ & $50(100 \%)$ \\
\hline Olfactory epithelium, atrophy & $4(8 \%)$ & $44(88 \%)$ & $39(78 \%)$ & $24(48 \%)$ \\
\hline Olfactory epithelium, hyperplasia & $1(2 \%)$ & $22(44 \%)$ & $16(32 \%)$ & $8(16 \%)$ \\
\hline $\begin{array}{l}\text { Olfactory epithelium, metaplasia, } \\
\text { respiratory }\end{array}$ & $1(2 \%)$ & $26(52 \%)$ & $44(88 \%)$ & $50(100 \%)$ \\
\hline $\begin{array}{l}\text { Olfactory epithelium, metaplasia, } \\
\text { squamous }\end{array}$ & - & - & - & $1(2 \%)$ \\
\hline $\begin{array}{l}\text { Olfactory epithelium, respiratory } \\
\text { metaplasia, atypical }\end{array}$ & - & $18(36 \%)$ & $14(28 \%)$ & $1(2 \%)$ \\
\hline $\begin{array}{l}\text { Respiratory epithelium, } \\
\text { accumulation, hyaline droplet }\end{array}$ & $12(24 \%)$ & $38(76 \%)$ & $40(80 \%)$ & $10(20 \%)$ \\
\hline Respiratory epithelium, hyperplasia & $43(86 \%)$ & $40(80 \%)$ & $40(80 \%)$ & $9(18 \%)$ \\
\hline $\begin{array}{l}\text { Respiratory epithelium, } \\
\text { hyperplasia, histiocytic }\end{array}$ & $1(2 \%)$ & - & - & - \\
\hline $\begin{array}{l}\text { Respiratory epithelium, metaplasia, } \\
\text { squamous }\end{array}$ & - & $49(98 \%)$ & $49(98 \%)$ & $50(100 \%)$ \\
\hline $\begin{array}{l}\text { Respiratory epithelium, } \\
\text { vacuolization cytoplasmic }\end{array}$ & - & $40(80 \%)$ & $47(94 \%)$ & $47(94 \%)$ \\
\hline Squamous epithelium, erosion & - & $7(14 \%)$ & $1(2 \%)$ & $5(10 \%)$ \\
\hline Turbinate, atrophy & - & $44(88 \%)$ & $50(100 \%)$ & $50(100 \%)$ \\
\hline Pleura & $(0)$ & (1) & $(0)$ & (1) \\
\hline Trachea & $(48)$ & $(50)$ & (48) & $(49)$ \\
\hline Inflammation, suppurative & - & $1(2 \%)$ & - & $1(2 \%)$ \\
\hline $\begin{array}{l}\text { Epithelium, vacuolization } \\
\text { cytoplasmic }\end{array}$ & - & $26(52 \%)$ & $37(77 \%)$ & $39(80 \%)$ \\
\hline
\end{tabular}




\section{Cobalt Metal, NTP TR 581}

\begin{tabular}{|c|c|c|c|c|}
\hline & $\begin{array}{l}\text { Chamber } \\
\text { Control }\end{array}$ & $1.25 \mathrm{mg} / \mathrm{m}^{3}$ & $2.5 \mathrm{mg} / \mathrm{m}^{3}$ & $5 \mathrm{mg} / \mathrm{m}^{3}$ \\
\hline \multicolumn{5}{|l|}{ Special Senses System } \\
\hline Eye & $(46)$ & $(46)$ & (48) & (48) \\
\hline Phthisis bulbi & - & $2(4 \%)$ & - & - \\
\hline $\begin{array}{l}\text { Cornea, inflammation, chronic } \\
\text { active }\end{array}$ & - & $2(4 \%)$ & $1(2 \%)$ & - \\
\hline Harderian gland & (49) & (49) & (49) & (50) \\
\hline Hyperplasia & $4(8 \%)$ & $1(2 \%)$ & $1(2 \%)$ & - \\
\hline Zymbal's gland & $(0)$ & (1) & (1) & $(0)$ \\
\hline \multicolumn{5}{|l|}{ Urinary System } \\
\hline Kidney & $(50)$ & (50) & (50) & (50) \\
\hline Amyloid deposition & $1(2 \%)$ & - & - & - \\
\hline Cyst & $1(2 \%)$ & - & - & - \\
\hline Infarct & - & $2(4 \%)$ & $2(4 \%)$ & $1(2 \%)$ \\
\hline Nephropathy & $35(70 \%)$ & $31(62 \%)$ & $20(40 \%)$ & $19(38 \%)$ \\
\hline Renal tubule, hyperplasia & $1(2 \%)$ & - & - & - \\
\hline Renal tubule, necrosis & $1(2 \%)$ & $1(2 \%)$ & - & - \\
\hline Urinary bladder & (49) & (49) & (48) & (49) \\
\hline
\end{tabular}

${ }^{\mathrm{a}}$ Number of animals examined microscopically at the site and the number of animals with lesion. 


\section{Appendix E. Genetic Toxicology}

\section{Table of Contents}

E.1. Bacterial Mutagenicity Test Protocol .................................................................................2-2

E.2. Mouse Peripheral Blood Micronucleus Test Protocol......................................................E-2

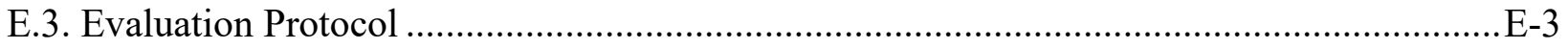

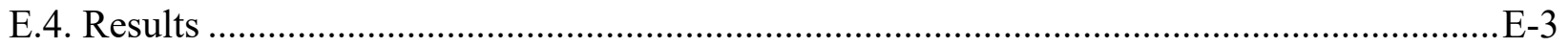

\section{Tables}

Table E-1. Mutagenicity of Cobalt Metal in Bacterial Tester Strains ...................................... E-4

Table E-2. Mutagenicity of Cobalt Metal in Bacterial Tester Strains (Escherichia coli)............E-5

Table E-3. Frequency of Micronuclei in Peripheral Blood Erythrocytes of Mice

Following Exposure to Cobalt Metal by Inhalation for Three Months ...................... E-6 


\section{E.1. Bacterial Mutagenicity Test Protocol}

Testing procedures were modified from those reported by Zeiger et al. ${ }^{262}$. Coded samples of cobalt metal [the same chemical lot (P32 3040-1) that was used in the 2-week, 3-month, and 2year studies] were incubated with the Salmonella typhimurium (TA98, TA100) and Escherichia coli (WP2 uvrA/pKM101) tester strains either in buffer or S9 mix (metabolic activation enzymes and cofactors from Aroclor 1254-induced male Sprague Dawley rat liver) for 20 minutes at $37^{\circ} \mathrm{C}$. Top agar supplemented with L-histidine and $d$-biotin was added, and the contents of the tubes were mixed and poured onto the surfaces of minimal glucose agar plates. Histidineindependent mutant colonies arising on these plates were counted following 2 days incubation at $37^{\circ} \mathrm{C}$.

Each trial consisted of triplicate plates of concurrent positive and negative controls and of five doses of cobalt metal. The highest dose was limited by toxicity. All trials were repeated.

In this assay, a positive response is defined as a reproducible, dose-related increase in histidineindependent (revertant) colonies in any one strain/activation combination. An equivocal response is defined as an increase in revertants that is not dose related, is not reproducible, or is not of sufficient magnitude to support a determination of mutagenicity. A negative response is obtained when no increase in revertant colonies is observed following chemical treatment. There is no minimum percentage or fold increase required for a chemical to be judged positive or weakly positive, although positive calls are typically reserved for increases in mutant colonies that are at least twofold over background.

\section{E.2. Mouse Peripheral Blood Micronucleus Test Protocol}

A detailed discussion of this assay is presented by MacGregor et al. ${ }^{263}$. At the end of the 3-month toxicity study, peripheral blood samples were obtained from male and female mice. Smears were immediately prepared and fixed in absolute methanol. The methanol-fixed slides were stained with acridine orange and coded. Slides were scanned to determine the frequency of micronuclei in 2,000 normochromatic erythrocytes (NCEs; mature erythrocytes) in each of five animals per exposure group. In addition, the percentage of circulating polychromatic erythrocytes (PCEs; reticulocytes) in 1,000 total erythrocytes per animal was scored for each exposure group as a measure of bone marrow toxicity.

The results were tabulated as the mean of the pooled results from all animals within a treatment group plus or minus the standard error of the mean. The frequency of micronucleated cells among NCEs was analyzed by a statistical software package that tested for increasing trend over exposure groups with a one-tailed Cochran-Armitage trend test, followed by pairwise comparisons between each exposed group and the control group. In the presence of excess binomial variation, as detected by a binomial dispersion test, the binomial variance of the Cochran-Armitage test was adjusted upward in proportion to the excess variation. In the micronucleus test, an individual trial is considered positive if the trend test $\mathrm{P}$ value is less than or equal to 0.025 or if the $\mathrm{P}$ value for any single exposed group is less than or equal to 0.025 divided by the number of exposed groups. A final call of positive for micronucleus induction is preferably based on reproducibly positive trials (as noted above). Results of the 3-month study were accepted without repeat tests, because additional test data could not be obtained. 
Ultimately, the final call is determined by the scientific staff after considering the results of statistical analyses, the reproducibility of any effects observed, and the magnitudes of those effects.

\section{E.3. Evaluation Protocol}

These are the basic guidelines for arriving at an overall assay result for assays performed by the National Toxicology Program. Statistical as well as biological factors are considered. For an individual assay, the statistical procedures for data analysis have been described in the preceding protocols. There have been instances, however, in which multiple samples of a chemical were tested in the same assay, and different results were obtained among these samples and/or among laboratories. Results from more than one aliquot or from more than one laboratory are not simply combined into an overall result. Rather, all the data are critically evaluated, particularly with regard to pertinent protocol variations, in determining the weight of evidence for an overall conclusion of chemical activity in an assay. In addition to multiple aliquots, the in vitro assays have another variable that must be considered in arriving at an overall test result. In vitro assays are conducted with and without exogenous metabolic activation. Results obtained in the absence of activation are not combined with results obtained in the presence of activation; each testing condition is evaluated separately. The summary table in the Abstract of this Technical Report presents a result that represents a scientific judgment of the overall evidence for activity of the chemical in an assay.

\section{E.4. Results}

Results of the bacterial mutagenicity tests conducted with cobalt metal are presented in Table E-1 and Table E-2. Cobalt metal (100 to 5,000 $\mu$ g/plate) gave an equivocal response in S. typhimurium strain TA100 in the absence of S9 activation mix; with 10\% rat liver S9, doses up to 7,500 $\mu \mathrm{g} /$ plate did not induce an increase in mutant colonies in TA100. In S. typhimurium strain TA98 without $\mathrm{S} 9$, cobalt metal (100 to 3,500 $\mu \mathrm{g} /$ plate) was mutagenic, although the responses observed were weak and not well correlated with dose level; with S9, no mutagenic activity was observed. In E. coli strain WP2 uvrA/pKM101, doses of cobalt metal up to $450 \mu \mathrm{g} /$ plate were not associated with mutagenic activity, with or without S9. No increases in the frequencies of NCEs were observed in peripheral blood of male or female mice exposed to cobalt metal $\left(0.625\right.$ to $\left.10 \mathrm{mg} / \mathrm{m}^{3}\right)$ for 3 months by inhalation (Table E-3). No significant alterations in the percentages of reticulocytes were seen in male or female mice, suggesting that exposure to cobalt metal under these conditions did not cause bone marrow toxicity. 
Cobalt Metal, NTP TR 581

Table E-1. Mutagenicity of Cobalt Metal in Bacterial Tester Strains ${ }^{\text {a }}$

\begin{tabular}{|c|c|c|c|c|c|c|c|}
\hline Strain & $\begin{array}{c}\text { Dose } \\
(\mu \mathrm{g} / \text { plate })\end{array}$ & Without S9 & Without S9 & Without S9 & $\begin{array}{c}\text { With } 10 \% \\
\text { Rat S9 }\end{array}$ & $\begin{array}{c}\text { With } 10 \% \\
\text { Rat S9 }\end{array}$ & $\begin{array}{c}\text { With } 10 \% \\
\text { Rat S9 }\end{array}$ \\
\hline \multicolumn{8}{|l|}{ TA100 } \\
\hline & 0 & $78 \pm 2$ & $85 \pm 7$ & $57 \pm 5$ & $96 \pm 4$ & $84 \pm 8$ & \\
\hline & 100 & $81 \pm 2$ & $77 \pm 12$ & $85 \pm 5$ & & & \\
\hline & 500 & $97 \pm 3$ & $146 \pm 10$ & $107 \pm 1$ & $101 \pm 5$ & $83 \pm 1$ & \\
\hline & 1,000 & $91 \pm 4$ & $165 \pm 4$ & $133 \pm 4$ & $97 \pm 2$ & $92 \pm 10^{b}$ & \\
\hline & 2,500 & $86 \pm 4$ & $128 \pm 11$ & $118 \pm 3$ & $80 \pm 9$ & $80 \pm 6^{b}$ & \\
\hline & 5,000 & $84 \pm 5$ & $25 \pm 16$ & $104 \pm 1$ & $85 \pm 7$ & $44 \pm 16^{\mathrm{b}}$ & \\
\hline & 7,500 & & & & $96 \pm 4$ & $55 \pm 9^{b}$ & \\
\hline Trial summary & & Negative & Equivocal & Equivocal & Negative & Negative & \\
\hline Positive control $^{\mathrm{c}}$ & & $304 \pm 1$ & $400 \pm 62$ & $206 \pm 2$ & $511 \pm 15$ & $949 \pm 38$ & \\
\hline \multicolumn{8}{|l|}{ TA98 } \\
\hline & 0 & $35 \pm 3$ & $22 \pm 3$ & $19 \pm 2$ & $34 \pm 2$ & $28 \pm 2$ & $29 \pm 2$ \\
\hline & 100 & $24 \pm 4$ & $43 \pm 7$ & $66 \pm 5$ & & & \\
\hline & 500 & $54 \pm 3$ & $47 \pm 7$ & $33 \pm 3$ & $22 \pm 2$ & $37 \pm 6$ & $18 \pm 1$ \\
\hline & 1,000 & $70 \pm 5$ & & & $20 \pm 1$ & $20 \pm 1^{b}$ & $12 \pm 1$ \\
\hline & 1,500 & & $52 \pm 8$ & $12 \pm 1$ & & & \\
\hline & 2,500 & $18 \pm 6$ & $11 \pm 1$ & $10 \pm 0$ & $26 \pm 2$ & $16 \pm 5^{\mathrm{b}}$ & $11 \pm 2$ \\
\hline & 3,500 & & $2 \pm 1$ & $0 \pm 0$ & & & \\
\hline & 5,000 & Toxic & & & $21 \pm 1$ & $12 \pm 4^{\mathrm{b}}$ & $12 \pm 2$ \\
\hline & 7,500 & & & & $15 \pm 2$ & $5 \pm 2^{b}$ & $2 \pm 1$ \\
\hline Trial summary & & Equivocal & Positive & Positive & Negative & Negative & Negative \\
\hline Positive control & & $517 \pm 30$ & $254 \pm 20$ & $461 \pm 18$ & $353 \pm 28$ & $751 \pm 72$ & $474 \pm 44$ \\
\hline
\end{tabular}


Cobalt Metal, NTP TR 581

Table E-2. Mutagenicity of Cobalt Metal in Bacterial Tester Strains (Escherichia coli)

\begin{tabular}{|c|c|c|c|c|c|}
\hline Strain & Dose $(\mu g / p l a t e)$ & Without S9 & Without S9 & Without S9 & Without S9 \\
\hline \multicolumn{6}{|c|}{ Escherichia coli WP2 uvrA/pKM101 (analogous to TA102) } \\
\hline & 0 & $201 \pm 7$ & $197 \pm 9$ & $272 \pm 4$ & $163 \pm 13$ \\
\hline & 5 & & & $283 \pm 13$ & \\
\hline & 25 & $148 \pm 9$ & $238 \pm 6$ & $297 \pm 9$ & \\
\hline & 50 & $90 \pm 8$ & $184 \pm 16$ & $268 \pm 1$ & $237 \pm 5$ \\
\hline & 75 & & & $243 \pm 3$ & \\
\hline & 100 & $134 \pm 9$ & $42 \pm 1$ & $224 \pm 2$ & $206 \pm 22$ \\
\hline & 150 & $0 \pm 0$ & $0 \pm 0$ & & $235 \pm 4$ \\
\hline & 200 & $0 \pm 0$ & $0 \pm 0$ & & \\
\hline & 300 & & & & Toxic \\
\hline & 450 & & & & Toxic \\
\hline & Trial summary & Negative & Negative & Negative & Negative \\
\hline & Positive control & $1,237 \pm 56$ & $1,531 \pm 43$ & $1,981 \pm 23$ & $1,485 \pm 205$ \\
\hline & & $\begin{array}{c}\text { With } 10 \% \\
\text { Rat S9 }\end{array}$ & $\begin{array}{c}\text { With } 10 \% \\
\text { Rat S9 }\end{array}$ & $\begin{array}{c}\text { With 10\% } \\
\text { Rat S9 }\end{array}$ & $\begin{array}{c}\text { With } 10 \% \\
\text { Rat S9 }\end{array}$ \\
\hline & 0 & $233 \pm 4$ & $250 \pm 10$ & $291 \pm 2$ & $220 \pm 37$ \\
\hline & 25 & & & $319 \pm 9$ & \\
\hline & 50 & $58 \pm 6$ & $238 \pm 9$ & $278 \pm 10$ & $239 \pm 6$ \\
\hline & 75 & & & $276 \pm 10$ & \\
\hline & 100 & $41 \pm 3$ & $175 \pm 4$ & $255 \pm 3$ & $130 \pm 7$ \\
\hline & 150 & $16 \pm 4$ & $73 \pm 6$ & $261 \pm 4$ & $220 \pm 18$ \\
\hline & 300 & $1 \pm 1$ & $4 \pm 1$ & & Toxic \\
\hline & 450 & $1 \pm 1$ & $1 \pm 0$ & & Toxic \\
\hline & Trial summary & Negative & Negative & Negative & Negative \\
\hline & Positive control & $855 \pm 5$ & $886 \pm 9$ & $1,172 \pm 36$ & $1,017 \pm 151$ \\
\hline
\end{tabular}


Cobalt Metal, NTP TR 581

Table E-3. Frequency of Micronuclei in Peripheral Blood Erythrocytes of Mice Following Exposure to Cobalt Metal by Inhalation for Three Months ${ }^{\mathrm{a}}$

\begin{tabular}{|c|c|c|c|c|c|}
\hline & $\begin{array}{c}\text { Dose } \\
\left(\mathrm{mg} / \mathrm{m}^{3}\right)\end{array}$ & $\begin{array}{l}\text { Number of Mice } \\
\text { with Erythrocytes } \\
\text { Scored }\end{array}$ & $\begin{array}{c}\text { Micronucleated } \\
\text { NCEs/1,000 NCEs }\end{array}$ & P Value ${ }^{c}$ & $\operatorname{PCEs}^{\mathrm{b}}(\%)$ \\
\hline \multicolumn{6}{|l|}{ Male } \\
\hline $\operatorname{Air}^{\mathrm{d}}$ & 0 & 5 & $2.40 \pm 0.33$ & & $2.56 \pm 0.17$ \\
\hline \multirow[t]{6}{*}{ Cobalt metal } & 0.625 & 5 & $2.40 \pm 0.33$ & 0.5000 & $3.04 \pm 0.29$ \\
\hline & 1.25 & 5 & $2.30 \pm 0.37$ & 0.5581 & $2.64 \pm 0.14$ \\
\hline & 2.5 & 5 & $3.10 \pm 0.19$ & 0.1723 & $2.70 \pm 0.15$ \\
\hline & 5 & 5 & $2.80 \pm 0.34$ & 0.2893 & $2.34 \pm 0.02$ \\
\hline & 10 & 5 & $2.80 \pm 0.37$ & 0.2893 & $2.64 \pm 0.07$ \\
\hline & & & $\mathrm{P}=0.236^{\mathrm{e}}$ & & \\
\hline \multicolumn{6}{|l|}{ Female } \\
\hline Air & 0 & 5 & $2.50 \pm 0.35$ & & $2.62 \pm 0.18$ \\
\hline \multirow[t]{6}{*}{ Cobalt metal } & 0.625 & 5 & $2.60 \pm 0.29$ & 0.4442 & $2.64 \pm 0.29$ \\
\hline & 1.25 & 5 & $2.00 \pm 0.22$ & 0.7722 & $2.54 \pm 0.16$ \\
\hline & 2.5 & 5 & $2.80 \pm 0.30$ & 0.3399 & $2.32 \pm 0.15$ \\
\hline & 5 & 5 & $2.00 \pm 0.32$ & 0.7722 & $2.30 \pm 0.11$ \\
\hline & 10 & 5 & $2.30 \pm 0.34$ & 0.6137 & $2.22 \pm 0.20$ \\
\hline & & & $P=0.664$ & & \\
\hline
\end{tabular}

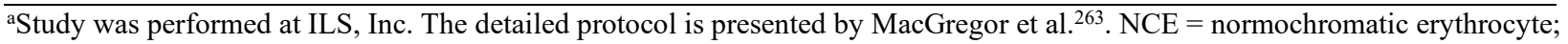
$\mathrm{PCE}=$ polychromatic erythrocyte.

${ }^{b}$ Mean \pm standard error.

'Pairwise comparison with the chamber control group; exposed group values are significant at $\mathrm{P} \leq 0.005$.

${ }^{\mathrm{d}}$ Chamber control.

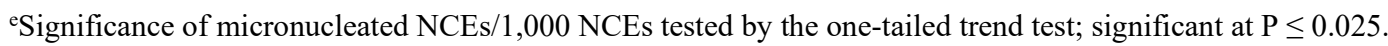


Cobalt Metal, NTP TR 581

\section{Appendix F. Clinical Pathology Results}

\section{Tables}

Table F-1. Hematology and Clinical Chemistry Data for Rats in the Three-month Inhalation Study of Cobalt Metal ................................................................F-2

Table F-2. Hematology Data for Mice in the Three-month Inhalation Study of Cobalt Metal... F-10 
Cobalt Metal, NTP TR 581

Table F-1. Hematology and Clinical Chemistry Data for Rats in the Three-month Inhalation Study of Cobalt Metal ${ }^{\mathrm{a}}$

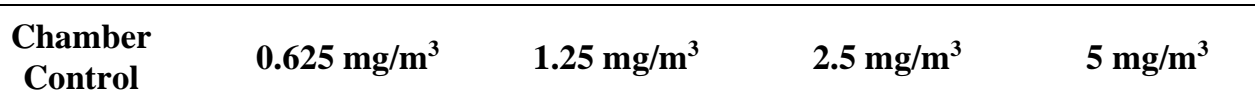

Male

Hematology

n

\begin{tabular}{|c|c|c|c|c|c|}
\hline Day 3 & 10 & 10 & 10 & 10 & 10 \\
\hline Day 23 & 10 & 10 & 10 & 9 & 10 \\
\hline Week 14 & 10 & 10 & 10 & 10 & 10 \\
\hline
\end{tabular}

Hematocrit (spun) (\%)

Day 3

Day 23

Week 14

\begin{abstract}
$46.5 \pm 0.2$
\end{abstract}
$47.9 \pm 0.4$

$49.6 \pm 0.4$

Packed cell volume (mL/dL)

Day 3

Day 23

Week 14

$$
45.1 \pm 0.2
$$

$46.8 \pm 0.4$

$49.6 \pm 0.5$

$46.4 \pm 0.2$
$48.5 \pm 0.4$
$51.6 \pm 0.4^{* *}$

$47.4 \pm 0.2^{*}$

$47.7 \pm 0.3^{* *}$

$51.2 \pm 0.4^{* *}$

$49.9 \pm 0.5^{* *}$

$51.1 \pm 0.2^{* *}$

$53.3 \pm 0.4^{* *}$

$59.2 \pm 0.4^{* *}$

$61.8 \pm 0.2^{* *}$

$63.9 \pm 0.3^{* *}$

Hemoglobin $(\mathrm{g} / \mathrm{dL})$

$\begin{array}{cc}44.5 \pm 0.3 & 45.7 \pm 0.3 \\ 47.3 \pm 0.4 & 49.0 \pm 0.6^{* *} \\ 51.7 \pm 0.4^{* *} & 58.3 \pm 0.4^{* *}\end{array}$

$46.1 \pm 0.4^{*}$

$49.7 \pm 0.4^{* *}$

$49.7 \pm 0.2 * *$

$51.5 \pm 0.5^{* *}$

$60.7 \pm 0.1 * *$

$62.9 \pm 0.3^{* *}$

Day 3

Day 23

Week 14

$13.7 \pm 0.1$

$15.0 \pm 0.1$

$15.6 \pm 0.1$

Erythrocytes $\left(10^{6} / \mu \mathrm{L}\right)$
Day 3

Day 23

Week 14

Reticulocytes $\left(10^{3} / \mu \mathrm{L}\right)$

Day 3

Day 23

Week 14
$7.30 \pm 0.06$

$7.97 \pm 0.09$

$9.19 \pm 0.10$

$13.8 \pm 0.1$

$$
14.9 \pm 0.1
$$

$16.3 \pm 0.1^{* *}$

$$
14.1 \pm 0.1 * *
$$

$15.4 \pm 0.2$

$18.7 \pm 0.1^{* *}$

$7.27 \pm 0.09$

$8.05 \pm 0.09$

$9.67 \pm 0.09^{* *}$

$7.46 \pm 0.05$

$8.36 \pm 0.11^{*}$

$11.20 \pm 0.06^{* *}$

$14.2 \pm 0.1 * *$

$15.8 \pm 0.1^{* *}$

$19.7 \pm 0.1^{* *}$

$15.5 \pm 0.1 * *$

$16.1 \pm 0.2^{* *}$

$20.3 \pm 0.1^{* *}$

Nucleated erythrocytes/100 leukocytes

$$
\text { Day } 3
$$

Day 23

Week 14

$612.0 \pm 42.3$

$562.3 \pm 24.5^{\mathrm{b}}$

$613.0 \pm 30.8$

$261.3 \pm 17.8$

$280.9 \pm 14.3$

$191.7 \pm 19.9$

$189.3 \pm 13.1$

$1.6 \pm 0.3$

$0.3 \pm 0.2$

$0.3 \pm 0.2$

$0.3 \pm 0.2$

Total nucleated cells $\left(10^{3} / \mu \mathrm{L}\right)$

Day 3

$8.4 \pm 0.4$

$9.5 \pm 0.3$

$7.0 \pm 0.6$

$6.8 \pm 0.3$

$7.0 \pm 0.4$
$2.6 \pm 0.6$

$0.6 \pm 0.2$

$0.1 \pm 0.1$

$8.9 \pm 0.4$

$7.4 \pm 0.6$

$7.2 \pm 0.5$
$7.57 \pm 0.08^{*}$

$8.44 \pm 0.06^{* *}$

$11.80 \pm 0.06^{* *}$

$622.1 \pm 25.6$

$291.6 \pm 18.6$

$272.7 \pm 18.7$
$8.22 \pm 0.08 * *$

$9.27 \pm 0.14^{* *}$

$11.90 \pm 0.08 * *$

$873.8 \pm 53.4^{* *}$

$339.6 \pm 25.1 *$

$360.0 \pm 35.9 *$
Day 23

$7.3 \pm 0.3$

$7.0 \pm 0.4$

$7.2 \pm 0.5$
$2.4 \pm 0.5$
$0.3 \pm 0.2$
$0.2 \pm 0.1$

$1.0 \pm 0.3$

$0.4 \pm 0.2$

$0.4 \pm 0.2$

Week 14

(a)


Cobalt Metal, NTP TR 581

\begin{tabular}{|c|c|c|c|c|c|}
\hline & $\begin{array}{c}\text { Chamber } \\
\text { Control }\end{array}$ & $0.625 \mathrm{mg} / \mathrm{m}^{3}$ & $1.25 \mathrm{mg} / \mathrm{m}^{3}$ & $2.5 \mathrm{mg} / \mathrm{m}^{3}$ & $5 \mathrm{mg} / \mathrm{m}^{3}$ \\
\hline \multicolumn{6}{|c|}{ Mean cell volume (fL) } \\
\hline Day 3 & $61.8 \pm 0.3$ & $61.2 \pm 0.5$ & $61.3 \pm 0.2$ & $61.0 \pm 0.4$ & $60.5 \pm 0.4^{*}$ \\
\hline Day 23 & $58.8 \pm 0.2$ & $58.7 \pm 0.2$ & $58.7 \pm 0.4$ & $58.9 \pm 0.3$ & $55.7 \pm 0.7^{* *}$ \\
\hline Week 14 & $54.0 \pm 0.3$ & $53.5 \pm 0.2$ & $52.1 \pm 0.2 * *$ & $51.4 \pm 0.2^{* *}$ & $52.9 \pm 0.2 * *$ \\
\hline \multicolumn{6}{|c|}{ Mean cell hemoglobin (pg) } \\
\hline Day 3 & $18.8 \pm 0.1$ & $19.0 \pm 0.1$ & $18.9 \pm 0.1$ & $18.8 \pm 0.1$ & $18.9 \pm 0.1$ \\
\hline Day 23 & $18.8 \pm 0.1$ & $18.5 \pm 0.1$ & $18.5 \pm 0.1$ & $18.7 \pm 0.1$ & $17.4 \pm 0.2^{* *}$ \\
\hline Week 14 & $17.0 \pm 0.1$ & $16.9 \pm 0.0$ & $16.7 \pm 0.0 * *$ & $16.7 \pm 0.1^{*}$ & $17.1 \pm 0.1$ \\
\hline \multicolumn{6}{|c|}{ Mean cell hemoglobin concentration $(\mathrm{g} / \mathrm{dL})$} \\
\hline Day 3 & $30.4 \pm 0.1$ & $31.1 \pm 0.2^{* *}$ & $30.9 \pm 0.2 * *$ & $30.9 \pm 0.2 *$ & $31.2 \pm 0.1 * *$ \\
\hline Day 23 & $31.9 \pm 0.1$ & $31.5 \pm 0.1$ & $31.5 \pm 0.2$ & $31.7 \pm 0.1$ & $31.3 \pm 0.1 * *$ \\
\hline Week 14 & $31.6 \pm 0.1$ & $31.6 \pm 0.1$ & $32.1 \pm 0.1 * *$ & $32.5 \pm 0.2^{* *}$ & $32.3 \pm 0.1 * *$ \\
\hline \multicolumn{6}{|c|}{ Platelets $\left(10^{3} / \mu \mathrm{L}\right)$} \\
\hline Day 3 & $899.7 \pm 14.0$ & $883.5 \pm 23.9$ & $939.4 \pm 14.4$ & $903.8 \pm 22.8$ & $1,123.7 \pm 34.2 * *$ \\
\hline Day 23 & $740.8 \pm 16.2$ & $712.0 \pm 16.3$ & $732.1 \pm 22.7$ & $682.0 \pm 44.5$ & $796.7 \pm 12.8$ \\
\hline Week 14 & $682.5 \pm 27.7$ & $646.8 \pm 8.7$ & $611.2 \pm 13.8^{*}$ & $548.2 \pm 22.3 * *$ & $573.2 \pm 15.3 * *$ \\
\hline \multicolumn{6}{|c|}{ Leukocytes $\left(10^{3} / \mu \mathrm{L}\right)$} \\
\hline Day 3 & $8.22 \pm 0.34$ & $9.38 \pm 0.28$ & $8.63 \pm 0.37$ & $8.57 \pm 0.64$ & $8.10 \pm 0.32$ \\
\hline Day 23 & $6.93 \pm 0.58$ & $6.77 \pm 0.32$ & $7.31 \pm 0.58$ & $7.66 \pm 0.57$ & $6.44 \pm 0.44$ \\
\hline Week 14 & $7.25 \pm 0.34$ & $6.98 \pm 0.42$ & $7.19 \pm 0.48$ & $6.96 \pm 0.39$ & $7.27 \pm 0.24$ \\
\hline \multicolumn{6}{|c|}{ Segmented neutrophils $\left(10^{3} / \mu \mathrm{L}\right)$} \\
\hline Day 3 & $0.86 \pm 0.03$ & $1.02 \pm 0.06$ & $1.01 \pm 0.04$ & $0.98 \pm 0.05$ & $0.98 \pm 0.09$ \\
\hline Day 23 & $0.83 \pm 0.04$ & $0.96 \pm 0.03 *$ & $1.02 \pm 0.03 * *$ & $1.19 \pm 0.04 * *$ & $1.13 \pm 0.08 * *$ \\
\hline Week 14 & $1.36 \pm 0.06$ & $1.54 \pm 0.07$ & $1.52 \pm 0.09$ & $1.48 \pm 0.05$ & $1.41 \pm 0.05$ \\
\hline \multicolumn{6}{|c|}{ Bands $\left(10^{3} / \mu \mathrm{L}\right)$} \\
\hline Day 3 & $0.00 \pm 0.00$ & $0.00 \pm 0.00$ & $0.00 \pm 0.00$ & $0.00 \pm 0.00$ & $0.00 \pm 0.00$ \\
\hline Day 23 & $0.00 \pm 0.00$ & $0.00 \pm 0.00$ & $0.00 \pm 0.00$ & $0.00 \pm 0.00$ & $0.00 \pm 0.00$ \\
\hline Week 14 & $0.00 \pm 0.00$ & $0.00 \pm 0.00$ & $0.00 \pm 0.00$ & $0.00 \pm 0.00$ & $0.00 \pm 0.00$ \\
\hline \multicolumn{6}{|c|}{ Lymphocytes $\left(10^{3} / \mu \mathrm{L}\right)$} \\
\hline Day 3 & $7.04 \pm 0.33$ & $7.99 \pm 0.26$ & $7.40 \pm 0.36$ & $7.34 \pm 0.58$ & $6.88 \pm 0.25$ \\
\hline Day 23 & $5.92 \pm 0.55$ & $5.68 \pm 0.31$ & $6.10 \pm 0.56$ & $6.28 \pm 0.56$ & $5.13 \pm 0.35$ \\
\hline Week 14 & $5.46 \pm 0.33$ & $5.08 \pm 0.38$ & $5.18 \pm 0.45$ & $5.15 \pm 0.39$ & $5.61 \pm 0.20$ \\
\hline \multicolumn{6}{|c|}{ Monocytes $\left(10^{3} / \mu \mathrm{L}\right)$} \\
\hline Day 3 & $0.23 \pm 0.03$ & $0.26 \pm 0.04$ & $0.12 \pm 0.04$ & $0.16 \pm 0.05$ & $0.15 \pm 0.05$ \\
\hline Day 23 & $0.12 \pm 0.04$ & $0.07 \pm 0.02$ & $0.12 \pm 0.03$ & $0.11 \pm 0.04$ & $0.13 \pm 0.04$ \\
\hline
\end{tabular}


Cobalt Metal, NTP TR 581

\begin{tabular}{|c|c|c|c|c|c|}
\hline & $\begin{array}{l}\text { Chamber } \\
\text { Control }\end{array}$ & $0.625 \mathrm{mg} / \mathrm{m}^{3}$ & $1.25 \mathrm{mg} / \mathrm{m}^{3}$ & $2.5 \mathrm{mg} / \mathrm{m}^{3}$ & $5 \mathrm{mg} / \mathrm{m}^{3}$ \\
\hline Week 14 & $0.30 \pm 0.07$ & $0.23 \pm 0.06$ & $0.36 \pm 0.07$ & $0.17 \pm 0.06$ & $0.12 \pm 0.05$ \\
\hline \multicolumn{6}{|c|}{ Basophils $\left(10^{3} / \mu \mathrm{L}\right)$} \\
\hline Day 3 & $0.008 \pm 0.002$ & $0.007 \pm 0.002$ & $0.006 \pm 0.002$ & $0.005 \pm 0.002$ & $0.010 \pm 0.001$ \\
\hline Day 23 & $0.010 \pm 0.003$ & $0.007 \pm 0.002$ & $0.009 \pm 0.003$ & $0.008 \pm 0.003$ & $0.008 \pm 0.004$ \\
\hline Week 14 & $0.009 \pm 0.003$ & $0.005 \pm 0.002$ & $0.010 \pm 0.005$ & $0.003 \pm 0.002$ & $0.002 \pm 0.001$ \\
\hline \multicolumn{6}{|c|}{ Eosinophils $\left(10^{3} / \mu \mathrm{L}\right)$} \\
\hline Day 3 & $0.08 \pm 0.01$ & $0.11 \pm 0.01$ & $0.09 \pm 0.01$ & $0.08 \pm 0.01$ & $0.08 \pm 0.01$ \\
\hline Day 23 & $0.06 \pm 0.01$ & $0.05 \pm 0.01$ & $0.06 \pm 0.01$ & $0.07 \pm 0.01$ & $0.05 \pm 0.01$ \\
\hline Week 14 & $0.12 \pm 0.01$ & $0.13 \pm 0.02$ & $0.11 \pm 0.02$ & $0.16 \pm 0.02$ & $0.13 \pm 0.02$ \\
\hline
\end{tabular}

\section{Clinical Chemistry}

\begin{tabular}{lccccc}
$\mathbf{n}$ & 10 & 10 & 10 & 10 & 10 \\
\hline Urea nitrogen $(\mathrm{mg} / \mathrm{dL})$ & & & & & \\
$\quad$ Day 3 & $8.0 \pm 0.4$ & $8.0 \pm 0.5$ & $8.1 \pm 0.4$ & $8.9 \pm 0.4$ & $16.5 \pm 1.1^{* *}$ \\
Day 23 & $11.0 \pm 0.5$ & $10.9 \pm 0.4$ & $9.7 \pm 0.4$ & $9.9 \pm 0.5$ & $8.6 \pm 0.4^{* *}$ \\
$\quad$ Week 14 & $13.4 \pm 0.3$ & $13.9 \pm 0.3$ & $14.5 \pm 0.5$ & $14.1 \pm 0.6$ & $13.2 \pm 0.5$ \\
Creatinine (mg/dL) & & & & \\
$\quad$ Day 3 & $0.23 \pm 0.02$ & $0.25 \pm 0.02$ & $0.26 \pm 0.02$ & $0.25 \pm 0.02$ & $0.22 \pm 0.01$ \\
Day 23 & $0.28 \pm 0.01$ & $0.26 \pm 0.02$ & $0.30 \pm 0.00$ & $0.32 \pm 0.01$ & $0.30 \pm 0.00$ \\
Week 14 & $0.34 \pm 0.02$ & $0.38 \pm 0.01$ & $0.36 \pm 0.02$ & $0.37 \pm 0.02$ & $0.42 \pm 0.01^{* *}$
\end{tabular}

Glucose (mg/dL)

Day 3

$135 \pm 2$

$138 \pm 1$

$134 \pm 2$

$136 \pm 1$

$129 \pm 4$

Day 23

$132 \pm 3$

$132 \pm 4$

$143 \pm 9$

$139 \pm 8$

$120 \pm 2$

Week 14

$126 \pm 3$

$128 \pm 3$

$118 \pm 5^{*}$

$111 \pm 3^{* *}$

$104 \pm 4^{* *}$

Total protein $(\mathrm{g} / \mathrm{dL})$

Day 3

$6.1 \pm 0.1$

$5.9 \pm 0.0$

$6.0 \pm 0.0$

$6.1 \pm 0.1$

$6.3 \pm 0.1^{*}$

Day 23

$6.5 \pm 0.1$

$6.4 \pm 0.1$

$6.5 \pm 0.1$

$6.5 \pm 0.1$

$6.4 \pm 0.1$

Week 14

$7.4 \pm 0.1$

$7.3 \pm 0.0$

$7.4 \pm 0.1$

$7.3 \pm 0.1$

$7.1 \pm 0.1^{* *}$

Albumin (g/dL)

Day 3

$4.3 \pm 0.0$

$4.1 \pm 0.0^{* *}$

$4.2 \pm 0.0$

$4.3 \pm 0.0$

$4.3 \pm 0.0$

Day 23

$4.6 \pm 0.1$

$4.5 \pm 0.0$

$4.5 \pm 0.1$

$4.5 \pm 0.0$

$4.4 \pm 0.0$

Week 14

$4.9 \pm 0.1$

$4.8 \pm 0.0$

$4.8 \pm 0.0$

$4.8 \pm 0.0$

$4.8 \pm 0.0$

Globulin (g/dL)

Day 3

$1.8 \pm 0.0$

$1.7 \pm 0.0$

$1.8 \pm 0.0$

$1.9 \pm 0.1$

$2.0 \pm 0.0^{* *}$

Day 23

$2.0 \pm 0.0$

$2.0 \pm 0.0$

$2.1 \pm 0.1$

$2.0 \pm 0.0$

$2.0 \pm 0.0$

Week 14

$2.5 \pm 0.0$

$2.5 \pm 0.0$

$2.6 \pm 0.0$

$2.5 \pm 0.0$

$2.3 \pm 0.0 * *$ 
Cobalt Metal, NTP TR 581

\begin{tabular}{|c|c|c|c|c|c|}
\hline & $\begin{array}{c}\text { Chamber } \\
\text { Control }\end{array}$ & $0.625 \mathrm{mg} / \mathrm{m}^{3}$ & $1.25 \mathrm{mg} / \mathrm{m}^{3}$ & $2.5 \mathrm{mg} / \mathrm{m}^{3}$ & $5 \mathrm{mg} / \mathrm{m}^{3}$ \\
\hline \multicolumn{6}{|c|}{ Albumin to globulin ratio } \\
\hline Day 3 & $2.4 \pm 0.3$ & $2.4 \pm 0.0$ & $2.3 \pm 0.0$ & $2.3 \pm 0.1$ & $2.1 \pm 0.0^{* *}$ \\
\hline Day 23 & $2.3 \pm 0.1$ & $2.3 \pm 0.0$ & $2.2 \pm 0.1$ & $2.2 \pm 0.0$ & $2.3 \pm 0.0$ \\
\hline Week 14 & $1.9 \pm 0.0$ & $2.0 \pm 0.0$ & $1.9 \pm 0.0$ & $1.9 \pm 0.0$ & $2.1 \pm 0.0$ \\
\hline \multicolumn{6}{|c|}{ Cholesterol (mg/dL) } \\
\hline Day 3 & $95 \pm 2$ & $87 \pm 1^{*}$ & $84 \pm 1 * *$ & $79 \pm 2 * *$ & $81 \pm 2 * *$ \\
\hline Day 23 & $79 \pm 1$ & $76 \pm 2$ & $70 \pm 1^{* *}$ & $73 \pm 2 * *$ & $63 \pm 1^{* *}$ \\
\hline Week 14 & $91 \pm 1$ & $91 \pm 1$ & $88 \pm 2$ & $79 \pm 2 * *$ & $67 \pm 1^{* *}$ \\
\hline \multicolumn{6}{|c|}{ Triglycerides $(\mathrm{mg} / \mathrm{dL})$} \\
\hline Day 3 & $52 \pm 3$ & $53 \pm 3$ & $57 \pm 3$ & $61 \pm 4$ & $95 \pm 6^{* *}$ \\
\hline Day 23 & $61 \pm 5$ & $63 \pm 8$ & $54 \pm 5$ & $69 \pm 5$ & $56 \pm 4$ \\
\hline Week 14 & $112 \pm 7$ & $122 \pm 8$ & $137 \pm 10$ & $141 \pm 8$ & $130 \pm 20$ \\
\hline \multicolumn{6}{|c|}{ Alanine aminotransferase (IU/L) } \\
\hline Day 3 & $55 \pm 1$ & $52 \pm 1$ & $50 \pm 1^{*}$ & $44 \pm 1 * *$ & $39 \pm 1 * *$ \\
\hline Day 23 & $47 \pm 3$ & $41 \pm 1$ & $41 \pm 1$ & $45 \pm 1$ & $40 \pm 1$ \\
\hline Week 14 & $88 \pm 7$ & $99 \pm 8$ & $106 \pm 8$ & $97 \pm 6$ & $77 \pm 2$ \\
\hline \multicolumn{6}{|c|}{ Alkaline phosphatase (IU/L) } \\
\hline Day 3 & $579 \pm 6$ & $586 \pm 8$ & $601 \pm 6$ & $609 \pm 10$ & $518 \pm 12$ \\
\hline Day 23 & $387 \pm 7$ & $392 \pm 9$ & $410 \pm 8$ & $415 \pm 10$ & $458 \pm 9^{* *}$ \\
\hline Week 14 & $236 \pm 8$ & $238 \pm 5$ & $238 \pm 4$ & $249 \pm 10$ & $235 \pm 5$ \\
\hline \multicolumn{6}{|c|}{ Creatine kinase (IU/L) } \\
\hline Day 3 & $402 \pm 29$ & $521 \pm 55$ & $482 \pm 29$ & $467 \pm 30$ & $480 \pm 65$ \\
\hline Day 23 & $378 \pm 113$ & $418 \pm 70$ & $569 \pm 98$ & $630 \pm 114$ & $467 \pm 54$ \\
\hline Week 14 & $224 \pm 47$ & $202 \pm 33$ & $266 \pm 44$ & $231 \pm 25$ & $254 \pm 44$ \\
\hline \multicolumn{6}{|c|}{ Sorbitol dehydrogenase (IU/L) } \\
\hline Day 3 & $15 \pm 0$ & $13 \pm 1^{*}$ & $14 \pm 1$ & $14 \pm 0$ & $12 \pm 1^{* *}$ \\
\hline Day 23 & $15 \pm 2$ & $13 \pm 1$ & $13 \pm 0$ & $14 \pm 1$ & $14 \pm 1$ \\
\hline Week 14 & $23 \pm 1$ & $27 \pm 1$ & $28 \pm 1^{*}$ & $26 \pm 1$ & $24 \pm 1$ \\
\hline \multicolumn{6}{|c|}{ Bile salts $(\mu \mathrm{mol} / \mathrm{L})$} \\
\hline Day 3 & $5.3 \pm 0.4$ & $5.7 \pm 1.3$ & $5.3 \pm 0.3$ & $5.6 \pm 0.6$ & $7.3 \pm 0.8$ \\
\hline Day 23 & $4.2 \pm 0.4$ & $3.9 \pm 0.2$ & $5.3 \pm 1.2$ & $4.6 \pm 0.6$ & $4.1 \pm 0.2$ \\
\hline Week 14 & $3.2 \pm 0.1$ & $3.4 \pm 0.2$ & $3.3 \pm 0.3$ & $3.6 \pm 0.4$ & $5.2 \pm 1.2$ \\
\hline
\end{tabular}

\section{Female}

n

10

10

10

10

10

Hematology 
Cobalt Metal, NTP TR 581

\begin{tabular}{|c|c|c|c|c|c|}
\hline & $\begin{array}{c}\text { Chamber } \\
\text { Control }\end{array}$ & $0.625 \mathrm{mg} / \mathrm{m}^{3}$ & $1.25 \mathrm{mg} / \mathrm{m}^{3}$ & $2.5 \mathrm{mg} / \mathrm{m}^{3}$ & $5 \mathrm{mg} / \mathrm{m}^{3}$ \\
\hline \multicolumn{6}{|c|}{ Hematocrit (spun) (\%) } \\
\hline Day 3 & $49.0 \pm 0.6$ & $47.8 \pm 0.7$ & $47.6 \pm 0.5$ & $48.9 \pm 0.3$ & $49.9 \pm 0.5$ \\
\hline Day 23 & $51.6 \pm 0.5$ & $50.9 \pm 0.3$ & $51.0 \pm 0.3$ & $52.0 \pm 0.2$ & $53.3 \pm 1.9^{* *}$ \\
\hline Week 14 & $48.3 \pm 0.5$ & $48.7 \pm 0.4$ & $52.6 \pm 0.6^{* *}$ & $57.3 \pm 0.5^{* *}$ & $59.5 \pm 0.3 * *$ \\
\hline \multicolumn{6}{|c|}{ Packed cell volume (mL/dL) } \\
\hline Day 3 & $47.6 \pm 0.6$ & $46.7 \pm 0.6$ & $46.5 \pm 0.5$ & $47.6 \pm 0.3$ & $48.3 \pm 0.5$ \\
\hline Day 23 & $51.3 \pm 0.5$ & $50.2 \pm 0.3$ & $50.5 \pm 0.3$ & $51.0 \pm 0.3$ & $51.7 \pm 1.7$ \\
\hline Week 14 & $49.1 \pm 0.5$ & $49.5 \pm 0.4$ & $53.1 \pm 0.5^{* *}$ & $57.1 \pm 0.5^{* *}$ & $60.0 \pm 0.4^{* *}$ \\
\hline \multicolumn{6}{|c|}{ Hemoglobin (g/dL) } \\
\hline Day 3 & $14.8 \pm 0.2$ & $14.4 \pm 0.2$ & $14.5 \pm 0.2$ & $14.7 \pm 0.1$ & $15.2 \pm 0.2$ \\
\hline Day 23 & $16.1 \pm 0.1$ & $15.8 \pm 0.1$ & $16.0 \pm 0.1$ & $16.2 \pm 0.1$ & $16.4 \pm 0.6^{*}$ \\
\hline Week 14 & $15.5 \pm 0.2$ & $15.8 \pm 0.1^{*}$ & $16.9 \pm 0.2 * *$ & $18.3 \pm 0.1 * *$ & $19.1 \pm 0.1 * *$ \\
\hline \multicolumn{6}{|c|}{ Erythrocytes $\left(10^{6} / \mu \mathrm{L}\right)$} \\
\hline Day 3 & $7.79 \pm 0.12$ & $7.62 \pm 0.13$ & $7.65 \pm 0.09$ & $7.77 \pm 0.08$ & $8.07 \pm 0.12$ \\
\hline Day 23 & $8.49 \pm 0.09$ & $8.24 \pm 0.07$ & $8.43 \pm 0.10$ & $8.56 \pm 0.05$ & $8.75 \pm 0.31^{*}$ \\
\hline Week 14 & $8.55 \pm 0.09$ & $8.69 \pm 0.06$ & $9.30 \pm 0.10^{* *}$ & $10.05 \pm 0.07 * *$ & $10.47 \pm 0.09 * *$ \\
\hline \multicolumn{6}{|c|}{ Reticulocytes $\left(10^{3} / \mu \mathrm{L}\right)$} \\
\hline Day 3 & $489.3 \pm 22.4$ & $497.7 \pm 30.8$ & $544.1 \pm 28.1$ & $511.9 \pm 25.5$ & $520.0 \pm 23.6$ \\
\hline Day 23 & $184.1 \pm 11.1$ & $244.2 \pm 9.9 * *$ & $212.9 \pm 12.4^{*}$ & $276.7 \pm 18.9^{* *}$ & $386.6 \pm 20.4^{* *}$ \\
\hline Week 14 & $202.5 \pm 13.0$ & $224.5 \pm 13.0$ & $222.2 \pm 8.8$ & $246.5 \pm 10.8^{*}$ & $316.7 \pm 25.0 * *$ \\
\hline \multicolumn{6}{|c|}{ Nucleated erythrocytes/100 leukocytes } \\
\hline Day 3 & $1.3 \pm 0.4$ & $1.6 \pm 0.3$ & $1.3 \pm 0.7$ & $2.5 \pm 0.3$ & $1.5 \pm 0.3$ \\
\hline Day 23 & $0.6 \pm 0.2$ & $0.9 \pm 0.3$ & $0.7 \pm 0.3$ & $0.6 \pm 0.2$ & $1.2 \pm 0.4$ \\
\hline Week 14 & $0.4 \pm 0.2$ & $0.2 \pm 0.2$ & $0.6 \pm 0.3$ & $0.2 \pm 0.2$ & $0.3 \pm 0.2$ \\
\hline \multicolumn{6}{|c|}{ Total nucleated cells $\left(10^{3} / \mu \mathrm{L}\right)$} \\
\hline Day 3 & $11.7 \pm 0.7$ & $9.2 \pm 0.4^{*}$ & $10.0 \pm 0.5$ & $9.6 \pm 0.4$ & $9.1 \pm 0.6^{*}$ \\
\hline Day 23 & $12.7 \pm 0.5$ & $12.8 \pm 0.3$ & $12.7 \pm 0.4$ & $11.3 \pm 0.5$ & $10.5 \pm 0.4^{* *}$ \\
\hline Week 14 & $6.2 \pm 0.3$ & $6.3 \pm 0.3$ & $7.0 \pm 0.3$ & $6.8 \pm 0.3$ & $6.6 \pm 0.2$ \\
\hline \multicolumn{6}{|c|}{ Mean cell volume (fL) } \\
\hline Day 3 & $61.1 \pm 0.3$ & $61.4 \pm 0.4$ & $60.7 \pm 0.3$ & $61.3 \pm 0.2$ & $59.8 \pm 0.4$ \\
\hline Day 23 & $60.4 \pm 0.3$ & $60.9 \pm 0.3$ & $59.9 \pm 0.4$ & $59.5 \pm 0.4$ & $59.2 \pm 0.3^{*}$ \\
\hline Week 14 & $57.4 \pm 0.2$ & $56.9 \pm 0.2$ & $57.2 \pm 0.2$ & $56.8 \pm 0.3$ & $57.2 \pm 0.2$ \\
\hline \multicolumn{6}{|c|}{ Mean cell hemoglobin (pg) } \\
\hline Day 3 & $18.9 \pm 0.1$ & $18.9 \pm 0.1$ & $18.9 \pm 0.1$ & $19.0 \pm 0.1$ & $18.8 \pm 0.1$ \\
\hline Day 23 & $19.0 \pm 0.1$ & $19.2 \pm 0.1$ & $19.0 \pm 0.1$ & $19.0 \pm 0.1$ & $18.7 \pm 0.1$ \\
\hline
\end{tabular}


Cobalt Metal, NTP TR 581

\begin{tabular}{|c|c|c|c|c|c|}
\hline & $\begin{array}{l}\text { Chamber } \\
\text { Control }\end{array}$ & $0.625 \mathrm{mg} / \mathrm{m}^{3}$ & $1.25 \mathrm{mg} / \mathrm{m}^{3}$ & $2.5 \mathrm{mg} / \mathrm{m}^{3}$ & $5 \mathrm{mg} / \mathrm{m}^{3}$ \\
\hline Week 14 & $18.1 \pm 0.1$ & $18.2 \pm 0.1$ & $18.1 \pm 0.1$ & $18.2 \pm 0.1$ & $18.3 \pm 0.1$ \\
\hline \multicolumn{6}{|c|}{ Mean cell hemoglobin concentration $(\mathrm{g} / \mathrm{dL})$} \\
\hline Day 3 & $31.0 \pm 0.1$ & $30.7 \pm 0.2$ & $31.2 \pm 0.2$ & $31.0 \pm 0.1$ & $31.5 \pm 0.2$ \\
\hline Day 23 & $31.5 \pm 0.1$ & $31.5 \pm 0.1$ & $31.8 \pm 0.1$ & $31.9 \pm 0.2$ & $31.7 \pm 0.2$ \\
\hline Week 14 & $31.6 \pm 0.1$ & $32.0 \pm 0.1^{*}$ & $31.8 \pm 0.1$ & $32.1 \pm 0.2$ & $31.9 \pm 0.1$ \\
\hline \multicolumn{6}{|c|}{ Platelets $\left(10^{3} / \mu \mathrm{L}\right)$} \\
\hline Day 3 & $905.6 \pm 27.8$ & $841.4 \pm 22.5$ & $914.7 \pm 18.8$ & $912.4 \pm 20.1$ & $875.0 \pm 27.4$ \\
\hline Day 23 & $783.1 \pm 20.5$ & $799.2 \pm 13.2$ & $763.0 \pm 25.7$ & $800.7 \pm 21.5$ & $807.9 \pm 22.2$ \\
\hline Week 14 & $702.1 \pm 8.0$ & $660.2 \pm 14.7^{*}$ & $646.6 \pm 17.3^{* *}$ & $575.3 \pm 16.7^{* * b}$ & $608.0 \pm 16.8^{* *}$ \\
\hline \multicolumn{6}{|c|}{ Leukocytes $\left(10^{3} / \mu \mathrm{L}\right)$} \\
\hline Day 3 & $11.57 \pm 0.66$ & $9.09 \pm 0.45^{*}$ & $9.85 \pm 0.52$ & $9.41 \pm 0.38$ & $9.00 \pm 0.57^{*}$ \\
\hline Day 23 & $12.66 \pm 0.52$ & $12.72 \pm 0.30$ & $12.61 \pm 0.44$ & $11.21 \pm 0.50$ & $10.37 \pm 0.40^{* *}$ \\
\hline Week 14 & $6.16 \pm 0.32$ & $6.26 \pm 0.29$ & $6.93 \pm 0.35$ & $6.80 \pm 0.30$ & $6.58 \pm 0.21$ \\
\hline
\end{tabular}

Segmented neutrophils $\left(10^{3} / \mu \mathrm{L}\right)$

\begin{tabular}{lccccc} 
Day 3 & $0.93 \pm 0.06$ & $0.88 \pm 0.07$ & $0.98 \pm 0.05$ & $1.06 \pm 0.03$ & $0.98 \pm 0.09$ \\
Day 23 & $1.16 \pm 0.07$ & $1.28 \pm 0.09$ & $1.37 \pm 0.10$ & $1.07 \pm 0.05$ & $1.25 \pm 0.13$ \\
Week 14 & $1.00 \pm 0.07$ & $1.20 \pm 0.09$ & $1.32 \pm 0.10$ & $1.25 \pm 0.06$ & $1.17 \pm 0.04$ \\
Bands $\left(10^{3} / \mu \mathrm{L}\right)$ & & & & \\
Day 3 & $0.00 \pm 0.00$ & $0.00 \pm 0.00$ & $0.00 \pm 0.00$ & $0.00 \pm 0.00$ & $0.00 \pm 0.00$ \\
Day 23 & $0.00 \pm 0.00$ & $0.00 \pm 0.00$ & $0.00 \pm 0.00$ & $0.00 \pm 0.00$ & $0.00 \pm 0.00$ \\
Week 14 & $0.00 \pm 0.00$ & $0.00 \pm 0.00$ & $0.00 \pm 0.00$ & $0.00 \pm 0.00$ & $0.00 \pm 0.00$ \\
Lymphocytes $\left(10^{3} / \mu \mathrm{L}\right)$ & & & & \\
Day 3 & $10.33 \pm 0.61$ & $7.93 \pm 0.45^{*}$ & $8.56 \pm 0.48$ & $8.10 \pm 0.36$ & $7.80 \pm 0.54^{*}$ \\
Day 23 & $11.18 \pm 0.52$ & $11.12 \pm 0.32$ & $10.89 \pm 0.43$ & $9.84 \pm 0.44$ & $8.82 \pm 0.30^{* *}$ \\
Week 14 & $4.83 \pm 0.26$ & $4.64 \pm 0.20$ & $5.36 \pm 0.28$ & $5.17 \pm 0.24$ & $4.94 \pm 0.22$ \\
Monocytes $\left(10^{3} / \mu \mathrm{L}\right)$ & & & & \\
Day 3 & $0.16 \pm 0.03$ & $0.14 \pm 0.03$ & $0.18 \pm 0.05$ & $0.11 \pm 0.03$ & $0.09 \pm 0.02$ \\
Day 23 & $0.14 \pm 0.03$ & $0.15 \pm 0.03$ & $0.17 \pm 0.03$ & $0.15 \pm 0.03$ & $0.15 \pm 0.03$ \\
Week 14 & $0.24 \pm 0.07$ & $0.32 \pm 0.06$ & $0.14 \pm 0.05$ & $0.25 \pm 0.08$ & $0.34 \pm 0.05$ \\
Basophils $\left(10^{3} / \mu \mathrm{L}\right)$ & & & & \\
Day 3 & $0.016 \pm 0.004$ & $0.012 \pm 0.004$ & $0.017 \pm 0.004$ & $0.014 \pm 0.003$ & $0.011 \pm 0.003$ \\
Day 23 & $0.012 \pm 0.003$ & $0.024 \pm 0.002^{*}$ & $0.022 \pm 0.003$ & $0.015 \pm 0.003$ & $0.012 \pm 0.004$ \\
Week 14 & $0.006 \pm 0.003$ & $0.006 \pm 0.002$ & $0.002 \pm 0.001$ & $0.007 \pm 0.003$ & $0.008 \pm 0.002$ \\
Eosinophils $\left(10^{3} / \mu \mathrm{L}\right)$ & & & & & \\
Day 3 & $0.13 \pm 0.02$ & $0.13 \pm 0.02$ & $0.11 \pm 0.01$ & $0.11 \pm 0.01$ & $0.12 \pm 0.02$ \\
\hline
\end{tabular}


Cobalt Metal, NTP TR 581

\begin{tabular}{lccccc}
\hline & $\begin{array}{c}\text { Chamber } \\
\text { Control }\end{array}$ & $\mathbf{0 . 6 2 5} \mathbf{~ m g / \mathbf { m } ^ { \mathbf { 3 } }}$ & $\mathbf{1 . 2 5} \mathbf{~ m g / \mathbf { m } ^ { \mathbf { 3 } }}$ & $\mathbf{2 . 5} \mathbf{~ \mathbf { g }} / \mathbf{m}^{\mathbf{3}}$ & $\mathbf{5} \mathbf{~ m g} / \mathbf{m}^{\mathbf{3}}$ \\
\hline Day 23 & $0.17 \pm 0.02$ & $0.15 \pm 0.01$ & $0.15 \pm 0.01$ & $0.15 \pm 0.01$ & $0.14 \pm 0.01$ \\
Week 14 & $0.09 \pm 0.01$ & $0.09 \pm 0.01$ & $0.12 \pm 0.01$ & $0.12 \pm 0.01$ & $0.11 \pm 0.01$ \\
\hline
\end{tabular}

\section{Clinical Chemistry}

Urea nitrogen (mg/dL)

$\begin{array}{lccccc}\text { Day 3 } & 10.7 \pm 0.6 & 8.7 \pm 0.6^{*} & 8.7 \pm 0.5 & 9.3 \pm 0.4 & 10.8 \pm 0.2 \\ \text { Day 23 } & 11.8 \pm 0.4 & 12.5 \pm 0.8 & 12.4 \pm 0.4 & 10.3 \pm 0.3^{*} & 10.1 \pm 0.4^{*} \\ \text { Week 14 } & 15.0 \pm 0.4 & 16.7 \pm 0.4 & 15.0 \pm 0.4 & 15.6 \pm 0.6 & 13.5 \pm 0.5 \\ \text { Creatinine }(\mathrm{mg} / \mathrm{dL}) & & & & \\ \text { Day 3 } & 0.25 \pm 0.02 & 0.24 \pm 0.02 & 0.23 \pm 0.02 & 0.23 \pm 0.02 & 0.22 \pm 0.01 \\ \text { Day 23 } & 0.26 \pm 0.02 & 0.28 \pm 0.01 & 0.28 \pm 0.02 & 0.29 \pm 0.01 & 0.29 \pm 0.02 \\ \text { Week 14 } & 0.39 \pm 0.01 & 0.37 \pm 0.02 & 0.37 \pm 0.02 & 0.39 \pm 0.01 & 0.41 \pm 0.01\end{array}$

Glucose (mg/dL)

\begin{tabular}{|c|c|c|c|c|c|}
\hline Day 3 & $137 \pm 3$ & $136 \pm 3$ & $139 \pm 2$ & $136 \pm 1$ & $125 \pm 3$ \\
\hline Day 23 & $140 \pm 4$ & $141 \pm 2$ & $147 \pm 7$ & $141 \pm 3$ & $132 \pm 5$ \\
\hline Week 14 & $130 \pm 7$ & $133 \pm 7$ & $131 \pm 11$ & $112 \pm 3$ & $123 \pm 10$ \\
\hline
\end{tabular}

Total protein $(\mathrm{g} / \mathrm{dL})$

$$
\text { Day } 3
$$

Day 23

Week 14

Albumin (g/dL)

Day 3

Day 23

Week 14

Globulin (g/dL)

Day 3

Day 23

Week 14

Albumin to globulin ratio

Day 3

Day 23

Week 14

Cholesterol (mg/dL)

Day 3

Day 23

Week 14
$6.2 \pm 0.1$

$6.5 \pm 0.1$

$7.6 \pm 0.1$

$4.6 \pm 0.1$

$4.7 \pm 0.0$

$5.3 \pm 0.1$

$1.6 \pm 0.0$

$1.8 \pm 0.0$

$2.3 \pm 0.0$

$2.8 \pm 0.0$

$2.6 \pm 0.0$

$2.3 \pm 0.0$

$98 \pm 2$

$102 \pm 3$

$102 \pm 3$
$6.0 \pm 0.1$

$6.5 \pm 0.1$

$7.5 \pm 0.1$

$4.4 \pm 0.0$

$4.7 \pm 0.0$

$5.2 \pm 0.1$

$1.6 \pm 0.0$

$1.8 \pm 0.0$

$2.3 \pm 0.0$

$2.8 \pm 0.0$

$2.6 \pm 0.0$

$2.3 \pm 0.0$

$95 \pm 2$

$97 \pm 2$

$100 \pm 2$
$6.1 \pm 0.1$

$6.6 \pm 0.1$

$7.4 \pm 0.1$

$4.4 \pm 0.1$

$4.7 \pm 0.0$

$5.2 \pm 0.1$

$1.7 \pm 0.1$

$1.9 \pm 0.1$

$2.3 \pm 0.1$

$2.6 \pm 0.1$

$2.5 \pm 0.1$

$2.3 \pm 0.0$

$91 \pm 2 *$

$97 \pm 2$

$92 \pm 3 *$
$6.1 \pm 0.1$

$6.5 \pm 0.1$

$7.4 \pm 0.1 *$

$4.5 \pm 0.0$

$4.7 \pm 0.0$

$5.2 \pm 0.0$

$1.6 \pm 0.0$

$1.9 \pm 0.0$

$2.3 \pm 0.0$

$2.7 \pm 0.0$

$2.5 \pm 0.1$

$2.3 \pm 0.0$

$91 \pm 2 *$

$93 \pm 2$

$93 \pm 3 *$

$89 \pm 3 * *$

$91 \pm 3^{* *}$
$6.2 \pm 0.0$

$6.5 \pm 0.1$

$7.0 \pm 0.1^{* *}$

$4.4 \pm 0.0$

$4.7 \pm 0.1$

$5.0 \pm 0.1^{* *}$

$1.8 \pm 0.0^{* *}$

$1.9 \pm 0.1$

$2.1 \pm 0.1 * *$

$2.5 \pm 0.0^{* *}$

$2.5 \pm 0.1$

$2.4 \pm 0.0$ 
Cobalt Metal, NTP TR 581

\begin{tabular}{|c|c|c|c|c|c|}
\hline & $\begin{array}{c}\text { Chamber } \\
\text { Control }\end{array}$ & $0.625 \mathrm{mg} / \mathrm{m}^{3}$ & $1.25 \mathrm{mg} / \mathrm{m}^{3}$ & $2.5 \mathrm{mg} / \mathrm{m}^{3}$ & $5 \mathrm{mg} / \mathrm{m}^{3}$ \\
\hline \multicolumn{6}{|c|}{ Triglycerides (mg/dL) } \\
\hline Day 3 & $63 \pm 4$ & $60 \pm 5$ & $59 \pm 4$ & $63 \pm 4$ & $78 \pm 7$ \\
\hline Day 23 & $79 \pm 6$ & $79 \pm 4$ & $89 \pm 10$ & $67 \pm 6$ & $80 \pm 7$ \\
\hline Week 14 & $98 \pm 10$ & $134 \pm 15$ & $91 \pm 9$ & $93 \pm 8$ & $71 \pm 3$ \\
\hline \multicolumn{6}{|c|}{ Alanine aminotransferase (IU/L) } \\
\hline Day 3 & $49 \pm 2$ & $49 \pm 1$ & $48 \pm 1$ & $46 \pm 1$ & $43 \pm 1^{*}$ \\
\hline Day 23 & $39 \pm 2$ & $38 \pm 1$ & $40 \pm 1$ & $39 \pm 1$ & $37 \pm 1$ \\
\hline Week 14 & $68 \pm 6$ & $75 \pm 6$ & $65 \pm 6$ & $65 \pm 5$ & $58 \pm 6$ \\
\hline \multicolumn{6}{|c|}{ Alkaline phosphatase (IU/L) } \\
\hline Day 3 & $506 \pm 13$ & $539 \pm 9$ & $521 \pm 10$ & $530 \pm 10$ & $487 \pm 15$ \\
\hline Day 23 & $336 \pm 10$ & $359 \pm 7$ & $332 \pm 10$ & $354 \pm 9$ & $348 \pm 13$ \\
\hline Week 14 & $203 \pm 7$ & $216 \pm 7$ & $208 \pm 5$ & $225 \pm 6$ & $202 \pm 7$ \\
\hline \multicolumn{6}{|c|}{ Creatine kinase (IU/L) } \\
\hline Day 3 & $538 \pm 60$ & $375 \pm 36^{*}$ & $519 \pm 74$ & $367 \pm 18$ & $421 \pm 39$ \\
\hline Day 23 & $431 \pm 67$ & $323 \pm 42$ & $411 \pm 65$ & $340 \pm 39$ & $393 \pm 45$ \\
\hline Week 14 & $189 \pm 15$ & $209 \pm 25$ & $172 \pm 18$ & $195 \pm 20$ & $225 \pm 32$ \\
\hline \multicolumn{6}{|c|}{ Sorbitol dehydrogenase (IU/L) } \\
\hline Day 3 & $15 \pm 1$ & $14 \pm 0$ & $14 \pm 1$ & $14 \pm 0$ & $13 \pm 0 * *$ \\
\hline Day 23 & $17 \pm 1$ & $18 \pm 0$ & $19 \pm 1^{*}$ & $17 \pm 1$ & $16 \pm 1$ \\
\hline Week 14 & $19 \pm 1$ & $20 \pm 1$ & $19 \pm 1$ & $20 \pm 1$ & $18 \pm 1$ \\
\hline \multicolumn{6}{|c|}{ Bile salts $(\mu \mathrm{mol} / \mathrm{L})$} \\
\hline Day 3 & $5.3 \pm 0.6$ & $5.8 \pm 0.8$ & $5.5 \pm 0.4$ & $5.0 \pm 0.3$ & $6.1 \pm 0.7$ \\
\hline Day 23 & $4.4 \pm 1.0$ & $4.0 \pm 0.5$ & $5.6 \pm 1.3$ & $4.7 \pm 0.6$ & $4.9 \pm 0.6$ \\
\hline Week 14 & $4.8 \pm 0.3$ & $5.4 \pm 0.5$ & $5.4 \pm 0.8$ & $4.6 \pm 0.3$ & $10.6 \pm 2.5$ \\
\hline
\end{tabular}

*Significantly different $(\mathrm{P} \leq 0.05)$ from the chamber control group by Dunn's or Shirley's test. $* * \mathrm{P} \leq 0.01$.

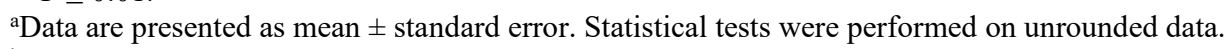
${ }^{b} \mathrm{n}=9$. 
Table F-2. Hematology Data for Mice in the Three-month Inhalation Study of Cobalt Metal ${ }^{\text {a }}$

\begin{tabular}{|c|c|c|c|c|c|c|}
\hline & Chamber Control & $0.625 \mathrm{mg} / \mathrm{m}^{3}$ & $1.25 \mathrm{mg} / \mathrm{m}^{3}$ & $2.5 \mathrm{mg} / \mathrm{m}^{3}$ & $5 \mathrm{mg} / \mathrm{m}^{3}$ & $10 \mathrm{mg} / \mathrm{m}^{3}$ \\
\hline \multicolumn{7}{|l|}{ Male } \\
\hline $\mathbf{n}$ & 10 & 10 & 10 & 10 & 10 & 10 \\
\hline Hematocrit (spun) (\%) & $50.1 \pm 0.4$ & $50.6 \pm 0.4$ & $50.7 \pm 0.4$ & $51.0 \pm 0.3$ & $50.7 \pm 0.3$ & $51.3 \pm 0.5$ \\
\hline Packed cell volume $(\%)$ & $51.3 \pm 0.5$ & $51.8 \pm 0.3$ & $51.9 \pm 0.5$ & $52.3 \pm 0.3$ & $51.8 \pm 0.3$ & $52.0 \pm 0.5$ \\
\hline Hemoglobin (g/dL) & $15.7 \pm 0.1$ & $15.9 \pm 0.1$ & $15.9 \pm 0.1$ & $16.0 \pm 0.1$ & $16.0 \pm 0.1$ & $16.2 \pm 0.1 * *$ \\
\hline Erythrocytes $(106 / \mu \mathrm{L})$ & $10.51 \pm 0.08$ & $10.59 \pm 0.05$ & $10.54 \pm 0.08$ & $10.61 \pm 0.07$ & $10.63 \pm 0.07$ & $10.91 \pm 0.14^{*}$ \\
\hline Reticulocytes $(103 / \mu \mathrm{L})$ & $238.60 \pm 13.00$ & $211.70 \pm 16.50$ & $227.70 \pm 21.50$ & $254.00 \pm 15.30$ & $192.90 \pm 23.40$ & $230.50 \pm 16.30$ \\
\hline Nucleated erythrocytes/ 100 leukocytes & $0.00 \pm 0.00$ & $0.00 \pm 0.00$ & $0.00 \pm 0.00$ & $0.00 \pm 0.00$ & $0.00 \pm 0.00$ & $0.00 \pm 0.00$ \\
\hline Total nucleated erythrocytes $\left(10^{3} / \mu \mathrm{L}\right)$ & $2.9 \pm 0.3$ & $3.4 \pm 0.3$ & $3.3 \pm 0.4^{*}$ & $2.8 \pm 0.3$ & $3.5 \pm 0.3$ & $3.3 \pm 0.2$ \\
\hline Howell-Jolly bodies (\% erythrocytes) & $0.1 \pm 0.0$ & $0.1 \pm 0.0$ & $0.1 \pm 0.0$ & $0.1 \pm 0.0$ & $0.1 \pm 0.0$ & $0.1 \pm 0.0$ \\
\hline Mean cell volume (fL) & $48.8 \pm 0.2$ & $48.9 \pm 0.3$ & $49.2 \pm 0.2$ & $49.4 \pm 0.2$ & $48.7 \pm 0.1$ & $47.6 \pm 0.2$ \\
\hline Mean cell hemoglobin (pg) & $14.9 \pm 0.1$ & $15.0 \pm 0.0$ & $15.1 \pm 0.1$ & $15.1 \pm 0.1$ & $15.0 \pm 0.1$ & $14.8 \pm 0.1$ \\
\hline Mean cell hemoglobin concentration (g/dL) & $30.5 \pm 0.1$ & $30.6 \pm 0.1$ & $30.6 \pm 0.1$ & $30.5 \pm 0.1$ & $30.8 \pm 0.1$ & $31.0 \pm 0.1 * *$ \\
\hline Platelets $\left(10^{3} / \mu \mathrm{L}\right)$ & $903.7 \pm 19.5$ & $929.8 \pm 18.5$ & $883.7 \pm 13.6$ & $919.5 \pm 11.7$ & $894.7 \pm 10.0$ & $834.2 \pm 11.6^{* *}$ \\
\hline Leukocytes $\left(10^{3} / \mu \mathrm{L}\right)$ & $2.85 \pm 0.27$ & $3.38 \pm 0.25$ & $3.33 \pm 0.36$ & $2.76 \pm 0.32$ & $3.46 \pm 0.33$ & $3.33 \pm 0.23$ \\
\hline Segmented neutrophils $\left(10^{3} / \mu \mathrm{L}\right)$ & $0.34 \pm 0.04$ & $0.42 \pm 0.04$ & $0.37 \pm 0.06$ & $0.36 \pm 0.05$ & $0.59 \pm 0.09 *$ & $0.49 \pm 0.03 *$ \\
\hline Bands $\left(10^{3} / \mu \mathrm{L}\right)$ & $0.00 \pm 0.00$ & $0.00 \pm 0.00$ & $0.00 \pm 0.00$ & $0.00 \pm 0.00$ & $0.00 \pm 0.00$ & $0.00 \pm 0.00$ \\
\hline Lymphocytes $\left(10^{3} / \mu \mathrm{L}\right)$ & $2.44 \pm 0.24$ & $2.86 \pm 0.22$ & $2.88 \pm 0.34$ & $2.27 \pm 0.27$ & $2.74 \pm 0.31$ & $2.74 \pm 0.22$ \\
\hline Monocytes $\left(10^{3} / \mu \mathrm{L}\right)$ & $0.04 \pm 0.01$ & $0.04 \pm 0.01$ & $0.03 \pm 0.01$ & $0.08 \pm 0.02$ & $0.07 \pm 0.02$ & $0.03 \pm 0.01$ \\
\hline Basophils $\left(10^{3} / \mu \mathrm{L}\right)$ & $0.013 \pm 0.002$ & $0.020 \pm 0.005$ & $0.014 \pm 0.003$ & $0.024 \pm 0.003$ & $0.019 \pm 0.003$ & $0.019 \pm 0.005$ \\
\hline Eosinophils $\left(10^{3} / \mu \mathrm{L}\right)$ & $0.02 \pm 0.01$ & $0.03 \pm 0.01$ & $0.04 \pm 0.01$ & $0.03 \pm 0.01$ & $0.04 \pm 0.01$ & $0.05 \pm 0.01$ \\
\hline
\end{tabular}


Cobalt Metal, NTP TR 581

\begin{tabular}{|c|c|c|c|c|c|c|}
\hline & Chamber Control & $0.625 \mathrm{mg} / \mathrm{m}^{3}$ & $1.25 \mathrm{mg} / \mathrm{m}^{3}$ & $2.5 \mathrm{mg} / \mathrm{m}^{3}$ & $5 \mathrm{mg} / \mathrm{m}^{3}$ & $10 \mathrm{mg} / \mathrm{m}^{3}$ \\
\hline \multicolumn{7}{|l|}{ Female } \\
\hline $\mathbf{n}$ & 10 & 10 & 10 & 9 & 10 & 10 \\
\hline Hematocrit spun $(\%)$ & $49.8 \pm 0.7$ & $50.2 \pm 0.5$ & $50.2 \pm 0.4$ & $50.2 \pm 0.6$ & $50.2 \pm 0.3$ & $51.2 \pm 0.5$ \\
\hline Packed cell volume (\%) & $50.9 \pm 0.6$ & $51.6 \pm 0.4$ & $51.8 \pm 0.4$ & $51.4 \pm 0.6$ & $51.1 \pm 0.3$ & $52.3 \pm 0.4$ \\
\hline Hemoglobin $(\mathrm{g} / \mathrm{dL})$ & $15.7 \pm 0.2$ & $15.9 \pm 0.1$ & $16.0 \pm 0.1$ & $15.9 \pm 0.2$ & $15.9 \pm 0.1$ & $16.1 \pm 0.1$ \\
\hline Erythrocytes $\left(10^{6} / \mu \mathrm{L}\right)$ & $10.12 \pm 0.15$ & $10.24 \pm 0.08$ & $10.25 \pm 0.07$ & $10.24 \pm 0.11$ & $10.37 \pm 0.06$ & $10.60 \pm 0.09 * *$ \\
\hline Reticulocytes $\left(10^{3} / \mu \mathrm{L}\right)$ & $290.00 \pm 19.00$ & $289.10 \pm 24.90$ & $250.00 \pm 15.30$ & $259.40 \pm 16.30$ & $260.30 \pm 13.20$ & $281.70 \pm 21.20$ \\
\hline Nucleated erythrocytes/100 leukocytes & $0.00 \pm 0.00$ & $0.00 \pm 0.00$ & $0.00 \pm 0.00$ & $0.00 \pm 0.00$ & $0.00 \pm 0.00$ & $0.00 \pm 0.00$ \\
\hline Total nucleated erythrocytes $\left(10^{3} / \mu \mathrm{L}\right)$ & $2.8 \pm 0.3$ & $3.0 \pm 0.4$ & $3.2 \pm 0.3$ & $3.3 \pm 0.3$ & $3.6 \pm 0.2$ & $3.3 \pm 0.2$ \\
\hline Howell-Jolly bodies ( $\%$ erythrocytes) & $0.1 \pm 0.0$ & $0.2 \pm 0.0$ & $0.1 \pm 0.0$ & $0.1 \pm 0.0$ & $0.1 \pm 0.0$ & $0.2 \pm 0.0$ \\
\hline Mean cell volume (fL) & $50.3 \pm 0.2$ & $50.4 \pm 0.1$ & $50.6 \pm 0.1$ & $50.1 \pm 0.1$ & $49.3 \pm 0.2 * *$ & $49.4 \pm 0.2 * *$ \\
\hline Mean cell hemoglobin (pg) & $15.5 \pm 0.1$ & $15.5 \pm 0.0$ & $15.6 \pm 0.1$ & $15.5 \pm 0.1$ & $15.3 \pm 0.1 *$ & $15.2 \pm 0.1 * *$ \\
\hline Mean cell hemoglobin concentration $(\mathrm{g} / \mathrm{dL})$ & $30.8 \pm 0.1$ & $30.8 \pm 0.1$ & $30.8 \pm 0.1$ & $30.9 \pm 0.1$ & $31.0 \pm 0.1$ & $30.8 \pm 0.1$ \\
\hline Platelets $\left(10^{3} / \mu \mathrm{L}\right)$ & $755.2 \pm 19.5$ & $826.6 \pm 7.4^{* *}$ & $819.1 \pm 9.9 *$ & $811.1 \pm 16.4$ & $801.1 \pm 15.0$ & $722.7 \pm 35.7$ \\
\hline Leukocytes $\left(10^{3} / \mu \mathrm{L}\right)$ & $2.75 \pm 0.30$ & $2.98 \pm 0.39$ & $3.16 \pm 0.29$ & $3.29 \pm 0.27$ & $3.57 \pm 0.21$ & $3.30 \pm 0.23$ \\
\hline Segmented neutrophils $\left(10^{3} / \mu \mathrm{L}\right)$ & $0.33 \pm 0.04$ & $0.38 \pm 0.08$ & $0.32 \pm 0.03$ & $0.41 \pm 0.06$ & $0.58 \pm 0.06^{* *}$ & $0.52 \pm 0.05 * *$ \\
\hline Bands $\left(10^{3} / \mu \mathrm{L}\right)$ & $0.00 \pm 0.00$ & $0.00 \pm 0.00$ & $0.00 \pm 0.00$ & $0.00 \pm 0.00$ & $0.00 \pm 0.00$ & $0.00 \pm 0.00$ \\
\hline Lymphocytes $\left(10^{3} / \mu \mathrm{L}\right)$ & $2.33 \pm 0.26$ & $2.52 \pm 0.31$ & $2.74 \pm 0.28$ & $2.79 \pm 0.23$ & $2.87 \pm 0.17$ & $2.65 \pm 0.23$ \\
\hline Monocytes $\left(10^{3} / \mu \mathrm{L}\right)$ & $0.04 \pm 0.01$ & $0.04 \pm 0.01$ & $0.05 \pm 0.01$ & $0.03 \pm 0.01$ & $0.04 \pm 0.01$ & $0.04 \pm 0.01$ \\
\hline Basophils $\left(10^{3} / \mu \mathrm{L}\right)$ & $0.014 \pm 0.003$ & $0.010 \pm 0.002$ & $0.018 \pm 0.002$ & $0.017 \pm 0.002$ & $0.019 \pm 0.002$ & $0.024 \pm 0.004 *$ \\
\hline Eosinophils $\left(10^{3} / \mu \mathrm{L}\right)$ & $0.04 \pm 0.01$ & $0.04 \pm 0.01$ & $0.03 \pm 0.01$ & $0.03 \pm 0.01$ & $0.06 \pm 0.01 *$ & $0.07 \pm 0.01 *$ \\
\hline
\end{tabular}

*Significantly different $(\mathrm{P} \leq 0.05)$ from the chamber control group by Dunn's or Shirley's test.

$* * \mathrm{P} \leq 0.01$.

aData are presented as mean \pm standard error. Statistical tests were performed on unrounded data. 


\section{Appendix G. Organ Weights and Organ-Weight-to-Body- Weight Ratios}

\section{Tables}

Table G-1. Organ Weights and Organ-Weight-to-Body-Weight Ratios for Rats in the Two-week Inhalation Study of Cobalt Metal G-2

Table G-2. Organ Weights and Organ-Weight-to-Body-Weight Ratios for Rats in the Three-month Inhalation Study of Cobalt Metal G-4

Table G-3. Organ Weights and Organ-Weight-to-Body-Weight Ratios for Mice in the Two-week Inhalation Study of Cobalt Metal G-6

Table G-4. Organ Weights and Organ-Weight-to-Body-Weight Ratios for Mice in the Three-month Inhalation Study of Cobalt Metal G-8 
Cobalt Metal, NTP TR 581

Table G-1. Organ Weights and Organ-Weight-to-Body-Weight Ratios for Rats in the Two-week Inhalation Study of Cobalt Metal ${ }^{\mathrm{a}}$

\begin{tabular}{|c|c|c|c|c|c|c|}
\hline & $\begin{array}{c}\text { Chamber } \\
\text { Control }\end{array}$ & $2.5 \mathrm{mg} / \mathrm{m}^{3}$ & $5 \mathrm{mg} / \mathrm{m}^{3}$ & $10 \mathrm{mg} / \mathrm{m}^{3}$ & $20 \mathrm{mg} / \mathrm{m}^{3}$ & $40 \mathrm{mg} / \mathrm{m}^{3}$ \\
\hline \multicolumn{7}{|l|}{ Male } \\
\hline $\mathbf{n}$ & 5 & 5 & 5 & 5 & 0 & 0 \\
\hline Necropsy body wt & $144 \pm 3$ & $144 \pm 2$ & $140 \pm 4$ & $115 \pm 6^{* *}$ & - & - \\
\hline \multicolumn{7}{|l|}{ Heart } \\
\hline Absolute & $0.55 \pm 0.01$ & $0.54 \pm 0.01$ & $0.53 \pm 0.01$ & $0.47 \pm 0.01 * *$ & - & - \\
\hline Relative & $3.84 \pm 0.07$ & $3.73 \pm 0.02$ & $3.79 \pm 0.14$ & $4.11 \pm 0.17$ & - & - \\
\hline \multicolumn{7}{|l|}{ L. Kidney } \\
\hline Absolute & $0.61 \pm 0.02$ & $0.61 \pm 0.01$ & $0.58 \pm 0.01$ & $0.52 \pm 0.02 * *$ & - & - \\
\hline Relative & $4.25 \pm 0.06$ & $4.26 \pm 0.08$ & $4.12 \pm 0.09$ & $4.57 \pm 0.10^{*}$ & - & - \\
\hline \multicolumn{7}{|l|}{ Liver } \\
\hline Absolute & $5.84 \pm 0.16$ & $5.10 \pm 0.09 * *$ & $5.08 \pm 0.15^{* *}$ & $4.29 \pm 0.24^{* *}$ & - & - \\
\hline Relative & $40.61 \pm 0.46$ & $35.40 \pm 0.28 * *$ & $36.35 \pm 0.63 * *$ & $37.43 \pm 0.86^{* *}$ & - & - \\
\hline \multicolumn{7}{|l|}{ Lung } \\
\hline Absolute & $1.14 \pm 0.10$ & $1.16 \pm 0.08$ & $1.19 \pm 0.04$ & $1.28 \pm 0.12$ & - & - \\
\hline Relative & $7.91 \pm 0.61$ & $8.07 \pm 0.55$ & $8.49 \pm 0.30$ & $11.13 \pm 0.50^{* *}$ & - & - \\
\hline \multicolumn{7}{|l|}{ L. Testis } \\
\hline Absolute & $0.886 \pm 0.040$ & $0.928 \pm 0.017$ & $0.852 \pm 0.035$ & $0.590 \pm 0.088^{* *}$ & - & - \\
\hline Relative & $6.165 \pm 0.246$ & $6.446 \pm 0.155$ & $6.103 \pm 0.248$ & $5.053 \pm 0.502$ & - & - \\
\hline \multicolumn{7}{|l|}{ Thymus } \\
\hline Absolute & $0.374 \pm 0.013$ & $0.358 \pm 0.025$ & $0.358 \pm 0.007$ & $0.284 \pm 0.008 * *$ & - & - \\
\hline Relative & $2.605 \pm 0.054$ & $2.485 \pm 0.161$ & $2.560 \pm 0.023$ & $2.498 \pm 0.112$ & - & - \\
\hline \multicolumn{7}{|l|}{ Thyroid gland } \\
\hline Absolute & $0.017 \pm 0.002$ & $0.019 \pm 0.002$ & $0.016 \pm 0.001$ & $0.015 \pm 0.001$ & - & - \\
\hline Relative & $0.115 \pm 0.014$ & $0.131 \pm 0.011$ & $0.115 \pm 0.010$ & $0.130 \pm 0.008$ & - & - \\
\hline
\end{tabular}

\section{Female}

\begin{tabular}{|c|c|c|c|c|c|c|}
\hline $\mathbf{n}$ & 5 & 5 & 5 & 5 & 2 & 0 \\
\hline Necropsy body wt & $112 \pm 4$ & $112 \pm 2$ & $107 \pm 3$ & $98 \pm 4^{* *}$ & $61 \pm 5^{* *}$ & - \\
\hline \multicolumn{7}{|l|}{ Heart } \\
\hline Absolute & $0.42 \pm 0.01$ & $0.47 \pm 0.01^{*}$ & $0.44 \pm 0.01$ & $0.42 \pm 0.01$ & $0.34 \pm 0.00 * *$ & - \\
\hline Relative & $3.75 \pm 0.06$ & $4.17 \pm 0.07^{* *}$ & $4.15 \pm 0.06^{* *}$ & $4.25 \pm 0.10^{* *}$ & $5.59 \pm 0.41 * *$ & - \\
\hline \multicolumn{7}{|l|}{ L. Kidney } \\
\hline Absolute & $0.52 \pm 0.02$ & $0.50 \pm 0.01$ & $0.50 \pm 0.02$ & $0.46 \pm 0.01 *$ & $0.35 \pm 0.00 * *$ & - \\
\hline Relative & $4.66 \pm 0.11$ & $4.46 \pm 0.05$ & $4.63 \pm 0.08$ & $4.74 \pm 0.12$ & $5.75 \pm 0.42 * *$ & - \\
\hline \multicolumn{7}{|l|}{ Liver } \\
\hline Absolute & $4.07 \pm 0.16$ & $3.77 \pm 0.05$ & $3.61 \pm 0.13^{* *}$ & $3.44 \pm 0.05^{* *}$ & $2.57 \pm 0.06^{* *}$ & - \\
\hline Relative & $36.37 \pm 0.49$ & $33.59 \pm 0.16$ & $33.78 \pm 1.08$ & $35.17 \pm 1.00$ & $42.15 \pm 2.12 * *$ & - \\
\hline
\end{tabular}




\section{Cobalt Metal, NTP TR 581}

\begin{tabular}{|c|c|c|c|c|c|c|}
\hline & $\begin{array}{c}\text { Chamber } \\
\text { Control }\end{array}$ & $2.5 \mathrm{mg} / \mathrm{m}^{3}$ & $5 \mathrm{mg} / \mathrm{m}^{3}$ & $10 \mathrm{mg} / \mathrm{m}^{3}$ & $20 \mathrm{mg} / \mathrm{m}^{3}$ & $40 \mathrm{mg} / \mathrm{m}^{3}$ \\
\hline \multicolumn{7}{|l|}{ Lung } \\
\hline Absolute & $0.86 \pm 0.04$ & $0.83 \pm 0.01$ & $0.91 \pm 0.04$ & $1.03 \pm 0.06^{*}$ & $1.01 \pm 0.04^{*}$ & - \\
\hline Relative & $7.71 \pm 0.36$ & $7.44 \pm 0.07$ & $8.49 \pm 0.34$ & $10.54 \pm 0.69 * *$ & $16.54 \pm 0.56^{* *}$ & - \\
\hline \multicolumn{7}{|l|}{ Thymus } \\
\hline Absolute & $0.317 \pm 0.016$ & $0.324 \pm 0.011$ & $0.352 \pm 0.022$ & $0.289 \pm 0.011$ & $0.064 \pm 0.016^{* *}$ & - \\
\hline Relative & $2.842 \pm 0.167$ & $2.895 \pm 0.126$ & $3.289 \pm 0.201$ & $2.948 \pm 0.092$ & $1.024 \pm 0.178^{* *}$ & - \\
\hline \multicolumn{7}{|c|}{ Thyroid gland } \\
\hline Absolute & $0.011 \pm 0.002$ & $0.017 \pm 0.002$ & $0.019 \pm 0.003$ & $0.014 \pm 0.003$ & $0.017 \pm 0.005$ & - \\
\hline Relative & $0.099 \pm 0.012$ & $0.151 \pm 0.018$ & $0.174 \pm 0.024$ & $0.140 \pm 0.026$ & $0.266 \pm 0.054 * *$ & - \\
\hline
\end{tabular}

*Significantly different $(\mathrm{P} \leq 0.05)$ from the chamber control group by Williams' or Dunnett's test.

$* * \mathrm{P} \leq 0.01$.

${ }^{\mathrm{a}}$ Organ weights (absolute weights) and body weights are given in grams; organ-weight-to-body-weight ratios (relative weights) are given as $\mathrm{mg}$ organ weight $/ \mathrm{g}$ body weight (mean \pm standard error). No data available for $20 \mathrm{mg} / \mathrm{m}^{3} \mathrm{males}$ or $40 \mathrm{mg} / \mathrm{m}^{3} \mathrm{males}$ or females due to $100 \%$ mortality. 
Cobalt Metal, NTP TR 581

Table G-2. Organ Weights and Organ-Weight-to-Body-Weight Ratios for Rats in the Three-month Inhalation Study of Cobalt Metal ${ }^{\mathrm{a}}$

\begin{tabular}{|c|c|c|c|c|c|}
\hline & Chamber Control & $0.625 \mathrm{mg} / \mathrm{m}^{3}$ & $1.25 \mathrm{mg} / \mathrm{m}^{3}$ & $2.5 \mathrm{mg} / \mathrm{m}^{3}$ & $5 \mathrm{mg} / \mathrm{m}^{3}$ \\
\hline $\mathbf{n}$ & 10 & 10 & 10 & 10 & 10 \\
\hline \multicolumn{6}{|l|}{ Male } \\
\hline Necropsy body wt & $319 \pm 5$ & $336 \pm 6$ & $327 \pm 7$ & $326 \pm 6$ & $297 \pm 5^{*}$ \\
\hline \multicolumn{6}{|l|}{ Heart } \\
\hline Absolute & $0.86 \pm 0.02$ & $0.89 \pm 0.02$ & $0.86 \pm 0.02$ & $0.86 \pm 0.02$ & $0.81 \pm 0.01 *$ \\
\hline Relative & $2.707 \pm 0.036$ & $2.650 \pm 0.035$ & $2.624 \pm 0.040$ & $2.636 \pm 0.020$ & $2.717 \pm 0.038$ \\
\hline \multicolumn{6}{|l|}{ R. Kidney } \\
\hline Absolute & $0.95 \pm 0.01$ & $1.00 \pm 0.02$ & $0.98 \pm 0.02$ & $0.99 \pm 0.02$ & $0.91 \pm 0.02$ \\
\hline Relative & $2.988 \pm 0.037$ & $2.970 \pm 0.036$ & $3.014 \pm 0.021$ & $3.044 \pm 0.041$ & $3.074 \pm 0.036$ \\
\hline \multicolumn{6}{|l|}{ Liver } \\
\hline Absolute & $9.97 \pm 0.19$ & $10.89 \pm 0.33$ & $10.08 \pm 0.31$ & $10.22 \pm 0.20$ & $9.07 \pm 0.18^{*}$ \\
\hline Relative & $31.232 \pm 0.479$ & $32.362 \pm 0.443$ & $30.808 \pm 0.467$ & $31.374 \pm 0.401$ & $30.596 \pm 0.389$ \\
\hline \multicolumn{6}{|l|}{ Lung } \\
\hline Absolute & $1.63 \pm 0.03$ & $1.99 \pm 0.05 * *$ & $2.14 \pm 0.08 * *$ & $2.04 \pm 0.06^{* *}$ & $2.05 \pm 0.05^{* *}$ \\
\hline Relative & $5.129 \pm 0.111$ & $5.932 \pm 0.146^{* *}$ & $6.549 \pm 0.174 * *$ & $6.259 \pm 0.115^{* *}$ & $6.915 \pm 0.138^{* *}$ \\
\hline \multicolumn{6}{|l|}{ R. Testis } \\
\hline Absolute & $1.292 \pm 0.014$ & $1.325 \pm 0.023$ & $1.311 \pm 0.015$ & $1.314 \pm 0.016$ & $1.319 \pm 0.020$ \\
\hline Relative & $4.052 \pm 0.051$ & $3.949 \pm 0.065$ & $4.025 \pm 0.061$ & $4.039 \pm 0.060$ & $4.462 \pm 0.117 * *$ \\
\hline \multicolumn{6}{|l|}{ Thymus } \\
\hline Absolute & $0.322 \pm 0.024$ & $0.335 \pm 0.018$ & $0.328 \pm 0.016$ & $0.294 \pm 0.019$ & $0.277 \pm 0.013$ \\
\hline Relative & $1.004 \pm 0.063$ & $0.995 \pm 0.045$ & $1.008 \pm 0.053$ & $0.905 \pm 0.064$ & $0.935 \pm 0.041$ \\
\hline \multicolumn{6}{|l|}{ Thyroid gland } \\
\hline Absolute & $0.019 \pm 0.001$ & $0.019 \pm 0.002$ & $0.020 \pm 0.002$ & $0.024 \pm 0.002$ & $0.021 \pm 0.001$ \\
\hline Relative & $0.060 \pm 0.002$ & $0.057 \pm 0.005$ & $0.060 \pm 0.006$ & $0.072 \pm 0.006$ & $0.070 \pm 0.004$ \\
\hline \multicolumn{6}{|l|}{ Female } \\
\hline Necropsy body wt & $201 \pm 3$ & $205 \pm 4$ & $198 \pm 4$ & $199 \pm 4$ & $187 \pm 3^{*}$ \\
\hline \multicolumn{6}{|l|}{ Heart } \\
\hline Absolute & $0.64 \pm 0.01$ & $0.62 \pm 0.01$ & $0.61 \pm 0.01$ & $0.63 \pm 0.01$ & $0.60 \pm 0.01$ \\
\hline Relative & $3.198 \pm 0.071$ & $3.049 \pm 0.030$ & $3.090 \pm 0.052$ & $3.156 \pm 0.042$ & $3.197 \pm 0.033$ \\
\hline \multicolumn{6}{|l|}{ R. Kidney } \\
\hline Absolute & $0.64 \pm 0.01$ & $0.63 \pm 0.02$ & $0.66 \pm 0.02$ & $0.67 \pm 0.02$ & $0.67 \pm 0.01$ \\
\hline Relative & $3.184 \pm 0.036$ & $3.068 \pm 0.082$ & $3.343 \pm 0.052$ & $3.349 \pm 0.047$ & $3.589 \pm 0.055^{* *}$ \\
\hline \multicolumn{6}{|l|}{ Liver } \\
\hline Absolute & $6.06 \pm 0.09$ & $6.37 \pm 0.18$ & $6.03 \pm 0.21$ & $6.06 \pm 0.19$ & $5.80 \pm 0.12$ \\
\hline Relative & $30.171 \pm 0.262$ & $31.073 \pm 0.519$ & $30.400 \pm 0.557$ & $30.444 \pm 0.511$ & $31.012 \pm 0.288$ \\
\hline
\end{tabular}




\section{Cobalt Metal, NTP TR 581}

\begin{tabular}{|c|c|c|c|c|c|}
\hline & Chamber Control & $0.625 \mathrm{mg} / \mathrm{m}^{3}$ & $1.25 \mathrm{mg} / \mathrm{m}^{3}$ & $2.5 \mathrm{mg} / \mathrm{m}^{3}$ & $5 \mathrm{mg} / \mathrm{m}^{3}$ \\
\hline \multicolumn{6}{|l|}{ Lung } \\
\hline Absolute & $1.05 \pm 0.02$ & $1.31 \pm 0.02 * *$ & $1.33 \pm 0.05 * *$ & $1.32 \pm 0.03 * *$ & $1.36 \pm 0.02 * *$ \\
\hline Relative & $5.237 \pm 0.087$ & $6.388 \pm 0.107 * *$ & $6.687 \pm 0.149 * *$ & $6.650 \pm 0.131 * *$ & $7.298 \pm 0.111^{* *}$ \\
\hline \multicolumn{6}{|l|}{ Thymus } \\
\hline Absolute & $0.347 \pm 0.013$ & $0.363 \pm 0.011$ & $0.337 \pm 0.009$ & $0.354 \pm 0.014$ & $0.318 \pm 0.009$ \\
\hline Relative & $1.725 \pm 0.053$ & $1.775 \pm 0.059$ & $1.715 \pm 0.071$ & $1.785 \pm 0.069$ & $1.704 \pm 0.045$ \\
\hline \multicolumn{6}{|c|}{ Thyroid gland } \\
\hline Absolute & $0.021 \pm 0.001$ & $0.018 \pm 0.002$ & $0.019 \pm 0.001$ & $0.019 \pm 0.001$ & $0.021 \pm 0.001$ \\
\hline Relative & $0.103 \pm 0.007$ & $0.090 \pm 0.008$ & $0.097 \pm 0.006$ & $0.096 \pm 0.007$ & $0.109 \pm 0.005$ \\
\hline
\end{tabular}

*Significantly different $(\mathrm{P} \leq 0.05)$ from the chamber control group by Williams' or Dunnett's test.

$* * \mathrm{P} \leq 0.01$.

${ }^{a}$ Organ weights (absolute weights) and body weights are given in grams; organ-weight-to-body-weight ratios (relative weights) are given as $\mathrm{mg}$ organ weight/g body weight (mean \pm standard error). 
Cobalt Metal, NTP TR 581

Table G-3. Organ Weights and Organ-Weight-to-Body-Weight Ratios for Mice in the Two-week Inhalation Study of Cobalt Metal ${ }^{\mathrm{a}}$

\begin{tabular}{|c|c|c|c|c|c|c|}
\hline & $\begin{array}{c}\text { Chamber } \\
\text { Control }\end{array}$ & $2.5 \mathrm{mg} / \mathrm{m}^{3}$ & $5 \mathrm{mg} / \mathrm{m}^{3}$ & $10 \mathrm{mg} / \mathrm{m}^{3}$ & $20 \mathrm{mg} / \mathrm{m}^{3}$ & $40 \mathrm{mg} / \mathrm{m}^{3}$ \\
\hline $\mathbf{n}$ & 5 & 5 & 5 & 5 & 5 & 2 \\
\hline \multicolumn{7}{|l|}{ Male } \\
\hline Necropsy body wt & $25.7 \pm 0.5$ & $25.0 \pm 0.5$ & $25.9 \pm 0.3$ & $25.3 \pm 0.5$ & $23.4 \pm 0.4^{* *}$ & $18.9 \pm 1.1^{* *}$ \\
\hline \multicolumn{7}{|l|}{ Heart } \\
\hline Absolute & $0.12 \pm 0.01$ & $0.13 \pm 0.00$ & $0.12 \pm 0.00$ & $0.12 \pm 0.00$ & $0.11 \pm 0.00$ & $0.11 \pm 0.01 *$ \\
\hline Relative & $4.83 \pm 0.16$ & $5.14 \pm 0.21$ & $4.79 \pm 0.12$ & $4.68 \pm 0.20$ & $4.79 \pm 0.11$ & $5.57 \pm 0.05$ \\
\hline \multicolumn{7}{|l|}{ L. Kidney } \\
\hline Absolute & $0.21 \pm 0.01$ & $0.21 \pm 0.01$ & $0.20 \pm 0.01$ & $0.18 \pm 0.02$ & $0.16 \pm 0.01 * *$ & $0.13 \pm 0.00^{* *}$ \\
\hline Relative & $8.02 \pm 0.20$ & $8.24 \pm 0.45$ & $7.64 \pm 0.23$ & $7.02 \pm 0.52$ & $7.02 \pm 0.25$ & $6.92 \pm 0.39$ \\
\hline \multicolumn{7}{|l|}{ Liver } \\
\hline Absolute & $1.13 \pm 0.04$ & $0.98 \pm 0.04^{* *}$ & $0.98 \pm 0.04 * *$ & $0.99 \pm 0.02 * *$ & $0.89 \pm 0.02 * *$ & $0.83 \pm 0.01^{* *}$ \\
\hline Relative & $43.88 \pm 0.80$ & $39.18 \pm 1.35^{*}$ & $37.67 \pm 1.09^{* *}$ & $39.32 \pm 0.91 *$ & $37.93 \pm 0.51^{* *}$ & $44.20 \pm 2.99$ \\
\hline \multicolumn{7}{|l|}{ Lung } \\
\hline Absolute & $0.18 \pm 0.01$ & $0.21 \pm 0.01$ & $0.23 \pm 0.01 *$ & $0.24 \pm 0.01 * *$ & $0.29 \pm 0.01 * *$ & $0.36 \pm 0.05^{* *}$ \\
\hline Relative & $7.08 \pm 0.15$ & $8.33 \pm 0.32$ & $8.73 \pm 0.33$ & $9.61 \pm 0.62 *$ & $12.62 \pm 0.51^{* *}$ & $19.31 \pm 3.73^{* *}$ \\
\hline \multicolumn{7}{|l|}{ L. Testis } \\
\hline Absolute & $0.098 \pm 0.002$ & $0.104 \pm 0.001$ & $0.099 \pm 0.004$ & $0.084 \pm 0.009$ & $0.089 \pm 0.003$ & $0.070 \pm 0.002 * *$ \\
\hline Relative & $3.834 \pm 0.074$ & $4.180 \pm 0.114$ & $3.812 \pm 0.149$ & $3.322 \pm 0.311$ & $3.807 \pm 0.088$ & $3.731 \pm 0.314$ \\
\hline \multicolumn{7}{|l|}{ Thymus } \\
\hline Absolute & $0.049 \pm 0.004$ & $0.053 \pm 0.003$ & $0.046 \pm 0.008$ & $0.046 \pm 0.004$ & $0.045 \pm 0.002$ & $0.025 \pm 0.014^{*}$ \\
\hline Relative & $1.906 \pm 0.148$ & $2.137 \pm 0.136$ & $1.789 \pm 0.300$ & $1.851 \pm 0.210$ & $1.910 \pm 0.074$ & $1.264 \pm 0.646$ \\
\hline \multicolumn{7}{|l|}{ Thyroid gland } \\
\hline Absolute & $0.004 \pm 0.000$ & $0.004 \pm 0.001$ & $0.003 \pm 0.000$ & $0.003 \pm 0.000$ & $0.003 \pm 0.001$ & $0.003 \pm 0.001$ \\
\hline Relative & $0.141 \pm 0.011$ & $0.146 \pm 0.023$ & $0.131 \pm 0.015$ & $0.118 \pm 0.017$ & $0.144 \pm 0.020$ & $0.132 \pm 0.019$ \\
\hline \multicolumn{7}{|l|}{ Female } \\
\hline Necropsy body wt & $20.8 \pm 0.1$ & $20.3 \pm 0.5$ & $20.1 \pm 0.5$ & $20.0 \pm 0.6$ & $17.4 \pm 0.4^{* *}$ & $13.0 \pm 1.6^{* *}$ \\
\hline \multicolumn{7}{|l|}{ Heart } \\
\hline Absolute & $0.11 \pm 0.00$ & $0.11 \pm 0.00$ & $0.11 \pm 0.00$ & $0.10 \pm 0.00^{*}$ & $0.10 \pm 0.01^{* *}$ & $0.10 \pm 0.01 *$ \\
\hline Relative & $5.39 \pm 0.09$ & $5.32 \pm 0.10$ & $5.48 \pm 0.15$ & $5.02 \pm 0.16$ & $5.51 \pm 0.23$ & $7.49 \pm 1.28^{* *}$ \\
\hline \multicolumn{7}{|l|}{ L. Kidney } \\
\hline Absolute & $0.14 \pm 0.00$ & $0.14 \pm 0.01$ & $0.14 \pm 0.01$ & $0.12 \pm 0.00$ & $0.11 \pm 0.01 * *$ & $0.10 \pm 0.01 * *$ \\
\hline Relative & $6.74 \pm 0.16$ & $6.69 \pm 0.23$ & $6.86 \pm 0.29$ & $6.23 \pm 0.23$ & $6.31 \pm 0.27$ & $7.74 \pm 0.15$ \\
\hline \multicolumn{7}{|l|}{ Liver } \\
\hline Absolute & $0.93 \pm 0.03$ & $0.81 \pm 0.02 * *$ & $0.80 \pm 0.03^{* *}$ & $0.75 \pm 0.03 * *$ & $0.69 \pm 0.03 * *$ & $0.61 \pm 0.06^{* *}$ \\
\hline Relative & $44.56 \pm 1.13$ & $40.09 \pm 0.31 * *$ & $39.75 \pm 0.82 * *$ & $37.40 \pm 1.12 * *$ & $39.73 \pm 0.70^{* *}$ & $46.88 \pm 1.36$ \\
\hline
\end{tabular}




\section{Cobalt Metal, NTP TR 581}

\begin{tabular}{|c|c|c|c|c|c|c|}
\hline & $\begin{array}{c}\text { Chamber } \\
\text { Control }\end{array}$ & $2.5 \mathrm{mg} / \mathrm{m}^{3}$ & $5 \mathrm{mg} / \mathrm{m}^{3}$ & $10 \mathrm{mg} / \mathrm{m}^{3}$ & $20 \mathrm{mg} / \mathrm{m}^{3}$ & $40 \mathrm{mg} / \mathrm{m}^{3}$ \\
\hline \multicolumn{7}{|l|}{ Lung } \\
\hline Absolute & $0.19 \pm 0.01$ & $0.19 \pm 0.00$ & $0.22 \pm 0.01 *$ & $0.23 \pm 0.01 * *$ & $0.29 \pm 0.01 * *$ & $0.33 \pm 0.02 * *$ \\
\hline Relative & $9.34 \pm 0.38$ & $9.49 \pm 0.37$ & $11.14 \pm 0.24^{*}$ & $11.77 \pm 0.59 * *$ & $16.80 \pm 0.58^{* *}$ & $25.67 \pm 1.53^{* *}$ \\
\hline \multicolumn{7}{|l|}{ Thymus } \\
\hline Absolute & $0.078 \pm 0.000$ & $0.075 \pm 0.003$ & $0.074 \pm 0.003$ & $0.073 \pm 0.004$ & $0.059 \pm 0.006^{* *}$ & $0.016 \pm 0.008^{* *}$ \\
\hline Relative & $3.735 \pm 0.024$ & $3.699 \pm 0.199$ & $3.658 \pm 0.118$ & $3.668 \pm 0.182$ & $3.361 \pm 0.353$ & $1.144 \pm 0.442 * *$ \\
\hline \multicolumn{7}{|c|}{ Thyroid gland } \\
\hline Absolute & $0.003 \pm 0.001$ & $0.004 \pm 0.001$ & $0.003 \pm 0.000$ & $0.004 \pm 0.000$ & $0.003 \pm 0.001$ & $0.002 \pm 0.001$ \\
\hline Relative & $0.126 \pm 0.025$ & $0.208 \pm 0.037$ & $0.168 \pm 0.017$ & $0.202 \pm 0.025$ & $0.162 \pm 0.036$ & $0.147 \pm 0.060$ \\
\hline
\end{tabular}

*Significantly different $(\mathrm{P} \leq 0.05)$ from the chamber control group by Williams' or Dunnett's test.

$* * \mathrm{P} \leq 0.01$.

${ }^{a}$ Organ weights (absolute weights) and body weights are given in grams; organ-weight-to-body-weight ratios (relative weights) are given as $\mathrm{mg}$ organ weight/g body weight (mean \pm standard error). 
Cobalt Metal, NTP TR 581

Table G-4. Organ Weights and Organ-Weight-to-Body-Weight Ratios for Mice in the Three-month Inhalation Study of Cobalt Metal ${ }^{\mathrm{a}}$

\begin{tabular}{|c|c|c|c|c|c|c|}
\hline & $\begin{array}{c}\text { Chamber } \\
\text { Control }\end{array}$ & $0.625 \mathrm{mg} / \mathrm{m}^{3}$ & $1.25 \mathrm{mg} / \mathrm{m}^{3}$ & $2.5 \mathrm{mg} / \mathrm{m}^{3}$ & $5 \mathrm{mg} / \mathrm{m}^{3}$ & $10 \mathrm{mg} / \mathrm{m}^{3}$ \\
\hline \multicolumn{7}{|l|}{ Male } \\
\hline $\mathbf{n}$ & 10 & 10 & 10 & 10 & 10 & 10 \\
\hline Necropsy body wt & $37.7 \pm 0.8$ & $38.2 \pm 0.6$ & $37.9 \pm 0.8$ & $37.0 \pm 0.5$ & $37.0 \pm 0.9$ & $32.5 \pm 0.5^{* *}$ \\
\hline \multicolumn{7}{|l|}{ Heart } \\
\hline Absolute & $0.16 \pm 0.00$ & $0.16 \pm 0.00$ & $0.16 \pm 0.01$ & $0.16 \pm 0.00$ & $0.15 \pm 0.00$ & $0.14 \pm 0.00 * *$ \\
\hline Relative & $4.233 \pm 0.095$ & $4.219 \pm 0.067$ & $4.224 \pm 0.056$ & $4.187 \pm 0.063$ & $4.029 \pm 0.053$ & $4.385 \pm 0.157$ \\
\hline \multicolumn{7}{|l|}{ R. Kidney } \\
\hline Absolute & $0.31 \pm 0.01$ & $0.32 \pm 0.01$ & $0.32 \pm 0.01$ & $0.32 \pm 0.00$ & $0.29 \pm 0.01 * *$ & $0.23 \pm 0.01 * *$ \\
\hline Relative & $8.360 \pm 0.192$ & $8.441 \pm 0.122$ & $8.333 \pm 0.237$ & $8.507 \pm 0.047$ & $7.714 \pm 0.145^{* *}$ & $7.176 \pm 0.131^{* *}$ \\
\hline \multicolumn{7}{|l|}{ Liver } \\
\hline Absolute & $1.48 \pm 0.04$ & $1.53 \pm 0.04$ & $1.51 \pm 0.07$ & $1.49 \pm 0.04$ & $1.42 \pm 0.05$ & $1.15 \pm 0.03 * *$ \\
\hline Relative & $39.217 \pm 0.586$ & $40.049 \pm 0.671$ & $39.753 \pm 1.032$ & $40.301 \pm 0.698$ & $38.159 \pm 0.723$ & $35.457 \pm 0.668^{* *}$ \\
\hline \multicolumn{7}{|l|}{ Lung } \\
\hline Absolute & $0.20 \pm 0.01$ & $0.23 \pm 0.01$ & $0.22 \pm 0.01$ & $0.23 \pm 0.01 *$ & $0.27 \pm 0.01 * *$ & $0.30 \pm 0.01 * *$ \\
\hline Relative & $5.416 \pm 0.116$ & $6.051 \pm 0.235$ & $5.737 \pm 0.147$ & $6.234 \pm 0.088^{* *}$ & $7.436 \pm 0.262 * *$ & $9.142 \pm 0.177 * *$ \\
\hline \multicolumn{7}{|l|}{ R. Testis } \\
\hline Absolute & $0.118 \pm 0.002$ & $0.119 \pm 0.002$ & $0.114 \pm 0.002$ & $0.114 \pm 0.002$ & $0.104 \pm 0.003 * *$ & $0.033 \pm 0.001 * *$ \\
\hline Relative & $3.136 \pm 0.058$ & $3.131 \pm 0.037$ & $3.019 \pm 0.078$ & $3.073 \pm 0.056$ & $2.825 \pm 0.082 * *$ & $1.004 \pm 0.025 * *$ \\
\hline \multicolumn{7}{|l|}{ Thymus } \\
\hline Absolute & $0.043 \pm 0.002$ & $0.046 \pm 0.003$ & $0.051 \pm 0.003$ & $0.048 \pm 0.002$ & $0.048 \pm 0.003$ & $0.049 \pm 0.002$ \\
\hline Relative & $1.136 \pm 0.054$ & $1.190 \pm 0.078$ & $1.348 \pm 0.060$ & $1.290 \pm 0.057$ & $1.298 \pm 0.081$ & $1.507 \pm 0.048^{* *}$ \\
\hline \multicolumn{7}{|l|}{ Female } \\
\hline $\mathbf{n}$ & 10 & 10 & 10 & 9 & 10 & 10 \\
\hline Necropsy body wt & $30.9 \pm 1.0$ & $31.6 \pm 1.1$ & $31.4 \pm 0.9$ & $30.1 \pm 0.7$ & $29.0 \pm 1.1$ & $26.8 \pm 1.0^{* *}$ \\
\hline \multicolumn{7}{|l|}{ Heart } \\
\hline Absolute & $0.14 \pm 0.00$ & $0.14 \pm 0.00$ & $0.14 \pm 0.00$ & $0.13 \pm 0.00$ & $0.13 \pm 0.00 *$ & $0.13 \pm 0.00^{* *}$ \\
\hline Relative & $4.561 \pm 0.124$ & $4.549 \pm 0.092$ & $4.472 \pm 0.101$ & $4.477 \pm 0.082$ & $4.475 \pm 0.133$ & $4.753 \pm 0.195$ \\
\hline \multicolumn{7}{|l|}{ R. Kidney } \\
\hline Absolute & $0.21 \pm 0.01$ & $0.22 \pm 0.00$ & $0.21 \pm 0.01$ & $0.20 \pm 0.00$ & $0.17 \pm 0.00^{* *}$ & $0.16 \pm 0.00^{* *}$ \\
\hline Relative & $6.887 \pm 0.184$ & $6.849 \pm 0.155$ & $6.661 \pm 0.126$ & $6.689 \pm 0.254$ & $6.031 \pm 0.132 * *$ & $6.142 \pm 0.185^{* *}$ \\
\hline \multicolumn{7}{|l|}{ Liver } \\
\hline Absolute & $1.46 \pm 0.06$ & $1.51 \pm 0.07$ & $1.46 \pm 0.05$ & $1.30 \pm 0.03 *$ & $1.16 \pm 0.04 * *$ & $1.01 \pm 0.03^{* *}$ \\
\hline Relative & $47.051 \pm 0.808$ & $47.552 \pm 0.952$ & $46.455 \pm 1.046$ & $43.092 \pm 0.773^{* *}$ & $39.831 \pm 0.459^{* *}$ & $38.045 \pm 1.246^{* *}$ \\
\hline \multicolumn{7}{|l|}{ Lung } \\
\hline Absolute & $0.21 \pm 0.01$ & $0.22 \pm 0.00$ & $0.23 \pm 0.01$ & $0.23 \pm 0.01$ & $0.28 \pm 0.01 * *$ & $0.33 \pm 0.01 * *$ \\
\hline Relative & $6.904 \pm 0.227$ & $6.884 \pm 0.176$ & $7.300 \pm 0.274$ & $7.555 \pm 0.184$ & $9.787 \pm 0.241^{* *}$ & $12.602 \pm 0.487 * *$ \\
\hline
\end{tabular}




\section{Cobalt Metal, NTP TR 581}

\begin{tabular}{|c|c|c|c|c|c|c|}
\hline & $\begin{array}{c}\text { Chamber } \\
\text { Control }\end{array}$ & $0.625 \mathrm{mg} / \mathrm{m}^{3}$ & $1.25 \mathrm{mg} / \mathrm{m}^{3}$ & $2.5 \mathrm{mg} / \mathrm{m}^{3}$ & $5 \mathrm{mg} / \mathrm{m}^{3}$ & $10 \mathrm{mg} / \mathrm{m}^{3}$ \\
\hline \multicolumn{7}{|l|}{ Thymus } \\
\hline Absolute & $0.060 \pm 0.004$ & $0.064 \pm 0.004$ & $0.068 \pm 0.005$ & $0.062 \pm 0.003$ & $0.066 \pm 0.004$ & $0.064 \pm 0.003$ \\
\hline Relative & $1.960 \pm 0.106$ & $2.029 \pm 0.100$ & $2.163 \pm 0.113$ & $2.084 \pm 0.105$ & $2.257 \pm 0.110$ & $2.431 \pm 0.155^{* *}$ \\
\hline
\end{tabular}

*Significantly different $(\mathrm{P} \leq 0.05)$ from the chamber control group by Williams' test.

$* * \mathrm{P} \leq 0.01$

${ }^{\mathrm{a}}$ Organ weights (absolute weights) and body weights are given in grams; organ-weight-to-body-weight ratios (relative weights) are given as $\mathrm{mg}$ organ weight/g body weight (mean \pm standard error). 


\section{Appendix H. Reproductive Tissue Evaluations and Estrous Cycle Characterization}

\section{Tables}

Table H-1. Summary of Reproductive Tissue Evaluations for Male Rats in the Threemonth Inhalation Study of Cobalt Metal

Table H-2. Estrous Cycle Characterization for Female Rats in the Three-month Inhalation Study of Cobalt Metal

Table H-3. Results of Vaginal Cytology Study Using the Transition Matrix Approach in Female Rats Exposed to Cobalt Metal by Inhalation for Three Months

Table H-4. Summary of Reproductive Tissue Evaluations for Male Mice in the Threemonth Inhalation Study of Cobalt Metal

Table H-5. Estrous Cycle Characterization for Female Mice in the Three-month Inhalation Study of Cobalt Metal

Table H-6. Results of Vaginal Cytology Study Using the Transition Matrix Approach in Female Mice Exposed to Cobalt Metal by Inhalation for Three Months

\section{Figures}

Figure H-1. Vaginal Cytology Plots for Female Rats in the Three-month Inhalation Study of Cobalt Metal H-6

Figure H-2. Vaginal Cytology Plots for Female Mice in the Three-month Inhalation Study of Cobalt Metal 
Table H-1. Summary of Reproductive Tissue Evaluations for Male Rats in the Three-month Inhalation Study of Cobalt Metal ${ }^{\mathrm{a}}$

\begin{tabular}{|c|c|c|c|c|}
\hline & Chamber Control & $1.25 \mathrm{mg} / \mathrm{m}^{3}$ & $2.5 \mathrm{mg} / \mathrm{m}^{3}$ & $5 \mathrm{mg} / \mathrm{m}^{3}$ \\
\hline $\mathbf{n}$ & 10 & 10 & 10 & 10 \\
\hline \multicolumn{5}{|l|}{ Weights (g) } \\
\hline Necropsy body wt & $319 \pm 5$ & $327 \pm 7$ & $326 \pm 6$ & $297 \pm 5^{*}$ \\
\hline L. Cauda epididymis & $0.1741 \pm 0.0054$ & $0.1775 \pm 0.0043$ & $0.1853 \pm 0.0075$ & $0.1688 \pm 0.0038$ \\
\hline L. Epididymis & $0.4850 \pm 0.0095$ & $0.4999 \pm 0.0117$ & $0.4926 \pm 0.0146$ & $0.4846 \pm 0.0116$ \\
\hline L. Testis & $1.3700 \pm 0.0179$ & $1.3680 \pm 0.0147$ & $1.3778 \pm 0.0205$ & $1.3947 \pm 0.0124$ \\
\hline \multicolumn{5}{|l|}{ Spermatid measurements } \\
\hline Spermatid heads $\left(10^{6} /\right.$ testis $)$ & $174.00 \pm 10.16$ & $180.00 \pm 10.06$ & $176.50 \pm 5.81$ & $172.50 \pm 5.94$ \\
\hline Spermatid heads $\left(10^{6} / \mathrm{g}\right.$ testis $)$ & $141.3 \pm 8.7$ & $146.5 \pm 8.2$ & $142.5 \pm 3.7$ & $139.9 \pm 4.8$ \\
\hline \multicolumn{5}{|c|}{ Epididymal spermatozoal measurements } \\
\hline Sperm motility (\%) & $88.8 \pm 0.8$ & $86.0 \pm 1.1^{*}$ & $83.8 \pm 1.3^{* *}$ & $81.9 \pm 1.3^{* *}$ \\
\hline Sperm ( $10^{6} /$ cauda epididymis $)$ & $104.52 \pm 3.78$ & $98.40 \pm 3.13$ & $102.27 \pm 3.04$ & $94.15 \pm 3.18$ \\
\hline Sperm ( $10^{6} / \mathrm{g}$ cauda epididymis $)$ & $602.3 \pm 21.6$ & $556.4 \pm 19.3$ & $564.1 \pm 37.0$ & $559.4 \pm 19.6$ \\
\hline
\end{tabular}

*Significantly different $(\mathrm{P} \leq 0.05)$ from the chamber control group by Dunnett's test (body weight) or Shirley's test (sperm motility).

$* * \mathrm{P} \leq 0.01$.

aData are presented as mean \pm standard error. Differences from the chamber control group are not significant by Dunnett's test (tissue weights) or Dunn's test (spermatid, sperm/cauda epididymis, and sperm/g cauda epididymis).

Table H-2. Estrous Cycle Characterization for Female Rats in the Three-month Inhalation Study of Cobalt Metal ${ }^{\mathrm{a}}$

\begin{tabular}{|c|c|c|c|c|}
\hline & Chamber Control & $1.25 \mathrm{mg} / \mathrm{m}^{3}$ & $2.5 \mathrm{mg} / \mathrm{m}^{3}$ & $5 \mathrm{mg} / \mathrm{m}^{3}$ \\
\hline Number weighed at necropsy & 10 & 10 & 10 & 10 \\
\hline Necropsy body wt (g) & $201 \pm 3$ & $198 \pm 4$ & $199 \pm 4$ & $187 \pm 3^{*}$ \\
\hline Proportion of regular cycling females ${ }^{b}$ & $10 / 10$ & $10 / 10$ & $10 / 10$ & $10 / 10$ \\
\hline Estrous cycle length (days) & $5.0 \pm 0.0$ & $5.0 \pm 0.0$ & $5.0 \pm 0.0$ & $5.1 \pm 0.05$ \\
\hline \multicolumn{5}{|l|}{ Estrous stages ( $\%$ of cycle) } \\
\hline Diestrus & 50.0 & 52.5 & 45.0 & 60.8 \\
\hline Proestrus & 19.2 & 19.2 & 19.2 & 15.8 \\
\hline Estrus & 22.5 & 21.7 & 20.8 & 23.3 \\
\hline Metestrus & 8.3 & 6.7 & 15.0 & 0.0 \\
\hline
\end{tabular}

*Significantly different $(\mathrm{P} \leq 0.05)$ from the chamber control group by Dunnett's test.

${ }^{a}$ Necropsy body weights and estrous cycle length data are presented as mean \pm standard error. Differences from the chamber control group are not significant by Dunn's test (estrous cycle length). By multivariate analysis of variance, exposed females do not differ significantly from the chamber control females in the relative length of time spent in the estrous stages. Tests for equality of transition probability matrices among all groups and between the chamber control group and each exposed group indicated a significantly higher probability of extended diestrus in the $5 \mathrm{mg} / \mathrm{m}^{3}$ group compared to the chamber control group.

${ }^{b}$ Number of females with a regular cycle/number of females cycling. 
Table H-3. Results of Vaginal Cytology Study Using the Transition Matrix Approach in Female Rats Exposed to Cobalt Metal by Inhalation for Three Months

\begin{tabular}{|c|c|c|c|}
\hline Stage & Comparison & P Value & Trend $^{a}$ \\
\hline Overall Tests & Overall & 0.01 & \\
\hline Overall Tests & $1.25 \mathrm{mg} / \mathrm{m}^{3}$ vs. chamber controls & 0.944 & - \\
\hline Overall Tests & $2.5 \mathrm{mg} / \mathrm{m}^{3}$ vs. chamber controls & 0.163 & $\mathrm{~N}$ \\
\hline Overall Tests & $5 \mathrm{mg} / \mathrm{m}^{3}$ vs. chamber controls & 0.001 & - \\
\hline Extended Estrus & Overall & 0.407 & \\
\hline Extended Estrus & $1.25 \mathrm{mg} / \mathrm{m}^{3}$ vs. chamber controls & 0.601 & $\mathrm{~N}$ \\
\hline Extended Estrus & $2.5 \mathrm{mg} / \mathrm{m}^{3}$ vs. chamber controls & 0.601 & $\mathrm{~N}$ \\
\hline Extended Estrus & $5 \mathrm{mg} / \mathrm{m}^{3}$ vs. chamber controls & 0.128 & - \\
\hline Extended Diestrus & Overall & 0.06 & \\
\hline Extended Diestrus & $1.25 \mathrm{mg} / \mathrm{m}^{3}$ vs. chamber controls & 0.785 & - \\
\hline Extended Diestrus & $2.5 \mathrm{mg} / \mathrm{m}^{3}$ vs. chamber controls & 0.267 & $\mathrm{~N}$ \\
\hline Extended Diestrus & $5 \mathrm{mg} / \mathrm{m}^{3}$ vs. chamber controls & 0.011 & - \\
\hline Extended Metestrus & Overall & 1 & \\
\hline Extended Metestrus & $1.25 \mathrm{mg} / \mathrm{m}^{3}$ vs. chamber controls & 1 & - \\
\hline Extended Metestrus & $2.5 \mathrm{mg} / \mathrm{m}^{3}$ vs. chamber controls & 1 & - \\
\hline Extended Metestrus & $5 \mathrm{mg} / \mathrm{m}^{3} \mathrm{vs}$. chamber controls & 1 & - \\
\hline Extended Proestrus & Overall & 1 & \\
\hline Extended Proestrus & $1.25 \mathrm{mg} / \mathrm{m}^{3}$ vs. chamber controls & 1 & - \\
\hline Extended Proestrus & $2.5 \mathrm{mg} / \mathrm{m}^{3}$ vs. chamber controls & 1 & - \\
\hline Extended Proestrus & $5 \mathrm{mg} / \mathrm{m}^{3}$ vs. chamber controls & 1 & - \\
\hline Skipped Estrus & Overall & 1 & \\
\hline Skipped Estrus & $1.25 \mathrm{mg} / \mathrm{m}^{3}$ vs. chamber controls & 1 & - \\
\hline Skipped Estrus & $2.5 \mathrm{mg} / \mathrm{m}^{3}$ vs. chamber controls & 1 & - \\
\hline Skipped Estrus & $5 \mathrm{mg} / \mathrm{m}^{3}$ vs. chamber controls & 1 & - \\
\hline Skipped Diestrus & Overall & 1 & \\
\hline Skipped Diestrus & $1.25 \mathrm{mg} / \mathrm{m}^{3}$ vs. chamber controls & 1 & - \\
\hline Skipped Diestrus & $2.5 \mathrm{mg} / \mathrm{m}^{3}$ vs. chamber controls & 1 & - \\
\hline Skipped Diestrus & $5 \mathrm{mg} / \mathrm{m}^{3}$ vs. chamber controls & 1 & - \\
\hline \multicolumn{4}{|c|}{ Summary of Significant Groups } \\
\hline Overall Tests & $5 \mathrm{mg} / \mathrm{m}^{3}$ vs. chamber controls & 0.001 & - \\
\hline Extended Diestrus & $5 \mathrm{mg} / \mathrm{m}^{3}$ vs. Chamber Controls & 0.011 & - \\
\hline
\end{tabular}

${ }^{\mathrm{a}} \mathrm{N}$ means that the exposed group had a lower probability of transitioning to the relevant abnormal state (extended estrus, extended diestrus, extended metestrus, extended proestrus, skipped estrus, or skipped diestrus) than did the chamber control group. 
Table H-4. Summary of Reproductive Tissue Evaluations for Male Mice in the Three-month Inhalation Study of Cobalt Metal ${ }^{\mathrm{a}}$

\begin{tabular}{|c|c|c|c|c|c|}
\hline & $\begin{array}{l}\text { Chamber } \\
\text { Control }\end{array}$ & \multicolumn{2}{|c|}{$2.5 \mathrm{mg} / \mathrm{m}^{3}$} & $5 \mathrm{mg} / \mathrm{m}^{3}$ & $10 \mathrm{mg} / \mathrm{m}^{3}$ \\
\hline $\mathbf{n}$ & 10 & & 10 & 10 & 10 \\
\hline \multicolumn{6}{|l|}{ Weights (g) } \\
\hline Necropsy body wt & $37.7 \pm 0.8$ & \multicolumn{2}{|c|}{$37.0 \pm 0.5$} & $37.0 \pm 0.9$ & $32.5 \pm 0.5^{* *}$ \\
\hline L. Cauda epididymis & $0.0217 \pm 0.0014$ & \multicolumn{2}{|c|}{$0.0210 \pm 0.0008$} & $0.0231 \pm 0.0018$ & $0.0168 \pm 0.0006^{*}$ \\
\hline L. Epididymis & $0.0603 \pm 0.0022$ & \multicolumn{2}{|c|}{$0.0578 \pm 0.0019$} & $0.0614 \pm 0.0035$ & $0.0429 \pm 0.0021 * *$ \\
\hline L. Testis & $0.1185 \pm 0.0017$ & \multicolumn{2}{|c|}{$0.1132 \pm 0.0023$} & $0.1027 \pm 0.0036^{* *}$ & $0.0316 \pm 0.0014 * *$ \\
\hline \multicolumn{6}{|l|}{ Spermatid measurements } \\
\hline Spermatid heads ( $10^{6} /$ testis $)$ & $22.34 \pm 0.84$ & \multicolumn{2}{|c|}{$22.22 \pm 0.65$} & $18.90 \pm 1.20^{*}$ & $0.53 \pm 0.10^{* *}$ \\
\hline Spermatid heads ( $10^{6} / \mathrm{g}$ testis) & $210.84 \pm 6.85$ & \multicolumn{2}{|c|}{$227.74 \pm 7.16$} & $205.67 \pm 7.43$ & $24.27 \pm 4.78^{* *}$ \\
\hline \multicolumn{6}{|c|}{ Epididymal spermatozoal measurements } \\
\hline Sperm motility (\%) & $86.0 \pm 1.1$ & \multicolumn{2}{|c|}{$82.0 \pm 0.8^{*}$} & $82.2 \pm 1.1^{*}$ & $2.6 \pm 1.2 * *$ \\
\hline Sperm (10\%/cauda epididymis) & $11.55 \pm 0.39$ & \multicolumn{2}{|c|}{$10.53 \pm 0.43$} & $9.62 \pm 0.49 * *$ & $0.71 \pm 0.06^{* *}$ \\
\hline Sperm $\left(10^{6} / \mathrm{g}\right.$ cauda epididymis $)$ & $551.1 \pm 37.9$ & \multicolumn{2}{|c|}{$505.9 \pm 23.3$} & $439.9 \pm 40.3^{*}$ & $43.4 \pm 3.7 * *$ \\
\hline \multicolumn{6}{|c|}{$\begin{array}{l}\text { *Significantly different }(\mathrm{P} \leq 0.05) \text { from the chamber control group by Dunnett's test (cauda epididymis weight) or Shirley's test } \\
\text { (spermatid and epididymal spermatozoal measurements). } \\
\text { **Significantly different }(\mathrm{P} \leq 0.01) \text { from the chamber control group by Williams' test (body and tissue weights) or Shirley's test } \\
\text { (spermatid and epididymal spermatozoal measurements). } \\
\text { atata are presented as mean } \pm \text { standard error. }\end{array}$} \\
\hline \multicolumn{6}{|c|}{$\begin{array}{l}\text { Table H-5. Estrous Cycle Characterization for Female Mice in the Three-month Inhalation Study } \\
\text { of Cobalt Metal }^{\text {a }}\end{array}$} \\
\hline & \multicolumn{2}{|c|}{ Chamber Control } & $2.5 \mathrm{mg} / \mathrm{m}^{3}$ & $5 \mathrm{mg} / \mathrm{m}^{3}$ & $10 \mathrm{mg} / \mathrm{m}^{3}$ \\
\hline Number weighed at necropsy & \multicolumn{2}{|l|}{10} & 9 & 10 & 10 \\
\hline Necropsy body wt (g) & $30.9 \pm 1.0$ & & $30.1 \pm 0.7$ & $29.0 \pm 1.1$ & $26.8 \pm 1.0^{* *}$ \\
\hline Proportion of regular cycling female & $9 / 10$ & & $9 / 9$ & $9 / 10$ & $6 / 10$ \\
\hline Estrous cycle length (days) & $4.1 \pm 0.05$ & & $4.0 \pm 0.00$ & $4.1 \pm 0.13$ & $4.9 \pm 0.36^{*}$ \\
\hline Estrous stages (\% of cycle) & & & & & \\
\hline Diestrus & 28.3 & & 25.9 & 29.2 & 30.0 \\
\hline Proestrus & 0.0 & & 0.0 & 0.0 & 0.0 \\
\hline Estrus & 47.5 & & 50.0 & 48.3 & 49.2 \\
\hline Metestrus & 24.2 & & 24.1 & 22.5 & 20.8 \\
\hline
\end{tabular}

*Significantly different $(\mathrm{P} \leq 0.05)$ from the chamber control group by Dunn's test.

**Significantly different $(\mathrm{P} \leq 0.01)$ from the chamber control group by Williams' test.

${ }^{a}$ Necropsy body weights and estrous cycle length data are presented as mean \pm standard error. By multivariate analysis of variance, exposed females do not differ significantly from the chamber control females in the relative length of time spent in the estrous stages. Tests for equality of transition probability matrices among all groups and between the chamber control group and each exposed group indicated no significant differences in estrous cyclicity of the exposed and chamber control groups.

${ }^{b}$ Number of females with a regular cycle/number of females cycling. 
Table H-6. Results of Vaginal Cytology Study Using the Transition Matrix Approach in Female Mice Exposed to Cobalt Metal by Inhalation for Three Months

\begin{tabular}{|c|c|c|c|}
\hline Stage & Comparison & P Value & Trend $^{\mathrm{a}}$ \\
\hline Overall Tests & Overall & $<0.001$ & \\
\hline Overall Tests & $2.5 \mathrm{mg} / \mathrm{m}^{3}$ vs. chamber controls & 0.627 & $\mathrm{~N}$ \\
\hline Overall Tests & $5 \mathrm{mg} / \mathrm{m}^{3}$ vs. chamber controls & 0.107 & - \\
\hline Overall Tests & $10 \mathrm{mg} / \mathrm{m}^{3}$ vs. chamber controls & $<0.001$ & - \\
\hline Extended Estrus & Overall & 0.932 & \\
\hline Extended Estrus & $2.5 \mathrm{mg} / \mathrm{m}^{3}$ vs. chamber controls & 1 & - \\
\hline Extended Estrus & $5 \mathrm{mg} / \mathrm{m}^{3}$ vs. chamber controls & 0.596 & - \\
\hline Extended Estrus & $10 \mathrm{mg} / \mathrm{m}^{3}$ vs. chamber controls & 0.663 & - \\
\hline Extended Diestrus & Overall & 0.812 & \\
\hline Extended Diestrus & $2.5 \mathrm{mg} / \mathrm{m}^{3}$ vs. chamber controls & 0.627 & $\mathrm{~N}$ \\
\hline Extended Diestrus & $5 \mathrm{mg} / \mathrm{m}^{3}$ vs. chamber controls & 1 & $\mathrm{~N}$ \\
\hline Extended Diestrus & $10 \mathrm{mg} / \mathrm{m}^{3}$ vs. chamber controls & 0.361 & - \\
\hline Extended Metestrus & Overall & 1 & \\
\hline Extended Metestrus & $2.5 \mathrm{mg} / \mathrm{m}^{3}$ vs. chamber controls & 1 & - \\
\hline Extended Metestrus & $5 \mathrm{mg} / \mathrm{m}^{3}$ vs. chamber controls & 1 & - \\
\hline Extended Metestrus & $10 \mathrm{mg} / \mathrm{m}^{3}$ vs. chamber controls & 1 & - \\
\hline Extended Proestrus & Overall & 1 & \\
\hline Extended Proestrus & $2.5 \mathrm{mg} / \mathrm{m}^{3}$ vs. chamber controls & 1 & - \\
\hline Extended Proestrus & $5 \mathrm{mg} / \mathrm{m}^{3}$ vs. chamber controls & 1 & - \\
\hline Extended Proestrus & $10 \mathrm{mg} / \mathrm{m}^{3}$ vs. chamber controls & 1 & - \\
\hline Skipped Estrus & Overall & 1 & \\
\hline Skipped Estrus & $2.5 \mathrm{mg} / \mathrm{m}^{3}$ vs. chamber controls & 1 & - \\
\hline Skipped Estrus & $5 \mathrm{mg} / \mathrm{m}^{3}$ vs. chamber controls & 1 & - \\
\hline Skipped Estrus & $10 \mathrm{mg} / \mathrm{m}^{3}$ vs. chamber controls & 1 & - \\
\hline Skipped Diestrus & Overall & 1 & \\
\hline Skipped Diestrus & $2.5 \mathrm{mg} / \mathrm{m}^{3}$ vs. chamber controls & 1 & - \\
\hline Skipped Diestrus & $5 \mathrm{mg} / \mathrm{m}^{3}$ vs. chamber controls & 1 & - \\
\hline Skipped Diestrus & $10 \mathrm{mg} / \mathrm{m}^{3}$ vs. chamber controls & 1 & - \\
\hline \multicolumn{4}{|c|}{ Summary of Significant Groups } \\
\hline Overall Tests & $10 \mathrm{mg} / \mathrm{m}^{3}$ vs. Chamber Controls & $<0.001$ & \\
\hline
\end{tabular}

${ }^{a} \mathrm{~N}$ means that the exposed group had a lower probability of transitioning to the relevant abnormal state (extended estrus, extended diestrus, extended metestrus, extended proestrus, skipped estrus, or skipped diestrus) than did the chamber control group. 


\begin{tabular}{|c|c|c|c|c|c|c|c|c|c|c|c|c|c|c|c|c|c|}
\hline $\begin{array}{c}\text { Dose } \\
\left(\mathrm{mg} / \mathrm{m}^{3}\right)\end{array}$ & & & & & & & & & & & & & & & & & \\
\hline 0 & & & & & $\mathrm{E}$ & M & D & D & P & $\mathrm{E}$ & M & D & D & $\mathrm{E}$ & $\mathrm{E}$ & $\mathrm{M}$ & \\
\hline 0 & & & & & $\mathrm{E}$ & $\mathrm{D}$ & $\mathrm{D}$ & $\mathrm{D}$ & $\mathbf{P}$ & $\mathrm{E}$ & $\mathrm{D}$ & $\mathrm{D}$ & $\mathrm{D}$ & $P$ & $\bar{E}$ & $\bar{D}$ & \\
\hline 0 & & $\mathrm{D}$ & D & $\mathrm{P}$ & $E$ & $\mathrm{M}$ & $\mathrm{D}$ & $\mathrm{D}$ & $\mathrm{P}$ & $E$ & $\mathrm{D}$ & $\mathrm{D}$ & $\mathrm{D}$ & & 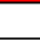 & & \\
\hline 0 & & D & D & $\mathrm{P}$ & $E$ & $\mathrm{M}$ & $\mathrm{D}$ & $\mathrm{D}$ & $\mathrm{P}$ & $E$ & $\mathrm{D}$ & $\mathrm{D}$ & $\mathrm{D}$ & & 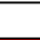 & & \\
\hline 0 & & & & $\mathrm{P}$ & $E$ & $\mathrm{M}$ & $\mathrm{D}$ & D & $\mathrm{P}$ & $E$ & $\mathrm{M}$ & $\mathrm{D}$ & $\mathrm{D}$ & $P$ & $\mathrm{E}$ & & \\
\hline 0 & & & & & $E$ & $\mathrm{M}$ & D & D & $\mathrm{P}$ & $\mathrm{E}$ & D & $\mathrm{D}$ & D & $P$ & $\mathrm{E}$ & M & \\
\hline 0 & & & $\bar{D}$ & $P$ & $E$ & $\mathrm{D}$ & $\mathrm{D}$ & $\mathrm{D}$ & $\mathbf{P}$ & $E$ & $\mathrm{M}$ & $\mathrm{D}$ & $\mathrm{D}$ & $P$ & 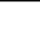 & & \\
\hline 0 & & & & $\mathrm{P}$ & $\mathrm{E}$ & D & D & D & P & $\mathrm{E}$ & D & D & D & P & $\mathrm{E}$ & & \\
\hline 0 & $\mathrm{D}$ & $\bar{D}$ & $\bar{D}$ & $P$ & $E$ & $\mathrm{D}$ & $\mathrm{D}$ & $\mathrm{D}$ & $\mathbf{P}$ & $\mathrm{E}$ & $\mathrm{D}$ & $\mathrm{D}$ & & & 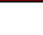 & & \\
\hline 0 & & & & $\mathrm{P}$ & $\mathrm{E}$ & $\mathrm{D}$ & $\mathrm{D}$ & $\mathrm{D}$ & $\mathrm{P}$ & $\mathrm{E}$ & $\mathrm{D}$ & $\mathrm{D}$ & $\mathrm{D}$ & $\mathrm{P}$ & $\mathrm{E}$ & & \\
\hline & & & & & & & & & & & & & & & & & \\
\hline 1.25 & & $\mathrm{D}$ & D & $P$ & $E$ & $\mathrm{D}$ & $\mathrm{D}$ & $\mathrm{D}$ & $\mathrm{P}$ & $E$ & $\mathrm{D}$ & $\mathrm{D}$ & $\mathrm{D}$ & & 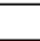 & & \\
\hline 1.25 & & & & $\mathrm{P}$ & $E$ & $\mathrm{M}$ & $\mathrm{D}$ & $\mathrm{D}$ & $\mathrm{P}$ & E & $\mathrm{M}$ & $\mathrm{D}$ & $\mathrm{D}$ & $\mathrm{P}$ & $E$ & & \\
\hline 1.25 & & & & & $\mathrm{E}$ & $\mathrm{D}$ & $\mathrm{D}$ & $\mathrm{D}$ & $\mathrm{P}$ & $\mathrm{E}$ & $\mathrm{D}$ & $\mathrm{D}$ & $\mathrm{D}$ & $\mathrm{P}$ & $\mathrm{E}$ & $\mathrm{D}$ & \\
\hline 1.25 & & & & & $E$ & $\mathrm{D}$ & $\mathrm{D}$ & $\mathrm{D}$ & $\mathbf{P}$ & $E$ & D & $\mathrm{D}$ & $\mathrm{D}$ & P & E & $\bar{D}$ & \\
\hline 1.25 & & $\mathrm{D}$ & D & $\mathrm{P}$ & $E$ & $\mathrm{D}$ & D & D & $\mathrm{P}$ & E & D & $\mathrm{D}$ & D & & 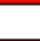 & & \\
\hline 1.25 & & & & $\mathrm{P}$ & $\mathrm{E}$ & $\mathrm{M}$ & $\mathrm{D}$ & $\mathrm{D}$ & $\mathrm{P}$ & $\mathrm{E}$ & $\mathrm{D}$ & $\mathrm{D}$ & $\mathrm{D}$ & $\mathrm{P}$ & $\mathrm{E}$ & & \\
\hline 1.25 & & & & $\mathrm{P}$ & $\mathrm{E}$ & D & D & D & $\mathrm{P}$ & $\mathrm{E}$ & D & D & D & P & E & & \\
\hline 1.25 & & & & & $E$ & $\mathrm{D}$ & $\mathrm{D}$ & $\mathrm{D}$ & $\mathrm{P}$ & $E$ & $\mathrm{D}$ & $\mathrm{D}$ & $\mathrm{D}$ & $\mathrm{P}$ & $\mathrm{E}$ & $\mathrm{D}$ & \\
\hline 1.25 & D & D & D & $P$ & $E$ & $\mathrm{M}$ & $\mathrm{D}$ & D & $\mathrm{P}$ & $\mathrm{E}$ & $\mathrm{M}$ & $\mathrm{D}$ & & & - & & \\
\hline 1.25 & $\mathrm{M}$ & $\mathrm{D}$ & $\mathrm{D}$ & $\mathrm{P}$ & $E$ & $\mathrm{M}$ & $\mathrm{D}$ & $\mathrm{D}$ & $\mathrm{P}$ & $\mathrm{E}$ & $\mathrm{M}$ & $\mathrm{D}$ & & & 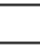 & & \\
\hline & & & & & 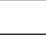 & & & & & 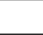 & & & & & & & \\
\hline 2.5 & $\mathrm{D}$ & $\mathrm{D}$ & D & $\bar{P}$ & $E$ & $\mathrm{D}$ & D & $\mathrm{D}$ & $\mathrm{P}$ & $\mathrm{E}$ & $\bar{M}$ & $\mathrm{D}$ & & & 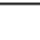 & & \\
\hline 2.5 & & & & & $E$ & $\mathrm{M}$ & D & $\mathrm{D}$ & $\mathrm{P}$ & $\mathrm{E}$ & $\mathrm{D}$ & $\mathrm{D}$ & $\mathrm{D}$ & $\mathrm{P}$ & E & $\mathrm{M}$ & \\
\hline 2.5 & & & & & $\mathrm{E}$ & $\mathrm{D}$ & $\mathrm{D}$ & $\mathrm{D}$ & $\mathrm{P}$ & $\mathrm{E}$ & $\mathrm{D}$ & $\mathrm{D}$ & $\mathrm{D}$ & $\mathrm{P}$ & $\mathrm{E}$ & $\mathrm{D}$ & \\
\hline 2.5 & & & & & $\mathrm{E}$ & $\mathrm{M}$ & $\mathrm{D}$ & $\mathrm{D}$ & $\mathrm{P}$ & $\mathrm{E}$ & $\mathrm{M}$ & $\mathrm{D}$ & D & P & $E$ & $\mathrm{M}$ & \\
\hline 2.5 & & & & $\mathrm{P}$ & $E$ & $\mathrm{M}$ & $\mathrm{D}$ & $\mathrm{D}$ & $\mathrm{P}$ & $\mathrm{E}$ & $\mathrm{M}$ & $\mathrm{D}$ & $\mathrm{D}$ & $\mathrm{P}$ & $\mathrm{E}$ & & \\
\hline 2.5 & & & $\mathrm{D}$ & $\mathrm{P}$ & $E$ & $\mathrm{M}$ & $\mathrm{D}$ & $\mathrm{D}$ & $\mathrm{P}$ & $\mathrm{E}$ & $\mathrm{M}$ & $\mathrm{D}$ & $\mathrm{D}$ & $\mathrm{P}$ & & & \\
\hline 2.5 & $\mathrm{M}$ & D & D & $\mathrm{P}$ & $\mathrm{E}$ & $\mathrm{M}$ & D & D & P & $\mathrm{E}$ & $\mathrm{M}$ & D & & & & & \\
\hline 2.5 & $\mathrm{M}$ & D & D & $\mathrm{P}$ & $E$ & $\mathrm{M}$ & $\mathrm{D}$ & D & $\mathrm{P}$ & $\mathrm{E}$ & $\mathrm{M}$ & $\mathrm{D}$ & & & 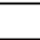 & & \\
\hline 2.5 & & & & & $E$ & $\mathrm{M}$ & D & $\mathrm{D}$ & $\mathrm{P}$ & $\mathrm{E}$ & $\mathrm{D}$ & $\mathrm{D}$ & $\mathrm{D}$ & $\mathrm{P}$ & E & $\mathrm{D}$ & \\
\hline 2.5 & & & $\mathrm{D}$ & $\mathrm{P}$ & $\mathrm{E}$ & $\mathrm{M}$ & $\mathrm{D}$ & $\mathrm{D}$ & $\mathrm{P}$ & $\mathrm{E}$ & $\mathrm{D}$ & $\mathrm{D}$ & $\mathrm{D}$ & $\mathrm{P}$ & & & \\
\hline & & & & & & & & & & 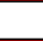 & & & & & & & \\
\hline 5.0 & & & & $\mathrm{P}$ & $E$ & $\mathrm{D}$ & $\mathrm{D}$ & $\mathrm{D}$ & $\mathrm{P}$ & $\mathrm{E}$ & $\mathrm{D}$ & $\mathrm{D}$ & $\mathrm{D}$ & $\mathrm{E}$ & $\mathrm{E}$ & & \\
\hline 5.0 & & & & & $\mathrm{E}$ & $\mathrm{D}$ & $\mathrm{D}$ & $\mathrm{D}$ & $\mathrm{P}$ & $\mathrm{E}$ & $\mathrm{D}$ & $\mathrm{D}$ & $\mathrm{D}$ & $\mathrm{E}$ & $\mathrm{E}$ & $\mathrm{D}$ & \\
\hline 5.0 & & D & $\mathrm{D}$ & $\mathrm{P}$ & $E$ & D & $\mathrm{D}$ & $\mathrm{D}$ & $\mathrm{P}$ & $\mathrm{E}$ & $\mathrm{D}$ & $\mathrm{D}$ & $\mathrm{D}$ & & & & \\
\hline 5.0 & & & $\mathrm{D}$ & $\mathrm{P}$ & $\mathrm{E}$ & $\mathrm{D}$ & $\mathrm{D}$ & $\mathrm{D}$ & $\mathbf{P}$ & $\mathrm{E}$ & $\mathrm{D}$ & $\mathrm{D}$ & $\mathrm{D}$ & $\mathrm{P}$ & & & \\
\hline 5.0 & & $\mathrm{D}$ & $\mathrm{D}$ & $\mathrm{D}$ & $\mathrm{P}$ & $\mathrm{E}$ & $\mathrm{D}$ & $\mathrm{D}$ & $\mathrm{D}$ & $\mathrm{E}$ & $\mathrm{E}$ & $\mathrm{D}$ & $\mathrm{D}$ & & & & \\
\hline 5.0 & $\mathrm{D}$ & $\mathrm{D}$ & $\mathrm{D}$ & $\mathrm{P}$ & $\mathrm{E}$ & $\mathrm{D}$ & $\mathrm{D}$ & $\mathrm{D}$ & $\mathrm{P}$ & $\mathrm{E}$ & $\mathrm{D}$ & $\mathrm{D}$ & & & & & \\
\hline 5.0 & $\mathrm{D}$ & $\mathrm{D}$ & D & $\mathrm{P}$ & $E$ & $\mathrm{D}$ & $\mathrm{D}$ & $\mathrm{D}$ & $\mathrm{P}$ & $\mathrm{E}$ & $\mathrm{D}$ & $\mathrm{D}$ & & & & & \\
\hline 5.0 & & & & & $E$ & $\mathrm{D}$ & $\mathrm{D}$ & $\mathrm{D}$ & $\mathrm{P}$ & $\mathrm{E}$ & $\mathrm{D}$ & $\mathrm{D}$ & $\mathrm{D}$ & $\mathrm{P}$ & $\mathrm{E}$ & $\mathrm{D}$ & \\
\hline 5.0 & & & & & $\mathrm{E}$ & $\mathrm{D}$ & $\mathrm{D}$ & $\mathrm{D}$ & $\mathrm{P}$ & $\mathrm{E}$ & $\mathrm{D}$ & $\mathrm{D}$ & $\mathrm{D}$ & $\mathrm{P}$ & $\mathrm{E}$ & $\mathrm{D}$ & \\
\hline 5.0 & & & & E & D & D & $\mathrm{D}$ & $\mathrm{D}$ & $\mathrm{P}$ & $\mathrm{E}$ & $\mathrm{D}$ & $\mathrm{D}$ & $\mathrm{D}$ & $\mathrm{P}$ & E & & \\
\hline
\end{tabular}

Figure H-1. Vaginal Cytology Plots for Female Rats in the Three-month Inhalation Study of Cobalt Metal

$\mathrm{D}=$ diestrus, $\mathrm{P}=$ proestrus, $\mathrm{E}=$ estrus, $\mathrm{M}=$ metestrus. 


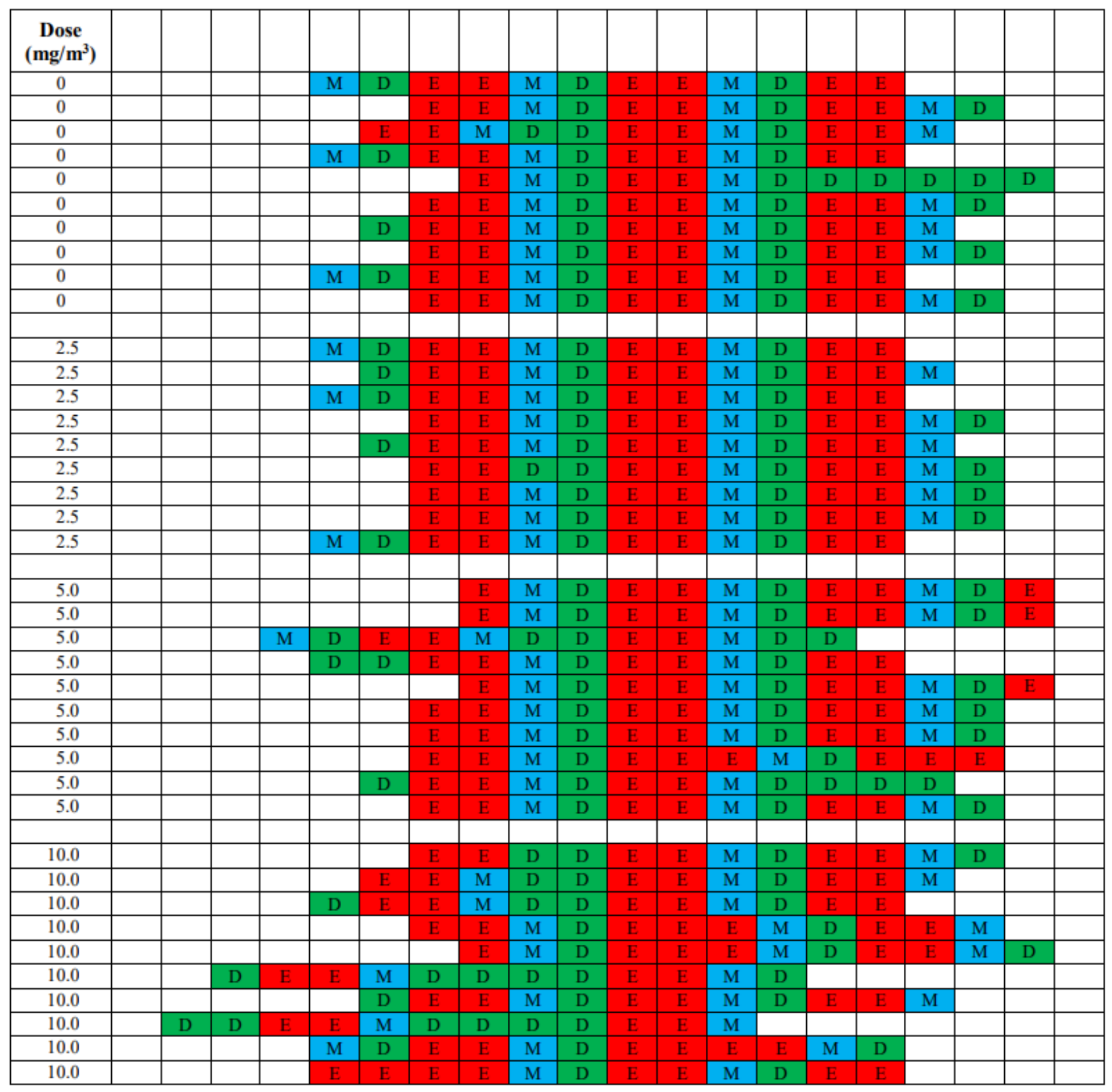

Figure H-2. Vaginal Cytology Plots for Female Mice in the Three-month Inhalation Study of Cobalt Metal

$\mathrm{D}=$ diestrus, $\mathrm{E}=$ estrus, $\mathrm{M}=$ metestrus. 


\section{Appendix I. Tissue Burden Results}

\section{Table of Contents}

I.1. Lung Deposition and Clearance Equations Used in the Two-week Inhalation Studies

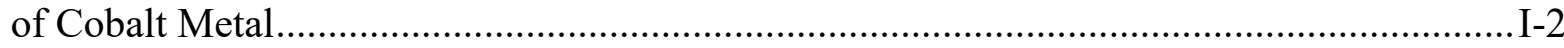

I.2. Lung Deposition and Clearance Equations Used in the Three-month and Two-year Inhalation Studies of Cobalt Metal.................................................................................. I-3

\section{Tables}

Table I-1. Tissue Weights, Cobalt Concentrations, and Cobalt Burdens for Rats in the Two-week Inhalation Study of Cobalt Metal

Table I-2. Deposition and Clearance Parameter Estimates for Female Rats in the Twoweek Inhalation Study of Cobalt Metal

Table I-3. Urine Volume, Creatinine and Cobalt Concentrations, and Cobalt Burdens for Rats on Day 12 in the Two-week Inhalation Study of Cobalt Metal

Table I-4. Tissue Weights, Cobalt Concentrations, and Cobalt Burdens for Female Rats in the Three-month Inhalation Study of Cobalt Metal

Table I-5. Lung Deposition and Clearance Parameter Estimates for Female Rats During the Recovery Period in the Three-month Inhalation Study of Cobalt Metal (Two-Compartment Model)

Table I-6. Lung Deposition and Clearance Parameter Estimates for Female Rats During the Exposure Period in the Three-month Inhalation Study of Cobalt Metal (One-Compartment Model)

Table I-7. Lung Weights, Cobalt Concentrations, and Cobalt Burdens for Female Rats in the Two-year Inhalation Study of Cobalt Metal

Table I-8. Lung Deposition and Clearance Parameter Estimates for Female Rats During the Exposure Periods in the Three-month and Two-year Inhalation Studies of Cobalt Metal (Two-Compartment Model)

Table I-9. Tissue Weights, Cobalt Concentrations, and Cobalt Burdens for Mice in the Two-week Inhalation Study of Cobalt Metal

Table I-10. Deposition and Clearance Parameter Estimates for Female Mice in the Twoweek Inhalation Study of Cobalt Metal

Table I-11. Tissue Weights, Cobalt Concentrations, and Cobalt Burdens for Female Mice in the Three-month Inhalation Study of Cobalt Metal.

Table I-12. Lung Deposition and Clearance Parameter Estimates for Female Mice During the Recovery Period in the Three-month Inhalation Study of Cobalt Metal (Two-Compartment Model)

Table I-13. Lung Deposition and Clearance Parameter Estimates for Female Mice During the Exposure Period in the Three-month Inhalation Study of Cobalt Metal (One-Compartment Model)

Table I-14. Lung Weights, Cobalt Concentrations, and Cobalt Burdens for Female Mice in the Two-year Inhalation Study of Cobalt Metal

Table I-15. Lung Deposition and Clearance Parameter Estimates for Female Mice During the Exposure Periods in the Three-month and Two-year Inhalation Studies of Cobalt Metal (Two-Compartment Model) 


\section{Figures}

Figure I-1. Lung Cobalt Burdens in 1.25 (top), 2.5 (middle), and 5 (bottom) $\mathrm{mg} / \mathrm{m}^{3}$ Female Rats in the Three-month and Two-year Inhalation Studies of Cobalt Metal.

Figure I-2. Lung Cobalt Burdens in 1.25 (top), 2.5 (middle), and 5 (bottom) mg/m $\mathrm{m}^{3}$ Female Mice in the Three-month and Two-year Inhalation Studies of Cobalt Metal 


\section{I.1. Lung Deposition and Clearance Equations Used in the Two-week Inhalation Studies of Cobalt Metal}

Lung clearance rates were calculated using postexposure data and Equation (1):

$$
\text { Equation (1): } \mathrm{A}_{(\mathrm{t})}=\mathrm{A}_{0}\left(\mathrm{e}^{-\mathrm{kt}}\right)
$$

where $A_{(\mathrm{t})}$ is the lung burden ( $\mu \mathrm{g}$ cobalt) at time $\mathrm{t}\left(\mathrm{t}=21\right.$ days postexposure), $A_{0}$ is the lung burden at $\mathrm{t}=0$ days postexposure, and $\mathrm{k}$ is the lung clearance rate constant (fraction cleared per day).

Lung clearance half-lives in days ( $t / 1 / 2)$ were calculated from Equation (2), where $\ln 2$ is the Napierian logarithm of 2:

Equation (2): $\mathrm{t}_{1 / 2}=\ln 2 / \mathrm{k}$

Deposition rates were calculated from lung cobalt burdens using Equation (3). The lung cobalt burden and time at terminal kill and the calculated lung clearance rate constant were used to solve for the deposition rate $\alpha(\mu \mathrm{g} /$ day).

$$
\text { Equation (3): } \mathrm{A}_{(\mathrm{t})}=(\alpha / \mathrm{k})\left(1-\mathrm{e}^{-\mathrm{kt}}\right)
$$

In Equation (3), $A_{(t)}$ is the lung burden ( $\mu \mathrm{g}$ cobalt ) at time $t[t=16$ (rats) or 17 (mice) days on study]; $\alpha$ is the amount of cobalt deposited $(\mu \mathrm{g} / \mathrm{day})$; and $\mathrm{k}$ is the first-order clearance rate constant derived from Equation (1). Steady-state or equilibrium lung burdens $\left(\mathrm{A}_{\mathrm{e}}, \mu \mathrm{g}\right.$ cobalt) were calculated according to Equation (4):

Equation (4): $A_{e}=\alpha / k$

Blood elimination rates and half-lives were calculated as described for lung cobalt burden, except blood cobalt concentration data were used.

\section{I.2. Lung Deposition and Clearance Equations Used in the Three- month and Two-year Inhalation Studies of Cobalt Metal}

Lung burdens during the recovery period initially decreased rapidly, followed by a slower clearance phase. The pattern of decrease fit a two-compartment, biexponential clearance model of the form shown in Equation (5):

$$
\text { Equation (5): } \mathrm{L}_{(\mathrm{t})}=\mathrm{Ae}^{-\mathrm{at}}+\mathrm{Be}^{-\mathrm{bt}}
$$

where $\mathrm{L}_{(\mathrm{t})}$ is the retained lung burden ( $\mu \mathrm{g}$ cobalt/lung) at any recovery time point $\mathrm{t}$ (days); $\mathrm{A}$ and $\mathrm{B}$ are the lung burdens ( $\mu \mathrm{g}$ cobalt $/ \mathrm{lung}$ ) at $\mathrm{t}=0$ days after exposure was terminated postexposure in the rapid and slow clearance compartments, respectively; and $\mathrm{a}$ and $\mathrm{b}$ are the lung clearance rate constants (in days ${ }^{-1}$ ) in the rapid and slow clearance compartments, respectively. This model was fitted to lung cobalt burdens normalized to exposure concentrations at 7, 14, 28, and 42 days after termination of exposures in the animals sampled. An exposure concentration term was included in Equation (5) to account for this normalization, and model output parameters A and B were subsequently converted to appropriate values by multiplying by exposure concentration. 
Half-lives for the rapid and slow clearance phases were calculated using Equation (6):

$$
\text { Equation (6): } \mathrm{t}_{1 / 2(\text { (rapid phase) }}=\ln 2 / \mathrm{a} \text { or } \mathrm{t}_{1 / 2(\text { slow phase })}=\ln 2 / \mathrm{b}
$$

where $t_{1} / 2$ is the rapid or slow phase lung clearance half-life (in days), $\ln 2$ is the Napierian logarithm of 2 , and $\mathrm{a}$ and $\mathrm{b}$ are the rapid phase and slow phase clearance rate constants, respectively, defined in Equation (5).

Given the nature of lung clearance observed with the recovery data, it was expected that a reasonable model for lung cobalt burden during exposure would incorporate a constant deposition rate and a two-compartment, biexponential clearance rate. Attempts were made to fit such a model to lung burden data collected during exposure. However, attempts to fit this model to the data were unsuccessful. The model fit to the data was poor, and standard errors for the parameter estimates were excessively large.

The inability of the two-compartment model to fit these data was possibly due to the fact that the data were inadequate to fully define the lung cobalt burden versus time curve, especially during the early part of the study. The rapid lung clearance half-lives calculated from the recovery period were on the order of 1 to 3 days. In examining plots of lung burden versus time during exposure, it was clear that lung burdens rapidly approached steady state. Within the first 5 days of exposure, lung burdens increased rapidly and were already approaching steady-state values. This was consistent with the rapid clearance half-life observed during the recovery period. Generally, after the first 5 to 40 days of exposure, lung burden values continued to increase slowly for the remainder of the exposure period as they asymptotically approached steady state over time. However, given that no data were collected until 5 days of exposure, there were insufficient data to define the early part of the lung burden versus the time curve during this period when lung burdens were rapidly changing. Thus, a possible explanation for the inability to fit the two-compartment model to lung burden data during exposure was the lack of data during the very early part of the study ( $<5$ days).

However, a model that assumes a constant cobalt deposition rate and a one-compartment, monoexponential clearance rate provided a reasonable fit to the data collected during exposure. This model is defined by Equations (7), (8), and (9):

$$
\begin{aligned}
& \text { Equation (7): } \mathrm{L}_{(\mathrm{t})}=(\mathrm{D} / \mathrm{k})\left(1-\mathrm{e}^{-\mathrm{kt}}\right) \\
& \text { Equation (8): } \mathrm{t}_{1 / 2}=\ln 2 / \mathrm{k} \\
& \text { Equation (9): } \mathrm{L}_{\mathrm{ss}}=\mathrm{D} / \mathrm{k}
\end{aligned}
$$

where $\mathrm{L}_{(\mathrm{t})}$ is the retained lung burden ( $\mu \mathrm{g}$ cobalt/lung) at any time $\mathrm{t}$ (days) during exposure; $\mathrm{D}$ is the deposition rate of cobalt in the lungs ( $\mu \mathrm{g}$ cobalt/lung per day); $\mathrm{k}$ is the lung clearance rate constant $\left(\right.$ days $\left.^{-1}\right) ; t_{1} / 2$ is the clearance half-life in days; $\ln 2$ is the Napierian logarithm of 2; and $L_{s s}$ is the predicted steady-state lung burden ( $\mu \mathrm{g}$ cobalt/lung).

This one-compartment model does not adequately define the two-phase lung clearance process that was seen during the recovery period. This model predicts only one clearance half-life, and it is expected that this half-life would be intermediate between the true rapid and slow clearance half-lives. However, it was found that this model did generally fit the data adequately to provide a reasonably precise estimate for the cobalt deposition rate, which is important in predicting the 
dose of cobalt to the lungs arising from the inhalation process. While this model does not adequately describe the true clearance process, it provides reasonable predictive power for determining the lung dose. The two-compartment model provides an adequate description of the lung clearance process during the recovery period. Thus, between the two models used for data collected during and after exposure, reasonable estimates of both deposition and clearance parameters were obtained.

Because the 3-month study postexposure lung cobalt burden data fit the model described by Equation (5) reasonably well, the model chosen for the 2-year studies also included both rapid and slow lung clearance phases. However, lung burden data collected during the 2-year studies were all collected during the in-life parts of the studies because there was not a recovery period. Accordingly, the model used for the 2-year studies had to account for deposition rates as well as clearance rates, because both deposition and clearance were occurring during the in-life part of the study.

The model used for the 2-year studies assumed zero-order (constant) deposition and first-order (with respect to lung burden) clearance rates and included rapid and slow clearance phases. This model is described by Equation (10):

$$
\text { Equation (10): } \mathrm{L}_{(\mathrm{t})}=\mathrm{A} / \mathrm{k}_{\mathrm{a}}\left(1-\mathrm{e}_{\mathrm{a}}^{-\mathrm{k}^{\mathrm{t}}}\right)+\mathrm{B} / \mathrm{k}_{\mathrm{b}}\left(1-\mathrm{e}^{\left.-\mathrm{k}_{\mathrm{b}}{ }^{t}\right)}\right.
$$

Equation (10) is essentially the sum of two processes that incorporate zero-order deposition and first-order clearance. A and B represent the deposition rates ( $\mu \mathrm{g}$ cobalt/day) in the rapid and slow phases, respectively, and $\mathrm{k}_{\mathrm{a}}$ and $\mathrm{k}_{\mathrm{b}}$ represent the lung clearance rate constants $\left(\right.$ days $^{-1}$ ) in the rapid and slow clearance phases, respectively.

This model was fit to the lung cobalt burden data collected during the in-life parts of the 3-month and 2-year studies using data collected in both studies from the $1.25,2.5$, and $5 \mathrm{mg} / \mathrm{m}^{3}$ exposure groups. The model was fit to the data from each exposure group using SAS PROC NLIN (SAS Institute Inc., Cary, NC). This fit provided direct estimates of $\mathrm{A}, \mathrm{k}_{\mathrm{a}}, \mathrm{B}$, and $\mathrm{k}_{\mathrm{b}}$ along with their asymptotic standard errors. These values were used to calculate the following quantities along with their approximate standard errors using propagation of error techniques:

- Fraction of deposition in the slow clearance phase: $F_{B}=B /(A+B)$

- Half-life of the rapid clearance phase: $t_{1 / 2 a}=\ln 2 / k_{a}$ (days)

- Half-life of the slow clearance phase: $\mathrm{t}_{2 / \mathrm{b}}=\ln 2 / \mathrm{k}_{\mathrm{b}}$ (days)

- Theoretical steady-state lung burden for the rapid phase: $\mathrm{L}_{\mathrm{SSa}}=\mathrm{A} / \mathrm{k}_{\mathrm{a}}$ ( $\mu \mathrm{g}$ cobalt $/$ lung)

- Theoretical steady-state lung burden for the slow phase: $\mathrm{L}_{\mathrm{SSb}}=\mathrm{B} / \mathrm{k}_{\mathrm{b}}$ ( $\mu \mathrm{g}$ cobalt $/ \mathrm{lung}$ )

Due to the potential for more uncertainty with relatively higher lung cobalt burdens, several weighting schemes were investigated, including unweighted, $1 /$ mean, and $1 /$ variance. Review of the results suggested that $1 /$ mean was the best choice, so all results presented are from model fits using $1 /$ mean weighting. 
Cobalt Metal, NTP TR 581

Table I-1. Tissue Weights, Cobalt Concentrations, and Cobalt Burdens for Rats in the Two-week Inhalation Study of Cobalt Metal ${ }^{\mathrm{a}}$

\begin{tabular}{|c|c|c|c|c|c|}
\hline & $\begin{array}{l}\text { Chamber } \\
\text { Control }\end{array}$ & $2.5 \mathrm{mg} / \mathrm{m}^{3}$ & $5 \mathrm{mg} / \mathrm{m}^{3}$ & $10 \mathrm{mg} / \mathrm{m}^{3}$ & $20 \mathrm{mg} / \mathrm{m}^{3}$ \\
\hline \multicolumn{6}{|l|}{ Male } \\
\hline $\mathbf{n}$ & 5 & 5 & 5 & 5 & $0^{\mathrm{b}}$ \\
\hline \multicolumn{6}{|l|}{ Blood } \\
\hline \multicolumn{6}{|c|}{$\mu \mathrm{g} \mathrm{Co} / \mathrm{g}$ blood } \\
\hline Day 16 & 0 & $0.14 \pm 0.01$ & $0.27 \pm 0.02$ & $0.45 \pm 0.02$ & - \\
\hline \multicolumn{6}{|l|}{ Serum } \\
\hline \multicolumn{6}{|c|}{$\mu \mathrm{g} \mathrm{Co} / \mathrm{g}$ serum } \\
\hline Day 16 & 0 & $0.28 \pm 0.01$ & $0.48 \pm 0.02$ & $0.72 \pm 0.04$ & - \\
\hline \multicolumn{6}{|l|}{ Right femur } \\
\hline \multicolumn{6}{|c|}{ Absolute right femur wt (g) } \\
\hline Day 16 & $0.142 \pm 0.008$ & $0.143 \pm 0.004$ & $0.135 \pm 0.003$ & $0.116 \pm 0.005^{*}$ & - \\
\hline \multicolumn{6}{|c|}{$\mu \mathrm{g} \mathrm{Co} / \mathrm{g}$ right femur } \\
\hline Day 16 & 0 & $0.58 \pm 0.04$ & $1.08 \pm 0.09$ & $2.24 \pm 0.13$ & - \\
\hline \multicolumn{6}{|c|}{$\mu \mathrm{g} \mathrm{Co} /$ right femur } \\
\hline Day 16 & 0 & $0.082 \pm 0.006$ & $0.146 \pm 0.014$ & $0.253 \pm 0.009$ & - \\
\hline \multicolumn{6}{|c|}{$\mu \mathrm{g} \mathrm{Co} /$ right femur per $\mathrm{mg} \mathrm{Co} / \mathrm{m}^{3}$} \\
\hline Day 16 & NA & $0.033 \pm 0.002$ & $0.029 \pm 0.003$ & $0.025 \pm 0.001$ & - \\
\hline \multicolumn{6}{|l|}{ Heart } \\
\hline \multicolumn{6}{|c|}{ Absolute heart wt (g) } \\
\hline Day 16 & $0.551 \pm 0.013$ & $0.540 \pm 0.007$ & $0.527 \pm 0.014$ & $0.468 \pm 0.014^{* *}$ & - \\
\hline \multicolumn{6}{|c|}{$\mu \mathrm{g} \mathrm{Co} / \mathrm{g}$ heart } \\
\hline Day 16 & 0 & $0.48 \pm 0.03$ & $0.73 \pm 0.06$ & $1.07 \pm 0.06$ & - \\
\hline \multicolumn{6}{|c|}{$\mu \mathrm{g} \mathrm{Co} /$ heart } \\
\hline Day 16 & 0 & $0.258 \pm 0.014$ & $0.384 \pm 0.034$ & $0.498 \pm 0.024$ & - \\
\hline \multicolumn{6}{|c|}{$\mu \mathrm{g} \mathrm{Co} /$ heart per $\mathrm{mg} \mathrm{Co} / \mathrm{m}^{3}$} \\
\hline Day 16 & NA & $0.103 \pm 0.006$ & $0.077 \pm 0.007$ & $0.050 \pm 0.002$ & - \\
\hline \multicolumn{6}{|c|}{ Right kidney } \\
\hline \multicolumn{6}{|c|}{ Absolute right kidney wt (g) } \\
\hline Day 16 & $0.586 \pm 0.017$ & $0.579 \pm 0.008$ & $0.566 \pm 0.016$ & $0.519 \pm 0.018$ & - \\
\hline \multicolumn{6}{|c|}{$\mu \mathrm{g} \mathrm{Co} / \mathrm{g}$ right kidney } \\
\hline Day 16 & 0 & $2.04 \pm 0.02$ & $3.26 \pm 0.18$ & $5.90 \pm 0.38$ & - \\
\hline \multicolumn{6}{|c|}{$\mu \mathrm{g}$ Co/right kidney } \\
\hline Day 16 & 0 & $1.177 \pm 0.018$ & $1.838 \pm 0.116$ & $3.031 \pm 0.146$ & - \\
\hline
\end{tabular}


Cobalt Metal, NTP TR 581

\begin{tabular}{|c|c|c|c|c|c|}
\hline & $\begin{array}{l}\text { Chamber } \\
\text { Control }\end{array}$ & $2.5 \mathrm{mg} / \mathrm{m}^{3}$ & $5 \mathrm{mg} / \mathrm{m}^{3}$ & $10 \mathrm{mg} / \mathrm{m}^{3}$ & $20 \mathrm{mg} / \mathrm{m}^{3}$ \\
\hline \multicolumn{6}{|c|}{$\mu \mathrm{g} \mathrm{Co} /$ right kidney per $\mathrm{mg} \mathrm{Co} / \mathrm{m}^{3}$} \\
\hline Day 16 & NA & $0.471 \pm 0.007$ & $0.368 \pm 0.023$ & $0.303 \pm 0.015$ & - \\
\hline \multicolumn{6}{|l|}{ Liver } \\
\hline \multicolumn{6}{|c|}{ Absolute liver wt (g) } \\
\hline Day 16 & $5.834 \pm 0.164$ & $5.102 \pm 0.094^{*}$ & $5.081 \pm 0.156^{*}$ & $4.292 \pm 0.238^{* *}$ & - \\
\hline \multicolumn{6}{|c|}{$\mu \mathrm{g} \mathrm{Co} / \mathrm{g}$ liver } \\
\hline Day 16 & 0 & $2.69 \pm 0.09$ & $5.14 \pm 0.46$ & $14.00 \pm 1.58$ & - \\
\hline \multicolumn{6}{|c|}{$\mu \mathrm{g} \mathrm{Co/liver}$} \\
\hline Day 16 & 0 & $13.638 \pm 0.335$ & $25.956 \pm 2.189$ & $58.632 \pm 4.098$ & - \\
\hline \multicolumn{6}{|c|}{$\mu \mathrm{g} \mathrm{Co} /$ liver per $\mathrm{mg} \mathrm{Co} / \mathrm{m}^{3}$} \\
\hline Day 16 & NA & $5.455 \pm 0.134$ & $5.191 \pm 0.438$ & $5.863 \pm 0.410$ & - \\
\hline \multicolumn{6}{|l|}{ Total lung ${ }^{\mathrm{c}}$} \\
\hline \multicolumn{6}{|c|}{ Absolute total lung wt (g) } \\
\hline \multicolumn{5}{|c|}{$\mu \mathrm{g} \mathrm{Co/total} \mathrm{lung}$} & - \\
\hline Day 16 & 0 & $10.806 \pm 0.619$ & $20.077 \pm 0.675$ & $23.518 \pm 1.647$ & - \\
\hline \multicolumn{6}{|c|}{$\mu \mathrm{g} \mathrm{Co} /$ total lung per $\mathrm{mg} \mathrm{Co} / \mathrm{m}^{3}$} \\
\hline Day 16 & NA & $4.322 \pm 0.247$ & $4.015 \pm 0.135$ & $2.352 \pm 0.165$ & - \\
\hline \multicolumn{6}{|l|}{ Right lung $^{\mathrm{d}}$} \\
\hline \multicolumn{6}{|c|}{ Absolute right lung wt (g) } \\
\hline Day 16 & $0.573 \pm 0.047$ & $0.570 \pm 0.055$ & $0.578 \pm 0.020$ & $0.636 \pm 0.063$ & - \\
\hline \multicolumn{6}{|c|}{$\mu \mathrm{g} \mathrm{Co} / \mathrm{g}$ right lung } \\
\hline Day 16 & 0 & $9.53 \pm 1.07$ & $16.95 \pm 0.38$ & $18.44 \pm 0.70$ & - \\
\hline \multicolumn{6}{|l|}{ Right testis } \\
\hline \multicolumn{6}{|c|}{ Absolute right testis wt (g) } \\
\hline Day 16 & $0.850 \pm 0.040$ & $0.870 \pm 0.016$ & $0.831 \pm 0.033$ & $0.563 \pm 0.089$ & - \\
\hline \multicolumn{6}{|c|}{$\mu \mathrm{g} \mathrm{Co} / \mathrm{g}$ right testis } \\
\hline Day 16 & 0 & $0.09 \pm 0.00$ & $0.20 \pm 0.02$ & $0.48 \pm 0.06$ & - \\
\hline \multicolumn{6}{|c|}{$\mu \mathrm{g} \mathrm{Co} /$ right testis } \\
\hline Day 16 & 0 & $0.086 \pm 0.003$ & $0.170 \pm 0.014$ & $0.245 \pm 0.011$ & - \\
\hline \multicolumn{6}{|c|}{$\mu \mathrm{g} \mathrm{Co} /$ right testis per $\mathrm{mg} \mathrm{Co} / \mathrm{m}^{3}$} \\
\hline Day 16 & NA & $0.034 \pm 0.001$ & $0.034 \pm 0.003$ & $0.024 \pm 0.001$ & - \\
\hline
\end{tabular}




\section{Cobalt Metal, NTP TR 581}

\begin{tabular}{|c|c|c|c|c|}
\hline $\begin{array}{c}\text { Chamber } \\
\text { Control }\end{array}$ & $2.5 \mathrm{mg} / \mathrm{m}^{3}$ & $5 \mathrm{mg} / \mathrm{m}^{3}$ & $10 \mathrm{mg} / \mathrm{m}^{3}$ & $20 \mathrm{mg} / \mathrm{m}^{3}$ \\
\hline
\end{tabular}

\section{Female}

n

$\begin{array}{llllll}\text { Day } 16 & 5 & 5 & 5 & 5 & 2 \\ \text { Week } 3 \text { PE } & 3 & 3 & 3 & 3 & 1^{\mathrm{e}}\end{array}$

Blood

$\mu \mathrm{g} \mathrm{Co} / \mathrm{g}$ blood

$\begin{array}{lllllc}\text { Day 16 } & 0^{\mathrm{f}} & 0.13 \pm 0.01^{\mathrm{f}} & 0.25 \pm 0.02^{\mathrm{f}} & 0.48 \pm 0.02^{\mathrm{f}} & 1.06 \pm 0.17^{\mathrm{g}} \\ \text { Week 3 PE } & 0 & 0.02 \pm 0.00 & 0.06 \pm 0.01 & 0.12 \pm 0.01 & 0.22\end{array}$

Serum

$\mu \mathrm{g} \mathrm{Co} / \mathrm{g}$ serum

$\begin{array}{lllllc}\text { Day 16 } & 0^{\mathrm{f}} & 0.25 \pm 0.01^{\mathrm{f}} & 0.44 \pm 0.04^{\mathrm{f}} & 0.76 \pm 0.04^{\mathrm{f}} & 1.88 \pm 0.21^{\mathrm{g}} \\ \text { Week 3 PE } & 0 & 0.00 \pm 0.00 & 0.00 \pm 0.00 & 0.00 \pm 0.00 & 0.01\end{array}$

Right femur

Absolute right femur wt (g)
Day 16
$0.105 \pm 0.006$
$0.115 \pm 0.004$
$0.108 \pm 0.003$
$0.100 \pm 0.006$
$0.070 \pm 0.005$

$\mu \mathrm{g} \mathrm{Co/g} \mathrm{right} \mathrm{femur}$
Day 16
0
$0.69 \pm 0.06$
$1.30 \pm 0.11$
$2.40 \pm 0.24$
$6.09 \pm 0.72$

$\mu \mathrm{g} \mathrm{Co/right} \mathrm{femur}$
Day 16
0
$0.080 \pm 0.005$

$0.139 \pm 0.007$

$0.235 \pm 0.012$

$0.418 \pm 0.018$

$\mu \mathrm{g} \mathrm{Co} /$ right femur per $\mathrm{mg} \mathrm{Co} / \mathrm{m}^{3}$
Day 16
NA
$0.032 \pm 0.002$
$0.028 \pm 0.001$
$0.023 \pm 0.001$
$0.021 \pm 0.001$

Heart

Absolute heart wt (g)

Day 16

$$
0.420 \pm 0.013
$$

$0.469 \pm 0.013$

$0.445 \pm 0.013$

$0.416 \pm 0.006$

$0.341 \pm 0.004$

$\mu \mathrm{g} \mathrm{Co} / \mathrm{g}$ heart

Day 16

0

$0.44 \pm 0.03$

$0.76 \pm 0.04$

$1.03 \pm 0.03$

$1.81 \pm 0.19$

$\mu \mathrm{g} \mathrm{Co} /$ heart

Day 16

0

$0.206 \pm 0.008$

$0.339 \pm 0.015$

$0.427 \pm 0.014$

$0.613 \pm 0.068$

$\mu \mathrm{g} \mathrm{Co} /$ heart per $\mathrm{mg} \mathrm{Co} / \mathrm{m}^{3}$

Day 16

NA

$0.083 \pm 0.003$

$0.068 \pm 0.003$

$0.043 \pm 0.001$

$0.031 \pm 0.003$

Right kidney

Absolute right kidney wt (g)

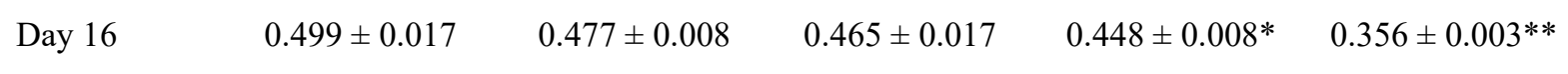
$\mu \mathrm{g}$ Co/g right kidney

Day 16

0

$1.78 \pm 0.07$

$3.01 \pm 0.21$

$5.74 \pm 0.23$

$13.87 \pm 0.51$ 


\section{Cobalt Metal, NTP TR 581}

\begin{tabular}{|c|c|c|c|c|c|}
\hline & $\begin{array}{l}\text { Chamber } \\
\text { Control }\end{array}$ & $2.5 \mathrm{mg} / \mathrm{m}^{3}$ & $5 \mathrm{mg} / \mathrm{m}^{3}$ & $10 \mathrm{mg} / \mathrm{m}^{3}$ & $20 \mathrm{mg} / \mathrm{m}^{3}$ \\
\hline \multicolumn{6}{|c|}{$\mu \mathrm{g}$ Co/right kidney } \\
\hline Day 16 & 0 & $0.844 \pm 0.025$ & $1.382 \pm 0.059$ & $2.556 \pm 0.111$ & $4.896 \pm 0.145$ \\
\hline \multicolumn{6}{|c|}{$\mu \mathrm{g} \mathrm{Co} /$ right kidney per $\mathrm{mg} \mathrm{Co} / \mathrm{m}^{3}$} \\
\hline Day 16 & NA & $0.338 \pm 0.010$ & $0.276 \pm 0.012$ & $0.256 \pm 0.011$ & $0.245 \pm 0.007$ \\
\hline \multicolumn{6}{|l|}{ Liver } \\
\hline \multicolumn{6}{|c|}{ Absolute liver wt (g) } \\
\hline Day 16 & $4.073 \pm 0.163$ & $3.768 \pm 0.046$ & $3.613 \pm 0.125^{*}$ & $3.440 \pm 0.049 * *$ & $2.567 \pm 0.061 * *$ \\
\hline \multicolumn{6}{|l|}{$\mu \mathrm{g} \mathrm{Co/g} \mathrm{liver}$} \\
\hline Day 16 & 0 & $2.15 \pm 0.13$ & $4.81 \pm 0.43$ & $15.93 \pm 1.59$ & $59.02 \pm 9.05$ \\
\hline \multicolumn{6}{|l|}{$\mu \mathrm{g} \mathrm{Co/liver}$} \\
\hline Day 16 & 0 & $8.064 \pm 0.403$ & $17.223 \pm 1.292$ & $54.542 \pm 5.203$ & $150.785 \pm 19.614$ \\
\hline \multicolumn{6}{|c|}{$\mu \mathrm{g} \mathrm{Co} /$ liver per $\mathrm{mg} \mathrm{Co} / \mathrm{m}^{3}$} \\
\hline Day 16 & NA & $3.226 \pm 0.161$ & $3.445 \pm 0.258$ & $5.454 \pm 0.520$ & $7.539 \pm 0.981$ \\
\hline \multicolumn{6}{|l|}{ Total lung ${ }^{\mathrm{c}}$} \\
\hline \multicolumn{6}{|c|}{ Absolute total lung wt (g) } \\
\hline Day 16 & $0.850 \pm 0.032^{\mathrm{f}}$ & $0.891 \pm 0.051^{\mathrm{f}}$ & $0.943 \pm 0.034^{\mathrm{f}}$ & $1.004 \pm 0.042 * \mathrm{f}$ & $0.996 \pm 0.026^{* g}$ \\
\hline Week 3 PE & $0.90 \pm 0.02$ & $0.96 \pm 0.07$ & $1.24 \pm 0.10^{*}$ & $1.17 \pm 0.04^{*}$ & 1.41 \\
\hline \multicolumn{6}{|c|}{$\mu \mathrm{g}$ Co/total lung } \\
\hline Day 16 & $0^{\mathrm{f}}$ & $9.713 \pm 0.431^{\mathrm{f}}$ & $17.289 \pm 0.805^{\mathrm{f}}$ & $20.710 \pm 1.070^{\mathrm{f}}$ & $49.842 \pm 11.212^{\mathrm{g}}$ \\
\hline Week 3 PE & 0 & $0.605 \pm 0.011$ & $1.288 \pm 0.113$ & $1.278 \pm 0.112$ & 1.514 \\
\hline \multicolumn{6}{|c|}{$\mu \mathrm{g} \mathrm{Co} /$ total lung per $\mathrm{mg} \mathrm{Co} / \mathrm{m}^{3}$} \\
\hline Day 16 & $\mathrm{NA}^{\mathrm{f}}$ & $3.885 \pm 0.173^{\mathrm{f}}$ & $3.458 \pm 0.161^{\mathrm{f}}$ & $2.071 \pm 0.107^{\mathrm{f}}$ & $2.492 \pm 0.561^{\mathrm{g}}$ \\
\hline Week 3 PE & NA & $0.242 \pm 0.004$ & $0.258 \pm 0.023$ & $0.128 \pm 0.011$ & 0.076 \\
\hline \multicolumn{6}{|l|}{ Left lung ${ }^{\mathrm{h}}$} \\
\hline \multicolumn{6}{|c|}{ Absolute left lung wt (g) } \\
\hline Day 16 & $0.237 \pm 0.048^{\mathrm{i}}$ & $0.333 \pm 0.061^{\mathrm{i}}$ & $0.299 \pm 0.009^{\mathrm{i}}$ & $0.275 \pm 0.003^{\mathrm{i}}$ & $0.299^{\mathrm{e}}$ \\
\hline Week 3 PE & $0.24 \pm 0.01$ & $0.28 \pm 0.02$ & $0.38 \pm 0.04 * *$ & $0.35 \pm 0.00 * *$ & 0.40 \\
\hline \multicolumn{6}{|c|}{$\mu \mathrm{g} \mathrm{Co} / \mathrm{g}$ left lung } \\
\hline Day 16 & $0^{\mathrm{i}}$ & $9.89 \pm 2.35^{\mathrm{i}}$ & $19.04 \pm 0.32^{\mathrm{i}}$ & $21.87 \pm 2.07^{\mathrm{i}}$ & $35.68^{e}$ \\
\hline Week 3 PE & 0 & $0.62 \pm 0.01$ & $0.93 \pm 0.17$ & $1.09 \pm 0.10$ & 0.96 \\
\hline \multicolumn{6}{|c|}{$\mu \mathrm{g} \mathrm{Co/left} \mathrm{lung}$} \\
\hline Day 16 & $0^{\mathrm{i}}$ & $3.150 \pm 0.178^{\mathrm{i}}$ & $5.698 \pm 0.267^{\mathrm{i}}$ & $6.000 \pm 0.514^{\mathrm{i}}$ & $10.669^{\mathrm{e}}$ \\
\hline Week 3 PE & 0 & $0.171 \pm 0.011$ & $0.335 \pm 0.027$ & $0.377 \pm 0.032$ & 0.386 \\
\hline \multicolumn{6}{|c|}{$\mu \mathrm{g} \mathrm{Co} /$ left lung per $\mathrm{mg} \mathrm{Co} / \mathrm{m}^{3}$} \\
\hline Day 16 & $\mathrm{NA}^{\mathrm{i}}$ & $1.260 \pm 0.071^{\mathrm{i}}$ & $1.140 \pm 0.053^{\mathrm{i}}$ & $0.600 \pm 0.051^{\mathrm{i}}$ & $0.533^{\mathrm{e}}$ \\
\hline
\end{tabular}


Cobalt Metal, NTP TR 581

\begin{tabular}{|c|c|c|c|c|c|}
\hline & $\begin{array}{l}\text { Chamber } \\
\text { Control }\end{array}$ & $2.5 \mathrm{mg} / \mathrm{m}^{3}$ & $5 \mathrm{mg} / \mathrm{m}^{3}$ & $10 \mathrm{mg} / \mathrm{m}^{3}$ & $20 \mathrm{mg} / \mathrm{m}^{3}$ \\
\hline Week 3 PE & NA & $0.069 \pm 0.004$ & $0.067 \pm 0.005$ & $0.038 \pm 0.003$ & 0.019 \\
\hline \multicolumn{6}{|l|}{ Right lung ${ }^{\mathrm{d}}$} \\
\hline \multicolumn{6}{|c|}{ Absolute right lung wt (g) } \\
\hline Day 16 & $0.404 \pm 0.018^{\mathrm{f}}$ & $0.446 \pm 0.036^{\mathrm{f}}$ & $0.471 \pm 0.025^{\mathrm{f}}$ & $0.519 \pm 0.022 * * \mathrm{f}$ & $0.539 \pm 0.018^{* * \mathrm{~g}}$ \\
\hline Week 3 PE & $0.44 \pm 0.01$ & $0.48 \pm 0.03$ & $0.63 \pm 0.05 * *$ & $0.60 \pm 0.01^{* *}$ & 0.77 \\
\hline \multicolumn{6}{|c|}{$\mu \mathrm{g} \mathrm{Co} / \mathrm{g}$ right lung } \\
\hline Day 16 & $0^{\mathrm{f}}$ & $11.06 \pm 0.66^{\mathrm{f}}$ & $18.43 \pm 0.92^{\mathrm{f}}$ & $20.82 \pm 1.31^{\mathrm{f}}$ & $49.55 \pm 9.81^{\mathrm{f}}$ \\
\hline Week 3 PE & 0 & $0.64 \pm 0.04$ & $1.06 \pm 0.16$ & $1.09 \pm 0.10$ & 1.07 \\
\hline \multicolumn{6}{|c|}{$\mu \mathrm{g} \mathrm{Co} /$ right lung } \\
\hline Day 16 & $0^{\mathrm{i}}$ & $5.501 \pm 0.323^{\mathrm{i}}$ & $10.283 \pm 0.593^{\mathrm{i}}$ & $11.304 \pm 1.402^{\mathrm{i}}$ & $17.765^{\mathrm{e}}$ \\
\hline Week 3 PE & 0 & $0.302 \pm 0.006$ & $0.652 \pm 0.057$ & $0.661 \pm 0.059$ & 0.823 \\
\hline \multicolumn{6}{|c|}{$\mu \mathrm{g} \mathrm{Co} /$ right lung per $\mathrm{mg} \mathrm{Co} / \mathrm{m}^{3}$} \\
\hline Day 16 & $\mathrm{NA}^{\mathrm{i}}$ & $2.200 \pm 0.129^{\mathrm{i}}$ & $2.057 \pm 0.119^{\mathrm{i}}$ & $1.130 \pm 0.140^{\mathrm{i}}$ & $0.888^{\mathrm{e}}$ \\
\hline Week 3 PE & NA & $0.121 \pm 0.002$ & $0.130 \pm 0.011$ & $0.066 \pm 0.006$ & 0.041 \\
\hline
\end{tabular}

*Significantly different $(\mathrm{P} \leq 0.05)$ from the chamber control group by Williams' or Shirley's test.

$* * \mathrm{P} \leq 0.01$.

${ }^{a}$ Data are presented as mean \pm standard error. All values except absolute tissue weights are control corrected. Statistical tests were performed only on absolute tissue weight data. All $40 \mathrm{mg} / \mathrm{m}^{3}$ rats died before the end of the study. NA = not applicable;

$\mathrm{PE}=$ postexposure.

${ }^{\mathrm{b}} \mathrm{All} 20 \mathrm{mg} / \mathrm{m}^{3}$ male rats died before the end of the study; no data are available for this group.

${ }^{\mathrm{c}}$ Total lung cobalt burden was calculated using the weight of the total lung with mainstem bronchi at collection and cobalt concentration measured in the right lung.

${ }^{\mathrm{d}}$ Right lung cobalt burden was calculated using the right lung weight and the concentration of cobalt measured in the right lung.

${ }^{e} \mathrm{n}=1$; No standard error was calculated; less than two measurements were available.

$\mathrm{f} n=7$.

$\mathrm{g}_{\mathrm{n}}=3$.

${ }^{h}$ Left lung cobalt burden was calculated using the left lung weight and the concentration of cobalt measured in the left lung.

${ }^{\mathrm{i}} \mathrm{n}=2$. 
Table I-2. Deposition and Clearance Parameter Estimates for Female Rats in the Two-week Inhalation Study of Cobalt Metal ${ }^{\text {a }}$

\begin{tabular}{|c|c|c|c|c|c|}
\hline Tissue & $\begin{array}{c}\text { Exposure } \\
\text { Concentration }\left(\mathbf{m g} / \mathbf{m}^{3}\right) \\
\end{array}$ & k $\left(\right.$ days $\left.^{-1}\right)$ & $\mathbf{t}_{1 / 2}$ (days) & $\alpha(\mu g$ Co/day) & $A_{e}\left(\mu g C_{0}\right)$ \\
\hline \multirow[t]{4}{*}{ Blood } & 2.5 & 0.07 & 9.45 & NA & NA \\
\hline & 5 & 0.06 & 11.09 & NA & NA \\
\hline & 10 & 0.07 & 10.35 & NA & NA \\
\hline & 20 & 0.08 & 9.15 & NA & NA \\
\hline \multirow[t]{4}{*}{ Serum } & 2.5 & $-^{\mathrm{b}}$ & NA & NA & NA \\
\hline & 5 & - & NA & NA & NA \\
\hline & 10 & 0.21 & 3.35 & NA & NA \\
\hline & 20 & 0.25 & 2.77 & NA & NA \\
\hline \multirow[t]{4}{*}{ Lung $^{\mathrm{c}}$} & 2.5 & 0.13 & 5.24 & 1.46 & 11.06 \\
\hline & 5 & 0.12 & 5.61 & 2.48 & 20.07 \\
\hline & 10 & 0.13 & 5.23 & 3.12 & 23.54 \\
\hline & 20 & 0.17 & 4.17 & 8.91 & 53.59 \\
\hline
\end{tabular}

a Statistical analyses of these data were not performed due to the limited number of time points and data points within some time points. $\mathrm{k}=$ first-order clearance rate constant; $\mathrm{t} / 2=$ clearance half-life; $\alpha=$ lung deposition rate; $\mathrm{A}_{\mathrm{e}}=$ steady-state lung burden; $\mathrm{NA}=$ not applicable.

bParameters not calculated when average concentration for a time point is zero.

${ }^{\mathrm{c}}$ Total lung cobalt burden was calculated using the weight of the total lung with mainstem bronchi at collection and cobalt concentration measured in the right lung. 
Cobalt Metal, NTP TR 581

Table I-3. Urine Volume, Creatinine and Cobalt Concentrations, and Cobalt Burdens for Rats on Day 12 in the Two-week Inhalation Study of Cobalt Metal ${ }^{\text {a }}$

\begin{tabular}{|c|c|c|c|c|c|}
\hline & $\begin{array}{l}\text { Chamber } \\
\text { Control }\end{array}$ & $2.5 \mathrm{mg} / \mathrm{m}^{3}$ & $5 \mathrm{mg} / \mathrm{m}^{3}$ & $10 \mathrm{mg} / \mathrm{m}^{3}$ & $20 \mathrm{mg} / \mathrm{m}^{3}$ \\
\hline \multicolumn{6}{|l|}{ Male } \\
\hline $\mathbf{n}$ & 5 & 5 & 5 & 5 & $1^{\mathrm{b}}$ \\
\hline Volume (mL/16 hours) & $19.54 \pm 2.70$ & $17.88 \pm 1.06$ & $17.42 \pm 0.43$ & $5.88 \pm 1.47 * *$ & 0.40 \\
\hline Creatinine (mg/dL) & $13.0 \pm 1.8$ & $13.0 \pm 0.8$ & $13.4 \pm 1.0$ & $23.4 \pm 2.0^{* *}$ & 46.1 \\
\hline$\mu \mathrm{g} \mathrm{Co} / \mathrm{mL}$ (control corrected) & NA & $3.23 \pm 0.33$ & $5.98 \pm 0.21$ & $19.61 \pm 2.97$ & 71.27 \\
\hline$\mu \mathrm{g} \mathrm{Co} / \mathrm{mg}$ creatinine & $0.2 \pm 0.1$ & $25.0 \pm 1.8^{* *}$ & $45.4 \pm 3.0^{* *}$ & $81.8 \pm 6.6^{* *}$ & 154.6 \\
\hline$\mu \mathrm{g} \mathrm{Co} / 16$ hours (control corrected) & NA & $56.87 \pm 4.24$ & $104.13 \pm 4.58$ & $100.18 \pm 8.95$ & 28.51 \\
\hline $\begin{array}{l}\mu \mathrm{g} \mathrm{Co} / 16 \text { hours per } \mathrm{mg} \mathrm{Co} / \mathrm{m}^{3} \\
\text { (control corrected) }\end{array}$ & NA & $22.721 \pm 1.691$ & $20.817 \pm 0.918$ & $9.990 \pm 0.897$ & 1.407 \\
\hline
\end{tabular}

\section{Female}

\begin{tabular}{lccccc}
$\mathbf{n}$ & 5 & 5 & 5 & 5 & 2 \\
\hline Volume (mL/16 hours) & $15.74 \pm 3.09$ & $17.90 \pm 2.68$ & $10.98 \pm 1.89$ & $5.62 \pm 2.23$ & $0.25 \pm 0.05^{*}$ \\
Creatinine (mg/dL) & $12.9 \pm 2.1$ & $11.4 \pm 1.3$ & $16.8 \pm 2.7$ & $25.0 \pm 4.3$ & $50.4 \pm 24.2^{*}$ \\
$\mu \mathrm{g} \mathrm{Co} / \mathrm{mL}$ (control corrected) & NA & $2.51 \pm 0.22$ & $6.76 \pm 1.10$ & $20.21 \pm 4.72$ & $70.27 \pm 22.35$ \\
$\mu \mathrm{g} \mathrm{Co} / \mathrm{mg}$ creatinine & $0.4 \pm 0.1$ & $22.7 \pm 1.1^{* *}$ & $40.8 \pm 2.5^{* *}$ & $76.9 \pm 6.6^{* *}$ & $153.6 \pm 29.4^{* *}$ \\
$\mu \mathrm{g} \mathrm{Co} / 16$ hours (control corrected) & NA & $42.71 \pm 1.86$ & $66.22 \pm 3.06$ & $77.85 \pm 5.27$ & $18.68 \pm 9.10$ \\
$\mu \mathrm{g} \mathrm{Co} / 16$ hours per mg Co/m & NA & $17.111 \pm 0.785$ & $13.213 \pm 0.621$ & $7.749 \pm 0.536$ & $0.906 \pm 0.455$ \\
$\quad($ control corrected) & & & & &
\end{tabular}

*Significantly different $(\mathrm{P} \leq 0.05)$ from the chamber control group by Shirley's test.

$* * \mathrm{P} \leq 0.01$.

a Data are presented as mean \pm standard error. Statistical tests were performed only on data that were not control corrected. All $40 \mathrm{mg} / \mathrm{m}^{3}$ rats died before day 12 ; no data are available for this group. NA = not applicable.

bNo standard error was calculated for data in this exposed group; less than two measurements were available. 
Cobalt Metal, NTP TR 581

Table I-4. Tissue Weights, Cobalt Concentrations, and Cobalt Burdens for Female Rats in the Three-month Inhalation Study of Cobalt Metal ${ }^{a}$

\begin{tabular}{|c|c|c|c|c|c|}
\hline & $\begin{array}{c}\text { Chamber } \\
\text { Control }\end{array}$ & $0.625 \mathrm{mg} / \mathrm{m}^{3}$ & $1.25 \mathrm{mg} / \mathrm{m}^{3}$ & $2.5 \mathrm{mg} / \mathrm{m}^{3}$ & $5 \mathrm{mg} / \mathrm{m}^{3}$ \\
\hline $\mathbf{n}$ & 3 & 3 & 3 & 3 & 3 \\
\hline \multicolumn{6}{|l|}{ Blood } \\
\hline \multicolumn{6}{|c|}{$\mu \mathrm{g} \mathrm{Co} / \mathrm{g}$ blood } \\
\hline Day 5 & $0.004 \pm 0.000$ & $0.066 \pm 0.006^{*}$ & $0.109 \pm 0.021^{* *}$ & $0.344 \pm 0.071 * *$ & $0.745 \pm 0.116^{* *}$ \\
\hline Day 12 & $0.004 \pm 0.000$ & $0.065 \pm 0.006^{*}$ & $0.109 \pm 0.014 * *$ & $0.235 \pm 0.035^{* *}$ & $0.512 \pm 0.009 * *$ \\
\hline Day 26 & $0.004 \pm 0.000$ & $0.060 \pm 0.002 *$ & $0.096 \pm 0.011^{* *}$ & $0.230 \pm 0.003^{* *}$ & $0.520 \pm 0.036^{* *}$ \\
\hline Day 40 & $0.004 \pm 0.000$ & $0.053 \pm 0.014^{*}$ & $0.115 \pm 0.020^{* *}$ & $0.199 \pm 0.026^{* *}$ & $0.479 \pm 0.046^{* *}$ \\
\hline Day 61 & $0.004 \pm 0.000$ & $0.047 \pm 0.011^{*}$ & $0.116 \pm 0.010^{* *}$ & $0.213 \pm 0.013 * *$ & $0.440 \pm 0.027 * *$ \\
\hline Day 89 & $0.004 \pm 0.000$ & $0.037 \pm 0.012 *$ & $0.097 \pm 0.013^{* *}$ & $0.164 \pm 0.024 * *$ & $0.401 \pm 0.012 * *$ \\
\hline PE day 7 & $0.004 \pm 0.000$ & $0.025 \pm 0.000^{*}$ & $0.025 \pm 0.000^{*}$ & $0.048 \pm 0.012 * *$ & $0.142 \pm 0.013^{* *}$ \\
\hline PE day 14 & $0.004 \pm 0.000$ & $0.018 \pm 0.007$ & $0.025 \pm 0.000^{*}$ & $0.038 \pm 0.013^{* *}$ & $0.119 \pm 0.010^{* *}$ \\
\hline PE day 28 & $0.004 \pm 0.000$ & $0.011 \pm 0.007$ & $0.025 \pm 0.000^{*}$ & $0.025 \pm 0.000^{*}$ & $0.067 \pm 0.003 * *$ \\
\hline PE day 42 & $0.004 \pm 0.000$ & $0.004 \pm 0.000$ & $0.018 \pm 0.007$ & $0.025 \pm 0.000^{*}$ & $0.025 \pm 0.000^{*}$ \\
\hline \multicolumn{6}{|c|}{$\mu \mathrm{g} \mathrm{Co} / \mathrm{g}$ blood per $\mathrm{mg} \mathrm{Co} / \mathrm{m}^{3}$} \\
\hline Day 5 & - & $0.106 \pm 0.009$ & $0.087 \pm 0.017$ & $0.138 \pm 0.029$ & $0.149 \pm 0.023$ \\
\hline Day 12 & - & $0.104 \pm 0.010$ & $0.087 \pm 0.011$ & $0.094 \pm 0.014$ & $0.102 \pm 0.002$ \\
\hline Day 26 & - & $0.095 \pm 0.004$ & $0.077 \pm 0.009$ & $0.092 \pm 0.001$ & $0.104 \pm 0.007$ \\
\hline Day 40 & - & $0.085 \pm 0.022$ & $0.092 \pm 0.016$ & $0.079 \pm 0.011$ & $0.096 \pm 0.009$ \\
\hline Day 61 & - & $0.076 \pm 0.018$ & $0.093 \pm 0.008$ & $0.085 \pm 0.005$ & $0.088 \pm 0.005$ \\
\hline Day 89 & - & $0.059 \pm 0.019$ & $0.078 \pm 0.010$ & $0.066 \pm 0.009$ & $0.080 \pm 0.002$ \\
\hline PE day 7 & - & $0.040 \pm 0.000$ & $0.020 \pm 0.000$ & $0.019 \pm 0.005$ & $0.028 \pm 0.003$ \\
\hline PE day 14 & - & $0.029 \pm 0.011$ & $0.020 \pm 0.000$ & $0.015 \pm 0.005$ & $0.024 \pm 0.002$ \\
\hline PE day 28 & - & $0.017 \pm 0.011$ & $0.020 \pm 0.000$ & $0.010 \pm 0.000$ & $0.013 \pm 0.001$ \\
\hline PE day 42 & - & $0.006 \pm 0.000$ & $0.014 \pm 0.006$ & $0.010 \pm 0.000$ & $0.005 \pm 0.000$ \\
\hline
\end{tabular}

Liver

Absolute liver wt (g)

Day 26

$5.601 \pm 0.170 \quad 4.917 \pm 0.119$

$5.342 \pm 0.163$

$5.324 \pm 0.111$

$4.188 \pm 0.281 *$

Day 40

$5.810 \pm 0.134$

$5.261 \pm 0.039$

$5.689 \pm 0.388$

$5.843 \pm 0.320$

$5.194 \pm 0.358$

$\mu \mathrm{g} \mathrm{Co/g} \mathrm{liver}$

Day 26

$0.025 \pm 0.000$

$0.801 \pm 0.069^{*}$

$1.360 \pm 0.146^{* *}$

$2.975 \pm 0.103^{* *}$

$6.762 \pm 0.100^{* *}$

Day 40

$0.025 \pm 0.000$

$0.666 \pm 0.128^{*}$

$1.322 \pm 0.268^{*}$

$2.070 \pm 0.084^{* *}$

$4.893 \pm 0.462 * *$

$\mu \mathrm{g} \mathrm{Co/liver}$

Day 26

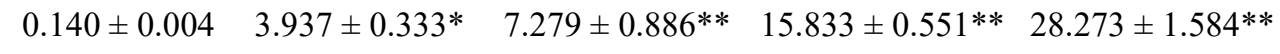

Day 40

$0.145 \pm 0.003 \quad 3.503 \pm 0.671 * \quad 7.324 \pm 1.093 * *$

$12.139 \pm 1.079 * * 25.095 \pm 0.995 * *$ 
Cobalt Metal, NTP TR 581

\begin{tabular}{|c|c|c|c|c|c|}
\hline & $\begin{array}{l}\text { Chamber } \\
\text { Control }\end{array}$ & $0.625 \mathrm{mg} / \mathrm{m}^{3}$ & $1.25 \mathrm{mg} / \mathrm{m}^{3}$ & $2.5 \mathrm{mg} / \mathrm{m}^{3}$ & $5 \mathrm{mg} / \mathrm{m}^{3}$ \\
\hline \multicolumn{6}{|c|}{$\mu \mathrm{g}$ Co per liver $\mathrm{Co} / \mathrm{m}^{3}$} \\
\hline Day 26 & - & $6.299 \pm 0.533$ & $5.823 \pm 0.709$ & $6.333 \pm 0.220$ & $5.655 \pm 0.317$ \\
\hline Day 40 & - & $5.605 \pm 1.074$ & $5.859 \pm 0.874$ & $4.856 \pm 0.432$ & $5.019 \pm 0.199$ \\
\hline \multicolumn{6}{|l|}{ Total lung ${ }^{\mathrm{b}}$} \\
\hline \multicolumn{6}{|c|}{ Absolute total lung wt (g) } \\
\hline Day 5 & $0.957 \pm 0.072$ & $0.626 \pm 0.057 *$ & $0.768 \pm 0.029$ & $0.711 \pm 0.097$ & $0.809 \pm 0.094$ \\
\hline Day 12 & $0.826 \pm 0.030$ & $0.944 \pm 0.170$ & $0.723 \pm 0.052$ & $0.763 \pm 0.031$ & $0.953 \pm 0.020$ \\
\hline Day 26 & $1.028 \pm 0.134$ & $0.741 \pm 0.019$ & $0.862 \pm 0.058$ & $0.965 \pm 0.021$ & $0.957 \pm 0.077$ \\
\hline Day 40 & $0.841 \pm 0.012$ & $0.817 \pm 0.014$ & $0.959 \pm 0.075$ & $1.111 \pm 0.064$ & $1.206 \pm 0.075^{*}$ \\
\hline Day 61 & $0.849 \pm 0.027$ & $1.046 \pm 0.019^{*}$ & $1.093 \pm 0.038^{*}$ & $1.129 \pm 0.056^{*}$ & $1.291 \pm 0.078^{* *}$ \\
\hline Day 89 & $0.816 \pm 0.034$ & $1.233 \pm 0.014$ & $1.224 \pm 0.095$ & $1.322 \pm 0.040^{* *}$ & $1.318 \pm 0.006^{* *}$ \\
\hline PE day 7 & $0.755 \pm 0.059$ & $1.176 \pm 0.034$ & $1.295 \pm 0.022 * *$ & $1.247 \pm 0.031$ & $1.206 \pm 0.065$ \\
\hline PE day 14 & $0.961 \pm 0.101$ & $1.369 \pm 0.152$ & $1.312 \pm 0.093$ & $1.165 \pm 0.131$ & $1.565 \pm 0.292$ \\
\hline PE day 28 & $0.904 \pm 0.069$ & $1.010 \pm 0.057$ & $1.065 \pm 0.047$ & $1.203 \pm 0.109^{*}$ & $1.181 \pm 0.076^{*}$ \\
\hline PE day 42 & $0.842 \pm 0.062$ & $1.151 \pm 0.074 *$ & $0.993 \pm 0.030$ & $0.948 \pm 0.030$ & $1.451 \pm 0.344$ \\
\hline \multicolumn{6}{|c|}{$\mu \mathrm{g} \mathrm{Co} / \mathrm{g}$ total lung } \\
\hline Day 5 & $0.040 \pm 0.000$ & $2.407 \pm 0.107 *$ & $3.829 \pm 0.349^{* *}$ & $7.433 \pm 0.773^{* *}$ & $12.976 \pm 1.766^{* *}$ \\
\hline Day 12 & $0.040 \pm 0.000$ & $2.407 \pm 0.377^{*}$ & $6.435 \pm 0.725^{* *}$ & $12.663 \pm 1.047^{* *}$ & $16.101 \pm 0.689^{* *}$ \\
\hline Day 26 & $0.040 \pm 0.000$ & $3.235 \pm 0.034 *$ & $6.456 \pm 0.314^{* *}$ & $11.423 \pm 0.814 * *$ & $21.581 \pm 0.734^{* *}$ \\
\hline Day 40 & $0.040 \pm 0.000$ & $3.135 \pm 0.117 *$ & $6.114 \pm 0.245^{* *}$ & $12.214 \pm 0.180^{* *}$ & $21.263 \pm 0.520^{* *}$ \\
\hline Day 61 & $0.040 \pm 0.000$ & $2.682 \pm 0.152 *$ & $5.978 \pm 0.303^{* *}$ & $11.146 \pm 0.301 * *$ & $22.517 \pm 1.509^{* *}$ \\
\hline Day 89 & $0.040 \pm 0.000$ & $2.741 \pm 0.131 *$ & $6.018 \pm 0.184^{* *}$ & $12.108 \pm 0.417^{* *}$ & $21.882 \pm 0.806^{* *}$ \\
\hline PE day 7 & $0.040 \pm 0.000$ & $0.601 \pm 0.011 *$ & $1.216 \pm 0.018^{* *}$ & $2.496 \pm 0.015^{* *}$ & $6.067 \pm 0.065^{* *}$ \\
\hline PE day 14 & $0.040 \pm 0.000$ & $0.339 \pm 0.035^{*}$ & $0.769 \pm 0.061 * *$ & $1.613 \pm 0.121^{* *}$ & $2.705 \pm 0.344^{* *}$ \\
\hline PE day 28 & $0.040 \pm 0.000$ & $0.277 \pm 0.010^{*}$ & $0.499 \pm 0.020^{* *}$ & $0.852 \pm 0.080^{* *}$ & $1.603 \pm 0.227^{* *}$ \\
\hline PE day 42 & $0.040 \pm 0.000$ & $0.100 \pm 0.000 *$ & $0.372 \pm 0.004 * *$ & $0.670 \pm 0.023 * *$ & $0.959 \pm 0.195^{* *}$ \\
\hline \multicolumn{6}{|c|}{$\mu \mathrm{g}$ Co/total lung } \\
\hline Day 5 & $0.038 \pm 0.003$ & $1.497 \pm 0.082 *$ & $2.923 \pm 0.173 * *$ & $5.132 \pm 0.107 * *$ & $10.304 \pm 1.063^{* *}$ \\
\hline Day 12 & $0.033 \pm 0.001$ & $2.144 \pm 0.024 *$ & $4.591 \pm 0.334 * *$ & $9.607 \pm 0.454 * *$ & $15.337 \pm 0.723 * *$ \\
\hline Day 26 & $0.041 \pm 0.005$ & $2.397 \pm 0.086^{*}$ & $5.530 \pm 0.125^{* *}$ & $10.993 \pm 0.592 * *$ & $20.545 \pm 3.555^{* *}$ \\
\hline Day 40 & $0.034 \pm 0.000$ & $2.559 \pm 0.051 *$ & $5.888 \pm 0.648^{* *}$ & $13.543 \pm 0.593 * *$ & $25.710 \pm 2.135^{* *}$ \\
\hline Day 61 & $0.034 \pm 0.001$ & $2.809 \pm 0.194 *$ & $6.512 \pm 0.146^{* *}$ & $12.603 \pm 0.859^{* *}$ & $28.860 \pm 0.770^{* *}$ \\
\hline Day 89 & $0.033 \pm 0.001$ & $3.379 \pm 0.162 *$ & $7.345 \pm 0.453^{* *}$ & $15.996 \pm 0.544 * *$ & $28.857 \pm 1.184 * *$ \\
\hline PE day 7 & $0.030 \pm 0.002$ & $0.706 \pm 0.014 *$ & $1.576 \pm 0.045^{* *}$ & $3.114 \pm 0.095^{* *}$ & $7.325 \pm 0.471 * *$ \\
\hline PE day 14 & $0.038 \pm 0.004$ & $0.453 \pm 0.011^{*}$ & $0.999 \pm 0.022 * *$ & $1.856 \pm 0.135^{* *}$ & $4.042 \pm 0.227^{* *}$ \\
\hline
\end{tabular}




\begin{tabular}{|c|c|c|c|c|c|}
\hline & $\begin{array}{l}\text { Chamber } \\
\text { Control }\end{array}$ & $0.625 \mathrm{mg} / \mathrm{m}^{3}$ & $1.25 \mathrm{mg} / \mathrm{m}^{3}$ & $2.5 \mathrm{mg} / \mathrm{m}^{3}$ & $5 \mathrm{mg} / \mathrm{m}^{3}$ \\
\hline PE day 28 & $0.036 \pm 0.003$ & $0.279 \pm 0.012 *$ & $0.531 \pm 0.026^{* *}$ & $1.007 \pm 0.013^{* *}$ & $1.864 \pm 0.164 * *$ \\
\hline PE day 42 & $0.034 \pm 0.002$ & $0.115 \pm 0.007^{*}$ & $0.370 \pm 0.015^{* *}$ & $0.634 \pm 0.021^{* *}$ & $1.261 \pm 0.019^{* *}$ \\
\hline \multicolumn{6}{|c|}{$\mu \mathrm{g} \mathrm{Co} /$ total lung per $\mathrm{mg} \mathrm{Co} / \mathrm{m}^{3}$ ) } \\
\hline Day 5 & - & $2.395 \pm 0.132$ & $2.338 \pm 0.139$ & $2.053 \pm 0.043$ & $2.061 \pm 0.213$ \\
\hline Day 12 & - & $3.430 \pm 0.038$ & $3.673 \pm 0.267$ & $3.843 \pm 0.182$ & $3.067 \pm 0.145$ \\
\hline Day 26 & - & $3.835 \pm 0.138$ & $4.424 \pm 0.100$ & $4.397 \pm 0.237$ & $4.109 \pm 0.711^{\mathrm{c}}$ \\
\hline Day 40 & - & $4.094 \pm 0.082$ & $4.710 \pm 0.519$ & $5.417 \pm 0.237$ & $5.142 \pm 0.427$ \\
\hline Day 61 & - & $4.494 \pm 0.310$ & $5.210 \pm 0.116$ & $5.041 \pm 0.343$ & $5.772 \pm 0.154$ \\
\hline Day 89 & - & $5.406 \pm 0.259$ & $5.876 \pm 0.363$ & $6.399 \pm 0.218$ & $5.771 \pm 0.237$ \\
\hline PE day 7 & - & $1.130 \pm 0.022$ & $1.260 \pm 0.036$ & $1.246 \pm 0.038$ & $1.465 \pm 0.094$ \\
\hline PE day 14 & - & $0.726 \pm 0.017$ & $0.799 \pm 0.017$ & $0.742 \pm 0.054$ & $0.808 \pm 0.045$ \\
\hline PE day 28 & - & $0.446 \pm 0.019$ & $0.425 \pm 0.021$ & $0.403 \pm 0.005$ & $0.373 \pm 0.033$ \\
\hline PE day 42 & - & $0.184 \pm 0.012$ & $0.296 \pm 0.012$ & $0.254 \pm 0.009$ & $0.252 \pm 0.004$ \\
\hline
\end{tabular}

*Significantly different $(\mathrm{P} \leq 0.05)$ from the chamber control group by Dunn's or Shirley's test.

$* * \mathrm{P} \leq 0.01$.

${ }^{a}$ Data are presented as mean \pm standard error. Values below the limit of detection (LOD) or below the experimental limit of quantitation (ELOQ) were replaced with $1 / 2$ the LOD or ELOQ value. Statistical tests were performed only on data that were not normalized. $\mathrm{PE}=$ postexposure.

${ }^{b}$ Total lung cobalt burden was calculated using the weight of the total lung with mainstem bronchi at collection and cobalt concentration measured in the right lung.

${ }^{c} n=2$.

Table I-5. Lung Deposition and Clearance Parameter Estimates for Female Rats During the Recovery Period in the Three-month Inhalation Study of Cobalt Metal (Two-Compartment Model) $)^{\mathbf{a}}$

\begin{tabular}{|c|c|c|c|c|}
\hline Parameter & $0.625 \mathrm{mg} / \mathrm{m}^{3}$ & $1.25 \mathrm{mg} / \mathrm{m}^{3}$ & $2.5 \mathrm{mg} / \mathrm{m}^{3}$ & $5 \mathrm{mg} / \mathrm{m}^{3}$ \\
\hline $\mathrm{A}(\mu \mathrm{g} \mathrm{Co} /$ total lung) & $2.69 \pm 0.15$ & $5.78 \pm 0.39$ & $13.0 \pm 0.69$ & $23.4 \pm 2.03$ \\
\hline $\mathrm{A}\left(\right.$ days $\left.^{-1}\right)$ & $0.39 \pm 0.04$ & $0.37 \pm 0.04$ & $0.38 \pm 0.03$ & $0.27 \pm 0.03$ \\
\hline $\mathrm{t}_{1 / 2 \text { (rapid) }}$ (days) & $1.78 \pm 0.02$ & $1.90 \pm 0.02$ & $1.81 \pm 0.01$ & $2.59 \pm 0.04$ \\
\hline $\mathrm{B}(\mu \mathrm{g} \mathrm{Co} /$ total lung) & $0.65 \pm 0.05$ & $1.45 \pm 0.15$ & $2.86 \pm 0.23$ & $4.95 \pm 0.95$ \\
\hline $\mathrm{b}\left(\right.$ days $\left.^{-1}\right)$ & $0.030 \pm 0.003$ & $0.034 \pm 0.003$ & $0.036 \pm 0.003$ & $0.033 \pm 0.006$ \\
\hline $\mathrm{t}_{1 / 2 \text { (slow) }}$ (days) & $23.5 \pm 0.2$ & $20.6 \pm 0.2$ & $19.1 \pm 0.1$ & $20.7 \pm 0.6$ \\
\hline
\end{tabular}

${ }^{\mathrm{a}}$ Data are presented as mean \pm standard error. $\mathrm{A}=$ lung burden in the rapid clearance compartment at $\mathrm{t}=0$ days postexposure; $\mathrm{a}=$ rapid phase lung clearance rate constant; $\mathrm{t} / 2($ rapid) $=$ rapid phase lung clearance rate constant; $\mathrm{B}=$ lung burden in the slow clearance compartment at $\mathrm{t}=0$ days postexposure; $\mathrm{b}=$ slow phase lung clearance rate constant; $\mathrm{t} / 2($ slow $)=$ slow phase lung clearance rate constant. 
Table I-6. Lung Deposition and Clearance Parameter Estimates for Female Rats During the Exposure Period in the Three-month Inhalation Study of Cobalt Metal (One-Compartment Model) ${ }^{\mathrm{a}}$

\begin{tabular}{|c|c|c|c|c|}
\hline Parameter & $0.625 \mathrm{mg} / \mathrm{m}^{3}$ & $1.25 \mathrm{mg} / \mathrm{m}^{3}$ & $2.5 \mathrm{mg} / \mathrm{m}^{3}$ & $5 \mathrm{mg} / \mathrm{m}^{3}$ \\
\hline $\begin{array}{l}\mathrm{D}(\mu \mathrm{g} \text { Co/total lung } \\
\text { per day) }\end{array}$ & $0.40 \pm 0.04$ & $0.72 \pm 0.07$ & $1.28 \pm 0.10$ & $2.06 \pm 0.20$ \\
\hline $\mathrm{k}\left(\right.$ days $\left.^{-1}\right)$ & $0.15 \pm 0.02$ & $0.12 \pm 0.01$ & $0.10 \pm 0.01$ & $0.08 \pm 0.01$ \\
\hline $\mathrm{t}_{1 / 2}$ (days) & $4.65 \pm 0.07$ & $5.94 \pm 0.08$ & $7.23 \pm 0.07$ & $8.99 \pm 0.16$ \\
\hline $\mathrm{L}_{\mathrm{ss}}(\mu \mathrm{g} \mathrm{Co} /$ total lung $)$ & $2.7 \pm 0.1$ & $6.2 \pm 0.2$ & $13.3 \pm 0.5$ & $26.8 \pm 1.5$ \\
\hline
\end{tabular}

${ }^{\mathrm{a}}$ Data are presented as mean \pm standard error. $\mathrm{D}=$ deposition rate; $\mathrm{k}=$ lung clearance rate constant; $\mathrm{t} 1 / 2=$ lung clearance half-life; $\mathrm{L}_{\mathrm{ss}}=$ steady-state lung burden.

Table I-7. Lung Weights, Cobalt Concentrations, and Cobalt Burdens for Female Rats in the Twoyear Inhalation Study of Cobalt Metal ${ }^{\mathrm{a}}$

\begin{tabular}{|c|c|c|c|c|}
\hline & Chamber Control & $1.25 \mathrm{mg} / \mathrm{m}^{3}$ & $2.5 \mathrm{mg} / \mathrm{m}^{3}$ & $5 \mathrm{mg} / \mathrm{m}^{3}$ \\
\hline $\mathbf{n}$ & 5 & 5 & 5 & 5 \\
\hline \multicolumn{5}{|c|}{ Absolute total lung wt $(\mathrm{g})^{\mathrm{b}}$} \\
\hline Day 1 & $0.509 \pm 0.023$ & $0.632 \pm 0.070$ & $0.497 \pm 0.027$ & $0.465 \pm 0.012$ \\
\hline Day 2 & $0.552 \pm 0.037$ & $0.523 \pm 0.021$ & $0.488 \pm 0.017$ & $0.539 \pm 0.016$ \\
\hline Day 3 & $0.539 \pm 0.035$ & $0.567 \pm 0.018$ & $0.616 \pm 0.074$ & $0.647 \pm 0.027$ \\
\hline Day 4 & $0.786 \pm 0.091$ & $0.877 \pm 0.041$ & $0.688 \pm 0.025$ & $0.693 \pm 0.018$ \\
\hline Day 184 & $1.125 \pm 0.237$ & $1.468 \pm 0.064$ & $1.682 \pm 0.075^{*}$ & $1.693 \pm 0.030^{*}$ \\
\hline Day 366 & $1.039 \pm 0.025$ & $1.801 \pm 0.064^{* *}$ & $2.103 \pm 0.066^{* *}$ & $2.333 \pm 0.070^{* *}$ \\
\hline Day 548 & $1.279 \pm 0.039$ & $2.678 \pm 0.187 * *$ & $2.691 \pm 0.145^{* c}$ & $3.815 \pm 0.323 * *$ \\
\hline \multicolumn{5}{|c|}{$\mu \mathrm{g} \mathrm{Co} / \mathrm{g}$ total lung } \\
\hline Day 1 & $0.040 \pm 0.000$ & $2.797 \pm 0.393 * *$ & $6.028 \pm 0.400 * *$ & $16.152 \pm 0.466^{* *}$ \\
\hline Day 2 & $0.040 \pm 0.000$ & $4.603 \pm 0.219^{* *}$ & $8.987 \pm 0.045^{* *}$ & $12.662 \pm 0.865^{* *}$ \\
\hline Day 3 & $0.040 \pm 0.000$ & $5.474 \pm 0.237 * *$ & $8.177 \pm 0.735^{* *}$ & $13.511 \pm 0.853^{* *}$ \\
\hline Day 4 & $0.040 \pm 0.000$ & $3.804 \pm 0.232 * *$ & $7.338 \pm 0.393 * *$ & $18.406 \pm 1.266^{* *}$ \\
\hline Day 184 & $0.040 \pm 0.000$ & $5.685 \pm 0.126^{* *}$ & $11.238 \pm 0.592 * *$ & $21.670 \pm 0.606^{* *}$ \\
\hline Day 366 & $0.040 \pm 0.000$ & $5.251 \pm 0.058^{* *}$ & $10.748 \pm 0.351^{* *}$ & $18.491 \pm 0.401 * *$ \\
\hline Day 548 & $0.052 \pm 0.012$ & $5.113 \pm 0.400^{* *}$ & $10.108 \pm 0.532 * * \mathrm{c}$ & $13.248 \pm 0.567^{* *}$ \\
\hline \multicolumn{5}{|c|}{$\mu \mathrm{g} \mathrm{Co/total} \mathrm{lung}$} \\
\hline Day 1 & $0.020 \pm 0.001$ & $1.663 \pm 0.081^{* *}$ & $2.962 \pm 0.114 * *$ & $7.505 \pm 0.253^{* *}$ \\
\hline Day 2 & $0.022 \pm 0.001$ & $2.404 \pm 0.136^{* *}$ & $4.382 \pm 0.154^{* *}$ & $6.792 \pm 0.379 * *$ \\
\hline Day 3 & $0.022 \pm 0.001$ & $3.103 \pm 0.165^{* *}$ & $4.829 \pm 0.147^{* *}$ & $8.705 \pm 0.522 * *$ \\
\hline Day 4 & $0.031 \pm 0.004$ & $3.306 \pm 0.137^{* *}$ & $5.018 \pm 0.124 * *$ & $12.780 \pm 1.038^{* *}$ \\
\hline Day 184 & $0.045 \pm 0.009$ & $8.327 \pm 0.279^{* *}$ & $18.758 \pm 0.484^{* *}$ & $36.678 \pm 1.061^{* *}$ \\
\hline Day 366 & $0.042 \pm 0.001$ & $9.454 \pm 0.309^{* *}$ & $22.578 \pm 0.923^{* *}$ & $43.068 \pm 0.985^{* *}$ \\
\hline Day 548 & $0.067 \pm 0.016$ & $13.419 \pm 0.454 * *$ & $26.998 \pm 0.677^{* * \mathrm{c}}$ & $49.954 \pm 2.876^{* *}$ \\
\hline
\end{tabular}


Cobalt Metal, NTP TR 581

\begin{tabular}{|c|c|c|c|c|}
\hline & Chamber Control & $1.25 \mathrm{mg} / \mathrm{m}^{3}$ & $2.5 \mathrm{mg} / \mathrm{m}^{3}$ & $5 \mathrm{mg} / \mathrm{m}^{3}$ \\
\hline \multicolumn{5}{|c|}{$\mu \mathrm{g} \mathrm{Co} /$ total lung per $\mathrm{mg} \mathrm{Co} / \mathrm{m}^{3}$ ) } \\
\hline Day 1 & - & $1.330 \pm 0.065$ & $1.185 \pm 0.046$ & $1.501 \pm 0.051$ \\
\hline Day 2 & - & $1.923 \pm 0.109$ & $1.753 \pm 0.062$ & $1.358 \pm 0.076$ \\
\hline Day 3 & - & $2.482 \pm 0.132$ & $1.932 \pm 0.059$ & $1.741 \pm 0.104$ \\
\hline Day 4 & - & $2.645 \pm 0.110$ & $2.007 \pm 0.050$ & $2.556 \pm 0.208$ \\
\hline Day 184 & - & $6.662 \pm 0.223$ & $7.503 \pm 0.194$ & $7.336 \pm 0.212$ \\
\hline Day 366 & - & $7.563 \pm 0.247$ & $9.031 \pm 0.369$ & $8.614 \pm 0.197$ \\
\hline Day 548 & - & $10.735 \pm 0.363$ & $10.799 \pm 0.271^{\mathrm{c}}$ & $9.991 \pm 0.575$ \\
\hline
\end{tabular}

*Significantly different $(\mathrm{P} \leq 0.05)$ from the chamber control group by Shirley's test.

$* * \mathrm{P} \leq 0.01$.

aata are presented as mean \pm standard error. Values below the limit of detection (LOD) or below the experimental limit of quantitation (ELOQ) were replaced with $1 / 2$ the LOD or ELOQ value. Statistical tests were performed only on data that were not normalized.

${ }^{\text {b}}$ Total lung cobalt burden was calculated using the weight of the total lung with mainstem bronchi at collection and cobalt concentration measured in the right lung.

${ }^{c} \mathrm{n}=4$. 
Table I-8. Lung Deposition and Clearance Parameter Estimates for Female Rats During the Exposure Periods in the Three-month and Two-year Inhalation Studies of Cobalt Metal (TwoCompartment Model) ${ }^{\mathrm{a}}$

\begin{tabular}{|c|c|c|c|}
\hline Parameter & $1.25 \mathrm{mg} / \mathrm{m}^{3}$ & $2.5 \mathrm{mg} / \mathrm{m}^{3}$ & $5 \mathrm{mg} / \mathrm{m}^{3}$ \\
\hline $\mathrm{A}(\mu \mathrm{g} \mathrm{Co} /$ day $)$ & $1.449 \pm 0.112$ & $2.128 \pm 0.158$ & $5.555 \pm 0.832$ \\
\hline $\mathrm{k}_{\mathrm{a}}\left(\right.$ days $\left.^{-1}\right)$ & $0.293 \pm 0.035$ & $0.236 \pm 0.032$ & $0.453 \pm 0.114$ \\
\hline $\mathrm{B}(\mu \mathrm{g} \mathrm{Co} /$ day $)$ & $0.0179 \pm 0.0043$ & $0.0778 \pm 0.0156$ & $0.2885 \pm 0.0530$ \\
\hline $\mathrm{k}_{\mathrm{b}}\left(\right.$ days $\left.^{-1}\right)$ & $0.00088 \pm 0.00097$ & $0.00414 \pm 0.00110$ & $0.00837 \pm 0.00156$ \\
\hline $\mathrm{F}_{\mathrm{B}}$ & $0.012 \pm 0.003$ & $0.035 \pm 0.007$ & $0.049 \pm 0.008$ \\
\hline $\mathrm{t}_{1 / 2 \mathrm{a}}$ (days) & $2.37 \pm 0.28$ & $2.94 \pm 0.39$ & $1.53 \pm 0.38$ \\
\hline $\mathrm{L}_{\mathrm{SSa}}(\mu \mathrm{g}$ Co/total lung) & $4.95 \pm 0.30$ & $9.02 \pm 0.75$ & $12.25 \pm 1.55$ \\
\hline $\mathrm{t}_{1 / 2 \mathrm{~b}}$ (days) & $789 \pm 874$ & $167 \pm 45$ & $83 \pm 15$ \\
\hline $\mathrm{L}_{\mathrm{sSb}}$ ( $\mu \mathrm{g}$ Co/total lung) & $20.4 \pm 18.0$ & $18.8 \pm 1.8$ & $34.5 \pm 2.1$ \\
\hline
\end{tabular}


Table I-9. Tissue Weights, Cobalt Concentrations, and Cobalt Burdens for Mice in the Two-week Inhalation Study of Cobalt Metal ${ }^{\mathrm{a}}$

\begin{tabular}{|c|c|c|c|c|c|c|}
\hline & Chamber Control & $2.5 \mathrm{mg} / \mathrm{m}^{3}$ & $5 \mathrm{mg} / \mathrm{m}^{3}$ & $10 \mathrm{mg} / \mathrm{m}^{3}$ & $20 \mathrm{mg} / \mathrm{m}^{3}$ & $40 \mathrm{mg} / \mathrm{m}^{3}$ \\
\hline \multicolumn{7}{|l|}{ Male } \\
\hline $\mathbf{n}$ & 5 & 5 & 5 & 5 & 5 & 2 \\
\hline \multicolumn{7}{|l|}{ Blood } \\
\hline \multicolumn{7}{|c|}{$\mu \mathrm{g} \mathrm{Co} / \mathrm{g}$ blood } \\
\hline Day 17 & $0^{\mathrm{b}}$ & $0.40 \pm 0.04$ & $0.74 \pm 0.06$ & $0.65 \pm 0.03$ & $1.44 \pm 0.22$ & $2.53 \pm 0.13$ \\
\hline \multicolumn{7}{|l|}{ Serum } \\
\hline \multicolumn{7}{|c|}{$\mu \mathrm{g} \mathrm{Co} / \mathrm{g}$ serum } \\
\hline Day 17 & 0 & $0.63 \pm 0.06$ & $1.38 \pm 0.12$ & $1.31 \pm 0.04$ & $2.72 \pm 0.38$ & $4.94 \pm 0.03$ \\
\hline \multicolumn{7}{|l|}{ Right femur } \\
\hline \multicolumn{7}{|c|}{ Absolute right femur wt (g) } \\
\hline Day 17 & $0.025 \pm 0.001$ & $0.026 \pm 0.001$ & $0.025 \pm 0.001$ & $0.025 \pm 0.001$ & $0.024 \pm 0.001$ & $0.021 \pm 0.001$ \\
\hline \multicolumn{7}{|c|}{$\mu \mathrm{g} \mathrm{Co} / \mathrm{g}$ right femur } \\
\hline Day 17 & 0 & $0.42 \pm 0.10$ & $0.56 \pm 0.06$ & $1.13 \pm 0.15$ & $1.65 \pm 0.23$ & $2.78 \pm 0.24$ \\
\hline \multicolumn{7}{|c|}{$\mu \mathrm{g} \mathrm{Co/right} \mathrm{femur}$} \\
\hline Day 17 & 0 & $0.011 \pm 0.002$ & $0.014 \pm 0.001$ & $0.029 \pm 0.003$ & $0.039 \pm 0.005$ & $0.057 \pm 0.007$ \\
\hline \multicolumn{7}{|c|}{$\mu \mathrm{g} \mathrm{Co} /$ right femur per $\mathrm{mg} \mathrm{Co} / \mathrm{m}^{3}$} \\
\hline Day 17 & NA & $0.004 \pm 0.001$ & $0.003 \pm 0.000$ & $0.003 \pm 0.000$ & $0.002 \pm 0.000$ & $0.001 \pm 0.000$ \\
\hline \multicolumn{7}{|l|}{ Heart } \\
\hline \multicolumn{7}{|c|}{ Absolute heart wt (g) } \\
\hline Day 17 & $0.125 \pm 0.005$ & $0.127 \pm 0.003$ & $0.124 \pm 0.003$ & $0.120 \pm 0.004$ & $0.110 \pm 0.003 *$ & $0.106 \pm 0.007^{*}$ \\
\hline \multicolumn{7}{|c|}{$\mu \mathrm{g} \mathrm{Co} / \mathrm{g}$ heart } \\
\hline Day 17 & 0 & $0.39 \pm 0.09$ & $0.98 \pm 0.20$ & $0.95 \pm 0.13$ & $1.65 \pm 0.21$ & $6.45 \pm 2.77$ \\
\hline \multicolumn{7}{|c|}{$\mu \mathrm{g} \mathrm{Co} /$ heart } \\
\hline Day 17 & 0 & $0.050 \pm 0.011$ & $0.123 \pm 0.028$ & $0.112 \pm 0.014$ & $0.178 \pm 0.018$ & $0.700 \pm 0.340$ \\
\hline \multicolumn{7}{|c|}{$\mu \mathrm{g} \mathrm{Co} /$ heart per $\mathrm{mg} \mathrm{Co} / \mathrm{m}^{3}$} \\
\hline Day 17 & NA & $0.020 \pm 0.004$ & $0.025 \pm 0.006$ & $0.011 \pm 0.001$ & $0.009 \pm 0.001$ & $0.017 \pm 0.008$ \\
\hline Right kidney & & & & & & \\
\hline
\end{tabular}


Cobalt Metal, NTP TR 581

\begin{tabular}{|c|c|c|c|c|c|c|}
\hline & Chamber Control & $2.5 \mathrm{mg} / \mathrm{m}^{3}$ & $5 \mathrm{mg} / \mathrm{m}^{3}$ & $10 \mathrm{mg} / \mathrm{m}^{3}$ & $20 \mathrm{mg} / \mathrm{m}^{3}$ & $40 \mathrm{mg} / \mathrm{m}^{3}$ \\
\hline \multicolumn{7}{|c|}{ Absolute right kidney wt (g) } \\
\hline Day 17 & $0.223 \pm 0.009$ & $0.216 \pm 0.011$ & $0.197 \pm 0.005$ & $0.199 \pm 0.007$ & $0.171 \pm 0.003 * *$ & $0.150 \pm 0.003 * *$ \\
\hline \multicolumn{7}{|c|}{$\mu \mathrm{g}$ Co/g right kidney } \\
\hline Day 17 & 0 & $0.91 \pm 0.06$ & $1.86 \pm 0.12$ & $2.02 \pm 0.06$ & $3.82 \pm 0.35$ & $4.97 \pm 1.76$ \\
\hline \multicolumn{7}{|c|}{$\mu \mathrm{g}$ Co/right kidney } \\
\hline Day 17 & 0 & $0.197 \pm 0.019$ & $0.360 \pm 0.019$ & $0.396 \pm 0.018$ & $0.642 \pm 0.058$ & $0.737 \pm 0.279$ \\
\hline \multicolumn{7}{|c|}{$\mu \mathrm{g} \mathrm{Co} /$ right kidney per $\mathrm{mg} \mathrm{Co} / \mathrm{m}^{3}$} \\
\hline Day 17 & NA & $0.079 \pm 0.007$ & $0.072 \pm 0.004$ & $0.040 \pm 0.002$ & $0.032 \pm 0.003$ & $0.018 \pm 0.007$ \\
\hline \multicolumn{7}{|l|}{ Liver } \\
\hline \multicolumn{7}{|c|}{ Absolute liver wt (g) } \\
\hline Day 17 & $1.127 \pm 0.037$ & $0.978 \pm 0.035^{*}$ & $0.976 \pm 0.037^{*}$ & $0.993 \pm 0.018^{*}$ & $0.888 \pm 0.019 * *$ & $0.830 \pm 0.013^{* *}$ \\
\hline \multicolumn{7}{|c|}{$\mu \mathrm{g} \mathrm{Co} / \mathrm{g}$ liver } \\
\hline Day 17 & 0 & $1.72 \pm 0.13$ & $4.79 \pm 0.70$ & $4.55 \pm 0.41$ & $12.84 \pm 2.07$ & $33.73 \pm 2.79$ \\
\hline \multicolumn{7}{|c|}{$\mu \mathrm{g} \mathrm{Co/liver}$} \\
\hline Day 17 & 0 & $1.669 \pm 0.138$ & $4.566 \pm 0.512$ & $4.516 \pm 0.437$ & $11.370 \pm 1.836$ & $28.007 \pm 2.751$ \\
\hline \multicolumn{7}{|c|}{$\mu \mathrm{g} \mathrm{Co} /$ liver per $\mathrm{mg} \mathrm{Co} / \mathrm{m}^{3}$} \\
\hline Day 17 & NA & $0.668 \pm 0.055$ & $0.913 \pm 0.102$ & $0.452 \pm 0.044$ & $0.568 \pm 0.092$ & $0.700 \pm 0.069$ \\
\hline \multicolumn{7}{|l|}{ Total lung ${ }^{\mathrm{c}}$} \\
\hline \multicolumn{7}{|c|}{ Absolute total lung wt (g) } \\
\hline Day 17 & $0.182 \pm 0.007$ & $0.210 \pm 0.009 *$ & $0.225 \pm 0.009 * *$ & $0.244 \pm 0.014 * *$ & $0.295 \pm 0.007 * *$ & $0.360 \pm 0.050 * *$ \\
\hline \multicolumn{7}{|c|}{$\mu \mathrm{g} \mathrm{Co/total} \mathrm{lung}$} \\
\hline Day 17 & 0 & $5.587 \pm 0.473$ & $10.451 \pm 0.448$ & $16.403 \pm 0.980$ & $20.356 \pm 0.666$ & $28.931 \pm 11.814$ \\
\hline \multicolumn{7}{|c|}{$\mu \mathrm{g} \mathrm{Co} /$ total lung per $\mathrm{mg} \mathrm{Co} / \mathrm{m}^{3}$} \\
\hline Day 17 & NA & $2.235 \pm 0.189$ & $2.090 \pm 0.090$ & $1.640 \pm 0.098$ & $1.018 \pm 0.033$ & $0.723 \pm 0.295$ \\
\hline \multicolumn{7}{|l|}{ Right lung $^{\mathrm{d}}$} \\
\hline \multicolumn{7}{|c|}{ Absolute right lung wt (g) } \\
\hline Day 17 & $0.078 \pm 0.006$ & $0.097 \pm 0.002$ & $0.110 \pm 0.003$ & $0.117 \pm 0.004$ & $0.152 \pm 0.005$ & $0.199 \pm 0.031^{* *}$ \\
\hline$\mu \mathrm{g} \mathrm{Co} / \mathrm{g} \mathrm{ri}$ & & & & & & \\
\hline
\end{tabular}


Cobalt Metal, NTP TR 581

\begin{tabular}{|c|c|c|c|c|c|c|}
\hline & Chamber Control & $2.5 \mathrm{mg} / \mathrm{m}^{3}$ & $5 \mathrm{mg} / \mathrm{m}^{3}$ & $10 \mathrm{mg} / \mathrm{m}^{3}$ & $20 \mathrm{mg} / \mathrm{m}^{3}$ & $40 \mathrm{mg} / \mathrm{m}^{3}$ \\
\hline Day 17 & 0 & $26.49 \pm 1.37$ & $46.36 \pm 67.26$ & $67.26 \pm 1.13$ & $69.14 \pm 2.93$ & $86.50 \pm 44.86$ \\
\hline \multicolumn{7}{|c|}{$\mu \mathrm{g} \mathrm{Co/right} \mathrm{lung}$} \\
\hline Day 17 & - & $0.942 \pm 0.048$ & $0.988 \pm 0.048$ & $0.730 \pm 0.151$ & 0.442 & $-^{\mathrm{e}}$ \\
\hline \multicolumn{7}{|c|}{$\mu \mathrm{g} \mathrm{Co/right} \mathrm{lung} \mathrm{per} \mathrm{mg} \mathrm{Co} / \mathrm{m}^{3}$} \\
\hline Day 17 & NA & $0.523 \pm 0.012$ & $0.590 \pm 0.006$ & $0.455 \pm 0.002$ & 0.309 & - \\
\hline \multicolumn{7}{|l|}{ Right testis } \\
\hline \multicolumn{7}{|c|}{ Absolute right testis wt (g) } \\
\hline Day 17 & $0.098 \pm 0.004$ & $0.100 \pm 0.002$ & $0.098 \pm 0.002$ & $0.097 \pm 0.002$ & $0.088 \pm 0.002 *$ & $0.076 \pm 0.005^{*}$ \\
\hline \multicolumn{7}{|c|}{$\mu \mathrm{g} \mathrm{Co} / \mathrm{g}$ right testis } \\
\hline Day 17 & 0 & $0.06 \pm 0.01$ & $0.15 \pm 0.01$ & $0.34 \pm 0.08$ & $0.58 \pm 0.06$ & $1.20 \pm 0.03$ \\
\hline \multicolumn{7}{|c|}{$\mu \mathrm{g} \mathrm{Co/right} \mathrm{testis}$} \\
\hline Day 17 & 0 & $0.005 \pm 0.001$ & $0.014 \pm 0.001$ & $0.033 \pm 0.009$ & $0.051 \pm 0.005$ & $0.090 \pm 0.008$ \\
\hline \multicolumn{7}{|c|}{$\mu \mathrm{g} \mathrm{Co} /$ right testis per $\mathrm{mg} \mathrm{Co} / \mathrm{m}^{3}$} \\
\hline Day 17 & NA & $0.002 \pm 0.000$ & $0.003 \pm 0.000$ & $0.003 \pm 0.001$ & $0.003 \pm 0.000$ & $0.002 \pm 0.000$ \\
\hline \multicolumn{7}{|l|}{ Female } \\
\hline \multicolumn{7}{|l|}{$\mathbf{n}$} \\
\hline Day 17 & 5 & 5 & 5 & 5 & 5 & 2 \\
\hline Week 3 PE & 3 & 3 & 3 & 3 & 3 & $1^{\mathrm{b}}$ \\
\hline \multicolumn{7}{|l|}{ Blood } \\
\hline \multicolumn{7}{|l|}{$\mu \mathrm{g} \mathrm{Co} / \mathrm{g}$ blood } \\
\hline Day 17 & $0^{\mathrm{g}}$ & $0.47 \pm 0.06^{\mathrm{f}}$ & $0.78 \pm 0.08^{\mathrm{f}}$ & $0.97 \pm 0.10^{\mathrm{f}}$ & $2.01 \pm 0.13^{\mathrm{g}}$ & $2.52 \pm 0.88$ \\
\hline Week 3 PE & 0 & $0.06 \pm 0.02$ & $0.07 \pm 0.05$ & $0.05 \pm 0.01$ & $0.05 \pm 0.01$ & 0.13 \\
\hline \multicolumn{7}{|l|}{ Serum } \\
\hline \multicolumn{7}{|l|}{$\mu \mathrm{g} \mathrm{Co} / \mathrm{g}$ serum } \\
\hline Day 17 & $0^{\mathrm{g}}$ & $0.99 \pm 0.13^{\mathrm{f}}$ & $1.52 \pm 0.18^{\mathrm{f}}$ & $1.83 \pm 0.20^{\mathrm{f}}$ & $3.92 \pm 0.28^{\mathrm{g}}$ & $5.10 \pm 1.57$ \\
\hline Week 3 PE & 0 & $0.00 \pm 0.00$ & $0.00 \pm 0.00$ & $0.00 \pm 0.00$ & $0.03 \pm 0.01$ & 0.07 \\
\hline \multicolumn{7}{|l|}{ Right femur } \\
\hline Absolute righ & $w t(g)$ & & & & & \\
\hline
\end{tabular}


Cobalt Metal, NTP TR 581

\begin{tabular}{|c|c|c|c|c|c|c|}
\hline & Chamber Control & $2.5 \mathrm{mg} / \mathrm{m}^{3}$ & $5 \mathrm{mg} / \mathrm{m}^{3}$ & $10 \mathrm{mg} / \mathrm{m}^{3}$ & $20 \mathrm{mg} / \mathrm{m}^{3}$ & $40 \mathrm{mg} / \mathrm{m}^{3}$ \\
\hline Day 17 & $0.024 \pm 0.001$ & $0.025 \pm 0.000$ & $0.025 \pm 0.001$ & $0.023 \pm 0.001$ & $0.020 \pm 0.001 *$ & $0.018 \pm 0.001 *$ \\
\hline \multicolumn{7}{|c|}{$\mu \mathrm{g} \mathrm{Co} / \mathrm{g}$ right femur } \\
\hline Day 17 & 0 & $0.71 \pm 0.05$ & $1.24 \pm 0.10$ & $1.80 \pm 0.14$ & $3.03 \pm 0.29$ & $3.92 \pm 1.07$ \\
\hline \multicolumn{7}{|c|}{$\mu \mathrm{g} \mathrm{Co/right} \mathrm{femur}$} \\
\hline Day 17 & 0 & $0.019 \pm 0.001$ & $0.030 \pm 0.002$ & $0.041 \pm 0.003$ & $0.058 \pm 0.009$ & $0.065 \pm 0.016$ \\
\hline \multicolumn{7}{|c|}{$\mu \mathrm{g} \mathrm{Co} /$ right femur per $\mathrm{mg} \mathrm{Co} / \mathrm{m}^{3}$} \\
\hline Day 17 & NA & $0.007 \pm 0.001$ & $0.006 \pm 0.000$ & $0.004 \pm 0.000$ & $0.003 \pm 0.000$ & $0.002 \pm 0.000$ \\
\hline \multicolumn{7}{|l|}{ Heart } \\
\hline \multicolumn{7}{|c|}{ Absolute heart wt (g) } \\
\hline Day 17 & $0.111 \pm 0.002$ & $0.110 \pm 0.003$ & $0.112 \pm 0.001$ & $0.100 \pm 0.002$ & $0.097 \pm 0.005$ & $0.099 \pm 0.004$ \\
\hline \multicolumn{7}{|c|}{$\mu \mathrm{g} \mathrm{Co} / \mathrm{g}$ heart } \\
\hline Day 17 & 0 & $0.70 \pm 0.12$ & $0.99 \pm 0.08$ & $1.33 \pm 0.12$ & $2.53 \pm 0.13$ & $3.26 \pm 1.11$ \\
\hline \multicolumn{7}{|c|}{$\mu \mathrm{g} \mathrm{Co} /$ heart } \\
\hline Day 17 & 0 & $0.076 \pm 0.012$ & $0.111 \pm 0.009$ & $0.131 \pm 0.009$ & $0.243 \pm 0.012$ & $0.316 \pm 0.097$ \\
\hline \multicolumn{7}{|c|}{$\mu \mathrm{g} \mathrm{Co} /$ heart per $\mathrm{mg} \mathrm{Co} / \mathrm{m}^{3}$} \\
\hline Day 17 & NA & $0.030 \pm 0.005$ & $0.02 \pm 0.002$ & $0.013 \pm 0.001$ & $0.012 \pm 0.001$ & $0.008 \pm 0.002$ \\
\hline \multicolumn{7}{|c|}{ Right kidney } \\
\hline \multicolumn{7}{|c|}{ Absolute right kidney wt (g) } \\
\hline Day 17 & $0.151 \pm 0.002$ & $0.147 \pm 0.004$ & $0.142 \pm 0.005$ & $0.132 \pm 0.004 * *$ & $0.113 \pm 0.003 * *$ & $0.098 \pm 0.006^{* *}$ \\
\hline \multicolumn{7}{|c|}{$\mu \mathrm{g}$ Co/g right kidney } \\
\hline Day 17 & 0 & $1.44 \pm 0.15$ & $2.08 \pm 0.17$ & $2.76 \pm 0.16$ & $5.45 \pm 0.32$ & $8.63 \pm 0.69$ \\
\hline \multicolumn{7}{|c|}{$\mu \mathrm{g}$ Co/right kidney } \\
\hline Day 17 & 0 & $0.213 \pm 0.028$ & $0.291 \pm 0.017$ & $0.355 \pm 0.015$ & $0.600 \pm 0.028$ & $0.826 \pm 0.117$ \\
\hline \multicolumn{7}{|c|}{$\mu \mathrm{g} \mathrm{Co} /$ right kidney per $\mathrm{mg} \mathrm{Co} / \mathrm{m}^{3}$} \\
\hline Day 17 & NA & $0.085 \pm 0.011$ & $0.058 \pm 0.003$ & $0.035 \pm 0.001$ & $0.030 \pm 0.001$ & $0.021 \pm 0.003$ \\
\hline \multicolumn{7}{|l|}{ Liver } \\
\hline \multicolumn{7}{|c|}{ Absolute liver wt (g) } \\
\hline Day 17 & $0.928 \pm 0.026$ & $0.814 \pm 0.021 *$ & $0.799 \pm 0.029 *$ & $0.748 \pm 0.030 * *$ & $0.692 \pm 0.026^{* *}$ & $0.605 \pm 0.057 * *$ \\
\hline
\end{tabular}


Cobalt Metal, NTP TR 581

\begin{tabular}{|c|c|c|c|c|c|c|}
\hline & Chamber Control & $2.5 \mathrm{mg} / \mathrm{m}^{3}$ & $5 \mathrm{mg} / \mathrm{m}^{3}$ & $10 \mathrm{mg} / \mathrm{m}^{3}$ & $20 \mathrm{mg} / \mathrm{m}^{3}$ & $40 \mathrm{mg} / \mathrm{m}^{3}$ \\
\hline \multicolumn{7}{|l|}{$\mu \mathrm{g} \mathrm{Co} / \mathrm{g}$ liver } \\
\hline Day 17 & 0 & $2.74 \pm 0.28$ & $4.94 \pm 0.63$ & $7.21 \pm 0.74$ & $21.98 \pm 1.62$ & $35.83 \pm 5.10$ \\
\hline \multicolumn{7}{|l|}{$\mu \mathrm{g} \mathrm{Co/liver}$} \\
\hline Day 17 & 0 & $2.213 \pm 0.229$ & $3.884 \pm 0.426$ & $5.329 \pm 0.474$ & $15.155 \pm 1.123$ & $21.941 \pm 5.131$ \\
\hline \multicolumn{7}{|c|}{$\mu \mathrm{g} \mathrm{Co} /$ liver per $\mathrm{mg} \mathrm{Co} / \mathrm{m}^{3}$} \\
\hline Day 17 & NA & $0.885 \pm 0.092$ & $0.777 \pm 0.085$ & $0.533 \pm 0.047$ & $0.758 \pm 0.056$ & $0.549 \pm 0.128$ \\
\hline \multicolumn{7}{|l|}{ Total lung ${ }^{\mathrm{c}}$} \\
\hline \multicolumn{7}{|c|}{ Absolute total lung wt (g) } \\
\hline Day 17 & $0.189 \pm 0.007^{\mathrm{e}}$ & $0.194 \pm 0.004^{\mathrm{e}}$ & $0.223 \pm 0.006^{* * f}$ & $0.234 \pm 0.006^{* * \mathrm{f}}$ & $0.290 \pm 0.010^{* * \mathrm{~g}}$ & $0.329 \pm 0.020 * *$ \\
\hline Week 3 PE & $0.18 \pm 0.01$ & $0.16 \pm 0.01$ & $0.19 \pm 0.02$ & $0.18 \pm 0.01$ & $0.24 \pm 0.03$ & 0.20 \\
\hline \multicolumn{7}{|c|}{$\mu \mathrm{g} \mathrm{Co/total} \mathrm{lung}$} \\
\hline Day 17 & $0^{\mathrm{f}}$ & $4.511 \pm 0.159^{f}$ & $9.630 \pm 0.327^{\mathrm{f}}$ & $14.495 \pm 0.855^{\mathrm{f}}$ & $16.303 \pm 0.843^{g}$ & $8.205 \pm 4.106$ \\
\hline Week 3 PE & 0 & $0.483 \pm 0.029$ & $0.947 \pm 0.112$ & $1.484 \pm 0.187$ & $1.123 \pm 0.144$ & 1.927 \\
\hline \multicolumn{7}{|c|}{$\mu \mathrm{g} \mathrm{Co} /$ total lung per $\mathrm{mg} \mathrm{Co} / \mathrm{m}^{3}$} \\
\hline Day 17 & $0^{\mathrm{f}}$ & $1.804 \pm 0.064^{\mathrm{f}}$ & $1.926 \pm 0.065^{\mathrm{f}}$ & $1.450 \pm 0.085^{\mathrm{f}}$ & $0.815 \pm 0.042^{\mathrm{g}}$ & $0.205 \pm 0.103$ \\
\hline Week 3 PE & 0 & $0.193 \pm 0.012$ & $0.189 \pm 0.022$ & $0.148 \pm 0.019$ & $0.056 \pm 0.007$ & 0.048 \\
\hline \multicolumn{7}{|l|}{ Left lung ${ }^{g}$} \\
\hline \multicolumn{7}{|c|}{ Absolute left lung wt (g) } \\
\hline Day 17 & $0.103 \pm 0.006^{\mathrm{i}}$ & $0.088 \pm 0.004^{\mathrm{i}}$ & $0.091 \pm 0.006^{\mathrm{i}}$ & $0.099 \pm 0.002^{\mathrm{i}}$ & $0.113^{\mathrm{b}}$ & - \\
\hline Week 3 PE & $0.04 \pm 0.00$ & $0.04 \pm 0.00$ & $0.04 \pm 0.00$ & $0.05 \pm 0.00$ & $0.07 \pm 0.01 *$ & 0.06 \\
\hline \multicolumn{7}{|c|}{$\mu \mathrm{g} \mathrm{Co/g} \mathrm{left} \mathrm{lung}$} \\
\hline Day 17 & $\mathrm{NA}^{\mathrm{h}}$ & $14.88 \pm 0.34^{\mathrm{i}}$ & $32.72 \pm 2.34^{\mathrm{i}}$ & $45.98 \pm 1.16^{\mathrm{i}}$ & $54.72^{\mathrm{b}}$ & - \\
\hline Week 3 PE & NA & $3.05 \pm 0.06$ & $5.66 \pm 0.49$ & $8.00 \pm 0.20$ & $5.75 \pm 1.06$ & 9.71 \\
\hline \multicolumn{7}{|c|}{$\mu \mathrm{g}$ Co/left lung } \\
\hline Day 17 & $\mathrm{NA}^{\mathrm{h}}$ & $1.308 \pm 0.030^{\mathrm{i}}$ & $2.949 \pm 0.032^{\mathrm{i}}$ & $4.550 \pm 0.024^{\mathrm{i}}$ & $6.183^{b}$ & - \\
\hline Week 3 PE & NA & $0.123 \pm 0.004$ & $0.246 \pm 0.030$ & $0.360 \pm 0.017$ & $0.365 \pm 0.028$ & 0.566 \\
\hline \multicolumn{7}{|c|}{$\mu \mathrm{g} \mathrm{Co} /$ left lung per $\mathrm{mg} \mathrm{Co} / \mathrm{m}^{3}$} \\
\hline Day 17 & $\mathrm{NA}^{\mathrm{h}}$ & $0.523 \pm 0.012^{\mathrm{i}}$ & $0.590 \pm 0.006^{\mathrm{i}}$ & $0.455 \pm 0.002^{\mathrm{i}}$ & $0.309^{b}$ & - \\
\hline
\end{tabular}


Cobalt Metal, NTP TR 581

\begin{tabular}{|c|c|c|c|c|c|c|}
\hline & Chamber Control & $2.5 \mathrm{mg} / \mathrm{m}^{3}$ & $5 \mathrm{mg} / \mathrm{m}^{3}$ & $10 \mathrm{mg} / \mathrm{m}^{3}$ & $20 \mathrm{mg} / \mathrm{m}^{3}$ & $40 \mathrm{mg} / \mathrm{m}^{3}$ \\
\hline Week 3 PE & NA & $0.049 \pm 0.002$ & $0.049 \pm 0.006$ & $0.036 \pm 0.002$ & $0.018 \pm 0.001$ & 0.014 \\
\hline \multicolumn{7}{|l|}{ Right lung $^{\mathrm{d}}$} \\
\hline \multicolumn{7}{|c|}{ Absolute right lung wt (g) } \\
\hline Day 17 & $0.090 \pm 0.005^{\mathrm{f}}$ & $0.098 \pm 0.002^{\mathrm{f}}$ & $0.112 \pm 0.003 * * \mathrm{f}$ & $0.120 \pm 0.004 * * \mathrm{f}$ & $0.159 \pm 002 * * \mathrm{~g}$ & $0.186 \pm 0.006$ \\
\hline Week 3 PE & $0.09 \pm 0.00$ & $0.08 \pm 0.00$ & $0.09 \pm 0.00$ & $0.09 \pm 0.00$ & $0.12 \pm 0.01 *$ & 0.11 \\
\hline \multicolumn{7}{|c|}{$\mu \mathrm{g} \mathrm{Co} / \mathrm{g}$ right lung } \\
\hline Day 17 & $N A^{f}$ & $23.28 \pm 0.86^{\mathrm{f}}$ & $43.24 \pm 1.18^{\mathrm{f}}$ & $61.99 \pm 3.66^{\mathrm{f}}$ & $56.24 \pm 2.78^{\mathrm{g}}$ & $24.28 \pm 11.05$ \\
\hline Week 3 PE & NA & $3.10 \pm 0.03$ & $5.04 \pm 0.15$ & $8.06 \pm 0.48$ & $4.99 \pm 1.14$ & 9.68 \\
\hline \multicolumn{7}{|c|}{$\mu \mathrm{g} \mathrm{Co/right} \mathrm{lung}$} \\
\hline Day 17 & $N A^{h}$ & $2.356 \pm 0.120 \mathrm{i}$ & $4.939 \pm 0.241^{\mathrm{i}}$ & $7.303 \pm 1.512^{\mathrm{i}}$ & $8.848^{b}$ & NA \\
\hline Week 3 PE & NA & $0.253 \pm 0.014$ & $0.473 \pm 0.036$ & $0.751 \pm 0.053$ & $0.553 \pm 0.086$ & 1.017 \\
\hline \multicolumn{7}{|c|}{$\mu \mathrm{g} \mathrm{Co} /$ right lung per $\mathrm{mg} \mathrm{Co} / \mathrm{m}^{3}$} \\
\hline Day 17 & $\mathrm{NA}^{\mathrm{i}}$ & $0.942 \pm 0.048^{\mathrm{i}}$ & $0.988 \pm 0.048^{\mathrm{i}}$ & $0.730 \pm 0.151^{\mathrm{h}}$ & $0.442^{\mathrm{b}}$ & NA \\
\hline Week 3 PE & NA & $0.101 \pm 0.005$ & $0.095 \pm 0.007$ & $0.075 \pm 0.005$ & $0.028 \pm 0.004$ & 0.025 \\
\hline
\end{tabular}

*Significantly different $(\mathrm{P} \leq 0.05)$ from the chamber control group by Dunnett's or Shirley's test.

$* * \mathrm{P} \leq 0.01$.

${ }^{a}$ Data are presented as mean \pm standard error. All values except absolute tissue weights are control corrected. Statistical tests were performed only on absolute tissue weight data.

$\mathrm{NA}=$ not applicable; $\mathrm{PE}=$ postexposure.

$\mathrm{b}_{\mathrm{n}}=1$; No standard error was calculated; less than two measurements were available.

${ }^{\mathrm{c}}$ Total lung cobalt burden was calculated using the weight of the total lung with mainstem bronchi at collection and cobalt concentration measured in the right lung.

${ }^{\mathrm{d}}$ Right lung cobalt burden was calculated using the right lung weight and the concentration of cobalt measured in the right lung.

eNo data are available.

$\mathrm{f} n=7$.

$\mathrm{g}_{\mathrm{n}}=6$.

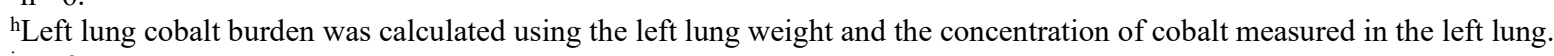

${ }^{\mathrm{i}} \mathrm{n}=2$. 
Cobalt Metal, NTP TR 581

Table I-10. Deposition and Clearance Parameter Estimates for Female Mice in the Two-week Inhalation Study of Cobalt Metal ${ }^{a}$

\begin{tabular}{|c|c|c|c|c|c|}
\hline Tissue & $\begin{array}{c}\text { Exposure } \\
\text { Concentration } \\
\left(\mathbf{m g} / \mathrm{m}^{3}\right)\end{array}$ & $k\left(\right.$ days $\left.^{-1}\right)$ & $\mathbf{t}_{1 / 2}$ (days) & $\alpha(\mu g$ Co/day) & $A_{e}\left(\mu g C_{0}\right)$ \\
\hline \multirow[t]{4}{*}{ Blood } & 2.5 & 0.10 & 7.26 & NA & NA \\
\hline & 5 & 0.11 & 6.19 & NA & NA \\
\hline & 10 & 0.14 & 4.81 & NA & NA \\
\hline & 20 & 0.17 & 4.11 & NA & NA \\
\hline \multirow[t]{4}{*}{ Serum } & 2.5 & 0.19 & 3.71 & NA & NA \\
\hline & 5 & 0.24 & 2.89 & NA & NA \\
\hline & 10 & 0.22 & 3.22 & NA & NA \\
\hline & 20 & 0.22 & 3.17 & NA & NA \\
\hline \multirow[t]{4}{*}{ Lung $^{\mathrm{b}}$} & 2.5 & 0.11 & 6.57 & 0.57 & 5.44 \\
\hline & 5 & 0.11 & 6.30 & 1.25 & 11.41 \\
\hline & 10 & 0.11 & 6.40 & 1.87 & 17.26 \\
\hline & 20 & 0.13 & 5.46 & 2.34 & 18.45 \\
\hline
\end{tabular}

${ }^{a}$ Statistical analyses of these data were not performed due to the limited number of time points and data points within some time points. $\mathrm{k}=$ first order clearance rate constant; $\mathrm{t}_{1 / 2}=$ clearance half-life; $\alpha=$ lung deposition rate; $\mathrm{Ae}_{\mathrm{e}}=$ steady-state lung burden; $\mathrm{NA}=$ not applicable.

${ }^{\mathrm{b}}$ Total lung cobalt burden was calculated using the weight of total lung with mainstem bronchi at collection and cobalt concentration measured in the right lung. 
Cobalt Metal, NTP TR 581

Table I-11. Tissue Weights, Cobalt Concentrations, and Cobalt Burdens for Female Mice in the Three-month Inhalation Study of Cobalt Metal $^{\mathrm{a}}$

\begin{tabular}{|c|c|c|c|c|c|c|}
\hline Parameter & $\begin{array}{c}\text { Chamber } \\
\text { Control }\end{array}$ & $0.625 \mathrm{mg} / \mathrm{m}^{3}$ & $1.25 \mathrm{mg} / \mathrm{m}^{3}$ & $2.5 \mathrm{mg} / \mathrm{m}^{3}$ & $5 \mathrm{mg} / \mathrm{m}^{3}$ & $10 \mathrm{mg} / \mathrm{m}^{3}$ \\
\hline $\mathbf{n}$ & 3 & 3 & 3 & 3 & 3 & 3 \\
\hline \multicolumn{7}{|l|}{ Blood } \\
\hline \multicolumn{7}{|l|}{$\mu \mathrm{g} \mathrm{Co} / \mathrm{g}$ blood } \\
\hline Day 5 & $0.009 \pm 0.000$ & $0.056 \pm 0.015^{*}$ & $0.154 \pm 0.030 * *$ & $0.412 \pm 0.087 * *$ & $0.857 \pm 0.114^{* *}$ & $1.073 \pm 0.029 * *$ \\
\hline Day 12 & $0.009 \pm 0.000$ & $0.134 \pm 0.012^{*}$ & $0.250 \pm 0.045^{* *}$ & $0.352 \pm 0.025^{* *}$ & $0.949 \pm 0.078 * *$ & $1.540 \pm 0.097 * *$ \\
\hline Day 26 & $0.009 \pm 0.000$ & $0.077 \pm 0.010^{*}$ & $0.154 \pm 0.011^{* *}$ & $0.421 \pm 0.023 * *$ & $0.874 \pm 0.113^{* *}$ & $1.634 \pm 0.169^{* *}$ \\
\hline Day 40 & $0.009 \pm 0.000$ & $0.155 \pm 0.014^{*}$ & $0.263 \pm 0.036^{* *}$ & $0.520 \pm 0.026^{* *}$ & $0.941 \pm 0.119^{* *}$ & $1.619 \pm 0.053 * *$ \\
\hline Day 61 & $0.009 \pm 0.000$ & $0.111 \pm 0.028 *$ & $0.188 \pm 0.008^{* *}$ & $0.359 \pm 0.046^{* *}$ & $0.916 \pm 0.071^{* *}$ & $2.576 \pm 0.944 * *$ \\
\hline Day 89 & $0.009 \pm 0.000$ & $0.093 \pm 0.013^{*}$ & $0.155 \pm 0.007 * *$ & $0.308 \pm 0.008^{* *}$ & $0.660 \pm 0.035^{* *}$ & $1.257 \pm 0.088^{* *}$ \\
\hline PE day 7 & $0.009 \pm 0.000$ & $0.009 \pm 0.000$ & $0.014 \pm 0.005$ & $0.025 \pm 0.000 *$ & $0.063 \pm 0.004 * *$ & $0.115 \pm 0.002 * *$ \\
\hline PE day 14 & $0.009 \pm 0.000$ & $0.009 \pm 0.000$ & $0.009 \pm 0.000$ & $0.009 \pm 0.000$ & $0.025 \pm 0.000 * *$ & $0.069 \pm 0.008 * *$ \\
\hline PE day 28 & $0.009 \pm 0.000$ & $0.009 \pm 0.000$ & $0.009 \pm 0.000$ & $0.009 \pm 0.000$ & $0.009 \pm 0.000$ & $0.025 \pm 0.000 * *$ \\
\hline PE day 42 & $0.009 \pm 0.000$ & $0.009 \pm 0.000$ & $0.009 \pm 0.000$ & $0.009 \pm 0.000$ & $0.009 \pm 0.000$ & $0.009 \pm 0.000$ \\
\hline \multicolumn{7}{|c|}{$\mu \mathrm{g} \mathrm{Co} / \mathrm{g}$ blood per $\mathrm{mg} \mathrm{Co} / \mathrm{m}^{3}$} \\
\hline Day 5 & - & $0.089 \pm 0.025$ & $0.123 \pm 0.024$ & $0.165 \pm 0.035$ & $0.171 \pm 0.023$ & $0.107 \pm 0.003$ \\
\hline Day 12 & - & $0.214 \pm 0.019$ & $0.200 \pm 0.036$ & $0.141 \pm 0.010$ & $0.190 \pm 0.016$ & $0.154 \pm 0.010$ \\
\hline Day 26 & - & $0.123 \pm 0.016$ & $0.123 \pm 0.009$ & $0.168 \pm 0.009$ & $0.175 \pm 0.023$ & $0.163 \pm 0.017$ \\
\hline Day 40 & - & $0.248 \pm 0.023$ & $0.211 \pm 0.029$ & $0.208 \pm 0.010$ & $0.188 \pm 0.024$ & $0.162 \pm 0.005$ \\
\hline Day 61 & - & $0.178 \pm 0.045$ & $0.151 \pm 0.006$ & $0.144 \pm 0.019$ & $0.183 \pm 0.014$ & $0.258 \pm 0.094$ \\
\hline Day 89 & - & $0.149 \pm 0.020$ & $0.124 \pm 0.006$ & $0.123 \pm 0.003$ & $0.132 \pm 0.007$ & $0.126 \pm 0.009$ \\
\hline PE day 7 & - & $0.014 \pm 0.000$ & $0.011 \pm 0.004$ & $0.010 \pm 0.000$ & $0.013 \pm 0.001$ & $0.011 \pm 0.000$ \\
\hline PE day 14 & - & $0.014 \pm 0.000$ & $0.007 \pm 0.000$ & $0.004 \pm 0.000$ & $0.005 \pm 0.000$ & $0.007 \pm 0.001$ \\
\hline PE day 28 & - & $0.014 \pm 0.000$ & $0.007 \pm 0.000$ & $0.004 \pm 0.000$ & $0.002 \pm 0.000$ & $0.003 \pm 0.000$ \\
\hline
\end{tabular}


Cobalt Metal, NTP TR 581

\begin{tabular}{|c|c|c|c|c|c|c|}
\hline Parameter & $\begin{array}{l}\text { Chamber } \\
\text { Control }\end{array}$ & $0.625 \mathrm{mg} / \mathrm{m}^{3}$ & $1.25 \mathrm{mg} / \mathrm{m}^{3}$ & $2.5 \mathrm{mg} / \mathrm{m}^{3}$ & $5 \mathrm{mg} / \mathrm{m}^{3}$ & $10 \mathrm{mg} / \mathrm{m}^{3}$ \\
\hline PE day 42 & - & $0.014 \pm 0.000$ & $0.007 \pm 0.000$ & $0.004 \pm 0.000$ & $0.002 \pm 0.000$ & $0.001 \pm 0.000$ \\
\hline \multicolumn{7}{|l|}{ Liver } \\
\hline \multicolumn{7}{|l|}{ Absolute liver wt (g) } \\
\hline Day 26 & $0.969 \pm 0.055^{\prime}$ & $0.970 \pm 0.024$ & $0.902 \pm 0.019$ & $0.873 \pm 0.056$ & $0.813 \pm 0.031^{*}$ & $0.754 \pm 0.022 *$ \\
\hline Day 40 & $1.011 \pm 0.011$ & $1.046 \pm 0.021$ & $1.082 \pm 0.008$ & $0.989 \pm 0.032$ & $0.880 \pm 0.052$ & $0.802 \pm 0.019$ \\
\hline \multicolumn{7}{|l|}{$\mu \mathrm{g} \mathrm{Co} / \mathrm{g}$ liver } \\
\hline Day 26 & $0.220 \pm 0.000$ & $0.500 \pm 0.000^{*}$ & $0.956 \pm 0.240 * *$ & $2.494 \pm 0.247 * *$ & $5.768 \pm 0.593 * *$ & $12.002 \pm 1.095^{* *}$ \\
\hline Day 40 & $0.220 \pm 0.000^{\mathrm{b}}$ & $0.722 \pm 0.222$ & $1.808 \pm 0.289^{*}$ & $3.307 \pm 0.101^{* *}$ & $6.676 \pm 1.189 * *$ & $11.583 \pm 0.434^{* *}$ \\
\hline \multicolumn{7}{|l|}{$\mu \mathrm{g}$ Co/liver } \\
\hline Day 26 & $0.213 \pm 0.012$ & $0.485 \pm 0.012 *$ & $0.866 \pm 0.220^{*}$ & $2.157 \pm 0.149^{* *}$ & $4.699 \pm 0.538^{* *}$ & $9.065 \pm 0.943^{* *}$ \\
\hline Day 40 & $0.222 \pm 0.004^{\mathrm{b}}$ & $0.747 \pm 0.214$ & $1.952 \pm 0.297^{*}$ & $3.263 \pm 0.022 * *$ & $5.963 \pm 1.311^{* *}$ & $9.310 \pm 0.560^{* *}$ \\
\hline \multicolumn{7}{|c|}{$\mu \mathrm{g} \mathrm{Co} /$ liver per $\mathrm{mg} \mathrm{Co} / \mathrm{m}^{3}$} \\
\hline Day 26 & - & $0.776 \pm 0.019$ & $0.693 \pm 0.176$ & $0.863 \pm 0.060$ & $0.940 \pm 0.108$ & $0.906 \pm 0.094$ \\
\hline Day 40 & - & $1.195 \pm 0.343$ & $1.561 \pm 0.237$ & $1.305 \pm 0.009$ & $1.193 \pm 0.262$ & $0.931 \pm 0.056$ \\
\hline \multicolumn{7}{|l|}{ Total lung ${ }^{\mathrm{c}}$} \\
\hline \multicolumn{7}{|c|}{ Absolute total lung wt (g) } \\
\hline Day 5 & $0.144 \pm 0.006$ & $0.138 \pm 0.012$ & $0.151 \pm 0.007$ & $0.158 \pm 0.007$ & $0.167 \pm 0.011$ & $0.178 \pm 0.023$ \\
\hline Day 12 & $0.144 \pm 0.005$ & $0.158 \pm 0.010$ & $0.168 \pm 0.015$ & $0.177 \pm 0.012 *$ & $0.200 \pm 0.006^{* *}$ & $0.238 \pm 0.023^{* *}$ \\
\hline Day 26 & $0.145 \pm 0.006$ & $0.157 \pm 0.002$ & $0.163 \pm 0.009$ & $0.169 \pm 0.008$ & $0.203 \pm 0.014 * *$ & $0.251 \pm 0.015^{* *}$ \\
\hline Day 40 & $0.168 \pm 0.008$ & $0.165 \pm 0.007$ & $0.183 \pm 0.006$ & $0.191 \pm 0.002 *$ & $0.247 \pm 0.015^{* *}$ & $0.259 \pm 0.006^{* *}$ \\
\hline Day 61 & $0.188 \pm 0.023$ & $0.170 \pm 0.002$ & $0.227 \pm 0.025$ & $0.189 \pm 0.003$ & $0.255 \pm 0.005^{*}$ & $0.279 \pm 0.015^{*}$ \\
\hline Day 89 & $0.189 \pm 0.022$ & $0.187 \pm 0.006$ & $0.197 \pm 0.002$ & $0.206 \pm 0.003$ & $0.260 \pm 0.010^{*}$ & $0.300 \pm 0.014 * *$ \\
\hline PE day 7 & $0.174 \pm 0.015$ & $0.178 \pm 0.013$ & $0.203 \pm 0.019$ & $0.201 \pm 0.019$ & $0.237 \pm 0.014 *$ & $0.289 \pm 0.014^{* *}$ \\
\hline PE day 14 & $0.176 \pm 0.011$ & $0.186 \pm 0.009$ & $0.190 \pm 0.006$ & $0.183 \pm 0.004$ & $0.209 \pm 0.009^{*}$ & $0.249 \pm 0.016^{* *}$ \\
\hline PE day 28 & $0.174 \pm 0.008$ & $0.192 \pm 0.013$ & $0.168 \pm 0.006$ & $0.184 \pm 0.010$ & $0.184 \pm 0.007$ & $0.209 \pm 0.006$ \\
\hline
\end{tabular}


Cobalt Metal, NTP TR 581

\begin{tabular}{|c|c|c|c|c|c|c|}
\hline Parameter & $\begin{array}{c}\text { Chamber } \\
\text { Control }\end{array}$ & $0.625 \mathrm{mg} / \mathrm{m}^{3}$ & $1.25 \mathrm{mg} / \mathrm{m}^{3}$ & $2.5 \mathrm{mg} / \mathrm{m}^{3}$ & $5 \mathrm{mg} / \mathrm{m}^{3}$ & $10 \mathrm{mg} / \mathrm{m}^{3}$ \\
\hline PE day 42 & $0.169 \pm 0.002$ & $0.169 \pm 0.005$ & $0.181 \pm 0.007$ & $0.178 \pm 0.002 *$ & $0.196 \pm 0.005 * *$ & $0.213 \pm 0.014^{* *}$ \\
\hline \multicolumn{7}{|l|}{$\mu \mathrm{g} \mathrm{Co} / \mathrm{g}$ total lung } \\
\hline Day 5 & $0.200 \pm 0.000$ & $5.920 \pm 0.456^{*}$ & $10.292 \pm 0.874 * *$ & $20.877 \pm 1.371 * *$ & $28.959 \pm 2.874 * *$ & $15.697 \pm 2.720 * *$ \\
\hline Day 12 & $0.200 \pm 0.000$ & $6.770 \pm 0.817^{*}$ & $12.310 \pm 1.396 * *$ & $22.624 \pm 1.973 * *$ & $46.133 \pm 3.142 * *$ & $47.334 \pm 19.444^{* *}$ \\
\hline Day 26 & $0.200 \pm 0.000^{\mathrm{b}}$ & $5.505 \pm 0.313$ & $12.957 \pm 1.029^{*}$ & $27.070 \pm 0.926^{* *}$ & $72.553 \pm 3.071 * *$ & $109.153 \pm 3.027^{* *}$ \\
\hline Day 40 & $0.200 \pm 0.000$ & $7.038 \pm 0.531^{*}$ & $16.986 \pm 0.344 * *$ & $28.659 \pm 1.896^{* *}$ & $85.895 \pm 5.226^{* *}$ & $141.694 \pm 2.904^{* *}$ \\
\hline Day 61 & $0.200 \pm 0.000$ & $6.517 \pm 0.412^{*}$ & $12.891 \pm 1.662 * *$ & $32.787 \pm 2.120^{* *}$ & $77.234 \pm 6.030 * *$ & $143.304 \pm 6.747^{* *}$ \\
\hline Day 89 & $0.200 \pm 0.000$ & $6.944 \pm 0.431^{*}$ & $18.504 \pm 1.996 * *$ & $39.129 \pm 0.956^{* *}$ & $108.420 \pm 3.413 * *$ & $141.003 \pm 5.456^{* *}$ \\
\hline PE day 7 & $0.200 \pm 0.000$ & $2.342 \pm 0.041^{*}$ & $6.392 \pm 0.619 * *$ & $17.275 \pm 2.533^{* *}$ & $44.664 \pm 1.910^{* *}$ & $65.663 \pm 3.483 * *$ \\
\hline PE day 14 & $0.200 \pm 0.000$ & $1.565 \pm 0.160^{*}$ & $5.147 \pm 0.114^{* *}$ & $15.226 \pm 0.822 * *$ & $42.632 \pm 3.321 * *$ & $48.445 \pm 4.201 * *$ \\
\hline PE day 28 & $0.200 \pm 0.000$ & $0.670 \pm 0.170^{*}$ & $3.548 \pm 0.174^{* *}$ & $9.758 \pm 0.484 * *$ & $30.466 \pm 0.878 * *$ & $42.285 \pm 1.482 * *$ \\
\hline PE day 42 & $0.200 \pm 0.000$ & $0.500 \pm 0.000^{*}$ & $2.762 \pm 0.027 * *$ & $7.583 \pm 0.446^{* *}$ & $24.409 \pm 1.234^{* *}$ & $31.551 \pm 2.958^{* *}$ \\
\hline \multicolumn{7}{|l|}{$\mu \mathrm{g}$ Co/total lung } \\
\hline Day 5 & $0.029 \pm 0.001$ & $0.809 \pm 0.050^{*}$ & $1.539 \pm 0.072 * *$ & $3.273 \pm 0.100^{* *}$ & $4.791 \pm 0.286^{* *}$ & $2.671 \pm 0.206^{* *}$ \\
\hline Day 12 & $0.029 \pm 0.001$ & $1.051 \pm 0.054^{*}$ & $2.031 \pm 0.080 * *$ & $3.996 \pm 0.460 * *$ & $9.219 \pm 0.537 * *$ & $10.431 \pm 3.867^{* *}$ \\
\hline Day 26 & $0.030 \pm 0.001^{b}$ & $0.864 \pm 0.044$ & $2.094 \pm 0.060^{*}$ & $4.561 \pm 0.054 * *$ & $14.723 \pm 1.257^{* *}$ & $27.397 \pm 1.454^{* *}$ \\
\hline Day 40 & $0.034 \pm 0.002$ & $1.154 \pm 0.071 *$ & $3.110 \pm 0.141^{* *}$ & $5.479 \pm 0.334 * *$ & $21.033 \pm 0.324 * *$ & $36.623 \pm 0.529 * *$ \\
\hline Day 61 & $0.038 \pm 0.005$ & $1.104 \pm 0.060^{*}$ & $2.849 \pm 0.109 * *$ & $6.193 \pm 0.388 * *$ & $19.777 \pm 1.877 * *$ & $39.990 \pm 2.606^{* *}$ \\
\hline Day 89 & $0.038 \pm 0.004$ & $1.293 \pm 0.067^{*}$ & $3.644 \pm 0.413 * *$ & $8.070 \pm 0.150 * *$ & $28.293 \pm 1.946^{* *}$ & $42.263 \pm 1.680^{* *}$ \\
\hline PE day 7 & $0.035 \pm 0.003$ & $0.417 \pm 0.033^{*}$ & $1.274 \pm 0.012 * *$ & $3.384 \pm 0.327 * *$ & $10.517 \pm 0.251^{* *}$ & $18.903 \pm 0.681 * *$ \\
\hline PE day 14 & $0.035 \pm 0.002$ & $0.290 \pm 0.030^{*}$ & $0.977 \pm 0.051 * *$ & $2.798 \pm 0.207 * *$ & $8.857 \pm 0.430 * *$ & $11.943 \pm 0.455^{* *}$ \\
\hline PE day 28 & $0.035 \pm 0.002$ & $0.126 \pm 0.026^{*}$ & $0.598 \pm 0.050 * *$ & $1.801 \pm 0.150 * *$ & $5.594 \pm 0.064^{* *}$ & $8.864 \pm 0.515^{* *}$ \\
\hline PE day 42 & $0.034 \pm 0.000$ & $0.085 \pm 0.003^{*}$ & $0.499 \pm 0.015^{* *}$ & $1.347 \pm 0.079 * *$ & $4.765 \pm 0.137 * *$ & $6.678 \pm 0.517 * *$ \\
\hline \multicolumn{7}{|c|}{$\mu \mathrm{g} \mathrm{Co} /$ total lung per $\mathrm{mg} \mathrm{Co} / \mathrm{m}^{3}$} \\
\hline Day 5 & - & $1.294 \pm 0.080$ & $1.231 \pm 0.058$ & $1.309 \pm 0.040$ & $0.958 \pm 0.057$ & $0.267 \pm 0.021$ \\
\hline
\end{tabular}


Cobalt Metal, NTP TR 581

\begin{tabular}{|c|c|c|c|c|c|c|}
\hline Parameter & $\begin{array}{c}\text { Chamber } \\
\text { Control }\end{array}$ & $0.625 \mathrm{mg} / \mathrm{m}^{3}$ & $1.25 \mathrm{mg} / \mathrm{m}^{3}$ & $2.5 \mathrm{mg} / \mathrm{m}^{3}$ & $5 \mathrm{mg} / \mathrm{m}^{3}$ & $10 \mathrm{mg} / \mathrm{m}^{3}$ \\
\hline Day 12 & - & $1.682 \pm 0.086$ & $1.625 \pm 0.064$ & $1.599 \pm 0.184$ & $1.844 \pm 0.107$ & $1.043 \pm 0.387$ \\
\hline Day 26 & - & $1.382 \pm 0.070$ & $1.675 \pm 0.048$ & $1.824 \pm 0.022$ & $2.945 \pm 0.251$ & $2.740 \pm 0.145$ \\
\hline Day 40 & - & $1.847 \pm 0.114$ & $2.488 \pm 0.112$ & $2.191 \pm 0.134$ & $4.207 \pm 0.065$ & $3.662 \pm 0.053$ \\
\hline Day 61 & - & $1.767 \pm 0.096$ & $2.279 \pm 0.087$ & $2.477 \pm 0.155$ & $3.955 \pm 0.375$ & $3.999 \pm 0.261$ \\
\hline Day 89 & - & $2.069 \pm 0.106$ & $2.915 \pm 0.330$ & $3.228 \pm 0.060$ & $5.659 \pm 0.389$ & $4.226 \pm 0.168$ \\
\hline PE day 7 & - & $0.667 \pm 0.052$ & $1.019 \pm 0.010$ & $1.354 \pm 0.131$ & $2.103 \pm 0.050$ & $1.890 \pm 0.068$ \\
\hline PE day 14 & - & $0.464 \pm 0.048$ & $0.782 \pm 0.041$ & $1.119 \pm 0.083$ & $1.771 \pm 0.086$ & $1.194 \pm 0.046$ \\
\hline PE day 28 & - & $0.201 \pm 0.042$ & $0.478 \pm 0.040$ & $0.721 \pm 0.060$ & $1.119 \pm 0.013$ & $0.886 \pm 0.051$ \\
\hline PE day 42 & - & $0.135 \pm 0.004$ & $0.399 \pm 0.012$ & $0.539 \pm 0.032$ & $0.953 \pm 0.027$ & $0.668 \pm 0.052$ \\
\hline
\end{tabular}

*Significantly different $(\mathrm{P} \leq 0.05)$ from the chamber control group by Shirley's test.

$* * \mathrm{P} \leq 0.01$.

${ }^{a}$ Data are presented as mean \pm standard error. Values below the limit of detection (LOD) or below the experimental limit of quantitation (ELOQ) were replaced with $1 / 2$ the LOD or ELOQ value. Statistical tests were performed only on data that were not normalized. PE $=$ postexposure.

$\mathrm{b}_{\mathrm{n}}=2$.

${ }^{\mathrm{c} T o t a l}$ lung cobalt burden was calculated using the weight of the total lung with mainstem bronchi at collection and cobalt concentration measured in the right lung. 
Table I-12. Lung Deposition and Clearance Parameter Estimates for Female Mice During the Recovery Period in the Three-month Inhalation Study of Cobalt Metal (Two-Compartment Model) $)^{\mathbf{a}}$

\begin{tabular}{|c|c|c|c|c|c|}
\hline Parameter & $0.625 \mathrm{mg} / \mathrm{m}^{3}$ & $1.25 \mathrm{mg} / \mathrm{m}^{3}$ & $2.5 \mathrm{mg} / \mathrm{m}^{3}$ & $5 \mathrm{mg} / \mathrm{m}^{3}$ & $10 \mathrm{mg} / \mathrm{m}^{3}$ \\
\hline $\mathrm{A}$ ( $\mu \mathrm{g} \mathrm{Co} /$ total lung) & $0.947 \pm 0.124$ & $2.302 \pm 0.341$ & $4.283 \pm 0.736$ & $16.746 \pm 1.876$ & $28.342 \pm 3.014$ \\
\hline $\mathrm{A}\left(\right.$ days $\left.^{-1}\right)$ & $0.271 \pm 0.060$ & $0.268 \pm 0.073$ & $0.509 \pm 0.454$ & $0.381 \pm 0.119$ & $0.215 \pm 0.042$ \\
\hline $\mathrm{t}_{1 / 2 \text { (rapid) }}$ (days) & $2.556 \pm 0.127$ & $2.592 \pm 0.194$ & $1.361 \pm 1.080$ & $1.818 \pm 0.177$ & $3.225 \pm 0.123$ \\
\hline $\mathrm{B}(\mu \mathrm{g} \mathrm{Co} /$ total lung $)$ & $0.324 \pm 0.070$ & $1.103 \pm 0.222$ & $3.776 \pm 0.458$ & $10.933 \pm 1.141$ & $13.811 \pm 2.331$ \\
\hline $\mathrm{b}\left(\right.$ days $\left.^{-1}\right)$ & $0.022 \pm 0.006$ & $0.020 \pm 0.006$ & $0.026 \pm 0.004$ & $0.021 \pm 0.003$ & $0.018 \pm 0.005$ \\
\hline $\mathrm{t}_{1 / 2 \text { (slow) }}$ (days) & $30.88 \pm 2.56$ & $34.66 \pm 3.11$ & $27.08 \pm 0.64$ & $33.01 \pm 0.82$ & $39.33 \pm 2.80$ \\
\hline
\end{tabular}

Table I-13. Lung Deposition and Clearance Parameter Estimates for Female Mice During the Exposure Period in the Three-month Inhalation Study of Cobalt Metal (One-Compartment Model) ${ }^{\mathrm{a}}$

\begin{tabular}{|c|c|c|c|c|c|}
\hline Parameter & $0.625 \mathrm{mg} / \mathrm{m}^{3}$ & $1.25 \mathrm{mg} / \mathrm{m}^{3}$ & $2.5 \mathrm{mg} / \mathrm{m}^{3}$ & $5 \mathrm{mg} / \mathrm{m}^{3}$ & $10 \mathrm{mg} / \mathrm{m}^{3}$ \\
\hline $\begin{array}{l}\mathrm{D}(\mu \mathrm{g} \text { Co/total lung } \\
\text { per day })\end{array}$ & $0.301 \pm 0.062$ & $0.381 \pm 0.061$ & $0.737 \pm 0.129$ & $0.971 \pm 0.076$ & $0.710 \pm 0.094$ \\
\hline $\mathrm{k}\left(\right.$ days $\left.^{-1}\right)$ & $0.287 \pm 0.065$ & $0.141 \pm 0.027$ & $0.132 \pm 0.028$ & $0.041 \pm 0.006$ & $0.006 \pm 0.005$ \\
\hline$t_{1 / 2}$ (days) & $2.412 \pm 0.122$ & $4.903 \pm 0.183$ & $5.249 \pm 0.235$ & $16.996 \pm 0.321$ & $121.975 \pm 104.527$ \\
\hline $\begin{array}{l}\mathrm{L}_{\mathrm{ss}}(\mu \mathrm{g} \mathrm{Co} / \text { total } \\
\text { lung) }\end{array}$ & $1.048 \pm 0.045$ & $2.691 \pm 0.162$ & $5.578 \pm 0.375$ & $23.810 \pm 1.853$ & $124.948 \pm 103.207$ \\
\hline
\end{tabular}

${ }^{\mathrm{a}}$ Data are presented as mean \pm standard error. $\mathrm{D}=$ deposition rate; $\mathrm{k}=$ lung clearance rate constant; $\mathrm{t} / 2=$ lung clearance half-life; $\mathrm{L}_{\mathrm{ss}}=$ steady-state lung burden. 
Cobalt Metal, NTP TR 581

Table I-14. Lung Weights, Cobalt Concentrations, and Cobalt Burdens for Female Mice in the Two-year Inhalation Study of Cobalt Metal ${ }^{a}$

\begin{tabular}{|c|c|c|c|c|}
\hline & Chamber Control & $1.25 \mathrm{mg} / \mathrm{m}^{3}$ & $2.5 \mathrm{mg} / \mathrm{m}^{3}$ & $5 \mathrm{mg} / \mathrm{m}^{3}$ \\
\hline $\mathbf{n}$ & 5 & 5 & 5 & 5 \\
\hline \multicolumn{5}{|c|}{ Absolute total lung wt $(\mathrm{g})^{\mathrm{b}}$} \\
\hline Day 1 & $0.130 \pm 0.005$ & $0.134 \pm 0.002$ & $0.133 \pm 0.004$ & $0.130 \pm 0.002$ \\
\hline Day 2 & $0.139 \pm 0.003$ & $0.130 \pm 0.004$ & $0.139 \pm 0.004$ & $0.139 \pm 0.003$ \\
\hline Day 3 & $0.132 \pm 0.006$ & $0.140 \pm 0.002$ & $0.136 \pm 0.005$ & $0.146 \pm 0.006$ \\
\hline Day 4 & $0.130 \pm 0.004$ & $0.138 \pm 0.007$ & $0.156 \pm 0.002 * *$ & $0.163 \pm 0.007 * *$ \\
\hline Day 184 & $0.180 \pm 0.015$ & $0.175 \pm 0.005$ & $0.208 \pm 0.006^{*}$ & $0.283 \pm 0.005^{* *}$ \\
\hline Day 366 & $0.170 \pm 0.003$ & $0.218 \pm 0.004^{* *}$ & $0.249 \pm 0.004 * *$ & $0.418 \pm 0.005^{* *}$ \\
\hline Day 548 & $0.208 \pm 0.015$ & $0.279 \pm 0.024^{\mathrm{c}}$ & $0.380 \pm 0.077 * *$ & $0.508 \pm 0.029^{* *}$ \\
\hline \multicolumn{5}{|c|}{$\mu \mathrm{g} \mathrm{Co} / \mathrm{g}$ total lung } \\
\hline Day 1 & $0.200 \pm 0.000^{\mathrm{c}}$ & $5.527 \pm 0.184 * \mathrm{c}$ & $11.246 \pm 0.403 * *$ & $21.869 \pm 0.910^{* *}$ \\
\hline Day 2 & $0.200 \pm 0.000$ & $7.797 \pm 0.396^{* *}$ & $14.953 \pm 0.393 * *$ & $19.443 \pm 1.488^{* *}$ \\
\hline Day 3 & $0.200 \pm 0.000$ & $9.994 \pm 0.340^{* *}$ & $18.467 \pm 0.502 * *$ & $25.041 \pm 0.392 * *$ \\
\hline Day 4 & $0.200 \pm 0.000$ & $11.009 \pm 0.391 * *$ & $20.554 \pm 0.638^{* *}$ & $26.425 \pm 0.767 * *$ \\
\hline Day 184 & $0.200 \pm 0.000$ & $32.370 \pm 2.691 * *$ & $58.677 \pm 2.508^{* *}$ & $120.993 \pm 6.170 * *$ \\
\hline Day 366 & $0.200 \pm 0.000$ & $44.922 \pm 2.798 * *$ & $82.778 \pm 8.031 * *$ & $126.628 \pm 4.668^{* *}$ \\
\hline Day 548 & $0.200 \pm 0.000$ & $38.918 \pm 0.471 * * \mathrm{c}$ & $53.982 \pm 9.362 * *$ & $93.214 \pm 3.957 * *$ \\
\hline \multicolumn{5}{|c|}{$\mu \mathrm{g} \mathrm{Co/total} \mathrm{lung}$} \\
\hline Day 1 & $0.026 \pm 0.001^{\mathrm{c}}$ & $0.743 \pm 0.017^{* c}$ & $1.499 \pm 0.084^{* *}$ & $2.839 \pm 0.085^{* *}$ \\
\hline Day 2 & $0.028 \pm 0.001$ & $1.020 \pm 0.072^{* *}$ & $2.075 \pm 0.081^{* *}$ & $2.698 \pm 0.191^{* *}$ \\
\hline Day 3 & $0.026 \pm 0.001$ & $1.399 \pm 0.057^{* *}$ & $2.512 \pm 0.157^{* *}$ & $3.654 \pm 0.144^{* *}$ \\
\hline Day 4 & $0.026 \pm 0.001$ & $1.524 \pm 0.118^{* *}$ & $3.191 \pm 0.056^{* *}$ & $4.303 \pm 0.143^{* *}$ \\
\hline Day 184 & $0.036 \pm 0.003$ & $5.663 \pm 0.475^{* *}$ & $12.134 \pm 0.285^{* *}$ & $34.246 \pm 1.933 * *$ \\
\hline Day 366 & $0.034 \pm 0.001$ & $9.834 \pm 0.698^{* *}$ & $20.538 \pm 1.833^{* *}$ & $52.896 \pm 1.698^{* *}$ \\
\hline Day 548 & $0.042 \pm 0.003$ & $10.883 \pm 0.995^{* c}$ & $17.680 \pm 1.790^{* *}$ & $47.218 \pm 2.652^{* *}$ \\
\hline \multicolumn{5}{|c|}{$\mu \mathrm{g} \mathrm{Co} /$ total lung per $\mathrm{mg} \mathrm{Co} / \mathrm{m}^{3}$} \\
\hline Day 1 & - & $0.595 \pm 0.014^{\mathrm{c}}$ & $0.600 \pm 0.033$ & $0.568 \pm 0.017$ \\
\hline Day 2 & - & $0.816 \pm 0.058$ & $0.830 \pm 0.032$ & $0.540 \pm 0.038$ \\
\hline Day 3 & - & $1.119 \pm 0.046$ & $1.005 \pm 0.063$ & $0.731 \pm 0.029$ \\
\hline Day 4 & - & $1.220 \pm 0.095$ & $1.276 \pm 0.022$ & $0.861 \pm 0.029$ \\
\hline Day 184 & - & $4.530 \pm 0.380$ & $4.854 \pm 0.114$ & $6.849 \pm 0.387$ \\
\hline Day 366 & - & $7.867 \pm 0.559$ & $8.215 \pm 0.733$ & $10.579 \pm 0.340$ \\
\hline Day 548 & - & $8.706 \pm 0.796^{c}$ & $7.072 \pm 0.716$ & $9.444 \pm 0.530$ \\
\hline
\end{tabular}

*Significantly different $(\mathrm{P} \leq 0.05)$ from the chamber control group by Shirley's test.

$* * \mathrm{P} \leq 0.01$.

aData are presented as mean \pm standard error. Values below the limit of detection (LOD) or below the experimental limit of quantitation (ELOQ) were replaced with $1 / 2$ the LOD or ELOQ value. Statistical tests were performed only on data that were not normalized.

${ }^{\mathrm{b}}$ Total lung cobalt burden was calculated using the weight of the total lung with mainstem bronchi at collection and cobalt concentration measured in the right lung.

${ }^{c} n=4$ 
Table I-15. Lung Deposition and Clearance Parameter Estimates for Female Mice During the Exposure Periods in the Three-month and Two-year Inhalation Studies of Cobalt Metal (TwoCompartment Model) ${ }^{a}$

\begin{tabular}{|c|c|c|c|}
\hline Parameter & $1.25 \mathrm{mg} / \mathrm{m}^{3}$ & $2.5 \mathrm{mg} / \mathrm{m}^{3}$ & $5 \mathrm{mg} / \mathrm{m}^{3}$ \\
\hline $\mathrm{A}(\mu \mathrm{g} \mathrm{Co} /$ day $)$ & $0.870 \pm 0.161$ & $1.842 \pm 0.431$ & $1.175 \pm 0.147$ \\
\hline $\mathrm{k}_{\mathrm{a}}\left(\right.$ days $\left.^{-1}\right)$ & $0.559 \pm 0.146$ & $0.652 \pm 0.214$ & $0.132 \pm 0.040$ \\
\hline $\mathrm{B}(\mu \mathrm{g} \mathrm{Co} /$ day $)$ & $0.0274 \pm 0.0034$ & $0.0750 \pm 0.0103$ & $0.2516 \pm 0.0499$ \\
\hline $\mathrm{k}_{\mathrm{b}}\left(\right.$ days $\left.^{-1}\right)$ & $0.00169 \pm 0.00060$ & $0.00404 \pm 0.00083$ & $0.00586 \pm 0.00119$ \\
\hline $\mathrm{F}_{\mathrm{B}}$ & $0.031 \pm 0.005$ & $0.039 \pm 0.008$ & $0.176 \pm 0.033$ \\
\hline $\mathrm{t}_{1 / 2 \mathrm{a}}$ (days) & $1.24 \pm 0.32$ & $1.06 \pm 0.35$ & $5.24 \pm 1.57$ \\
\hline $\mathrm{L}_{\mathrm{SSa}}(\mu \mathrm{g} \mathrm{Co} /$ total lung) & $1.56 \pm 0.16$ & $2.82 \pm 0.37$ & $8.89 \pm 2.20$ \\
\hline $\mathrm{t}_{1 / 2 \mathrm{~b}}$ (days) & $409 \pm 145$ & $172 \pm 35$ & $118 \pm 24$ \\
\hline $\mathrm{L}_{\mathrm{sSb}}$ ( $\mu \mathrm{g}$ Co/total lung) & $16.2 \pm 3.9$ & $18.6 \pm 1.7$ & $42.9 \pm 2.5$ \\
\hline
\end{tabular}



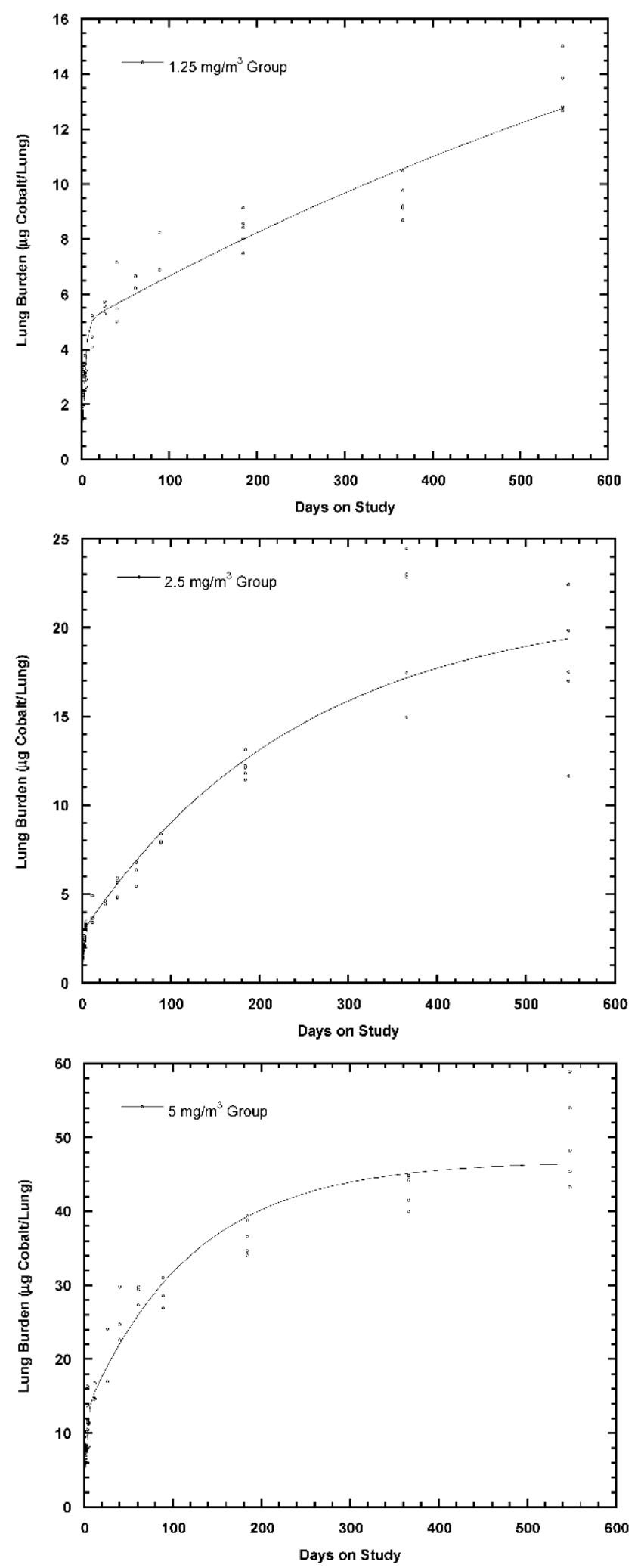

Figure I-1. Lung Cobalt Burdens in 1.25 (top), 2.5 (middle), and 5 (bottom) $\mathrm{mg} / \mathrm{m}^{3}$ Female Rats in the Three-month and Two-year Inhalation Studies of Cobalt Metal

The lines represent the fit of the lung deposition and clearance model to the data. 

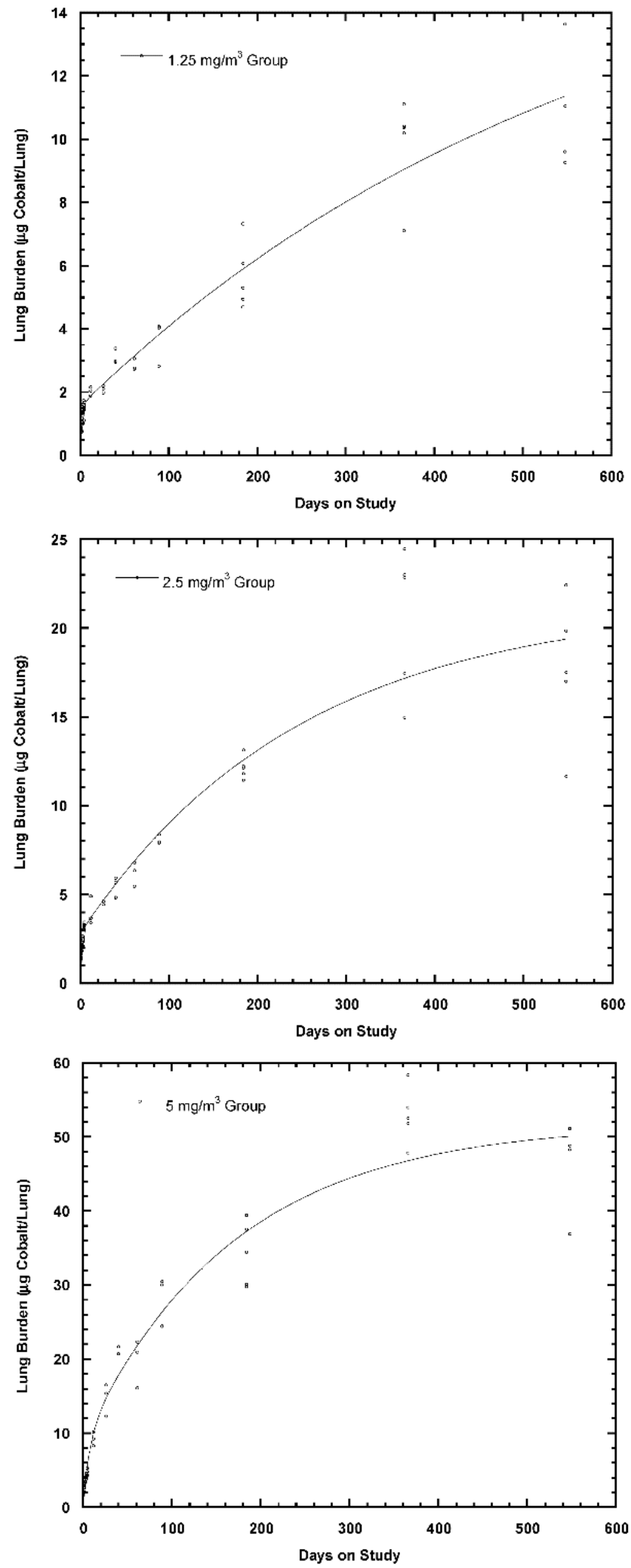

Figure I-2. Lung Cobalt Burdens in 1.25 (top), 2.5 (middle), and 5 (bottom) mg/m $\mathrm{m}^{3}$ Female Mice in the Three-month and Two-year Inhalation Studies of Cobalt Metal

The lines represent the fit of the lung deposition and clearance model to the data. 
Cobalt Metal, NTP TR 581

\section{Appendix J. Liver Cytochrome P450 Data}

\section{Tables}

Table J-1. Liver Cytochrome P450 Data for Female Rats in the Three-month Inhalation

Study of Cobalt Metal ${ }^{\mathrm{a}}$

Table J-2. Liver Cytochrome P450 Data for Special Study Female Mice in the Three-

month Inhalation Study of Cobalt Metal ${ }^{\mathrm{a}}$ 
Cobalt Metal, NTP TR 581

Table J-1. Liver Cytochrome P450 Data for Female Rats in the Three-month Inhalation Study of Cobalt Metal $^{\mathrm{a}}$

\begin{tabular}{|c|c|c|c|c|c|}
\hline & $\begin{array}{l}\text { Chamber } \\
\text { Control }\end{array}$ & $0.625 \mathrm{mg} / \mathrm{m}^{3}$ & $1.25 \mathrm{mg} / \mathrm{m}^{3}$ & $2.5 \mathrm{mg} / \mathrm{m}^{3}$ & $5 \mathrm{mg} / \mathrm{m}^{3}$ \\
\hline $\mathbf{n}$ & 3 & 3 & 3 & 3 & 3 \\
\hline \multicolumn{6}{|c|}{ Acetanilide-4-hydroxylase (A4H) (nmol/minute per mg microsomal protein) } \\
\hline Day 26 & $0.571 \pm 0.022$ & $0.707 \pm 0.022$ & $0.730 \pm 0.042 *$ & $0.615 \pm 0.039$ & $0.635 \pm 0.009$ \\
\hline Day 40 & $0.615 \pm 0.035$ & $0.703 \pm 0.048$ & $0.650 \pm 0.050$ & $0.592 \pm 0.047$ & $0.637 \pm 0.027$ \\
\hline
\end{tabular}

7-Ethoxyresorufin-O-deethylase (EROD) (pmol/minute per mg microsomal protein)

$\begin{array}{llllll}\text { Day 26 } & 44.185 \pm 2.751 & 41.774 \pm 1.129 & 36.922 \pm 1.376^{*} & 36.933 \pm 0.636^{*} & 36.119 \pm 2.635^{*} \\ \text { Day 40 } & 34.291 \pm 1.548 & 37.698 \pm 4.281 & 36.055 \pm 2.482 & 32.852 \pm 2.045 & 38.460 \pm 3.395\end{array}$

7-Pentoxyresorufin- $O$-deethylase (PROD) ( $\mathrm{pmol} /$ minute per $\mathrm{mg}$ microsomal protein)

\begin{tabular}{llllll} 
Day 26 & $4.766 \pm 0.122$ & $4.918 \pm 0.094$ & $4.489 \pm 0.214$ & $4.089 \pm 0.161^{*}$ & $3.899 \pm 0.247^{*}$ \\
Day 40 & $3.905 \pm 0.154$ & $4.334 \pm 0.311$ & $4.283 \pm 0.071$ & $3.721 \pm 0.230$ & $3.747 \pm 0.241$ \\
\hline
\end{tabular}

*Significantly different $(\mathrm{P} \leq 0.05)$ from the chamber control group by Dunn's or Shirley's test.

${ }^{\text {aData }}$ are presented as mean \pm standard error.

Table J-2. Liver Cytochrome P450 Data for Special Study Female Mice in the Three-month Inhalation Study of Cobalt Metal ${ }^{\mathrm{a}}$

\begin{tabular}{|c|c|c|c|c|c|c|}
\hline & $\begin{array}{l}\text { Chamber } \\
\text { Control }\end{array}$ & $0.625 \mathrm{mg} / \mathrm{m}^{3}$ & $1.25 \mathrm{mg} / \mathrm{m}^{3}$ & $2.5 \mathrm{mg} / \mathrm{m}^{3}$ & $5 \mathrm{mg} / \mathrm{m}^{3}$ & $10 \mathbf{m g} / \mathbf{m}^{3}$ \\
\hline n & 3 & 3 & 3 & 3 & 3 & 3 \\
\hline \multicolumn{7}{|c|}{ Acetanilide-4-hydroxylase (A4H) (nmol/minute per mg microsomal protein) } \\
\hline Day 26 & $0.515 \pm 0.018$ & $0.434 \pm 0.017$ & $0.521 \pm 0.043$ & $0.525 \pm 0.016$ & $0.752 \pm 0.081$ & $0.659 \pm 0.014$ \\
\hline Day 40 & $0.483 \pm 0.031$ & $0.557 \pm 0.041$ & $0.515 \pm 0.025$ & $0.526 \pm 0.052$ & $0.656 \pm 0.044^{* *}$ & $0.736 \pm 0.070^{* *}$ \\
\hline \multicolumn{7}{|c|}{ 7-Ethoxyresorufin-O-deethylase (EROD) (pmol/minute per mg microsomal protein) } \\
\hline Day 26 & $52.375 \pm 4.334$ & $53.046 \pm 0.746$ & $63.475 \pm 6.343$ & $58.552 \pm 3.113$ & $65.036 \pm 0.191 *$ & $70.237 \pm 4.834^{*}$ \\
\hline Day 40 & $52.914 \pm 3.989$ & $53.358 \pm 0.936$ & $60.768 \pm 2.310$ & $58.398 \pm 1.156$ & $67.890 \pm 2.157^{* *}$ & $73.859 \pm 7.616^{*}$ \\
\hline \multicolumn{7}{|c|}{ 7-Pentoxyresorufin-O-deethylase (PROD) ( $\mathrm{pmol} /$ minute per mg microsomal protein) } \\
\hline Day 26 & $9.992 \pm 0.740$ & $10.329 \pm 0.517$ & $10.349 \pm 0.676$ & $9.577 \pm 0.800$ & $11.013 \pm 0.651$ & $9.737 \pm 0.337$ \\
\hline Day 40 & $10.212 \pm 0.388$ & $10.150 \pm 0.404$ & $11.274 \pm 0.558$ & $10.234 \pm 0.252$ & $10.539 \pm 0.492$ & $9.148 \pm 0.503$ \\
\hline
\end{tabular}

*Significantly different $(\mathrm{P} \leq 0.05)$ from the chamber control group by Shirley's test.

$* * \mathrm{P} \leq 0.01$.

${ }^{\text {aDData }}$ are presented as mean \pm standard error. 


\title{
Appendix K. Analysis of Kras, Egfr, and Tp53 Mutations in F344/NTac Rat and B6C3F1/N Mouse Alveolar/Bronchiolar Carcinomas Resulting from Chronic Inhalation Exposure to Cobalt Metal
}

\author{
A.R. Pandiri ${ }^{1,2}$, H.H. Hong ${ }^{1}$, M.J. Hooth ${ }^{3}$, M.V. Behl ${ }^{3}$, G. Kissling ${ }^{4}$, R.A. Herbert ${ }^{1}$, R.C. Sills ${ }^{1}$, \\ M.J. Hoenerhoff ${ }^{1}$ \\ ${ }^{1}$ Cellular and Molecular Pathology Branch, Division, NTP, NIEHS, Research Triangle Park, NC \\ 27709
}

${ }^{2}$ Experimental Pathology Laboratories, Inc., Durham, NC 27709

${ }^{3}$ Toxicology Branch, NTP, NIEHS, Research Triangle Park, NC 27709

${ }^{4}$ Biostatistics Branch, NIEHS, Research Triangle Park, NC 27709

\section{Table of Contents}

K.1. Introduction $\mathrm{K}-3$

K.2. Materials and Methods......................................................................................... K-4

K.3. Results .................................................................................................................. K-5

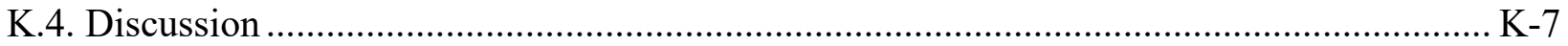

\section{Tables}

Table K-1. Primers Used to Amplify the Hot Spot Regions of Rat Kras, Tp53, and Egfr Genes K-9

Table K-2. Primers Used to Amplify the Hot Spot Regions of Mouse Kras, TP53, and Egfr Genes

Table K-3. Kras Mutations in Alveolar/bronchiolar Carcinomas from Male and Female F344/NTac Rats in the Two-year Inhalation Study of Cobalt Metal K-11

Table K-4. Kras and Egfr Mutations in Alveolar/bronchiolar Carcinomas from Male F344/NTac Rats in the Two-year Inhalation Study of Cobalt Metal

Table K-5. Kras and Egfr Mutations in Alveolar/bronchiolar Carcinomas from Female F344/NTac Rats in the Two-year Inhalation Study of Cobalt Metal K-13

Table K-6. Egfr Mutations in Alveolar/bronchiolar Carcinomas from Male and Female F344/NTac Rats in the Two-year Inhalation Study of Cobalt Metal ....

Table K-7. Tp53 Mutations in Alveolar/bronchiolar Carcinomas from Male and Female F344/NTac Rats in the Two-year Inhalation Study of Cobalt Metal

Table K-8. Tp53 Mutations in Alveolar/bronchiolar Carcinomas from Male F344/NTac Rats in the Two-year Inhalation Study of Cobalt Metal

Table K-9. Tp53 Mutations in Alveolar/bronchiolar Carcinomas from Female F344/NTac Rats in the Two-year Inhalation Study of Cobalt Metal

Table K-10. Kras Mutations in Alveolar/bronchiolar Carcinomas from Male and Female B6C3F1/N Mice in the Two-year Inhalation Study of Cobalt Metal K-18 
Table K-11. Kras and Egfr Mutations in Alveolar/bronchiolar Carcinomas from Male B6C3F1/N Mice in the Two-year Inhalation Study of Cobalt Metal K-19

Table K-12. Kras and Egfr Mutations in Alveolar/bronchiolar Carcinomas from Female B6C3F1/N Mice in the Two-year Inhalation Study of Cobalt Metal

Table K-13. Egfr Mutations in Alveolar/bronchiolar Carcinomas from Male and Female B6C3F1/N Mice in the Two-year Inhalation Study of Cobalt Metal

Table K-14. Tp53 Mutations in Alveolar/bronchiolar Carcinomas from Male and Female B6C3F1/N Mice in the Two-year Inhalation Study of Cobalt Metal

Table K-15. Tp53 Mutations in Alveolar/bronchiolar Carcinomas from Male B6C3F1/N Mice in the Two-year Inhalation Study of Cobalt Metal

Table K-16. Tp53 Mutations in Alveolar/bronchiolar Carcinomas from Female B6C3F1/N Mice in the Two-year Inhalation Study of Cobalt Metal

Table K-17. Summary of Kras Mutations in Alveolar/bronchiolar Neoplasms from Male and Female B6C3F1/N Mice in Selected Two-year NTP Studies 


\section{K.1. Introduction}

Spontaneous incidences of alveolar/bronchiolar adenoma or carcinoma (combined) in control B6C3F1/N mice range from $8.4 \%$ (female) to $26.2 \%$ (male) (all routes, all vehicles, NTP historical control database, May 2011), and the incidences of these lung neoplasms after exposure to certain chemicals can be considerably higher in 2-year NTP studies. The incidences of spontaneous alveolar/bronchiolar adenoma or carcinoma (combined) in control F344/N rats are considerably lower $[2.3 \%$ females, $3.6 \%$ males (all routes, all vehicles, NTP historical control database, May 2011)] than in the B6C3F1/N mouse. In the concurrent 2-year cobalt metal studies, there were significantly increased incidences of alveolar/bronchiolar neoplasms in both B6C3F $1 / \mathrm{N}$ mice and F344/NTac rats exposed by inhalation compared to those in the chamber controls. The rodent lung neoplasms that arise either spontaneously or from chronic exposure to chemicals morphologically resemble non-small cell lung cancer in humans ${ }^{264}$.

The carcinogenic process involves the alteration of four broad categories of cancer-associated genes: proto-oncogenes, tumor suppressor genes, apoptosis genes, and repair genes ${ }^{265}$. At least 80 genetic alterations in cancer genes, a dozen of which are considered "driver" mutations, are involved in the cancer process. Proto-oncogene and tumor suppressor gene mutation analysis has revealed mechanistic insights in chemical carcinogenesis. Mutations may be due to direct interaction of the chemical or its metabolite with DNA, or indirectly via altering DNA replicative or repair (epigenetic) processes. A change in the frequency and spectrum of cancer gene mutations reveals chemical-specific pathways that either override or include promotion of endogenous events. Interpretation of these data is complicated because mutation profiles are dependent on species, strain, gender, tumor differentiation, dose of carcinogen, as well as dosing regimen. Our main objective was to assess whether mutation frequencies and spectra in alveolar/bronchiolar carcinomas in treated rats and mice differed from published literature on spontaneous rodent and human lung cancers.

Lung cancer is a complex disease with variable clinical presentations and behavior. Genome sequencing of lung cancers in humans has identified several "driver" mutations that may play an important role in lung carcinogenesis. These cancer genes include KRAS, EGFR, TP53, EML4ALK, HER2 (or ERBB2), BRAF, PIK3CA, PTEN, STK11, FGFR1, DDR2, AKT1, MAP2K1, and $M E T^{266}$. These genes encode proteins that are critical for cellular proliferation and survival, as well as cellular transformation and tumorigenesis. We performed extensive mutation analyses on the most commonly altered cancer genes in human lung cancer (KRAS, EGFR, and TP53) in alveolar/bronchiolar carcinomas from F344/NTac rats and B6C3F1/N mice exposed to various concentrations of cobalt metal by inhalation for 2 years.

In human non-small cell lung cancer, the incidence of KRAS mutations is approximately $26 \%$ $(67 / 254)^{267}$. Of these, $86 \%$ of the mutations arise within codon 12 , and $14 \%$ occur in codon 13 . Point mutations in codons 12, 13, and 61 of KRAS are activating mutations that result in constitutive activation of the KRAS protein, making it refractory to the inhibitory GTPase activating proteins, which results in stimulus-independent, persistent activation of downstream effectors, in particular the RAF-MEK-ERK cascade. Constitutive activation of this kinase cascade results in promotion of cellular proliferation and transformation ${ }^{268 ; 269}$.

The incidence of EGFR mutations in the non-small cell lung cancer subtype of adenocarcinoma in humans is about $9 \%(22 / 254)$ with the majority $(70 \%)$ of the mutations located within codons 
19 and $21^{267}$. EGFR is a transmembrane receptor, which upon ligand binding, dimerizes and activates the cytosolic kinase domain of the receptor tyrosine kinase resulting in activation of signaling pathways that support cancer development and progression. These signaling pathways include the PI3K pathway, which when activated leads to AKT activation and apoptosis inhibition, and the GRB2 and SOS pathways, which lead to activation of P21RAS, resulting in cell cycle progression ${ }^{270}$.

The incidence of TP53 mutations in human non-small cell lung cancer is approximately $50 \%$; the frequency of this mutation is relatively increased in smokers ${ }^{271}$. TP53 is regarded as a master regulator gene that is frequently altered in a wide range of cancers, and as a tumor suppressor, its critical roles in cell cycle control, apoptosis, and DNA repair are compromised when the gene is mutated. Tobacco-associated lung cancer has a high frequency of transversions (e.g., purine to pyrimidine or pyrimidine to purine) in TP53, whereas lung cancers in humans who have never smoked are associated with transitions (e.g., pyrimidine to pyrimidine or purine to purine), especially in women ${ }^{272 ; 273}$. In contrast to KRAS and EGFR mutations, which are predominantly noted only in the non-small cell lung cancer subtype of adenocarcinoma, mutations in TP53 are observed equally in both squamous cell carcinoma and adenocarcinoma subtypes of non-small cell lung cancer ${ }^{274}$.

\section{K.2. Materials and Methods}

\section{K.2.1. Animals and Tissue Sampling}

Alveolar/bronchiolar carcinoma tissue with adjacent nontumor tissue was obtained from F344/NTac rats and $\mathrm{B} 6 \mathrm{C} 3 \mathrm{~F} 1 / \mathrm{N}$ mice exposed to cobalt metal by inhalation for 2 years; spontaneous alveolar/bronchiolar carcinomas were obtained from chamber control mice at terminal kill. There were no spontaneous alveolar/bronchiolar carcinomas in the F344/NTac rats in the concurrent cobalt metal study. Hence, the spontaneous alveolar/bronchiolar carcinomas $(\mathrm{n}=10)$ were sourced from F344 rat vehicle control groups in various NTP chronic bioassays. These studies include sodium azide (VM55), elmiron (VM17), ginseng (VM46), tert-butyl alcohol (VM10) (all gavage, water vehicle); probenecid (VF23), isoeugenol (VM3, VM33) (all gavage, corn oil vehicle); trimethylolpropane triacrylate (VM63) (topical application in acetone vehicle); lauric acid diethanolamine condensate (VF163) (topical application in ethanol vehicle); and ethylbenzene (VM460) (inhalation of filtered air). These spontaneous alveolar/bronchiolar carcinoma samples were sourced from eight male and two female F344 rats. The mouse tumors that were selected for mutation analysis were generally greater than $5 \mathrm{~mm}$ in diameter, whereas the rat tumors were smaller and scattered throughout the pulmonary parenchyma. Normal lungs without tumors from age-matched chamber control rats and mice were also collected for histologic evaluation and molecular analysis. After formalin fixation, the tissues were transferred to $70 \%$ ethanol and were routinely processed in graded alcohols, embedded in paraffin, sectioned, and stained with hematoxylin and eosin (H\&E) for microscopic analysis. The formalin fixed paraffin embedded (FFPE) tissues were used for mutation analysis. The alveolar/bronchiolar carcinomas were selected for molecular biology analysis based on their overall size and viability (minimal to no necrosis/hemorrhage microscopically) in order to maximize the amount and quality of DNA obtained from FFPE sections. DNA quality was measured using a Nanodrop spectrophotometer (Thermo Scientific, Wilmington DE) to calculate the $260 / 280 \mathrm{~nm}$ absorbance ratio, and DNA samples with a purity range of 1.7 to 2.0 were used 
for analysis. Samples falling outside of this range were reisolated from FFPE sections until a suitable purity measure was obtained or discarded.

\section{K.2.2. DNA Extraction, Polymerase Chain Reaction, Autosequencing, and Mutation Analysis}

Alveolar/bronchiolar carcinoma samples (48 from F344/NTac rats and 69 from B6C3F1/N mice) representing all cobalt metal exposed groups and spontaneous alveolar/bronchiolar carcinomas (10 each from chamber control rats and mice) were evaluated for hot spot mutations within specific codons or exons in Kras, Egfr, and Tp53 genes that are relevant in human lung cancer. FFPE sections at $10 \mu \mathrm{m}$ thickness were collected on glass slides. The neoplasms (single or multiple from the same lung) were dissected with a sharp microtome blade and collected into screw top tubes. In some cases (especially in rats) where the alveolar/bronchiolar carcinomas had a miliary distribution and were scattered throughout the pulmonary parenchyma, the entire lung section was used for DNA isolation. DNA was isolated from these FFPE dissected neoplasm tissue sections with the DNeasy ${ }^{\circledR}$ Tissue Kit (Qiagen, Valencia, CA). Amplification reactions were carried out by semi-nested polymerase chain reaction (PCR) using primer sets designed for Kras (exons 1 and 2), Tp53 (exons 5 through 8), and Egfr (exons 18 through 21) (Table K-1 and Table K-2). Controls lacking DNA were run with all sets of reactions. PCR products were purified using a QIAquick ${ }^{\circledR}$ Gel Extraction Kit (Qiagen). The purified products were cycled with Terminal Ready Reaction Mix-Big Dye ${ }^{\circledR}$ (Perkin-Elmer, Foster City, CA), and the extension products were purified with the DyeEx ${ }^{\circledR} 2.0$ SpinKit (Qiagen). The lyophilized PCR products were sequenced with an automatic sequencer (Perkin-Elmer ABI Model 3100). The resulting electropherograms were compared to identify mutations in alveolar/bronchiolar carcinomas that either arise spontaneously or due to exposure to cobalt metal ${ }^{275}$.

\section{K.2.3. Statistics}

To test for significance of exposure concentration-related trends in the incidences of mutations, a one-sided Cochran-Armitage test was conducted. A Fisher exact test was conducted to test for significant differences in the number of mutations between the control and various exposure groups.

\section{K.3. Results}

\section{K.3.1. Rats}

The incidence of Kras mutations in lung alveolar/bronchiolar carcinomas from F344/NTac rats exposed to cobalt metal by inhalation for 2 years was 31\% (15/48) (Table K-3, supplemented by Table K-4 and Table K-5). The majority of Kras mutations in the rat were within codon 12 [29\% (14/48)], followed by codon 13 [2\% (1/48)]. Surprisingly there were no mutations within codon 61. The most common codon 12 mutations in rat alveolar/bronchiolar carcinomas from all groups exposed to cobalt metal were $\mathrm{G} \rightarrow \mathrm{T}$ transversions $[57 \%(8 / 14)]$ and $\mathrm{G} \rightarrow \mathrm{A}$ transitions $[43 \%(6 / 14)]$. The frequencies of Kras mutations were 14\% (2/14), 35\% (6/17), and 41\% (7/17) in alveolar/bronchiolar carcinomas from the $1.25,2.5$, and $5 \mathrm{mg} / \mathrm{m}^{3}$ groups, respectively. Kras mutations were not detected in spontaneous alveolar/bronchiolar carcinomas from chamber control rats in this study. 
The incidence of Egfr mutations in alveolar/bronchiolar carcinomas from F344/NTac rats exposed to cobalt metal by inhalation for 2 years was $17 \%(8 / 48)$ (Table K-3, supplemented by Table K-4 and Table K-5). The majority of Egfr mutations in alveolar/bronchiolar carcinomas from rats exposed to cobalt metal for 2 years were present in exon 20 (codons 768, 770, 771, $785,790,791$, and 806) [13\% (6/48)], followed by exon 21 (codons 845 and 875) [4\% (2/48)] and exon 19 (codon 750) [2\% (1/48)]. The frequencies of Egfr mutations in alveolar/bronchiolar carcinomas from rats exposed to cobalt metal were $14 \%(2 / 14), 18 \%(3 / 17)$, and $18 \%(3 / 17)$ from the $1.25,2.5$, and $5 \mathrm{mg} / \mathrm{m}^{3}$ groups, respectively. A majority of Egfr mutations in rat alveolar/bronchiolar carcinomas were transitions such as $\mathrm{G} \rightarrow \mathrm{A}[50 \%(5 / 10)]$ or $\mathrm{C} \rightarrow \mathrm{T}[30 \%$ (3/10)]. Egfr mutations were not detected in spontaneous alveolar/bronchiolar carcinomas from chamber control rats in this study.

The incidence of Tp53 mutations in alveolar/bronchiolar carcinomas from F344/NTac rats exposed to cobalt metal for 2 years was 23\% (11/48) (Table K-7, supplemented by Table K-8 and Table K-9). The majority of Tp53 mutations in alveolar/bronchiolar carcinomas from exposed rats were present within exon 6 (codons 203, 212, 242, and 247) [10\% (5/48)], followed by exon 7 (codons 266, 277, and 278) [6\% (3/48)] and exon 8 (codons 314 and 321) [6\% (3/48)]. The frequencies of Tp53 mutations were $21 \%(3 / 14), 35 \%(6 / 17)$, and $12 \%(2 / 17)$ in the alveolar/bronchiolar carcinomas from the $1.25,2.5$, and $5 \mathrm{mg} / \mathrm{m}^{3}$ groups, respectively. The majority of $\mathrm{Tp} 53$ mutations in rats were transitions [85\% (11/13)] such as $\mathrm{C} \rightarrow \mathrm{T}[38 \%(5 / 13)]$ or $\mathrm{G} \rightarrow \mathrm{A}[31 \%$ (4/13)]. Tp53 mutations were not observed in spontaneous alveolar/bronchiolar carcinomas from chamber control rats in this study.

\section{K.3.2. Mice}

The incidence of Kras mutations in lung alveolar/bronchiolar carcinomas from B6C3F1/N mice exposed to cobalt metal for 2 years was 67\% (46/69) (Table K-10; supplemented by Table K-11 and Table K-12). The majority of Kras mutations were localized within codon 12 [43\% (30/69)], followed by codons 61 [20\% (14/69)] and 13 [6\% (4/69)]. The most common codon 12 mutations in alveolar/bronchiolar carcinomas from all groups exposed to cobalt metal were $\mathrm{G} \rightarrow \mathrm{T}$ transversions $(\mathrm{GGT} \rightarrow \mathrm{GTT}$ or $\mathrm{TGT})[80 \%(24 / 30)]$ and $\mathrm{G} \rightarrow \mathrm{A}$ transitions $(\mathrm{GGT} \rightarrow \mathrm{G} \underline{\mathrm{AT}}$ ) [17\% (5/30)]. The frequencies of Kras mutations were 69\% (11/16), 48\% (11/23), and $80 \%$ $(24 / 30)$ in alveolar/bronchiolar carcinomas from the $1.25,2.5$, and $5 \mathrm{mg} / \mathrm{m}^{3}$ groups, respectively. Kras mutations were not detected in spontaneous alveolar/bronchiolar carcinomas in this study. However, according to historical control data, Kras mutation incidence in spontaneous alveolar/bronchiolar carcinomas is $27 \%(34 / 124)$, and these mutations are localized within codon $12[16 \%(20 / 124)]$, followed by codons $61[6 \%(8 / 124)]$ and $13[5 \%(6 / 124)]^{275}$. The most common mutation in codon 12 is a $\mathrm{G} \rightarrow \mathrm{A}$ transition $(\mathrm{GGT} \rightarrow \mathrm{GAT}$ ) [70\% (14/20)].

The incidence of Egfr mutations in alveolar/bronchiolar carcinomas from B6C3F1/N mice exposed to cobalt metal by inhalation was relatively low. The incidence of Egfr mutations in mouse alveolar/bronchiolar carcinomas due to exposure to cobalt metal for 2 years was $17 \%$ (12/69) (Table K-13, supplemented by Table K-11 and Table K-12). All regions of DNA that were queried for mutations (exons 18 through 21 ) encode the tyrosine kinase domain. The majority of Egfr mutations within alveolar/bronchiolar carcinomas from groups exposed to cobalt metal were present within exon 20 (codons 780, 791, 801, 812, and 817) [9\% (6/69)], followed by exons 21 (codons 846, 861, 870, and 873) [6\% (4/69)], 18 (codon 721) and 19 (codon 735) [each 1\% (1/69)]. The frequencies of Egfr mutations in mouse alveolar/bronchiolar 
carcinomas exposed to cobalt metal were $13 \%(2 / 16), 30 \%(7 / 23)$, and $10 \%(3 / 30)$ from the 1.25 , 2.5 , and $5 \mathrm{mg} / \mathrm{m}^{3}$ groups, respectively. A majority of Egfr mutations in mouse alveolar/bronchiolar carcinomas were transition mutations such as $\mathrm{G} \rightarrow \mathrm{A}[42 \%(5 / 12)]$ or $\mathrm{C} \rightarrow \mathrm{T}$ [17\% (2/12)]. Egfr mutations were not detected in spontaneous alveolar/bronchiolar carcinomas from chamber control mice in this study.

The incidence of Tp53 mutations in alveolar/bronchiolar carcinomas from B6C3F1/N mice exposed to cobalt metal for 2 years was 19\% (13/69) (Table K-14, supplemented by Table K-15 and Table K-16). The majority of Tp53 mutations in alveolar/bronchiolar carcinomas from mice exposed to cobalt metal was found within exon 5 (codons 155, 156, 158, and 179) [10\% (7/69)], followed by exons 6 (codons 194 and 212) [4\% (3/69)] and 7 (codons 230, 232, 239, and 257) $[6 \%(4 / 69)]$. The majority of mutations were transversions $[60 \%(9 / 15)]$, most of which were $\mathrm{G} \rightarrow \mathrm{C}$. The frequencies of Tp53 mutations were 19\% (3/16), 13\% (3/23), and 20\% (6/30) in alveolar/bronchiolar carcinomas from the $1.25,2.5$, and $5 \mathrm{mg} / \mathrm{m}^{3}$ groups, respectively. Tp53 mutations were not detected in spontaneous alveolar/bronchiolar carcinomas from chamber control mice in this study.

According to the historical control database (all routes, all vehicles, NTP historical control database, May 2011), male B6C3F1 mice have a higher incidence (12.5\%) of spontaneous alveolar/bronchiolar carcinomas than $\mathrm{B} 6 \mathrm{C} 3 \mathrm{~F} 1$ female mice $(3.7 \%)$. However, there were no significant differences in the number or type of mutations evaluated in Kras, Egfr, or Tp53 genes between male and female B6C3F1/N mice chronically exposed to cobalt metal by inhalation.

\section{K.4. Discussion}

Kras mutations were more frequent than Egfr and Tp53 mutations within the lung alveolar/bronchiolar carcinomas from $\mathrm{F} 344 / \mathrm{NTac}$ rats and $\mathrm{B} 6 \mathrm{C} 3 \mathrm{~F} 1 / \mathrm{N}$ mice exposed to cobalt metal for 2 years in the concurrent studies. It is interesting to note that in mice, Kras mutations were observed in both spontaneous alveolar/bronchiolar carcinomas [27\% (34/124); Hong et al. $\left.{ }^{275}\right]$ as well as in alveolar/bronchiolar carcinomas from mice exposed to cobalt metal $[67 \%$ (46/69)]. However, alveolar/bronchiolar carcinomas from mice exposed to cobalt metal had predominantly $\mathrm{G} \rightarrow \mathrm{T}$ transversions [80\% (24/30)], whereas the spontaneous alveolar/bronchiolar carcinomas had $\mathrm{G} \rightarrow \mathrm{A}$ transitions $[70 \%$ (14/20)] in codon 12. Not surprisingly, $\mathrm{G} \rightarrow \mathrm{T}$ transversions were also the most predominant $[67 \%(6 / 9)]$ mutations in alveolar/bronchiolar carcinomas from mice exposed to cobalt sulfate heptahydrate aerosols for 2 years (Table K-17; $\mathrm{NTP}^{122}$ ). Incidentally, $\mathrm{G} \rightarrow \mathrm{T}$ transversions are one of the most common Kras mutations in human lung cancer ${ }^{260}$. G $\rightarrow$ T Kras mutations appear to correlate with 8-hydroxydeoxyguanine adducts that result from oxidative stress. $\mathrm{G} \rightarrow \mathrm{T}$ transversions in codon 12 of Kras were also noted previously in several lung neoplasms in mice exposed to various other chemicals such as tobacco-specific nitrosamines, aflatoxin B1, vanadium pentoxide, ozone, cumene, ethylene oxide, and transplacental AZT exposure ${ }^{276-279}$. In the current study, it is not surprising that these mutations were seen almost exclusively in alveolar/bronchiolar carcinomas from mice exposed to cobalt metal and not in spontaneous alveolar/bronchiolar carcinomas. It has been demonstrated that cobalt can induce hypoxia and upregulate HIF-1 $\alpha$ signaling, thereby modulating inflammatory responses and inducing oxidative stress ${ }^{154}$. Thus, the $\mathrm{G} \rightarrow \mathrm{T}$ transversions that were observed in mice, primarily within codon 12 of Kras, may be attributable to oxidative stress resulting from chronic cobalt metal exposure. 
EGFR, together with KRAS, plays an important role in initiating and maintaining the MAPK signaling cascade and other signaling pathways that are relevant to cancer. Kras mutations in non-small cell lung cancer are observed more frequently in smokers, while Egfr mutations in lung cancers are most frequently observed in women and people who have never smoked ${ }^{267}$. The incidence of Egfr mutations in alveolar/bronchiolar carcinomas from rats and mice exposed to cobalt metal was lower than the incidence of Kras mutations. In contrast to the Kras mutations which are predominantly transversions, Egfr mutations in both rat and mouse alveolar/bronchiolar carcinomas were $\mathrm{G} \rightarrow \mathrm{A}$ or $\mathrm{C} \rightarrow \mathrm{T}$ transitions. This is the first study in which Egfr mutations were examined in the context of chemically induced rodent lung neoplasms. Mutations within Egfr and Kras are mutually exclusive in human lung and colon cancers, and the treatment modalities depend on Egfr and Kras mutation status of the respective neoplasm. Surprisingly, in alveolar/bronchiolar carcinomas from rodents exposed to cobalt metal, $38 \%$ $(3 / 8)$ of rats and 25\% (3/12) of mice that harbored Egfr mutations also had Kras mutations.

Mutations in Tp53 in chemically induced rodent models are considered a late event, especially in neoplasms that are initiated by mutations in $\mathrm{Kras}^{256}$. The incidences of Tp53 mutations resulting from exposure to cobalt metal for 2 years were similar in rats and mice, but the nature of the mutations was different; Tp53 mutations in rats were predominantly transitions, whereas in mice, they were predominantly transversions. Interestingly, in humans, Tp53 transversions are commonly observed in non-small cell lung cancer from smokers, while Tp53 transitions are often noted in non-small cell lung cancer from people who have never smoked. The significance of this differential mutation spectrum in rats and mice and its relationship to human lung cancer needs to be further explored.

In summary, there was a significantly higher incidence of Kras mutations, accompanied by a lower incidence of Egfr and Tp53 mutations, in alveolar/bronchiolar carcinomas from rats and mice exposed to cobalt metal for 2 years. Several of the observed mutations in Kras are comparable to mutations observed in other NTP studies in which a significant increase in the incidence of lung neoplasms in response to chronic chemical exposure was evident. In addition, these mutations arise within the hotspot regions of Kras, Egfr, and Tp53 genes, and are thus comparable to the mutations observed in human non-small cell lung cancer. 
Cobalt Metal, NTP TR 581

Table K-1. Primers Used to Amplify the Hot Spot Regions of Rat Kras, Tp53, and Egfr Genes

\begin{tabular}{|c|c|c|c|c|}
\hline Exon & Codon & Primer & Strand & Sequence \\
\hline \multirow[t]{3}{*}{1} & Kras-12-13 & RK12F25927 & Sense & 5'-ACTTGTGGTAGTTGGAGC-3' \\
\hline & & RK12R26069 & Antisense & 5'-CTGCCACCCTTTACAAATTG-3' \\
\hline & & RK12R26034 & Antisense & 5'-GCAGCATTTACCTCTATCGT-3' \\
\hline \multirow[t]{3}{*}{2} & Kras-61 & RK61F14325 & Sense & 5'-ATCCAGACTGTGTTTCTACC-3' \\
\hline & & RK61R13986 & Antisense & 5'-CAGGAATTCTACATACTTGACAC-3' \\
\hline & & RK61R14035 & Antisense & 5'-TGCAGGCCTAACAACTAGC-3' \\
\hline \multirow[t]{4}{*}{5} & Tp53-124-184 & Rp53Ex5OF1366 & Sense & 5'-CCTAGTTGGCTTGTCCG-3' \\
\hline & & Rp53Ex5OR1671 & Antisense & 5'-AGCAAGAATAAGTCAGAGGC-3' \\
\hline & & Rp53Ex5IF1382 & Sense & 5'-CGCTGACCTTTGATTCTTTCTCC-3' \\
\hline & & Rp53Ex5IR1639 & Antisense & 5'-GACAACCAGTTCTAAACCCCACAG-3' \\
\hline \multirow[t]{4}{*}{6} & Tp53-185-259 & Rp53Ex6OF1620 & Sense & 5'-TGGGGTTAGAACTGGTTG-3' \\
\hline & & Rp53Ex6OR1963 & Antisense & 5'-GAACAAAAACAGGCCGAG-3' \\
\hline & & Rp53Ex6IF1645 & Sense & 5'-TCTCCCGGCCTCTGACTTATTC-3' \\
\hline & & Rp53Ex6IR1927 & Antisense & $5^{\prime}$-CAGCCCAACCTGGCACAC-3' \\
\hline \multirow[t]{4}{*}{7} & Tp53-260-304 & Rp53Ex7OF2101 & Sense & 5'-AGCTCCAGATAGGACAAG-3' \\
\hline & & Rp53Ex7OR2434 & Antisense & 5'-TGGGCAGTGCTATGGAAG-3' \\
\hline & & Rp53Ex7IF2166 & Sense & 5'-AGCTTTCTTACTGCCTTGTG-3' \\
\hline & & Rp53Ex7IR2402 & Antisense & 5'-TGACTTTGGGGTGAAGCTG-3' \\
\hline \multirow[t]{4}{*}{8} & Tp53-305-329 & Rp53Ex8OF2333 & Sense & 5'-GGAGTGCAAAGAGAGGTG-3' \\
\hline & & Rp53Ex8OR2602 & Antisense & 5'-TGCGCTCTGACGATAATG-3' \\
\hline & & Rp53Ex8IF2386 & Sense & 5'-GCTTCACCCCAAAGTCAC-3' \\
\hline & & Rp53Ex8IR2549 & Antisense & 5'-GCGTTTTGTGTCCTAGACTTAG-3' \\
\hline \multirow[t]{3}{*}{18} & Egfr-689-729 & REgfr18F144544 & Sense & 5'-ACACATTGCTCCTTTGATCAC-3' \\
\hline & & REgfr18R144922 & Antisense & 5'-AACACGAGTTCCTACATAAACC-3' \\
\hline & & REgfr18R145009 & Antisense & 5'-CACTCCCAAGTTTATGCCTC-3' \\
\hline \multirow[t]{3}{*}{19} & Egfr-730-762 & REgfr19F146377 & Sense & 5'-ACAAGGCAACATGCTGCTG-3' \\
\hline & & REgfr19R146775 & Antisense & 5'-TGACTTTACTCTTCCCТCCCC-3' \\
\hline & & REgfr19R146605 & Antisense & 5'-ACACAAACTAAGGAAGCAAGAC-3' \\
\hline \multirow[t]{3}{*}{20} & Egfr-763-824 & REgfr20F150643 & Sense & 5'-ACATGTGTTGTCCTTACC-3' \\
\hline & & REgfr20R150924 & Antisense & 5'-ATTCATCCTGCTTCTGAAACC-3' \\
\hline & & REgfr20R150905 & Antisense & 5'-CCTGCTATTGGCTCTTTG-3' \\
\hline \multirow[t]{3}{*}{21} & Egfr-825-876 & REgfr21F162620 & Sense & 5'-TCACTCCCTCACTGAAGC-3' \\
\hline & & REgfr21R162881 & Antisense & 5'-CTACAGCTGACACATAGG-3' \\
\hline & & REgfr21R162852 & Antisense & 5'-GGGCTGTCAGGAAAATGC-3' \\
\hline
\end{tabular}


Table K-2. Primers Used to Amplify the Hot Spot Regions of Mouse Kras, TP53, and Egfr Genes

\begin{tabular}{|c|c|c|c|c|}
\hline Exon & Codon & Primer & Strand & Sequence \\
\hline \multirow[t]{4}{*}{1} & Kras-12-13 & K12AOS & Sense & 5'-TTATTGTAAGGCCTGCTGAA-3' \\
\hline & & $\mathrm{K} 12 \mathrm{AOA}$ & Antisense & 5'-GCAGCGTTACCTCTATCGTA-3' \\
\hline & & K12AIS & Sense & 5'-ATGACTGAGTATAAACTTGT-3' \\
\hline & & K12AIA & Antisense & 5'-TCGTACTCATCCACAAAGTG-3' \\
\hline \multirow[t]{4}{*}{2} & Kras-61 & K61OS & Sense & 5'-TTCTCAGGACTCCTACAGGA-3' \\
\hline & & K61OA & Antisense & 5'-ACCCACCTATAATGGTGAAT-3' \\
\hline & & APK61IS & Sense & 5'-TACAGGAAACAAGTAGTAATTGATGGAGAA-3' \\
\hline & & APK61IA & Antisense & 5'-ATAATGGTGAATATCTTCAAATGATTTAGT-3' \\
\hline \multirow[t]{3}{*}{$5-6$} & Tp53-123-221 & Mp53F1407 & Sense & 5'-TCCCCACCTTGACACCTG-3' \\
\hline & & Mp53R1885 & Antisense & 5'-GTCTCTAAGACGCACAAACC-3' \\
\hline & & Mp53F1453 & Sense & 5'-GTTCTCTCTCCTCTCTTCCAG-3' \\
\hline \multirow[t]{4}{*}{7} & Tp53-222-258 & Mp7FO & Sense & 5'-GGTCACCTGTAGTGAGGTAG-3' \\
\hline & & Mp7RO & Antisense & 5'-GGAACAGAAACAGGCAGAAG-3' \\
\hline & & Mp7FI & Sense & 5'-TGTAGTGAGGTAGGGAGCGAC-3' \\
\hline & & Mp7RI & Antisense & 5'-AAGCTGGGGAAGAAACAGGC-3' \\
\hline \multirow[t]{4}{*}{8} & Tp53-259-303 & Mp8FO & Sense & 5'-GTTTACACACAGTCAGGATGG-3' \\
\hline & & Mp8RO & Antisense & 5'-TGTGGAAGGAGAGAGCAAG-3' \\
\hline & & Mp8FI & Sense & 5'-AGCTTTCTTACTGCCTTGTGC-3' \\
\hline & & Mp8RI & Antisense & 5'-TGAAGCTCAACAGGCTCCTC-3' \\
\hline \multirow[t]{3}{*}{18} & Egfr-688-728 & Egfr18F138824 & Sense & 5'-CCACTGCTCCTTTGAACAC-3' \\
\hline & & Egfr18F138836 & Sense & 5'-TGAACACATTGCTCCTTTGAAC-3' \\
\hline & & Egfr18R139212 & Antisense & 5'-TGGAGAGCACAGCAAACAC-3' \\
\hline \multirow[t]{3}{*}{19} & Egfr-729-761 & Egfr19F140636 & Sense & 5'-GAGCTTGATAGCTAAGAACCTC-3' \\
\hline & & Egfr19F140659 & Sense & 5'-GAAATATGAAGAGTCCCAGCAC-3' \\
\hline & & Egfr19R141071 & Antisense & 5'-CCAGAATACTTCCAAACAGTCC-3' \\
\hline \multirow[t]{3}{*}{20} & Egfr-762-823 & Egfr20F144483 & Sense & 5'-TCC TTTTAACATGCAACATCCC-3' \\
\hline & & Egfr20F144598 & Sense & 5'-GGGGGGGCATTTCATTTTAC-3' \\
\hline & & Egfr20R145114 & Antisense & 5'-CAGACACACACACCTATCATC-3' \\
\hline \multirow[t]{3}{*}{21} & Egfr-824-875 & Egfr21F151895 & Sense & 5'-TGTCTTGTCATTCATGCCAG-3' \\
\hline & & Egfr21F151978 & Sense & 5'-TGTTGAGCAGCCTAGAGATTC-3' \\
\hline & & Egfr21R152444 & Antisense & 5'-TCСТССТТАСТАСТСССАCС-3' \\
\hline
\end{tabular}


Table K-3. Kras Mutations in Alveolar/bronchiolar Carcinomas from Male and Female F344/NTac Rats in the Two-year Inhalation Study of Cobalt Metal

\begin{tabular}{|c|c|c|c|c|c|c|c|c|}
\hline \multirow{2}{*}{\multicolumn{2}{|c|}{$\begin{array}{c}\text { Cobalt Metal } \\
\text { Concentration }\end{array}$}} & \multirow{2}{*}{$\begin{array}{l}\text { Mutation } \\
\text { Frequency }\end{array}$} & \multicolumn{4}{|c|}{ Codon 12 (GGT) } & \multirow{2}{*}{$\begin{array}{c}\text { Codon } 13 \\
\text { (GGC) } \\
\text { (CGC) }\end{array}$} & \multirow[t]{2}{*}{$\begin{array}{l}\text { Codon } 61 \\
\text { (CAA) }\end{array}$} \\
\hline & & & (GAT) & (TGT) & (GTT) & (TTT) & & \\
\hline \multicolumn{2}{|l|}{$0 \mathrm{mg} / \mathrm{m}^{3 \#}$} & $0 / 10(0 \%)^{* *}$ & 0 & 0 & 0 & 0 & 0 & 0 \\
\hline \multicolumn{2}{|c|}{$1.25 \mathrm{mg} / \mathrm{m}^{3}$} & $2 / 14(14 \%)$ & 1 & 0 & 1 & 0 & 0 & 0 \\
\hline \multicolumn{2}{|l|}{$2.5 \mathrm{mg} / \mathrm{m}^{3}$} & $6 / 17(35 \%)^{*}$ & 4 & 1 & 0 & 0 & 1 & 0 \\
\hline \multicolumn{2}{|l|}{$5 \mathrm{mg} / \mathrm{m}^{3}$} & $7 / 17(41 \%)^{*}$ & 1 & 2 & 3 & 1 & 0 & 0 \\
\hline \multicolumn{2}{|c|}{$\begin{array}{l}\text { All exposed groups } \\
\text { combined }\end{array}$} & $15 / 48(31 \%)^{*}$ & 6 & 3 & 4 & 1 & 1 & 0 \\
\hline \multicolumn{9}{|c|}{$\begin{array}{l}\text { There were no spontaneous alveolar/bronchiolar carcinomas in the cobalt metal study. Hence, the spontaneous } \\
\text { alveolar/bronchiolar carcinomas }(\mathrm{n}=10) \text { were sourced from vehicle or chamber control groups in various NTP chronic } \\
\text { bioassays. These spontaneous alveolar/bronchiolar samples were sourced from eight male and two female F344/N rats. }\end{array}$} \\
\hline \multicolumn{9}{|c|}{$\begin{array}{l}\text { Table K-4. Kras and Egfr Mutations in Alveolar/bronchiolar Carcinomas from Male F344/NTac } \\
\text { Rats in the Two-year Inhalation Study of Cobalt Metal }\end{array}$} \\
\hline \multirow{2}{*}{$\begin{array}{l}\text { Sample/ } \\
\text { Animal \# }\end{array}$} & \multirow{2}{*}{$\begin{array}{l}\text { Cobalt } \\
\text { Conc. } \\
\left(\mathbf{m g} / \mathrm{m}^{3}\right)\end{array}$} & \multicolumn{3}{|c|}{ Kras } & \multicolumn{4}{|c|}{ Egfr } \\
\hline & & Codon 12 & Codon 13 & Codon 61 & Exon 18 & Exon 19 & Exon 20 & Exon 21 \\
\hline 1/M55 & 0 & none & none & none & none & none & none & none \\
\hline 2/M46 & 0 & none & none & none & none & none & none & none \\
\hline 3/M17 & 0 & none & none & none & none & none & none & none \\
\hline 4/M10 & 0 & none & none & none & none & none & none & none \\
\hline 5/M03 & 0 & none & none & none & none & none & none & none \\
\hline 6/M33 & 0 & none & none & none & none & none & none & none \\
\hline 7/M63 & 0 & none & none & none & none & none & none & $\begin{array}{l}\text { Codon } 837 \\
\text { CGT } \rightarrow \text { CGC } \\
(\text { Arg } \rightarrow \text { Arg })\end{array}$ \\
\hline 8/M46 & 0 & none & none & none & none & none & none & $\begin{array}{l}\text { Codon } 863 \\
\text { CTT } \rightarrow \text { CTA } \\
(\text { Leu } \rightarrow \text { Leu })\end{array}$ \\
\hline 9/M215 & 1.25 & none & none & none & none & none & none & none \\
\hline 10/M230 & 1.25 & none & none & none & none & none & none & $\begin{array}{l}\text { Codon } 875 \\
\text { GGC } \rightarrow \text { GGT } \\
(\text { Gly } \rightarrow \text { Gly })\end{array}$ \\
\hline 11/M249 & 1.25 & none & none & none & none & none & none & none \\
\hline 12/M202 & 1.25 & none & none & none & none & none & none & none \\
\hline 13/M226 & 1.25 & none & none & none & none & none & none & none \\
\hline 14/M233 & 1.25 & none & none & none & none & none & none & none \\
\hline 15/M241 & 1.25 & none & none & none & none & none & none & none \\
\hline
\end{tabular}


Cobalt Metal, NTP TR 581

\begin{tabular}{|c|c|c|c|c|c|c|c|c|}
\hline \multirow{2}{*}{$\begin{array}{l}\text { Sample/ } \\
\text { Animal \# }\end{array}$} & \multirow{2}{*}{$\begin{array}{l}\text { Cobalt } \\
\text { Conc. } \\
\left(\mathbf{m g} / \mathbf{m}^{3}\right)\end{array}$} & \multicolumn{3}{|c|}{ Kras } & \multicolumn{4}{|c|}{ Egfr } \\
\hline & & Codon 12 & Codon 13 & Codon 61 & Exon 18 & Exon 19 & Exon 20 & Exon 21 \\
\hline 16/M247 & 1.25 & none & none & none & none & none & none & none \\
\hline 17/M248 & 1.25 & none & none & none & none & none & none & $\begin{array}{c}\text { Codon } 845 \\
\text { CTG } \rightarrow \text { CAG } \\
(\mathrm{Leu} \rightarrow \mathrm{Gln})\end{array}$ \\
\hline 18/M406 & 2.5 & none & none & none & none & none & none & none \\
\hline 19/M408 & 2.5 & none & $\begin{array}{c}\text { GGC } \rightarrow \text { CGC } \\
(\text { Gly } \rightarrow \text { Arg })\end{array}$ & none & none & none & $\begin{array}{c}\text { Codon } 791 \\
\text { ACA } \rightarrow \text { ATA } \\
(\mathrm{Thr} \rightarrow \text { Ile) } \\
\text { Codon } 806 \\
\text { CAT } \rightarrow \text { TAT } \\
(\text { His } \rightarrow \text { Tyr })\end{array}$ & none \\
\hline 20/M413 & 2.5 & none & none & none & none & none & none & none \\
\hline 21/M425 & 2.5 & none & none & none & none & none & none & none \\
\hline 22/M438 & 2.5 & none & none & none & none & none & none & none \\
\hline 23/M448 & 2.5 & $\begin{array}{r}\text { GGT } \rightarrow \text { TGT } \\
(\text { Gly } \rightarrow \text { Cys })\end{array}$ & none & none & none & none & none & none \\
\hline 24/M417 & 2.5 & $\begin{array}{r}\text { GGT } \rightarrow \text { GAT } \\
(\text { Gly } \rightarrow \text { Asp })\end{array}$ & none & none & none & none & none & none \\
\hline 25/M418 & 2.5 & $\begin{array}{r}\text { GGT } \rightarrow \text { GAT } \\
(\text { Gly } \rightarrow \text { Asp })\end{array}$ & none & none & none & none & none & none \\
\hline 26/M411 & 5 & $\begin{array}{l}\mathrm{GGT} \rightarrow \mathrm{TGT} \\
(\mathrm{Gly} \rightarrow \mathrm{Cys})\end{array}$ & none & none & none & none & none & none \\
\hline 27/M641 & 5 & none & none & none & none & none & none & none \\
\hline 28/M649 & 5 & none & none & none & none & none & none & none \\
\hline 29/M605 & 5 & none & none & none & none & none & none & none \\
\hline 30/M608 & 5 & $\begin{array}{c}\mathrm{GGT} \rightarrow \mathrm{GTT} \\
(\mathrm{Gly} \rightarrow \mathrm{Val})\end{array}$ & none & none & none & none & none & none \\
\hline 31/M611 & 5 & none & none & none & none & none & none & none \\
\hline 32/M615 & 5 & none & none & none & none & none & none & $\begin{array}{c}\text { Codon } 875 \\
\text { GGC } \rightarrow \text { AGC } \\
(\text { Gly } \rightarrow \text { Ser })\end{array}$ \\
\hline 33/M616 & 5 & none & none & none & none & none & none & none \\
\hline 34/M618 & 5 & none & none & none & none & none & none & none \\
\hline
\end{tabular}


Table K-5. Kras and Egfr Mutations in Alveolar/bronchiolar Carcinomas from Female F344/NTac Rats in the Two-year Inhalation Study of Cobalt Metal

\begin{tabular}{|c|c|c|c|c|c|c|c|c|}
\hline \multirow{2}{*}{$\begin{array}{c}\text { Sample/ } \\
\text { Animal } \\
\#\end{array}$} & \multirow{2}{*}{$\begin{array}{l}\text { Cobalt } \\
\text { Conc. } \\
\left(\mathbf{m g} / \mathbf{m}^{3}\right)\end{array}$} & \multicolumn{3}{|c|}{ Kras } & \multicolumn{4}{|c|}{$E g f r$} \\
\hline & & Codon 12 & Codon 13 & Codon 61 & Exon 18 & Exon 19 & Exon 20 & Exon 21 \\
\hline $1 / \mathrm{F} 224$ & 0 & none & none & none & none & none & none & none \\
\hline 2/F163 & 0 & none & none & none & none & none & none & none \\
\hline $3 / F 314$ & 1.25 & $\begin{array}{c}\mathrm{GGT} \rightarrow \mathrm{GTT} \\
(\mathrm{Gly} \rightarrow \mathrm{Val})\end{array}$ & none & none & none & none & none & none \\
\hline 4/F337 & 1.25 & none & none & none & none & none & none & none \\
\hline 5/F338 & 1.25 & none & none & none & none & none & none & none \\
\hline 6/F342 & 1.25 & none & none & none & none & none & $\begin{array}{c}\text { Codon } 790 \\
\text { ATT } \rightarrow \text { ACT } \\
(\mathrm{Ile} \rightarrow \mathrm{Thr})\end{array}$ & none \\
\hline 7/F348 & 1.25 & $\begin{array}{r}\text { GGT } \rightarrow \text { GAT } \\
(\text { Gly } \rightarrow \text { Asp })\end{array}$ & none & none & none & none & none & $\begin{array}{c}\text { Codon } 844 \\
\text { GTA } \rightarrow \text { GTG } \\
(\text { Val } \rightarrow \text { Val) }\end{array}$ \\
\hline 8/F504 & 2.5 & none & none & none & none & none & none & none \\
\hline 9/F505 & 2.5 & $\begin{array}{l}\text { GGT } \rightarrow \text { GAT } \\
(\text { Gly } \rightarrow \text { Asp })\end{array}$ & none & none & none & none & none & $\begin{array}{c}\text { Codon } 838 \\
\text { GAC } \rightarrow \text { GAT } \\
(\mathrm{Asp} \rightarrow \text { Asp })\end{array}$ \\
\hline 10/F513 & 2.5 & none & none & none & none & none & $\begin{array}{c}\text { Codon } 770 \\
\text { GTG } \rightarrow \text { ATG } \\
\text { (Val } \rightarrow \text { Met })\end{array}$ & none \\
\hline 11/F514 & 2.5 & $\begin{array}{c}\text { GGT } \rightarrow \text { GAT } \\
(\text { Gly } \rightarrow \text { Asp })\end{array}$ & none & none & none & none & none & none \\
\hline 12/F519 & 2.5 & none & none & none & none & none & none & none \\
\hline 13/F520 & 2.5 & none & none & none & none & $\begin{array}{c}\text { Codon } 750 \\
\text { GAA } \rightarrow \text { AAA } \\
(\text { Glu } \rightarrow \text { Lys })\end{array}$ & $\begin{array}{c}\text { Codon } 771 \\
\text { GAC } \rightarrow \text { AAC } \\
(\mathrm{Asp} \rightarrow \text { Asn })\end{array}$ & none \\
\hline 14/F539 & 2.5 & none & none & none & none & none & none & none \\
\hline 15/F543 & 2.5 & none & none & none & none & none & none & none \\
\hline 16/F548 & 2.5 & none & none & none & none & none & none & none \\
\hline 17/F707 & 5 & $\begin{array}{c}\mathrm{GGT} \rightarrow \mathrm{GTT} \\
(\mathrm{Gly} \rightarrow \mathrm{Val})\end{array}$ & none & none & none & none & none & none \\
\hline 18/F723 & 5 & $\begin{array}{r}\text { GGT } \rightarrow \text { GAT } \\
(\text { Gly } \rightarrow \text { Asp })\end{array}$ & none & none & none & none & $\begin{array}{c}\text { Codon } 768 \\
\text { GCC } \rightarrow \text { ACC } \\
(\mathrm{Ala} \rightarrow \mathrm{Thr})\end{array}$ & none \\
\hline 19/F728 & 5 & none & none & none & none & none & none & none \\
\hline 20/F746 & 5 & none & none & none & none & none & none & none \\
\hline 22/F703 & 5 & $\begin{array}{c}\text { GGT } \rightarrow \text { TGT } \\
(\text { Gly } \rightarrow \text { Cys })\end{array}$ & none & none & none & none & none & none \\
\hline
\end{tabular}


Cobalt Metal, NTP TR 581

\begin{tabular}{|c|c|c|c|c|c|c|c|c|}
\hline \multirow{2}{*}{$\begin{array}{c}\text { Sample/ } \\
\text { Animal } \\
\#\end{array}$} & \multirow{2}{*}{$\begin{array}{l}\text { Cobalt } \\
\text { Conc. } \\
\left(\mathbf{m g} / \mathbf{m}^{3}\right)\end{array}$} & \multicolumn{3}{|c|}{ Kras } & \multicolumn{4}{|c|}{ Egfr } \\
\hline & & Codon 12 & Codon 13 & Codon 61 & Exon 18 & Exon 19 & Exon 20 & Exon 21 \\
\hline 21/F701 & 5 & $\begin{array}{c}\mathrm{GGT} \rightarrow \mathrm{GTT} \\
(\mathrm{Gly} \rightarrow \mathrm{Val})\end{array}$ & none & none & none & none & $\begin{array}{l}\text { Codon } 785 \\
\text { TCC } \rightarrow \text { TTC } \\
(\text { Ser } \rightarrow \text { Phe })\end{array}$ & none \\
\hline 23/F711 & 5 & none & none & none & none & none & none & none \\
\hline 24/F717 & 5 & $\begin{array}{c}\mathrm{GGT} \rightarrow \mathrm{TTT} \\
(\mathrm{Gly} \rightarrow \mathrm{Phe})\end{array}$ & none & none & none & none & none & none \\
\hline
\end{tabular}

Table K-6. Egfr Mutations in Alveolar/bronchiolar Carcinomas from Male and Female F344/NTac Rats in the Two-year Inhalation Study of Cobalt Metal ${ }^{\mathrm{a}}$

\begin{tabular}{lccccc}
\hline $\begin{array}{c}\text { Cobalt Metal } \\
\text { Concentration }\end{array}$ & $\begin{array}{c}\text { Mutation } \\
\text { Frequency }\end{array}$ & Exon 18 & Exon 19 & Exon 20 & Exon 21 \\
\hline $0 \mathrm{mg} / \mathrm{m}^{3 \#}$ & $0 / 10(0 \%)$ & 0 & 0 & 0 & 0 \\
$1.25 \mathrm{mg} / \mathrm{m}^{3}$ & $2 / 14(14 \%)$ & 0 & 0 & 1 & 1 \\
$2.5 \mathrm{mg} / \mathrm{m}^{3}$ & $3 / 17(18 \%)$ & 0 & $1^{\mathrm{b}}$ & $3^{\mathrm{b}}$ & 0 \\
$5 \mathrm{mg} / \mathrm{m}^{3}$ & $3 / 17(18 \%)$ & 0 & 0 & 2 & 1 \\
$\begin{array}{l}\text { All exposed } \\
\text { groups combined }\end{array}$ & $8 / 48(17 \%)$ & 0 & $1^{\mathrm{b}}$ & $6^{\mathrm{b}}$ & 2 \\
\hline
\end{tabular}

\#There were no spontaneous alveolar/bronchiolar carcinomas in the cobalt metal study. Hence, the spontaneous alveolar/bronchiolar carcinomas $(\mathrm{n}=10)$ were sourced from vehicle or chamber control groups in various NTP chronic bioassays. These spontaneous alveolar/bronchiolar samples were sourced from eight male and two female F344/N rats.

aSilent mutations are not included.

bSame animal with multiple mutations.

Table K-7. Tp53 Mutations in Alveolar/bronchiolar Carcinomas from Male and Female F344/NTac Rats in the Two-year Inhalation Study of Cobalt Metal ${ }^{a}$

\begin{tabular}{lccccc}
\hline $\begin{array}{c}\text { Cobalt Metal } \\
\text { Concentration }\end{array}$ & $\begin{array}{c}\text { Mutation } \\
\text { Frequency }\end{array}$ & Exon 5 & Exon 6 & Exon 7 & Exon 8 \\
\hline $0 \mathrm{mg} / \mathrm{m}^{3 \#}$ & $0 / 10(0 \%)$ & 0 & 0 & 0 & 0 \\
$1.25 \mathrm{mg} / \mathrm{m}^{3}$ & $3 / 14(21 \%)$ & 1 & $1^{\mathrm{b}}$ & $1^{\mathrm{b}}$ & 1 \\
$2.5 \mathrm{mg} / \mathrm{m}^{3}$ & $6 / 17(35 \%)^{*}$ & 0 & $4^{\mathrm{b}}$ & $1^{\mathrm{b}}$ & 2 \\
$5 \mathrm{mg} / \mathrm{m}^{3}$ & $2 / 17(12 \%)$ & 1 & 0 & 1 & 0 \\
$\begin{array}{l}\text { All exposed groups } \\
\text { combined }\end{array}$ & $11 / 48(23 \%)$ & 2 & $5^{\mathrm{b}}$ & $3^{\mathrm{b}}$ & 3 \\
\hline
\end{tabular}

*Significantly different $(\mathrm{P} \leq 0.05)$ from the chamber control group by the Fisher exact test.

\#There were no spontaneous alveolar/bronchiolar carcinomas in the cobalt metal study. Hence, the spontaneous alveolar/bronchiolar carcinomas $(\mathrm{n}=10)$ were sourced from vehicle or chamber control groups in various NTP chronic bioassays. These spontaneous alveolar/bronchiolar samples were sourced from eight male and two female F344/N rats. a Silent mutations are not included.

bSame animal with double mutations. 
Cobalt Metal, NTP TR 581

Table K-8. Tp53 Mutations in Alveolar/bronchiolar Carcinomas from Male F344/NTac Rats in the Two-year Inhalation Study of Cobalt Metal

\begin{tabular}{|c|c|c|c|c|c|}
\hline Sample/Animal \# & $\begin{array}{c}\text { Cobalt Metal } \\
\text { Concentration } \\
\left(\mathbf{m g} / \mathbf{m}^{3}\right)\end{array}$ & Exon 5 & Exon 6 & Exon 7 & Exon 8 \\
\hline 1/M55 & 0 & none & none & none & none \\
\hline 2/M46 & 0 & none & none & none & none \\
\hline 3/M17 & 0 & none & none & none & none \\
\hline 4/M10 & 0 & none & none & none & none \\
\hline 5/M03 & 0 & none & $\begin{array}{c}\text { Codon } 205 \\
\mathrm{GAC} \rightarrow \mathrm{GAT} \\
(\mathrm{Asp} \rightarrow \mathrm{Asp})\end{array}$ & none & none \\
\hline 6/M33 & 0 & none & none & none & none \\
\hline 7/M63 & 0 & none & none & none & none \\
\hline 8/M46 & 0 & none & none & none & none \\
\hline 9/M215 & 1.25 & $\begin{array}{l}\text { Codon } 134 \\
\text { CAG } \rightarrow \text { TAG } \\
(\mathrm{Gln} \rightarrow \text { Stop })\end{array}$ & none & none & none \\
\hline 10/M230 & 1.25 & $\begin{array}{c}\text { Codon } 160 \\
\text { ATC } \rightarrow \text { ATT } \\
(\text { Ile } \rightarrow \text { Ile })\end{array}$ & none & none & none \\
\hline 11/M249 & 1.25 & none & none & none & none \\
\hline 12/M202 & 1.25 & none & none & none & none \\
\hline 13/M226 & 1.25 & none & none & none & none \\
\hline 14/M233 & 1.25 & none & none & none & none \\
\hline 15/M241 & 1.25 & none & none & none & none \\
\hline 16/M247 & 1.25 & none & none & none & none \\
\hline 17/M248 & 1.25 & none & none & none & none \\
\hline 18/M406 & 2.5 & none & $\begin{array}{c}\text { Codon } 247 \\
\mathrm{CGG} \rightarrow \mathrm{CAG} \\
(\mathrm{Arg} \rightarrow \mathrm{Gln})\end{array}$ & none & none \\
\hline 19/M408 & 2.5 & none & none & none & $\begin{array}{c}\text { Codon } 321 \\
\text { CTC } \rightarrow \text { TTC } \\
(\text { Leu } \rightarrow \text { Phe })\end{array}$ \\
\hline 20/M413 & 2.5 & none & $\begin{array}{c}\text { Codon } 206 \\
(\mathrm{GAC} \rightarrow \mathrm{GAT} \\
(\mathrm{Asp} \rightarrow \mathrm{Asp}) \\
\text { Codon } 242 \\
\mathrm{GGG} \rightarrow \mathrm{GAG} \\
(\mathrm{Gly} \rightarrow \mathrm{Gln})\end{array}$ & none & none \\
\hline 21/M425 & 2.5 & none & none & none & none \\
\hline 22/M438 & 2.5 & none & none & none & none \\
\hline 23/M448 & 2.5 & none & none & none & none \\
\hline
\end{tabular}


Cobalt Metal, NTP TR 581

\begin{tabular}{|c|c|c|c|c|c|}
\hline Sample/Animal\# & $\begin{array}{c}\text { Cobalt Metal } \\
\text { Concentration } \\
\left(\mathrm{mg} / \mathrm{m}^{3}\right)\end{array}$ & Exon 5 & Exon 6 & Exon 7 & Exon 8 \\
\hline 24/M417 & 2.5 & none & none & none & none \\
\hline 25/M418 & 2.5 & none & none & none & none \\
\hline 26/M411 & 5 & none & none & none & none \\
\hline 27/M641 & 5 & none & none & none & none \\
\hline 28/M649 & 5 & none & $\begin{array}{c}\text { Codon } 258 \\
\text { TCC } \rightarrow \text { TCT } \\
\text { (Ser } \rightarrow \text { Ser })\end{array}$ & none & none \\
\hline 29/M605 & 5 & none & $\begin{array}{c}\text { Codon } 242 \\
\text { GGG } \rightarrow \text { GGA } \\
(\text { Gly } \rightarrow \text { Gly })\end{array}$ & $\begin{array}{c}\text { Codon } 277 \\
\text { GGG } \rightarrow \text { GAG } \\
(\mathrm{Gly} \rightarrow \mathrm{Glu})\end{array}$ & none \\
\hline 30/M608 & 5 & none & none & none & none \\
\hline 31/M611 & 5 & none & none & none & none \\
\hline 32/M615 & 5 & none & none & none & none \\
\hline 33/M616 & 5 & none & none & none & none \\
\hline 34/M618 & 5 & $\begin{array}{c}\text { Codon } 135 \\
\text { CTG } \rightarrow \text { CGG } \\
(\text { Leu } \rightarrow \text { Arg })\end{array}$ & none & none & none \\
\hline
\end{tabular}


Cobalt Metal, NTP TR 581

Table K-9. Tp53 Mutations in Alveolar/bronchiolar Carcinomas from Female F344/NTac Rats in the Two-year Inhalation Study of Cobalt Metal

\begin{tabular}{|c|c|c|c|c|c|}
\hline Sample/Animal \# & $\begin{array}{c}\text { Cobalt Metal } \\
\text { Concentration } \\
\left(\mathbf{m g} / \mathbf{m}^{3}\right)\end{array}$ & Exon 5 & Exon 6 & Exon 7 & Exon 8 \\
\hline $1 / \mathrm{F} 224$ & 0 & none & none & none & none \\
\hline $2 / F 163$ & 0 & none & none & none & none \\
\hline 3/F314 & 1.25 & none & $\begin{array}{c}\text { Codon } 203 \\
\text { TAT } \rightarrow \text { CAT } \\
(\mathrm{Tyr} \rightarrow \text { His })\end{array}$ & $\begin{array}{c}\text { Codon } 278 \\
\mathrm{AGA} \rightarrow \text { GGA } \\
(\mathrm{Arg} \rightarrow \mathrm{Gly})\end{array}$ & none \\
\hline 4/F337 & 1.25 & none & none & none & none \\
\hline $5 / \mathrm{F} 338$ & 1.25 & none & none & none & none \\
\hline 6/F342 & 1.25 & none & none & none & $\begin{array}{c}\text { Codon } 314 \\
\text { CCC } \rightarrow \text { CTC } \\
(\text { Pro } \rightarrow \text { Leu })\end{array}$ \\
\hline 7/F348 & 1.25 & none & none & none & none \\
\hline $8 / F 504$ & 2.5 & none & $\begin{array}{c}\text { Codon } 242 \\
\text { GGG } \rightarrow \text { AGG } \\
(\mathrm{Gly} \rightarrow \mathrm{Arg})\end{array}$ & $\begin{array}{c}\text { Codon } 266 \\
\text { GAC } \rightarrow \text { TAC } \\
(\mathrm{Asp} \rightarrow \mathrm{Tyr})\end{array}$ & none \\
\hline 9/F505 & 2.5 & none & none & none & none \\
\hline 10/F513 & 2.5 & none & none & none & $\begin{array}{c}\text { Codon } 314 \\
\text { CCC } \rightarrow \text { CTC } \\
(\text { Pro } \rightarrow \text { Leu })\end{array}$ \\
\hline 11/F514 & 2.5 & none & $\begin{array}{c}\text { Codon } 212 \\
\text { CAC } \rightarrow \text { TAC } \\
(\text { His } \rightarrow \text { Tyr })\end{array}$ & none & none \\
\hline $12 / \mathrm{F} 519$ & 2.5 & none & none & none & none \\
\hline $13 / \mathrm{F} 520$ & 2.5 & none & none & none & none \\
\hline $14 / \mathrm{F} 539$ & 2.5 & none & none & none & none \\
\hline $15 /$ F543 & 2.5 & none & none & none & none \\
\hline $16 /$ F548 & 2.5 & none & none & none & none \\
\hline 17/F707 & 5 & none & none & none & none \\
\hline $18 /$ F723 & 5 & none & none & none & none \\
\hline 19/F728 & 5 & none & none & none & none \\
\hline 20/F746 & 5 & none & none & none & none \\
\hline 21/F701 & 5 & none & none & none & none \\
\hline 22/F703 & 5 & none & none & none & none \\
\hline 23/F711 & 5 & none & none & none & none \\
\hline 24/F717 & 5 & none & none & none & none \\
\hline
\end{tabular}


Table K-10. Kras Mutations in Alveolar/bronchiolar Carcinomas from Male and Female B6C3F1/N Mice in the Two-year Inhalation Study of Cobalt Metal

\begin{tabular}{|c|c|c|c|c|c|c|c|c|c|}
\hline \multirow{2}{*}{$\begin{array}{l}\text { Cobalt Metal } \\
\text { Concentration }\end{array}$} & \multirow{2}{*}{$\begin{array}{l}\text { Mutation } \\
\text { Frequency }\end{array}$} & \multicolumn{4}{|c|}{ Codon 12 (GGT) } & \multirow{2}{*}{$\begin{array}{c}\begin{array}{c}\text { Codon } 13 \\
\text { (GGC) }\end{array} \\
\text { (CGC) }\end{array}$} & \multicolumn{3}{|c|}{ Codon 61 (CAA) } \\
\hline & & (GAT) & (TGT) & (GTT) & (CGT) & & (CGA) & (CAT) & (CAC) \\
\hline Historical Control $^{\mathrm{a}}$ & $34 / 124(27 \%)$ & 14 & 5 & 1 & 0 & 6 & 3 & 4 & 1 \\
\hline $0 \mathrm{mg} / \mathrm{m}^{3}$ & $0 / 10(0 \%)^{\# \#}$ & 0 & 0 & 0 & 0 & 0 & 0 & 0 & 0 \\
\hline $1.25 \mathrm{mg} / \mathrm{m}^{3}$ & $11 / 16(69 \%)^{* * *}$ & 1 & 0 & 7 & 0 & 0 & 1 & 2 & 0 \\
\hline $2.5 \mathrm{mg} / \mathrm{m}^{3}$ & $11 / 23(48 \%)^{* *}$ & 2 & 1 & 5 & 0 & 0 & 1 & 0 & 2 \\
\hline $5 \mathrm{mg} / \mathrm{m}^{3}$ & $24 / 30(80 \%)^{* * *}$ & 2 & 0 & $11^{\mathrm{b}}$ & $1^{\mathrm{b}}$ & 4 & $3^{\mathrm{b}}$ & 2 & 3 \\
\hline $\begin{array}{l}\text { All exposed groups } \\
\text { combined }\end{array}$ & $46 / 69(67 \%)^{* * *}$ & 5 & 1 & $23^{\mathrm{b}}$ & $1^{\mathrm{b}}$ & 4 & $5^{\mathrm{b}}$ & 4 & 5 \\
\hline
\end{tabular}


Table K-11. Kras and Egfr Mutations in Alveolar/bronchiolar Carcinomas from Male B6C3F1/N Mice in the Two-year Inhalation Study of Cobalt Metal

\begin{tabular}{|c|c|c|c|c|c|c|c|c|}
\hline \multirow{2}{*}{$\begin{array}{l}\text { Sample/ } \\
\text { Animal \# }\end{array}$} & \multirow{2}{*}{$\begin{array}{l}\text { Cobalt } \\
\text { Conc. } \\
\left(\mathrm{mg} / \mathrm{m}^{3}\right)\end{array}$} & \multicolumn{3}{|c|}{ Kras } & \multicolumn{4}{|c|}{$E g f r$} \\
\hline & & Codon 12 & Codon 13 & Codon 61 & Exon 18 & Exon 19 & Exon 20 & Exon 21 \\
\hline $1 / \mathrm{M} 42$ & 0 & none & none & none & none & none & none & none \\
\hline 2/M12 & 0 & none & none & none & none & none & none & none \\
\hline 3/M30 & 0 & none & none & none & none & none & none & none \\
\hline 4/M36 & 0 & none & none & none & none & none & none & none \\
\hline 5/M21 & 0 & none & none & none & none & none & none & none \\
\hline 6/M24 & 0 & none & none & none & none & none & none & none \\
\hline 7/M201 & 1.25 & none & none & none & none & none & $\begin{array}{c}\text { Codon } 807 \\
\text { CAC } \rightarrow \text { CAT } \\
(\text { His } \rightarrow \text { His })\end{array}$ & none \\
\hline 8/M203 & 1.25 & $\begin{array}{r}\mathrm{GGT} \rightarrow \mathrm{GTT} \\
(\mathrm{Gly} \rightarrow \mathrm{Val})\end{array}$ & none & none & none & none & none & none \\
\hline 9/M209 & 1.25 & $\begin{array}{c}\mathrm{GGT} \rightarrow \text { GTT } \\
(\mathrm{Gly} \rightarrow \text { Val })\end{array}$ & none & none & none & none & none & none \\
\hline 10/M211 & 1.25 & none & none & none & none & none & none & none \\
\hline 11/M216 & 1.25 & none & none & none & $\begin{array}{c}\text { Codon } 721 \\
\text { GGT } \rightarrow \text { AGT } \\
(\text { Gly } \rightarrow \text { Ser })\end{array}$ & none & none & none \\
\hline 12/M219 & 1.25 & $\begin{array}{c}\mathrm{GGT} \rightarrow \mathrm{GAT} \\
(\mathrm{Gly} \rightarrow \mathrm{Asp})\end{array}$ & none & none & none & none & none & $\begin{array}{c}\text { Codon } 846 \\
\mathrm{CTG} \rightarrow \mathrm{CAG} \\
(\mathrm{Leu} \rightarrow \mathrm{Gln})\end{array}$ \\
\hline 13/M204 & 1.25 & none & none & $\begin{array}{c}\mathrm{CAA} \rightarrow \mathrm{CAT} \\
(\mathrm{Gln} \rightarrow \mathrm{His})\end{array}$ & none & none & none & none \\
\hline 14/M217 & 1.25 & $\begin{array}{c}\mathrm{GGT} \rightarrow \mathrm{GTT} \\
(\mathrm{Gly} \rightarrow \mathrm{Val})\end{array}$ & none & none & none & none & none & none \\
\hline 15/M218 & 1.25 & none & none & $\begin{array}{c}\mathrm{CAA} \rightarrow \mathrm{CGA} \\
(\mathrm{Gln} \rightarrow \mathrm{Arg})\end{array}$ & none & none & none & none \\
\hline 16/M401 & 2.5 & none & none & none & none & none & none & $\begin{array}{c}\text { Codon } 870 \\
\text { GAA } \rightarrow \text { AAA } \\
(\mathrm{Glu} \rightarrow \text { Lys })\end{array}$ \\
\hline 17/M403 & 2.5 & $\begin{array}{c}\mathrm{GGT} \rightarrow \mathrm{GTT} \\
(\mathrm{Gly} \rightarrow \mathrm{Val})\end{array}$ & none & none & none & none & none & none \\
\hline 18/M405 & 2.5 & none & none & none & none & $\begin{array}{c}\text { Codon } 735 \\
\mathrm{CCA} \rightarrow \text { CTA } \\
(\text { Pro } \rightarrow \text { Leu })\end{array}$ & none & none \\
\hline 19/M407 & 2.5 & none & none & none & none & none & $\begin{array}{c}\text { Codon } 812 \\
\text { GGC } \rightarrow \text { GAC } \\
(\text { Gly } \rightarrow \text { Asp })\end{array}$ & none \\
\hline 20/M409 & 2.5 & none & none & none & none & none & none & none \\
\hline 21/M404 & 2.5 & none & none & none & none & none & none & none \\
\hline 22/M411 & 2.5 & $\begin{array}{c}\mathrm{GGT} \rightarrow \mathrm{GTT} \\
(\mathrm{Gly} \rightarrow \mathrm{Val})\end{array}$ & none & none & none & none & none & none \\
\hline
\end{tabular}


Cobalt Metal, NTP TR 581

\begin{tabular}{|c|c|c|c|c|c|c|c|c|}
\hline \multirow{2}{*}{$\begin{array}{l}\text { Sample/ } \\
\text { Animal \# }\end{array}$} & \multirow{2}{*}{$\begin{array}{c}\text { Cobalt } \\
\text { Conc. } \\
\left(\mathrm{mg} / \mathrm{m}^{3}\right)\end{array}$} & \multicolumn{3}{|c|}{ Kras } & \multicolumn{4}{|c|}{ Egfr } \\
\hline & & Codon 12 & Codon 13 & Codon 61 & Exon 18 & Exon 19 & Exon 20 & Exon 21 \\
\hline 23/M413 & 2.5 & $\begin{array}{c}\mathrm{GGT} \rightarrow \mathrm{GTT} \\
(\mathrm{Gly} \rightarrow \mathrm{Val})\end{array}$ & none & $\begin{array}{c}\mathrm{CAA} \rightarrow \mathrm{CGA} \\
(\mathrm{Gln} \rightarrow \mathrm{Arg})\end{array}$ & none & none & none & none \\
\hline 24/M417 & 2.5 & $\begin{array}{r}\mathrm{GGT} \rightarrow \mathrm{TGT} \\
(\mathrm{Gly} \rightarrow \mathrm{Cys})\end{array}$ & none & none & none & none & none & none \\
\hline 25/M418 & 2.5 & none & none & none & none & none & none & none \\
\hline 26/M618 & 5 & none & none & $\begin{array}{c}\mathrm{CAA} \rightarrow \mathrm{CAT} \\
(\mathrm{Gln} \rightarrow \mathrm{His})\end{array}$ & none & none & none & none \\
\hline 27/M627 & 5 & none & none & $\begin{array}{c}\mathrm{CAA} \rightarrow \mathrm{CAT} \\
(\mathrm{Gln} \rightarrow \mathrm{His})\end{array}$ & none & none & none & none \\
\hline 28/M629 & 5 & none & none & $\begin{array}{l}\text { CAACAC } \\
(\mathrm{Gln} \rightarrow \mathrm{His})\end{array}$ & & & & \\
\hline 29/M606 & 5 & $\begin{array}{r}\mathrm{GGT} \rightarrow \mathrm{GTT} \\
(\mathrm{Gly} \rightarrow \mathrm{Val})\end{array}$ & none & none & none & none & none & none \\
\hline 30/M648 & 5 & none & $\begin{aligned} \mathrm{GGC} & \rightarrow \mathrm{CGC} \\
(\mathrm{Gly} & \rightarrow \text { Arg })\end{aligned}$ & none & none & none & none & none \\
\hline 31/M632 & 5 & $\begin{array}{c}\mathrm{GGT} \rightarrow \mathrm{GTT} \\
(\mathrm{Gly} \rightarrow \text { Val })\end{array}$ & none & none & none & none & none & none \\
\hline 32/M601 & 5 & none & none & none & none & none & none & none \\
\hline 33/M602 & 5 & none & none & none & none & none & none & $\begin{array}{c}\text { Codon } 861 \\
\text { GCC } \rightarrow \text { ACC } \\
(\text { Ala } \rightarrow \text { Thr })\end{array}$ \\
\hline 34/M605 & 5 & $\begin{array}{c}\mathrm{GGT} \rightarrow \mathrm{GTT} \\
(\mathrm{Gly} \rightarrow \text { Val })\end{array}$ & none & $\begin{array}{c}\mathrm{CAA} \rightarrow \mathrm{CGA} \\
(\mathrm{Gln} \rightarrow \mathrm{Arg})\end{array}$ & none & none & $\begin{array}{c}\text { Codon } 791 \\
\text { ATT } \rightarrow \text { GTT } \\
(\mathrm{Ile} \rightarrow \text { Val })\end{array}$ & none \\
\hline 35/M609 & 5 & none & $\begin{array}{c}\mathrm{GGC} \rightarrow \mathrm{CGC} \\
(\mathrm{Gly} \rightarrow \text { Arg })\end{array}$ & none & none & none & $\begin{array}{c}\text { Codon } 804 \\
\text { GTC } \rightarrow \text { GTA } \\
(\text { Val } \rightarrow \text { Val })\end{array}$ & none \\
\hline 36/M611 & 5 & $\begin{array}{c}\mathrm{GGT} \rightarrow \mathrm{GAT} \\
(\mathrm{Gly} \rightarrow \mathrm{Asp})\end{array}$ & none & none & none & none & none & none \\
\hline 37/M604 & 5 & none & none & none & none & none & none & none \\
\hline 38/M607 & 5 & none & none & $\begin{array}{c}\mathrm{CAA} \rightarrow \mathrm{CAC} \\
(\mathrm{Gln} \rightarrow \mathrm{His})\end{array}$ & none & none & none & none \\
\hline 39/M608-12a & 5 & $\begin{array}{r}\mathrm{GGT} \rightarrow \mathrm{GTT} \\
(\mathrm{Gly} \rightarrow \mathrm{Val})\end{array}$ & none & none & none & none & none & none \\
\hline 40/M608-13a & 5 & $\begin{array}{c}\mathrm{GGT} \rightarrow \mathrm{GTT} \\
(\mathrm{Gly} \rightarrow \text { Val })\end{array}$ & none & none & none & none & none & none \\
\hline
\end{tabular}

aSame animal with different blocks. 
Table K-12. Kras and Egfr Mutations in Alveolar/bronchiolar Carcinomas from Female B6C3F1/N Mice in the Two-year Inhalation Study of Cobalt Metal

\begin{tabular}{|c|c|c|c|c|c|c|c|c|}
\hline \multirow{2}{*}{$\begin{array}{l}\text { Sample/ } \\
\text { Animal \# }\end{array}$} & \multirow{2}{*}{$\begin{array}{c}\text { Cobalt } \\
\text { Conc. } \\
\left(\mathbf{m g} / \mathbf{m}^{3}\right)\end{array}$} & \multicolumn{3}{|c|}{ Kras } & \multicolumn{4}{|c|}{ Egfr } \\
\hline & & Codon 12 & Codon 13 & Codon 61 & Exon 18 & Exon 19 & Exon 20 & Exon 21 \\
\hline $1 / \mathrm{F} 145$ & 0 & none & none & none & none & none & none & none \\
\hline $2 / F 125$ & 0 & none & none & none & none & none & none & none \\
\hline 3/F106 & 0 & none & none & none & none & none & none & none \\
\hline 4/F131 & 0 & none & none & none & none & none & none & $\begin{array}{c}\text { Codon } 832 \\
\text { GAT } \rightarrow \text { GAC } \\
(\text { Asp } \rightarrow \text { Asp) } \\
\text { Codon } 859 \\
\text { GGG } \rightarrow \text { GGA } \\
(\text { Gly } \rightarrow \text { Gly })\end{array}$ \\
\hline 5/F308 & 1.25 & none & none & none & none & none & none & none \\
\hline 6/F318 & 1.25 & $\begin{array}{c}\mathrm{GGT} \rightarrow \mathrm{GTT} \\
(\mathrm{Gly} \rightarrow \mathrm{Val})\end{array}$ & none & none & none & none & none & none \\
\hline 7/F322 & 1.25 & none & none & none & none & none & none & none \\
\hline 8/F306 & 1.25 & $\begin{array}{c}\mathrm{GGT} \rightarrow \mathrm{GTT} \\
(\mathrm{Gly} \rightarrow \mathrm{Val})\end{array}$ & none & none & none & none & none & none \\
\hline 9/F307 & 1.25 & none & none & $\begin{array}{c}\text { CAA } \rightarrow \text { CAT } \\
(\text { Gln } \rightarrow \text { His })\end{array}$ & none & none & none & none \\
\hline 10/F321 & 1.25 & $\begin{array}{c}\mathrm{GGT} \rightarrow \mathrm{GTT} \\
(\mathrm{Gly} \rightarrow \mathrm{Val})\end{array}$ & none & none & none & none & none & none \\
\hline 11/F326 & 1.25 & $\begin{array}{c}\mathrm{GGT} \rightarrow \mathrm{GTT} \\
(\mathrm{Gly} \rightarrow \mathrm{Val})\end{array}$ & none & none & none & none & none & none \\
\hline 12/F502 & 2.5 & $\begin{array}{c}\mathrm{GGT} \rightarrow \mathrm{GAT} \\
(\mathrm{Gly} \rightarrow \mathrm{Asp})\end{array}$ & none & none & none & none & none & none \\
\hline 13/F506 & 2.5 & none & none & none & none & none & $\begin{array}{c}\text { Codon } 780 \\
\mathrm{CTG} \rightarrow \mathrm{CAG} \\
(\mathrm{Leu} \rightarrow \mathrm{Gln})\end{array}$ & none \\
\hline 14/F508 & 2.5 & none & none & none & none & none & $\begin{array}{c}\text { Codon } 817 \\
\text { CTC } \rightarrow \text { TTC } \\
(\text { Leu } \rightarrow \text { Phe })\end{array}$ & none \\
\hline 15/F509 & 2.5 & none & none & none & none & none & none & none \\
\hline 16/F511 & 2.5 & $\begin{array}{c}\mathrm{GGT} \rightarrow \mathrm{GTT} \\
(\mathrm{Gly} \rightarrow \mathrm{Val})\end{array}$ & none & none & none & none & none & none \\
\hline 17/F512 & 2.5 & none & none & none & none & none & $\begin{array}{c}\text { Codon } 801 \\
\text { CTG } \rightarrow \text { CAG } \\
(\mathrm{Leu} \rightarrow \mathrm{Gln})\end{array}$ & none \\
\hline 18/F517 & 2.5 & $\begin{array}{c}\mathrm{GGT} \rightarrow \mathrm{GAT} \\
(\mathrm{Gly} \rightarrow \mathrm{Asp})\end{array}$ & none & none & none & none & none & $\begin{array}{c}\text { Codon } 873 \\
\text { GCC } \rightarrow \text { ACC } \\
(\text { Ala } \rightarrow \text { Thr })\end{array}$ \\
\hline 19/F504 & 2.5 & none & none & none & none & none & none & none \\
\hline
\end{tabular}


Cobalt Metal, NTP TR 581

\begin{tabular}{|c|c|c|c|c|c|c|c|c|}
\hline \multirow{2}{*}{$\begin{array}{l}\text { Sample/ } \\
\text { Animal \# }\end{array}$} & \multirow{2}{*}{$\begin{array}{l}\text { Cobalt } \\
\text { Conc. } \\
\left(\mathbf{m g} / \mathbf{m}^{3}\right)\end{array}$} & \multicolumn{3}{|c|}{ Kras } & \multicolumn{4}{|c|}{$E g f r$} \\
\hline & & Codon 12 & Codon 13 & Codon 61 & Exon 18 & Exon 19 & Exon 20 & Exon 21 \\
\hline 20/F505 & 2.5 & $\begin{array}{c}\mathrm{GGT} \rightarrow \mathrm{GTT} \\
(\mathrm{Gly} \rightarrow \mathrm{Val})\end{array}$ & none & none & none & none & none & none \\
\hline 21/F507 & 2.5 & none & none & $\begin{array}{c}\mathrm{CAA} \rightarrow \mathrm{CAC} \\
(\mathrm{Gln} \rightarrow \mathrm{His})\end{array}$ & none & none & none & none \\
\hline 22/F514 & 2.5 & none & none & none & none & none & none & none \\
\hline 23/F515 & 2.5 & none & none & $\begin{array}{c}\mathrm{CAA} \rightarrow \mathrm{CAC} \\
(\mathrm{Gln} \rightarrow \mathrm{His})\end{array}$ & none & none & none & none \\
\hline 24/F517 & 2.5 & none & none & none & none & none & none & none \\
\hline 25/F746 & 5 & none & $\begin{array}{c}\mathrm{GGC} \rightarrow \mathrm{CGC} \\
(\mathrm{Gly} \rightarrow \text { Arg })\end{array}$ & none & none & none & none & none \\
\hline 26/F717 & 5 & none & $\begin{array}{c}\mathrm{GGC} \rightarrow \mathrm{CGC} \\
(\mathrm{Gly} \rightarrow \mathrm{Arg})\end{array}$ & none & none & none & none & none \\
\hline 27/F720 & 5 & none & none & none & none & none & none & none \\
\hline 28/F732 & 5 & none & none & $\begin{array}{c}\mathrm{CAA} \rightarrow \mathrm{CAC} \\
(\mathrm{Gln} \rightarrow \mathrm{His})\end{array}$ & none & none & none & none \\
\hline 29/F718 & 5 & $\begin{array}{c}\mathrm{GGT} \rightarrow \text { GTT } \\
(\mathrm{Gly} \rightarrow \text { Val })\end{array}$ & none & none & none & none & none & none \\
\hline 30/F714 & 5 & $\begin{array}{c}\mathrm{GGT} \rightarrow \mathrm{GAT} \\
(\mathrm{Gly} \rightarrow \mathrm{Asp})\end{array}$ & none & none & none & none & none & none \\
\hline 31/F723 & 5 & $\begin{array}{c}\mathrm{GGT} \rightarrow \mathrm{GTT} \\
(\mathrm{Gly} \rightarrow \mathrm{Val})\end{array}$ & none & none & none & none & none & none \\
\hline $32 /$ F710 & 5 & $\begin{array}{c}\mathrm{GGT} \rightarrow \mathrm{CGT} \\
(\mathrm{Gly} \rightarrow \mathrm{Arg})\end{array}$ & none & $\begin{array}{c}\mathrm{CAA} \rightarrow \mathrm{CGA} \\
(\mathrm{Gln} \rightarrow \mathrm{Arg})\end{array}$ & none & none & none & none \\
\hline 33/F729 & 5 & $\begin{array}{c}\mathrm{GGT} \rightarrow \mathrm{GTT} \\
(\mathrm{Gly} \rightarrow \mathrm{Val})\end{array}$ & none & none & none & none & none & none \\
\hline 34/F747 & 5 & none & none & none & none & none & none & none \\
\hline 35/F711 & 5 & none & none & none & none & none & $\begin{array}{c}\text { Codon } 780 \\
\mathrm{CTG} \rightarrow \mathrm{CAG} \\
(\mathrm{Leu} \rightarrow \mathrm{Gln})\end{array}$ & none \\
\hline 36/F712 & 5 & $\begin{array}{c}\mathrm{GGT} \rightarrow \mathrm{GTT} \\
(\mathrm{Gly} \rightarrow \mathrm{Val})\end{array}$ & none & none & none & none & none & none \\
\hline 37/F713 & 5 & $\begin{array}{c}\mathrm{GGT} \rightarrow \mathrm{GTT} \\
(\mathrm{Gly} \rightarrow \mathrm{Val})\end{array}$ & none & none & none & none & none & none \\
\hline 38/F715 & 5 & $\begin{array}{c}\mathrm{GGT} \rightarrow \mathrm{GTT} \\
(\mathrm{Gly} \rightarrow \mathrm{Val})\end{array}$ & none & none & none & none & none & none \\
\hline 39/F710 & 5 & none & none & $\begin{array}{c}\mathrm{CAA} \rightarrow \mathrm{CGA} \\
(\mathrm{Gln} \rightarrow \mathrm{Arg})\end{array}$ & none & none & none & none \\
\hline
\end{tabular}


Table K-13. Egfr Mutations in Alveolar/bronchiolar Carcinomas from Male and Female B6C3F1/N Mice in the Two-year Inhalation Study of Cobalt Metal ${ }^{a}$

\begin{tabular}{lccccc}
\hline $\begin{array}{c}\text { Cobalt Metal } \\
\text { Concentration }\end{array}$ & $\begin{array}{c}\text { Mutation } \\
\text { Frequency }\end{array}$ & Exon 18 & Exon 19 & Exon 20 & Exon 21 \\
\hline $0 \mathrm{mg} / \mathrm{m}^{3}$ & $0 / 10(0 \%)$ & 0 & 0 & 0 & 0 \\
$1.25 \mathrm{mg} / \mathrm{m}^{3}$ & $2 / 16(13 \%)$ & 1 & 0 & 0 & 1 \\
$2.5 \mathrm{mg} / \mathrm{m}^{3}$ & $7 / 23(30 \%)$ & 0 & 1 & 4 & 2 \\
$5 \mathrm{mg} / \mathrm{m}^{3}$ & $3 / 30(10 \%)$ & 0 & 0 & 2 & 1 \\
$\begin{array}{l}\text { All exposed } \\
\text { groups combined }\end{array}$ & $12 / 69(17 \%)$ & 1 & 1 & 6 & 4 \\
a & & & &
\end{tabular}

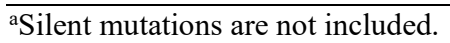

Table K-14. Tp53 Mutations in Alveolar/bronchiolar Carcinomas from Male and Female B6C3F1/N Mice in the Two-year Inhalation Study of Cobalt Metal ${ }^{\mathrm{a}}$

\begin{tabular}{lcccc}
\hline \multicolumn{1}{c}{$\begin{array}{c}\text { Cobalt Metal } \\
\text { Concentration }\end{array}$} & $\begin{array}{c}\text { Mutation } \\
\text { Frequency }\end{array}$ & Exon 5 & Exon 6 & Exon 7 \\
\hline $0 \mathrm{mg} / \mathrm{m}^{3}$ & $0 / 10(0 \%)$ & 0 & 0 & 0 \\
$1.25 \mathrm{mg} / \mathrm{m}^{3}$ & $3 / 16(19 \%)$ & 1 & 0 & 2 \\
$2.5 \mathrm{mg} / \mathrm{m}^{3}$ & $3 / 23(13 \%)$ & $2^{\mathrm{b}}$ & 1 & $1^{\mathrm{b}}$ \\
$5 \mathrm{mg} / \mathrm{m}^{3}$ & $6 / 30(20 \%)$ & 4 & 2 & 1 \\
All exposed groups & $13 / 69(19 \%)$ & $7^{\mathrm{b}}$ & 3 & $4^{\mathrm{b}}$ \\
combined & & & & \\
\hline
\end{tabular}

asilent mutations are not included. No mutations were detected in exon 8 .

${ }^{\mathrm{b} S a m e}$ animal with double mutations. 
Cobalt Metal, NTP TR 581

Table K-15. Tp53 Mutations in Alveolar/bronchiolar Carcinomas from Male B6C3F1/N Mice in the Two-year Inhalation Study of Cobalt Metal

\begin{tabular}{|c|c|c|c|c|c|}
\hline $\begin{array}{c}\text { Sample/Animal } \\
\#\end{array}$ & $\begin{array}{c}\text { Cobalt Metal } \\
\text { Concentration } \\
\left(\mathbf{m g} / \mathbf{m}^{3}\right)\end{array}$ & Exon 5 & Exon 6 & Exon 7 & Exon 8 \\
\hline 1/M42 & 0 & none & none & none & none \\
\hline 2/M12 & 0 & none & none & none & none \\
\hline 3/M30 & 0 & none & none & none & none \\
\hline 4/M36 & 0 & none & none & none & none \\
\hline $5 / \mathrm{M} 21$ & 0 & none & none & none & none \\
\hline 6/M24 & 0 & none & none & none & none \\
\hline 7/M201 & 1.25 & none & $\begin{array}{c}\text { Codon } 203 \\
\text { CTG } \rightarrow \text { CTA } \\
(\text { Leu } \rightarrow \text { Leu })\end{array}$ & none & none \\
\hline 8/M203 & 1.25 & none & none & none & none \\
\hline 9/M209 & 1.25 & none & none & $\begin{array}{c}\text { Codon } 230 \\
\text { CAC } \rightarrow \text { TAC } \\
(\text { His } \rightarrow \text { Tyr })\end{array}$ & none \\
\hline 10/M211 & 1.25 & none & none & none & none \\
\hline 11/M216 & 1.25 & $\begin{array}{c}\text { Codon } 156 \\
\text { GCC } \rightarrow \text { CCC } \\
(\text { Ala } \rightarrow \text { Pro })\end{array}$ & none & none & none \\
\hline 12/M219 & 1.25 & none & none & none & none \\
\hline 13/M204 & 1.25 & none & none & none & none \\
\hline 14/M217 & 1.25 & none & none & none & none \\
\hline $15 / \mathrm{M} 218$ & 1.25 & none & none & none & none \\
\hline 16/M401 & 2.5 & none & none & none & none \\
\hline 17/M403 & 2.5 & none & none & none & none \\
\hline 18/M405 & 2.5 & $\begin{array}{l}\text { Codon } 179 \\
\text { TGC } \rightarrow \text { TAC } \\
(\text { Cys } \rightarrow \text { Tyr })\end{array}$ & none & none & none \\
\hline 19/M407 & 2.5 & none & none & none & none \\
\hline 20/M409 & 2.5 & none & $\begin{array}{c}\text { Codon } 194 \\
\mathrm{GTG} \rightarrow \mathrm{GAG} \\
(\mathrm{Val} \rightarrow \mathrm{Glu})\end{array}$ & none & none \\
\hline 21/M404 & 2.5 & none & none & none & none \\
\hline 22/M411 & 2.5 & none & none & none & none \\
\hline 23/M413 & 2.5 & $\begin{array}{c}\text { Codon } 156 \\
\mathrm{GCC} \rightarrow \mathrm{CCC} \\
(\mathrm{Ala} \rightarrow \text { Pro })\end{array}$ & none & $\begin{array}{c}\text { Codon } 232 \\
\mathrm{AAG} \rightarrow \mathrm{ACG} \\
(\mathrm{Lys} \rightarrow \mathrm{Thr})\end{array}$ & none \\
\hline 24/M417 & 2.5 & none & none & none & none \\
\hline
\end{tabular}


Cobalt Metal, NTP TR 581

\begin{tabular}{|c|c|c|c|c|c|}
\hline $\begin{array}{c}\text { Sample/Animal } \\
\#\end{array}$ & $\begin{array}{c}\text { Cobalt Metal } \\
\text { Concentration } \\
\left(\mathbf{m g} / \mathrm{m}^{3}\right)\end{array}$ & Exon 5 & Exon 6 & Exon 7 & Exon 8 \\
\hline 25/M418 & 2.5 & none & none & $\begin{array}{l}\text { Codon } 231 \\
\text { TAC } \rightarrow \text { TAT } \\
(\text { Tyr } \rightarrow \text { Tyr })\end{array}$ & none \\
\hline 26/M618 & 5 & none & none & none & none \\
\hline 27/M627 & 5 & none & none & none & none \\
\hline 28/M629 & 5 & none & $\begin{array}{c}\text { Codon } 212 \\
\mathrm{AGC} \rightarrow \mathrm{GGC} \\
(\mathrm{Ser} \rightarrow \mathrm{Gly})\end{array}$ & none & none \\
\hline 29/M606 & 5 & none & none & none & none \\
\hline 30/M648 & 5 & none & none & none & none \\
\hline 31/M632 & 5 & none & none & none & none \\
\hline 32/M601 & 5 & none & $\begin{array}{c}\text { Codon } 192 \\
\text { ATC } \rightarrow \text { ATT } \\
(\text { Ile } \rightarrow \text { Ile })\end{array}$ & none & none \\
\hline 33/M602 & 5 & none & none & none & none \\
\hline 34/M605 & 5 & $\begin{array}{c}\text { Codon } 155 \\
\text { CGC } \rightarrow \text { CCC } \\
(\text { Arg } \rightarrow \text { Pro })\end{array}$ & none & none & none \\
\hline 35/M609 & 5 & $\begin{array}{c}\text { Codon } 155 \\
\text { CGC } \rightarrow \text { CCC } \\
(\text { Arg } \rightarrow \text { Pro })\end{array}$ & none & none & none \\
\hline 36/M611 & 5 & none & none & none & none \\
\hline 37/M604 & 5 & none & none & none & none \\
\hline 38/M607 & 5 & none & none & none & none \\
\hline 39/M608-12a & 5 & none & none & none & none \\
\hline 40/M608-13a & 5 & none & none & none & none \\
\hline
\end{tabular}

aSame animal with different blocks. 
Cobalt Metal, NTP TR 581

Table K-16. Tp53 Mutations in Alveolar/bronchiolar Carcinomas from Female B6C3F1/N Mice in the Two-year Inhalation Study of Cobalt Metal

\begin{tabular}{|c|c|c|c|c|c|}
\hline $\begin{array}{c}\text { Sample/Animal } \\
\#\end{array}$ & $\begin{array}{c}\text { Cobalt Metal } \\
\text { Concentration } \\
\left(\mathbf{m g} / \mathbf{m}^{3}\right)\end{array}$ & Exon 5 & Exon 6 & Exon 7 & Exon 8 \\
\hline $1 / \mathrm{F} 145$ & 0 & none & none & none & none \\
\hline $2 / \mathrm{F} 125$ & 0 & none & none & none & none \\
\hline 3/F106 & 0 & none & none & none & none \\
\hline 4/F131 & 0 & none & none & none & none \\
\hline 5/F308 & 1.25 & none & none & $\begin{array}{l}\text { Codon } 257 \\
\text { TCC } \rightarrow \text { TTC } \\
(\text { Ser } \rightarrow \text { Phe })\end{array}$ & none \\
\hline 6/F318 & 1.25 & none & none & none & none \\
\hline 7/F322 & 1.25 & none & none & none & none \\
\hline 8/F306 & 1.25 & none & none & none & none \\
\hline 9/F307 & 1.25 & none & none & none & none \\
\hline 10/F321 & 1.25 & none & none & none & none \\
\hline 11/F326 & 1.25 & none & none & none & none \\
\hline 12/F502 & 2.5 & none & none & none & none \\
\hline 13/F506 & 2.5 & none & none & none & none \\
\hline 14/F508 & 2.5 & none & none & none & none \\
\hline 15/F509 & 2.5 & none & none & none & none \\
\hline 16/F511 & 2.5 & none & none & none & none \\
\hline 17/F512 & 2.5 & none & none & none & none \\
\hline 18/F517 & 2.5 & none & none & none & none \\
\hline 19/F504 & 2.5 & none & none & none & none \\
\hline 20/F505 & 2.5 & none & none & none & none \\
\hline 21/F507 & 2.5 & none & none & none & none \\
\hline 22/F514 & 2.5 & none & none & none & none \\
\hline 23/F515 & 2.5 & none & none & none & none \\
\hline 24/F517 & 2.5 & none & none & none & none \\
\hline 25/F746 & 5 & none & none & none & none \\
\hline 26/F717 & 5 & none & none & none & none \\
\hline 27/F720 & 5 & none & none & none & none \\
\hline 28/F732 & 5 & none & $\begin{array}{c}\text { Codon } 212 \\
\mathrm{AGC} \rightarrow \mathrm{GGC} \\
(\mathrm{Ser} \rightarrow \mathrm{Gly})\end{array}$ & none & none \\
\hline 29/F718 & 5 & none & none & none & none \\
\hline 30/F714 & 5 & none & none & none & none \\
\hline
\end{tabular}


Cobalt Metal, NTP TR 581

\begin{tabular}{|c|c|c|c|c|c|}
\hline $\begin{array}{c}\text { Sample/Animal } \\
\#\end{array}$ & $\begin{array}{c}\text { Cobalt Metal } \\
\text { Concentration } \\
\left(\mathbf{m g} / \mathbf{m}^{3}\right)\end{array}$ & Exon 5 & Exon 6 & Exon 7 & Exon 8 \\
\hline 31/F723 & 5 & none & none & none & none \\
\hline 32/F710 & 5 & none & none & none & none \\
\hline 33/F729 & 5 & none & none & none & none \\
\hline 34/F747 & 5 & none & none & none & none \\
\hline 35/F711 & 5 & $\begin{array}{c}\text { Codon } 161 \\
\text { AAG } \rightarrow \text { AAA } \\
(\text { Lys } \rightarrow \text { Lys) } \\
\text { Codon } 179 \\
\text { TGC } \rightarrow \text { TTC } \\
\text { (Cys } \rightarrow \text { Gly) }\end{array}$ & none & none & none \\
\hline 36/F712 & 5 & $\begin{array}{c}\text { Codon } 158 \\
\text { GCC } \rightarrow \text { ACC } \\
(\text { Ala } \rightarrow \text { Thr }) \\
\text { Codon } 179 \\
\text { TGC } \rightarrow \text { GGC } \\
(\text { Cys } \rightarrow \text { Gly) }\end{array}$ & none & none & none \\
\hline 37/F713 & 5 & none & none & none & none \\
\hline 38/F715 & 5 & none & none & $\begin{array}{c}\text { Codon } 239 \\
\text { TGC } \rightarrow \text { TGG } \\
(\text { Cys } \rightarrow \text { Trp })\end{array}$ & none \\
\hline 39/F710 & 5 & none & none & none & none \\
\hline
\end{tabular}


Table K-17. Summary of Kras Mutations in Alveolar/bronchiolar Neoplasms from Male and Female B6C3F1/N Mice in Selected Twoyear NTP Studies

\begin{tabular}{|c|c|c|c|c|c|c|c|c|c|c|c|c|c|c|c|c|}
\hline \multirow{2}{*}{$\begin{array}{c}\text { Chemical } \\
\text { (Study Type) }\end{array}$} & \multirow{2}{*}{ Mutations } & \multicolumn{5}{|c|}{ Codon 12 GGT } & \multicolumn{5}{|c|}{ Codon 13 GGC } & \multicolumn{5}{|c|}{ Codon 61 CAA } \\
\hline & & GAT & TGT & GTT & CGT & Other & CGC & GAC & CGA & CAT & Other & CAC & CTA & CGA & CAT & CAC \\
\hline \multicolumn{17}{|c|}{ Historical controls } \\
\hline & $34 / 124(27 \%)$ & 14 & 5 & 1 & 0 & - & 6 & 0 & 0 & 0 & - & 0 & 0 & 3 & 4 & 1 \\
\hline \multicolumn{17}{|c|}{ Concurrent chamber controls (inhalation) } \\
\hline & $0 / 10(0 \%)$ & 0 & 0 & 0 & 0 & - & 0 & 0 & 0 & 0 & - & 0 & 0 & 0 & 0 & 0 \\
\hline \multicolumn{17}{|c|}{ Cobalt metal (inhalation) ${ }^{\mathrm{a}}$} \\
\hline & $46 / 69(67 \%)$ & 5 & 1 & 23 & 1 & - & 4 & 0 & 0 & 0 & - & 0 & 0 & 5 & 4 & 5 \\
\hline \multicolumn{17}{|c|}{ Cobalt sulfate heptahydrate (inhalation) } \\
\hline & $9 / 26(35 \%)$ & 2 & 1 & 5 & 0 & - & 1 & 0 & 0 & 0 & - & 0 & 0 & 0 & 0 & 0 \\
\hline \multicolumn{17}{|c|}{ Ozone (inhalation) } \\
\hline & $19 / 26(73 \%)$ & 3 & 2 & 5 & 0 & - & 0 & 1 & 0 & 0 & - & 0 & 8 & 0 & 0 & 0 \\
\hline \multicolumn{17}{|c|}{ 1,3,Butadiene (inhalation) } \\
\hline & $20 / 24(83 \%)$ & 1 & 2 & 0 & 0 & - & 8 & 0 & 0 & 0 & - & 0 & 6 & 3 & 0 & 0 \\
\hline \multicolumn{17}{|c|}{ Methylene chloride (inhalation) } \\
\hline & $11 / 54(20 \%)$ & 1 & 1 & 1 & 0 & - & 1 & 0 & 0 & 0 & - & 4 & 1 & 1 & 1 & 0 \\
\hline \multicolumn{17}{|c|}{ Ethylene oxide (inhalation) ${ }^{\mathrm{a}}$} \\
\hline & $23 / 23(100 \%)$ & 2 & 0 & 21 & 0 & - & 0 & 0 & 0 & 0 & AGC & 0 & 0 & 1 & 0 & 0 \\
\hline \multicolumn{17}{|c|}{ Cumene (inhalation) } \\
\hline & $45 / 52(87 \%)$ & 6 & 5 & 11 & 3 & - & 4 & 0 & 13 & 0 & - & 2 & 1 & 0 & 0 & 0 \\
\hline \multicolumn{17}{|c|}{ 2,2-Bis(bromomethyl-1,3-propanediol (feed) } \\
\hline & $29 / 51(57 \%)$ & 20 & 1 & 7 & 0 & - & 1 & 0 & 0 & 0 & - & 0 & 0 & 0 & 0 & 0 \\
\hline \multicolumn{17}{|c|}{ Tetranitromethane (inhalation) $^{\mathrm{a}}$} \\
\hline & $14 / 26(54 \%)$ & 13 & 0 & 0 & 0 & - & 2 & 0 & 0 & 0 & - & 0 & 0 & 0 & 0 & 0 \\
\hline \multicolumn{17}{|c|}{ Isoprene (inhalation) } \\
\hline & $11 / 11(100 \%)$ & 0 & 0 & 0 & 0 & - & 1 & 0 & 0 & 0 & - & 0 & 10 & 0 & 0 & 0 \\
\hline \multicolumn{17}{|c|}{ Chloroprene (inhalation) } \\
\hline & $37 / 46(80 \%)$ & 5 & 0 & 2 & 1 & $\begin{array}{l}\text { ATT } \\
\text { CTT }\end{array}$ & 2 & 0 & 0 & 0 & - & 0 & 22 & 3 & 0 & 0 \\
\hline
\end{tabular}

If the same neoplasm had two point mutations, it was counted as one. 


\section{Appendix L. Chemical Characterization and Generation of Chamber Concentrations}

\section{Table of Contents}

L.1. Procurement and Characterization of Cobalt Metal ......................................................2

L.2. Aerosol Generation and Exposure System ....................................................................

L.3. Aerosol Concentration Monitoring ........................................................................

L.4. Chamber Atmosphere Characterization .........................................................................

\section{Tables}

Table L-1. Summary of Chamber Concentrations in the Two-week Inhalation Studies of Cobalt Metal

Table L-2. Summary of Chamber Concentrations in the Three-month Inhalation Studies of Cobalt Metal

Table L-3. Summary of Chamber Concentrations in the Two-year Inhalation Studies of Cobalt Metal

Table L-4. Summary of Aerosol Size Measurements for the Rat and Mouse Exposure Chambers in the Two-week Inhalation Studies of Cobalt Metal....

Table L-5. Summary of Aerosol Size Measurements for the Rat and Mouse Exposure Chambers in the Three-month Inhalation Studies of Cobalt Metal.

Table L-6. Summary of Aerosol Size Measurements for the Rat Exposure Chambers in the Two-year Inhalation Study of Cobalt Metal

Table L-7. Summary of Aerosol Size Measurements for the Mouse Exposure Chambers in the Two-year Inhalation Study of Cobalt Metal....

\section{Figures}

Figure L-1. X-ray Diffraction Pattern of Cobalt Metal

Figure L-2. Schematic of the Aerosol Generation and Delivery System in the Two-week Inhalation Studies of Cobalt Metal.

Figure L-3. Schematic of the Aerosol Generation and Delivery System in the Threemonth and Two-year Inhalation Studies of Cobalt Metal

Figure L-4. Schematic of the Linear Feed Generator in the Fill Position Used in the Three-month and Two-year Inhalation Studies of Cobalt Metal 


\section{L.1. Procurement and Characterization of Cobalt Metal}

Cobalt metal was produced by OMG Kokkola Chemicals Oy (Kokkola, Finland) and was provided by the Cobalt Development Institute via PEL Technologies in one lot (P32 3040-1) that was used in the 2-week, 3-month, and 2-year studies. Identity and purity analyses were performed by the study laboratory at Battelle Toxicology Northwest [Richland, WA; inductively coupled plasma/atomic emission spectroscopy (ICP/AES) analysis] and by the analytical chemistry laboratories at Pacific Northwest National Laboratory [Richland, WA; X-ray diffraction (XRD) and proton-induced X-ray emission (PIXE) analyses], AMIA Laboratories (The Woodlands, TX; XRD using Rietveld analysis), H\&M Analytical Services, Inc. (Allentown, NJ; XRD with and without Rietveld analysis), Elemental Analysis, Inc. (Lexington, KY; PIXE), and Galbraith Laboratories (Knoxville, TN; coulometry for total carbon). Reports on analyses performed in support of the cobalt metal studies are on file at the National Institute of Environmental Health Sciences.

Lot P32 3040-1 of the chemical, a silver-gray powder, was identified as cobalt metal by the analytical chemistry laboratories using XRD. XRD patterns were consistent with library reference patterns (Joint Center for Powder Diffraction Studies/International Centre for Diffraction Data) for cubic and hexagonal phases of cobalt; Rietveld analysis indicated two crystalline forms of cobalt metal, cubic at $13.9 \%$ and hexagonal at $85.9 \%$, and cobalt oxide at $0.2 \%$. A representative XRD pattern is presented in Figure L-1.

The purity of lot P32 3040-1 was determined by the analytical chemistry laboratories by determination of the carbon content using combustion/coulometric analysis by induction furnace (Leco Corporation, St. Joseph, MI) with a carbon dioxide coulometer (Coulometrics, Inc., Wheat Ridge, $\mathrm{CO}$ ) and by PIXE analyses using system A to determine the presence of cobalt metal and trace element impurities with atomic numbers from 11 (sodium) to 53 (iodine) or 92 (uranium). The study laboratory quantitated the purity of the bulk chemical using ICP/AES by system B.

A) The PIXE systems included the use of purchased elemental standards, a $2.5 \mathrm{MeV} \mathrm{H}^{+}$ion beam with an incident angle of $45^{\circ}$ and an exit angle of $39^{\circ}$, a $160 \mu \mathrm{m}$ thick graphite disk attenuator, a $0.05 \mu \mathrm{C}$ charge, and a beam current of $0.2 \mathrm{nA}$.

B) For ICP/AES, samples were dissolved in trace metal grade concentrated $\mathrm{HCl}: \mathrm{HNO}_{3}(1: 1)$ and analyzed for cobalt $(230.786 \mathrm{~nm})$ and yttrium (internal standard) $(224.306 \mathrm{~nm})$. Analyses were performed on a Thermo Elemental IRIS Intrepid Inductively Coupled Plasma-Atomic Emission Spectrometer (Thermo Elemental, Franklin, MA), and the results were normalized against those of cobalt reference standards obtained from the National Institute of Standards and Technology (NIST).

For lot P32 3040-1, the carbon content was $0.09 \% \pm 0.01 \%$. PIXE analysis by system A indicated trace elements of aluminum, sulfur, calcium, chromium, and iron. Chromium was consistently current at approximately $84 \mathrm{ppm}$; the other impurities were below the minimum detection limits. ICP/AES analysis by system B indicated a purity of $98.2 \% \pm 0.6 \%$ relative to a NIST standard reference material [(SRM); cobalt SRM 3113, Gaithersburg, MD]. The overall purity of cobalt metal was determined to be greater than $98 \%$. 
To ensure stability, the bulk chemical was stored at room temperature in safety-coated amber glass containers with Teflon ${ }^{\circledR}$-lined caps under a nitrogen headspace. Periodic reanalyses of the bulk chemical were performed by the study laboratory using ICP/AES by system B; no degradation of the bulk chemical was detected.

\section{L.2. Aerosol Generation and Exposure System}

Schematic diagrams of the cobalt metal generation and distribution systems used during the 2week studies and the 3-month and 2-year studies are shown in Figure L-2 and Figure L-3, respectively. During the 2-week studies, an auger feed device (Tuf-Flex, Model 102 powder feeder; Schenk AccuRate, Inc., Whitewater, WI) was used to meter cobalt metal into a Trost jet mill (Garlock, Inc., Newtown, PA) for aerosolization and particle size reduction. For the 3-month and 2-year studies, the generation system used a linear feed device (Figure L-4) designed and built by Battelle to meter cobalt metal into the jet mill. The linear feed device consisted of a slide bar, a body, a delivery tube, and a test article reservoir. A motor-driven brush (not shown in Figure L-3 and Figure L-4), mounted above and extending into the reservoir, gently rotated and continually stirred the cobalt metal held within the reservoir; this action aided the metering port filling process.

The compressed air driven slide bar slid back and forth during generation. As the slide bar moved to the filling position, the metering port on the shuttle bar was aligned with the reservoir opening and was filled with a small metered amount of test article. A stainless steel screen at the bottom of the metering port held the material within the port. A slight vacuum was applied to the metering port to assist with filling. As the slide bar moved to the dispersing position, the metering port was aligned with a compressed air port in the body. A puff of air from the port dispersed the test article from the metering port. The output of the linear feeder was regulated by adjusting the shuttle bar cadence.

Initial particle size reduction was accomplished within the Trost jet mill. Opposing nitrogen and air gas streams drove the jet mill. All components of the generation system were housed within a glove box located within the exposure control center.

From the jet mill, aerosol was directed to the main distribution line where it was diluted with humidified air then conveyed from the exposure control center to the exposure room where it passed through a cyclone separator to further reduce particle size. On exiting the cyclone, the aerosol-laden air was directed to either of two smaller branch lines. The main distribution and branch lines were made of stainless steel, bonded and grounded to prevent the buildup of electrostatic charge. The distribution line pressure was continuously monitored and maintained slightly negative to the exposure room.

From the branch line, aerosol was delivered to each exposure chamber by a sampling tube. The flow through the sampling tube was induced by a stainless steel ejector pump designed and fabricated at Battelle. The flow rate and configuration of the ejector pumps were chosen to optimize the efficiency of the delivery system. The aerosol then entered the chamber inlet duct where it was further diluted with conditioned chamber air to achieve the desired exposure concentration. 
During exposure periods, there was a small excess of aerosol in each branch line over that needed to maintain chamber concentrations. This additional aerosol was available for making adjustments to the chamber aerosol delivery flow rates and was controlled using house vacuum regulated by a filter-protected flow meter. A second flow control system was available during off-exposure periods. This system consisted of a vacuum transducer pump (Air-Vac Engineering Company, Inc., Seymour, CT) of higher flow capacity positioned in parallel with each branch line flow meter control assembly that became operational only during critical shut-down periods. This backup pump was intended to create sufficient vacuum in the branch line to overcome the negative pressure in the chambers and prevent the flow of aerosol-laden air from the branch line to the chambers as the air supply to each chamber ejector pump was shut off. A high-efficiency particulate air (HEPA) filter was placed before the endline flow control assembly of each branch to remove aerosol from the airstream prior to exhausting from the room.

The study laboratory designed the inhalation exposure chambers (Lab Products, Inc., Seaford, DE) so that uniform aerosol concentrations could be maintained throughout the chambers with the catch pans in place. The total volume of the chamber was $2.3 \mathrm{~m}^{3}$ with an active mixing volume of $1.7 \mathrm{~m}^{3}$. There were three levels of caging, each level split into two tiers that were offset from each other and from the chamber walls. Drawer-like stainless steel cage units composed of individual animal cages were suspended in the space above each tier. Stainless steel catch pans for the collection of urine and feces were suspended below each cage unit.

Incoming air that contained a uniform mixture of test chemical was diverted so that it flowed vertically along the inner surfaces of the chambers. Eddies were formed at each tier as the aerosol flowed past the catch pans. Stagnant zones that would normally exist above each pair of catch pans were cleared by exhaust flow through the space between the tiers. Aerosol reaching the lowest level was deflected across the bottom tiers by metal strips in the space between the catch pan and the wall. Tests showed that aerosol concentration could be reliably maintained homogenous within $8 \%$ throughout the chambers, provided the aerosol was uniformly mixed before passing through the chamber inlet and provided the test material did not react to a significant extent with animals, animal excrement, or the chamber interior ${ }^{164}$.

\section{L.3. Aerosol Concentration Monitoring}

Summaries of the chamber aerosol concentrations are given in Table L-1 through Table L-3. The concentration of cobalt metal in the exposure chambers and room air was monitored using three real-time aerosol monitors (RAMs) (Model RAM-1; MIE, Inc., Bedford, MA). The monitors were connected to the chambers by a sampling system designed by Battelle incorporating a valve that multiplexed each RAM to a $0 \mathrm{mg} / \mathrm{m}^{3}$ chamber or the room, a HEPA-filtered room air blank, and two exposure chambers. The output (voltage) of the RAM was recorded by a program designed by Battelle (Battelle Exposure Data Acquisition and Control) to select the correct sample stream and acquire a raw voltage signal from each RAM. Equations for the calibration curves resided within the program and were used to convert the measured RAM voltages to exposure chamber concentrations. Concentration control limits within the program were compared to each measured concentration and, if limits were exceeded, an audible alarm was triggered or, in extreme cases, exposure was terminated. 
Each RAM was calibrated by constructing a response curve using the measured RAM voltages (voltage readings were corrected by subtracting the RAM zero-offset voltage from measured RAM voltages) and cobalt metal concentrations that were determined by analyzing tandem Pallflex TX40HI20WW (Pall Corporation, Ann Arbor, MI) Teflon ${ }^{\circledR}$-coated, glass-fiber filters collected daily from the exposure chambers. Cobalt was extracted from the filters with 1:1 $\mathrm{HCl}: \mathrm{HNO}_{3}$ and analyzed using ICP/AES by system $\mathrm{B}$.

The ICP/AES instrument was calibrated against serially diluted NIST-traceable $10 \mathrm{mg} / \mathrm{mL}$ spectrometric standards of cobalt and the internal standard yttrium. Quality control standards and a reagent blank were analyzed after calibration, after approximately every tenth sample, and at the end of the analysis to determine accuracy and calibration drift during analysis.

\section{L.4. Chamber Atmosphere Characterization}

Particle size distribution was determined once prior to the 3-month and 2-year studies, once during the 2-week studies, twice during the 3-month studies, and monthly during the 2-year studies. Impactor samples were taken from each exposure chamber using a Mercer-style sevenstage impactor (In-Tox Products, Moriarty, NM) and the stages (glass coverslips lightly coated with silicone to prevent particle bounce) were analyzed using ICP/AES by system B after cobalt was extracted from the slides with 1:1 $\mathrm{HCl}: \mathrm{HNO}_{3}$. The relative mass collected on each stage was analyzed by the CASPACT impactor analysis program developed at Battelle based on probit analysis $^{165}$. The resulting estimates of the mass median aerodynamic particle diameter and the geometric standard deviation of each set of samples are given in Table L-4 through Table L-7. All samples were within the 1 to $3 \mu \mathrm{m}$ range required by the protocol.

Buildup and decay rates for chamber aerosol concentrations were determined with and without animals present in the chambers. At a chamber airflow rate of 15 air changes per hour, the theoretical value for the time to achieve $90 \%$ of the target concentration after the beginning of aerosol generation $\left(\mathrm{T}_{90}\right)$ and the time for the chamber concentration to decay to $10 \%$ of the target concentration after aerosol generation was terminated $\left(\mathrm{T}_{10}\right)$ was approximately 9.4 minutes. For rats and mice in the 2-week studies, T90 values ranged from 9 to 11 minutes with animals present; $T_{10}$ values ranged from 7 to 10 minutes. For rats and mice in the 3 -month studies, $T_{90}$ values ranged from 10 to 14 minutes without animals present and from 11 to 14 minutes with animals present; $\mathrm{T}_{10}$ values ranged from 8 to 10 minutes without animals and were 9 minutes with animals present. For rats in the 2-year studies, T90 values ranged from 10 to 13 minutes without animals present and from 9 to 10 minutes with animals present; $\mathrm{T}_{10}$ values ranged from 7 to 10 minutes without animals present and from 9 to 10 minutes with animals present. For mice, T90 values ranged from 10 to 13 minutes without animals present and from 11 to 13 minutes with animals present; $T_{10}$ values ranged from 6 to 9 minutes without animals present and from 9 to 10 minutes with animals present. A T90 value of 12 minutes was selected for all studies.

The uniformity of aerosol concentration in the inhalation exposure chambers without animals was evaluated before the 3-month and 2-year studies began; in addition, concentration uniformity with animals present in the chambers was measured once during the 2-week and 3month studies and every 3 to 4 months during the 2-year studies. Aerosol concentrations were measured using the on-line monitor with the stream-selection valve fixed in one position to allow continuous monitoring from a single input line. Concentrations were measured at 12 chamber 
sample ports; one in front and one in back for each of six possible cage unit positions per chamber. Chamber concentration uniformity was maintained throughout the studies.

The persistence of cobalt metal in the exposure chambers after aerosol delivery ended was determined by monitoring the concentration overnight in the $40 \mathrm{mg} / \mathrm{m}^{3}$ rat and mouse chambers in the 2 -week studies, the $5 \mathrm{mg} / \mathrm{m}^{3}$ rat and $10 \mathrm{mg} / \mathrm{m}^{3}$ mouse chambers in the 3 -month studies, and the $5 \mathrm{mg} / \mathrm{m}^{3}$ rat and mouse chambers in the 2-year studies, with and without (except for the 2 -week studies) animals present in the chambers. The average cobalt metal concentration decreased to $1 \%$ of the target concentration within 15 (2-week studies), 17 to 18 (3-month studies), or 19 (2-year studies) minutes.

Stability studies of the test material in the generation and exposure system were performed before and during the studies by the study laboratory and the analytical chemistry laboratories. Before the start of each study, a cobalt powder sample was taken from the aerosol distribution line using a Gore-Tex lined polyester filter bag (Sturtevant, Inc., Hanover, MA). Before (except for the 2-week studies) and during each study, microporous filters were collected from the 2.5 and $40 \mathrm{mg} / \mathrm{m}^{3}$ (2-week studies), 0.625, 5, and 10 (mice only) $\mathrm{mg} / \mathrm{m}^{3}$ (3-month studies), or 1.25 and $5 \mathrm{mg} / \mathrm{m}^{3}$ (2-year studies) exposure chambers and the aerosol distribution line. All microporous filters were obtained from Pall Corporation; samples for all XRD and ICP/AES analyses were collected on $25 \mathrm{~mm} \mathrm{~A}$ /E glass-fiber filters ( $1 \mu \mathrm{m}$ pore size:330 $\mu \mathrm{m}$ thickness $)$ and those for PIXE analyses were collected on $25 \mathrm{~mm}$ Zefluor $^{\mathrm{TM}}$ polytetrafluoroethylene filters ( $1 \mu \mathrm{m}$ pore size: $165 \mu \mathrm{m}$ thickness) for the 3-month studies or $25 \mathrm{~mm}$ GH Polypro filters for the 2-year studies. On each sample collection day, a sample of the bulk cobalt metal was collected before filling the reservoir, and a sample of the test article from the generator reservoir was collected at the end of the generation day; additional test material was added to the generator each day. In-system test article stability was assessed using multiple but similar XRD systems as well as PIXE by system A and ICP/AES by system B. The XRD systems included a Siemens D5000 ${ }^{\Theta / \Theta}$ (Siemens, Munich, Germany) or Philips PW1800 or 3020 (Panalytical, Inc., Westborough, PA) diffractometer using $\mathrm{Cu}$ radiation at 40 to $50 \mathrm{KV} / 30$ to $300 \mathrm{~mA}$. Angular ranges varied from $15^{\circ}, 20^{\circ}, 30^{\circ}$, or $35^{\circ}$ to $65^{\circ}, 70^{\circ}, 78^{\circ}, 80^{\circ}$, or $90^{\circ}$; stepsizes were $0.01^{\circ}, 0.02^{\circ}$, or $0.05^{\circ}$, and counting times were $1.2,4.8,10,70$, or 100 seconds.

For the 2-week studies, XRD analyses of the bulk chemical, filter samples, and material from the generator reservoir indicated two primary crystal forms of cobalt, cubic and hexagonal, with no detection of known cobalt oxides. XRD with Rietveld analysis of samples from the distribution line, bulk chemical, and generator reservoir indicated the presence of two crystal forms of cobalt, approximately $15 \%$ to $18 \%$ cubic and $78 \%$ to $79 \%$ hexagonal, and approximately $4 \%$ to $5 \%$ cubic cobalt oxide. Due to the unexpected presence of significant amounts of cobalt oxide in these samples, they were reanalyzed using XRD with standards containing cobalt metal as well as cobalt oxide $(\mathrm{CoO})$ added at $0 \%, 1 \%$, or $2 \%$ by weight. No cobalt oxide was detected in the distribution line or bulk chemical samples; cobalt oxide was detected in the generator reservoir sample, but it was less than $1 \%$ relative to the cobalt oxide standard. Bulk chemical, generator reservoir, and filter samples were analyzed using ICP/AES by system B to determine if inorganic impurities were introduced into the test atmosphere by the exposure generation system. Samples were analyzed for aluminum, arsenic, beryllium, cadmium, chromium, cobalt, copper, iron, manganese, molybdenum, nickel, lead, and tin. All measured trace element impurities were present at less than $0.05 \%$ by weight relative to cobalt. 
Before the 3-month studies, XRD analyses indicated two crystal forms of cobalt (cubic and hexagonal) in all analyzed samples. Cobalt oxides were not detected and were less than $1 \%$ in the powder and filter samples relative to cobalt oxide standards. XRD coupled with Rietveld analysis of similar samples collected during the 3-month studies with animals in the exposure chambers detected cobalt oxide $\left(\mathrm{Co}_{3} \mathrm{O}_{4}\right)$ at approximately $1.6 \%$ and $1.7 \%$, respectively, with an uncertainty of approximately $\pm 2 \%$. Comparison of the XRD patterns of these samples relative to those of cobalt oxide standards analyzed at the same time indicated less than $1 \% \mathrm{CoO}$ and less than $1 \% \mathrm{Co}_{3} \mathrm{O}_{4}$. No cobalt oxides were detected in the filter samples.

Before the 3-month studies, PIXE analysis for trace elements using system A indicated no inorganic impurities in the distribution line or exposure chamber filter samples or in the generator reservoir bulk samples. In similar PIXE analyses conducted during the 3-month studies with animals in the exposure chambers, no inorganic impurities were detected in the bulk chemical samples from the generator reservoir. Relative to cobalt concentrations in the same samples, chromium was detected in the aerosol distribution line and the 0.625 and $5 \mathrm{mg} / \mathrm{m}^{3}$ exposure chamber filter samples at levels of $0.13 \%, 0.21 \%$, and $0.11 \%$, respectively, and iron was detected in the $0.625 \mathrm{mg} / \mathrm{m}^{3}$ chamber filter sample at a level of $0.25 \%$.

Before the 2-year studies, XRD with Rietveld analysis indicated the presence of two crystal forms of cobalt in all of the analyzed samples; cubic at $14 \%$ to $25 \%$ and hexagonal at $74 \%$ to $84 \%$. Cobalt oxide $\left(\mathrm{Co}_{3} \mathrm{O}_{4}\right)$ was detected at $1 \%$ to $2 \%$ in the bulk and generator samples and at $2 \%$ to $3 \%$ in the chamber and distribution line filter samples (with an uncertainty of approximately $\pm 2 \%$ ). Comparison of the XRD patterns of these samples to those of cobalt oxide standards analyzed at the same time indicated less than $1 \% \mathrm{CoO}$ and less than $1 \% \mathrm{Co}_{3} \mathrm{O}_{4}$. Powder (bulk and generator reservoir) and filter (exposure chamber and distribution line) samples were collected for PIXE analyses for trace element impurities using system A; analyses detected chromium in three filter samples (distribution line, $5 \mathrm{mg} / \mathrm{m}^{3}$ rat chamber, and $1.25 \mathrm{mg} / \mathrm{m}^{3}$ mouse chamber) and chlorine in one filter sample $\left(1.25 \mathrm{mg} / \mathrm{m}^{3}\right.$ mouse chamber $)$ at less than $0.1 \%$ relative to cobalt. An additional set of powder (bulk, generator reservoir, and distribution line) samples were collected and similarly analyzed by PIXE; chlorine was detected in all three of these powder samples and silicon was detected in the powder from the distribution line at less than $0.1 \%$ relative to cobalt.

At the beginning of the 2-year studies, XRD with Rietveld analysis indicated the presence of two crystal forms of cobalt in all of the analyzed samples, cubic at approximately $27 \%$ to $31 \%$ and hexagonal at $66 \%$ to $72 \%$. Cobalt oxide $\left(\mathrm{Co}_{3} \mathrm{O}_{4}\right)$ was detected at approximately $2 \%$ and $3 \%$ (with an uncertainty of approximately $\pm 3 \%$ ) in the bulk and generator reservoir samples and chamber and distribution line filter samples, respectively. Comparison of the XRD patterns of these samples to those of cobalt oxide standards analyzed at the same time indicated less than $1 \% \mathrm{CoO}$ and less than $1 \% \mathrm{Co}_{3} \mathrm{O}_{4}$. PIXE analysis for trace element impurities by system $\mathrm{A}$ indicated the presence of silicon in the generator reservoir powder samples at $0.4 \%$ relative to cobalt. The bulk and generator reservoir samples were reanalyzed using ICP/AES by system $\mathrm{B}$ following microwave acid digestion in $\mathrm{HNO}_{3} / \mathrm{HCl} / \mathrm{HF}$. The following element impurities were detected at less than $0.1 \%$ relative to cobalt: silicon in the bulk and generator powder samples, chlorine in all powder and filter samples, vanadium in the $5 \mathrm{mg} / \mathrm{m}^{3}$ mouse chamber filter sample, and potassium and chromium in the $1.25 \mathrm{mg} / \mathrm{m}^{3}$ rat chamber filter sample. 
XRD reanalyses of test chemical stability in the exposure system were performed at 1year and at the end of the 2-year studies. Two crystal forms of cobalt were present in all analyzed samples, cubic at $21 \%$ to $24 \%$ and hexagonal at $73 \%$ to $78 \%$. Cobalt oxide $\left(\mathrm{Co}_{3} \mathrm{O}_{4}\right)$ was detected in the bulk and generator samples at approximately $2 \%$ to $3 \%$ and in the chamber and distribution line filter samples at approximately $2 \%$ to $5 \%$ with an uncertainty of approximately $\pm 2 \%$;

comparison of the XRD patterns of these samples to those of cobalt oxide standards analyzed at the same time indicated less than $1 \% \mathrm{CoO}$ and less than $1 \% \mathrm{Co}_{3} \mathrm{O}_{4}$. 
Table L-1. Summary of Chamber Concentrations in the Two-week Inhalation Studies of Cobalt Metal

\begin{tabular}{|c|c|c|}
\hline Target Concentration $\left(\mathrm{mg} / \mathrm{m}^{3}\right)$ & Total Number of Readings & Average Concentration ${ }^{\mathrm{a}}\left(\mathrm{mg} / \mathrm{m}^{3}\right)$ \\
\hline \multicolumn{3}{|l|}{ Rat Chambers } \\
\hline 2.5 & 134 & $2.5 \pm 0.2$ \\
\hline 5 & 131 & $4.9 \pm 0.4$ \\
\hline 10 & 134 & $9.7 \pm 1.1$ \\
\hline 20 & 131 & $19.7 \pm 2.0$ \\
\hline 40 & 57 & $40.1 \pm 3.4$ \\
\hline \multicolumn{3}{|l|}{ Mouse Chambers } \\
\hline 2.5 & 145 & $2.5 \pm 0.2$ \\
\hline 5 & 141 & $4.9 \pm 0.4$ \\
\hline 10 & 145 & $9.7 \pm 1.3$ \\
\hline 20 & 141 & $19.6 \pm 2.0$ \\
\hline 40 & 145 & $40.1 \pm 4.2$ \\
\hline
\end{tabular}

${ }^{\mathrm{a}}$ Mean \pm standard deviation.

Table L-2. Summary of Chamber Concentrations in the Three-month Inhalation Studies of Cobalt Metal

Target Concentration $\left(\mathbf{m g} / \mathbf{m}^{3}\right)$

Rat Chambers

$\begin{array}{cc}0.625 & 760 \\ 1.25 & 750 \\ 2.5 & 760 \\ 5 & 749\end{array}$

Mouse Chambers

\begin{tabular}{cc}
0.625 & 782 \\
1.25 & 772 \\
2.5 & 782 \\
5 & 771 \\
10 & 782 \\
\hline${ }^{a}$ Mean \pm standard deviation. &
\end{tabular}

${ }^{\mathrm{a}}$ Mean \pm standard deviation.
Total Number of Readings

Average Concentration ${ }^{\mathrm{a}}\left(\mathrm{mg} / \mathrm{m}^{3}\right)$

$$
\begin{gathered}
0.61 \pm 0.04 \\
1.23 \pm 0.07 \\
2.5 \pm 0.1 \\
5.0 \pm 0.3
\end{gathered}
$$

$10.0 \pm 0.5$ 
Table L-3. Summary of Chamber Concentrations in the Two-year Inhalation Studies of Cobalt Metal

\begin{tabular}{|c|c|c|}
\hline Target Concentration $\left(\mathrm{mg} / \mathrm{m}^{3}\right)$ & Total Number of Readings & Average Concentration ${ }^{\mathrm{a}}\left(\mathrm{mg} / \mathrm{m}^{3}\right)$ \\
\hline \multicolumn{3}{|l|}{ Rat Chambers } \\
\hline 1.25 & 5,644 & $1.24 \pm 0.07$ \\
\hline 2.5 & 5,647 & $2.50 \pm 0.10$ \\
\hline 5 & 5,707 & $5.01 \pm 0.18$ \\
\hline \multicolumn{3}{|l|}{ Mouse Chambers } \\
\hline 1.25 & 5,723 & $1.24 \pm 0.06$ \\
\hline 2.5 & 5,719 & $2.49 \pm 0.11$ \\
\hline 5 & 5,664 & $5.01 \pm 0.20$ \\
\hline
\end{tabular}

${ }^{\mathrm{a} M e a n} \pm$ standard deviation.

Table L-4. Summary of Aerosol Size Measurements for the Rat and Mouse Exposure Chambers in the Two-week Inhalation Studies of Cobalt Metal

\begin{tabular}{ccc}
\hline Target Concentration $\left(\mathbf{m g} / \mathbf{m}^{\mathbf{3}}\right)$ & Mass Median Aerodynamic Diameter $(\boldsymbol{\mu m})$ & Geometric Standard Deviation \\
\hline 2.5 & 1.86 & 1.76 \\
5 & 1.79 & 1.73 \\
10 & 1.92 & 1.74 \\
20 & 1.94 & 1.77 \\
40 & 1.92 & 1.79 \\
\hline
\end{tabular}

Table L-5. Summary of Aerosol Size Measurements for the Rat and Mouse Exposure Chambers in the Three-month Inhalation Studies of Cobalt Metal

\begin{tabular}{cccc}
\hline Date of Test & $\begin{array}{c}\text { Target Concentration } \\
\left(\mathbf{m g} / \mathbf{m}^{3}\right)\end{array}$ & $\begin{array}{c}\text { Mass Median Aerodynamic } \\
\text { Diameter }(\boldsymbol{\mu m})\end{array}$ & $\begin{array}{c}\text { Geometric Standard } \\
\text { Deviation }\end{array}$ \\
\hline March 2005 & 0.625 & 1.69 & 1.94 \\
& 1.25 & 1.73 & 1.84 \\
2.5 & 1.84 & 1.76 \\
& 5 & 1.86 & 1.76 \\
April 2005 & $10^{\mathrm{a}}$ & 2.00 & 1.79 \\
& 0.625 & 1.61 & 1.97 \\
& 1.25 & 1.71 & 1.83 \\
& 2.5 & 1.76 & 1.75 \\
May 2005 & 5 & 1.77 & 1.74 \\
& 10 & 1.98 & 1.80 \\
& 0.625 & 1.66 & 1.97 \\
& 1.25 & 1.72 & 1.81 \\
& 2.5 & 1.96 & 1.78 \\
5 & 1.90 & 1.78 \\
\hline
\end{tabular}

${ }^{\mathrm{a} M i c e}$ only. 
Table L-6. Summary of Aerosol Size Measurements for the Rat Exposure Chambers in the Twoyear Inhalation Study of Cobalt Metal

\begin{tabular}{|c|c|c|c|}
\hline Date of Test & $\begin{array}{c}\text { Target Concentration } \\
\left(\mathbf{m g} / \mathbf{m}^{3}\right)\end{array}$ & $\begin{array}{c}\text { Mass Median Aerodynamic } \\
\text { Diameter }(\mu \mathrm{m})\end{array}$ & $\begin{array}{c}\text { Geometric Standard } \\
\text { Deviation }\end{array}$ \\
\hline \multirow[t]{3}{*}{ May 2006} & 1.25 & 1.7 & 1.7 \\
\hline & 2.5 & 1.8 & 1.7 \\
\hline & 5 & 2.0 & 1.7 \\
\hline \multirow[t]{3}{*}{ June 2006} & 1.25 & 1.6 & 1.7 \\
\hline & 2.5 & 1.7 & 1.7 \\
\hline & 5 & 1.7 & 1.7 \\
\hline \multirow[t]{3}{*}{ July 2006} & 1.25 & 1.6 & 1.9 \\
\hline & 2.5 & 1.7 & 1.7 \\
\hline & 5 & 1.8 & 1.7 \\
\hline \multirow[t]{3}{*}{ August 2006} & 1.25 & 1.7 & 1.7 \\
\hline & 2.5 & 1.9 & 1.7 \\
\hline & 5 & 1.8 & 1.7 \\
\hline \multirow[t]{3}{*}{ September 2006} & 1.25 & 1.7 & 1.7 \\
\hline & 2.5 & 1.8 & 1.7 \\
\hline & 5 & 1.9 & 1.7 \\
\hline \multirow[t]{3}{*}{ October 2006} & 1.25 & 1.7 & 1.7 \\
\hline & 2.5 & 2.0 & 1.7 \\
\hline & 5 & 1.8 & 1.7 \\
\hline \multirow[t]{3}{*}{ November 2006} & 1.25 & 1.7 & 1.7 \\
\hline & 2.5 & 1.8 & 1.6 \\
\hline & 5 & 1.9 & 1.7 \\
\hline \multirow[t]{3}{*}{ December 2006} & 1.25 & 1.6 & 1.7 \\
\hline & 2.5 & 1.9 & 1.7 \\
\hline & 5 & 1.8 & 1.7 \\
\hline \multirow[t]{3}{*}{ January 2007} & 1.25 & 1.7 & 1.7 \\
\hline & 2.5 & 1.9 & 1.7 \\
\hline & 5 & 1.7 & 1.7 \\
\hline \multirow[t]{3}{*}{ February 2007} & 1.25 & 1.5 & 1.8 \\
\hline & 2.5 & 1.7 & 1.7 \\
\hline & 5 & 1.6 & 1.7 \\
\hline \multirow[t]{3}{*}{ March 2007} & 1.25 & 1.7 & 1.7 \\
\hline & 2.5 & 2.0 & 1.8 \\
\hline & 5 & 1.8 & 1.8 \\
\hline
\end{tabular}




\section{Cobalt Metal, NTP TR 581}

\begin{tabular}{|c|c|c|c|}
\hline Date of Test & $\begin{array}{c}\text { Target Concentration } \\
\left(\mathbf{m g} / \mathrm{m}^{3}\right)\end{array}$ & $\begin{array}{c}\text { Mass Median Aerodynamic } \\
\text { Diameter }(\mu \mathrm{m})\end{array}$ & $\begin{array}{c}\text { Geometric Standard } \\
\text { Deviation }\end{array}$ \\
\hline \multirow[t]{3}{*}{ April 2007} & 1.25 & 1.5 & 1.8 \\
\hline & 2.5 & 1.8 & 1.7 \\
\hline & 5 & 1.7 & 1.7 \\
\hline \multirow[t]{3}{*}{ May 2007} & 1.25 & 1.6 & 1.7 \\
\hline & 2.5 & 1.8 & 1.7 \\
\hline & 5 & 1.7 & 1.7 \\
\hline \multirow[t]{3}{*}{ June 2007} & 1.25 & 1.6 & 1.7 \\
\hline & 2.5 & 1.7 & 1.7 \\
\hline & 5 & 1.7 & 1.7 \\
\hline \multirow[t]{3}{*}{ July 2007} & 1.25 & 1.5 & 1.7 \\
\hline & 2.5 & 1.8 & 1.7 \\
\hline & 5 & 1.7 & 1.7 \\
\hline \multirow[t]{3}{*}{ August 2007} & 1.25 & 1.7 & 1.7 \\
\hline & 2.5 & 1.7 & 1.7 \\
\hline & 5 & 1.8 & 1.7 \\
\hline \multirow[t]{3}{*}{ September 2007} & 1.25 & 1.6 & 1.8 \\
\hline & 2.5 & 1.7 & 1.7 \\
\hline & 5 & 1.7 & 1.7 \\
\hline \multirow[t]{3}{*}{ October 2007} & 1.25 & 1.7 & 1.7 \\
\hline & 2.5 & 1.8 & 1.8 \\
\hline & 5 & 1.8 & 1.7 \\
\hline \multirow[t]{3}{*}{ November 2007} & 1.25 & 1.6 & 1.8 \\
\hline & 2.5 & 1.7 & 1.7 \\
\hline & 5 & 1.6 & 1.8 \\
\hline \multirow[t]{3}{*}{ December 2007} & 1.25 & 1.6 & 1.8 \\
\hline & 2.5 & 1.6 & 1.7 \\
\hline & 5 & 1.8 & 1.7 \\
\hline \multirow[t]{3}{*}{ January 2008} & 1.25 & 1.5 & 1.8 \\
\hline & 2.5 & 1.7 & 1.7 \\
\hline & 5 & 1.7 & 1.7 \\
\hline \multirow[t]{3}{*}{ February 2008} & 1.25 & 1.4 & 1.8 \\
\hline & 2.5 & 1.7 & 1.7 \\
\hline & 5 & 1.7 & 1.7 \\
\hline
\end{tabular}




\section{Cobalt Metal, NTP TR 581}

\begin{tabular}{lccc}
\hline Date of Test & $\begin{array}{c}\text { Target Concentration } \\
\left(\mathbf{m g} / \mathbf{m}^{\mathbf{3}}\right)\end{array}$ & $\begin{array}{c}\text { Mass Median Aerodynamic } \\
\text { Diameter }(\boldsymbol{\mu m})\end{array}$ & $\begin{array}{c}\text { Geometric Standard } \\
\text { Deviation }\end{array}$ \\
\hline March 2008 & 1.25 & 1.5 & 1.7 \\
& 2.5 & 1.7 & 1.7 \\
April 2008 & 5 & 1.7 & 1.7 \\
& 1.25 & 1.6 & 1.9 \\
May 2008 & 2.5 & 1.7 & 1.7 \\
& 5 & 1.8 & 1.8 \\
Range & 1.25 & 1.6 & 1.7 \\
& 2.5 & 1.7 & 1.7 \\
& 5 & 1.8 & 1.7 \\
& 1.25 & $1.4-1.7$ & $1.7-1.9$ \\
\hline
\end{tabular}


Table L-7. Summary of Aerosol Size Measurements for the Mouse Exposure Chambers in the Twoyear Inhalation Study of Cobalt Metal

\begin{tabular}{|c|c|c|c|}
\hline Date of Test & $\begin{array}{c}\text { Target Concentration } \\
\left(\mathbf{m g} / \mathrm{m}^{3}\right)\end{array}$ & $\begin{array}{c}\text { Mass Median Aerodynamic } \\
\text { Diameter }(\mu \mathrm{m})\end{array}$ & $\begin{array}{c}\text { Geometric Standard } \\
\text { Deviation }\end{array}$ \\
\hline \multirow[t]{3}{*}{ May 2006} & 1.25 & 1.8 & 1.7 \\
\hline & 2.5 & 2.1 & 1.7 \\
\hline & 5 & 2.0 & 1.7 \\
\hline \multirow[t]{3}{*}{ June 2006} & 1.25 & 1.7 & 1.7 \\
\hline & 2.5 & 1.7 & 1.7 \\
\hline & 5 & 1.7 & 1.7 \\
\hline \multirow[t]{3}{*}{ July 2006} & 1.25 & 1.7 & 1.7 \\
\hline & 2.5 & 1.8 & 1.7 \\
\hline & 5 & 2.0 & 1.7 \\
\hline \multirow[t]{3}{*}{ August 2006} & 1.25 & 1.7 & 1.7 \\
\hline & 2.5 & 1.8 & 1.7 \\
\hline & 5 & 1.9 & 1.7 \\
\hline \multirow[t]{3}{*}{ September 2006} & 1.25 & 1.7 & 1.7 \\
\hline & 2.5 & 1.9 & 1.6 \\
\hline & 5 & 2.0 & 1.7 \\
\hline \multirow[t]{3}{*}{ October 2006} & 1.25 & 1.7 & 1.7 \\
\hline & 2.5 & 1.8 & 1.7 \\
\hline & 5 & 2.0 & 1.7 \\
\hline \multirow[t]{3}{*}{ November 2006} & 1.25 & 1.6 & 1.7 \\
\hline & 2.5 & 1.8 & 1.7 \\
\hline & 5 & 2.0 & 1.6 \\
\hline \multirow[t]{3}{*}{ December 2006} & 1.25 & 1.7 & 1.7 \\
\hline & 2.5 & 1.6 & 1.7 \\
\hline & 5 & 1.7 & 1.7 \\
\hline \multirow[t]{3}{*}{ January 2007} & 1.25 & 1.7 & 1.7 \\
\hline & 2.5 & 1.7 & 1.7 \\
\hline & 5 & 1.9 & 1.7 \\
\hline \multirow[t]{3}{*}{ February 2007} & 1.25 & 1.7 & 1.7 \\
\hline & 2.5 & 1.9 & 1.7 \\
\hline & 5 & 2.0 & 1.7 \\
\hline \multirow[t]{3}{*}{ March 2007} & 1.25 & 1.7 & 1.7 \\
\hline & 2.5 & 2.0 & 1.7 \\
\hline & 5 & 1.9 & 1.7 \\
\hline
\end{tabular}




\section{Cobalt Metal, NTP TR 581}

\begin{tabular}{|c|c|c|c|}
\hline Date of Test & $\begin{array}{c}\text { Target Concentration } \\
\left(\mathbf{m g} / \mathrm{m}^{3}\right)\end{array}$ & $\begin{array}{c}\text { Mass Median Aerodynamic } \\
\text { Diameter }(\mu \mathrm{m})\end{array}$ & $\begin{array}{c}\text { Geometric Standard } \\
\text { Deviation }\end{array}$ \\
\hline \multirow[t]{3}{*}{ April 2007} & 1.25 & 1.5 & 1.7 \\
\hline & 2.5 & 1.8 & 1.7 \\
\hline & 5 & 1.7 & 1.7 \\
\hline \multirow[t]{3}{*}{ May 2007} & 1.25 & 1.6 & 1.7 \\
\hline & 2.5 & 1.8 & 1.8 \\
\hline & 5 & 1.8 & 1.7 \\
\hline \multirow[t]{3}{*}{ June 2007} & 1.25 & 1.7 & 1.7 \\
\hline & 2.5 & 1.8 & 1.7 \\
\hline & 5 & 1.9 & 1.7 \\
\hline \multirow[t]{3}{*}{ July 2007} & 1.25 & 1.7 & 1.7 \\
\hline & 2.5 & 1.9 & 1.7 \\
\hline & 5 & 1.9 & 1.8 \\
\hline \multirow[t]{3}{*}{ August 2007} & 1.25 & 1.7 & 1.7 \\
\hline & 2.5 & 1.7 & 1.7 \\
\hline & 5 & 1.8 & 1.7 \\
\hline \multirow[t]{3}{*}{ September 2007} & 1.25 & 1.6 & 1.8 \\
\hline & 2.5 & 1.7 & 1.8 \\
\hline & 5 & 1.7 & 1.7 \\
\hline \multirow[t]{3}{*}{ October 2007} & 1.25 & 1.6 & 1.8 \\
\hline & 2.5 & 1.9 & 1.7 \\
\hline & 5 & 1.8 & 1.8 \\
\hline \multirow[t]{3}{*}{ November 2007} & 1.25 & 1.7 & 1.7 \\
\hline & 2.5 & 1.7 & 1.7 \\
\hline & 5 & 1.8 & 1.7 \\
\hline \multirow[t]{3}{*}{ December 2007} & 1.25 & 1.6 & 1.9 \\
\hline & 2.5 & 1.8 & 1.7 \\
\hline & 5 & 1.9 & 1.7 \\
\hline \multirow[t]{3}{*}{ January 2008} & 1.25 & 1.6 & 1.8 \\
\hline & 2.5 & 1.7 & 1.7 \\
\hline & 5 & 1.7 & 1.7 \\
\hline \multirow[t]{3}{*}{ February 2008} & 1.25 & 1.5 & 1.8 \\
\hline & 2.5 & 1.7 & 1.8 \\
\hline & 5 & 1.8 & 1.8 \\
\hline
\end{tabular}


Cobalt Metal, NTP TR 581

\begin{tabular}{lccc}
\hline Date of Test & $\begin{array}{c}\text { Target Concentration } \\
\left(\mathbf{m g} / \mathbf{m}^{3}\right)\end{array}$ & $\begin{array}{c}\text { Mass Median Aerodynamic } \\
\text { Diameter }(\boldsymbol{\mu m})\end{array}$ & $\begin{array}{c}\text { Geometric Standard } \\
\text { Deviation }\end{array}$ \\
\hline March 2008 & 1.25 & 1.7 & 1.8 \\
& 2.5 & 1.8 & 1.8 \\
April 2008 & 5 & 1.9 & 1.7 \\
& 1.25 & 1.5 & 1.8 \\
May 2008 & 2.5 & 1.7 & 1.7 \\
& 5 & 1.8 & 1.7 \\
Range & 1.25 & 1.7 & 1.9 \\
& 2.5 & 1.7 & 1.7 \\
& 5 & 1.7 & 1.8 \\
& 1.25 & $1.5-1.8$ & $1.7-1.9$ \\
& 2.5 & $1.6-2.1$ & $1.6-1.8$ \\
\hline
\end{tabular}

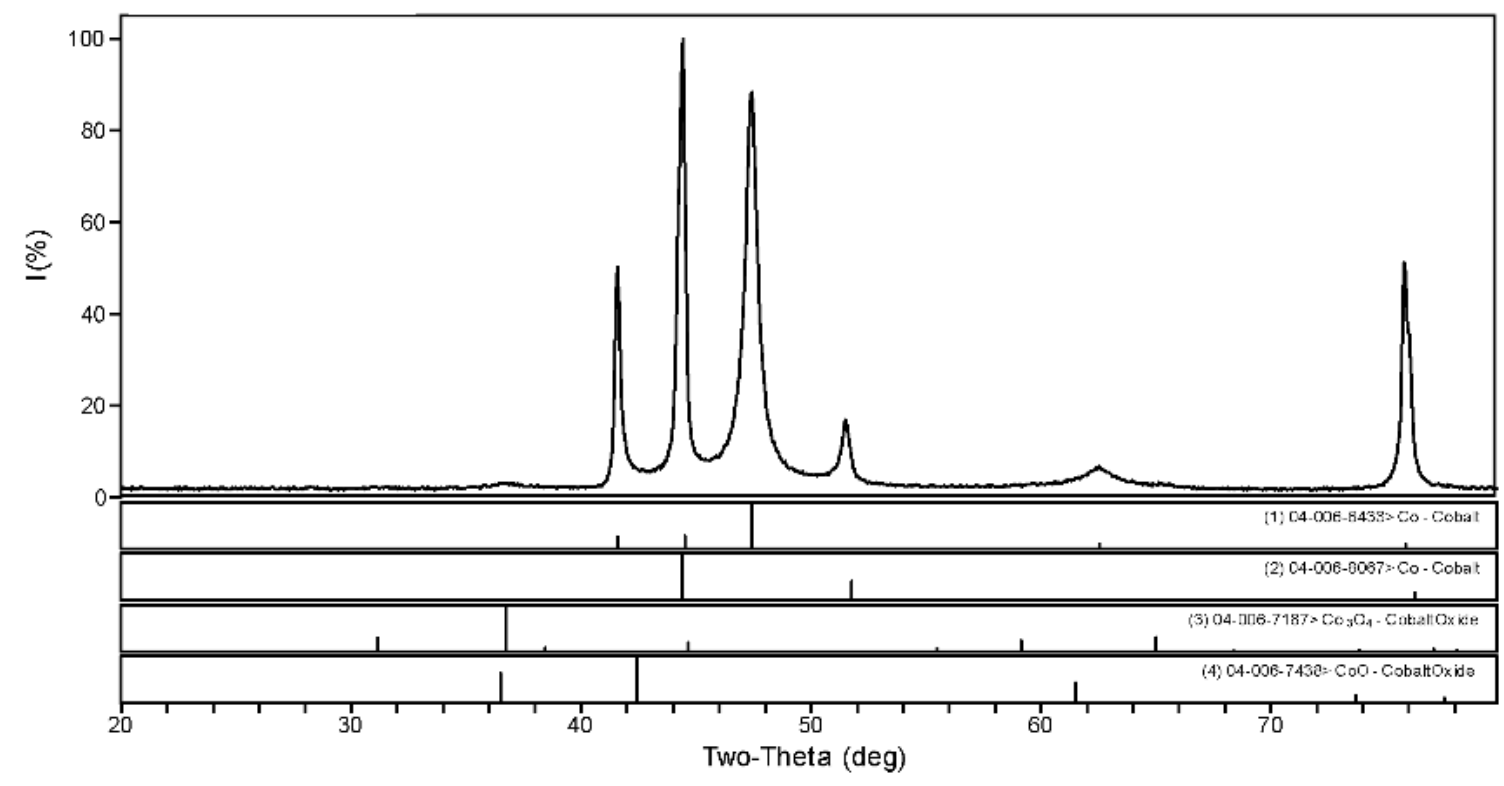

Figure L-1. X-ray Diffraction Pattern of Cobalt Metal 


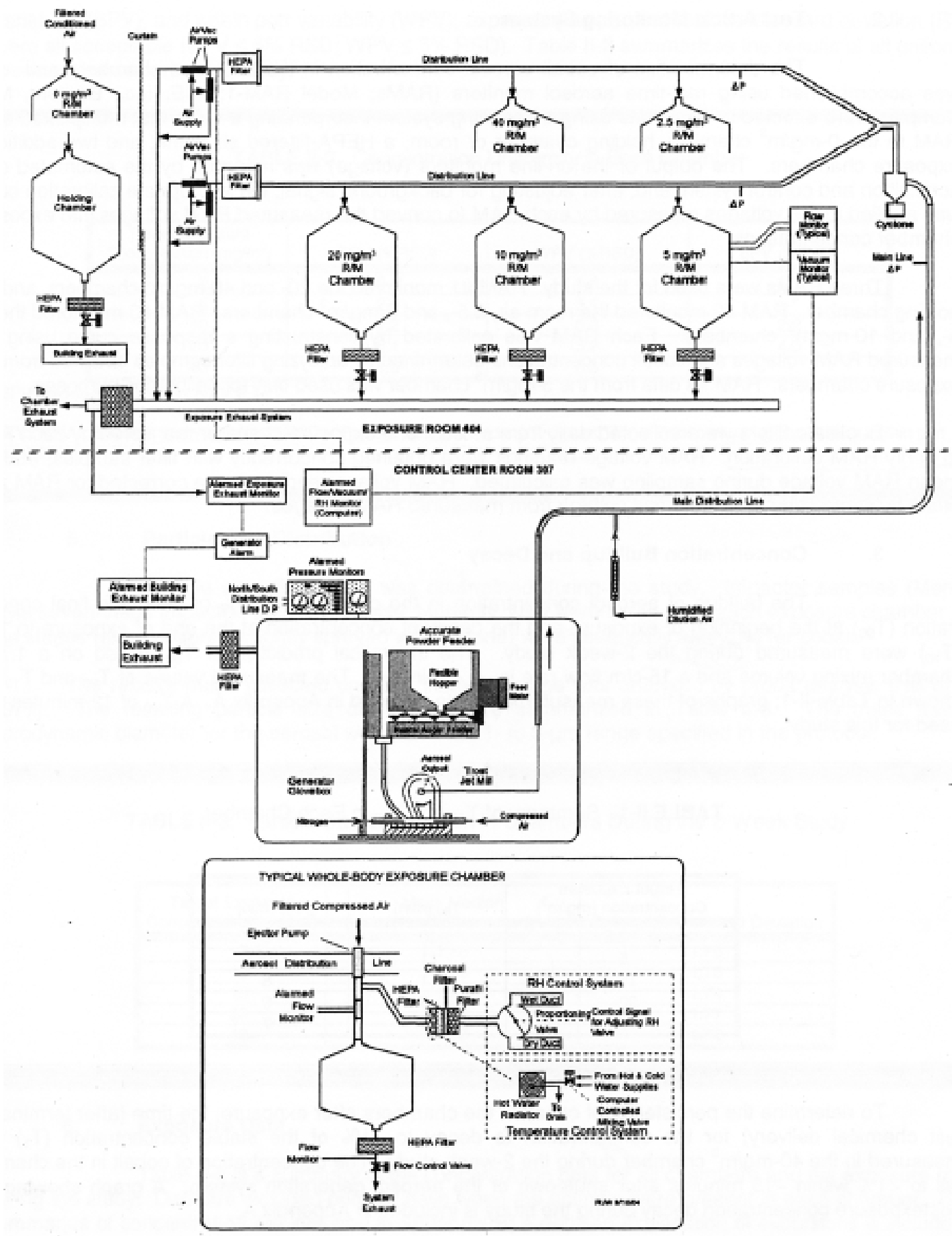

Figure L-2. Schematic of the Aerosol Generation and Delivery System in the Two-week Inhalation Studies of Cobalt Metal 


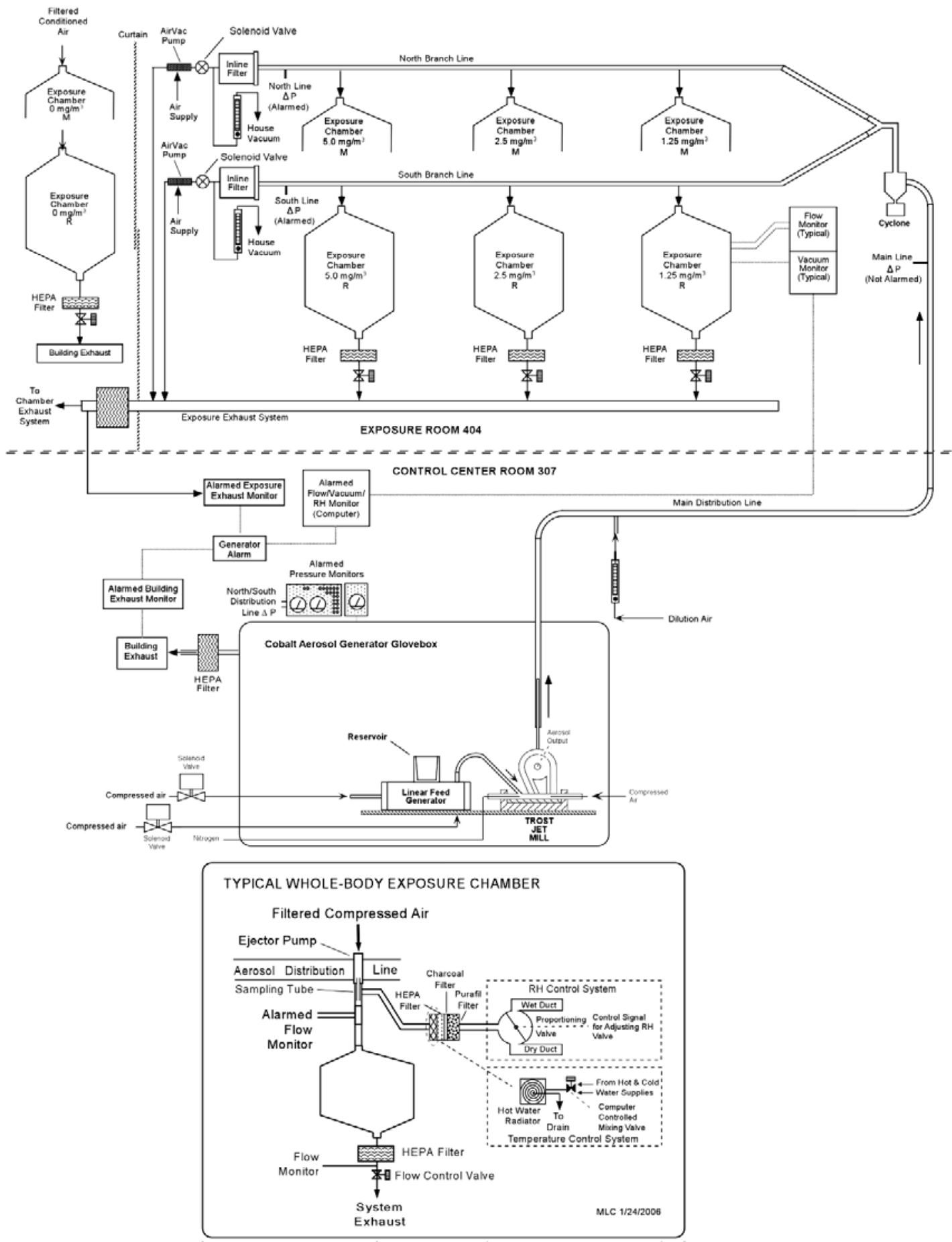

Figure L-3. Schematic of the Aerosol Generation and Delivery System in the Three-month and Two-year Inhalation Studies of Cobalt Metal 
Cobalt Metal, NTP TR 581

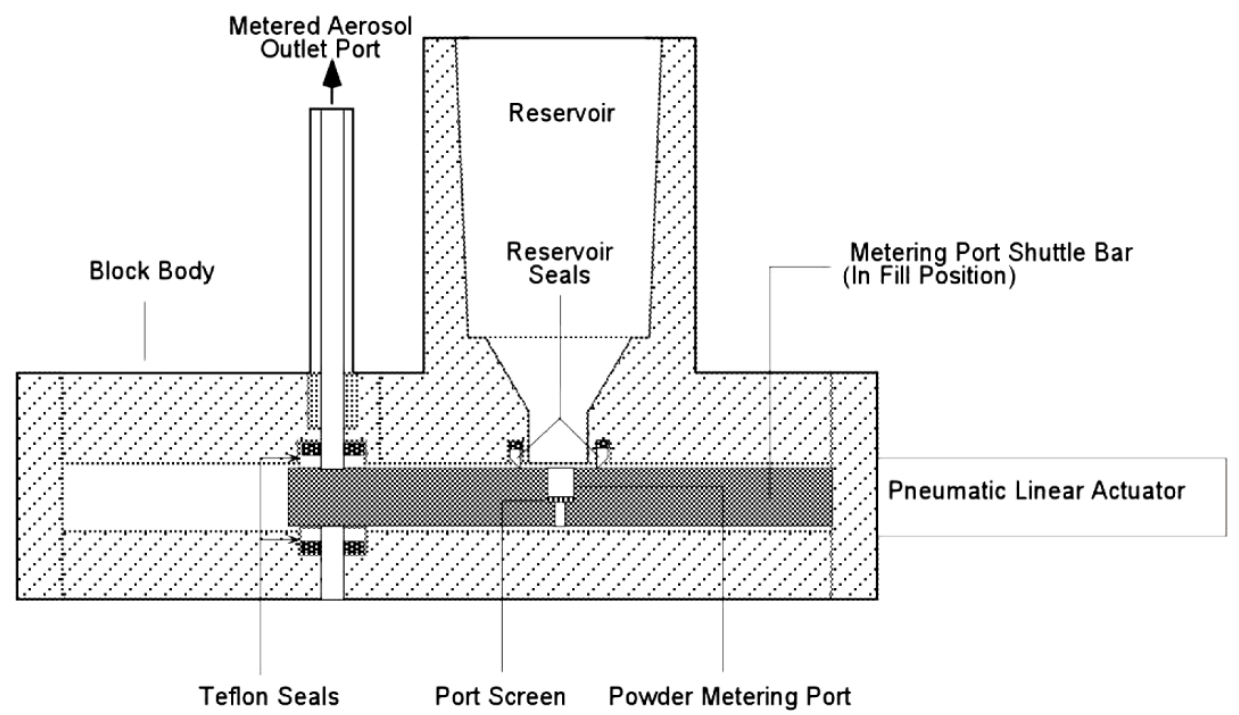

Figure L-4. Schematic of the Linear Feed Generator in the Fill Position Used in the Three-month and Two-year Inhalation Studies of Cobalt Metal 


\section{Appendix M. Ingredients, Nutrient Composition, and Contaminant Levels in NTP-2000 Rat and Mouse Ration}

\section{Tables}

Table M-1. Ingredients of NTP-2000 Rat and Mouse Ration

$\mathrm{M}-2$

Table M-2. Vitamins and Minerals in NTP-2000 Rat and Mouse Ration

Table M-3. Nutrient Composition of NTP-2000 Rat and Mouse Ration M-3

Table M-4. Contaminant Levels in NTP-2000 Rat and Mouse Ration M-4 
Table M-1. Ingredients of NTP-2000 Rat and Mouse Ration

\begin{tabular}{|c|c|c|}
\hline Ingredients & & Percent by Weight \\
\hline Ground hard winter wheat & & 22.26 \\
\hline Ground $\# 2$ yellow shelled corn & & 22.18 \\
\hline Wheat middlings & & 15.0 \\
\hline Oat hulls & & 8.5 \\
\hline Alfalfa meal (dehydrated, $17 \%$ protein) & & 7.5 \\
\hline Purified cellulose & & 5.5 \\
\hline Soybean meal (49\% protein) & & 5.0 \\
\hline Fish meal $(60 \%$ protein $)$ & & 4.0 \\
\hline Corn oil (without preservatives) & & 3.0 \\
\hline Soy oil (without preservatives) & & 3.0 \\
\hline Dried brewer's yeast & & 1.0 \\
\hline Calcium carbonate (USP) & & 0.9 \\
\hline Vitamin premix ${ }^{a}$ & & 0.5 \\
\hline Mineral premix ${ }^{b}$ & & 0.5 \\
\hline Calcium phosphate, dibasic (USP) & & 0.4 \\
\hline Sodium chloride & & 0.3 \\
\hline Choline chloride ( $70 \%$ choline) & & 0.26 \\
\hline Methionine & & 0.2 \\
\hline \multicolumn{3}{|l|}{$\begin{array}{l}\text { a Wheat middlings as carrier. } \\
\text { bCalcium carbonate as carrier. }\end{array}$} \\
\hline \multicolumn{3}{|c|}{ Table M-2. Vitamins and Minerals in NTP-2000 Rat and Mouse Ration ${ }^{\mathrm{a}}$} \\
\hline & Amount & Source \\
\hline \multicolumn{3}{|l|}{ Vitamins } \\
\hline A & 4,000 IU & Stabilized vitamin A palmitate or acetate \\
\hline $\mathrm{D}$ & $1,000 \mathrm{IU}$ & D-activated animal sterol \\
\hline $\mathrm{K}$ & $1.0 \mathrm{mg}$ & Menadione sodium bisulfite complex \\
\hline$\alpha$-Tocopheryl acetate & $100 \mathrm{IU}$ & - \\
\hline Niacin & $23 \mathrm{mg}$ & - \\
\hline Folic acid & $1.1 \mathrm{mg}$ & - \\
\hline$d$-Pantothenic acid & $10 \mathrm{mg}$ & $d$-Calcium pantothenate \\
\hline Riboflavin & $3.3 \mathrm{mg}$ & - \\
\hline Thiamine & $4 \mathrm{mg}$ & Thiamine mononitrate \\
\hline $\mathrm{B}_{12}$ & $52 \mu \mathrm{g}$ & - \\
\hline Pyridoxine & $6.3 \mathrm{mg}$ & Pyridoxine hydrochloride \\
\hline Biotin & $0.2 \mathrm{mg}$ & $d$-Biotin \\
\hline
\end{tabular}




\begin{tabular}{lll}
\hline & Amount & \multicolumn{1}{c}{ Source } \\
\hline Minerals & & \\
Magnesium & $514 \mathrm{mg}$ & Magnesium oxide \\
Iron & $35 \mathrm{mg}$ & Iron sulfate \\
Zinc & $12 \mathrm{mg}$ & Zinc oxide \\
Manganese & $10 \mathrm{mg}$ & Manganese oxide \\
Copper & $2.0 \mathrm{mg}$ & Copper sulfate \\
Iodine & $0.2 \mathrm{mg}$ & Calcium iodate \\
Chromium & $0.2 \mathrm{mg}$ & Chromium acetate \\
\hline aPer $k g$ of finished product. &
\end{tabular}

Per kg of finished product.

Table M-3. Nutrient Composition of NTP-2000 Rat and Mouse Ration

\begin{tabular}{lccc}
\hline \multicolumn{1}{c}{ Nutrient } & $\begin{array}{c}\text { Mean } \pm \text { Standard } \\
\text { Deviation }\end{array}$ & Range & Number of Samples \\
\hline Protein (\% by weight) & $14.6 \pm 0.61$ & $13.5-15.9$ & 24 \\
Crude fat (\% by weight) & $8.2 \pm 0.31$ & $7.7-8.9$ & 24 \\
Crude fiber (\% by weight) & $9.1 \pm 0.57$ & $8.1-10.3$ & 24 \\
Ash (\% by weight) & $4.9 \pm 0.22$ & $4.4-5.2$ & 24 \\
\hline Amino Acids (\% of total diet) & & & 22 \\
Arginine & $0.783 \pm 0.70$ & $0.67-0.97$ & 22 \\
Cystine & $0.220 \pm 0.024$ & $0.15-0.25$ & 22 \\
Glycine & $0.701 \pm 0.041$ & $0.62-0.80$ & 22 \\
Histidine & $0.352 \pm 0.077$ & $0.27-0.68$ & 22 \\
Isoleucine & $0.546 \pm 0.044$ & $0.43-0.66$ & 22 \\
Leucine & $1.095 \pm 0.067$ & $0.96-1.24$ & 22 \\
Lysine & $0.711 \pm 0.114$ & $0.31-0.86$ & 22 \\
Methionine & $0.409 \pm 0.046$ & $0.26-0.49$ & 22 \\
Phenylalanine & $0.628 \pm 0.040$ & $0.54-0.72$ & 22 \\
Threonine & $0.505 \pm 0.043$ & $0.43-0.61$ & 22 \\
Tryptophan & $0.150 \pm 0.028$ & $0.11-0.20$ & 22 \\
Tyrosine & $0.401 \pm 0.061$ & $0.28-0.54$ & 22 \\
Valine & $0.665 \pm 0.043$ & $0.55-0.73$ & \\
\hline Essential Fatty Acids (\% of total diet) & $3.49-4.55$ & $0.21-0.35$ \\
Linoleic & $3.95 \pm 0.259$ & & 22 \\
Linolenic & $0.30 \pm 0.032$ & $-340-4,780$ & - \\
\hline Vitamins & & & 22 \\
Vitamin A (IU/kg) & $1,000^{\mathrm{a}}$ & & \\
Vitamin D (IU/kg) & & & 22 \\
\hline & & & 22 \\
\hline
\end{tabular}




\begin{tabular}{|c|c|c|c|}
\hline Nutrient & $\begin{array}{l}\text { Mean } \pm \text { Standard } \\
\text { Deviation }\end{array}$ & Range & Number of Samples \\
\hline$\alpha$-Tocopherol (ppm) & $80.6 \pm 22.03$ & $27.0-124.0$ & 22 \\
\hline Thiamine (ppm) ${ }^{\mathrm{b}}$ & $7.2 \pm 1.12$ & $5.1-9.3$ & 24 \\
\hline Riboflavin (ppm) & $7.6 \pm 2.89$ & $4.20-17.50$ & 22 \\
\hline Niacin (ppm) & $78.9 \pm 9.08$ & $66.4-98.2$ & 22 \\
\hline Pantothenic acid (ppm) & $26.9 \pm 12.63$ & $17.4-81.0$ & 22 \\
\hline Pyridoxine $(\mathrm{ppm})^{\mathrm{b}}$ & $9.54 \pm 1.99$ & $6.44-13.7$ & 22 \\
\hline Folic acid (ppm) & $1.62 \pm 0.48$ & $1.15-3.27$ & 22 \\
\hline Biotin (ppm) & $0.32 \pm 0.10$ & $0.20-0.704$ & 22 \\
\hline Vitamin $B_{12}(\mathrm{ppb})$ & $53.6 \pm 39.6$ & $18.3-174.0$ & 22 \\
\hline Choline $(\mathrm{ppm})^{\mathrm{b}}$ & $2,846 \pm 485$ & $1,820-3,790$ & 22 \\
\hline \multicolumn{4}{|l|}{ Minerals } \\
\hline Calcium (\%) & $0.932 \pm 0.055$ & $0.808-1.030$ & 24 \\
\hline Phosphorus (\%) & $0.538 \pm 0.030$ & $0.471-0.592$ & 24 \\
\hline Potassium (\%) & $0.666 \pm 0.030$ & $0.626-0.733$ & 22 \\
\hline Chloride (\%) & $0.386 \pm 0.039$ & $0.300-0.474$ & 22 \\
\hline Sodium (\%) & $0.189 \pm 0.016$ & $0.160-0.222$ & 22 \\
\hline Magnesium (\%) & $0.216 \pm 0.062$ & $0.185-0.490$ & 22 \\
\hline Sulfur $(\%)$ & $0.170 \pm 0.029$ & $0.116-0.209$ & 14 \\
\hline Iron (ppm) & $186 \pm 39.2$ & $135-311$ & 22 \\
\hline Manganese (ppm) & $51.4 \pm 10.28$ & $21.0-73.1$ & 22 \\
\hline Zinc (ppm) & $53.4 \pm 8.46$ & $43.3-78.5$ & 22 \\
\hline Copper (ppm) & $7.01 \pm 2.562$ & $3.21-16.3$ & 22 \\
\hline Iodine ( $\mathrm{ppm})$ & $0.503 \pm 0.206$ & $0.158-0.972$ & 22 \\
\hline Chromium (ppm) & $0.694 \pm 0.276$ & $0.330-1.380$ & 22 \\
\hline Cobalt (ppm) & $0.256 \pm 0.164$ & $0.098-0.864$ & 22 \\
\hline \multicolumn{4}{|c|}{$\begin{array}{l}\text { aFrom formulation. } \\
{ }^{\mathrm{b}} \text { As hydrochloride (thiamine and pyridoxine) or chloride (choline). }\end{array}$} \\
\hline \multicolumn{4}{|c|}{ Table M-4. Contaminant Levels in NTP-2000 Rat and Mouse Ration ${ }^{a}$} \\
\hline & $\begin{array}{l}\text { Mean } \pm \text { Standard } \\
\text { Deviation }^{\mathrm{b}}\end{array}$ & Range & Number of Samples \\
\hline \multicolumn{4}{|l|}{ Contaminants } \\
\hline Arsenic (ppm) & $0.24 \pm 0.054$ & $0.16-0.40$ & 24 \\
\hline Cadmium (ppm) & $0.06 \pm 0.010$ & $0.04-0.08$ & 24 \\
\hline Lead (ppm) & $0.10 \pm 0.020$ & $0.08-0.16$ & 24 \\
\hline Mercury (ppm) & $<0.02$ & - & 24 \\
\hline Selenium (ppm) & $0.33 \pm 0.248$ & $0.16-1.02$ & 24 \\
\hline
\end{tabular}


Cobalt Metal, NTP TR 581

\begin{tabular}{|c|c|c|c|}
\hline & $\begin{array}{c}\text { Mean } \pm \text { Standard } \\
\text { Deviation }^{b}\end{array}$ & Range & Number of Samples \\
\hline Aflatoxins (ppb) & $<5.00$ & - & 24 \\
\hline Nitrate nitrogen $(\mathrm{ppm})^{\mathrm{c}}$ & $15.32 \pm 7.22$ & $5.09-36.8$ & 24 \\
\hline Nitrite nitrogen $(\mathrm{ppm})^{\mathrm{c}}$ & $0.80 \pm 0.68$ & $0.30-3.04$ & 24 \\
\hline BHA $(p p m)^{d}$ & $1.17 \pm 0.82$ & $1.0-5.0$ & 24 \\
\hline BHT $(\mathrm{ppm})^{\mathrm{d}}$ & $1.17 \pm 0.82$ & $1.0-5.0$ & 24 \\
\hline Aerobic plate count (CFU/g) & $10 \pm 0$ & 10 & 24 \\
\hline Coliform (MPN/g) & $3.0 \pm 0.0$ & 3.0 & 24 \\
\hline Escherichia coli (MPN/g) & $<10$ & - & 24 \\
\hline Salmonella (MPN/g) & Negative & - & 24 \\
\hline Total nitrosamines $(\mathrm{ppb})^{\mathrm{e}}$ & $7.3 \pm 6.10$ & $2.0-28.0$ & 24 \\
\hline$N$-Nitrosodimethylamine (ppb) ${ }^{\mathrm{e}}$ & $2.5 \pm 2.15$ & $1.0-10.3$ & 24 \\
\hline$N$-Nitrosopyrrolidine $(\mathrm{ppb})^{\mathrm{e}}$ & $4.7 \pm 4.69$ & $1.0-17.7$ & 24 \\
\hline \multicolumn{4}{|l|}{ Pesticides (ppm) } \\
\hline$\alpha-\mathrm{BHC}$ & $<0.01$ & - & 24 \\
\hline$\beta$-BHC & $<0.02$ & - & 24 \\
\hline$\gamma$-BHC & $<0.01$ & - & 24 \\
\hline$\delta$-BHC & $<0.01$ & - & 24 \\
\hline Heptachlor & $<0.01$ & - & 24 \\
\hline Aldrin & $<0.01$ & - & 24 \\
\hline Heptachlor epoxide & $<0.01$ & - & 24 \\
\hline DDE & $<0.01$ & - & 24 \\
\hline DDD & $<0.01$ & - & 24 \\
\hline DDT & $<0.01$ & - & 24 \\
\hline $\mathrm{HCB}$ & $<0.01$ & - & 24 \\
\hline Mirex & $<0.01$ & - & 24 \\
\hline Methoxychlor & $<0.05$ & - & 24 \\
\hline Dieldrin & $<0.01$ & - & 24 \\
\hline Endrin & $<0.01$ & - & 24 \\
\hline Telodrin & $<0.01$ & - & 24 \\
\hline Chlordane & $<0.05$ & - & 24 \\
\hline Toxaphene & $<0.10$ & - & 24 \\
\hline Estimated PCBs & $<0.20$ & - & 24 \\
\hline Ronnel & $<0.01$ & - & 24 \\
\hline Ethion & $<0.02$ & - & 24 \\
\hline Trithion & $<0.05$ & - & 24 \\
\hline
\end{tabular}


Cobalt Metal, NTP TR 581

\begin{tabular}{lccc}
\hline & $\begin{array}{c}\text { Mean } \pm \text { Standard } \\
\text { Deviation }^{\mathbf{b}}\end{array}$ & Range & Number of Samples \\
\hline Diazinon & $<0.10$ & - & 24 \\
Methyl chlorpyrifos & $0.058 \pm 0.038$ & $0.02-0.139$ & 24 \\
Methyl parathion & $<0.02$ & - & 24 \\
Ethyl parathion & $<0.02$ & - & 24 \\
Malathion & $0.116 \pm 0.137$ & $0.020-0.581$ & 24 \\
Endosulfan I & $<0.01$ & - & 24 \\
Endosulfan II & $<0.01$ & - & 24 \\
Endosulfan sulfate & $<0.03$ & - & 24 \\
\hline
\end{tabular}

${ }^{\mathrm{a}}$ All samples were irradiated. $\mathrm{CFU}=$ colony-forming units; MPN $=$ most probable number; $\mathrm{BHC}=$ hexachlorocyclohexane or benzene hexachloride.

${ }^{b}$ For values less than the limit of detection, the detection limit is given as the mean.

'Sources of contamination: alfalfa, grains, and fish meal.

dSources of contamination: soy oil and fish meal.

${ }^{\mathrm{e}}$ All values were corrected for percent recovery. 
Cobalt Metal, NTP TR 581

\section{Appendix N. Sentinel Animal Program \\ Table of Contents}

N.1. Methods

$\mathrm{N}-2$

N.2. Results ....

N-6

\section{Tables}

Table N-1. Laboratory Methods and Agents Tested for in the Sentinel Animal Program ......... N-2 


\section{N.1. Methods}

Rodents used in the National Toxicology Program are produced in optimally clean facilities to eliminate potential pathogens that may affect study results. The Sentinel Animal Program is part of the periodic monitoring of animal health that occurs during the toxicological evaluation of test compounds. Under this program, the disease state of the rodents is monitored via sera or feces from extra (sentinel) or dosed animals in the study rooms. The sentinel animals and the study animals are subject to identical environmental conditions. Furthermore, the sentinel animals come from the same production source and weanling groups as the animals used for the studies of test compounds.

Blood samples were collected, allowed to clot, and the serum was separated. Additionally, fecal samples were collected and tested for Helicobacter species. All samples were processed appropriately and tested in-house during week 2 of the study or sent to BioReliance Corporation (Rockville, MD) (end of the 3-month studies and at 6,12 and 18 months of the 2-year studies) or the Research Animal Diagnostic Laboratory (RADIL), University of Missouri (Columbia, MO) (end of 2-year studies) for determination of the presence of pathogens. The laboratory methods and agents for which testing was performed are tabulated below; the times at which samples were collected during the studies are also listed.

Blood was collected from five animals per sex except at the following collection time points:

Two-week study (rats): Start of study collections-four males and five females

Two-year study (rats): 6, 12, 18 month collections- -0 males and 10 females

Fecal samples were collected from five male and five female mice.

Table N-1. Laboratory Methods and Agents Tested for in the Sentinel Animal Program

\begin{tabular}{ll}
\hline \multicolumn{1}{c}{ Method and Test } & Time of Collection \\
\hline Rats & \\
Two-week Study & \\
In-House Antibody Testing & \\
Mycoplasma pulmonis & Study termination \\
PVM (Pneumonia virus of mice) & Study termination \\
RCV/SDA (Rat coronavirus/sialodacryoadenitis virus) & Study termination \\
RPV (Rat parvovirus) & Study termination \\
Sendai & Study termination \\
Three-month Study & \\
In-House Antibody Testing & \\
M. pulmonis & 2 weeks \\
PVM & 2 weeks \\
RCV/SDA & 2 weeks \\
RPV & 2 weeks \\
\hline
\end{tabular}


Cobalt Metal, NTP TR 581

\begin{tabular}{|c|c|}
\hline Method and Test & Time of Collection \\
\hline Sendai & 2 weeks \\
\hline \multicolumn{2}{|l|}{ ELISA } \\
\hline PVM & Study termination \\
\hline $\mathrm{RCV} / \mathrm{SDA}$ & Study termination \\
\hline Sendai & Study termination \\
\hline \multicolumn{2}{|l|}{ Immunofluorescence Assay } \\
\hline Parvovirus & Study termination \\
\hline \multicolumn{2}{|l|}{ Two-year Study } \\
\hline \multicolumn{2}{|l|}{ In-House Antibody Testing } \\
\hline M. pulmonis & 2 weeks \\
\hline PVM & 2 weeks \\
\hline $\mathrm{RCV} / \mathrm{SDA}$ & 2 weeks \\
\hline RPV & 2 weeks \\
\hline Sendai & 2 weeks \\
\hline \multicolumn{2}{|l|}{ ELISA } \\
\hline Mycoplasma arthritidis & 18 months \\
\hline M. pulmonis & 18 months \\
\hline PVM & 6,12 , and 18 months \\
\hline $\mathrm{RCV} / \mathrm{SDA}$ & 6,12 , and 18 months \\
\hline Sendai & 6,12 , and 18 months \\
\hline \multicolumn{2}{|l|}{ Immunofluorescence Assay } \\
\hline KRV (Kilham's rat virus) & Study termination \\
\hline \multicolumn{2}{|l|}{ Multiplex Fluorescent Immunoassay } \\
\hline M. pulmonis & Study termination \\
\hline Parvo NS-1 & Study termination \\
\hline Parvovirus & 6,12 , and 18 months \\
\hline PVM & Study termination \\
\hline $\mathrm{RCV} / \mathrm{SDA}$ & Study termination \\
\hline RMV (Rat minute virus) & Study termination \\
\hline RPV & Study termination \\
\hline RTV (Rat theilovirus) & Study termination \\
\hline Sendai & Study termination \\
\hline $\begin{array}{l}\text { TMEV GDVII (Theiler's murine encephalomyelitis } \\
\text { virus- mouse poliovirus, strain GDVII) }\end{array}$ & Study termination \\
\hline H-1 (Toolan’s H-1) & Study termination \\
\hline
\end{tabular}




\section{Mice}

Two-week Study

In-House Antibody Testing

MHV (Mouse hepatitis virus)

Study termination

MPV (Mouse parvovirus)

Study termination

M. pulmonis

Study termination

PVM

Study termination

Sendai

Study termination

TMEV GDVII

Study termination

\section{Three-month Study}

In-House Antibody Testing

$\begin{array}{ll}\text { MHV } & 2 \text { weeks } \\ \text { MPV } & 2 \text { weeks } \\ \text { M. pulmonis } & 2 \text { weeks } \\ \text { PVM } & 2 \text { weeks } \\ \text { Sendai } & 2 \text { weeks } \\ \text { TMEV GDVII } & 2 \text { weeks }\end{array}$

ELISA

Ectromelia virus

Study termination

EDIM (epizootic diarrhea of infant mice)

Study termination

LCM (lymphocytic choriomeningitis virus)

Study termination

MAd-FL (Mouse adenovirus)

Study termination

MHV

Study termination

MMV VP2 (Mouse minute virus viral protein 2)

Study termination

MPV VP2 (Mouse parvovirus viral protein 2)

Study termination

PVM

Study termination

Reovirus

Study termination

Sendai

Study termination

TMEV GDVII

Study termination

Immunofluorescence Assay

Ectromelia virus

Study termination

EDIM

Study termination

Two-year Study

In-House Antibody Testing

GDVII

2 weeks

MHV

2 weeks 
Cobalt Metal, NTP TR 581

\begin{tabular}{|c|c|}
\hline Method and Test & Time of Collection \\
\hline MPV & 2 weeks \\
\hline M. pulmonis & 2 weeks \\
\hline PVM & 2 weeks \\
\hline Sendai & 2 weeks \\
\hline \multicolumn{2}{|l|}{ ELISA } \\
\hline Ectromelia virus & 6,12 , and 18 months \\
\hline EDIM & 6,12 , and 18 months \\
\hline LCM & 6,12 , and 18 months \\
\hline MAd-1 & 6,12 , and 18 months \\
\hline MHV & 6,12 , and 18 months \\
\hline MMV VP2 & 6,12 , and 18 months \\
\hline MPV VP2 & 6,12 , and 18 months \\
\hline PVM & 6,12 , and 18 months \\
\hline Reovirus & 6,12 , and 18 months \\
\hline Sendai & 6,12 , and 18 months \\
\hline TMEV GDVII & 6,12 , and 18 months \\
\hline \multicolumn{2}{|l|}{ Immunofluorescence Assay } \\
\hline EDIM & 6 months and study termination \\
\hline LCM & 6 months \\
\hline MHV & 18 months \\
\hline PVM & 6 months \\
\hline Reovirus & 6 and 18 months \\
\hline TMEV GDVII & 6 months \\
\hline \multicolumn{2}{|l|}{ Multiplex Fluorescent Immunoassay } \\
\hline Ectromelia virus & Study termination \\
\hline EDIM & Study termination \\
\hline LCM & Study termination \\
\hline MHV & Study termination \\
\hline MMV & Study termination \\
\hline Mouse norovirus & Study termination \\
\hline MPV & Study termination \\
\hline M. pulmonis & Study termination \\
\hline Parvo NS-1 & Study termination \\
\hline PVM & Study termination \\
\hline Reovirus & Study termination \\
\hline Sendai & Study termination \\
\hline
\end{tabular}


Cobalt Metal, NTP TR 581

\begin{tabular}{ll}
\hline \multicolumn{1}{c}{ Method and Test } & \multicolumn{1}{c}{ Time of Collection } \\
\hline TMEV GDVII & Study termination \\
Polymerase Chain Reaction & \\
Helicobacter species & 18 months \\
\hline
\end{tabular}

\section{N.2. Results}

All test results were negative. 


\section{Appendix O. Summary of Peer Review Panel Comments}

On October 29, 2013, the draft Technical Report on the toxicology and carcinogenesis studies of cobalt metal received public review by the National Toxicology Program's Board of Scientific Counselors' Technical Reports Peer Review Panel. The review meeting was held at the National Institute of Environmental Health Sciences, Research Triangle Park, NC.

Dr. M. Behl, NIEHS, introduced the studies on cobalt metal. The United Auto Workers and the Cobalt Development Institute nominated cobalt metal for toxicology and carcinogenesis studies, with support from OSHA and NIOSH. The nomination was based on widespread occupational exposure and occurrence of hard metal disease associated with exposure to cobalt and its compounds. Dr. Behl presented results of mutagenicity studies and described the nonneoplastic and neoplastic lesions observed in the studies.

The proposed conclusions for the 2-year inhalation studies were clear evidence of carcinogenic activity of cobalt metal in male and female F344/NTac rats and male and female B6C3F1/N mice.

NTP contract pathologist Dr. A.R. Pandiri of Experimental Pathology Laboratories, Inc., described the molecular analyses of Kras, Egfr, and Tp53 mutations in rat and mouse alveolar/bronchiolar carcinomas in these studies. He presented data demonstrating that mutations within Kras were higher than within Egfr and Tp53 genes in both rat and mouse alveolar/bronchiolar carcinomas resulting from chronic inhalation exposure to cobalt metal.

Dr. Cullen noted receipt and distribution to the panel of written comments from Dr. S. Verberckmoes of Umicore S.A. and Dr. R. Danzeisen of the Cobalt Development Institute. Dr. Cullen opened the floor for oral public comments.

Dr. Danzeisen commented on the draft Technical Report by telephone. Dr. Danzeisen noted that the Cobalt Development Institute nominated cobalt metal for NTP testing. She anticipated that NTP studies on cobalt metal would lead to an industry self-classification of cobalt metal by the inhalation route, pending the outcome of the Peer Review Panel's deliberations. She felt that the study was well designed and conducted, but the particle size used was very small compared to that in typical human exposure scenarios. She said the high concentration used was relatively high as reflected in the early reductions in body weights. There was no no-observed-adverseeffect level, which made it more difficult to extrapolate the findings for risk assessment. Her group agreed with the NTP conclusion that there was clear evidence of carcinogenicity, but suggested limiting the conclusions to indicate that the evidence was by inhalation exposure and in the respiratory tract. The NTP findings were in line with her group's thinking and evidence from past human epidemiologic studies that cobalt causes cancer in the lung by causing local inflammation leading to reparative mechanisms. She noted that the systemic cancers were seen only in rats and not in mice, and are not relevant for humans and human risk assessment. She remarked that the systemic cobalt levels were highest in the liver, at times even exceeding lung levels, but the liver had no neoplasms. The cobalt levels achieved in the tissues seemed disconnected with adverse effects, particularly neoplasms. She found this supported her group's hypothesis that there is local inflammation leading to reparative mechanisms, hyperplasia, and subsequently cancer. She stressed that the Cobalt Development Institute has strong evidence that 
cobalt is not a mutagen and agrees with the NTP review that oxidative stress causes interaction with the DNA.

Dr. Gordon, the first primary reviewer, indicated that the NTP's cobalt metal particle inhalation studies were very well designed and conducted and yielded important information regarding the carcinogenicity of a less soluble form of cobalt that complements the previous NTP studies with the soluble form. He said considerable data suggest that the soluble and insoluble forms can have differences in long-term toxicity or potency; therefore, he commended the testing of cobalt metal in the NTP bioassay program. He found the choices of exposure concentrations to be generally acceptable, but an additional low concentration would have been appropriate for both the rat and mouse 2-year studies. On the basis of some of the 3-month endpoints (e.g., larynx), there may have been sufficient data to justify using a lower exposure-concentration, which would enhance the relevance of the exposure concentrations. The multiple alveolar/bronchiolar carcinomas with exposure-concentration response in rats and mice, and the statistical significance of the tumors, provide additional evidence of the carcinogenicity of the particles in the lung. He indicated that if the concentration was lower, a clearer exposure concentration-related response might have been seen. He suggested particle size be addressed earlier in the report. He noted that a stainless steel jet mill was used to break up the cobalt metal into respirable particle sizes; the report should explain the resulting chromium contamination of the bulk chemical and note contamination was minimal. The potential for cobalt metal to be carcinogenic is strengthened by the similar lung tumors seen with the soluble form of cobalt. He inquired about a potential miscalculation in the normalization of the exposure concentration of cobalt sulfate heptahydrate to elemental cobalt. He noted that the cobalt metal particles might have been more potent than the soluble form, which would support the mode of action suggested by the public commenter. He agreed with the conclusion of clear evidence in the lung. He noted that the other conclusions, except for the cystic keratinizing epitheliomas, may not warrant the higher ratings and should perhaps be lowered.

Dr. Cory-Slechta, the second primary reviewer, noted that the studies were very well designed and conducted. Given that cobalt can be taken up by the nasal mucosa and into the brain, she said there should have been analysis of brain tissue.

Dr. Regan, the third primary reviewer, said the studies were well designed and had no interpretation differences at the clear evidence of carcinogenicity level. Regarding the equivocal evidence of carcinogenicity in the renal tubule adenomas and carcinomas, she asked about the lack of preneoplastic lesions in the kidneys. She asked what triggered the extended evaluation of the kidneys. She asked whether there was any evidence of the amphophilic-vacuolar carcinomas that have been found to be spontaneous; if so, then that should be taken into account. Regarding the pancreatic islet tumors, she said there was an increase in incidence compared to the historical control data, but the historical control data were not appropriate for this particular study because of the strain used. Thus, she proposed that the pancreatic islet tumors were ranked too high but agreed with the conclusions for all of the other tumor types.

Dr. Zacharewski, the fourth primary reviewer, asked whether the Kras, Egfr, and Tp53 mutations mapped to any specific consequences in terms of the activity of those proteins subsequent to the mutation. He also inquired whether any additional studies could have been done to demonstrate that the mutation actually had functional significance to the protein itself. He asked whether 
there was any correlation between a mutation and a tumor outcome in terms of aggressiveness, metastatic ability, etc.

Dr. Barlow asked why no mutations were found in the concurrent controls, despite a robust response in the historical animals. He also noted that there was a well-known and direct mechanism for the development of adrenal medullary tumors and asked for comment from the study pathologist.

Dr. Parker endorsed more large-scale sequencing efforts to allow for more accurate identification of genetic mutations and assessment of other types of mutations, such as indels, as opposed to just point mutations.

Dr. Cullen asked if cardiomyopathy was observed in the study. Dr. Behl replied that there was no evidence of cardiomyopathy in these studies. She said that cardiomyopathy was observed in a female rat in the 13-week cobalt sulfate heptahydrate study.

Responding to Dr. Gordon's review, Dr. Behl agreed with his comments about nonneoplastic lesions in the larynx. She explained that when NTP has different exposure concentrations in two species in studies involving inhalation chambers, it has elected to go with one less concentration rather than adding an additional group. Hence, a lower concentration was not used. She noted that the particle size used was consistent with the rat respirable range (see pages 29, 30, and 275). Regarding the dosing calculation Dr. Gordon had questioned, she explained that the exposure concentration in the cobalt sulfate heptahydrate study was based on the mass percentage of cobalt in anhydrous cobalt sulfate. Dr. Gordon asked for better justification for the conclusion related to cystic keratinizing epitheliomas, suggesting perhaps that it should have been equivocal. Dr. Behl explained the basis for the some evidence call in the females. Because cystic keratinizing epitheliomas are rare and are part of a continuum of lung lesions, their occurrence was included as a chemical-related effect in the conclusions. Dr. R.A. Herbert, NIEHS, added that in the nonneoplastic lesions, there was some evidence of squamous cell hyperplasia within the lung and evidence of a progression from nonneoplastic lesions to benign lesions to carcinomas, leading to the some evidence conclusion.

Responding to Dr. Cory-Slechta's comment, Dr. Behl said the brain tissue was examined, and there was no evidence of neoplasms. Dr. Cory-Slechta said neoplasms might not have been expected, although there were likely nonneoplastic lesions, such as white matter injury. Dr. Herbert said there was no evidence of nonneoplastic or neoplastic lesions. Dr. D.E. Malarkey, NIEHS, said NTP is very interested in improving evaluation of the brain, having recently invoked a new method for its analysis.

Dr. Herbert responded first to Dr. Regan's question about what triggers an extended review in the kidney. He said the renal tubule adenomas are usually small tumors, and an extended review is triggered if there is an indication from the data that there could be an effect. Dr. Regan asked if there was a specific level used. Dr. Herbert said there was not. Dr. Barlow asked how often the extended review yields additional results that affect the conclusions. Dr. Herbert did not have data on that issue at the time. Dr. Regan asked whether any amphophilic-vacuolated renal tubule neoplasms were observed in the male rat study. Dr. Herbert said none were seen in this study and indicated that NTP has not traditionally made a distinction between the amphophilic-vacuolated type and other types of renal tubular neoplasms in studies. He noted that one publication indicates such tumors are spontaneous, but the toxicologic pathology community does not 
generally accept this distinction. Dr. Regan mentioned that there are other publications on the topic. She asked how NTP could know that such a tumor type did not occur, if NTP does not distinguish that tumor type. Dr. Herbert indicated he had looked at all of the tumors, and that no amphophilic-vacuolated renal tubular neoplasms occurred in the study. Dr. Behl responded to Dr. Regan's comments about pancreatic islet tumors in the females and said the call equivocal evidence of carcinogenicity was primarily based on the increase in malignant neoplasms at the $5 \mathrm{mg} / \mathrm{m}^{3}$ dose, as well as supporting evidence from the males (e.g., significant trend and pairwise comparisons in the top two exposure concentrations).

Dr. Pandiri responded to Dr. Zacharewski's comments. Dr. Pandiri said the selection of the hot spot exons in all three genes was based on extensive literature review of human lung cancer as well as rodent models of chemical-induced pulmonary carcinogenesis. Dr. Pandiri indicated that immunohistochemistry could be used to demonstrate the alterations in protein expression within the molecular pathways associated with the mutated genes.

Regarding Dr. Barlow's question about why no Kras mutations were observed in the spontaneous alveolar/bronchiolar carcinomas from the concurrent chamber controls, Dr. Pandiri speculated that there were in fact mutations present, but perhaps not in the exons examined.

In response to Dr. Parker's question about the primary focus being on point mutations, Dr. Pandiri said point mutations account for the majority of genetic changes seen in some of the well-known carcinogenesis studies in the literature. Dr. Pandiri agreed that massive parallel sequencing of the cancer genes in tumor tissue is a more powerful tool for detecting mutations and differentiating chemical-induced tumors from spontaneous tumors. He also informed the committee that NTP is currently running a pilot project using exome sequencing and RNA-seq technologies for evaluating chemically induced and spontaneous hepatocellular carcinomas from previous NTP chronic bioassays.

Dr. Malarkey responded to Dr. Gordon's comments regarding the cystic keratinizing epitheliomas and indicated that they are very rare in most species, so it is a significant finding when present. Even though they are benign, this tumor type would be considered in the levels of evidence of carcinogenic activity, especially because it can progress to a malignant tumor. Dr. Malarkey also responded to Dr. Barlow's question concerning how extended reviews of the kidneys are triggered and how often the extended reviews yielded additional results that affect the conclusions. He noted that when the response is weak, follow-up serial sections might confirm a finding.

Dr. Cory-Slechta moved to accept the conclusions as written, and Dr. Gordon seconded. The Peer Review Panel voted unanimously to accept the conclusions on cobalt metal as written in the draft report. 


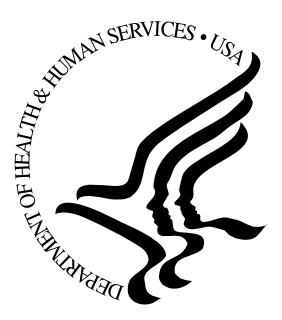

\title{
National Toxicology Program
}

\author{
NTP Central Data Management, MD EC-03
}

National Institute of Environmental Health Sciences

P.O. Box 12233

Research Triangle Park, NC 27709

http://ntp.niehs.nih.gov 